
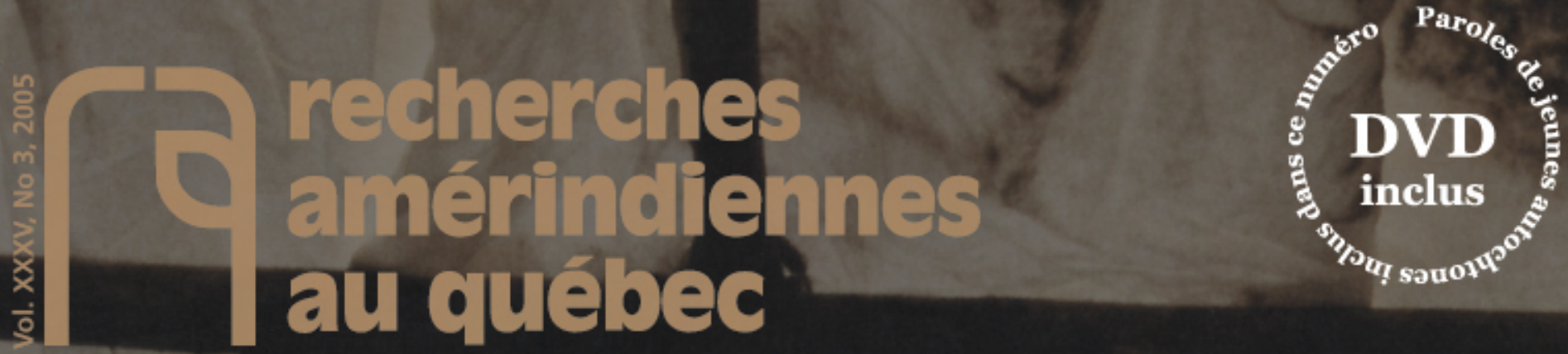

\title{
Jeunes autochtones
}

Espaces et expressions d'affirmation 


\section{Recherches amérindiennes au Québec}

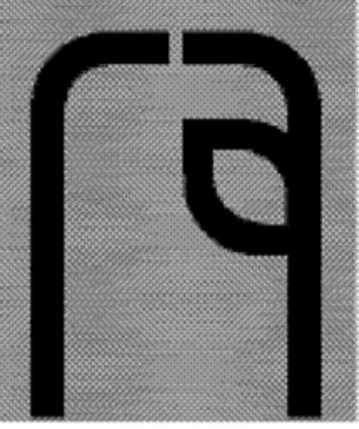

\section{Conseil international}

BERNARd ARCAND, Université Laval,

Québec, Canada

Philuppe Descola, Collège de France, Paris, France

Emanuel Désveaux, E.H.E.S.S. et L.A.S. Paris, France

Harvey Fert, McMaster University, Hamilton, Ont., Canada

JACQUES GALINIER, CNRS et Labethno, Université Paris-X, France

STEPHEN HUGH-JONES, University of Cambridge, Cambridge, Grande-Bretagne

PATRICK MENGET, EPHE, CNRS et Labethno, Université Paris X, France

Thomas C. PATterson, University of California, Riverside (CA), USA

JOELIE ROSTKOWSKI, Programme PREGANEM, EHESS / UNESCO, Paris, France

REMI SAVARD, Université de Montréal, Montréal, Canada

JUDITH R. SHAPIRO, President, Barnard College, Columbia University, New York, U.S.A.

Adriano Santlemma, Università di Cassino et Università di Roma "La Sapienza", Italia

ISABEUIE SCHULTE-TENCKHOFF, Institut universitaire d'études du développement, Genève, Suisse

Wimam SturTevant, National Museum of Natural History, Smithsonian Institution, Washington D.C., U.S.A.

\section{Directeur de la revue}

Michel LAVOIE, historien, Université Laval (mlci@globetrotter.net)

JEAN-PHIIIPPE WARREN, sociologue, Université Concordia (jphwarren@aol.com)

\section{Comité de rédaction}

NiCOLE BEAUDRY, ethnomusicologue, Université du Québec à Montréal

ALEXANDRINE BOUDREAULT-FOURNIER, anthropologue, University of Manchester (U.K.)

Éric ChaLIFoux, archéologue

DENYS DELAGE, sociologue, Université Laval

Christlan Gates Saint-PIERre, archéologue

CLAUDE GEunAs, anthropologue, Université de Sherbrooke

MARTIN HéBERT, anthropologue, Université Laval

Michel. LAVOIE, historien, Université Laval

TOBY MORANTZ, ethnohistorienne, McGill University

PierRe Trudel, anthropologue, Cégep du Vieux-Montréal

JEAN-PHILIPPE WARREN, sociologue, Université Concordia

$R^{\mathrm{n}}$ EVUE INTERNATIONAIE de langue française éditée au Québec, Recherches amérindiennes au Québec porte sur les premieres nations de l'Amérique latine, des États-Unis, du Canada et du Québec. Elle publie trois numéros par année; certains sont à thème, d'autres réunissent des articles hors theme.

Les principales disciplines auxquelles la revue s'intéresse sont l'anthropologie sociale et culturelle, l'anthropologie appliquée, l'archéologie sociale et culturelle, l'ethnohistoire, l'ethnographie, l'ethnolinguistique et l'ethnoscience amérindienne et, enfin, les sciences juridiques politiques et économiques relatives aux affaires amérindiennes. RAQ est ouverte à tous les courants théoriques et à toutes les traditions intellectuelles. Les positions prises par les auteurs ne refletent donc pas nécessairement celles du comité de rédaction, du conseil d’administration, de la Revue elle-même ou celles de la Société Recherches amérindiennes au Québec.

Le comité de rédaction invite les chercheurs a lui soumettre des projets de numéros à thème, soit d'ordre théorique, soit d'ordre régional - ou des articles soit dans le cadre des thèmes en préparation (voir en couverture 3) ou sur d'autres sujets. Chaque article est évalué par trois chercheurs qui ignorent le nom de l'auteur de l'article. Deux de ces chercheurs sont indépendants du comité de rédaction.

Avant de contacter le directeur de la revue, les chercheurs sont priés de consulter les Directives aux auteurs figurant en page 3 de couverture de chaque numéro.

Conseil d'administration

Présidente : Sylvie Vincent

Vice-président : Gérald McKenzie

Trêsorier : Laurent Girouard

\section{Adjoint à la Rédaction}

Éric Chalifoux

\section{Révision des textes}

Marcelle Roy

Responsable des comptes rendus

Alexandrine Boudreault-Fournier

\section{Monographies}

Collection *Paléo-Québec ": Daniel Chevrier. Archéotec inc.

Collection "Signes des Amériques ": Claude Gélinas, Université de Sherbrooke

\section{Documents audio-visuels}

Laurent Girouard

\section{Site Internet}

Laurent Girouard

\section{Service aux abonnés}

François Girard

\section{Graphisme}

Luc Mercier

\section{Impression}

AGMV-Marquis

\section{Organismes subventionnaires}

Ministère de la Culture et des

Communications du Québec

Fonds de recherche sur la société et la culture

Conseil de recherches en sciences humaines du Canada

Programme d'aide aux publications (PAP) Convention de la poste-publications no 40025159 No d'enregistrement 07634

Nous reconnaissons l'appui du gouvernement du Canada par l'entremise du Programme d'aide aux publications pour nos dépenses d'envoi postal.

\section{Canadä}

\section{Indexée dans :}

Point de repère; Abstracts in Anthropology; Historical Abstracts; America: History and Life; Anthropological Index; Anthropological Literature; Bibliography of Native North Americans; Bulletin signalétique (Ethnologie); Francis; Sociological Abstracts (SA); Social Planning/Policy E Development Abstracts (SOPODA); Linguistic and Behavior Abstracts (LLBA).

Dépôt légal : quatrième trimestre 1971 , Bibliothèque nationale du Québec.

ISSN 0318-4137

(C) Recherches amérindiennes au Québec

\section{Correspondance}

6742, rue Saint-DenisMontréal (Québec)

Canada $\mathrm{H} 2 \mathrm{~S} 2 \mathrm{~S} 2$

Tél. (514) 277-6178

Courriel : reamqu@globetrotter.net www.recherches-amerindiennes.qc.ca 


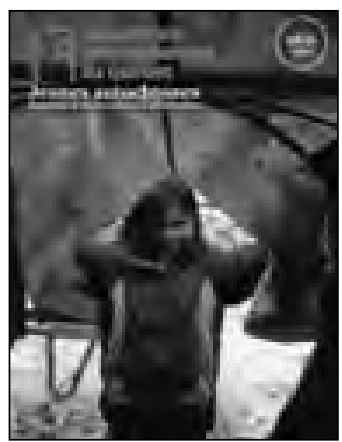

Sur la couverture :

Jeune fille atikamekw soulevant la porte d'une tente ronde au site du

"Poisson Blanc », un campement d'hiver situé à quelques kilomètres de Wemotaci, novembre 2003

Jeunes autochtones

Espaces et expressions d'affirmation

3 Présentation

Jeunes autochtones. Espaces et expressions d'affirmation Laurent Jérôme

7 Les jeunes Algonquins sont-ils biculturels ? Modèles de transmission et innovations dans quelques réserves Marie-Pierre Bousquet

19 Musique, tradition et parcours identitaire de jeunes Atikamekw : la pratique du tewehikan dans un processus de convocation culturelle Laurent Jérôme

31 Les chansons et musiques populaires innues: contexte, signification et pouvoir dans les expériences sociales de jeunes Innus Véronique Audet

39 L'espérance politique et économique chez les jeunes Tzeltals et Tlapanèques du Mexique Martin Hébert

49 Comment flirter avec la modernité pour conforter son identité : projet éducatif d'une communauté métisse au Manitoba Thibault Martin et Brieg Capitaine

59 Être jeune et maori aujourd'hui : l'université comme site de (ré)affirmation et de coexistence Natacha Gagné

71 L'espérance passe de la forêt au milieu scolaire : clivage et continuité dans les valeurs entre générations de Dènès Tha Jean-Guy A. Goulet et Kim Harvey-Trigoso

85 Regards croisés sur une éducation relative à l'environnement en milieu autochtone Lucie Sauvé, Hélène Godmaire, Marie Saint-Arnaud, Renée Brunelle et Françoise Lathoud

95 L'importance de l'aspect relationnel dans l'auto-(re)présentation de jeunes Innus de la communauté de Uashat mak Mani-Utenam Karoline Truchon

Ce numéro a été réalisé sous la direction de Laurent Jérôme
In Memoriam

107 James B. Petersen (1954-2005) L'inspirant respect d'une étoile filante Roland Tremblay

107 Pierre Dumais (1951-2006)

LE MONDE AUTOCHTONE EN CHIFFRES

107 Autochtones canadiens : combien sont-ils?

Pierre Trudel

ChroniQue JURIDIQUe

111 Quelques réflexions au sujet de la future politique de consultation des peuples autochtones du Québec Paul Dionne

\section{COMPTES RENDUS}

114 Dominique Raby : L'Épreuve fleurie. Symboliques du genre dans la littérature des Nahua du Mexique préhispanique Paul Roy

115 Jonathan C. Lainey : La « monnaie des Sauvages ". Les colliers de wampum d'hier à aujourd'hui Kathryn V. Muller

117 Rémi Savard : La forêt vive. Récits fondateurs du peuple innu Frédéric Laugrand

119 Publications québécoises récentes

120 THÈsES ET MÉMOIRES

121 LivRes Reçus

122 Colloques et congrès

122 Résumés / Abstracts

125 NOTES BIO-BIBLIOGRAPHIQUES

\section{DVD}

Paroles de jeunes autochtones Un document audiovisuel comprenant 3 films, 2 vidéoclips et 2 chansons (inclus dans ce numéro) 
COLLECTION SIGNES DES AMÉRIQUES

PUBLIÉE PAR RECHERCHES AMÉRINDIENNES AU QUÉBEC

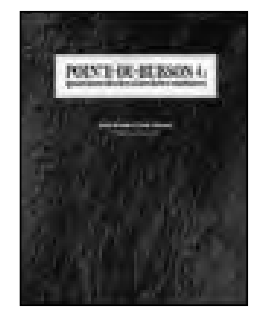

ÉPUISÉ :

Pointe-du-Buisson 4 :

QUARANTE SIÈCLES

D'ARCHIVES OUBLIÉES

Norman CleRmont

et Claude Chapdelaine

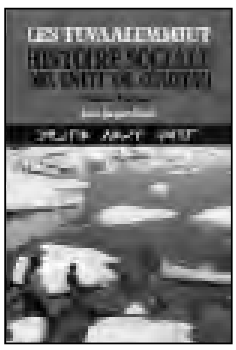

4

ÉPUISÉ :

Les TuvaAlummiut :

Histoire sociale des

INUIT DE QUAQTAQ

(QUÉbec ARCTIQUe)

LOUIS-JACQUES DORAIS

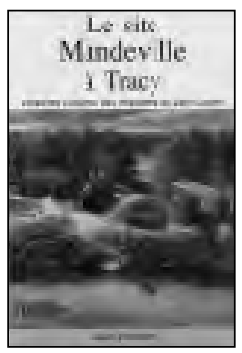

7

Le site Mandeville

À TraCY :

VARIAbilité CULTURELLe

DES IROQUOIENS DU

SAINT-LAURENT

Claude Chapdelaine

295 pages, 131 tableaux,

91 figures et 46 planches.

Prix : 16,05\$

(TPS incluse)

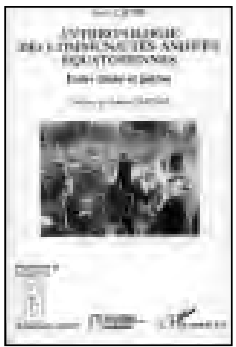

10

Anthropologie des COMMUNAUTÉS ANDINES ÉQUATORIENNES

Paul Cliche

Préface de Roberto

SANTANA

284 pages.

Prix : $31,60 \$$

(TPS incluse)

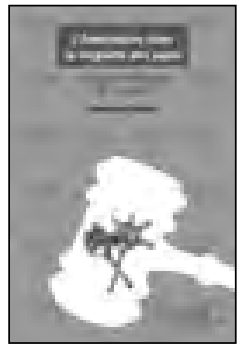

13

L'AMÉRINDIEN DANS

LA LORGNETTE DES

JUGES :

LE MIROIR DÉFORMANT

DE LA JUSTICE

Geneviève Mativat

96 pages

Prix : $16,05 \$$

(TPS incluse)

\section{Consultez notre site}

Canada H2S 2S2

Nom:

Adresse :

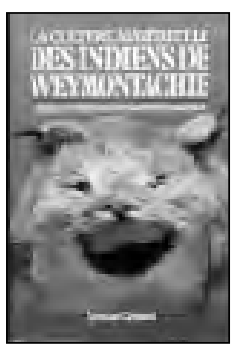

2

LA CULTURE MATÉRIELLE DES INDIENS DE

WeYmontachie :

IMAGES D'HIER DANS UNE

SOCIÉTÉ EN MUTATION

Norman Clermont

Préface de Camil Guy

160 pages, 39 planches,

30 figures, 7 tableaux.

Prix : $8,55 \$$

(TPS incluse)

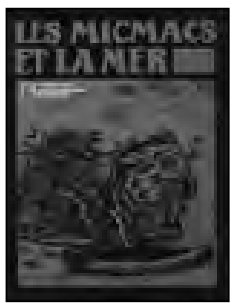

\section{5}

ÉPUISÉ :

Les Micmacs

ET LA MER

Charles A. Martijn, dir.

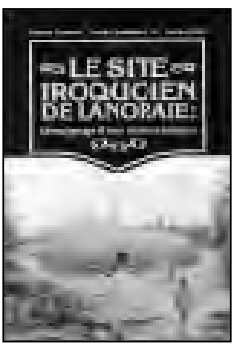

LE SITE IROQUOIEN DE LANORAIE : TÉmoIgnage

D'UNE MAISON LONGUE

Norman Clermont,

Claude Chapdelaine et

GEORGES BARRÉ

204 pages, 32 tableaux

33 figures et 52 planches.

Prix : $10,70 \$$

(TPS incluse)

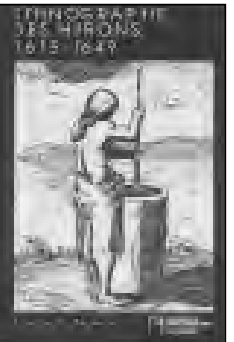

ÉPUISÉ :

EthNOGRAPHIE DES

Hurons, 1615-1649

ELISABETH TOOKER
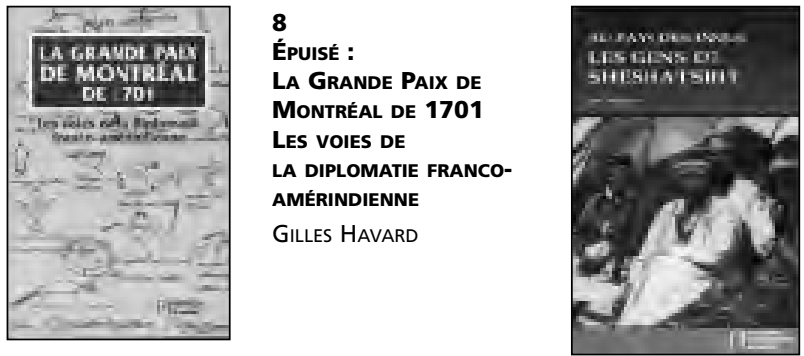

9

ÉPUISÉ :

La Grande Paix de Montréal de 1701

LES VOIES $D E$

LA DIPLOMATIE FRANCO-

AMÉRINDIENNE

Gilles Havard

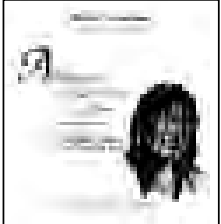

11

Affiquets, matachias et VERMILLON :

EthNOGRAPHIE ILLUSTRÉE

des Algonquiens du

NORD-EST DE L'AMÉRIQUE

AUX XVIE, XVIIE

ET XVIIIE SIÈCLES

MARC LABERGe

227 pages, 115 illustrations

de François Girard.

Prix : 26,75\$

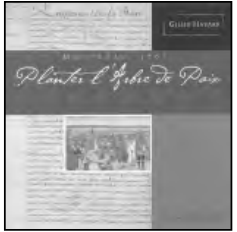

MONTRÉAL, 1701 :

Planter l'Arbre

DE PaIX

Gilles Havard

Coédition

Musée McCord

d'histoire canadienne

73 pages, illustrations et

cartes. Aussi disponible

en anglais.

Prix: 10,70\$

(TPS incluse)

www.recherches-amerindiennes.qc.ca

Faites parvenir votre commande accompagnée d'un chèque à :

Recherches amérindiennes au Québec

6742, rue Saint-Denis, Montréal, Québec,

Cochez $(\sqrt{ })$ :

Pointe-du-Buisson $\square \quad$ Weymontachie $\square \quad$ Lanoraie $\square \quad$ Tuvaalummiut $\square$ Mandeville $\square$

Sheshatshit $\square$ Communautés andines $\square$ Affiquets $\square$ Montréal $1701 \square$ Lorgnette

Frais de port : Ajoutez $3 \$$ pour le premier titre, et 1,10\$ par titre additionnel Ci-inclus : __ $\$$ 


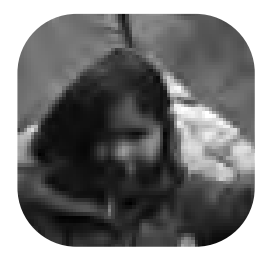

Présentation

Jeunes autochtones

Espaces et expressions d'affirmation

Laurent

Jérôme

Département

d'anthropologie,

Ciéra, Université

Laval,

et

Département d'ethnologie, Erase, Université Paul Verlaine de

Metz
CI LES « JEUNES » constituent une catégorie sociale privilégiée en recherche au point d'en faire une tradition de l'anthropologie (Bucholtz 2002), de la sociologie (Galland 2001) et plus largement des sciences sociales, force est de constater qu'ils sont encore négligés dans les études autochtones au Québec. La parole des jeunes est pourtant devenue incontournable dès que l'on s'intéresse aux enjeux et aux défis sociaux, politiques et culturels que soulèvent les réalités autochtones contemporaines. En 2001, l'âge médian des populations autochtones du Canada (Indiens de l'Amérique du Nord, Inuits et Métis) était de 24,7 ans contre 37,7 ans pour les non-autochtones. Toujours en 2001, le tiers de cette population était âgée de 14 ans et moins, contre $19 \%$ chez les non-autochtones (Canada 2003). Chez les Atikamekw par exemple, en 2004 le groupe des 15-35 ans, retenu comme définition politique de la jeunesse (voir plus loin les propos de Christian Coocoo), représentait $37 \%$ de la population totale (CNA 2004). Un recensement local à Wemotaci pour l'année 2000 faisait état de $69 \%$ de moins de trente ans pour une population totale de 1300 habitants.

Derrière ces chiffres, que signifie être jeune et autochtone aujourd'hui? Ce numéro de Recherches amérindiennes au Québec ne prétend pas livrer un regard homogénéisant et exhaustif sur la situation contemporaine des jeunes des premières nations du Québec. Il s'agit plutôt de poser une question encore marginalisée dans les recherches actuelles et de s'affranchir de lieux communs véhiculés dans l'espace public. Les représentations sur les jeunes alimentent en effet les discours misérabilistes : ils sont alcooliques, drogués et délinquants. Dans les réserves, le manque de loisir, la surpopulation dans les logements et les maisons, les taux de chômage élevés, le décrochage scolaire, les suicides... apparaissent comme des réalités qui alimentent des représentations réductrices: évoquer les réalités des jeunes autochtones, c'est parler des problèmes sociaux.

Et que dire des enfants, abandonnés, drogués, violés, méprisés, prisonniers d'une structure qui leur donne envie de se tuer à dix ou douze ans? [...] Les jeunes autochtones, exposés aux médias comme les autres, se sentent frustrés devant la vitrine du système de consommation qui est le nôtre. Ils sont frustrés, humiliés, aliénés. L'alcool, la drogue et une violence aussi gratuite que tragique demeurent leur seul mode d'expression. (Bombardier 2005)

Tous les jeunes subiraient passivement le contexte actuel de transition sociale, victimes contemporaines d'un héritage où s'entremêlent tentatives d'assimilation et négations de droits et d'identités. Ils seraient perdus entre deux références, celles de leurs aîné(e)s, gardiens et gardiennes des traditions et celle de la société dominante, lieu de la modernité avancée. Cette « crise d'identité » relève pour certains d'une incapacité de choisir entre l'une ou l'autre, pour d'autres, c'est le bricolage de la culture qui pose problème: «Le problème des jeunes aujourd'hui, c'est qu'ils font avec la culture ce qu'ils font avec la télé : ils font 
du zapping ${ }^{1}$. » La relation entre tradition et modernité revient souvent dans les discours qui tentent d'expliquer le contexte actuel de transition sociale en milieux autochtones contemporains (Boudreault 2005; Collin 1988, 1994; Sabev 2005). Dans ce numéro, cette relation sera perçue dans sa dimension dialectique. Elle servira à éclairer des initiatives plutôt que des crises. Elle sera envisagée en termes de « et » plutôt que de « ou » pour faire valoir un dynamisme et une continuité que masque l'emploi de ces deux concepts pour le moins flous et fourre-tout.

Outre une volonté de s'affranchir de représentations misérabilistes, ce numéro trouve sa pertinence scientifique dans certaines tendances des recherches actuelles. Lanthropologie en général et les recherches sur les savoirs locaux au Québec en particulier, ont posé et continuent de poser au centre de leur analyse la parole et les savoirs d'une autre catégorie sociale, celle des aîné(e)s, comme mode privilégié de connaissance. Enregistrer, compiler et valoriser les récits des aîné(e)s pour documenter une perception de l'Histoire, des relations à l'environnement, des visions du monde, des pratiques liées à l'éducation des enfants..., tels sont les apports scientifiques de ces travaux qui répondent par ailleurs à des préoccupations locales liées à la valorisation de l'histoire orale, de la mémoire sociale et des dynamiques de transmission des savoirs (voir Oosten et Laugrand 1999). Des spécialistes reconnus dans les études autochtones au Québec ont publié encore récemment des récits innus, récoltés dans les années 70, qui s'inscrivent en quelque sorte dans ce processus de «patrimonialisation » des savoirs autochtones (Bouchard 2004 ; Savard 2004; Vincent et Bacon 2003).

$\mathrm{Au}$ Québec et au Canada tout au moins, les processus d'objectivation de la «culture» (Poirier 2004) et de mise en valeur d'un « avoir culturel » ont sans doute pris une tout autre ampleur voilà trente-cinq ans, à une époque où publier un numéro de Recherches amérindiennes au Québec sur le thème des jeunes autochtones aurait permis d'interroger un « activisme indien » sur la scène nationale et internationale. Marqués par l'expérience de la scolarisation obligatoire et forcée des pensionnats catholiques et anglicans et dans la continuité de la montée des revendications aux États-Unis, ces jeunes autochtones des années 70 ont créé et investi des expressions et des espaces d'affirmation pour faire valoir des spécificités identitaires, des langues, des territoires, des pratiques culturelles, des droits ancestraux dans un contexte historique d'assimilation. Ces jeunes autochtones avaient des responsabilités. Ils occupent encore aujourd'hui la plupart des postes clés dans les domaines du politique, de l'intervention sociale, de l'éducation, de la préservation des langues et de la mise en valeur de la culture... En rappelant l'exemple de l'auteure maorie de Decolonizing methodologies, Linda Tuhiwai Smith (1999), laquelle faisait partie d'un groupe très actif dans les années 70 (Ngaa Tamatoa, 'les jeunes guerriers'), Natacha Gagné (ce numéro) renvoie le contexte nord-américain vers des réalités partagées par d'autres groupes autochtones dans le monde.

Aujourd'hui, parler des jeunes autochtones revient à interroger une majorité de moins en moins silencieuse qui aspire à une reconnaissance de réalités qui lui sont propres. Ces jeunes adultes, adolescents et enfants investissent et créent à leur tour des espaces et des expressions d'affirmation: conseils des jeunes, conseils des jeunes femmes, associations des étudiants autochtones dans les universités, innovations artistiques et musicales, discours de dénonciation sociale... Certains jeunes ne manquent pas de remettre en question le regard infantilisant que l'on porte sur eux et revendiquent parfois des responsabilités accrues, celles-là mêmes qui ont marqué la jeunesse de leurs parents. Considérer des personnes de vingt-huit ans comme des « jeunes », c'est nier toute une génération, nous dit Patrick Moar (voir encadré). C'est d'ailleurs tout le problème de l'utilisation d'une telle catégorie : quand commence et quand s'arrête la jeunesse? La jeunesse, "c'est une création de l'esprit», a souligné Johnny Jean Pierre (voir encadré). Que signifie « être jeune » dans des contextes où la scolarisation et la fin des rites marquant les étapes de la vie ont remis en question l'apprentissage et l'acquisition des savoirs, des compétences et des responsabilités par l'expérience, contribuant du même coup à faire apparaître une catégorie sociale relativement récente? Certaines pratiques rituelles sont d'ailleurs parfois (re)valorisées et convoquées dans le contexte contemporain pour (re)définir ces liens intergénérationnels (Laurent Jérôme, ce numéro). La catégorie «jeune » n'est pas plus ni moins problématique que la catégorie «aîné ». À partir de quel moment est-on considéré comme « aîné » et comment le devient-on?

Nous ne manquerons pas d'avancer prudemment dans le jeu des catégories «jeune» et «autochtone». À travers certaines contributions, le lecteur pourra entrevoir comment les liens de parenté et l'appartenance à une famille, à une communauté ou à une nation prévalent sur ces catégories comme expressions et représentations identitaires. La question des jeunes autochtones n'est pas soulevée à travers une catégorie sociale rigide et fermée. Elle sera plutôt envisagée de manière dynamique et fluide dans la relation que de jeunes Algonquins, Atikamekw, Innus, Dènès Tha, Métis, Maoris ou Tzeltals entretiennent avec la famille et les autres générations, l'école et l'université, la communauté et la ville, et ce à travers la pluralité des expériences de vie. La dimension comparative permettra de comprendre comment le fait de se sentir «jeune » dépend étroitement des contextes sociaux et politiques au sein desquels émergent et se formulent des discours et des représentations sur la jeunesse. Plus qu'une catégorie, il s'agit de valoriser des pratiques, des expériences et des aspirations qui se négocient à partir d'une position sociale particulière dans la société.

Il n'est pas question dans ce numéro de nier ou de dissimuler la série de pathologies sociales qui marquent l'expérience contemporaine de nombreux jeunes autochtones. Lobjectif est plutôt de livrer un regard sur ce que ces réalités peuvent engendrer comme créativités, innovations et ajustements au sein de différents espaces d'affirmation et à travers divers outils d'expression. Ces espaces sont révélateurs d'une originalité qui s'inscrit pourtant bien dans la continuité des initiatives mises de l'avant par les générations précédentes. Il ne s'agit pas de ruptures générationnelles mais bien d'une solidarité qui s'exprime par des références à une mémoire collective tout en composant avec les défis des contextes contemporains.

\section{LES ARTICLES}

À partir de données ethnographiques récoltées auprès de jeunes Algonquins, Marie-Pierre Bousquet ouvre ce numéro en interrogeant cette position «duale » qui marquerait les expériences contemporaines de jeunes Algonquins : les jeunes sont-ils «bi-culturels?» Loin de faire émerger un fossé intergénérationnel, le contexte contemporain de la vie dans les réserves que l'auteure nomme la «culture des réserves »- peut parfois valoriser au contraire une tension productrice d'innovations.

Les deux articles suivants aborderont le pouvoir de la musique dans les expériences sociales et individuelles de 
jeunes Atikamekw et Innus. La production musicale s'inscrit dans les deux cas dans des dynamiques d'affirmation identitaire et de guérison sociale qui dépassent les frontières du local par des références culturelles et sociales plus larges. À travers la pratique du tambour, Laurent Jérôme déconstruit ainsi l'idée de «musique traditionnelle» en tant que reproduction à l'identique de génération en génération. Dans le cas des chanteurs innus, l'engagement social s'exprime moins par des questions et réflexions ouvertement politiques, écrit Véronique Audet, mais bien plus par des discours d'espoir souvent guidés par des parcours personnels marqués de ruptures et de déséquilibres.

Martin Hébert utilise le concept « d'images-souhaits » pour faire valoir les aspirations à un «monde meilleur» de jeunes autochtones originaires de deux régions des États du Chiapas et du Guerrero (Mexique) malgré une crise sociale qui ne peut être niée. Lauteur montre en outre comment cette espérance se construit et se négocie entre appartenance communautaire et possibilités urbaines, entre ce qui « est » et ce qui « devrait être ».

Le milieu scolaire et les pratiques relatives à l'éducation sont tout autant porteurs de messages d'espérance dans un contexte social perturbé. Il en sera question dans les quatre textes suivants. À travers un projet éducatif dans une communauté métisse du Manitoba, Thibault Martin et Brieg Capitaine réfléchissent sur l'école comme lieu de synthèse culturelle dans laquelle le politique rencontre l'éducation et où les savoirs locaux se positionnent aux côtés de dynamiques contemporaines.

Natacha Gagné fait valoir le «bi-culturalisme » à propos d'un autre contexte national (l'État néo-zélandais en a fait une politique officielle) mais aussi local. Pour les étudiants maoris, la question identitaire se pose non pas dans leur communauté, mais à l'université et dans le milieu urbain. Les jeunes Maoris doivent, au quotidien, y justifier leur « maoritude ». Ce constat de l'auteure n'est pas sans rappeler des discours communs en Amérique du Nord à travers lesquels s'opèrent des distinctions entre « vrais » et «faux » « Indiens », renvoyant constamment l'« être autochtone » dans le faux-débat que représente la question de l'« authenticité » (Laurent Jérôme, ce numéro).

Deux articles abordent les principes épistémologiques, ontologiques mais aussi éthiques que sous-tendent les pratiques de l'éducation en milieux autochtones. Jean-Guy Goulet et Kim Harvey Trigoso nous emmèneront dans la perception dènèe tha de l'éducation à travers une recherche qui a mis en valeur une relation étroite entre communauté/milieu scolaire et forêt/activités traditionnelles. La dynamique familiale se pose en filigrane de ce texte: plus les enfants sont amenés par leurs familles à s'investir dans les activités traditionnelles dans la forêt, plus ils développent une attitude positive face à la vie dans la communauté.

Cette relation entre éducation et environnement est également au cœur de l'article de Lucie Sauvé et al. À travers deux expériences de recherche participative et d'intervention, l'une sur la question des contaminants au Labrador et l'autre sur la déforestation en Abitibi, les auteures évoquent les réussites, les défis, les enjeux, l'intérêt mais aussi les difficultés qu'ont pu susciter une éducation relative à l'environnement chez de jeunes Innus et Algonquins et dans l'ensemble des communautés partenaires. Laisser la caméra aux jeunes pour qu'ils expriment eux-mêmes leurs perceptions de l'environnement (les auteures conjuguent d'ailleurs ce concept au pluriel) est un choix méthodologique qui peut révéler des représentations à ce point significatives qu'elles peuvent déranger. La vérité

\section{QUelques OPINIONS DE JeUnes AUtOChtONes*}

Patrick Moar, Atikamekw de Manawan, 28 ans, étudiant À L'UNIVERSITÉ LAVAL

«Cela fait très longtemps que je réfléchis de mon bord sur la jeunesse. Actuellement, j'ai un dilemme intérieur. Je me dis que j'aimerais rester jeune toute ma vie. Aujourd'hui j'ai 28 ans et on me considère encore comme un jeune par chez nous alors que moi j'aurais le goût de m'affirmer et d'être reconnu comme un adulte, c'est ce que je me dis. [...] Si on a 28 ans et qu'on est encore considéré comme jeune dans nos communautés, je crois qu'il y a un petit problème de négation d'une génération...»

\section{JohnNy JeAn PierRe, InNu de UAShat, 24 ANS, Étudiant EN THÉÂTRE}

« Au-delà de 30 ans dans la politique, on laissait les jeunes dormir, dans un berceau, on ne parlait pas de jeunes, les jeunes, ça n'avait aucun sens. Depuis 30 ans, on entend tout le temps dans la politique, les jeunes, faut aider les jeunes. [...] C'est quoi un jeune? Hein? [...] C'est plus nos parents qui sont les leaders, ils sont perdus nos parents, nos parents nous appellent, ils nous ont réveillés et c'est à nous autres de faire le travail. Ne venez pas nous traiter d'incompétents, parce que vous êtes perdus, on va vous donner de l'aide, on va vous tendre la main. Vous nous avez bercés, maintenant c'est à notre tour, je suis réveillé, on va vous bercer à notre tour. "

«... Moi je suis jeune, est-ce que je vais être capable de m'exprimer un jour? Je suis en train de m'exprimer, mais vraiment ce qui se passe avec la jeunesse c'est dur, oui c'est dur, parce qu'on ne sait pas qui est la jeunesse, ça peut être la jeunesse à 5 ans, la jeunesse à 50 ans, la jeunesse à 100 ans. C'est beau de voir une personne âgée de 100 qui vit sa jeunesse encore. C'est la création de l'esprit. »

\section{ChRISTIAN COOCOO, 30 ANS, EN TANT QUE COORDONNATEUR RÉGIONAL AU DÉVELOPPEMENT DE LA JEUNESSE, CONSEIL DE LA NATION ATIKAMEKW}

" Ça fait environ un an que je travaille pour les jeunes, pour les trois communautés atikamekw et pour nous [...] la jeunesse c'est les 15-35 ans. [...]. Ce que j'ai remarqué chez les jeunes, c'est qu'il y a un discours qui commence à émerger quand ils parlent de l'avenir ou de la situation dans la communauté. Ils commencent à avoir des discours plus positifs que négatifs. Ils commencent à parler plus positifs : 'c'est ça qu'on devrait faire' au lieu de dire 'on est comme ça', 'on fait pitié', 'il faudrait'... Un discours vraiment négatif ou défaitiste. J'ai senti ça depuis que je travaille avec eux : 'faut faire des choses', 'faut changer des choses'... Et ça c'est vraiment un discours qui commence à ressortir. On voit de plus en plus de jeunes qui se présentent au niveau de la politique. Je parle du côté atikamekw, là, je pourrais pas généraliser partout au Québec, c'est ce que je vois du côté atikamekw, c'est ce que je vois du côté des jeunes. »

* Colloque tenu par le Centre interuniversitaire d'études et de recherches autochtones (Ciéra), printemps 2005. L'intégralité de ces points de vue se retrouve dans le DVD joint à ce numéro. 
s'exprime par les photographies des enfants, mais toutes les vérités ne sont pas bonnes à montrer.

Pour Piette (1996 : 150), deux arguments décisifs jouent en faveur de l'utilisation de la photographie comme mode de connaissance: la force de la représentation par rapport à l'inadéquation de l'écriture face aux exigences d'une vie sociale complexe et sa capacité à attirer le regard sur « des détails constitutifs d'une modalité non encore étudiée de la vie sociale ». Karoline Truchon montre comment un autre «fragment de vérité », celui d'images et de représentations positives sur le milieu de vie de jeunes Innus venant rompre avec les discours de victimisation, a émergé de son projet «La photographie comme bâton de parole ». Ce qui retient l'attention de l'auteure dans son article correspond à ce qui a retenu l'attention des jeunes dans leurs commentaires sur les photographies qu'ils ont prises : ces derniers ont plus insisté sur les liens et la relation les unissant au sujet représenté que sur la représentation (la photographie comme objet esthétique) en tant que telle.

\section{LE SUPPORT AUDIOVISUEL}

Le DVD qui accompagne ce numéro de Recherches amérindiennes au Québec appuie, sous diverses formes, les questions soulevées dans les articles. Il présente quelques-uns des documents réalisés lors de la première année du projet Wapikoni Mobile, un studio itinérant de formation et de réalisation cinématographique destiné aux jeunes autochtones du Québec. Encadrés par des formateurs, de jeunes Atikamekw et Algonquins ont tenu la caméra et mis en image, sous formes de documentaires et de clips vidéo, des réalités touchant ou interpellant leur quotidien.

Nous présentons dans ce DVD trois courts documentaires et deux clips musicaux réalisés dans le cadre de ce projet :

- En 1998, le documentaire L'Erreur boréale du chanteur québécois Richard Desjardins révélait au grand jour et de manière percutante les ravages des coupes à blanc. La forêt de mon grand-père est en quelque sorte une vision atikamekw de la déforestation et des problèmes de communication entre les différents utilisateurs de la forêt québécoise.

- Initialement, le documentaire La lettre devait aborder, de manière générale, la vie quotidienne de jeunes Atikamekw de Wemotaci. Une question de l'un des formateurs a donné à ce témoignage une tout autre dimension.

- J'ai déjà marché sur cette terre évoque le quotidien de Pierre à travers des interrogations sur ses rapports sociaux dans sa communauté depuis qu'un accident de motoneige l'a privé de l'usage de ses jambes.

- Awin-ni-nin (Qui suis-je?) exprime sous forme de vidéo-clip cette question de la dualité des références et des influences chez les jeunes: Cathy danse dans les powwows et chante sur la musique punk, Louis-Philippe est chanteur et joueur de tambour mais aussi batteur pour de nombreux groupes innus et atikamekw de musique pop rock.

- La musique rap fait partie des choix et des goûts musicaux des jeunes autochtones. Samien, jeune Algonquin de Pikogan, a pu réaliser ces premiers vidéo-clips dans le cadre du projet Wapikoni Mobile.

- Le DVD valorisera par ailleurs des artistes Atikamekw et Innus (Shauit Aster, Rod Pilot et Laura Niquay) à travers des morceaux choisis tirés de leurs albums.

- En plus de ces réalisations, le DVD contient des cartes afin de situer le contexte local et national de ces réalisations.

\section{Notes}

1. Propos recueilli à Wemotaci, en juin 2001, auprès d'une personne ressource en éducation, langue et culture. Nous préférons ici garder son anonymat.

\section{Remerciements}

La réalisation de ce numéro n'aurait pas été possible sans le soutien et la confiance de nombreuses personnes et institutions. Je tiens à remercier Claude Gélinas, ancien directeur de la revue, Véronique Duval, qui a participé avec énergie aux premières étapes du projet, Jean-Philippe Warren, Michel Lavoie, Eric Chalifoux et Sylvie Poirier pour leurs commentaires, Laurent Girouard pour la réalisation du support audiovisuel. Merci à l'Office national du film et à l'équipe du Wapikoni Mobile, particulièrement à Manon Barbeau, Marie-Claude Desrochers et Gilles Péloquin, pour avoir autorisé et facilité la diffusion de certaines réalisations du Wapikoni. La réalisation et l'insertion du DVD ont été rendues possibles grâce au soutien financier du Secrétariat à la jeunesse, du ministère de l'Éducation, du Loisir et du Sport et du Secrétariat aux affaires autochtones du Québec.

\section{Ouvrages cités}

BOMBARDIER, Denise, 2005: «Honte au Canada!» 26 et 27 novembre, Le Devoir, B5.

BOUCHARD, Serge, 2004 : Récits de Mathieu Mestokosho, chasseur innu. Boréal, Montréal.

BOUDREAULT, René, 2005: «Conjuguer tradition et modernité ». Relations $698: 21-22$.

BUCHOLTZ, Mary, 2002 : « Youth and Cultural Practice ». Annual Review of Anthropology $31: 525-552$.

CANADA, 2003 : Peuples autochtones du Canada. Un profil démographique. Statistique Canada.

CNA, 2004: Population Atikamekw. La Tuque, Développement jeunesse, Conseil de la Nation Atikamekw.

COLLIN, Dominique, 1988 : « L'ethno-ethnocentrisme : représentations d'identité chez de jeunes autochtones du Québec ». Anthropologie et Sociétés 12(1) : 59-76.

—, 1994: «Modernité et tradition dans le discours identitaire autochtone ». Recherches sociographiques 35(3): 477-505.

GALLAND, Olivier, 2001 [1993] : Sociologie de la jeunesse, PUF, Paris

OOSTEN, Jarich, et LAUGRAND, Frédéric, 1999: Interviewing Inuit Elders. Language and Culture Program of Nunavut Arctic College, Iqaluit, Nunavut.

PIETTE, Albert, 1996: Ethnographie de l'action: l'observation des détails. Métailié, Paris.

POIRIER, Sylvie, 2004 : « Présentation : Réflexions sur un concept pluriel ». Anthropologie et Sociétés 28(1) : 3-21.

SABEV, Dessislav, 2005 : " La modernité de la tradition : une analyse de la géométrie culturelle dans 'Nous, les Premières Nations', Musée de la civilisation, Québec ». Recherches amérindiennes au Québec 35(2): 49-60.

SAVARD, Rémi, 2004: La Forêt vive: récits fondateurs du peuple innu. Boréal, Montréal.

SMITH, Linda Tuhiwai, 1999 : Decolonizing Methodologies: Research and Indigenous Peoples. Zed Books et University of Otago Press, Dunedin et Londres.

VINCENT, Sylvie, et BACON, Joséphine, 2003: Le Récit de Uepishtikueiau: l'arrivée des Français à Québec selon la tradition orale innue. Recherches amérindiennes au Québec, Montréal. 
Marie-Pierre

Bousquet

Département d'anthropologie,

Université de

Montréal,

Montréal
T ORS DU COLlOQUE «Autochtonie et gouvernance » tenu à Montréal en octobre 2004, un chef innu commença sa communication par une anecdote. Reconnaissant dans la salle un anthropologue à la retraite, il raconta qu'il se souvenait, enfant, des séjours de celui-ci dans sa communauté. Lanthropologue demandait alors où il pouvait trouver les aînés. Le chef conclut en s'amusant du fait que les nouveaux chercheurs venaient maintenant le voir, lui qui était devenu un aîné.

Lanthropologie a bâti une partie de sa tradition de recherche sur une gérontoethnographie. S'adresser aux plus âgés est un réflexe de terrain, comme si les récits de ces informateurs, précieux au demeurant, garantissaient que les données soient plus riches et plus « authentiques». Ainsi, la phrase célèbre «Un vieillard qui meurt, c'est une bibliothèque qui brûle », dont plusieurs revendiquent la paternité (il s'agirait d'un dicton africain, popularisé par l'écrivain malien Hampâté Bâ), a aussi été attribuée à Marcel Griaule, figure française de la discipline. Si l'on tient compte du fait que, d'une part, cette phrase traduit une forte préoccupation amérindienne contemporaine et que, d'autre part, les anthropologues portent une attention coutumière aux personnes âgées, on trouve une explication au manque de documentation sur les jeunes Amérindiens, du Québec pour le cas qui nous intéresse, et ce pour n'importe quelle époque.

Ce travail ne prétend pas combler le manque relevé. Il ne peut non plus refléter les points de vue de tous les jeunes Algonquins. Si l'on aspire seulement à rendre compte de discours tenus par des «jeunes», encore faut-il pouvoir définir le profil de ce groupe d'âge. Rien n'est plus mouvant que cette catégorie, qui par essence n'est pas figée. J'ai donc choisi de retenir pour les fins de la présente recherche les discours d'hommes et de femmes nés entre 1974 et $1986^{1}$, qui sont membres de la nation algonquine et qui vivent dans des communautés ${ }^{2}$. Aujourd'hui tous majeurs, ils suivent mes recherches depuis plusieurs années et, face à mes interrogations, ont fait part de leurs points de vue spontanément. Ce suivi est appréciable, car il permet de rendre compte de l'évolution des idées des individus au fil du temps. En effet, une réflexion sur les jeunes ne peut que se périmer rapidement, puisque les personnes concernées sont destinées à passer, dans un avenir proche, à un autre stade de leur vie. Et d'année en année, il semble naturel que ces mêmes personnes changent d'opinions. Il importe donc de souligner que cette recherche se situe dans un contexte historique précis, celui du début du xxie siècle. Cette limite temporelle remet en question la traditionnelle ambition ethnologique d'accéder à un niveau de généralisation d'une relative pérennité. Ainsi, contrairement à l'habitude consacrée, il s'agit de rester conscient du caractère mobile des données qui vont être exposées.

Les stéréotypes tendent à présenter les jeunes autochtones, tant les Amérindiens que les Inuits, comme des individus « à problèmes », c'est-à-dire ayant et 
causant des problèmes issus d'un mal-être identitaire. En effet, ces jeunes se voient taxés d'être « coincés » entre deux cultures, la leur propre et la culture eurocanadienne. Dans un article sur les autochtones d'origine algonquine à Val-d'Or, Christiane Montpetit notait en 1993 qu'« à propos de l'Amérindien en milieu urbain, une caricature des plus grossières subsiste: ivrogne qui passe son temps à boire en groupe dans les bars ou les rues du centre-ville; culturellement déphasé, friand de nature, de gibier et de liberté, il ne peut être à l'aise sur du béton » (1993 : 119). Plus de dix après, force est de constater que ces stéréotypes se perpétuent et qu'il est pertinent de les extrapoler aux Algonquins résidant dans des réserves. Les jeunes seraient supposés vivre une tension permanente entre la culture algonquine et une culture «blanche », transmise par l'école, la télévision et les relations interpersonnelles avec des Québécois. Ce faisant, ils auraient de grandes difficultés d'adaptation. Ce type de discours s'entend aussi bien dans les communautés que dans le discours populaire canadien où, à côté des « deux solitudes » culturelles, française et anglaise, les autochtones en constitueraient une troisième (Cabana 2005).

La question qui se pose alors est celle-ci: les jeunes Algonquins sont-ils biculturels? Après une présentation de la nation algonquine et du poids démographique des jeunes en son sein, nous examinerons les implications qu'entraînerait l'application du concept de biculturalisme pour définir ces jeunes. Pour ce faire, nous retiendrons trois thèmes, qui ont surgi de façon récurrente dans la recherche : la thèse du fossé entre les générations, qui sous-entend d'être en conflit avec les plus âgés, dont on ne partage plus la même «culture »; la tension engendrée par le fait de devoir redéfinir la culture algonquine, dans un contexte de vie dans une réserve; et enfin l'illusion de biculturalisme que crée la Loi sur les Indiens, en particulier la loi C-31. Cela permettra de voir quelles réponses apportent les jeunes Algonquins à ces idées, en prêtant attention aux différences de réactions entre les hommes et les femmes.

\section{LES COMMUNAUTÉS ALGONQUINES ET LEURS JEUNES}

Dix bandes sont enregistrées comme algonquines par le gouvernement fédéral canadien. Neuf se trouvent au Québec, en Outaouais et en Abitibi-Témiscamingue: Kitigan Zibi, Kitiganik (Lac-Barrière), Kitcisakik (Grand-Lac-Victoria), LacSimon, Pikogan, Kipawa (Kebaowek), Wolf Lake, Winneway et Timiskaming. La dizième, Pikwakanagan (Golden Lake), dont nous ne parlerons pas ici, est en Ontario, dans le comté de Renfrew. Le classement fédéral sous cet ethnonyme peut sembler impropre, puisque le mot Algonquin n'est pas vernaculaire. Les locuteurs de la langue algonquine, surtout les aînés, se définissent comme Anicinabek ('personnes', ceux dont on comprend la langue). Le mot Anicinabe apparaît également dans les discours politiques pour mettre l'accent sur le vrai nom des Algonquins. Mais les jeunes utilisent plutôt le terme Algonquin. Ils ont donc intégré une nomenclature restreinte (Algonquins, contrairement à Anicinabek, ne s'applique pas à tous les Algonquiens dont on comprend la langue) et inexacte du point de vue historique et culturel (le classement administratif des onze bandes a été cristallisé de manière relativement aléatoire). En fait, les jeunes n'adoptent le terme Anicinabe qu'en prenant de l'âge et en se politisant.

Dans ces communautés, l'importance démographique des jeunes est à souligner. Selon Statistique Canada, lors du recensement de 2001, les groupes d'âge les plus représentés dans les neuf bandes concernées étaient la tranche des 5-14 ans et celle des 25-44 ans. Lâge médian oscillait entre 17 et 31 ans, pour une moyenne de 24-25 ans. En fait, en Abitibi-Témiscamingue, plus d'un autochtone sur deux avait moins de 30 ans en 2001. Ces faits parlent d'eux-mêmes : dans des villages comptant de quatre cents à un millier d'habitants, à forte densité de population étant donné la surface réduite de la plupart des réserves, le poids des jeunes est énorme. Les communautés constituent donc des réservoirs de dynamisme, peu encadré au point de vue économique car les revenus moyens sont, à l'instar de bien d'autres nations autochtones, parmi les plus faibles du Canada. Les taux de chômage sont très élevés : le taux de Kitigan Zibi était de 33\% en 2001, celui de Kitcisakik de $80 \%$. En outre, les taux de diplomation des plus de 25 ans au niveau postsecondaire demeurent très bas. Il faut cependant noter que le nombre d'entreprises et le niveau de scolarisation chez les Algonquins, comme chez les autres autochtones, sont en constante augmentation depuis quinze ans. Enfin, le nombre de mères adolescentes chez les autochtones est deux fois plus élevé que la moyenne québécoise. Il n'est pas rare de voir, dans les réserves algonquines, de très jeunes femmes ayant des enfants. Si ce fait est souvent interprété de manière négative selon les standards eurocanadiens, il est au contraire considéré comme positif du point de vue de ces jeunes femmes. En effet, celles-ci disent en général que les enfants les «sauvent » (de l'alcool, de la délinquance), soutenues en cela par leurs communautés, pour lesquelles les enfants représentent l'avenir.

La vitalité démographique des communautés a une incidence sur les conditions de logement. En 1988, François Larose notait à Lac-Simon le surpeuplement et l'insalubrité de nombreuses maisons. Il avait compté jusqu'à 32 habitants dans une surface prévue pour cinq à sept personnes (1988 : 285-286). Si la situation s'est améliorée, grâce aux programmes de rénovation et de construction dans les réserves (quand la surface le permet), les taux de natalité élevés obligent souvent les jeunes ayant des enfants à demeurer dans un foyer déjà établi, en attendant de se voir attribuer un logement. Les lieux de résidence des jeunes, dans une communauté, sont d'ailleurs changeants. Il arrive souvent que, de l'adolescence jusqu'au seuil de la trentaine, les individus soient mobiles d'une maison à l'autre. Il est donc fréquent que trois ou quatre générations cohabitent sous le même toit, pour des durées variables. On pourrait alors dire que le modèle algonquin de la famille étendue persiste (dans une réserve, les gens sont tous apparentés de près ou de loin). De fait, la prise en charge des mineurs ou des jeunes majeurs, communément acceptée, continue à jouer un rôle dans la reproduction du corps social. Cependant, on observe que le modèle de la famille nucléaire comme unité domiciliaire est considéré comme idéal. Qu'on impute ce fait à la promotion des standards nord-américains ou à une imposition coloniale d'un canon d'urbanisme eurocanadien, comme le suggérait Rémi Savard (1975), il est en tout cas certain que les plans de maisons préparés par les architectes du ministère des Affaires indiennes et du Nord canadien ont été prévus suivant ce modèle.

\section{LE FOSSÉ ENTRE LES GÉNÉRATIONS : CHANGEMENT SPATIAL, CHANGEMENT SOCIAL}

Après ce portrait général, il importe de situer avec plus de précision la position des jeunes par rapport aux autres générations, en expliquant comment chaque groupe d'âge se conçoit par rapport à des temps et à des espaces sociaux.

Lidée de génération suppose que des groupes d'âge se succèdent les uns les autres, les derniers venant remplacer les 
précédents au fil du temps. L'existence d'une génération particulière est identifiée, souvent a posteriori, à partir de différents paramètres: l'appartenance à un même cadre historico-social, la distanciation avec la ou les générations antérieures (qui s'opère aussi bien par référence que par opposition), une association d'idées avec des événements ou des conceptions du monde particulières, un regard sélectif sur le passé (Mannheim 1990 : 49-54; Attias-Donfut 1988: 169 et suiv.). Ainsi, selon Claudine Attias-Donfut (1988 : 172), « les discours sociaux sur les générations s'inscrivent dans la production par la société de sa propre mémoire: les représentations collectives associant des tranches d'histoire à des générations données sont autant de marqueurs du temps vécu, structuré et mémorisé ».

Les Algonquins articulent leur mémoire sur leur récent passé en retenant plusieurs événements, ou plutôt plusieurs processus qui, selon eux, ont changé la dynamique de leur organisation sociale et les modalités de transmission de l'héritage culturel. Ils structurent leur conception du temps social autour d'un axe qui constitue un point zéro, à partir duquel est pensé un «avant » et un « après »: le passage à la sédentarité, qui a officiellement commencé dans les années 1850 (pour les bandes de Kitigan Zibi et de Timiskaming) et qui s'est achevé dans les années 1960. Entre ces deux périodes, les Algonquins ont vécu une série de faits qui, progressivement, a altéré leur mode de vie semi-nomade jusqu'à ce qu'ils soient obligés de devenir sédentaires: les coupes forestières, le défrichement et la colonisation de leurs terres, l'industrialisation, l'expansion démographique eurocanadienne, l'urbanisation, la pollution de l'environnement. Avec la baisse des ressources fauniques subséquentes, leur économie a sombré. Ajoutons que l'entreprise d'évangélisation par les missionnaires oblats à partir des années 1840 a grandement affecté leur système de représentation du monde, au point de vue culturel, religieux et éthique (Leroux 1995). En outre, la sédentarisation a été concomitante, pour la plupart des bandes, avec la période des pensionnats indiens, les Algonquins ayant principalement été envoyés, entre 1955 et 1972, à l'école résidentielle de Saint-Marc-de-Figuery ${ }^{3}$. Ainsi, la période des années 1950-1960 est considérée comme la limite entre « avant », temps du semi-nomadisme et du mode de vie traditionnel, et « après », temps de la sédentarité, de la fin de l'autonomie, du bien-être social et de la perte de la culture.

D'après les Algonquins, ces changements ont créé une profonde cassure, qui se manifeste par un fossé entre les générations (concernant les différences générationnelles, voir Bousquet 2001). Ce fossé, présenté comme nouveau, signe d'une rupture telle qu'elle paraît inédite dans leur histoire, est pensé comme une source de danger pour la survie de l'identité algonquine. La génération "zéro » de cette discontinuité est appelée " génération du pensionnat », en référence à ceux qui y sont allés. Celle qui la précède est la génération " des aînés », nés sur les territoires et ayant connu la vie semi-nomade. Celles qui lui succèdent, fait notable, ne sont pas vraiment nommées, du moins pas encore. Or, comme Attias-Donfut le fait remarquer, «nommer une génération, n'est-ce pas produire un symbole historique et créer une mémoire?» (1988: 176). Les jeunes d'aujourd'hui, nouvelle génération, n'ont pour l'instant pas de charge symbolique. Encore difficiles à caractériser, ils constituent les « gens de l'avenir », pour reprendre une expression de Sylvie Vincent (1991: 131)4.

En somme, les générations que circonscrivent les Algonquins correspondent à des repères définis dans le temps, à des manières de vivre qui auraient façonné différents types de mentalités. À la dernière extrémité de la chaîne intergénérationnelle, les jeunes sont héritiers d'un processus de profonde perturbation de la société algonquine. Ils forment un ou des groupes d'âge descendant et résultant d'un changement social accéléré (par la sédentarisation, qui a sapé les fondements de ce que les Algonquins considèrent comme ayant été l'essence de leur culture, le semi-nomadisme). En outre, comme le sousentend la notion de "fossé » entre les générations, qui implique l'existence de conflits, les jeunes sont légataires des tensions résultant des difficultés d'ajustement entre, d'une part, leur société et celle des allochtones, et d'autre part les générations coexistant dans leurs propres communautés.

\section{« ON N'EST PLUS DES NOMADES "}

La notion de biculturalisme suppose celle d'un fossé entre les générations. Souvent appliqué aux migrants, le concept est associé à l'idée d'un ajustement social : les migrants, en contact permanent avec une autre culture, développeraient une « double identité », se servant de l'un ou de l'autre système de représentation en fonction des contextes pour adapter leurs comportements (Thompson 2003; Benet-Martínez et al. 2002). Dans la même lignée, et d'après Anderson (1999), les jeunes se verraient devenir les agents d'une « créolisation » de la culture familiale, d'une « réconciliation » des deux systèmes en compétition. Mais le pont culturel qu'ils forment peut entraîner des heurts avec les plus âgés. Ces hypothèses tiennent-elles pour les jeunes Algonquins?

Moi, je sais rien de la culture algonquine. Je suis née sur la réserve. Enfin en ville, mais je connais que la réserve. J'aime ça le bois, tu vois, mais je le connais pas vraiment. Le bois, c'est les vacances.

On n'est plus des nomades maintenant. Nous autres [les jeunes], on est des Indiens de réserve. [...] Je sais pas si j'ai envie de vivre ailleurs. Je crois pas. (Ikweses, 19 ans, août 2003)

Lidée de ne « rien savoir sur la culture » est une constante dans les discours de tous les informateurs interrogés. Pourtant, même si on part d'une définition anti-dynamique de la culture, que renieraient la plupart des anthropologues actuels mais selon laquelle une culture est un ensemble caractéristique de traits spécifiques à un peuple et qui en constituent ses normes, force est de constater que les jeunes font état de connaissances et de représentations du monde, qu'ils ont en commun avec les générations précédentes, considérées dans les bandes comme «typiquement algonquines » et répertoriées comme telles dans la littérature anthropologique. À titre d'exemples, mais qu'il serait possible de multiplier, nous avons retenu trois traits, dont la valeur est attestée dans la culture algonquine, ainsi d'ailleurs que dans toutes les cultures algonquiennes du nord-est du Canada. Le premier est la croyance en Kokodi, ou Windigo (Mac Pherson 1930; Beck 1947; Aubin 1982), ce monstre cannibale qui dévore les êtres humains, qui vit habituellement en forêt mais que les récits contemporains situent maintenant également à la périphérie des villages. Le deuxième est l'importance du rêve (Hallowell 1976; Vincent 1976), par lequel le monde invisible se manifeste aux vivants du monde visible pour dispenser des enseignements et des avertissements qu'il faut suivre. Enfin, le troisième est l'attention portée aux signes envoyés par la nature sous forme d'un événement anormal qui met généralement en scène un animal ou un être humain décédé, et annonciateurs de mauvais augures (Beck 1947).

Ainsi, ce sont souvent les jeunes qui enseignent à l'anthropologue les histoires de Kokodi, présenté dans leurs 


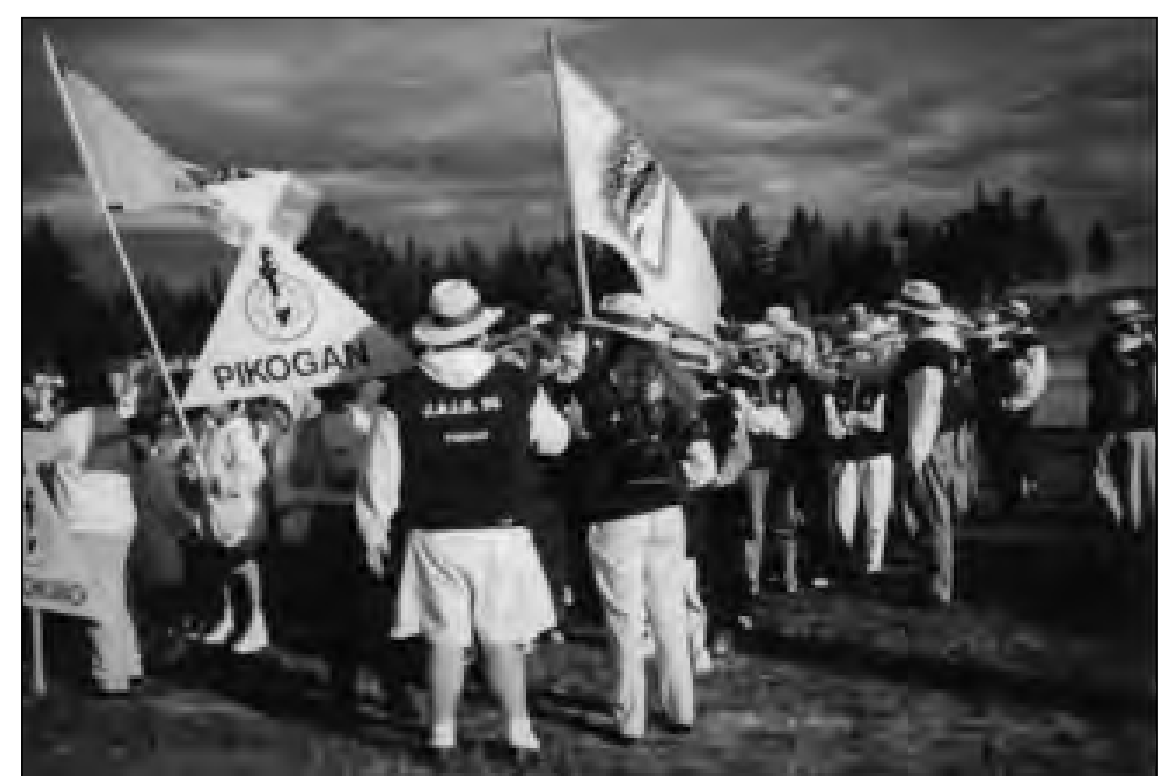

Délégation de Pikogan aux Jeux autochtones inter-bandes, juillet 1996, Uashat-Maliotenam (Photo Marie-Pierre Bousquet)

récits comme un croquemitaine qui emporte les enfants n'ayant pas été sages, pour les manger. Comme les gens plus âgés, les jeunes se posent des questions sur la signification de leurs rêves, pour en respecter les principes édictés. Ils connaissent les signes auxquels il faut prêter attention: un oiseau qui s'écrase contre une vitre ${ }^{5}$, un ours qui sort trop tôt d'hibernation, un renard qui s'approche d'êtres humains (alors que cet animal fuit leur compagnie), un mort dont on fait la rencontre. Ces messagers du monde surnaturel viennent dispenser des avertissements (un décès prochain ou autre événement tragique) ou réconforter (dire au revoir, assurer une protection). La perception d'un lien entre deux univers, visible et invisible, qui interagissent, est donc bien présent chez les jeunes. Pourquoi pensent-ils donc «ne rien savoir de la culture»? D'après leurs dires, ils ne pensent pas être habilités à en parler parce qu'ils n'en savent pas suffisamment. Ils estiment que l'étendue de leurs savoirs n'est rien en comparaison de ceux des aînés, considérés comme des autorités en matière de connaissances traditionnelles.

Il est surtout intéressant de relever que les jeunes n'incluent guère dans la culture algonquine l'expérience de leur mode de vie contemporain. Séparer une culture, qui paraît essentialisée, d'une autre, celle du quotidien, va ici dans le sens d'une biculture. Collin, dans un article sur les représentations de l'identité chez les jeunes autochtones du Québec, remarquait que ses répondants définissaient le traditionnel par une sélection de traits spécifiques

Seront considérés comme "traditionnels » les activités de trappe, la religion catholique (ou anglicane), I'épaisse toile [...] dont sont faites les tentes, le thé pris dans la toundra, le hors-bord, bref tout ce qui est relié à la vie en forêt des anciens [...]. On y oppose les réalités « non traditionnelles » comme les maisons du village, l'aide sociale [...], l'alcool et les drogues: tout ce qui est associé aux contraintes et à l'ennui de la vie sédentaire imposée par les «Blancs ». (1988: 71)

Ces discours, similaires à ceux de nos informateurs, figent la culture, non dans une acception passéiste, mais dans des catégories spatialisées. En effet, la rupture entre la culture «traditionnelle » et la vie moderne «détraditionalisée » ne se situe pas par rapport au strict temps révolu des anciens semi-nomades. S'il en était ainsi, les jeunes n'incluraient pas les quatre-roues, les tronçonneuses ou les hors-bord dans leurs descriptions du mode de vie représentatif de la culture. Laccent est plutôt mis sur la référence à l'espace: le bois symbolise à la fois un « hier», où les jeunes ne peuvent pas retourner, et un « là-bas », contexte du souvenir qu'on ne peut plus qu'évoquer. Par opposition, la réserve est le lieu du présent et de l'avenir et le lieu de l' «ici » concret où les jeunes ne peuvent plus se conformer au modèle des ancêtres. Dans la lignée de Speck qui avait qualifié les Algonquins de "forest-minded makers" (1941 : 262) dont la mentalité était marquée par une expérience approfondie de l'environnement écologique au quotidien (ibid.: 268), les Algonquins actuels continuent à identifier leur culture à cette expérience, même s'ils ne peuvent plus la mettre en pratique autrement que pendant de courtes périodes de l'année.

Les Blancs, ils disent qu'on n'est plus des Indiens, parce qu'on ne vit plus de la trappe. (Adam, 22 ans, août 2003)

Les aînés, ils disent que la culture est en train de se perdre, qu'on va pas assez dans le bois, qu'on parle plus la langue. Puis quand qu'on va dans le bois, on n'écoute pas assez, on se couche trop tard, on se lève pas assez tôt, on fait pas les choses comme il faut. Puis qu'on fait juste courailler, que dans le temps c'était pas de même. Des fois, je trouve que c'est décourageant. Ils voient pas que les choses ont changé. [...] Ou ben ils le voient, mais ça les décourage. Mais c'est comme ça, on va pas retourner dans le bois. (Simon, 23 ans, août 2003)

Tous les jeunes interrogés sont déjà allés en forêt, dans les camps de chasse de membres de leur famille. Certains y vont régulièrement, pendant les semaines de relâche, d'autres y vont moins souvent. "Le bois, c'est les vacances», disait Ikweses, et les aînés déplorent que les jeunes puissent qualifier ainsi ces séjours. Pour ces derniers, le bois est un lieu de travail et d'apprentissage de la «vraie » vie algonquine. Comment les jeunes considèrent-ils cette vie? Se reconnaissent-ils encore dans ce modèle? En fait, dans leurs discours, le fait qu'ils aiment ou non y aller peut aller de pair avec l'attachement de leur propre famille aux activités traditionnelles. Mais cela semble dépendre surtout de leur sexe.

Pour le comprendre, examinons une journée type dans un camp. Le réveil est très matinal, surtout en période de rut de l'orignal, au début de l'automne, quand les chasseurs se lèvent avant l'aube pour attendre l'animal. Après le déjeuner, le début de la matinée est consacré à préparer les appâts et les filets pour les poissons, à poser des collets et à ébrancher des piquets de tremble pour le piégeage des castors. Pendant que les hommes partent en canot avec les fusils et les pièges, les femmes restent au camp pour ranger la cabane, faire la vaisselle, ramasser du petit bois, chercher de l'eau et préparer le repas de midi. Cette division des tâches n'est pas toujours respectée, sauf quand il y 
a des enfants dans le groupe, que les femmes surveillent. Laprès-midi, hommes et femmes débitent des bûches, réparent et entretiennent les installations et retournent en forêt pour continuer à piéger et à chasser. Puis le groupe rentre au camp pour le repas du soir, suivi de parties de cartes ou de lecture de comics, bandes dessinées de poche, jusqu'à l'extinction des feux.

La division sexuelle des tâches liées à la vie en forêt, même si elle est relative, paraît influencer les perceptions des jeunes. En théorie, les rôles sont interchangeables, en fonction du degré de compétence de chacun. Mais les filles sont souvent moins motivées à aller en forêt que les garçons. En effet, quand elles accompagnent des femmes adultes, les activités qui leur sont dévolues (s'occuper de la cabane et de ses alentours) sont perçues comme étant moins valorisantes. La chasse et le piégeage sont des activités plus masculines que féminines, dans la mesure où on prête plus d'attention au fait que les jeunes hommes sachent tuer un animal qu'aux habiletés des jeunes femmes en la matière. Les jeunes hommes deviennent ainsi des adultes accomplis, alors que les femmes acquièrent ce statut en ayant leur premier enfant. Les jeunes filles qui associent la vie dans le bois à des tâches ménagères qui les rebutent préféreront partir, quand elles en ont le loisir, dans un groupe où le programme sera centré autour de la chasse, c'est-à-dire sur l'extérieur du camp. Elles choisiront donc un groupe où il y aura le moins possible de femmes, surtout celles qui sont attachées à leur montrer « les bonnes manières de faire ».

La vie en forêt transmet ainsi des modèles par genre, dans lesquels les filles ne veulent plus forcément se reconnaître, mais qui demeurent adéquats pour un certain nombre de garçons. Pour eux, améliorer leur habileté de chasseurs présente la double qualité d'être prestigieux à leurs propres yeux et à ceux des membres de la collectivité. Les garçons attirés par ce prestige ont d'ailleurs tendance à chercher à être invités dans un groupe comptant des chasseurs seniors reconnus pour leur expérience. En effet, la composition des groupes en partance pour le bois est disparate, n'étant plus mue par la nécessité impérieuse de la subsistance. Or, cette composition a une influence sur le nombre et la durée des activités. Si l'idéal est de constituer une unité de coopération équilibrée, avec une répartition équitable des âges et des sexes, il arrive que des groupes comptent une majorité d'hommes, ou de femmes, ou encore d'adolescents. Mais plus il y a d'adolescents, plus on se couche et se lève tard, ce qui compromet le succès des activités cynégétiques.

Ladolescence comporte souvent, pour les deux sexes, des moments de refus total d'aller en forêt, quitte à changer d'attitude plus tard. En 1996, certains jeunes allaient jusqu'à avancer : "ça m'intéresse pas la culture », alors qu'en 2003 et en 2004 ils déclaraient, surtout les garçons il est vrai, vouloir y passer le plus de temps possible.

Le prestige que représente la capacité, pour un homme, de se débrouiller en forêt, de savoir s'orienter, se nourrir, reconnaître les espèces d'arbres, détermine chez certains jeunes les

choix de métiers qu'ils posent. C'est le cas pour les décrocheurs scolaires. Joachim, 24 ans en 2003, en fait partie. Il attribue son manque d'intérêt pour l'école à l'incompatibilité du modèle de la réussite scolaire avec celui que lui a offert son père, peu scolarisé mais qui a travaillé toute sa vie en forêt à faire de l'arpentage et de la coupe de bois. Joachim, qui l'admirait, tire lui-même la majorité de son revenu des contrats qu'il effectue pour des compagnies forestières, de façon saisonnière. Il préférerait replanter plutôt que de couper, ce qui lui semble plus proche des idéaux algonquins, mais, explique-t-il, les salaires sont plus élevés dans la coupe. Comme les autres décrocheurs, il arrive que Joachim effectue aussi à l'occasion d'autres travaux manuels, dans la construction, métier également valorisé dans la société algonquine.

Les jeunes filles, elles, ne songent guère à des métiers où elles seraient en contact direct avec la forêt. Elles ont parfois occupé des emplois peu qualifiés dans les différentes entreprises publiques de leur réserve (conseil de bande, garderie, école, centre de santé, service de police, service des travaux publics, service de tourisme). Celles qui se destinent à une carrière stable se réfèrent, en général, comme les jeunes hommes, à la génération de leurs parents. Par exemple, les filles qui désirent devenir enseignantes ont le plus souvent une mère ou une tante enseignante dont elles sont proches. Ces femmes plus âgées appartiennent en effet à un groupe d'âge où les femmes se sont réapproprié un pouvoir que leurs aînées avaient perdu. Elles ont été de jeunes adultes dans les années 1970. Elles ont connu le pensionnat ou fait partie de la première cohorte à fréquenter l'école secondaire de la ville voisine. Elles ont participé à toutes les luttes pour contrer les fléaux sociaux et développer les services de leur communauté (notamment en matière d'éducation, de protection du patrimoine culturel et de communications).

Notons en passant que la perte du pouvoir des femmes algonquines, voire amérindiennes, est une question qui mériterait d'être approfondie. On peut supposer que la traite des fourrures, centrée autour de négociations avec des chefs de famille masculins, a dépossédé les femmes de leurs rôles décisionnaires 


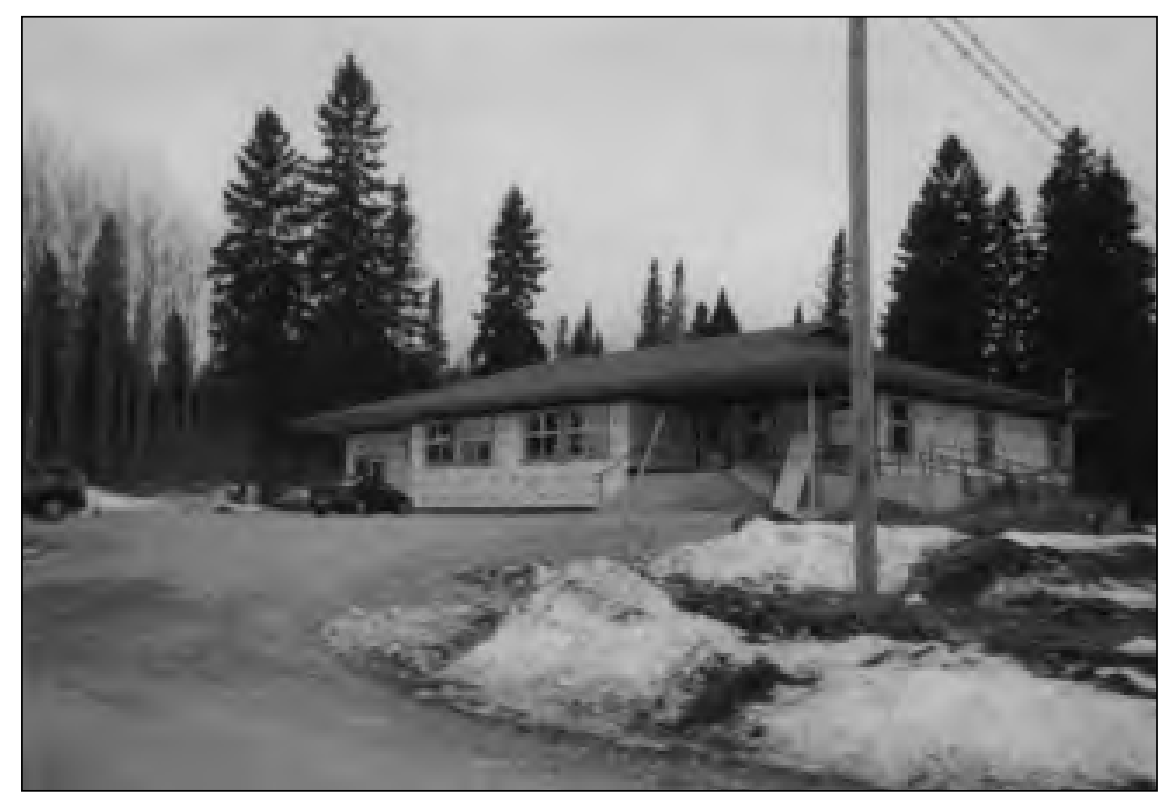

Garderie de Pikogan, en construction, avril 2002

(Photo Marie-Pierre Bousquet)

dans la gestion générale des activités traditionnelles. Également, les femmes relient leur perte d'autorité, en matière spirituelle, à l'évangélisation, puisque dans le catholicisme tous les rôles de leadership sont assumés par des hommes.

Comme le notait en 2003 un Abitibiwinni (nom vernaculaire des membres de la bande de Pikogan) de 43 ans, «la transmission ne saute pas une génération ». Quand lui-même était jeune, explique-t-il, il ne se préoccupait guère de la protection de la culture, de la langue et du territoire, ce qui aujourd'hui le passionne. Les aînés restent des modèles quand les Algonquins se réfèrent à leur tradition, mais les jeunes semblent plus influencés, pour construire leurs projets de vie, par la génération de ceux qui les ont mis au monde. C'est d'ailleurs ces derniers qui pourraient être vus comme biculturels dans des bandes comme Pikogan, puisqu'ils ont vécu le choc de l'acculturation forcée au pensionnat et de la sédentarisation, qui ont appris une deuxième langue et qui font le pont entre les aînés et les jeunes.

Il est possible que les jeunes actuels se tournent plus tard vers l'idéal traditionnel, en privilégiant des domaines qui les en rapprocheront, comme le tourisme de plein air, secteur en plein essor, ou la foresterie, où les Algonquins comptent déjà des diplômés. Ladaptation de modèles existants, en tout cas pour les bandes attachées aux activités traditionnelles, suggère que, plutôt que de réconcilier deux systèmes, les jeunes tentent de concilier avec leurs aspirations ce que leur propre système leur transmet.

\section{Culture de réserve et innovation}

Si la tradition est essentialisée, les jeunes peuvent-ils changer la mesure de référence de leur indianité? La manière dont leur biculturalisme est énoncé, un état d'entre deux mondes dans lequel ils ne pourraient pas se situer, présuppose qu'une des cultures présente des incohérences avec l'autre. Des écrivains amérindiens dénoncent cette thèse depuis la fin des années 1960. Vine Deloria, Sioux, dans un réquisitoire contre les anthropologues, s'en prend à la théorie qui prétend que les Amérindiens modernes sont «biculturels » (1969 79 et suiv.). En effet, ce paradigme explicatif bloque toute tentative de trouver un compromis culturellement acceptable. Pourtant, il a été intégré par les membres des communautés algonquines, qui ont une conscience aiguë des effets de l'acculturation. François Boudreau, pour les Ojibwas, en résume ainsi les conséquences:

L'impact global du contact a été tel que, dans certaines communautés, il a eu pour effet d'effacer la mémoire collective - la culture accumulée et transmise par le langage - le sens que donnaient les ancêtres à plusieurs pratiques sociales [...]. Dans d'autres communautés, il a effacé jusqu'au souvenir de certaines pratiques, y compris celle du langage [...] $(2000: 71)$

Cette analyse peut être étendue, à bien des égards, aux Algonquins. D'après Norris (1998), environ $30 \%$ des Algonquins seraient des locuteurs maternels de leur langue vernaculaire. Mais la situation est différente selon les bandes: certaines comprennent moins de $2 \%$ de locuteurs (comme Timiskaming), d'autres se servent de l'algonquin comme première langue du quotidien (comme Kitcisakik). En outre, la deuxième langue véhiculaire (ou unique, pour ceux qui ne parlent pas algonquin) peut être soit l'anglais, soit le français. Cette absence d'homogénéité linguistique, résultat des aléas de la colonisation, ne facilite pas les communications entre les bandes. Ajoutons que les ruptures de transmission de nombreuses pratiques (Bousquet 2002) et des versions endogènes de l'histoire ont pour conséquence une méconnaissance de l'héritage culturel, à divers degrés selon les communautés. Comment donc réorganiser une culture socialement cohérente, sachant qu'il n'est pas possible de faire abstraction de l'omniprésence d'une culture « autre »? Les jeunes sont ainsi mis en situation de gérer une tension qui existe parce que deux parties se reconnaissent comme différentes et l'affirment, et sont aussi mis en situation de redéfinir la culture algonquine.

Cette tension est mise en action par le fait de vivre dans une réserve, un monde fermé et spécifique où la gestion de la collectivité est sous tutelle fédérale. Larose, qui a travaillé à Lac-Simon, qualifie l'environnement de la réserve de « pathogène, tant sur le plan médical que psychologique » (1989 : 41). Il s'aperçoit que les jeunes nés dans des réserves « doivent apprendre à développer un sentiment d'identification et d'appartenance non pas au monde réel de la société autochtone tel qu'il est perceptible dans la réserve, mais plutôt à un monde légendaire et idéalisé correspondant à l'image véhiculée de "l'Indien des bois" ». Il ajoute que, pour se définir en tant qu'individu et en tant qu'Indien, ces jeunes auront le choix entre l'univers de la forêt, difficilement accessible, et une réalité (la réserve) présentée comme économiquement non viable, ou encore le monde extérieur, également peu accessible (ibid. : 39).

Les réserves algonquines, en général, se présentent comme des ensembles de maisons qui se ressemblent toutes, alignées le long de rues tracées au cordeau et pas toujours bitumées. On trouve toujours une église catholique, parfois une église 
pentecôtiste. Hormis les services gérés par le conseil de bande, les lieux de travail sont rares : les réserves disposent de très peu de commerces, souvent au mieux un dépanneur. Les lieux de loisir sont encore plus rares : certaines réserves ont des maisons de jeunes, mais celles-ci dépendent, outre de fonds spécifiques, de la disponibilité d'animateurs. À part ces bâtisses, ainsi que les salles communautaires qui ne sont ouvertes que pour les fêtes, les assemblées des conseils et les bingos, les jeunes doivent se rendre dans les villes les plus proches pour avoir accès aux lieux de loisir les plus courants en région (les bars, les salles de quilles et de billard, les cinémas quand il y en a).

Ainsi, quelle que soit leur génération, les gens qui y vivent voient volontiers la réserve comme un lieu à part, engendrant l'inertie, la passivité, la délinquance et la tendance à abuser de l'alcool. La marginalité juridique et socio-économique des Amérindiens, qui « résulte d'une domination exercée par l'État, domination maintenue, produite et reproduite dans le cadre des institutions sociales et politiques de la société dominante » (Jaccoud 1995: 95), se manifeste concrètement. Cette perception est liée aux problèmes de chômage. Si l'on regarde une réserve sous un autre angle, celle de la circulation constante des individus, elle ne paraît certes pas inerte.

La réserve est le point de référence des jeunes. Alors que la génération des aînés continue à se définir par rapport au territoire et que celle du pensionnat associe la réserve à une mentalité classée comme allochtone, l'individualisme, les jeunes affirment clairement s'y sentir chez eux. Tous les jeunes interrogés disent « être nés dans la réserve » (même si, dans les faits, ils sont nés à l'hôpital de la ville voisine). Certains, comme Ikweses, vont jusqu'à dire qu'ils sont « des Indiens de réserve ». Mais ils ne s'en sentent pas moins Algonquins. En fait, ils ont tendance à s'estimer plus Algonquins que les jeunes élevés en ville (voir Montpetit 1993 : 125-126). Ainsi, la troisième ou quatrième génération à connaître la sédentarité a fait de la communauté le centre des références spatiales.

Cette hypothèse se dessine quand on parcourt la réserve avec des jeunes. En déambulant à travers les rues de Pikogan avec des personnes qui avaient entre 25 et 35 ans en 1996, on pouvait reconstituer l'évolution des espaces de rencontre et de passe-temps : là, il y avait un bout de terrain vague qui constituait une aire de jeux, là un monticule de sable où l'on allait faire des glissades, là des carcasses de voitures qui offraient des cachettes géniales mais oh! combien dangereuses, là on traînait sans rien faire, à jouer au ballon, à boire de l'alcool et à « sniffer du naphta ». On peut d'ailleurs se rendre compte, pendant ces discours, de l'amélioration de l'entretien des villages au fil des années, car la plupart des lieux peu sécuritaires ont disparu, remplacés par des terrains de jeux. En contrepartie, refaire les mêmes parcours anodins avec des gens plus âgés suscite rarement des commentaires similaires. C'est donc peut-être les individus de la génération née au début des années 1970 qui se sont vraiment approprié les premiers le terrain de la réserve, car ils ont pu y créer des repères de mémoire en y passant leur enfance. Bien que n'ayant pas été interrogés, il est probable que les enfants actuels auront de semblables repères, car ils jouent dans les rues à toute heure du jour, et parfois de la nuit, en toute saison.

Il est frappant de constater que les jeunes de 1996, comme ceux de 2003, font très souvent allusion à l'oisiveté et à l'absorption d'alcool et d'intoxicants quand ils parlent de la réserve. "Ici, y a rien à faire », disent tous les informateurs. J'ai déjà débattu ailleurs du travail effectué par les bandes pour que la réserve soit, progressivement, identifiée comme un lieu sain (Bousquet 2005). Il faut souligner que les jeunes participent à ce processus. Ceux-ci sont souvent membres de « comités des jeunes », des groupes d'adolescents et d'individus dans la vingtaine qui s'engagent dans la vie de leur village et ont voix au chapitre devant les conseils. D'autres se sentent simplement sensibilisés aux messages pédagogiques diffusés, à longueur d'année, à l'école, à la radio communautaire et sur les panneaux qui jalonnent les rues. Ainsi, pour la fête de Halloween de 1996 à Pikogan, des adolescents de 14 à 17 ans (en majorité des filles) avaient été invités à décorer la salle communautaire, lieu des réjouissances collectives. Outre les guirlandes et les ballons, ils avaient choisi de placarder sur les murs des affiches à slogans éducatifs: " le vandalisme, c'est plate pour tout le monde », «pas la peine d'être gelé pour tripper» (pas la peine d'être drogué pour s'amuser) et autres phrases contre l'alcool, la violence et la délinquance. En 2003 et 2004, le noyau de ceux qui avaient participé à cette initiative continuait à affirmer leur volonté de lutter contre ces fléaux.

En résumé, la culture algonquine de l'avenir se dessine dans l'accumulation de repères et de souvenirs concernant la réserve, qui crée une nouvelle strate dans la mémoire. Dans leur milieu de vie quotidien, les jeunes Algonquins regardent les émissions de télé-réalité et les matchs de hockey, comme les autres jeunes au Québec. Ils écoutent de la musique de rock et de rap. Ils sont influencés par les modes (vestimentaires ou autres). Ils aiment le fast-food, les jeux vidéo et sortir en ville. Ils n'ont pas de choix à faire entre leur culture et la culture allochtone : ils savent qu'ils sont Algonquins, tout en ayant des manières de penser et des pratiques issues de représentations nord-américaines. Si on doit les définir comme biculturels, ils le sont selon des niveaux d'analyse que Ron Carpenter (2004) oppose. Il dénonce le premier problème : «Bien que les images populaires, aujourd'hui comme autrefois, postulent souvent qu'elles sont dichotomiques, les cultures euroaméricaines et autochtones ne sont des catégories ni mutuellement exclusives, ni antithétiques (ibid.: 1)». Même si, effectivement, cette dichotomie est non avenue, il n'en demeure pas moins que les jeunes Algonquins y sont confrontés, de par les perceptions traversant leurs communautés et l'extérieur de celles-ci. Mais on pourrait avancer qu'ils vivent une tension, non pas parce qu'ils seraient pris entre deux cultures qui seraient antithétiques (tels que les plus vieux les supposent), mais parce qu'ils se heurtent à un conflit de générations, similaire dans les « deux» mondes. Dans le même temps, ils sont biculturels au sens où l'entend Carpenter dans le cas de l'écrivaine yankton Zitkala-Sa :

Par biculturelle, je veux dire qu'elle s'exprime dans un contexte qui est inséparablement anglo et yankton; un contexte dans lequel elle est irréductible à une culture comme à l'autre et étrangère aux deux. [...] Les ressources euroaméricaines et yanktones coexistent et opèrent simultanément. (ibid. : 1).

La contradiction des perspectives d'approche du supposé biculturalisme des jeunes conduit à penser que cette théorie est peu appropriée pour définir leur identité. Afin de dépasser l'idée qu'ils puissent être bloqués entre deux modèles antinomiques, les informateurs proposent une solution : « il faut innover» (conception partagée, par ailleurs, par certains de leurs parents). Intervient alors une donnée importante pour saisir en quoi ce désir d'innovation n'est pas facile à mettre en pratique : dans une réserve, certaines choses se font et d'autres ne se font pas. Les conceptions de ce qui ne se fait pas évoluent 
au fil du temps, à mesure que les initiatives finissent par rentrer dans les normes, faisant basculer le poids du conformisme. Les exemples suivants ne sont ainsi qu'indicatifs, ayant été relevés depuis 1996 au cours de différents terrains : il est bizarre d'aller dans un restaurant dont on ne connaît pas la nourriture parce que « les Algonquins ne mangent pas ça », il est bizarre de faire un jardin fleuri devant chez soi parce que «les Algonquins ne sont pas des cultivateurs », il est bizarre de mettre du parfum (surtout dans les communautés encore attachées à la chasse, car porter du parfum est incompatible avec cette activité), il est bizarre de pratiquer un sport « que les Algonquins ne pratiquent pas ». S'il est difficile de passer outre à l'opinion collective, ce qui peut être un motif de départ de la réserve, les jeunes qui pensent que «la culture doit évoluer» n'hésitent pas à bousculer les préjugés.

Ainsi, peu à peu, le bagage de normes de ce qui est acceptable s'élargit. Mais si, comme l'avance Schwimmer (2001 : 141), « dans le biculturalisme, l'autre culture est toujours l'obstacle que nous contournons et que nous mettons au service de la nôtre », dans la réalité la menace de l'acculturation est toujours considérée comme présente, et les Algonquins continuent de porter une grande attention sur ce qu'il est possible de faire jusqu'à ce qu'on finisse par «virer Blanc».

Il en est de même pour les choix d'études. Les jeunes Algonquins ont commencé à investir des domaines où les membres de leur nation n'étaient jusque-là que peu, voire pas du tout, représentés: le droit, l'ingéniérie, l'anthropologie. Mais ceux et celles qui ont entamé des études supérieures l'ont fait dans l'idée que cela répondrait à des besoins de leurs communautés: les "survivants du système », comme les appelle Larose, «semblent le faire par identification aux intérêts communautaires plutôt que par souci d'améliorer leur situation individuelle » (1988: 255), assertion qui reste valable en 2005 . Chez les moins de 60 ans, ce paramètre entre dans la définition de ce qu'est un vrai Indien : il doit mettre ses connaissances au service des siens. Les discours sur l'insertion dans le monde du travail suivent la même logique. Notons quand même que les jeunes commencent à percevoir le travail comme un facteur d'épanouissement personnel.

\section{UNE ILLUSION DE BICULTURALISME : LES CATÉGORIES DE LA LOI SUR LES INDIENS}

Ce que les jeunes peuvent mettre au service de leur propre culture comporte des limites, floues, à ne pas dépasser pour ne pas trahir ce qu'ils sont. Mais leur latitude pour se définir et être reconnus comme Algonquins dépend aussi de la Loi sur les Indiens, qui détermine depuis 1876 les critères d'appartenance à une nation ou à une bande amérindienne. Les catégories de cette loi, qui reconnaît des degrés de métissage, ne correspondent pas toujours à leurs images de ce qu'est un Indien. La loi a-t-elle donc créé des biculturels, dans le sens où ces personnes décriraient leur identité comme «double» («ces individus s'identifient aux deux cultures », selon Benet-Martínez et al. $2002:$ 495)?

Tous les informateurs sont des Indiens inscrits. Mais ils ne détiennent pas tous leur statut selon les mêmes alinéas de la loi. Certains, la majorité du groupe de référence, sont des Indiens enregistrés qui auraient été admissibles à l'inscription même avant 1985. D'autres sont des enfants de femmes réinscrites en vertu de l'amendement C-31. Rappelons que la loi C-31, en 1985, a permis aux femmes qui avaient perdu leur statut par mariage avec un non-autochtone de le recouvrer et de le transmettre à leurs enfants. A contrario, les femmes allochtones épousant un Indien inscrit ne deviennent plus légalement indiennes, mais leurs enfants peuvent l'être. La loi suscite ainsi un imbroglio identitaire.

Elle a commencé, semble-t-il, à n'avoir de sens pour les Algonquins qu'au moment de leur sédentarisation. Les résidents d'une réserve sont des Indiens inscrits, à quelques exceptions près. Or, on constate que les enfants et les adolescents ont intégré les modalités de la Loi sur les Indiens, qu'ils utilisent comme critères de regroupement et comme motifs d'ostracisme. Ainsi, lors de disputes entre jeunes, ceux qui se considèrent comme « Indiens purs » ont tendance à discriminer les autres. D'après une aînée de Pikogan, née de mère « demie » 6 , cette distinction entre «Algonquins purs » et « moins purs » est assez récente. Choquée de voir une de ses petites-filles, dont le père est un Blanc, rejetée par les autres enfants, elle a tenu ce discours

De mon temps, ce n'était pas de même. Si tu vivais comme un Anicinabe, que tu parlais la langue et que tu savais survivre dans le bois, le monde te voyait comme un Anicinabe. [...] Je ne me suis jamais considérée comme une Abitawisi [voir note 6]. (Yvonne, 60 ans, avril 1996, Pikogan)

Depuis les années 1980, les Algonquins ont mis sur pied des programmes d'enseignement scolaire de la langue algonquine, allant de pair avec l'élaboration de matériel pédagogique. Afin de rendre plus efficaces et cohérents les différents projets dispersés, ils ont créé depuis le début des années 2000 un comité de valorisation de l'algonquin, qui réunit des représentants de toutes les communautés. Pourtant, les jeunes parlent de plus en plus rarement l'algonquin. Les signes de reconnaissance de l'identité algonquine ont changé et les jeunes ne vivent pas en forêt. Ils ont donc assimilé les catégorisations issues de diverses moutures de la Loi sur les Indiens pour se définir les uns par rapport aux autres, ainsi que pour se démarquer de la population allochtone.

Une de ces catégorisations est l'importance d'avoir été élevé dans une réserve. Le cas de Rémi l'illustre bien. Sa mère est une «C-31 », une femme née de deux parents anicinabek, qui avait épousé un Blanc et donc perdu son statut, retrouvé en 1985. Après le divorce de ses parents, Rémi, qui avait alors dans les 7-8 ans, est venu vivre à Pikogan :

J'ai eu de la misère à m'intégrer. Quand tu n'es pas né sur la réserve, c'est difficile. Le monde se méfie de toi, ils disent que tu es Blanc parce que tu viens de la ville. Tu dois faire des efforts pour devenir comme plus Algonquin que les autres, savoir mieux parler la langue, savoir mieux trapper, s'impliquer dans les activités communautaires. Maintenant, ça va, mais au début, je te dis que j'ai mangé de la marde. (Rémi, 27 ans, Pikogan, août 1996)

Habiter dans une réserve est une des façons de revendiquer son identité d'Algonquin, car obtenir le droit d'y résider constitue un enjeu politique important: les conseils n'ont le pouvoir de contrôler l'appartenance à la bande que depuis 1985 (Dupuis 1991 : 45).

Être né(e) d'une mère blanche ayant obtenu un statut indien par mariage, donc avant 1985, peut également être un facteur d'exclusion. C'est le cas de Johanne, qui a passé son enfance à Pikogan :

On prenait l'autobus scolaire pour aller de la réserve à l'école, à Amos. Puis moi, quand je montais dans le bus, tous les petits Indiens m'écœuraient, ils me traitaient de " sale Blanche », puis ils me frappaient. Moi, j'avais peur, puis je voulais pas monter dans le 
bus. [...] Je suis partie de la réserve dès que j'ai pu. Maintenant, des fois je dis que je suis Indienne, d'autres fois je me sens Québécoise. (Johanne, 28 ans, Amos, 19 juillet 1996)

Des femmes blanches «indianisées » se sont intégrées à la bande, pour peu qu'elles aient appris l'algonquin, qu'elles participent à la vie communautaire et qu'elles éduquent leurs enfants selon les valeurs algonquines. Elles sont alors considérées comme des Indiennes à part entière. Mais certaines, comme la mère de Johanne, même mariées depuis trente ou quarante ans à un Algonquin, restent à jamais des intruses. Contrairement à Rémi, qui se sent totalement algonquin, Johanne pourrait donc être qualifiée de biculturelle.

La question du statut a fait l'objet de maintes discussions au sein des réserves, notamment à partir des années 1970, quand les femmes autochtones se sont regroupées, à travers le Canada, pour faire pression afin que la loi soit modifiée. À partir de 1985, le retour des femmes C-31 et de leurs enfants dans les réserves ne s'est pas effectué sans heurts (certaines étaient revenues avant, mais elles étaient alors en général séparées de leur mari blanc). Outre le manque de maisons disponibles, ces familles ont dû prouver auprès des membres de leurs bandes qu'elles étaient restées fidèles à leur identité. Le métissage provoque donc des conflits dans les villages algonquins, bien qu'il soit une réalité sociale ancienne.

Accoler la qualification « C-31 n'est pas un artifice d'analyse. Cette définition légale est entrée dans le lexique d'appellations, soit pour se désigner soi-même $e^{7}$ ( je suis un/une C-31»), soit pour discriminer quelqu'un. Dans le deuxième cas, il apparaît que ce sont les jeunes qui ont transformé en répertoire d'insultes les références au statut. Les gens de plus de 30 ans (en 2004) ne se souviennent pas d'avoir agi ainsi dans leur enfance. Ils employaient plutôt la dichotomie Blanc/Indien (qui n'a pas disparu). Détenir une carte d'Indien n'est pas une garantie identitaire suffisante. "T'es qu'un C-31» ou «T'es qu'un métis », utilisés comme injures chez les jeunes, servent à exclure.

Les connaissances de la loi C-31 peuvent être plus raffinées. En effet, celle-ci a créé différents niveaux de statut. Larticle 6, qui se subdivise en deux sections, définit qui peut transmettre le statut (Frideres 1988 : 12-14). Cela dépend de la section par laquelle on a récupéré ou acquis le statut. Les « 6.1 » et les « $6.2 »$ n'ont pas les mêmes droits. Les « $6.1 »$ (par exemple les femmes ayant retrouvé leur statut perdu par mariage avec un Blanc) qui se marient avec un non-autochtone peuvent faire inscrire leurs enfants comme Indiens, contrairement aux «6.2» (par exemple les enfants de ces mêmes femmes ${ }^{8}$ ). Il arrive donc, tel qu'on me l'a rapporté en 2004 et 2005, que des jeunes traitent d'autres jeunes de « $6.2 »$, pour les outrager.

Enfin, les enfants inscrits dont le métissage est particulièrement visible, parce qu'ils sont blonds, qu'ils ont les yeux bleus, bref qu'ils n'ont pas « une tête d'Indien », se plaignent souvent d'être rejetés par ceux qui sont plus «typés ». Ils le sont d'autant plus quand ils habitent en ville. Les jeunes tendraient ainsi à associer des critères phénotypiques à leur conception d'une identité authentique (conception non partagée par ceux qui avaient plus de 25 ans en 2003). Ils ont donc la notion d'une intégrité raciale liée à la vie en réserve et celle d'une solidarité entre Algonquins contre l'extérieur, dans cette frontière mentale qui sépare le monde «blanc» du monde «indien ». Dans cette guerre des injures racistes, où les ripostes le sont tout autant («t'es qu'un Indien, donc t'es alcoolique »), les jeunes semblent exprimer une idéologie dans laquelle ils baignent depuis leur naissance, mélange de mise en marge par la loi, de stéréotypes et de peur de la disparition de la culture algonquine au profit d'un mode de vie «blanc».

Cette utilisation des catégories légales à des fins injurieuses inquiète les adultes, notamment les femmes, qui ont été sensibilisées au poids de la Loi sur les Indiens sur leur vie et sur celles de leurs enfants, en particulier à son impact dans les catégories de genres. En fait, si ces catégories ont pu créer des cas de biculturalisme, elles en ont le plus souvent créé l'illusion. Ceux qui se font insulter déploient des stratégies pour asseoir leur identité et ils n'en développent que rarement une «double». Peut-on, en fait, être à la fois Blanc et Indien? Il semble qu'il faille choisir son camp, car même la Loi constitutionnelle a mis à part la catégorie des métis.

\section{CONCLUSION}

Les jeunes Algonquins du début du Xxi ${ }^{\mathrm{e}}$ siècle composent avec plusieurs modèles, qui leur ont été transmis ou qu'ils ont créés, pour se projeter vers l'avenir. Certes, ils ont du mal à communiquer avec leurs aînés, ils connaissent peu leur héritage culturel, ils ont des problèmes et peut-être plus que les jeunes Québécois. Mais la différence avec leurs contemporains allochtones réside dans la brutalité et le caractère récent du bouleversement majeur qu'a été la sédentarisation. Le système social algonquin, désorganisé par la colonisation, est, pourrait-on dire, en voie de restructuration. La principale difficulté des jeunes ne semble pas consister, en fait, dans les conflits qu'ils peuvent avoir avec les générations plus âgées. De tels conflits ont sans doute existé à des époques plus anciennes. Bien qu'on manque de données sur le phénomène chez les Algonquins, Goulet (2000 : 61) l'atteste en citant la documentation amassée sur plus de soixante-dix ans chez les Gitksans de ColombieBritannique: les jeunes, observés en 1930, qui étaient les détracteurs des traditions, en étaient les défenseurs en 1970. Quoique les jeunes Algonquins ne semblent guère se poser comme des opposants à la tradition, ils se distancent des autres générations en essayant de se positionner vis-à-vis d'elles.

Leur position majeure paraît être le désir d'innover, en investissant la réserve de nouveaux repères, mémoriels, légaux, sociaux. Leurs parents se trouvaient déjà, du temps de leur jeunesse, dans la nécessité de repenser les modèles. Mais ce sont les générations nées à partir des années 1970 qui ont vraiment produit une "culture de réserve». Non seulement ils s'identifient à leur communauté et, par là, à leur réserve, ce qui, selon Weaver (2001 : 245), est souvent le cas chez les Amérindiens, mais ils y associent un (court) héritage collectif, qui se superpose à un héritage ancien, une entité sociale que forme leur entourage, des valeurs en transformation, des symboles et une faculté à se projeter dans l'avenir.

À l'issue de cette recherche, que penser du biculturalisme? Le problème de cette théorie est qu'elle est utilisée autant dans les cas de cultures opposées que compatibles (Benet-Martínez et al. 2002). Quand les jeunes empruntent, ils ne s'en sentent pas moins Algonquins, tout en opérant des distinctions entre ce qui est algonquin et ce qui ne l'est pas. Leurs prédécesseurs n'ont pas quitté leur pays, mais leur territoire pour la réserve. Si biculturalisme il y a eu, il s'est peut-être terminé avec la génération du pensionnat, qui a vécu avec acuité un passage entre deux mondes. De nouveaux codes, modèles et normes sont devenus algonquins. Ce que les jeunes construiront fera 
autant partie de la culture algonquine que ce que leurs ancêtres ont bâti.

\section{Notes}

1. Cet article est fondé sur des données recueillies au fil de séjours réguliers dans des communautés algonquines de l'ouest du Québec depuis 1996, surtout à Pikogan en Abitibi. J'ai aussi rencontré, dans leurs communautés ou lors de fêtes comme les pow-wows, des jeunes de Lac-Simon, Timiskaming et Kitigan Zibi. Kitci migwetc, merci beaucoup, aux jeunes Anicinabek qui acceptent de me parler de leur vie et de leurs aspirations depuis toutes ces années. J'ai surtout retenu ici les discours provenant de douze informateurs principaux, six hommes et six femmes, énoncés lors d'entretiens informels, en forêt, en réserve ou lors de passages en ville. Afin de respecter leur anonymat, les noms qui apparaissent dans le texte sont fictifs.

2. Bien que le mot « communauté » ne soit pas forcément le plus approprié, au sens littéral du terme, j'entends par là un village qui constitue une réserve ou un établissement. Je n'emploierai d'ailleurs pas le mot «établissement », préférant celui de " réserve » et ce, même si les villages algonquins n'ont pas tous le statut de réserve. Certains des jeunes dont il va être question ont connu des expériences urbaines, mais je centrerai mon attention sur une "culture de réserve», expression qui sera définie ultérieurement.

3. D'après d'anciens pensionnaires de Pikogan, les enfants ayant fréquenté ce pensionnat venaient de Pikogan, Lac-Simon, Kitcisakik, Low Bush (Wagoshig), quelques-uns de Winneway, de Timiskaming et de Kipawa. Il y avait aussi des Atikamekw, de Sanmaur (Wemotaci).

4. Sylvie Vincent, dans un article sur l'impact de la présence occidentale dans la tradition orale innue, fait état d'une terminologie parentale similaire à celle des Algonquins. Elle note que « le bassin sémantique à l'intérieur duquel se transmet la tradition [est constitué de cinq générations] (les grands-parents, les parents, Ego, ses enfants et ses petits-enfants). Avant ce noyau central et à partir des arrière-grands-parents, qui sont une génération frontière, on se trouve dans le monde des Anciens; après ce noyau et à partir des arrière-petits-enfants, autre génération frontière, on se trouve dans le monde des Gens de l'avenir [...]» (1991: 131).

5. Lécrasement d'un oiseau sur le pare-brise de l'autobus conduisant la délégation de Pikogan aux Jeux autochtones interbandes de 1996 (jeux pour les 9 à 17 ans) avait ainsi suscité de vives réactions chez tous les jeunes, car ce signe annonce la mort. Louise Tassé relate une interprétation similaire chez un aîné de Kitigan Zibi qui avait perdu sa petite-fille dans un accident de voiture. Or, un mois auparavant, un oiseau était mort après avoir heurté une vitre de sa maison (1995 : 47-48).

6. On pourrait considérer que les enfants de couples mixtes (Blanc/Indien) sont métis, et certains se désignent de fait comme tels. Mais comme la Loi constitutionnelle de 1982 reconnaît le statut spécifique de Métis, je n'emploie pas le terme pour parler de gens métissés mais ayant le statut d'Indien. À la place, j'ai choisi le mot « demi », qui traduit la notion algonquine de abitawisi (" être à demi », à moitié blanc, à moitié algonquin).

7. Je précise que, parmi mes informatrices, aucune femme née dans une bande, avec le statut, élevée en forêt ou en réserve, ayant perdu son statut par mariage et l'ayant retrouvé en 1985 , ne s'identifie spontanément comme « C-31».

8. Précisons qu'un 6.2 qui épouse un(e) allochtone aura des enfants non indiens. Mais deux personnes 6.2 qui se marient auront des enfants 6.1. Enfin, les hommes indiens ayant épousé des femmes allochtones ont des enfants inscrits comme 6.1.

\section{Ouvrages cités}

ANDERSON, Michael, 1999 : « Children In-Between: Constructing Identities in the Bicultural Family». Journal of the Royal Anthropological Institute 5(1): 13-26.
ATTIAS-DONFUT, Claudine, 1988 : Sociologie des générations. PUF, Le Sociologue, Paris.

AUBIN, George F., 1982 : «Ethnographic Notes from Golden Lake », in William Cowan (dir.), Papers of the Thirteenth Algonquian Conference: 47-52. Carleton University, Ottawa.

BECK, Horace P., 1947 : « Algonquin Folklore from Maniwaki ». Journal of American Folklore 60(237) : 259-264.

BENET-MARTÍNEZ, Verónica, Janxin LEU, Fiona LEE et Michael W. NORRIS, 2002 : « Negotiating Biculturalism. Cultural Frame Switching in Biculturals With Oppositional Versus Compatible Cultural Identities ». Journal of Cross-Cultural Psychology 33(5): 492-516.

BOUDREAU, François, 2000 : «Identité, politique et spiritualité. Entretiens avec quelques leaders ojibwas du nord du lac Huron ». Recherches amérindiennes au Québec XXX(1): 71-85.

BOUSQUET, Marie-Pierre, 2001 : « Quand nous vivions dans le bois », le changement spatial et sa dimension générationnelle: l'exemple des Algonquins du Canada. Thèse de doctorat en cotutelle France-Québec, département d'anthropologie, Université de Paris X - Nanterre et Université Laval .

—, 2002 : «Les Algonquins ont-ils toujours besoin des animaux indiens? Réflexions sur le bestiaire contemporain ». Théologiques 10(1): 63-87.

_, 2005 : «La production d'un réseau de sur-parenté : histoire de l'alcool et désintoxication chez les Algonquins ». Drogues, santé et société 3(2) : 63-85.

CABANA, Serge (dir.), 2005 : «Amérindiens : troisième solitude?» Réseau : 17-27 (dossier spécial).

CARPENTER, Ron, 2004: «Zitkala-Sa and Bicultural Subjectivity ». Studies in American Indian Literatures 16(3) : 1-28.

COLLIN, Dominique, 1988 : « Lethno-ethnocentrisme : représentations d'identité chez de jeunes autochtones du Québec ». Anthropologie et Sociétés 12(1) : 59-76.

DELORIA, Vine, 1969 : «Anthropologists and Other Friends », in Custer Died for Your Sins: 78-100. University of Oklahoma Press, Norman.

DUPUIS, Renée, 1991 : La Question indienne au Canada. Boréal, Boréal Express, Montréal.

FRIDERES, James S., 1988 : Natives Peoples in Canada. Contemporary Conflicts. Prentice-Hall Canada Inc., Scarborough, Ontario.

GOULET, Jean-Guy, 2000 : « Cérémonies, prières et médias : perspectives autochtones $»$. Recherches amérindiennes au Québec $\mathrm{XXX}(1): 59-70$

HALLOWELL, A. Irving, 1976 : Contributions to Anthropology, University of Chicago Press, Chicago et London.

JACCOUD, Mylène, 1995 : «L'exclusion sociale et les Autochtones ». Lien social et politiques - RIAC 34 : 93-100.

LAROSE, François, 1988, Éducation indienne au Québec et prise en charge scolaire: de l'assimilation à la souveraineté économique et culturelle. Thèse de doctorat (2 vol.), Faculté de psychologie et des sciences de l'éducation, Université de Genève.

—, 1989 : «L'environnement des réserves indiennes est-il pathogène? Réflexions sur le suicide et l'identification des facteurs de risque en milieu amérindien québécois ». Revue québécoise de psychologie 10(1) : 31-44.

LEROUX, Jacques, 1995 : « Les métamorphoses du pacte dans une communauté algonquine ». Recherches amérindiennes au Québec XXV(1): 51-69.

MAC PHERSON, John T., 1930 : An Ethnological Study of the Abitibi Indians. Musée canadien des civilisations, bibliothèque, document d'ethnologie III-G-38M, miméo.

MANNHEIM, Karl, 1990 : Le Problème des générations. Nathan (1ère éd. en allemand, 1928), Paris. 
MONTPETIT, Christiane, 1993 : « Les Autochtones d'origine algonquine à Val-d'Or : des migrants ou des citadins ». Recherches amérindiennes au Québec XXIII (2-3): 119-130.

NORRIS, Mary Jane, 1998: «Les langues autochtones du Canada ». Tendances sociales canadiennes $51: 8-18$.

SAVARD, Rémi, 1975: «Des tentes aux maisons à SaintAugustin ». Recherches amérindiennes au Québec V(2) : 53-62.

SCHWIMMER, Éric, 2001 : «La langue basque et le biculturalisme ». Anthropologie et Sociétés 25(1) : 141-150.

SPECK, Frank G., 1941: Art Processes in Birchbark of the River Desert Algonquin, a Circumboreal Trait. Bureau of American Ethnology, Bulletin 128, Anthropological Papers 17 : 231-274.

TASSÉ, Louise, 1995 : «Représentations de la santé mentale et de la filiation chez les Algonquins âgés de Kitigan Zibi ». Recherches amérindiennes au Québec XXV(1): 41-50.
THOMPSON, Richard H., March 2003: «Basing Educational Anthropology on the Education of Anthropologists: Can Bilingualism and Biculturalism Promote the Fundamental Goals of Anthropology Better than Multiculturalism? » Anthropology and Education Quarterly 34(1): 96-107.

VINCENT, Sylvie, 1976 : « Les bonnes et les mauvaises alliances ». Recherches amérindiennes au Québec VI(1) : 22-35.

—, 1991 : «La présence des gens du large dans la version montagnaise de l'histoire ». Anthropologie et Sociétés 15(1) : 125-143.

WEAVER, Hilary N., 2001 : « Indigenous Identity. What Is It, and Who Really Has It ? » American Indian Quarterly 25(2) : 240-255. 


\section{REVUE D'HISTOIRE \\ DE L'AMÉRIQUE FRANÇAISE}

\section{La consommation}

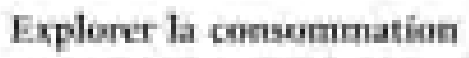

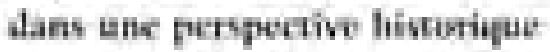

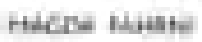

Le recur et l'ambrobite

Lu contominution

Jes bolsons alcooliyous che

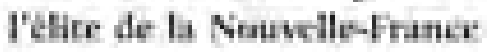

au sver stble

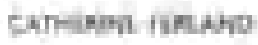

Entre l'art a la science:

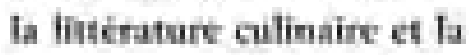
usmoformation do hohinulis allmentalies au Queher

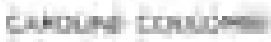

Le voyage viruel.

Lo fonsommatrices le momio de l"Araigh a Hatan a

Monirail 1RRI-1460

uchaing comomi

La contmonation bridect

Concolle des pria fet

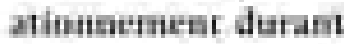

La Beuxikme Guerne mondiale

mE. Tirmin

Balinque de la constmandint.

Ducstions nationales

Sane les therers brits sus

Thisuire de h consommation

paikt =nt: 


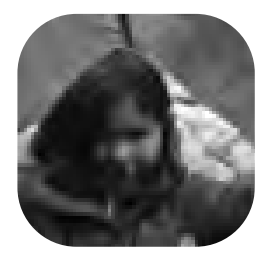

Musique, tradition et parcours identitaire de jeunes Atikamekw

La pratique du tewehikan dans un processus

de convocation culturelle

\section{Laurent Jérôme}

Département d'anthropologie, Ciéra, Université Laval,

et

Département d'ethnologie, Erase, Université Paul Verlaine de Metz $\beta_{\text {entre }}^{\text {IEN }}$ EN QU'ELLE FASSE actuellement l'objet d'une attention renouvelée, la relation entre musiques et sociétés reste encore peu appréhendée au sein des sciences sociales. C'est en tout cas le constat qui se dégage des travaux récents qui tentent d'élever la musique au statut d'objet anthropologique. Qu'il s'agisse d'études sur la danse (Terrain 2000), sur le jazz (L'Homme 2001), ou sur la relation entre musique et anthropologie (LHomme 2004) ou entre musique et émotions (Terrain 2001), ces différents numéros thématiques de revues scientifiques témoignent d'une volonté de rendre plus visibles des recherches qui saisissent la musique comme objet culturel "bon à penser », pour reprendre les termes de Jamin et Williams à propos du jazz (2001 : 11). Dans La Musique et la transe, Rouget (1980) réhabilitait le terme "musiquer » (Rousseau, Diderot). Pour Rouget, ce terme fait référence autant au produit de l'action musicale (l'œuvre, la pièce) qu'à l'action (modalités d'expression des acteurs musicaux ${ }^{1}$ ) proprement dite. Or, si l'on suit la perspective de Blum (2004), cette action musicale est inscrite dans le temps et ne peut s'appréhender en dehors de son contexte de création et de transmission.

Dans les études autochtones, de nombreuses recherches en ethnomusicologie ont montré l'importance de la musique, des chants et des danses dans l'histoire et les réalités contemporaines des premières nations du Québec, du Canada et plus largement de l'Amérique du Nord. Recherches amérindiennes au Québec a d'ailleurs consacré deux numéros à ces questions (Beaudry 1985, 1988; voir également 1992). Peu d'études en revanche ont tenté de faire le lien entre production musicale, contexte de création et pouvoir de la musique dans les parcours identitaires. Que révèle l'acte de « musiquer » le tambour sur ces processus?

Cet article est une réflexion sur la relation entre musique et « tradition» en milieux autochtones contemporains. À travers les expériences sociales et individuelles et les parcours identitaires d'un groupe de jeunes joueurs de tambour (drummers) de la communauté de Wemotaci (Haute-Mauricie, centre du Québec), j'interroge ici les dynamiques actuelles à la fois de continuités et de transformations sociale et culturelle chez les Atikamekw².

Dans le paysage sonore des premières nations du Québec et du Canada (voir Audet, ce numéro, et 2005), qu'en est-il de la musique du tambour (tewehikan) telle que pratiquée par un groupe de jeunes adultes de la communauté atikamekw de Wemotaci? Quelles dynamiques sociales, culturelles et intergénérationnelles cette pratique éclaire-t-elle?

Cet article se base sur des retours réguliers à Wemotaci depuis juin 2001 et une présence dans la communauté entre le printemps 2003 et l'hiver 2004. À travers des discussions informelles et des échanges plus formels avec les drummers, leurs amis, leur famille et d'autres Atikamekw, dans les camps familiaux ou dans les maisonnées, lors des voyages du groupe à l'extérieur de la communauté, j'ai partagé les expériences de ce groupe de jeunes joueurs 
de tambour. Le savoir inscrit dans cette pratique ne s'est pas donné à voir au sein du seul cadre spatial de la communauté. Celle-ci n'a représenté qu'un point de départ vers d'autres lieux et sites que les membres du groupe ont investis de leur expérience au monde. Cette dynamique méthodologique rayonne ici dans toute sa dimension ethnographique puisqu'elle renvoie à la métaphore de «suivre » ou de « pister» (following or tracking) utilisée par Marcus (1998) pour évoquer cette logique d'espace mouvant de l'ethnographie issue des confins du travail de terrain.

Conformément à un engagement pris en début de recherche, ce texte a été proposé à la lecture de certains interlocuteurs. Cette démarche s'inscrit dans la continuité des politiques actuelles d'éthiques de la recherche en accordant plus de transparence au travail anthropologique dans ces différentes étapes (ici, la présentation de données et de réflexions par l'écriture). Il semble pourtant que les reconsidérations éthiques et déontologiques de la discipline et du travail de terrain ne règlent pas le problème de la méfiance exprimée par de nombreuses personnes face à la présence de l'anthropologue et du chercheur en général. Cette méfiance est le résultat d'une combinaison de facteurs beaucoup trop complexes et nombreux pour que l'on puisse s'y attarder ici (nombreux passages des experts, propriété intellectuelle, absence de retour des données, de retombées des recherches...). Lors de cette recherche, cette méfiance s'est parfois exprimée en termes d'oppositions et de rejets, révélant des attitudes de fermeture et de repli sur soi considérées par certaines personnes comme des conditions essentielles à la persistance de la «culture » atikamekw. Il n'est pas dans mon intention de porter un jugement sur cette vision protectrice qui a sans aucun doute participé à la continuité de certaines pratiques et de savoirs atikamekw dans le contexte contemporain.

Il est par contre dans mes objectifs d'en considérer et d'en valoriser une autre, celle qui s'exprime par ce groupe de drummers. Leur expérience démontre en fait une tout autre vision de la persistance culturelle envisagée, au contraire, à travers une ouverture et un partage des savoirs. La perception identitaire se construit et se négocie au fil des rencontres interculturelles, des échanges avec des membres d'autres nations mais aussi avec les emitcikocic (les Blancs). Ces deux perceptions (ouverture/repli sur soi) renvoient à une ancienne intuition qui revient à vouloir découper les communautés autochtones entre «traditionalistes » et «modernistes». Certes, ce découpage rend compte de la diversité des points de vue au sein des communautés sur les stratégies actuelles d'affirmation identitaire et culturelle. Mais la réalité ne peut se réduire à ce découpage réducteur sous peine de ne pas considérer d'autres diversités, celles existant à l'intérieur même de ces deux catégories. De la même manière, il existe chez les drummers, au sein même du groupe, des stratégies et des opinions divergentes. Ces débats, loin des discours officiels de l'élite des communautés, montrent finalement l'importance de la négociation et de l'échange dans l'élaboration d'une continuité sociale en tenant compte également des points de vue moins « officiels», moins « institutionnels »

\section{QUESTIONS DE « TRADITION " ET DE MUSIQUE « TRADITIONNELLE »}

Dans un numéro de Recherches amérindiennes au Québec consacré aux «pouvoirs des sons », Preston (1985) insistait sur les transformations intervenues dans l'utilisation et la signification de la musique chez les Cris de l'est de la Baie James. Il livrait une analyse sur différentes formes de musique qui composaient alors le «paysage " musical de cette population: chants et danses traditionnels cris, danses et airs de violon européen, hymnes anglicans, musique country, chants religieux des chrétiens fondamentalistes, musique rock et chants et danses «traditionnels » pan-amérindiens. Dans son approche des transformations musicales, Preston n'accordait que quelques lignes à la musique rock en amenant l'idée que cette musique représentait une rupture avec la tradition (1985 : 26). «Le rock, réservé aux jeunes, exprime les ambitions et les sentiments de rejet qu'ils ressentent généralement et n'a pas de contenu cri que je puisse reconnaître, mis à part un léger accent plaintif ajouté à la voix de rock typique. » Lauteur notait que, dans ce paysage sonore des Cris de l'Est, la musique rock était de celles qui traduisait nettement le fossé entre les générations autant par le contenu du message que par les attitudes qu'elle véhicule. À plusieurs égards, la musique rock est pour lui synonyme « d'une identité et d'une culture cries transformées de manière significative, qui s'inspire d'une 'culture des médias' essentielle à son existence transculturelle, plus que de la continuité avec la culture crie elle-même » (ibid.).

Dans cet article, j'interroge une forme d'expression musicale «plus traditionnelle » que le style musical rock. Elle est «plus traditionnelle » puisqu'elle véhicule et mobilise un ensemble de savoirs locaux qui régit la pratique mais aussi la relation dialogique à ce qui est considéré comme plus qu'un simple « instrument musical ». Par savoirs, j'entends des règles et des codes sociaux, des savoir-faire et des manières d'être au monde, des valeurs et des significations culturelles inscrites dans la relation à l'environnement et à ce qui le constitue (entités humaines et non humaines). Suivant cette perspective, et si l'on compare la place de la musique de tewehikan chez les Atikamekw avec celle de la musique rock chez les Cris telle qu'évoquée par Preston, la première révèle une rupture moins évidente avec la «culture » atikamekw que la seconde ne la révèle avec la «culture » crie. Le tewehikan serait plus traditionnel que la guitare, la musique traditionnelle se positionnant face à la musique populaire (voir Audet, op. cit.).

Pourtant, il semblerait que la musique pop-rock-folk soit devenue une «tradition » dans le paysage sonore innu (Philippe McKenzie, Kashtin, Florent Vollant, Maten, Brian André...). Le festival Innu Nikamu est organisé chaque année depuis vingt ans pour nous le rappeler. D'un autre côté, la musique de tewehikan, telle qu'elle est pratiquée par ce groupe de drummers atikamekw, n'échappe pas à un phénomène grandissant de professionnalisation de la pratique, comme une musique populaire. Cette pratique, tout autant que ses espaces privilégiés d'expression que constituent les powwows, rencontre en effet un certain succès au Canada et aux États-Unis, succès grandissant au Québec, que ce soit dans les communautés ou en milieux urbains. Peut-on parler pour autant d'une popularisation de cette pratique? Et si c'était le cas, cette popularisation représenterait-elle pour autant une rupture avec son caractère traditionnel et avec la «culture » atikamekw?

Avec ces concepts de culture et de tradition, envisagées en termes de rupture, l'analyse des transformations musicales subit le même enfermement épistémologique que l'analyse des transformations des sociétés. Comment attribuer les coefficients de « traditionalité » ou d'authenticité à une pratique culturelle ou à un style musical? 
Le concept de tradition est un concept occidental. Si I'on considère l'ontologie, I'opposition entre musique populaire et musique traditionnelle est une distinction technique inacceptable. (C.C., octobre 2005, en réaction à ce texte)

Évoquer les concepts de culture ou de tradition en termes de rupture revient à nier leur caractère dynamique, créatif et adaptatif dans le temps et dans l'espace. Et nier ce caractère dynamique revient à rechercher l'image de cet « Indien authentique » qui renforce un point de vue encore fortement ancré chez les observateurs occidentaux : celui de la disparition des traditions et de l'occidentalisation complète des autochtones. De nombreux travaux s'intéressant aux usages de «la tradition » dans différents contextes ont pourtant démontré que la « tradition » ne peut pas être perçue comme un héritage stable reçu du passé mais s'inscrivant bien dans une vision dynamique (Hocart 1927). Ce dynamisme, Lenclud (1994: 33) l'exprime en écrivant qu'une tradition « est inventée et recréée... traditionnellement ». Pour Lindstrom (1982 : 317), la tradition est une tentative de «lire le présent dans les termes du passé en écrivant le passé dans les termes du présent ». Ces auteurs ont déjà depuis longtemps déconstruit cette idée de la transmission, véhiculée dans le concept même de tradition (Lenclud 1987), comme une reproduction à l'identique. La tradition, dans sa relation au passé, s'inscrit même pour Pouillon $(1975,1997)$ dans une filiation inversée : les pères n'engendrent pas les fils, les pères naissent des fils (Lenclud 1987). Lenclud suit ainsi Pouillon en affirmant que « ce n'est pas le passé qui produit le présent mais le présent qui façonne son passé » (1987: 118). Ces processus procèdent d'un échange entre " passé interprété » et « présent interprétant ${ }^{3}$ », consacrant la tradition comme une œuvre du présent qui se cherche une validité, une référence dans le passé (voir aussi Goody 1977; Harkin 1997; Linnekin 1983; Mauzé 1997). Les formules ne manquent pas pour insister sur le caractère dynamique de la tradition, dynamisme commun à toutes les sociétés.

Cette perception dynamique semble pourtant voler en éclat dans les stratégies actuelles d'affirmation identitaire autochtones et des nationalismes. Les premières nations actuellement engagées dans des processus de revendication face aux États qui les englobent doivent amener la preuve que ce qui définit l'essence de l'« être autochtone » est fidèle à un passé ancestral pour que soit simplement reconnu le droit d'entamer des négociations à propos de leurs spécificités culturelle, identitaire et territoriale. Cette essence de l'«être autochtone» n'est pas perçue à travers ce que les autochtones sont aujourd'hui mais en regard de ce qu'ils font avec ce qu'ils sont. Par exemple, les « activités traditionnelles » sont réduites par les systèmes juridique et politique, canadiens notamment, à un sens strict et minimum d'« activités de subsistance ».

Les " activités traditionnelles » renvoient clairement à des savoirs, à un code éthique, à des règles sociales, à des valeurs culturelles, à des systèmes symboliques et à une tradition orale qui s'étendent bien au-delà des besoins qui concernent la subsistance. (Poirier 2001 : 111)

Il y a là un découpage entre savoir-être et savoir-faire, les savoir-faire étant considérés comme une façade du savoir-être. Selon cette perspective, l'attention est plus portée sur les transformations que sur les continuités, et non pas sur les transformations et sur les continuités, toute transformation dans les savoir-faire courant le danger d'être considérée comme une rupture avec le savoir-être, celui-ci ne correspondant plus, du coup, à la perception du « devoir-être » imposée et véhiculée entre autres par les processus de négociation.

Dans les contextes (post)coloniaux actuels, ce « devoir-être autochtone » et ce que ce "devoir-être » implique comme savoir-faire, n'est pas tant le fruit d'une transformation à l'interne des sociétés autochtones mais plutôt de la persistance d'une perception ethnostalgique (Nelson 1996) fortement ancrée dans les imaginaires québécois, qu'ils s'expriment par les gouvernements ou la population, obligeant les autochtones à démontrer que ce qu'ils font est ce qu'ils ont toujours fait pour être. Selon cette perception figée dans le temps, les pratiques passées doivent encore être les pratiques présentes, sans aucune rupture.

Les exemples allant dans ce sens ne manquent pas et s'expriment tout autant par les gouvernements et la population non autochtone qu'au sein même des milieux autochtones: l'autochtone urbain est moins authentique que l'autochtone de la communauté, entend-on parfois dire, l'autochtone de la communauté est moins authentique que l'autochtone de la forêt et finalement les jeunes autochtones sont moins authentiques que leurs aînés. La pomme, rouge à l'extérieur, blanc à l'intérieur, est l'expression autochtone la plus répandue pour qualifier l'« inauthenticité » (ou disqualifier l'« authenticité ») d'un autochtone. La question de l'identité autochtone est donc rattachée à l'authenticité des savoir-faire: des activités traditionnelles, un territoire, une langue, une culture. Que penser alors des autochtones vivant en milieux urbains, lesquels représentent aujourd'hui près de $50 \%$ de la population au Canada? La question identitaire des autochtones urbains est ici très problématique puisqu'elle ne se rattache pas aux pratiques traditionnelles ni à un territoire. Les autochtones en milieux urbains sont-ils pour autant «moins» autochtones que les autres? Quel regard porter sur les jeunes autochtones, qui, pour diverses raisons (économiques, matérielles, travail, études) ne peuvent s'investir avec la même force que leurs aînés dans les activités traditionnelles? La pratique de tewehikan n'échappe pas à cette représentation idéalisée du passé : « Les Atikamekw ne sont pas des joueurs de tambour, ce sont des chasseurs. » (P.M., juin 2005, rapportant des propos entendus dans sa communauté). Pourtant, puisque les Atikamekw sont des chasseurs, ils sont aussi des joueurs de tambour (on le verra dans une section suivante). La pratique intime et personnelle de tewehikan associée à l'activité cynégétique et à certains contextes rituels fait l'objet, elle aussi, d'une attention renouvelée.

Puisqu'il est devenu indispensable pour les premières nations de valoriser des pratiques reproduites à lidentique de générations en générations pour que soit reconnue une spécificité, il n'est pas étonnant de constater un lien très fort entre culture et politique. Selon Babadzan (1999a: 7), culture et politique sont indissociables dans toutes les sociétés où la légitimation s'appuie sur une représentation idéalisée d'une « culture » nationale, situation présente partout où l'appartenance politique est basée sur une appartenance culturelle, partout où la nation est conçue comme une communauté de «culture ».

Dans ce cas, la sacralisation du passé culturel et des traditions (Babadzan 1982, 1984, 1999b) pose l'inévitable question de l'instrumentalisation politique des traditions et de la « culture ». Comme « la tradition », la « culture » est présente partout. « Même les aborigènes australiens qui ignoraient jusqu'à tout récemment avoir une 'culture', écrit Poirier (2004:9), sont maintenant fréquemment invités à donner des 'prestations culturelles' lors d'événements nationaux et internationaux. » 


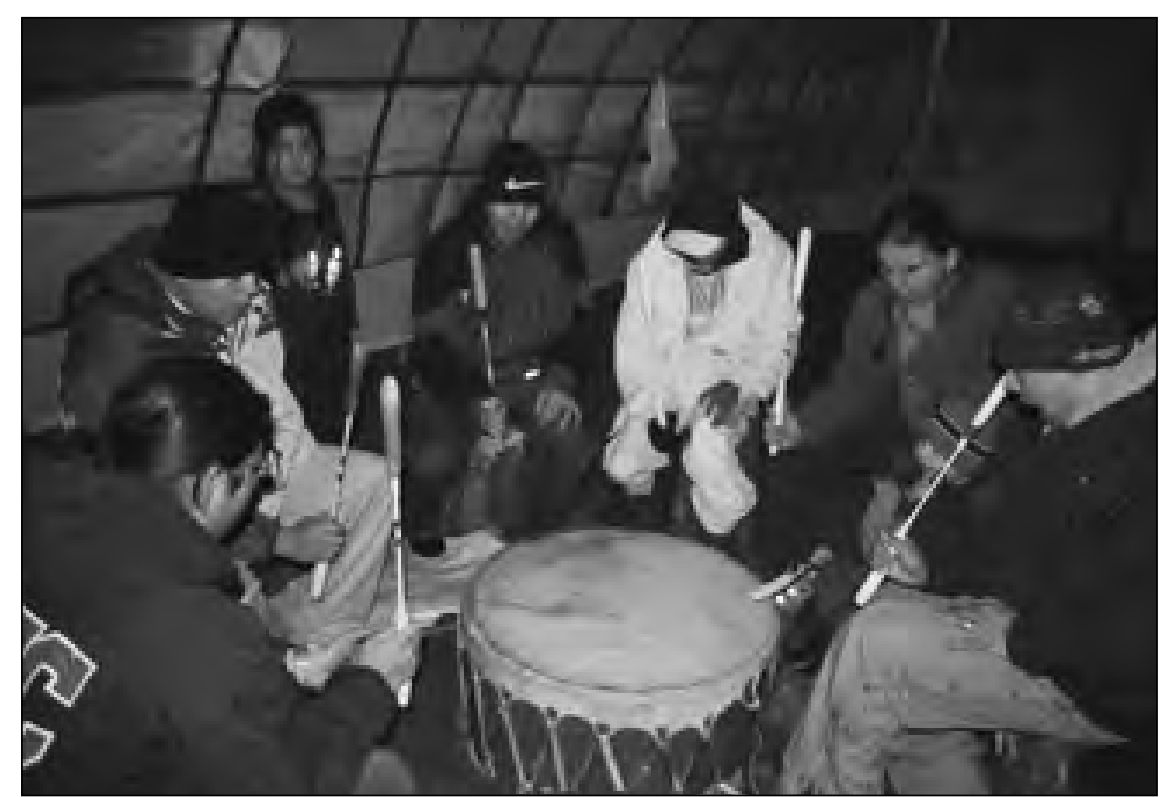

Une répétition sous le shaputuan du " poisson blanc ", un campement hivernal situé à quelques kilomètres de Wemotaci

(Photo de Laurent Jérôme)

«Tradition » et «culture » sont devenues des outils politiques privilégiés dans la reconnaissance d'une identité distincte, confirmant la pertinence du concept d'« essentialisme stratégique » de Spivak (1987). La musique n'échappe pas à ces stratégies. Reyniers (2000 : 401) montre par exemple en quoi, à travers un groupe de musique tzigane issu d'une communauté de Transylvanie, il y a eu moins transmission d'une tradition musicale que transmission par la musique et la danse d'une stratégie d'affirmation collective (Savishinski 1994).

Ces processus délibérés de traduction des traditions, dans le temps et dans l'espace, de sociétés et d'individus engagés dans des stratégies de mise en valeur d'ordres culturels et sociaux distincts ont été diversement conceptualisés. Wallace (1956) et Linton (1943) les ont nommés respectivement « mouvements de revitalisation » et "mouvements nativistes ». Lapproche de «l'invention des traditions " (Hobsbwam et Ranger 1983) interroge les processus de changements culturels en apportant un nouvel éclairage sur les processus d'affirmation identitaire et d'appropriation culturelle dans lesquels se sont engagés les groupes colonisés. Aujourd'hui et dans les études autochtones au Québec et au Canada, les termes de «traditionalisme » (voir par exemple Picard-Sioui 2003) et de "néo-traditionalisme » (voir Tanner 2004: 201) sont employés pour évoquer les réponses trouvées soit dans les savoirs locaux soit dans des réalités d'autres groupes, et formulées, traduites dans les termes du présent dans un contexte spécifique.

Si l'approche de l'invention des traditions a le mérite d'interroger ces dynamiques de changements dans la continuité en ne se focalisant pas uniquement sur le passé, elle soulève cependant des questions éthiques. Évoquer les transformations actuelles des sociétés autochtones en termes de rupture, de fabrication, de construction ou d'invention pose en effet des problèmes de compréhension au point d'engendrer et d'alimenter des relations difficiles entre les experts extérieurs (anthropologues et autres -logues) et les personnes avec lesquelles ils travaillent. «Est-il possible de parler de fabrication de la culture sans se faire des ennemis? » se demande Jackson (1989). Si le concept d'« invention » a émergé de la littérature anthropologique lorsque les groupes colonisés se sont engagés dans des mouvements de reconnaissance de leur identité distincte, il laisse transparaître, tout au moins chez les lecteurs autochtones, l'idée d'une construction dénuée de tout ancrage social, culturel, historique et ontologique. Les chercheurs, anthropologues notamment, sont confrontés depuis quelques années déjà à un retour critique qui ne manque pas de faire remarquer leur tendance à construire des concepts bien éloignés des pratiques et des expériences vécues.

Dans cet article, la pratique de tewehikan est considérée comme un processus qui répond exactement, dans sa transmission de génération en génération, à des mécanismes d'oubli, d'emprunts et d'adaptations. La musique de tewehikan, telle que pratiquée et vécue par un groupe de jeunes adultes atikamekw de Wemotaci, permet de regarder les négociations en cours dans l'élaboration d'une continuité malgré l'intensité et la fréquence des ruptures qui ont marqué et continuent de marquer la vie quotidienne de ces jeunes joueurs, en particulier, et des Atikamekw plus largement. Le concept de convocation est utilisé pour faire valoir le dynamisme et la capacité d'adaptation et de transformation des «savoir-faire » atikamekw étroitement liés à un « savoir-être » persistant dans le temps.

\section{LA PRATIQUe COLLective DE TEWEHIKAN DANS UN PROCESSUS DE CONVOCATION}

Par convocation, j'entends le processus par lequel sont traduits, réunis et intégrés des pratiques et des savoirs inspirés d'espaces relationnels multiples au sein des expériences contemporaines pour répondre à un contexte social en pleine mutation et transition. Pour la pratique de tewehikan, ce processus de convocation s'inspire, se construit et se négocie au sein de trois espaces relationnels :

1. Dans la relation entre les générations: pour les Atikamekw comme pour la plupart des groupes autochtones, la mémoire (nehiro kinokewin en atikamekw) et la transmission des savoirs par l'expérience sont perçues comme des dynamiques essentielles à la continuité sociale. Notion centrale en anthropologie en ce qu'elle représente l'instrument par excellence de la continuité sociale (Choron-Baix 2000: 357), la transmission est «largement imprévisible quant à ses effets, surtout lorsqu'elle touche à la dévolution d'un patrimoine immatériel et moral, toujours soumis, dans sa translation d'une classe d'âge à une autre, à de potentielles déperditions, réinterprétations et recompositions ».

2. Dans la relation aux groupes voisins: les nombreux échanges entre nations, s'ils ne sont pas nouveaux, continuent d'alimenter et d'influencer les pratiques et les savoirs contemporains. Les manifestations publiques pan-amérindiennes telles que les powwows et certaines pratiques rituelles n'en sont 


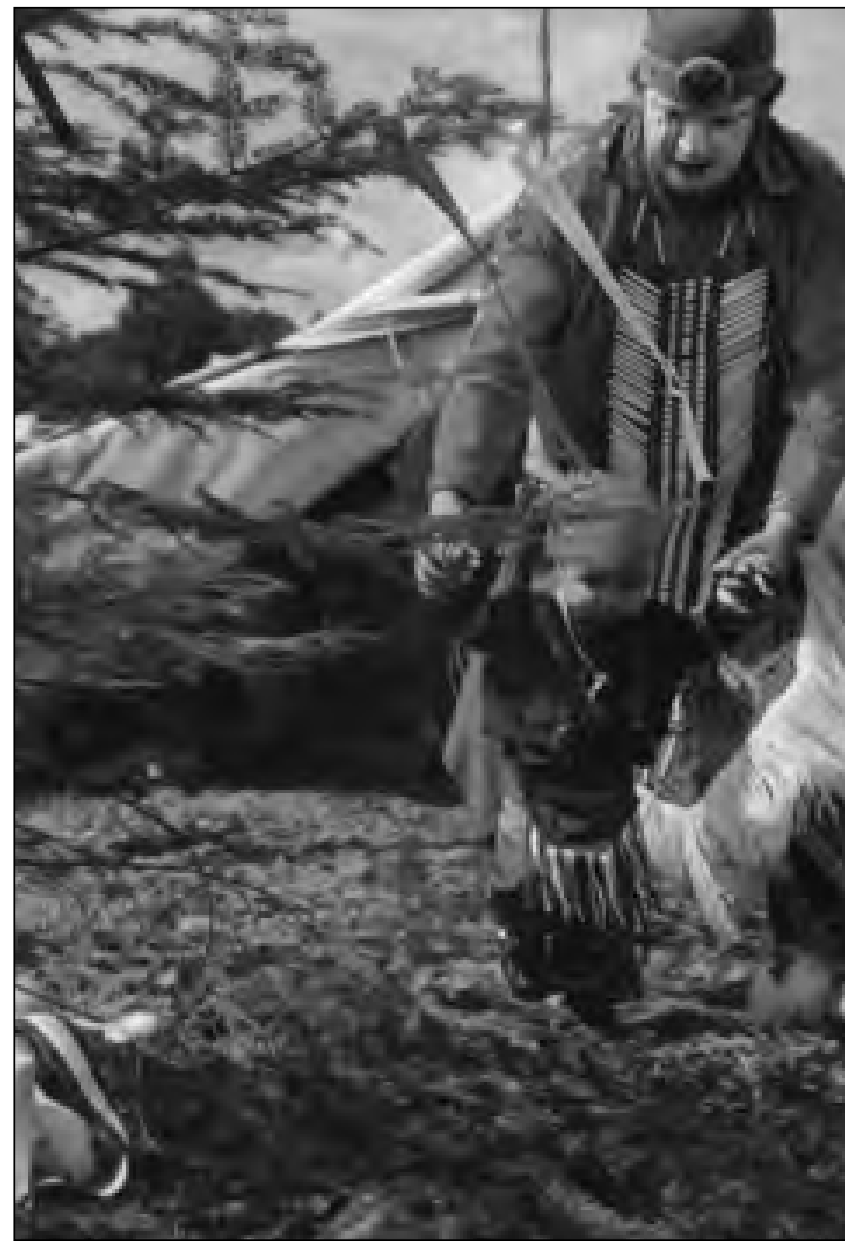

Certaines pratiques rituelles (loge à sudation) et cérémonies (du nouveau-né, des premiers pas) sont organisées en parallèle du powwow de Wemotaci. Ici, une cérémonie des premiers pas lors de l'édition 2004

(Photo de Laurent Jérôme)

qu'un exemple. Elles sont pourtant centrales dans l'évolution de la musique de tewehikan et dans les expériences contemporaines des drummers.

3. Dans la relation avec la société non-autochtone: les références à la mémoire collective atikamekw ainsi que les échanges entre groupes voisins contribuent aux processus actuels d'affirmation d'une identité distincte. Au contraire d'une fermeture à l'Autre et d'un repli sur soi, ce groupe de drummers démontre comment il est possible de s'investir dans une démarche d'affirmation identitaire tout en puisant énergie et inspiration dans la société non autochtone (médias, commercialisation et possibilités économiques, échanges interculturels).

Dans ce processus de convocation, la pratique de tewehikan se positionne aux côtés d'autres pratiques et initiatives valorisées à Wemotaci depuis le début des années 80 : les rites de la première fois, tels que la cérémonie du nouveau-né (Cawerimawasowin) ou des premiers pas (Orowitahawasowin), les loges à sudation (matotasiwin), l'attribution de prénoms atikamekw (Sikon, pré-printemps; Wapan, l'aube; Mikona, la plume; Atisoko, raconter les histoires), les offrandes de tabac ou encore l'utilisation de la roue médicinale.
Les Atikamekw (et nous parlons ici à un niveau national, soit celui des trois communautés) organisent par ailleurs des journées de réflexion sur différents enjeux : le Premier Sommet des jeunes Atikamekw (juillet 2005), le rassemblement spirituel (août 2004), les conférences des aînés (1999, 2001, 2003), le premier Sommet atikamekw sur le territoire (juillet 2005), sont autant d'initiatives qui dénotent une volonté de valoriser et de transmettre les savoirs relatifs au territoire, à l'éducation, à la transmission par l'expérience, à la protection de la langue..

Cette valorisation se réalise également dans le cadre des semaines culturelles, organisées deux fois par année, au printemps et à l'automne. Elles représentent une occasion supplémentaire de pratiquer certaines activités telles que la chasse, le piégeage, la pêche ou des pratiques rituelles sur les territoires familiaux.

Si ces différentes initiatives participent au processus actuel d'affirmation identitaire atikamekw, elles ne sont pas institutionnalisées dans un mouvement politique de résistance. Elles participent à la valorisation du concept de Nehiro Pimatisiwin, le mode de vie atikamekw, comme vision du monde atikamekw permettant, notamment, aux jeunes générations, mais aussi à l'ensemble des Atikamekw, de valoriser des pratiques et des savoirs ancestraux qui correspondent à une perception atikamekw des réalités contemporaines. Cette valorisation du concept de Nehiro Pimatisiwin a moins une visée politique d'affirmation identitaire face à la société dominante, même si elle y participe, qu'un objectif de guérison, ou pour reprendre le concept alternatif partagé par d'autres groupes, de mieux-être, miromatisiwin en atikamekw (littéralement, une vie meilleure). Dans la pluralité des pratiques et des initiatives du mieux-être communautaire, les initiatives évoquées plus haut agissent comme des outils (voir Tanner 2004; Waldram 1997; R.B., entrevue, juin 2001) qui puisent énergie et inspiration dans le savoir-faire et le savoir-être véhiculés par le Nehiro Pimatisiwin.

C'est d'ailleurs dans le cadre d'une initiative visant le mieuxêtre que cette pratique s'est donnée à voir et à entendre pour la première fois à Wemotaci.

\section{Histoire de MASKOPIRECIC, MÉMOIRE DE TEWEHIKAN}

Le groupe de drummers atikamekw dont il est question dans ce travail s'est formé en 1993, quelques mois après une visite d'un groupe de tambour ojibwa de Rama (Ontario) dans le cadre d'un colloque sur la violence organisé dans la communauté de Wemotaci en août 1993. Linitiative revient à deux frères alors âgés de 15 et 17 ans. La visite de ce groupe ojibwa n'a pas révélé une pratique jusqu'alors inconnue. L'un de ces deux frères l'avait d'ailleurs découverte pour la première fois en Alberta, en 1990, alors qu'il avait 13 ans, lors des jeux autochtones de l'Amérique du Nord. À la suite de ce colloque de l'été 1993 et après un mois de septembre de "réflexion » (D.B., octobre 2004), ces deux frères ont décidé de chanter devant leur grand-père pendant les semaines culturelles du mois d'octobre. Celui-ci leur a donné le nom de maskopirecic (oiseau ours).

II disait que c'était dans un temps où les tambours étaient interdits et brûlés. Dans ce temps là, il y avait un aîné qui ne voulait jamais danser pendant les fêtes et sur la musique du violon. II disait que l'aîné disait à tout le monde qu'il danserait le jour où il entendrait à nouveau le chant de l'oiseau ours. II pensait que cet aîné danserait aujourd'hui s'il avait pu nous entendre. (D.B. rapportant les propos de son grand-père, octobre 2003) 


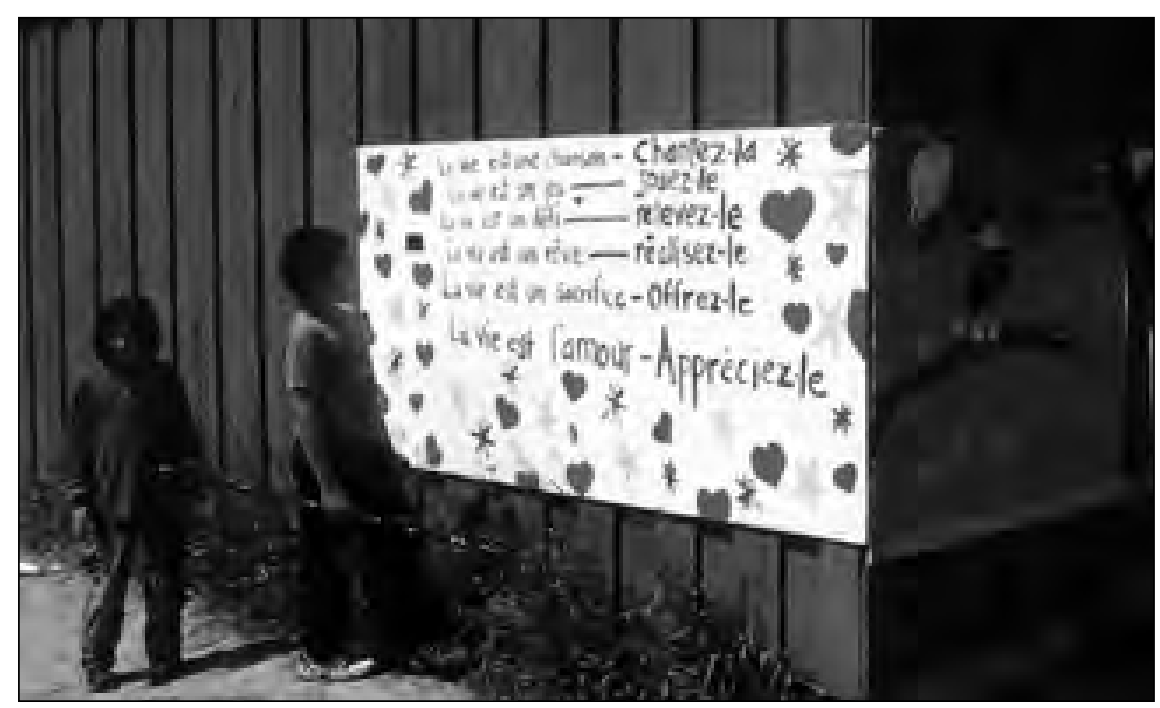

Les initiatives visant à combattre le suicide et la violence dans la communauté se multiplient depuis quelques années. Dans la continuité des programmes de guérison et de mieux-être, la communauté a formulé un énoncé de principes sur la non-violence, et des marches " pour la vie " sont parfois organisées. Ici, un message sur le mur de l'école primaire Seskitin décoré par les élèves après une série de suicides dans le village en juin 2003

(Photo de Laurent Jérôme)

Le contexte historique d'éradication de la pratique du tambour peut ici servir de clé de compréhension à cette histoire. À une époque où les Atikamekw étaient appelés «Têtes de boule », Speck (1977 : 178) avait relevé dans les Relations des Jésuites que

chaque Tête de boule, ou Indiens poissons blancs, avait un tambour de sorcier qui représentait tout pour lui, et le donner à un prêtre était considéré comme un acte de reddition inconditionnelle à la foi. Le Père Buteux s'attachait à mettre au feu les tambours qui lui avaient été remis. (notre trad.)

Les qualificatifs «diabolique », « sorcier », « magique », « superstitieux» abondent dans les Relations pour évoquer la pratique du tambour tout autant que d'autres pratiques telle que la loge à sudation (Paul Lejeune cité dans Laflèche 1973) : "Pour leurs chants superstitieux, ils s'en servent en mille actions » (ibid. : 43).

Ils se servent de ces chants, de ce tambour et de ces bruits ou tintamarres en leurs maladies, je le déclarai assez amplement l'an passé, mais depuis ce temps là, j'ai veu tant faire de sottises, de niaiseries, de badineries, de bruits, de tintamarres à ce malheureux Sorcier pour se pouvoir guérir... (ibid. : 44).

Lors de sa mission de 1651 , le père Buteux, premier missionnaire de la Mauricie, se réjouissait ainsi de constater la réussite de ses actions et la portée de sa parole : "On m'apportait les tambours et autres instruments superstitieux dont les Jongleurs, qui font métier de sortilège, se servent dans le recours qu'ils ont auz démons qu'ils invoquent. » (Buteux 1934 : 31)

Alors que ces archives dépeignent les Atikamekw comme d'irréductibles convertis, détruisant à coup de pied les tambours qui n'avaient pas été remis aux missionnaires (Speck 1977 : 178; Buteux 1934 : 31), l'histoire orale véhicule une autre vision qui peut éclairer la persistance des savoirs liés à la pratique de tewehikan: quelques aînés feignaient cette foi sans faille pour mieux continuer à pratiquer le tambour à l'extérieur de la communauté, dans le bois, à l'abri des missionnaires. Un toponyme atikamekw, tewehikan pawictikw (code toponyme T6.41, voir Dandenault et al. 1989), les rapides du tambour, situé à quelques centaines de mètres en aval de Wemotaci, appuie cette idée d'une perpétuation à l'abri du regard missionnaire. "On dit que c'est là que certains aînés s'échappaient pour jouer du tambour. » (Y.C., juin 2003) Selon certains interlocuteurs, une montagne située à proximité de ces rapides aurait également servi de refuge (nimihotcik matina, la montagne de la danse, A.C., octobre 2005, toponyme non répertorié).

De nombreux travaux ont évoqué les usages du tambour dans différents contextes (De Sales 1991; Armitage 1992; Feit 1994; Lamothe 1984). Ces travaux ont montré comment le tambour est utilisé par les officiants rituels (pour ne pas employer le terme « chaman ») ou par le chasseur pour établir une communication et un dialogue avec l'animal ou d'autres entités non humaines. Il a été perçu comme un attribut personnel renvoyant à un pouvoir et à une fonction particulière dans des actions et expressions rituelles spécifiques agissant comme un outil intermédiaire entre une réalité humaine et une autre non humaine. Brightman (2002 : 104) remarque par exemple que, pour les Cris du Nord, " jouer du tambour et chanter est une façon de prier et de demander aux animaux et aux autres entités non humaines des offrandes de nourriture » (notre trad.).

À travers le Canada, le tambour peut prendre diverses formes. Le quilaut inuit est ainsi bien différent du teueikan innu lui-même bien différent des tambours sur cadre des Athapascans du Nord (Keillor 1985 : 43). Il se décline en fonction de sa pratique qui peut être individuelle (tambour à mains) ou collective, comme pour ces drummers. Plus qu'un simple instrument de musique ou un outil de communication, tewehikan est un concept qui éclaire une cosmologie atikamekw que l'on retrouve également chez d'autres groupes autochtones de la grande famille linguistique algonquine semi-nomade.

Le tambour (dewe'igan en ojibwa, teueikan en innu ou tewehikan en atikamekw) comme voix, cris, chants, battements du cour et de l'âme, battements du cœur de la Terre-Mère, copie du cour, sont autant de tentatives d'interprétations qui m'ont été proposées pour me faire saisir la signification du terme. Selon différents interlocuteurs ${ }^{4}$, Tewehikan se construit avec la racine ote-, otehi désignant le cœur. Otehi, à l'instar de toutes les parties du corps (wiaw, le corps) entre dans la catégorie grammaticale du genre inanimé. À la différence de la racine qui le forme, le terme tewehikan fait partie de la catégorie des noms animés. Il faut alors non seulement tenir compte du suffixe -we ( - wehi), lequel fait référence à l'idée de vibration et de son (voir aussi Speck 1977 : 178) et du suffixe -kan (ou -kana) qui renvoie à cette idée de "servir à, permettre de » mais aussi de conserver et de contenir. Tewehikan serait, selon cette perspective et dans un français approximatif, le gardien des sons du cœur ou ce qui sert à diffuser les sons du cœur. 
Les savoirs sur le tewehikan renvoient à une perception qui s'étend au-delà du simple instrument de musique et de communication. Speck (1977: 176) notait que l'un de ses interlocuteurs percevait le tambour comme une entité vivante, avec une tête et un corps. La pratique individuelle du tambour (rituels, relation intime et personnelle) est à mettre en relation avec la pratique collective développée par les membres de ce groupe en ce qu'elle répond aux mêmes exigences de responsabilité culturelle. Jouer du tewehikan, que ce soit pour l'officiant du rituel ou pour ces drummers, c'est acquérir des savoirs véhiculés par une mémoire collective et transmis à celui qui démontre une certaine ouverture à la recevoir. Ce savoir contient des règles, des significations, des valeurs et des codes de réciprocité (nourrir, protéger, couvrir, fêter le tambour, ne pas le laisser seul en position couchée) qu'il faut valoriser pour être digne de la relation qui s'installe. La position de gardien de tambour vient explicitement rappeler cette question de la responsabilité (Jérôme 2004).

Malgré les diverses tentatives missionnaires d'éradication, le tewehikan s'est exprimé au sein de différentes sphères de la vie communautaire pendant cette recherche. À travers des manifestations publiques (colloques sur la violence, dans les écoles ou les garderies, pour ouvrir des consultations sur les revendications territoriales et politiques...) ou dans le cadre de contextes rituels particuliers (sur la demande des familles, comme ce fut le cas à l'hiver 2003 pour honorer la mémoire d'une personne défunte de la communauté).

La génération de ces drummers, âgés aujourd'hui entre 20 et 35 ans, semble incarner la génération transitoire entre deux références : celle d'une appréhension des dynamiques sociales et culturelles ancrées au sein d'un semi-nomadisme (nomadisme centralisé dans des territoires familiaux) et de ce qu'il implique comme relations, expériences, savoirs et valeurs, et celle qu'engendrent des relations sociales et familiales construites dans le cadre d'une communauté. «On a été les premiers enfants de cette communauté. » (L-P.B., mars 2003)

Ces deux références et les problèmes liés à leur conciliation ne sont pas sans engendrer des tensions intergénérationnelles, notamment en ce qui a trait à la question de l'expérience de transmission. Alors que la plupart des aînés (entre autres) considèrent que les jeunes ne sont pas prêts à recevoir certains savoirs en raison de leur manque d'attention, d'écoute et de respect, les jeunes ne se retrouvent ni en position traditionnelle de recevoir (seules les semaines culturelles représentent un cadre propice à la transmission par l'expérience) ni dans une démarche volontaire de recevoir (peu de jeunes questionnent leurs aînés même si, paradoxalement, la plupart regrettent cette absence de communication).

Le désir d'apprendre le tewehikan exprimé par ces drummers à leur grand-père a entraîné chez ce dernier un désir de transmettre (récit de transmission, attribution d'un nom). Pour beaucoup d'aîné(e)s cependant, le tewehikan est incompatible avec la pratique catholique de leur spiritualité, expliquant la réticence avec laquelle certains ont accueilli cette pratique. La réalité historique faite de diverses tentatives en vue d'éradiquer le tambour n'a donc pas été sans conséquence sur la réception de cette pratique dans la communauté. Selon plusieurs personnes, le powwow annuel de la communauté, organisé depuis bientôt dix ans, a représenté une étape marquante dans cette réception : «Au début, pour le premier powwow [1997], il y avait des aînés qui restaient loin en arrière, ils regardaient, cachés. Mais petit à petit, ils se sont rapprochés et aujourd'hui

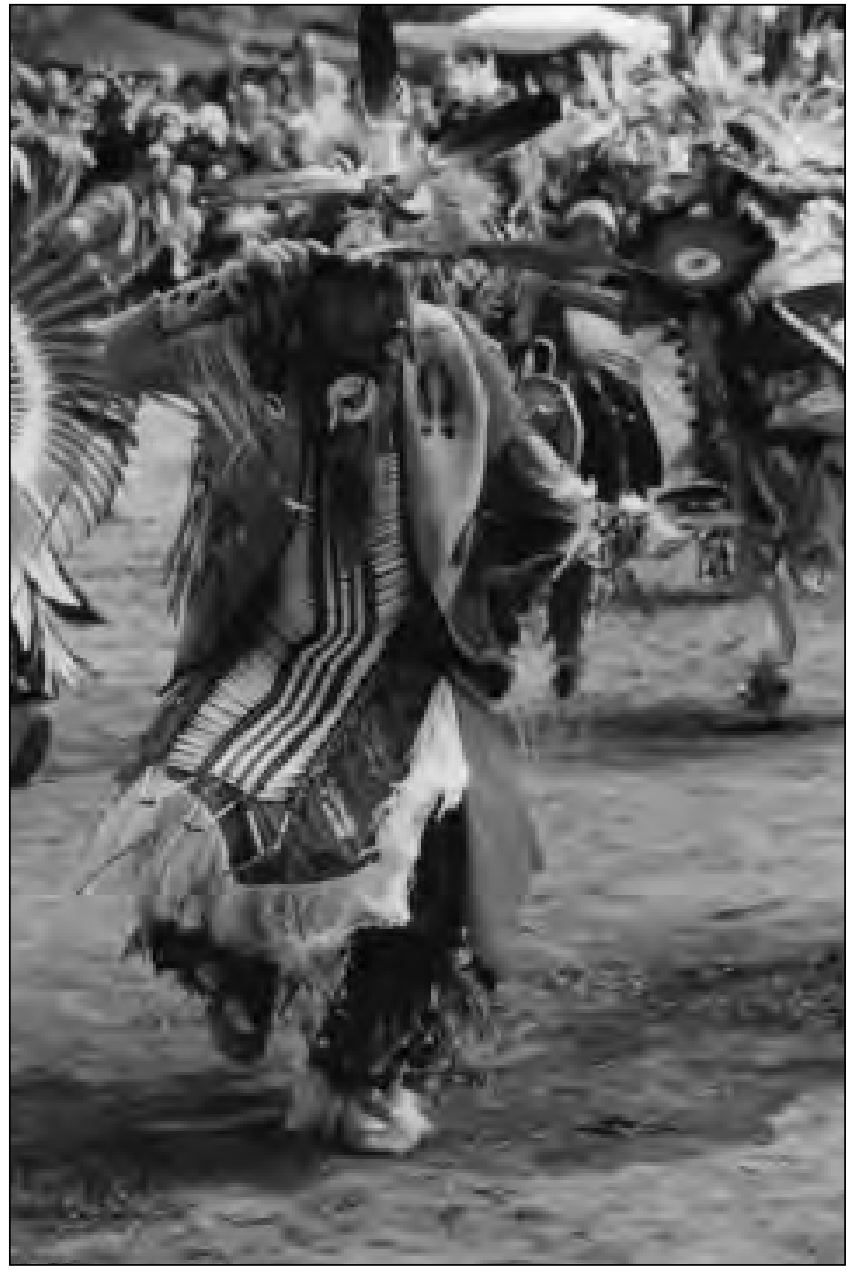

Dominique Boivin (surnommé " picture ") lors d'une danse de la catégorie " homme traditionnel " au powwow annuel de Kahnawake, juillet 2004

(Photo de Laurent Jérôme)

on peut en voir danser avec le monde. » (A.C., août 2003) Il existait un autre type de powwow dans les années 80 dans la communauté. Chaque été, pendant cinq jours, diverses activités était organisées (courses de canot et de portage, concours de bûcheurs...). À la différence de ces rassemblements festifs et ludiques, les powwows contemporains proscrivent toute consommation d'alcool et de drogues. La vente d'alcool est d'ailleurs interdite dans le village, durant toute la fin de semaine.

Si elle fait référence à un ensemble de savoirs locaux persistant dans le temps, la pratique collective de tewehikan ne peut se comprendre sans considérer la relation aux autres nations. C'est d'abord à travers elle que ces jeunes ont découvert le tewehikan. La musique de powwow révèle par ailleurs comment le «savoir-être avec tewehikan» s'exprime sous une autre forme de savoir-faire.

\section{" Powwow Music $"$ : PERformance et ReLAtions AUX AUTRES NATIONS}

Divers travaux ont documenté ces rassemblements panamérindiens venus des États-Unis et des prairies canadiennes, les powwows (opwakancimowin en atikamekw), qui se déroulent principalement entre mai et novembre de chaque année 


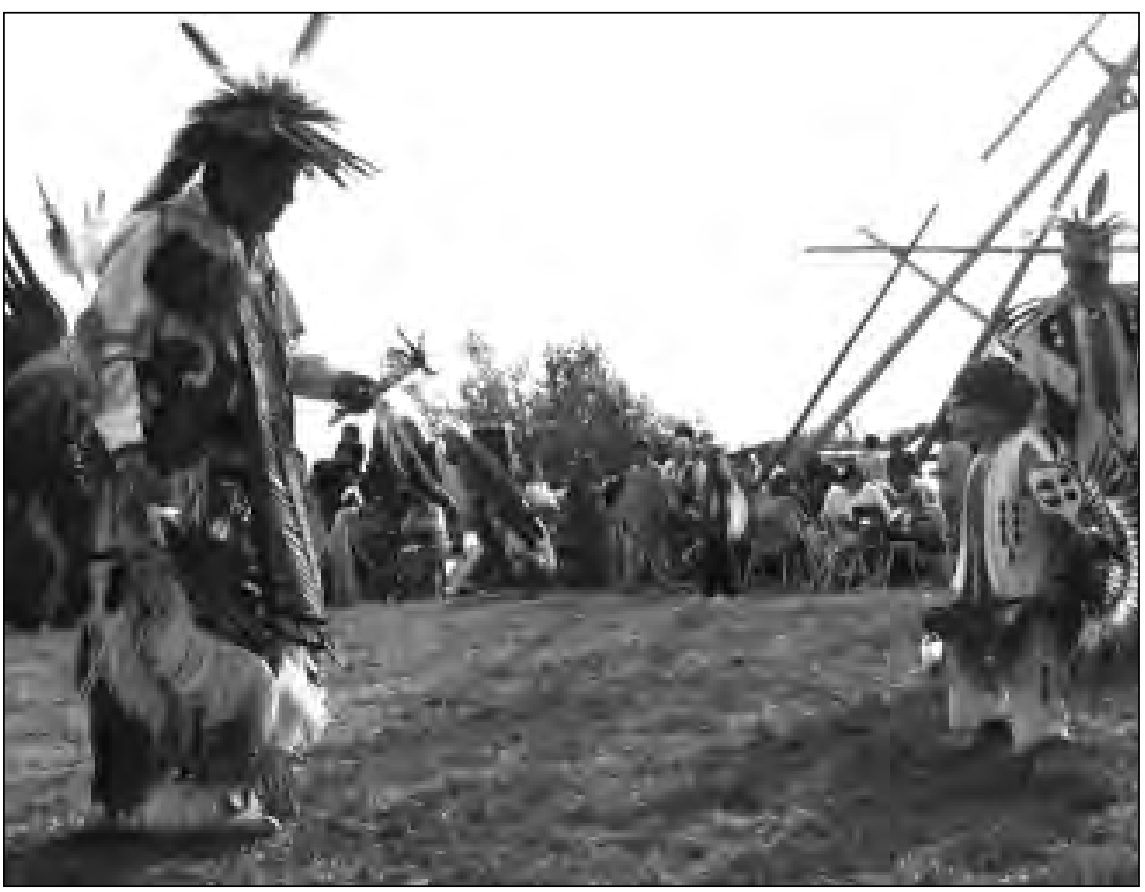

L'apprentissage des danses de powwows peut parfois commencer très tôt. Sikon, 2 ans, face à son grand oncle, Dominique Boivin

(Photo de Laurent Jérôme)

(voir entre autres Blundell 1985; Ellis 2003; Lassiter 1998; Preston 1985). Campisi (1975: 44) considérait le powwow comme un événement qui sert à « refléter ce que les Indiens pensent qu'un Blanc pense ce qu'est un Indien » (notre trad.), autrement dit, le powwow comme représentation de la représentation des Blancs à propos des Indiens. Plus qu'un rassemblement festif ou folklorique, plus que cette image ethnostalgique véhiculée a priori dans les vêtements utilisés (regalia), les peintures faciales de certains danseurs et les chants de tambour, le powwow contemporain est un espace d'échange et d'interaction (famille, convergence intergénérationnelle), de loisir et d'effort physique (artisanat dans la préparation des regalia, danses), de travail et d'économie (prix, kiosques artisanat, nourriture), de relations sociales et de rencontres entre autochtones et non-autochtones. Pour beaucoup, les powwows ont une grande valeur spirituelle et peuvent être aussi une occasion de débattre de différents enjeux de société (voir Buddle 2004).

Dans les powwows, la pratique du tambour est indissociable des chants et des danses (à chaque chant de tambour correspond un type particulier de danse), tout autant que d'autres instruments comme le hochet ou le sifflet d'aigle sacré. Les tambours, les danses et les chants apparaissent comme des éléments étroitement liés aux pratiques rituelles pan-amérindiennes, et la formule contemporaine du powwow ne fait pas exception. Preston (1985) note par exemple que ces pratiques musicales pan-amérindiennes, apparues vers la fin des années soixantedix, tout comme les formes anciennes de chants contenant un message spirituel, s'attachent à exprimer les relations avec des entités non humaines et s'inspirent de concepts véhiculés par des personnages spirituels antérieurs à l'époque missionnaire (kitcemanito, le grand esprit; kokotce, mauvais esprit, le monstre).

Les powwows peuvent être traditionnels (traditional powwow) ou de compétition (contest powwow). Le caractère traditionnel d'un powwow ne définit pas une sorte de respect sans faille pour des traditions ancestrales mais plutôt un type particulier de powwow centré sur les concepts de cohésion sociale et de mieux-être, lesquels s'inspirent des éléments spirituels partagés par un grand nombre de nations autochtones (offrandes régulières de tabac, purification à la sauge, danses de guérison). Il n'y a, dans les powwows traditionnels, que peu de (ou aucune) compétition, tout au plus la succession de danses qu'il est possible d'observer dans tous les powwows: danses intertribales, hommes traditionnels (traditional men), danses à clochettes (jingle dress)..

Des demandes spéciales visant à honorer un défunt ou à aider un malade sont souvent formulées par les familles. Le powwow de compétition n'est pas dénué de ces références spirituelles mais se démarque, comme son nom l'indique, par son caractère compétitif: danseurs et groupes de tambour doivent démontrer leurs qualités, compétences et habiletés respectives. L'évaluation par des juges porte tout autant sur les qualités performatives des danses et de la pratique du tambour que sur le respect de la relation contenue dans les savoirs sur le tambour: chants en accord avec le type de danse demandée, participation active de tous les chanteurs, synchronisation collective dans les battements et dans les voix, propreté autour du tambour..

La relation entre nations s'exprime à travers des échanges avec des chanteurs, des danseurs, des gardiens de tambour et des aînés originaires de diverses communautés du Québec, du Canada et des États-Unis, mais également avec des chanteurs issus des grandes régions urbaines canadiennes (Ottawa, Toronto...). Cette rencontre avec d'autres réalités a permis aux drummers de mesurer leur propre expérience en tant qu'Atikamekw, par exemple à travers les questions de la langue :

C'est en voyageant qu'on s'est rendu compte d'une certaine richesse. On s'est rendu compte qu'on avait encore notre langue, ce qui n'était pas le cas des autres chanteurs. Alors c'était deux surprises: pour nous, se rendre compte de cela, mais aussi pour eux, notamment les groupes des Plaines qui parlent souvent que I'anglais, ils découvraient qu'on parlait entre nous dans notre langue. (A.C., août 2003)

Ces nombreux échanges influencent leur manière de vivre cette pratique puisque c'est au contact des autres groupes de tambour qui parcourent les powwows que les chanteurs de Maskopirecic ont développé leur art. Mais à quoi fait référence la musique de tewehikan dans le cadre de ces powwows?

Il existe deux types de tambours collectifs: les tambours contemporains (dits aussi de powwows) et les tambours sacrés (dits aussi traditionnels). L'organisation musicale met en jeu quatre acteurs musicaux : le tambour, le bras, le bâton (ou drum stick, perçu comme le prolongement du bras) et la voix. Réunis en groupe de trois à douze membres assis en cercle autour du tambour, les chanteurs frappent le tambour par des battements 
soutenus et réguliers, accélérant parfois lentement au cours du chant. Ces battements déterminent le tempo à suivre pour les voix. Mais les premières notes du chant ne commencent pas exactement avec les battements de tambour, tombant plutôt entre deux battements. Ce décalage entre battements et voix représente un effort supplémentaire pour les chanteurs qui se situent dans des processus d'apprentissage. Cela ne veut pas dire que la mélodie s'organise de manière improvisée, la relation aux battements de tambour étant intrinsèque. Cette relation peut cependant être bien cachée et inaudible pour les néophytes. Plus que des mouvements synchronisés, il est possible d'entendre une sorte de lutte incessante entre les chants et le tambour qui détermine la spécificité de cette pratique. La forme standard d'un chant de tambour se caractérise par une succession de strophes avec des répétitions incomplètes au sein de chacune de ces strophes (push up). Cette organisa-

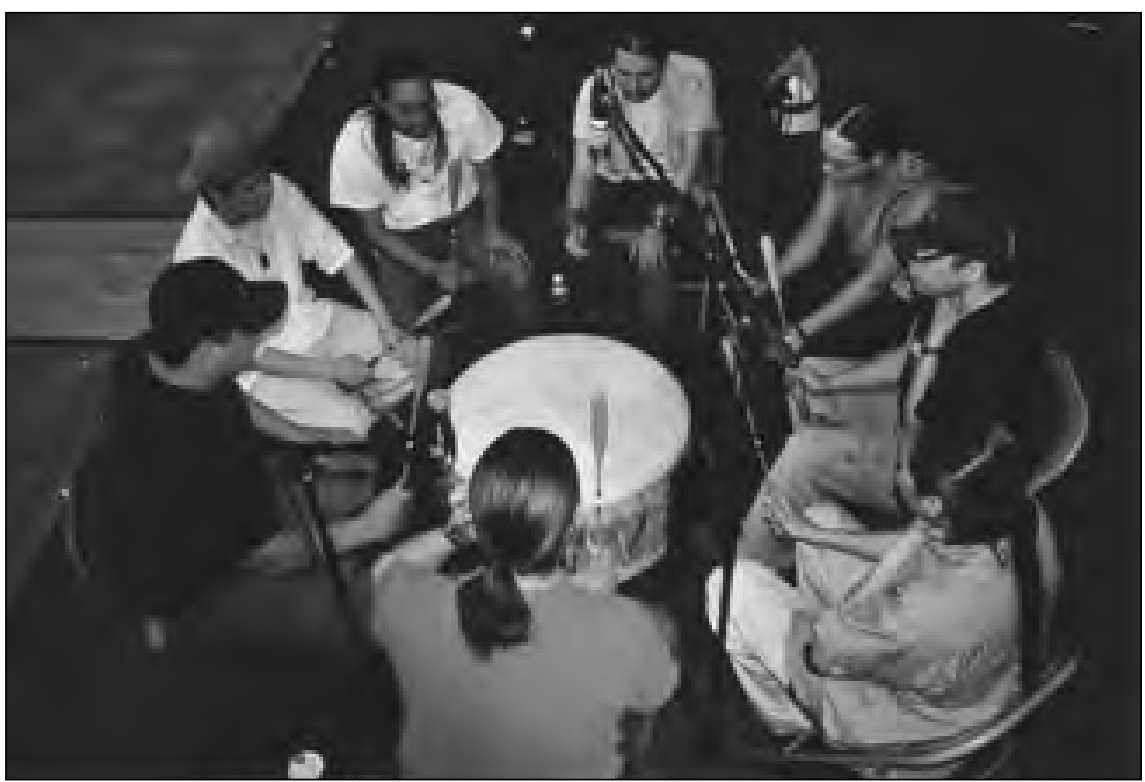

Le groupe Wemotashee Singers au festival Présence autochtone, Montréal, juin 2003 (Photo de Laurent Jérôme)

tion autorise cependant une certaine créativité dans la longueur et le rythme des strophes. Ces découpages mélodiques varient souvent de manière significative d'une chanson à l'autre et peuvent échapper à l'audience. La musique de tewehikan fait appel au corps tout entier, autant pour les chanteurs, les danseurs que les spectateurs. L'efficacité des sons produits par l'acte musical fait appel aux émotions, aux sensations, à une réaction qui dépasse largement le dispositif technique mobilisé et l'organisation générale de la performance musicale. Lénergie et le pouvoir attractif de la musique du tambour sur le public et les danseurs viennent moins de la forme de l'œuvre musicale et des mélodies que du rythme général et du timbre des voix des chanteurs. Cette organisation musicale se décline en différents styles qu'il n'est pas possible d'appréhender dans des catégories closes en raison de leur perpétuelle évolution.

Le style du Sud (Southern Style) se caractérise par des voix lourdes et graves. Les battements de tambour sont souvent moins rapides que les deux autres styles, et chaque strophe est découpée par trois battements d'honneur. Ces battements sont réalisés par un seul joueur, alors que les autres s'arrêtent de battre le tambour ou le battent de manière moins soutenue. Les octaves très graves du style du Sud sont souvent soutenues, à des moments précis pendant le chant, par les voix très aiguës de femmes qui se tiennent autour du tambour, en arrière du cercle formé par les joueurs. Les groupes Northern Style sont les plus nombreux au Canada. Les voix spectaculairement aiguës impressionnent le public et font écho à l'image populaire d'une musique autochtone qui a pris naissance dans l'Ouest et a été diffusée au tournant du siècle par les grandes épopées cinématographiques. Le profond pouvoir des sons rapides et réguliers du tambour et les fortes voix de falsetto donnent à ce style une grande énergie. Les chants comportent tout au plus quelques mots. Le style contemporain (contemporary style) est quant à lui très proche du Northern Style mais il s'en démarque notamment par des phrases plus longues.

Les powwows constituent des espaces de création dans lesquels évoluent les styles musicaux par une recherche constante d'originalité qui permet de se démarquer des autres groupes. Il n'est pas rare que les membres d'un groupe en rejoignent un autre le temps d'un chant. Ce qui est échangé dans ce cas concerne les techniques de voix ou les chants composés par l'autre groupe. Cette familiarisation avec des réalités parfois communes et partagées, d'autres fois originales, a grandement favorisé l'ouverture des drummers à d'autres réalités vécues en dehors de leur communauté et de leur nation. Les rencontres, les voyages, le désir de rompre avec le quotidien de la communauté et une reconnaissance toujours croissante sont autant de raisons qui ont poussé les drummers à continuer à parcourir les powwows. Cette reconnaissance constitue aujourd'hui un objectif de nombreux groupes de tambour et engendre un processus de professionnalisation de la pratique.

\section{De Maskopirecic à Wemotashee Singers}

Après la semaine culturelle de l'automne 1993, le groupe s'est progressivement élargi. La chaudière recouverte par le manteau de pluie a été successivement remplacée par un tronc d'arbre creux, puis par une caisse basse de batterie, avant que le groupe ne puisse réunir assez d'argent pour acquérir un tambour fabriqué par un aîné cri. Les espaces et les contextes d'expression se sont multipliés. Pour sa première prestation, le groupe s'est rendu à Québec dans le cadre d'une manifestation de sensibilisation culturelle. Il a ensuite rejoint Montréal pour jouer dans le cadre d'une conférence sur la jeunesse autochtone. En mai 1995, Maskopirecic participe à son premier powwow organisé à Ottawa. Quelques mois plus tard, c'est au Centre d'amitié autochtone de Montréal qu'ils sont demandés. Ces représentations culturelles, tout autant que les powwows amènent les drummers à voyager aux États-Unis, dans le New Hampshire et dans le Maine. En avril 1999, ils se rendent au powwow organisé par le Saskatchewan Indian Federated College (SIFC), institution académique créée en 1976 et rattachée à l'université de Regina pour favoriser la formation chez les autochtones et valoriser une perception autochtone de l'éducation. Pendant l'été 2000, ils rejoignent la Suisse dans le 


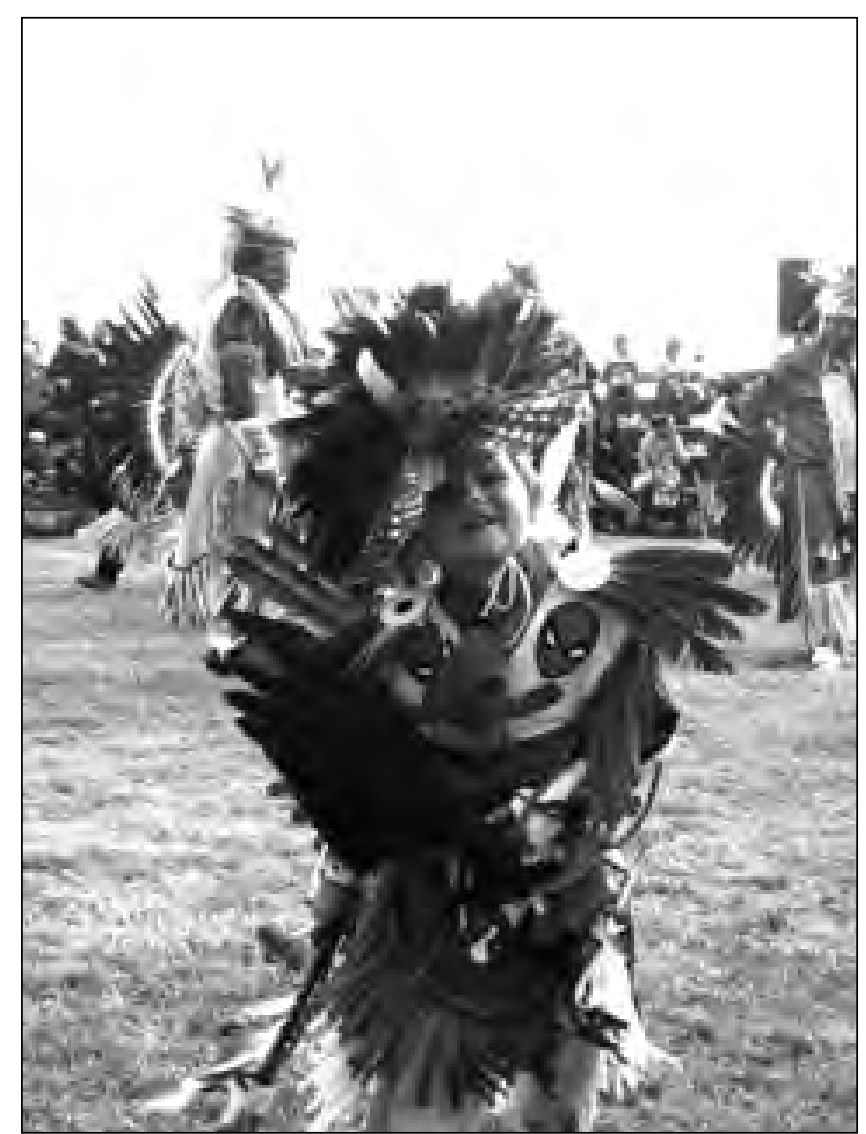

Deux ans après ses premiers pas de danse au powwow de Wemotaci, Sikon a voulu danser au powwow 2005 avec un héros de bande dessinée rendu célèbre par la télévision (Photo de Laurent Jérôme)

cadre d'une manifestation organisée pour célébrer la différence culturelle. Ils y retrouvent des représentants blackfoots, cheyennes et tchouktches. Aujourd'hui composé de onze chanteurs, pères de famille pour la plupart, et malgré des périodes « de baisse de motivation » (D.B., janvier 2004), le groupe a continué à parcourir les powwows au Canada (Fredericton en 2005, Kahnawake, 2003 et 2004, Ottawa, 2003), mais aussi en Europe dans le cadre de rencontres interculturelles (Norvège en 2003). Ils ont été appelés pour des journées ou des semaines de sensibilisation à la culture autochtone (TroisRivières, avril 2003; La Tuque, juin 2003).

Durant ces périodes de voyage à travers le Canada, les Étatsunis et l'Europe, le répertoire du groupe ne se composait que de chants repris à d'autres groupes (Red Bull, Black Lodge, Bear Creek...). En 2004, un premier album, contenant des chants composés en atikamekw, marque une étape significative dans le processus de création. Ce premier album Maskopirecic, éponyme du groupe, fait suite à une reconnaissance toujours grandissante de cette initiative artistique, laquelle a pris et prend de plus en plus la forme de contrats professionnels pour différentes manifestations culturelles: le festival de musique autochtone Innu Nikamu de Mani Utenam (2003, 2004), le gala Mishtapew (Québec, novembre 2003), le gala du régime des bénéfices autochtones (Québec, septembre 2004) ou le festival Présence autochtone (juin 2002, 2003 et 2004) sont autant d'espaces de représentations professionnelles qui sortent du cadre des powwows au sein duquel cette pratique se développe. En raison de cette sollicitation professionnelle toujours grandissante, les chanteurs ont décidé de changer de nom « pour toucher le plus de monde possible. Maskopirecic, c'est difficile à prononcer et à retenir. Wemotashee Singers, c'est plus facile » (P.F., août 2003).

Dans la recherche d'originalité, l'intégration d'un contenu linguistique atikamekw a représenté une démarche significative dans le processus de création. Les différents styles évoqués plus haut (Northern style, Southern style et contemporary style) ne sont plus restitués passivement à travers les différents chants du groupe mais intégrés dans un processus actif de création. Notamment en raison de la difficulté de la performance vocale, les paroles restent peu nombreuses. Elles sont, à l'image du powwow, « une célébration de la vie » (A.C, novembre 2003).

Cette créativité s'exprime également par un investissement des possibilités économiques permises pour toute création artistique. Être invités aux powwows en tant que host drum (tambour invité), remporter la première place des powwows de compétition, rechercher un caractère original pour se démarquer d'autres groupes, trouver les fonds pour enregistrer et vendre des CD représentent les principales préoccupations des groupes de tambour. À la responsabilité culturelle qu'implique cette pratique, s'ajoute une responsabilité professionnelle permise par la fréquence des powwows, les festivals autochtones, les journées interculturelles ou les galas.

Entre mai et novembre, s'engager sur le sentier des powwows (powwow trail) représente pour certains groupes de tambour et danseurs une activité professionnelle qu'ils partagent parfois avec toute leur famille. Certains n'hésitent pas à développer une économie liée à la pratique du tambour, comme peut le démontrer l'exemple du groupe MSR (Morning Star River), dont les membres vivent dans la grande région urbaine de Toronto. Dans un article consacré au groupe MSR dans la revue SAY (Spirit of Aboriginal Youth, printemps 2004) l'un des drummers constate que, lors des powwows, beaucoup de jeunes portent des vêtements de grande marque issus des influences hip hop directement reliées à leur expérience urbaine. Pour lui, la «culture » des powwows et la musique qui en découle représentent une influence similaire à cette influence urbaine hip hop dans les expériences sociales et individuelles et dans les parcours identitaires des plus jeunes générations. En partant de ce constat, le groupe MSR a décidé de se baser sur le pouvoir des powwows pour développer des lignes de vêtements associés au tambour et aux powwows. L'engouement pour la musique de tewehikan peut se mesurer à travers le développement de nombreux groupes au Québec (Cris, Atikamekw et Algonquins notamment) ou le succès annuel du championnat du monde de danses et de chants de Schemitzun (Connecticut) et d'Albuquerque (Nouveau Mexique).

Ces exemples montrent comment les concepts de loisir (goûts musicaux, voyage...) et de travail (professionnalisation de la pratique) se positionnent aujourd'hui aux côtés de règles et de codes sociaux, de savoir-faire et de savoir-être, de significations et de valeurs culturels dans la définition d'une pratique «traditionnelle ${ }^{6} »$. À travers leurs différentes représentations, les drummers sont allés à la rencontre de réalités qui dépassent largement les frontières de la communauté. Leur parcours identitaire s'est réalisé en cours de route, à partir d'une mémoire, d'une histoire et de savoirs atikamekw, et dans les relations avec d'autres groupes et nations autochtones et non autochtones avec lesquels ils ont mesuré différences et convergences. 
Ce parcours exprime en même temps une forme de persistance culturelle qui n'est pas envisagée par les membres du groupe en termes de repli sur soi. Il s'agit plutôt d'ouverture et de partage culturel, conférant au concept de résistance une dimension créative. "La résistance peut-être plus qu'une simple opposition, elle peut-être créative et transformative. » (Ortner 1995 : 191)

\section{Conclusion}

D'un côté il y a les powwows, traditionnels ou de compétitions, il y a les cérémonies dans la communauté, il y a notre rapport au monde, le respect du drum. De l'autre, il y a aussi le business: les colloques, les conférences, les festivals. Mais ces deux côtés-là, c'est notre mode de vie. Notre mode de vie, ce n'est pas seulement le bois. Il faut aussi gagner sa vie. Moi je chasse, je trappe et en même temps j'ai un pick up. Est-ce que je suis moins traditionnel, moins atikamekw pour ça? (A.C, février 2004)

Pour beaucoup à Wemotaci, les drummers sont considérés comme des «ambassadeurs » de leur communauté et de leur nation. Pour d'autres, l'évolution de la pratique dans une voie économique, professionnelle ou de loisir ne correspond plus aux savoirs locaux véhiculés par le terme même de tewehikan. La pratique du tambour révèle comment les membres de ce groupe négocient les changements et les transitions actuels par un processus de convocation de savoirs locaux et de pratiques pan-amérindiennes en les traduisant dans leur propre terme dans un contexte social et culturel en pleine mutation. Ces drummers ne sont pas des récepteurs passifs des transformations sociales et culturelles actuelles. Ils répondent au changement par une variété d'aspirations et d'influences qui se façonnent dans un processus complexe d'appropriation de signes, de symboles et de discours rendus accessibles par un engagement au sein de différents espaces relationnels (générationnels et familiaux, pan-amérindiens et non autochtones) et à travers des échanges sur différents thèmes (préférences et création musicales, créativité et imagination artistique, nouvelles technologies, participation sociale, engagement politique...). Chacun de ces espaces est en ce sens indissociable du contexte dans lequel se construit la pièce musicale, elle-même reflet d'une perception identitaire qui s'est construite à la mesure du processus de création. Ces espaces ne peuvent être dissociés l'un de l'autre puisqu'ils représentent les lieux de l'expérience contemporaine des drummers. Ceux-ci ne brandissent pas le tewehikan comme l'essence de leur identité atikamekw. Il leur a par contre permis de s'interroger « non pas sur ce qu'ils doivent se rappeler pour être mais sur ce que cela signifie, à l'expérience du passé, d'être ce qu'ils sont maintenant » (Létourneau 2000 : 39).

\section{Notes}

1. Par acteurs musicaux, j'entends l'ensemble des dispositifs mécaniques (positions et interactions des corps, voix) et techniques (instruments) qui sont mobilisés dans laction musicale.

2. Le territoire ancestral atikamekw (Nitaskinan) baigne le territoire de la Haute-Mauricie au centre du Québec. Les Atikamekw, au nombre de bientôt 6000 individus, habitent trois communautés : Wemotaci, Manawan et Opitciwan. Ils font partie de la grande famille linguistique algonquine semi-nomade et sont depuis vingt-cinq ans engagés dans un processus de revendications politique et territoriale avec les gouvernements.

3. Formulation que Lenclud emprunte à Ricœur.

4. Les informations concernant la construction étymologique du terme tewehikan proviennent d'un croisement de différentes sources: de certains drummers et d'autres personnes à
Wemotaci qui s'investissent dans les processus actuels de mise en valeur de pratiques et de savoirs, ainsi que de différents dictionnaires de langues autochtones, innues, ojibwas et algonquines notamment (Barraga 1992; Drapeau 1991; le plus pertinent pour ce terme étant Cuoc 1886: 317 et 399). Les technolinguistes atikamekw formé(e)s à la recherche linguistique et étymologique œuvrant à la préservation de la culture et de la langue atikamekw pourraient sans doute apporter certaines précisions quant à cette construction du terme. En espérant que ce futur soit proche, voilà pour le moment ce que ces différentes sources du savoir atikamekw et de la famille linguistique algonquine peuvent en dire.

5. Merci à Wapan pour cette information.

6. Voir l'article de Stern (2000) sur les relations entre les concepts de travail et loisir et les activités de subsistance chez les Inuits. Merci à Louis-Jacques Dorais pour cette référence.

\section{Remerciements}

Je tiens d'abord à remercier l'ensemble des personnes qui soutiennent cette recherche doctorale à Wemotaci et plus particulièrement Mary Coon, Marcel Boivin, Charles Coocoo, Simon Coocoo, les membres du groupe Wemotashee Singers ainsi que le Conseil des Atikamekw de Wemotaci. Ce texte a été enrichi des commentaires de Sylvie Poirier, Richard Lioger, Charles Coocoo, Christian Coocoo, David Boivin, Ayami Chilton et Yvon Chilton. Je les remercie pour leurs lectures attentives. Merci également aux lecteurs anonymes pour leurs commentaires et suggestions, ainsi qu'à Franck Michaud, Marcelle Roy et Éric Chalifoux pour la révision linguistique. Au Québec, cette recherche doctorale a bénéficié du soutien du CRSH (projet de Sylvie Poirier, «Pratiques et stratégies d'affirmation identitaire et culturelle en milieu autochtone postcolonial. Une analyse comparative d'exemples canadiens et australiens »). En France, le Centre international des études canadiennes à Paris, le ministère de l'éducation, de la Recherche et de la Technologie et le ministère des Affaires étrangères ont rendu cette recherche possible.

\section{Ouvrages cités}

ARMITAGE, Peter, 1992 : « Religious Ideology Among the Innu of Eastern Quebec and Labrador ». Religiologiques 6 : 63-110.

AUDET, Véronique, 2005: INNU NIKAMU. Expression musicale populaire, affirmation identitaire et guérison sociale en milieu innu contemporain. Mémoire de maîtrise, département d'anthropologie, Université Laval, Québec.

BABADZAN, Alain, 1982: Naissance d'une tradition: changement culturel et syncrétisme religieux aux îles australes (Polynésie française). Orstom, Paris.

_ 1984 : «Inventer des mythes, fabriquer des rites? » Archives européennes de sociologie 25 : 309-318.

_, 1999a : "Culture, coutume, nation : les enjeux d'un débat». Journal de la Société des océanistes 109(2) : 7-12.

- 1999b: "Linvention des traditions et le nationalisme». Journal de la Société des océanistes 109(2) : 13-35.

BARAGA, Frederic, 1992: A Dictionary of the Ojibway Language. Minnesota Historical Society Press, Minnesota.

BEAUDRY, Nicole, 1985 : «Présentation. Chants et tambours. Le pouvoir des sons ». Recherches amérindiennes au Québec 18(4) : 2-4.

—, 1988: «Présentation. Fêtes et musiques ». Recherches amérindiennes au Québec 18(4): 2-4.

—, 1992 : « La composition des chants amérindiens : création ou transmission? » Les Cahiers de l'ARMuQ 14: 1-13.

BLUNDELL, Valda, 1985: «Une approche sémiologique du powwow contemporain canadien ». Recherches amérindiennes au Québec 15(4): 53-66.

BLUM, Stephen, 2004: "Lacte musical: élément d'analyse». L'Homme (171-172) : 231-247. 
BRIGHTMAN, Robert A., 2002 [1973] : Grateful Prey: Rock Cree Human-Animal Relationship. Canadian Plains Research Center, University of Regina, Regina.

BUDDLE, Kathleen, 2004: "Media, Markets and Powwows. Matrices of Aboriginal Cultural Mediation in Canada ». Cultural dynamics 16(1): 29-69.

BUTEUX, Jacques, 1934 : Jacques Buteux, le premier évangélisateur de la région du Saint-Maurice (1634-1652). Les éditions du Bien public, Trois-Rivières.

CAMPISI, Jack, 1975 : «Powwow: A Study of Ethnic Boundary Maintenance ». Man in the Northeast 9:33-46.

CHORON-BAIX, Catherine, 2000 : «Transmettre et perpétuer aujourd'hui ». Ethnologie française 30(3) : 357-360.

CUOQ, Jean André, 1886: Lexique de la langue algonquine. J. Chapleau, Montréal.

DANDENAULT, André et al., 1989 : Aménagement hydro-électrique du Haut Saint Maurice. Toponymes Atikamekw, carte no 5 , editions Inter.

DE SALES, Anne, 1991 : Je suis né de vos jeux de tambours : la religion chamanique des Magar du Nord. Société d'ethnologie, Nanterre.

DRAPEAU, Lynn, 1991 : Dictionnaire montagnais français. Presses de l'Université du Québec, Sillery.

ELLIS, Clyde, 2003: A Dancing People. Powwow Culture on the Southern Plains. University Press of Kansas, Lawrence

FEIT, Harvey A., 1994: "Dreaming of Animals: the Waswanipi Cree Shaking Tent Ceremony in Relation to Environment, Hunting and Missionization », in Irimoto Takashi et Takako Yamada (dir.), Circumpolar Religion and Ecology. An anthropology of the North : 289-316. University of Tokyo Press, Tokyo.

GOODY, Jack, 1977 : "Mémoire et apprentissage dans les sociétés avec ou sans écriture: la transmission du Bagre». L'Homme 17(1) : 29-52.

HARKIN, Michael, 1997: «A Tradition of Invention: Modern Ceremonialism on the Northwest Coast », in Marie Mauzé (dir.), Present is Past. Some Uses of Tradition in Native Societies : 97-111. University Press of America, Oxford.

HOBSBAWM, Eric, et Terence RANGER (dir.), 1983 : The Invention of Tradition. Cambridge University Press, Cambridge.

HOCART, Arthur M., 1927: "Are Savages Custom-Bound?» Man $27: 220-221$

JACKSON, Jean, 1989 : «Is There A Way to Talk about Making Culture without Making Enemies? »Dialectical Anthropology 14: 127-143.

JAMIN, Jean, et Patrick WILLIAMS, 2001 : «Présentation. Jazz anthropologie ». L'Homme 158-159.

JÉRÔME, Laurent, 2004 : Circulation et transmission du savoir sur la relation avec une entité non-humaine. L'expérience d'un groupe de tambour atikamekw de Wemotaci (Haute-Mauricie, Québec). Communication présentée lors du colloque «La Nature des esprits. Humains et non-humains dans les cosmologies autochtones », Hôtel Clarendon, Québec, 29 avril - $1^{\text {er }}$ mai 2004.

KEILLOR, Elaine, 1985: "Les tambours des Athapascans du Nord». Recherches amérindiennes au Québec 15(4): 43-53.

LAFLĖCHE, Guy, 1973 : Le Missionnaire, l'apostat, le sorcier. Presses de l'Université de Montréal, Montréal.

LAMOTHE, Arthur, 1984: Mémoire battante. Les ateliers audiovisuels du Québec, Montréal.

LASSITER, Luke, E., 1998: The Power of Kiowa Songs: A Collaborative Ethnography. University of Arizona Press, Tucson.

LAUGRAND, Frédéric, 2002 : Mourir et renaître. La réception du christianisme par les Inuit de l'Arctique de l'Est canadien. Presses de l'Université Laval, Sainte-Foy.

LENCLUD, Gérard, 1987: « La tradition n'est plus ce qu'elle était ». Terrain 9: 110-123.

_, 1994 : «Qu'est-ce que la tradition? » in Marcel Détienne (dir.), Transcrire les mythologies : 25-44. Albin Michel, Paris.

LÉTOURNEAU, Jocelyn, 2000 : Passer à l'avenir. Histoire, mémoire, identité dans le Québec d'aujourd'hui. Boréal, Montréal.
L'HOMME (revue française d'anthropologie), 2001 : Jazz et anthropologie, 158-159.

-, 2004 : Musique et anthropologie, 171-172.

LINDSTROM, Lamont, 1982 : «Leftamap Kastom: the Political History of Tradition on Tanna (Vanuatu)». Mankind 13(4): 316-329.

LINNEKIN, Jocelyn, 1983 : « Defining Tradition: Variations on the Hawaian Identity ». American Ethnologist 10(2) : 241-252.

LINTON, Ralf, 1943: «Nativistic Movements». American Anthropologist 45(2) : 230-240.

MAUZÉ, Marie, 1997 : «On Concepts of Tradition: An Introduction », in Marie Mauzé (dir.), Present is Past. Some Uses of Tradition in Native Societies : 1-15. University Press of America, Oxford.

NELSON, Diane, 1996 : « Maya Hackers and the Cyberspatialized Nation-State: Modernity, Ethnostalgia, and a Lizard Queen in Guatemala ». Cultural Anthropology 11(3) : 287-308.

ORTNER, Sherry B., 1995: "Resistance and the Problem of Ethnographic Refusal». Comparative Studies in Society and History 37(1) : 173-193.

PICARD-SIOUI, Louis-Karl, 2003: "Rebuilding the Iroquois Confederacy ": discours et stratégies traditionalistes à Kanawake, 1890-1974. Mémoire de maîtrise, département d'anthropologie, Université Laval.

POIRIER, Sylvie, 2001 : " Territories, Identity, and Modernity among the Atikamekw (Haut St-Maurice, Québec) », in Colin H. Scott (dir.), Aboriginal Autonomy and Development in Northern Quebec and Labrador: 98-116. University of British Colombia Press, Vancouver.

_, 2004: «Présentation. La dépolitisation de la culture? Réflexion sur un concept pluriel »,.Anthropologie et Sociétés 28(1): 7-21

POUILLON, Jean, 1975 : «Tradition : transmission ou reconstruction », in Jean Pouillon (dir.), Fétiches sans fétichisme : 155-173, Maspero, Paris.

—, 1997 : «The Ambiguity of Tradition: Begetting the Father », in Marie Mauzé (dir.), Present is Past. Some Uses of Tradition in Native Societies : 17-21. University Press of America, Oxford.

PRESTON, Richard J., 1985 : « Traditions musicales et culturelles chez les Cris de l'Est ». Recherches amérindiennes au Québec 15(4) : 19-28.

REYNIERS, Alain, 2000 : «Musique et affirmation culturelle dans une communauté tsigane de Transylvanie ». Ethnologie française 30(3): 401-408.

ROUGET, Gilbert, 1980 : La Musique et la transe. Esquisse d'une théorie générale des relations de la musique et de la possession. Gallimard, Paris.

SAVISHINSKY, Joël, 1994 : « Rastafari in the Promised Land: the Spread of a Jamaican Socioreligious Movement Among the Youth of West Africa ». African Studies Review 37(3) : 19-50.

SPECK, Franck G., 1977 [1935] : Naskapi. The Savage Hunters of the Labrador Peninsula. University of Oklahoma Press, Norman.

SPIVAK, Gayatri, 1987 : In Other Worlds: Essays in Cultural Politics. Methuen, New York.

STERN, Pamela, 2000 : « Subsistence: Work and Leisure». Études/Inuit/Studies 24(1): 9-24.

TANNER, Adrian, 2004: "The Cosmology of Nature, Cultural Divergence, and the Metaphysics of Community Healing», in J. Clammer, S. Poirier et E. Schwimmer (dir.), Figured Worlds. Ontological Obstacles in Intercultural Relations: 189-222. University of Toronto Press, Toronto, Buffalo et London.

TERRAIN (revue d'ethnologie de l'Europe), 2000 : Danser, 35.

—, 2001: Musique et émotions, 37.

WALDRAM, James, B., 1997: "The Reification of Aboriginal Culture in Canadian Prison Spirituality Programs », in Marie Mauzé (dir.), Present is Past. Some Uses of Tradition in Native Societies : 131-143. University Press of America, Oxford

WALLACE, Anthony F.C, 1956: «Revitalization Movements: Some Theoretical Considerations for their Comparative Study ». American Anthropologist 58 : 264-281. 


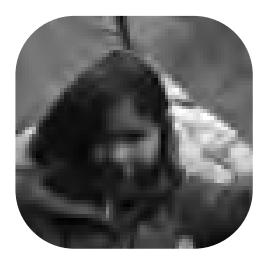

\section{Véronique}

Audet

CIÉRA, Université

Laval

\section{Les chansons et musiques populaires innues}

Contexte, signification et pouvoir dans les expériences

sociales de jeunes Innus'

Pour les jeunes autochtones, l'art - la sculpture, la peinture ou l'art sous toute autre forme - est une façon d'exister. (Florent Vollant, 2002)

U-DELÀ DU SUCCÈS MÉDIATIQUE du groupe folk-country-rock innu Kashtin, 1 sujet, on s'en souviendra, d'un grand engouement à l'été 1989 et au début des années 1990, on peut s'interroger sur la place et la signification de la musique chez les Innus, en particulier chez les jeunes ${ }^{2}$. Lécoute, la pratique et la création de chanson et musique populaires ${ }^{3}$ en langue innue ne sont-elles pas des phénomènes importants dans la plupart des communautés du Moyen Nord? Inspirés des traditions précolombiennes (innues) et chrétiennes, ces chants et musiques empruntent aussi au répertoire des violoneux, ainsi qu'aux courants country, folk, rock, etc. Dans les années 1960-1970, au moment où s'organisait le mouvement de la contre-culture, de nombreux chanteurs folk autochtones (tels Buffy Sainte-Marie, Willy Mitchell, Willy Dunn, Charlie Adams, Tom Jackson, Morley Loon et Philippe McKenzie) se saisirent de la musique comme d'une autre manière d'affirmer leur existence. Le groupe Kashtin ('tornade'), originaire de Mani-Utenam et composé de Florent Vollant et Claude McKenzie, n'a fait, en définitive, que confirmer cette tradition, tout en contribuant grandement à la consolidation, à la vivacité et à la reconnaissance de l'expression musicale autochtone, notamment à l'échelle nord-américaine.

Cet article se propose de dégager une meilleure compréhension de ces expressions musicales en tant qu'importantes voix et voies d'affirmation identitaire et de revitalisation sociale et culturelle. Nous verrons que les jeunes Innus sont les principaux porteurs de ce mouvement d'expression. Nous tâcherons ainsi de contextualiser l'émergence d'un mouvement artistique porté par la jeunesse innue depuis la deuxième moitié du $\mathrm{XX}^{\mathrm{e}}$ siècle, ce qui nous permettra de dégager leur portée et leur signification.

\section{QUELQUES DONNÉES SUR LES MUSIQUES POPULAIRES INNUES}

La chanson, le plus souvent accompagnée à la guitare, est le moyen privilégié de l'expression musicale populaire innue. Les groupes se forment autour des chansonniers, avec les instruments de base de la formation rock (quoique d'autres instruments s'y ajoutent aussi à l'occasion). Les Innus ne désignent-ils pas leurs musiques populaires par le terme général innunikamun, ce qui veut dire « chant innu »? Quant au terme tauapekaikan, qui peut se traduire par « musique instrumentale », il renvoie aux musiques et aux instruments d'origine allochtone (le terme tauapekaikan est couramment utilisé pour désigner la guitare). L'usage du teueikan, le tambour, fait l'objet de débat: traditionnellement investi de pouvoirs spirituels, son usage «hors contexte», «folklorique», constitue pour plusieurs un manque de respect de la culture ancestrale. Lorsqu'il est utilisé, son battement et sa résonance interpellent fortement le sentiment identitaire innu et incitent à danser le makusham, la danse traditionnelle. 


\section{Shauit}

Shauit Aster est un chanteur de la nouvelle génération, dans la vingtaine, qui démontre particulièrement bien la capacité des Innus à s'approprier de nouveaux styles musicaux et d'y faire résonner leur langue de façon surprenante, comme l'ont fait ses prédécesseurs. Le cheminement de ce métis innu-québécois est particulièrement intéressant, car c'est en interprétant des chansons innues à la guitare qu'il a appris la langue de ses proches, dans laquelle il compose aujourd'hui. Depuis quelques années, mais surtout avec la production de son album Shapatesh Nuna en 2004, Shauit intègre le reggae, le hip hop et le rap au répertoire de la musique populaire innue. On entend la langue innue se plier au rythme et à la musicalité de ce phénomène musical, très en vogue chez les adolescents innus, comme un peu partout dans le monde. En s'exprimant dans ce style, Shauit inscrit la jeunesse innue dans cette vague mondiale. Son originalité et son talent ont été remarqués au-delà des frontières ethnolinguistiques. Notamment, à l'automne 2004, il a été invité au spectacle télévisé «Belle et Bum ».

Sa chanson Nui kushpen raconte en détail son désir de monter dans le bois et ses motivations telles que vivre la vie de chasseur et fuir l'ennui, les problèmes et la consommation dans la communauté. Elle s'inscrit dans un répertoire de chansons qui renouvellent les thématiques du territoire et de la vie traditionnelle innue. Elles expriment les expériences contemporaines de ceux qui vont dans le bois selon des motifs et des moyens nouveaux, tout en poursuivant un mode d'être au monde proprement innu. Elles racontent alors le train, I'hydravion, la motoneige et les communications par CB (radios émetteurs à ondes courtes) plutôt que les rivières, portages, canots et teueikan, par exemple. La chanson Nui Kushpen parle de l'attrait de la montée à l'intérieur des terres, en s'adressant à ceux dont la vie est aujourd'hui basée dans les communautés. Elle valorise cette expérience, l'exalte et incite à agir afin de la mettre en œuvre, plutôt que de se laisser sombrer dans des problèmes identifiés comme étant liés au cloisonnement dans les "réserves ». Comme elle l'exprime, les jeunes, comme les moins jeunes, sont intéressés à monter dans le bois, que ce soit pour des jours, des semaines ou des mois.

\section{NUI KUSHPEN - JE VEUX MONTER DANS LE BOIS}

Shauit, album Shapatesh Nuna (2004)

Traduction des paroles innues par Sonny Hervieux

Ah-an-an-an-an-an / Je me réveille avant le soleil Je m'habille chaudement pour ne pas avoir froid / Je démarre mon ski-doo pour le réchauffer
Je prépare mon thé à l'avance / Je vais puiser mon eau et déjeuner Avant d'aller tuer le caribou / Je cherche à vivre, comme ces chasseurs

J'aime voir cela / Ne jamais abandonner, à chaque jour je vois

Ces caribous se lever, là dans le bois, là promenons-nous

J'aime aller dans le bois / À chaque fois que j'ai le temps / Je me prépare

Et j'attends de nouveau pour embarquer dans le train À chaque fois que j'embarque, j'ai hâte de débarquer

Et là je me vois là-bas, je vais aimer chasser le caribou / Je vais le faire comme je le pense

C'est comme ça que j'aime / C'est ce que je veux faire aujourd'hui Je me force à aller dans le bois / Afin d'apprendre à mieux chasser C'est tellement beau ici dans la forêt / Je m'attends à ce que...

Pourquoi encore, je suis tanné de tout le temps rester assis chez nous

Pourquoi je chante / Pourquoi je chante

II n'y aura personne / II n'y aura personne

Refrain (bis) :

Déjà là, je trouve quelque chose ici qui me passionne Je veux faire quelque chose afin d'aller souvent dans le bois Ça fait longtemps que je cherche cette vie que je nomme / Yo yo *

Tu ne te soucies de rien quand tu es là-bas / Tu sais ah ?

Tu ne te fatigues pas pour tes comptes / Tu sais ah?

Juste à aller chercher ton bois et ton eau / Tu sais ah ?

Juste à aller chercher quelque chose essentiel à la vie / Tu sais ah ?

De temps à autre je suis tanné de la vie

Je m'ennuie, je veux mourir, je me parle

Shauit! je me dis / Qu'est-ce que tu fais, qu'est-ce qui te tracasse? / Tu es juste là assis

Juste là à boire / Pour rien tu te fais mal, pour rien tu te malmènes Peu de temps tu monterais dans le bois / Ça fait longtemps que tu ne l'as pas vu

Qu'est-ce que tu attends, au lieu tu pourrais aller voir, avant que tu déprimes plus

Et tu te reposerais / Et tu prendrais soin de toi, avant que tu sois plus malheureux

Je te le dis vraiment / Ce n'est pas des mensonges / Ne va pas croire que tu n'aimerais pas ça

Tu me conteras ça / Tu me conteras ça

* Yo peut se comprendre comme iu : « il dit ».
Les musiques innues sont omniprésentes dans la vie des communautés. Les chansons des groupes et chanteurs les plus appréciés font ainsi l'objet de demandes spéciales sur les ondes des radios du réseau de la SOCAM (Société de communication atikamekw-montagnais). Les chanteurs, musiciens et groupes n'ayant pas encore enregistré un premier album se produisent en spectacles dans la sphère familiale ou communautaire, notamment dans les bars locaux. L'CEM (Institut culturel et éducatif montagnais) met à leur disposition certains moyens (limités) de financement afin de faciliter l'acquisition d'instruments de musique et d'encourager la création d'albums.
D'autres organismes et entreprises apportent aussi leur appui au développement de ce monde musical, notamment les radios de la SOCAM, les conseils de bande, les commanditaires locaux, le studio Makusham de Mani-Utenam et certaines écoles des communautés en offrant des cours et en mettant des instruments et des studios à la disposition des jeunes. Plusieurs jeunes font connaître leurs enregistrements «maison » sur Internet, notamment par le biais de Kazaa (logiciel de partage de musique sur Internet), où l'on peut trouver un vaste répertoire innu. Notons également le festival Innu Nikamu ('l'Innu chante') de Mani-Utenam, fondé en 1985 et qui est un creuset 


\section{Rod Pilot}

Aujourd'hui dans fin de la trentaine, Rod Pilot est chanteur depuis son jeune âge. Il est d'une famille où la musique est très présente, où tout le monde chante, compose ou joue de la guitare ou d'autres instruments. Plus que d'autres chanteurs de sa génération, il s'engage politiquement et met en valeur la culture traditionnelle et l'appartenance au territoire innu. Lors du festival Innu Nikamu 2004, il a démontré, comme à son habitude, sa vocation de chanteur engagé (c'est ainsi que l'a défini l'animatrice). II portait en cape symbolique I'ancien drapeau « national » innu, créé par la Naskapi-Montagnais Innu Association*. C'est sa sensibilité envers les questions identitaires, culturelles, politiques, juridiques et territoriales innues qui l'amène à composer sur ces thèmes. C'est aussi en écoutant et en discutant avec des aîné(e)s qu'il acquiert son inspiration, transmettant leurs messages à travers la chanson.

Sa chanson Eka ashuapatetau (Faut pas attendre) est une composition qui met de l'avant les thèmes revendicateurs et politisés propres à Philippe Mckenzie et à cette génération. Eka ashuapatetau vise la sensibilisation des Innus d'abord, mais aussi des Québécois et allochtones en général, à la responsabilité et aux droits des Innus envers le territoire. Elle appelle à la protection du territoire et à la solidarité.

C'est faire un éveil au peuple pour qu'il comprenne que c'est important le territoire, qu'il faut le sauvegarder, le protéger, et pour qu'on ne se laisse pas prendre nos droits. Je crie au peuple que ça serait le temps qu'on prenne nos droits, qu'on fasse attention aux animaux, aux arbres, aux rivières, que ça serait le temps qu'on se rassemble, qu'il ne faut pas attendre, qu'on mette nos forces ensemble. C'est un peu comme ce que fait Richard Desjardins. (Rod Pilot, entrevue, 2004)

C'est le mégaprojet d'aménagement hydroélectrique de Churchill Falls, au tournant du millénaire, qui a inspiré cette chanson que Rod a composée avec la collaboration de son frère Robert Pilot. Avec l'appui des cinq communautés innues qui se sont alliées pour négocier leurs droits et intérêts devant le mégaprojet de dérivation de rivières, Rod Pilot a été à l'origine d'un album politisé présentant la chanson Eka Ashuapatetau et des textes de sensibilisation en langue innue d'auteurs comme la poétesse Rita Mestokosho. Sur la pochette, on peut voir une représentation cartographique du territoire avec une raquette signifiant: " C'est nous autres qui avons marché là » (Rod Pilot, entrevue, 2004). Derrière, on peut voir une vieille photographie de Churchill Falls, avant que la chute retentissante ne soit réduite à un filet d'eau par les travaux d'aménagement hydroélectrique des années 1960. L'album Nitassinan Innu (Notre territoire innu), distribué gratuitement, a été lancé lors du Festival Innu Nikamu 2000.

\section{EKA ASHUAPATETAU - FAUT PAS ATTENDRE}

Rod Pilot (paroles de Robert Pilot), album Nitassinan Innu (2000)

Traduction des paroles innues par Sonny Hervieux

Déjà aujourd'hui, on est nombreux / Ce sera idéal pour se mettre ensemble

Déjà il parle (le gouvernement) de toutes nos terres / Faisons attention à ce qui nous appartient

Notre mère la terre (bis)

Tous les animaux et les poissons / Là où ils étaient, où irons-nous Par où pourrons-nous suivre les pistes de caribou s'ils sont détruits / Les chemins de portage qu'ils utilisaient Nos parents (bis)

N'attendons pas que tout soit désert / N'attendons pas que les lacs s'assèchent

N'attendons pas que les rivières s'assèchent / Si on est plus fort longtemps on va vivre innu

Déjà le gouvernement devrait nous reconnaître

N'allons pas n'importe où / N'attendons pas d'avoir de la misère C'est là qu'on va voir qu'on est fort / C'est là qu'on va voir quelle est notre force Innu (bis)

II a aussi composé une chanson sur la jeunesse innue, Uassat (Les jeunes, Les enfants), endisquée sur son album Nukum. II parle des enfants qui sont mal dans leur peau, souvent issus de familles dysfonctionnelles et laissés à eux-mêmes, et qui traîneront leur malaise et leurs problèmes affectifs et comportementaux jusqu'à l'âge adulte. II dit qu'il a pitié d'eux et qu'il faudrait que leur entourage leur parle afin qu'ils soient forts et qu'ils aillent loin.

* La Naskapi-Montagnais Innu Association, fondée au milieu des années 1970 et récemment renommée Innu Nation, avait originellement comme idéologie politique celle de rassembler tous les Innus en une seule nation souveraine, reconnue par le Canada et la communauté internationale. Des démarches furent menées par les politiciens innus du Labrador auprès de la Commission des droits de l'Homme à Genève dans les années 1980 (Mailhot 1999 : 59-60). La Innu Nation représente aujourd'hui les Innus du Labrador. de la musique populaire innue. Il fournit une scène pour les artistes autochtones de tout horizon et constitue un banc d'essai pour la relève locale. Il permet le rassemblement des Innus et des représentants d'autres nations autochtones et favorise ainsi l'expression d'un sentiment commun de fierté.

Les jeunes chanteurs et musiciens d'aujourd'hui ont pour la plupart grandi dans la musique, dans un milieu où les membres de la famille et de l'entourage jouent de divers instruments, chantent et improvisent, animant ainsi la vie quotidienne et les réunions festives. Les musiques populaires innues sont un phénomène familial et communautaire qui, au contraire des musiques traditionnelles au teueikan dont la pratique est le privilège de certains aînés, est porté et renouvelé par les jeunes. On peut faire remonter la première génération de chanteurs et musiciens populaires innus aux années 1950 (dont Émile Grégoire, surnommé par la suite Elvis dans son milieu), avec l'appropriation de la musique country et de la guitare. Le succès d'Elvis Presley et la révolution musicale des Beatles et des Rolling Stones ne feront que renforcer l'écoute et la pratique des musiques populaires alors même que les communautés, nouvellement sédentarisées, expérimentent des transformations radicales qui bouleversent la culture et la vie sociale 


\section{Maten}

Le groupe Maten, actif depuis la fin des années 1990, est formé de chanteurs et musiciens de la jeune vingtaine à la jeune trentaine. II a réalisé deux albums et de nombreux spectacles. C'est un groupe très apprécié du jeune public ainsi que de l'ensemble des générations innues, pour les thématiques abordées et l'innovation musicale. Ils sont représentatifs de la jeunesse musicale innue : on se réfère souvent à eux quand I'on parle de la musique des jeunes. Ils bénéficient de l'appui de Florent Vollant et ils ont fait quelques spectacles à l'extérieur du monde innu (comme lors de la commémoration de la Grande Paix de Montréal). Ils ont en outre participé à quelques émissions télévisées et sont impliqués dans leur milieu, notamment à travers la radio communautaire CKAU Kushapetshekan et le studio Makusham à Mani-Utenam. Leurs chansons, comme celles des autres jeunes, parlent des réalités de la vie actuelle, des problèmes d'amour, de rivalité, de consommation, de solitude et de la joie d'être entre ami(e)s. Certains ont souligné dans leurs textes l'importance de la référence à la prière et à Tshemanitu (Dieu, Créateur), à qui ils s'adressent pour demander de l'aide et acquérir de la force pour vivre et accomplir leurs rêves dans un monde qui n'est pas toujours facile.

La chanson Akua tutu (Fais-y attention) est la plus populaire de leur premier album réalisé en 1999. Elle exprime un phénomène important et préoccupant qui touche tout le monde: elle incite à faire attention à la drogue et au vendeur de drogue qui détruit les enfants, les familles et communautés. Elle a été beaucoup entendue à la radio et Kim Fontaine, bassiste et technicien de son du groupe Maten, soutient que cette chanson a pu changer la vision qu'ont certains Innus sur la drogue qu'ils consomment ou qu'ils voient consommer autour d'eux; elle a diffusé, observe-t-il, un angle d'observation critique sur cette expérience (Kim Fontaine, entrevue, 2003).

Samuel Pinette, auteur-compositeur-interprète de cette chanson, chanteur et guitariste dans le groupe Maten, exprime les expériences et les motivations qui l'ont inspiré :
Akua tutu, ça c'est une autre chanson qui a fait réfléchir le monde, je pense. [...] Dans mes chansons, j'essaye beaucoup de parler des problèmes qu'ont les enfants, les jeunes ici dans les réserves. Parce que ça consomme beaucoup ici, pis tu vois, juste au centre-ville*, tu vois le monde, ils sont tous en train de prendre une bière là, de fumer un joint juste à côté des enfants. [...] Pis les jeunes, il ont un gros problème de consommation, je pourrais dire, ici dans les réserves. [...] Je pense que c'est parce qu'ils ont toujours vécu dans ça. On voit notre grand-père, il marche, pis il est tout croche. Comment tu veux dire à ton enfant d'arrêter de consommer quand tu vois un grand-père, il est déjà ben croche là-bas. II le voit, tu ne peux pas cacher ça à tes enfants. [...] Avant, ils construisaient des affaires, des centres d'achat, où les jeunes ne pouvaient rien faire, il fallait que ça soit économique. [...] Ils commencent à comprendre que les jeunes ici, dans les réserves, ils n'ont pas grand chose à faire. Ils ont juste ça, consommer, pour être dans un autre monde, pour rêver, disons. » (Samuel Pinette, entrevue, 2003)

\section{AKUA TUTU - FAIS-Y ATTENTION}

Maten (composition originale de Samuel Pinette), album Akua tutu (1999)

Traduction des paroles innues par Sonny Hervieux

[1] Fais-y attention / Fais-y attention il est déjà proche / Et il va faire du mal à vos enfants

Le diable / Je l'appelle « le diable » / Vous, vous la nommez la drogue**

Fais-y attention / Fais-y attention il est déjà proche / Ne lui ouvrez pas s'il cogne à votre porte

[2] Je sais de quoi il est capable / Car moi je l'ai déjà vu De quoi il est capable, je vais vous le dire / Car moi je l'ai déjà rencontré

$[1]+[2]$

[3] De quoi il est capable, je vais vous le dire, ai ai / Déjà il revient encore pour les faire souffrir

Venez les parents pour penser à nous
(« on s'ennuyait au village », affirme Philippe McKenzie, entrevue, 2003). Mais si les pièces country, les rigodons et les musiques rock de la jeunesse occidentale d'après-guerre ont fait partie des premiers répertoires innus de chansons et de musiques à la guitare, les jeunes Innus, au cours des années 1970, ont peu à peu élaboré leurs propres créations musicales. Ces musiques sont alors devenues des instruments de construction et d'affirmation identitaires ainsi que de communication interculturelle.

Philippe Mckenzie, de Mani-Utenam, fut le pionnier de ce mouvement en composant des chansons fortement identitaires et revendicatrices en langue innue (voir Audet 2005: 85-93 et Keillor 1988). S'étant fait connaître grâce à ses enregistrements (réalisés par le Service québécois du Nord de Radio-Canada au cours de la seconde moitié des années 1970 et reproduits par Boot Records en 1982), il a su révéler à un large public, autochtone et allophone, le potentiel artistique des Innus, leur voix/voie et leurs aspirations. Il raconte qu'il avait été en cela inspiré par son ami, le chanteur folk cri Morley Loon, originaire de Mistissini, au nord de Chibougamau (Philippe Mckenzie, entrevue, 2003). Présent dans le milieu innu de la région de
Sept-îles au cours des années 1970, Morley Loon eut une grande influence sur l'expression musicale innue, en particulier à Mani-Utenam. Il fut l'un des premiers à faire enregistrer ses compositions par le Service du Nord de Radio-Canada. Ses musiques, fortement caractérisées par un rythme battant et des éléments de chant cri, ainsi que ses paroles chantées dans sa langue et exprimant la fragilisation du mode de vie traditionnel (Loon 1981), devinrent très populaires dans le monde autochtone.

Les musiques rock et folk semblaient annoncer une véritable révolution culturelle, sociale et politique (SRC 2004). La jeunesse autochtone des années 1960, 1970 et 1980 n'a pas échappé à cet emballement et s'est identifiée à la jeunesse mondiale en rupture avec un mode de vie dominant et qui prenait conscience de la richesse des différences culturelles. Selon Preston (1985), chez les Cris, c'est avec le rock, à partir des années 1970, que des changements majeurs sont survenus à la fois sur les plans musical, social et culturel

[S]i le fossé entre les générations est plus nettement prononcé dans ce genre de musique et dans les attitudes qu'elle véhicule, et si la 


\section{Maten (suite)}

La chanson Tshe shatshiek ${ }^{u}$ (Vous I'aimerez), figurant sur l'album Tshi metuatshen (2003), chantée en duo par Mathieu McKenzie et Alexandra Cormier-Vollant***, est caractéristique d'un parcours de guérison. Elle possède un grand pouvoir émotif et elle en fait pleurer plusieurs. La prestation d'Alexandra, alors âgée de 16 ans, contribue fortement à l'impact de cette chanson, car les femmes sont rares à prendre parole publiquement par la chanson; leur chant, surtout celui d'une jeune fille talentueuse, est vivement apprécié. L'auteurcompositeur de la chanson, Uapush Vollant, y exprime ses problèmes et son désir de changer pour avoir une vie meilleure ; il souhaite être aimé et chéri par ses proches malgré ses mauvais comportements. La chanson incite au pardon, à l'amour, au soutien et à l'acceptation entre ami(e)s, proches et membres de la communauté, ainsi qu'à la prise en charge de soi-même afin de gagner le chemin d'une vie meilleure. De nombreuses chansons innues exprimant les mêmes thèmes évoquent régulièrement le symbole du chemin de vie personnel qu'on doit suivre et soulignent l'importance de travailler à ce qu'il soit beau, plus lumineux. Le mot employé, meshkanu, signifie le chemin, la voie, le sentier, le parcours emprunté traditionnellement pour circuler dans le territoire, mais dans le langage actuel, il fait souvent référence au cheminement personnel. Par ses qualités émotives et suggestives, cette chanson acquiert un rôle thérapeutique pour plusieurs auditeurs à différents degrés; certains l'ont utilisée pour représenter et comprendre leurs problèmes en session de guérison.

\section{TSHE SHATSHIEK ${ }^{U}$ - VOUS L'AIMEREZ}

Maten (composition originale de Uapush Vollant), album Tshi metuatshen (2003)

Traduction des paroles innues par Sonny Hervieux

[1] Vous l'aimerez / Vous le chérirez / Malgré tout, comment il peut être (agir) dans sa vie

II a eu de la misère lui aussi / II ne se rend pas compte de son mauvais caractère
[2] Tout le monde, on cherchait / À l'aimer, à l'accepter comme il est

[3] II est très difficile, pour lui aussi, son chemin / II ne veut rien savoir

Qu'est-ce que je devrais faire, pour que j'aille bien / Afin que moi aussi, je me sente bien

Je suis en train de remarquer comment je suis / Ok, c'est le temps que je prie

Ok, moi aussi, c'est le temps que je lâche prise / Pour que moi aussi, je me sente bien dans ma peau

Afin de ne jamais désespérer / Afin que je rencontre dans mon chemin une belle vie

[4] Mes amis sont autour de moi / Pour savoir comment ça va [1] [2] [4]

* Le « centre-ville » de Mani-Utenam, c'est le lieu où se rencontrent et se rassemblent plusieurs personnes de la communauté, en particulier les fêtards, autour des deux dépanneurs situés côte à côte, en face de l'église et du poste de radio.

** L'auteur-compositeur joue poétiquement sur l'affinité étymologique entre Kamatshishit (le diable) et Kamatshikauat (la drogue), de matshi (mauvais).

*** Alexandra Cormier-Vollant est une jeune chanteuse innue qui, à 19 ans, est déjà grandement appréciée du public innu, depuis ses premiers enregistrements et spectacles sur scène à l'âge de 16 ans. Elle a d'ailleurs participé aux auditions de Star Académie 2005 pour la région de la Côte-Nord et du lac Saint-Jean et elle a été repêchée pour faire les auditions de Montréal, en reconnaissance de sa voix remarquable, de sa sensibilité et de l'univers innu qu'elle représente et qu'elle exprime en chantant dans sa langue innue. On peut retenir de cette expérience signifiante que le monde innu est totalement inscrit dans le monde médiatique et que Star Académie, quoiqu'on puisse en penser, est devenu un lieu d'expression et de reconnaissance convoité par les jeunes chanteurs autochtones. Pour une discussion de la place des femmes, relativement rares, dans le monde musical innu, se reporter à mon mémoire (Audet $2005: 124-127)$ jeunesse crie trouve cette musique appropriée à sa vie, c'est qu'elle fait partie d'une identité et d'une culture cries transformées de manière significative, qui s'inspire d'une "culture de médias» essentielle à son existence transculturelle, plus que de la continuité avec la culture crie elle-même. (Preston 1985 : 26)

Comme nous l'ont affirmé plusieurs Innus de cette génération, la Beatlesmania a vraiment changé leur perception de la musique et du monde, en transmettant un goût de l'innovation musicale et de la communication internationale à travers la musique. Cependant, tout en participant pleinement à ce mouvement mondial, plusieurs jeunes autochtones se sont approprié ces musiques en les « indigénisant » (voir Appadurai 2001), y trouvant un lieu d'expression de leur spécificité et de leur contribution originale au monde.

La musique est devenue un moyen de dire au monde: "Nous autres aussi, on existe. Nous autres aussi, on a une opinion, on a une culture, on a une langue, on a des rythmes, on a un soul qui est capable d'aller, pis de rejoindre les gens. Nous autres aussi, on a une ouverture, parce qu'on est influencés par la musique country, la musique des Beatles évidemment, des Eagles, toute cette époque-là, c'est très très fort. » (Florent Vollant, entrevue, 2003).

Comme l'a dit Tom Jackson, ils réagissaient et protestaient en chantant et en s'accompagnant à la guitare, plutôt qu'en brandissant un fusil (T. Jackson, dans LeBlanc 1994). Cette époque fut marquée par un ensemble d'événements déterminants pour les Innus, en particulier sur la Moyenne et la Basse-Côte-Nord, comme la sédentarisation dans les "réserves », la fin des pensionnats amérindiens religieux, la reconnaissance des peuples autochtones aux Nations unies, celle-ci favorisant notamment la création d'institutions sociales et politiques leur conférant un pouvoir accru au sein de la société dominante.

À partir de 13, 14, 15 ans, quand je suis sorti du pensionnat, j'ai appris qu'il y avait autre chose que la musique pouvait faire: on pouvait communiquer, puis on pouvait être identifiés. Là j'écoutais les Beatles évidemment, j'écoutais Bob Dylan, j'ai écouté toute cette période-là qui est folk, protest song, tout ce qui amène à une identité puis à une revendication, à tout ce phénomène-là. [...] C'était très fort. J'ai écouté aussi les folk-singers autochtones, puis 
avec Morley Loon, c'était la première fois que j'entendais un folksinger qui chantait en innu, en cri. (Florent Vollant, entrevue, 2003).

Cette constatation est encore plus vraie, s'il se peut, pour la jeunesse contemporaine. Comme le dit un artiste et homme politique de la communauté d'Ekuanitshit, les jeunes prennent la parole en chantant; ils s'affirment peu dans les assemblées, mais ils disent ce qu'ils ont à dire par la chanson (Vincent Napish, entrevue, 2005). Dans un monde où ils ont parfois peine à trouver leur place, la musique semble pour eux un lieu d'expression personnelle, sociale et culturelle qui les lie à leurs proches, leur communauté, la nation innue, le monde autochtone en général, ou toute autre communauté identitaire (par exemple celle du rap). Par cette pratique créatrice et profondément émotive, ils donnent un sens et de l'espoir à leur vie et à celle de leurs communautés. Comme le dit Florent Vollant (2002), ils « entretiennent le feu » pour maintenir la culture et la société innues en vie. Ils font battre le cœur culturel des Innus.

Les jeunes sont aussi de grands auditeurs et spectateurs de leurs pairs. Ils sont ceux qui connaissent le mieux l'univers contemporain des musiques populaires innues et qui comprennent le mieux le sens et la portée des chansons, généralement chantées dans la « jeune » langue innue 4 .

On remarque que, en milieu innu, l'apprentissage d'un instrument se fait très jeune, parfois dès la petite enfance. Les créateurs composent généralement leurs premières chansons dès l'adolescence, ou bien lors de périodes de réflexion et de remise en question. Le cheminement de Rod Pilot, auteurcompositeur-interprète de Mani-Utenam, est typique à cet égard : vers 9 ans, il a commencé à jouer de la guitare; vers 14 ans, il a commencé à composer des chansons sur ses premières amours d'adolescent; puis, plus tard, il a été inspiré par le thème de la perte de sa culture, de ses traditions, de sa langue et de son territoire, et par l'importance des valeurs innues pour vivre en équilibre et atteindre la guérison. Pour lui, le chant innu est directement lié à la question de la culture et de l'identité innues (Rod Pilot, entrevue, 2003). De manière plus générale, contentons-nous de souligner que, si les remises en question de l'adolescence et des premières années de l'âge adulte nourrissent le besoin de s'exprimer, ce qui offre à la musique un terreau fertile, les jeunes autochtones sont d'autant plus marqués par cette crise, cette quête identitaire, que l'ensemble de leur société est aspiré par ce besoin d'affirmation.

La pratique expressive des jeunes apparaît toutefois plus subjective et locale que celle de leurs prédécesseurs. Les jeunes chantent d'abord pour eux-mêmes et pour leur entourage immédiat; ils posent peu de questions ouvertement politiques. Ils expriment surtout leur amour et leurs problèmes d'amour, de vie sociale, ainsi que leur espoir d'un bel avenir. Cette tendance est liée à la fois à leur âge, à leur vécu et à l'époque actuelle, époque grandement imprégnée par des processus de guérison «psychosociale » en milieu autochtone, faisant suite notamment au Rapport de la Commission royale sur les peuples autochtones (CRPA-Canada 1996). Cela étant, entonner une chanson populaire en langue innue n'en demeure pas moins, en soi, un acte identitaire; c'est un acte de renouvellement culturel.

\section{UNE PARTICIPATION BÉNÉFIQUE À LA REVITALISATION DU MONDE INNU}

Lexpression musicale populaire innue est une forme d'affirmation identitaire. Les études ethnomusicologiques nous apprennent en effet que la musique est directement liée à un contexte social, culturel, historique et, partant, à la question de l'identité. Davantage, on a depuis longtemps remarqué que la musique joue un rôle important dans les mouvements d'affirmation identitaire et de revalorisation culturelle (Nettl 1992 : 386). Elle permet non seulement de renforcer la cohésion sociale, mais elle sert également à susciter la reconnaissance de la communauté par les membres de la société globale (ibid. : 384).

Selon Nettl, la musique et la danse apparaissent comme les marqueurs les plus signifiants des frontières culturelles des minorités autochtones en Amérique du Nord : «Quand les Indiens d'Amérique du Nord désirent représenter leur identité ethnique, à leur profit ou à celui des autres, ils le font typiquement par le biais des danses et des chants. » (Nettl 1992 : 394) Cependant, les musiques traditionnelles ou folkloriques sont utilisées dans des contextes nouveaux et, surtout, à des fins nouvelles.

C'est pourquoi, plutôt que de concevoir l'identité de façon statique et chercher ainsi à montrer « comment la musique reflète ou représente les gens qui l'interprètent » (ibid. : 11), nous préférons envisager la musique comme un moyen dynamique. La musique est pour nous un instrument de participation et de transformation du monde (Chernoff 2002: 397-398). Les musiques populaires innues contribuent directement et indirectement à « revitaliser» le monde. Davantage, nous dirons que l'expression musicale innue évoque la notion de guérison symbolique, utilisée principalement pour comprendre les rituels thérapeutiques. La notion de guérison symbolique (symbolic healing, Waldram 1997) renvoie à l'expérience vécue et à la conception du monde. Par exemple, la suggestion, entendue à la fois comme mode de stimulation et de canalisation de l'imagination et du comportement, réfère à cette capacité qu'ont les expressions artistiques de proposer de nouvelles façons de vivre et d'expérimenter son être au monde, de voir le monde et de s'y situer (Waldram 1997 : 74; Ingold 1996, 2004)5. Dans les communautés autochtones du Québec et du Canada, la guérison est considérée comme un processus holistique qui s'apparente au développement communautaire (Krawll 1994 : 1-2). Faite de façon autonome et responsable, elle vise à réparer les « blessures » résultant des rapports de colonisation et de subordination gouvernementale, générateurs de dysfonctionnements au sein des communautés (pauvreté, piètre alimentation, alcoolisme et toxicomanie, violence, négligence, décrochage, criminalité; érosion de la culture, de la langue, du mode de vie, du lien social et du système de valeurs) ${ }^{6}$. Le processus de revitalisation culturelle y est directement lié, car, dans leur quête de guérison, les autochtones valorisent ce qui leur est propre, soit leur héritage culturel (Frisbie 2001 : 492).

C'est ici que nous touchons à la question de la musique populaire contemporaine en langue innue. Quoique fortement acculturée en apparence, celle-ci favorise une " appropriation », une « indigénisation » (Appadurai 2001), qui s'inscrit dans un processus d'affirmation du «mode d'être » innu, ce «mode d'être » étant notamment orienté par l'oralité et un engagement actif dans le monde par l'expérience et l'expression personnelles au sein de la collectivité (Ingold 1996, 2004; Preston 1975; Krims 2000). Par la musique, les Innus peuvent vivre (et faire vivre) des expériences s'inscrivant dans un processus de revitalisation sociale et culturelle (Diamond et al. 1994 : 12, 180). La plupart des chanteurs-musiciens innus (et des Innus en général) soutiennent que la musique leur «fait du bien» (entrevues 
2003-2004). Pour certains, elle est une thérapie et même une raison de vivre :

Ça, ça me garde en vie. Ça nous donne envie de continuer de vivre. Faire de la musique, c'est ce qu'on aime le plus. (Mathieu McKenzie, groupe Maten, dans Meney 2001).

C'est un genre de thérapie, la musique. (Germain Hervieux, groupe Maten, dans Meney 2001)

Bien des Innus ont l'impression qu'être autochtone, c'est être victime: c'est être capable de vivre avec la souffrance. Si certaines chansons alimentent cette identité souffrante, plusieurs autres veulent participer à la prise en charge de l'être innu et de son avenir. Par exemple, Valmor Jourdain, guitariste, chanteur et auteur-compositeur, m'a expliqué qu'il chante pour réveiller le monde, pour faire prendre conscience de ce que chacun vit, en disant la vérité : "C'est comme mettre un miroir devant quelqu'un, en disant : 'Regarde-toi, tu es comme ça, c'est ça que tu vis. Est-ce que tu veux vraiment être de même ou tu veux changer?» (V. Jourdain, entrevue, 2003) Plusieurs chansonniers sont ainsi considérés comme des messagers et dans la langue innue, on dit katipatshimut, celui qui parle, qui raconte des histoires vécues ou des légendes, en livrant des messages.

Les musiques innues sont des moyens de manifester, d'affirmer une présence au monde. Alors que jadis, elles participaient surtout au monde spirituel, aujourd'hui, elles communiquent avec les mondes humains innus et non innus. Résonances humaines dans le monde, comme le souligne Ingold (2004), elles sont un moyen d'expression privilégié de l'affirmation identitaire de l'être innu et de la revitalisation de son « mode d'être au monde ». C'est en ce sens que l'on peut dire que le mouvement de chanson et de musique populaires innues, porté par les jeunes, est une expression culturelle significative (Friedman 2004) et qu'elle représente un des piliers de la vie culturelle et sociale innue contemporaine, ainsi que du sentiment identitaire innu.

\section{Notes}

1. Cet article repose sur mon mémoire de mâ̂trise dirigé par Sylvie Poirier du département d'anthropologie de l'université Laval et subventionné par le CRSH. Intitulé «Innu Nikamu (L'Innu chante). Expression musicale populaire, affirmation identitaire et guérison sociale en milieu innu contemporain », il concerne plus particulièrement les Innus des communautés d'Ekuanitshit et de Uashat mak Mani-Utenam, où j'ai séjourné et réalisé des recherches ethnographiques à l'été et à l'automne 2003 principalement. J'ai participé activement à la vie quotidienne et musicale du milieu, à la fois avec des musicien(ne)s et des auditeurs par exemple en écoutant la musique et en prenant part aux événements, notamment lors du festival de musique autochtone Innu Nikamu qui se tient tous les mois d'août dans la communauté de Mani-Utenam. Des rencontres et discussions avec des Innus de Pessamit, Nutashkuan Unamen Shipu, Matimekush et Sheshatshit ont également alimenté ma recherche. Une vingtaine d'Innus de 15 à 77 ans, principalement des chanteurs et musiciens, m'ont livré leurs paroles, expériences et réflexions en entrevue. Bien d'autres personnes que j'ai côtoyées et que je côtoie encore régulièrement ont partagé informellement avec moi leur compréhension et leur expérience. Je tiens à les remercier toutes, en particulier celles qui sont nommées dans cet article.

2. J'identifierai les jeunes selon un groupe d'âge très large, de 15 à 35 ans environ, soit, globalement, les générations suivant celle des pensionnats (voir les articles qui traitent des dimensions générationnelles dans ce numéro). Tel que je l'entends, les jeunes ne forment pas un groupe identitaire et générationnel homogène, quoique leur expression musicale et leur participation au monde musical innu présentent des traits communs. Ils sont la première, la deuxième, parfois la troisième génération issue de ceux qui sont nés et qui ont grandi dans les communautés nouvellement établies dans les années 1950-1960. Ils sont la première ou la deuxième génération issue de ceux qui sont allés au pensionnat de Sept-îles (Maliotenam) entre 1953 et 1970 environ, ou qui sont dans le groupe d'âge des 45-65 ans; ce sont généralement les petits-enfants, mais aussi les petits petits-enfants et parfois les enfants de ceux qui sont reconnus comme des aînés. En milieu innu, les générations se succèdent très rapidement et s'entrecroisent, notamment avec les familles nombreuses et l'adoption par des personnes d'une génération antérieure à celle des parents biologiques. Les jeunes qui ont participé à ma recherche, dont je livre certains exemples et extraits d'entretien, sont des Innus parlant couramment leur langue et habitant, pour la grande majorité, dans des communautés innues.

3. À l'instar de Pichette (2001), nous utilisons le terme « musique populaire » pour désigner les musiques «dont l'appréciation ne requiert de l'auditeur aucune compétence technique ou théorique » (Beaudry 1988: 4) et qui s'inspirent des courants plus commerciaux (pop). Nous les distinguons ainsi des musiques dites traditionnelles qui dénotent davantage une valeur spirituelle, comme les chants au teueikan, le tambour traditionnel innu.

4. Tout comme la langue innue varie selon les communautés, elle varie selon les générations. Avec le changement d'environnement et de mode de vie, elle se modifie grandement. Les jeunes générations développent un langage courant adapté à la vie dans les communautés; elles contractent les mots, leur ajoutent un sens nouveau, en inventent et en oublient... Les chansons sont un lieu d'expression du langage des jeunes, ainsi qu'un lieu d'innovation et de jeu linguistiques. Certains chanteurs se plaisent aussi à utiliser dans leurs chansons des «vieux mots » peu fréquents dans le langage courant (Kim Fontaine, entrevue, 2003).

5. D’autres techniques associées au processus de guérison symbolique, comme la catharsis et la restructuration sociale, interviennent de façon importante dans l'acte musical populaire innu (voir Waldram 1997 : 73-78; Audet 2005).

6. Les analyses de Samson (2004) et Tanner (2004) abordent la dynamique du rapport entre guérison et identité chez les Innus du Labrador. Ils suggèrent que le choc de la rencontre interculturelle et de la subordination des Innus dans le système social historique et actuel a provoqué et alimenté une "insécurité ontologique » et identitaire dont les conséquences pratiques se révèlent notamment dans les problèmes sociaux, la maladie, le suicide (Clammer et al. 2004: 149).

\section{Ouvrages cités}

APPADURAI, Arjun, 2001 : Après le colonialisme. Les conséquences culturelles de la globalisation. Paris, Payot.

AUDET, Véronique, 2005 : Innu Nikamu. Expression musicale populaire, affirmation identitaire et guérison sociale en milieu innu contemporain. Québec, Université Laval.

BEAUDRY, Nicole, 1988: «Présentation». Recherches amérindiennes au Québec 18(4) : 2-4.

BOUSQUET, Marie-Pierre, 2002: "Quand nous vivions dans le bois ", le changement spatial et sa dimension générationnelle: l'exemple des Algonquins du Canada. Québec et Paris, Université Laval et Université Paris X.

CHERNOFF, John, 2002 : «Ideas of Culture and the Challenge of Music », in J. MacClancy (dir.), Exotic No More: Anthropology on the Front Lines : 377-398. Chicago, The University of Chicago Press.

CLAMMER, John, POIRIER, Sylvie, SCHWIMMER, Eric (dir.), 2004: Figured Worlds: Ontological Obstacles in Intercultural Relations. Toronto, University of Toronto Press. 
COMMISSION ROYALE SUR LES PEUPLES AUTOCHTONESCANADA (CRPA), René DUSSAULT, Georges ERASMUS, 1996: Rapport de la Commission royale sur les peuples autochtones. Ottawa, La Commission.

DIAMOND, Beverley, CRONK, Sam M., VON ROSEN, Franziska, 1994: Visions of Sound: Musical Instruments of First Nations Communities in Northeastern America. Waterloo, Wilfrid Laurier University Press.

FRIEDMAN, Jonathan, 2004: "Culture et politique de la culture. Une dynamique durkheimienne ». Anthropologie et sociétés 28 (1): 23-43.

FRISBIE, Charlotte J., 2001: «American Indian Musical Repatriation », in E. Koskoff (dir.), The Garland Encyclopedia of World Music, United States and Canada, Volume 3: 491-501. New York, Garland Publishing Inc.

INGOLD, Tim, 1996: "Hunting and Gathering as Ways of Perceiving the Environment», in R. Ellen et K. Fukui, Redefining Nature: Ecology, Culture and Domestification: 117-155. Oxford et Washington, Berg.

—, 2004 : «A Circumpolar Night's Dream », in J. Clammer, S. Poirier et E. Schwimmer (dir.), Figured Worlds: Ontological Obstacles in Intercultural Relations : 25-57. Toronto, University of Toronto Press.

KEILLOR, Elaine, 1988: «La naissance d'un genre musical nouveau. Fusion du traditionnel et du 'country' ». Recherches amérindiennes au Québec 18(4): 65-74.

KRAWLL, Marcia B., 1994 : Comprendre le rôle de la guérison dans les collectivités autochtones. Ottawa, Solliciteur général du Canada.

KRIMS, Adam, 2000: Rap Music and the Poetics of Identity. Cambridge, Cambridge University Press.

LEBLANC, Larry, 1994: «Canada's Aboriginal Musicians Seek Mainstream Recognition ». Billboard, 3 septembre.

MAILHOT, José, 1999 : Au pays des Innus. Les Gens de Sheshatshit. Montréal, Recherches amérindiennes au Québec.

NETTL, Bruno, 1992 : « Recent Directions in Ethnomusicology », in H. Myers (dir.), Ethnomusicology: an Introduction: 375-399. New York, W.W. Norton.

PICHETTE, Marie-Hélène, 2001: Musique populaire et identité franco-ontariennes. La Nuit sur l'étang. Sudbury, Prise de parole.

PRESTON, Richard J., 1975 : Cree Narrative: Expressing the Personal Meanings of Events. Ottawa, Musée national de l'Homme.

—, 1985: "Transformations musicales et culturelles chez les Cris de l'Est ». Recherches amérindiennes au Québec 15(4): 19-28.

SAMSON, Colin, 2004 : «We Live This Experience’: Ontological Insecurity and the Colonial Domination of the Innu People of Northern Labrador», in J. Clammer, S. Poirier et E. Schwimmer (dir.), Figured Worlds: Ontological Obstacles in Intercultural Relations: 151-188. Toronto, University of Toronto Press.

SOCIÉTÉ RADIO-CANADA (SRC), 2004 : « Dossier : La génération hallucinée. 1960-1970». <http://www.archives.radio-canada.ca>.

TANNER, Adrian, 2004: "The Cosmology of Nature, Cultural Divergence, and the Metaphysics of Community Healing», in J. Clammer, S. Poirier et E. Schwimmer (dir.), Figured Worlds: Ontological Obstacles in Intercultural Relations : 189-222. Toronto, University of Toronto Press.

VOLLANT, Florent, 2002 : «Entretenir le feu». Rassemblement national sur l'expression artistique autochtone. <http://www.expressions.gc.ca/vollantpaper_f.htm>

WALDRAM, James B., 1997: The Way of the Pipe: Aboriginal Spirituality and Symbolic Healing in Canadian Prisons. Ontario, Broadview Press.

\section{Filmographie}

MENEY, Florence (SRC), 2002 : La fin d'un long silence. Les jeunes autochtones du Québec. Émission Enjeux, Société Radio-Canada.

\section{Musicographie}

KASHTIN, 1991 : Innu. Éditions Groupe concept Musique (Cd).

—, 1994 [1989] : Kashtin. Productions Tshinuau, Éditions Groupe concept Musique (Cd).

—, 1994: Akua tuta. Productions Tshinuau, Éditions Groupe concept Music / Uapukun Music (Cd).

LOON, Morley, 1981 : Northland, my Land / Cette terre du Nord qui est mienne. Boot Records NCB 503 (45 tours).

MATEN, 2001 : Akua tutu. Studio Makusham (Cd).

—, 2003 : Tshi metuatshen. Studio Makusham (Cd).

McKENZIE, Claude, 1996: Innu Town. Groupe concept Musique (Cd).

—, 2004 : Pishimuss. Production L-A Be (Cd).

McKENZIE, Philippe, 1982 : Mishtashipu. Boot Records SQN 103 (45 tours).

—, 2000 : Philippe Mckenzie. Studio Makusham, Éditions Uapan Nuta (Cd).

PILOT, Rod, s.d. : Nukum. Studio TSA (Cd).

PILOT, Rod et Robert PILOT, 2000 : Nitassinan Innu. Studio Makusham, Édition Eka ashuapatetau Nitassinan (Cd).

SHAUIT, 2004 : Shapatesh Nuna. Studio Makusham (Cd). 


\section{Martin Hébert}

Département d'anthropologie, Université Laval, Québec
N SCIENCES SOCIALES, il existe une tra- dition assez longue et complexe dans l'analyse des mobilisations politiques qui lie étroitement la participation politique à l'espoir que peuvent entretenir les acteurs quant à l'amélioration de la société dans son ensemble ou, à tout le moins, de leur sort individuel (Burridge 1969; Desroche 1973; Roy 2000). Cette espérance se manifeste souvent en contexte de crise sociale et a généralement une portée considérable auprès des jeunes adultes.

Il n'existe pas en anthropologie de définition formelle de ce qu'est la « jeunesse », mais les jeunes adultes dont il sera question ici ont en commun de se trouver à une période charnière de leur vie, située entre la dépendance de l'enfance et la prise en charge définitive d'une maisonnée et, par conséquent l'exercice d'une plus grande " agencéité » (agency) ou capacité d'exercer sa volonté dans le monde social. Cette capacité d'agir selon sa propre volonté est marquée et orientée par ce qu'Ernst Bloch, théoricien de l'espérance, a nommé des « imagessouhaits ». Il voit ces dernières comme un type de représentations matérialisées dans divers discours « d'une chose meilleure » (Bloch 1976 : 63) qui est, en quelque sorte, le précurseur de la volonté d'action et de l'action proprement dite (ibid.) chez des acteurs sociaux à part entière.

Les jeunes des communautés autochtones du Mexique entrent dans la vie adulte vers l'âge de 15 ans, c'est-à-dire au moment où ils sont en âge de se marier et d'assumer des positions dans le système de charges civiles et religieuses. Leur statut d'adulte se consolide graduellement à mesure qu'ils se marient, ont des enfants, établissent une maisonnée autonome et prennent des responsabilités de plus en plus grandes au sein du système de charge. Ce passage vers la capacité d'action et la responsabilité tire son sens, en grande partie, du rapport que les jeunes entretiennent avec une panoplie de ces images-souhaits, inscrites dans l'imaginaire social du groupe. Ces images permettent aux acteurs d'envisager non pas uniquement comment les choses sont mais également comment elles devraient être, et ce, même en dépit des possibilités réelles de réaliser cet état idéal.

Dans le présent article, je tenterai d'identifier quelques-unes des figures des espérances politiques et économiques que j'ai pu observer au cours de la dernière décennie chez la génération montante dans deux régions autochtones : la Selva Lacandona de l'État du Chiapas et la région de la Montaña dans la partie sudest de l'État du Guerrero. Ces deux régions ont connu, depuis mon premier séjour sur le terrain en 1996, une situation que l'on pourrait qualifier de « critique ». Face à cette crise qui sévit à la fois sur le front du politique, de l'économique, du religieux et de l'identitaire, ce sont les jeunes qui, prenant nouvellement conscience des contradictions de leur monde social, formulent souvent les espérances les plus explicites et les plus impatientes. Ils espèrent voir une résolution des contradictions qui éclatent au grand jour à travers cette crise. Les jeunes adultes rencontrés ici expriment 
parfois des souhaits complexes espérant la restauration d'une solidarité communautaire traditionnelle idéalisée, doublée de l'accès aux ressources matérielles et aux conforts de la société industrialisée. Mais ils espèrent surtout trouver leur place dans une société, parfois vaguement définie, qui semble leur en laisser peu. Dans les communautés de la Selva Lacandona comme dans les montagnes du Guerrero, cette espérance de trouver sa place s'entend souvent dans son sens le plus littéral, dans la mesure où les territoires exigus des communautés visitées ne suffisent plus pour accommoder les nouvelles familles qui tentent de s'établir.

Malgré un paysage social souvent alarmant, ou peut-être à cause des difficultés auxquelles ils font face, les jeunes autochtones espèrent. Ils espèrent un monde «autre », un monde " mieux », qui chevauche souvent les champs de l'onirique, de l'utopique et du projet politique, c'est-à-dire du rêve, de l'aspiration sociale et du projet d'action concrète. Cependant, comme j'ai pu l'observer dans les communautés dont il sera question ici, il peut arriver que les multiples espoirs, les diverses imagessouhaits entretenues par des jeunes plongés dans une période de grand dynamisme social et de transformations profondes, ne semblent pas toujours cohérentes entre elles. Il existe, après tout, de multiples façons d'envisager un "monde meilleur » pour soi et pour ses proches, particulièrement dans les périodes de grandes perturbations sociales (Brockett 2005 : 159).

Je vais ici tenter de rendre compte d'au moins deux tendances que l'on pourrait qualifier de «lourdes» dans les espoirs exprimés par les jeunes hommes du village tzeltal de Santa Elena (Chiapas) et de la communauté tlapanèque de Barranca Tigre (Guerrero), au Mexique, face aux transformations profondes et souvent houleuses vécues par leurs communautés depuis un peu plus d'une décennie. La raison pour laquelle cette étude se limite aux jeunes hommes est simple. Comme le souligne Brockett en parlant de l'Amérique centrale, l'expression des espoirs et désespoirs sociaux fait partie d'un « agencement complexe de sentiments et de croyances conflictuels qui ont peu de chances d'être partagés, sauf peut-être avec des intimes » (2005: 159). Or, si un chercheur masculin peut développer une intimité suffisante avec les jeunes hommes pour avoir accès à ces discours souvent chargés d'émotion, l'ambiguité d'un tel rapprochement est généralement mal vue lorsqu'il est question de jeunes femmes célibataires ou nouvellement mariées.

Tenant compte de cette limite, qui appelle certainement de futures recherches sur la question, je ferai état, dans un premier temps, de ce que l'on pourrait appeler l'espérance économique, vécue surtout, mais non exclusivement, sur un mode individuel et familial. Deuxièmement, je traiterai de l'espérance politique qui, elle, anticipe des solutions collectives aux problèmes vécus dans les communautés. Finalement, j'aborderai l'intersection de ces figures de l'espérance des jeunes hommes autochtones rencontrés en traitant de l'incidence importante qu'elles peuvent avoir sur leur niveau de participation politique.

\section{Crise ruRale et impact sur les JeUnes}

En conceptualisant l'espérance comme un élément particulièrement mobilisateur en contexte de crise, il faut se demander, d'abord, en quoi les deux régions étudiées peuvent être dites en « crise ». Il peut sembler à première vue que l'utilisation de la notion de crise, lorsque l'on parle du Mexique rural des dernières décennies, va de soi. La plupart des analyses macrostructurelles de l'économie politique de ce pays convergent, en effet, autour du récit généralement accepté selon lequel la crise du capitalisme global qui a affecté les pays industrialisés dans les années soixante-dix s'est étendue, au cours des années quatre-vingt, aux pays en voie de développement comme le Mexique sous la forme d'une crise de la dette externe (Vite Pérez et Rico Martínez 2001 : 15). Dans la foulée de cette crise, une panoplie de réformes structurelles volontaires et imposées aurait favorisé la montée d'un pouvoir « technocratique » d'idéologie néolibérale dans les échelons politiques et institutionnels du pays (Babb 2001; Lindau 1996). La somme de ces perturbations et des politiques qui en ont découlé aurait créé au Mexique une situation de «transition économique » (Williams 2001) qui a plongé plusieurs secteurs de l'économie rurale dans une situation de crise (Martínez Quezada 1995).

Cette lecture de l'histoire économique récente du Mexique, amplement supportée par des données empiriques et par des études détaillées décrivant les facettes de cette «crise » (par exemple Prud'homme 1995), est d'une grande utilité pour comprendre, à grands traits, la situation des campagnes mexicaines aujourd'hui. Cependant, il faut être prudent lorsque l'on tente de cerner l'articulation de ces phénomènes macrostructurels à des observations ethnographiques. Pierre Beaucage a bien illustré comment une crise socioéconomique, détectée par les chercheurs et par divers indicateurs macrosociaux, peut être perçue de manière totalement différente, voire ne pas être perçue du tout, par les habitants des campagnes (Beaucage 1992). Le récit de la «crise » construit par une communauté de chercheurs et d'analystes a donc une grande diversité de pendants locaux, et ce sont ces récits locaux qui constituent les véritables référents par rapport auxquels l'espérance des jeunes autochtones mexicains ruraux se mobilise.

Pour l'informateur de Beaucage, la crise caféière du milieu des années soixante-dix se résumait au fait que « le sucre était très cher en soixante-seize » (ibid. : 67). Étant donné la place si modeste accordée à la « crise » dans l'imaginaire de cet habitant de Cuetzalan, et probablement dans l'imaginaire de plusieurs de ses concitoyens, il a pu paraître, en effet, un peu facile et précipité d'utiliser la « crise » comme facteur explicatif des actions politiques observées dans cette communauté (ibid. : 68). Cet exemple, cependant, sert davantage de mise en garde pour le chercheur que de réfutation de la notion même de crise. Il nous rappelle que, même si une crise macrostructurelle est détectable de manière relativement rigoureuse, la prégnance de cette dernière au niveau local ne doit, elle, jamais être considérée comme allant de soi.

Les espérances politiques et économiques exprimées par les jeunes autochtones de la Selva Lacandona du Chiapas et de la Montaña du Guerrero reflètent, semble-t-il, une telle prégnance de la crise rurale dans les imaginaires locaux. Dans les années quatre-vingt-dix, les événements ayant incité les habitants de la Selva Lacandona et de la Montagne du Guerrero à percevoir leur situation comme critique furent assez marquants. Ils méritent que l'on s'y attarde, ne serait-ce que schématiquement, afin de mettre en contexte les images-souhaits politiques et économiques que nous avons pu observer à Santa Elena et à Barranca Tigre.

\section{Santa Elena, une communauté tzeltale de la Selva lacandona, Chiapas}

Le soulèvement autochtone du Chiapas de 1994 et les répressions militaire et paramilitaire qui l'ont suivi ont été abondamment décrits, médiatisés et étudiés (par exemple Nadal 1994; Harvey1998; Nash 2001). Plusieurs de ces sources cernent en 
détail les contours de cette crise à l'échelle nationale et régionale. Cependant, il est important ici d'établir dans quelle mesure et de quelle manière cette crise se traduisait pour les habitants de la communauté de Santa Elena lors de mon séjour en 1996.

Santa Elena peut, sans trop de doutes, être considérée comme la communauté la plus pauvre de son voisinage. Nommé la pista, ce dernier est composé de six villages tzeltals situés assez profondément dans le groupe de vallées (cañadas) OcosingoAltamirano, dans la région de la Selva Lacandona. Situé en altitude, ce petit village, qui compte environ 600 habitants, est ce que les gens de la région nomment une tierra fría, une terre froide. Le climat y rend pratiquement impossible la culture d'aliments très importants pour la subsistance des autochtones de la région. Il est particulièrement est propice à la culture du haricot noir. Un autre problème lié à l'altitude de Santa Elena est l'approvisionnement en eau. Alors que les communautés voisines bénéficient de systèmes de canalisation tubulaire branchés sur des sources proches, les femmes de Santa Elena doivent faire plusieurs voyages par jour au puits pour y trouver une eau sablonneuse. L'eau, semble-t-il, est l'une des principales préoccupations à Santa Elena. Comme plusieurs villages de sa région, celui-ci n'est pas un site de peuplement tzeltal ancestral. Son établissement remonte à la première moitié du vingtième siècle. Comme c'est le cas des communautés tzeltales voisines, la création de Santa Elena fut le produit de politiques de colonisation de la Selva mises en place par le gouvernement mexicain pour des raisons géostratégiques, et en particulier pour asseoir son droit à l'exploitation des ressources naturelles de la région (Collier et Quarantiello 1994).

Santa Elena, peut-être plus que ses voisines, a été créée sur un site arbitraire. Son seul véritable avantage était l'existence d'une petite aire relativement plane dans le relief accidenté des environs, permettant la construction d'une minuscule piste d'atterrissage qui a joué un rôle clé, pendant un moment, dans le processus de colonisation. Le climat et les sols des terres communales rendent la production de mais difficile. Lapprovisionnement en eau est grandement déficient, et la production caféière, dont l'introduction fut la contribution gouvernementale à la subsistance des villageois, est très peu rémunératrice, étant donné les deux heures de marche en montagne qui sont nécessaires avant d'atteindre le premier chemin carrossable. En somme, la crise de la subsistance fait partie de la réalité de Santa Elena depuis sa création et, par un effet combiné de la croissance démographique et de l'épuisement des sols, elle s'aggrave dans ce village comme dans l'ensemble des cañadas (Toledo 2000: 113).

Cette crise «permanente » s'est trouvée encore plus accentuée par le conflit chiapanèque. En 1993, le village s'est divisé en deux factions à propos de la question de se joindre ou non à l'EZLN. En 1994, c'est-à-dire dès la première année du soulèvement, le gouvernement a coupé la maigre aide qu'il avait jusqu'alors donnée au village. Plus d'agent de santé, plus de ravitaillement en médicaments (à l'exception, si l'on en croit la rumeur locale, de quelques cartons de Valium...), plus d'aide aux producteurs de café et plus d'instituteur pour la petite école du village. En février 1995, les choses empirent encore alors que l'armée mexicaine tente une offensive dans les communautés de la Selva. La piste d'atterrissage de Santa Elena est à nouveau mise à contribution par le gouvernement et, cette fois, ce sont des hélicoptères militaires qui se posent dans le village. La population fuit dans les montagnes avoisinantes, et les maigres possessions de la communauté sont vandalisées et pillées. Entre 1995 et 1998, ce sera la période de la militarisation autour des communautés. Chaque communauté autochtone, ou presque, se trouve flanquée d'un campement militaire. Celui de Santa Elena était à environ 400 mètres du centre du village. De fréquentes incursions de militaires en patrouille suivront, accompagnées d'une série d'abus contre les droits humains comme le vol, l'intimidation, la coupe d'arbres détruisant les caféières, et autres violations qui ont été répertoriées et documentées par des organismes de protection des droits humains chiapanèques.

Entrer dans la vie adulte à Santa Elena en 1994 fut sans doute une expérience mouvementée. Lâge minimal établi par les zapatistes pour devenir membre des milices de l'EZLN et prendre part aux combats était 15 ans. Il existe même au moins un cas d'un jeune homme de douze ans, originaire du voisinage de Santa Elena, ayant pris part aux affrontements de janvier 1994 et ayant péri au combat (Hébert 2000b). Pour ceux qui sont restés ou ont retourné au village, la vie ne fut pas facile non plus. Linsécurité liée à la militarisation régionale puis à la montée subséquente des milices paramilitaires pro-gouvernementales, le manque de ressources productives, de même que le factionnalisme communautaire offraient des perspectives d'avenir peu prometteuses aux jeunes hommes et femmes en voie de fonder une famille et de s'établir en maisonnée autonome.

Pour donner une idée de la fermeture des horizons économiques et des possibilités de pourvoir aux besoins de sa maisonnée, un habitant de Santa Elena, alors dans la jeune vingtaine et père de famille, raconte la vie sous le couvre-feu imposé par l'armée en 1995 et 1996 aux gens de la communauté. À dix-huit heures, tous devaient être rentrés des champs et ne pouvaient plus sortir de leur domicile.

Ce qui est arrivé est que l'armée a donné un horaire aux habitants de la communauté [...] car avant ils étaient ici à surveiller toutes les tâches de notre village et nous disaient quand aller dormir. À six heures [du soir] tu devais dormir, même si tu n'en avais pas envie. Ensuite ils nous dirent que nous devions leur demander la permission, le matin venu, pour aller travailler. [Tu devais demander la permission aux soldats] pour aller où que ce soit. Si ce n'était pas pour aller à la milpa, c'était pour aller chercher du bois ou chercher autre chose. Où que ce soit, tu dois demander la permission et dire où tu vas. [...] si nous revenions des champs à cinq heures ou à six heures, ils ne nous laissaient pas entrer, parce que l'heure était passée. (ma traduction)

Bien sûr, la situation de loi martiale décrite ici n'est pas représentative de la normalité de la vie à Santa Elena, elle relate la période particulièrement difficile d'entre février 1995 et le printemps 1996 qui a précédé mon séjour dans la communauté. Cependant, ce passage évoque une période qui est restée gravée dans les représentations des habitants de la communauté et qui a rendu la crise du Chiapas très réelle et très présente. La "crise» pour les habitants de Santa Elena, c'est d'avoir à fuir dans la montagne pour se sauver de la répression militaire, c'est de vivre sous la loi martiale et de ne pas pouvoir se rendre aux champs quand c'est nécessaire.

Face à cette crise, devenue si prégnante dans les consciences et indélébile dans la mémoire collective, surgit le souhait d'un « ailleurs » meilleur, l'image-souhait d'un village utopique où le soldat et la pauvreté seraient inexistants. Un récit qui circule dans la communauté parle d'un tel lieu où les souffrances économiques et politiques, qui ont peut-être culminé en 1995-1996 mais qui persistent jusqu'à ce jour, trouveront leur terme. Selon un informateur, il existerait près de la frontière du 
Guatemala une terre de 1400 hectares aux mains des zapatistes. Selon lui, un jour prochain les autochtones quitteront la Selva Lacandona où « il n'y a plus rien à espérer ». Les autochtones se dirigeront alors vers ce lieu où il y a une ville « de la grosseur de San Cristobál », nommée Juan Gómez, entièrement peuplée de Tzeltals. Cet homme dit que les préparatifs en vue de ce voyage sont déjà commencés et que l'on essaie d'apprendre certains métiers, comme faire du pain, aux habitants du village.

Cette ville autochtone dotée d'une école, de chemins et d'électricité constitue une image-souhait particulièrement forte dans la mesure où elle combine la représentation idéalisée que se font plusieurs Tzeltals d'une vie communautaire harmonieuse avec l'image de la prospérité matérielle « moderne ».

En un sens, cette vision, exprimée par un aîné de la communauté, marque une transition dans l'imaginaire entre les aspirations passées et les aspirations présentes. Comme il a été noté, la lutte actuelle des zapatistes:

[...] ne se fait pas contre les tyrans ladinos et vers la reconstruction d'une communauté indienne utopique, mais plutôt ce sont maintenant tant les structures communautaires que le contexte économique néolibéral qui maintiennent les peuples autochtones dans la misère, qui sont remis en question. (Zuñiga López et Parra Vázquez 1998 : 291)

Cette remise en question ne signifie pas, comme nous venons de le voir, un abandon des idéaux de coopération et d'unité qui, s'ils ne sont jamais vraiment réalisés, imprègnent néanmoins l'imaginaire social communautaire. Au contraire, l'épure utopique met bien en évidence ces valeurs. De plus, la critique des structures de domination néolibérale ne s'accompagne pas nécessairement, comme le montre l'image-souhait formulée par Marcello, d'un rejet systématique des biens et institutions associés à la société industrielle.

Cette juxtaposition d'espoirs communautaires et technologiques est assez présente dans les discours que j'ai pu recueillir. Juan, un jeune habitant de Santa Elena décrit ce qui constituerait, pour lui, une résolution « idéale » du conflit en cours, une résolution qui s'attaquerait à ses causes profondes, en des termes similaires. L'image qu'il entretient d'un village prospère et en paix en est une où une route pavée serait enfin construite jusqu'au village. Dans cette vision, le réseau électrique, à l'époque installé seulement dans la vallée où passait la route, s'étendrait jusqu'à Santa Elena, perchée dans les montagnes. Cette route et cette électricité ouvriraient la porte, dans sa vision d'une communauté prospère, aux compagnies forestières qui viendraient «sortir le bois » en échange d'emplois et de revenus monétaires qui assureraient la subsistance des familles du village.

Cette vision d'une industrialisation utopique de la communauté détonne par rapport à l'image de la prospérité que peuvent entretenir certains membres plus traditionnalistes de la communauté. Manuel, âgé d'environ 45 ans, illustre ce fossé, qui pourrait être en partie générationnel et en partie idéologique :

Est-ce que c'est écrit dans la Bible que l'important dans la vie est de s'enrichir? Non, l'important est ceci [il indique l'ensemble de la communauté d'un large geste]. Une bonne terre, un bon climat, une bonne milpa et la santé. Voilà ce dont un paysan a besoin, pas d'argent ou de tienda. (ma traduction)

Il existe un certain écart intergénérationnel entre les imagessouhaits qui donnent forme à l'action. Cet écart est marqué par l'opposition entre l'idée de prospérité monétaire et l'image d'une prospérité économique non monétaire inspirée des idéaux paysans colorés d'un certain édénisme chrétien. Il y a également une tension entre la modernité infrastructurelle (écoles, hôpitaux, routes) souhaitée par plusieurs jeunes habitants de Santa Elena et l'idée qu'une «bonne terre » et un «bon climat » suffisent à la prospérité, exprimée par les plus conservateurs de leurs aînés. Mais cette divergence ne devrait pas être lue comme une opposition entre deux ensembles d'aspirations monolithiques et mutuellement exclusifs. Au contraire, il s'agit plutôt de la rencontre de lignes de forces discursives, de grands axes de l'imaginaire, selon lesquels les désirs des individus infiniment plus complexes, et dont il ne serait pas possible de rendre compte ici - s'orientent et se façonnent. Cette complexité inclut en elle-même la possibilité qu'un individu ou le groupe dans son ensemble adhère sans problème à des imagessouhaits potentiellement contradictoires (Femia 1975). Louverture de la communauté au capital extérieur et aux biens matériels de la société de consommation, opérée concurremment à une restauration et une préservation de la solidarité communautaire idéalisée, semblent être deux images-souhaits particulièrement susceptibles d'entrer en contradiction.

La raison pour laquelle ces images sont particulièrement contradictoires dans le cas de Santa Elena est que la situation concrète de la communauté est loin d'indiquer une tendance générale à la réconciliation des idéaux de prospérité et d'unité sociale. En fait, ce qui caractérise certainement Santa Elena par rapport aux communautés voisines est son degré de division interne entre pro-zapatistes et pro-gouvernementaux, lesquels sont aussi appelés les « ARIC oficiales» à cause de leur participation soutenue à la Associación Rural de Interés Colectivo locale, demeurée fidèle au gouvernement mexicain après le soulèvement de 1994. Pamfilo, un zapatiste, décrit cette fracture importante de la manière suivante :

Ici, il y a vingt-trois familles zapatistes. Avant nous étions tous zapatistes au village, mais à un moment donné, le village s'est divisé. Plusieurs, las des zapatistes, sont devenus ARIC et ont décidé de collaborer avec le gouvernement. Ces gens-là disent que les zapatistes ne sont pas plus forts, qu'ils sont trop peu nombreux au village. Le dimanche, nous allons tous à la messe ensemble, car la chapelle est un lieu de rassemblement, mais nous nous assoyons chacun de notre côté : les ARIC d'un côté, les zapatistes de l'autre. Avant, nous mangions tous ensemble le dimanche, mais aujourd'hui nous sommes divisés et c'est chacun de son côté. La même chose pour l'école. Au mois de septembre nous avions soixante à soixante-dix élèves qui commençaient ensemble. Nous organisions alors une grande fiesta pour célébrer l'événement. Mais aujourd'hui, les ARIC ont leur école et nous avons la nôtre, et nous ne célébrons plus la rentrée. (ma traduction)

On constate que cette description du climat social au village est particulièrement marquée par une imagerie de la fracture. Cette fracture, très perceptible dans les interactions - ou évitements - , quotidiennes au village, est associée subjectivement à un manquement par rapport à la norme idéalisée d'une société harmonieuse. Comme le dit Pamfilo, « si tout le monde pouvait revenir du même côté et être zapatiste [...] les choses seraient beaucoup mieux ». Nous avons-là l'esquisse d'une image-souhait de réconciliation communautaire. La crise provoquée par la division profonde que vécurent les communautés de la Selva Lacandona à propos de la participation ou non au soulèvement zapatiste à partir de 1993 (Tello Díaz 1995) se cristallise dans l'imaginaire de cet habitant de Santa Elena 
dans l'image d'une division spatiale à l'église et dans l'abolition des célébrations de la rentrée scolaire. Si Pamfilo parle de réconciliation vague autour de l'idéal zapatiste, l'image-souhait d'unité que nous avons observée chez les jeunes s'exprime dans une résistance à un envahisseur externe, c'est-à-dire le militaire. Un exemple d'une telle image-souhait particulièrement développée m'a été offert par Antonio, un homme de Santa Elena qui venait de franchir le cap de la vingtaine, un matin où il décida de venir me raconter le rêve suivant, qu'il me dit avoir fait la veille :

J'ai rêvé que j'étais au village et que je me rendais chez ma sœur. Rendu chez elle, j'ai vu qu'il y avait là trois puits. Je ne sais pas si l'eau était potable ou non, mais de toute façon cela n'avait pas d'importance car les puits étaient gardés par deux soldats. Lorsque j'ai vu les soldats, je me suis aussitôt caché dans les hautes herbes, mais c'était déjà trop tard. Les soldats m'avaient vu. Aussitôt, je me suis mis à courir en direction de la montagne et les soldats me poursuivirent. Me voyant courir de la sorte, avec deux soldats à mes trousses, les habitants du village prirent aussitôt leurs machettes et leurs haches et, à leur tour, se lancèrent aux trousses des deux soldats qui me poursuivaient.

Rendus dans la montagne, les habitants du village, armés de machettes et de haches encerclèrent les deux soldats et les désarmèrent. Je ne sais pas pourquoi, mais il m'a semblé qu'à eux deux les soldats n'avaient pour toute arme qu'un seul pistolet. Les habitants du village menacèrent les deux soldats et s'emparèrent du pistolet. Mais à ce moment, d'autres soldats, très nombreux et bien armés cette fois, sortirent du bois et mirent les habitants du village en joue.

Nous nous tenions alors tous debout, épaule à épaule, de telle sorte que nous, les habitants du village, ne formions qu'une seule rangée, tous collés les uns aux autres. Le chef des soldats s'avança alors vers nous et nous ordonna de lui rendre le pistolet que nous avions pris aux soldats. Mais, toujours en une seule rangée, nous nous mîmes les mains derrière le dos de telle sorte que nous pûmes alors nous passer le pistolet sans que les soldats ne le voient. Lorsque le pistolet arrivait au bout de la rangée, il rebroussait chemin sans que le chef des soldats ne sache jamais où il se trouve. Chaque fois que le chef demandait à l'un de nous de lui montrer ses mains, il les lui montrait et, à chaque fois, elles étaient vides. (ma traduction)

Ce récit exprime le souhait de voir « les habitants du village », et non seulement les zapatistes, coopérer entre eux. La crise militaire vécue par Santa Elena, et qui apparaît ici sous la figure des soldats cherchant à désarmer la communauté, devient un catalyseur de cette espérance d'unité telle qu'entretenue par un jeune adulte dont l'accession au statut d'acteur social à part entière se fait dans un contexte de conflit armé. Ce contexte, différent de celui dans lequel les aînés de la communauté ont développé leurs propres attentes et espérances politiques, colore, semble-t-il, de manière toute particulière la philosophie politique des jeunes hommes de Santa Elena. Le « soldat » fait pour eux partie du paysage, il est un repoussoir contre lequel se définit leur identité, tout comme les biens et infrastructures de la modernité économique sont eux aussi des points de référence importants contre lesquels ou en faveur desquels se définissent les aspirations de la jeune génération de cette communauté.

Sans proposer une équivalence complète entre les deux cas considérés ici, nous retrouvons des balises semblables - c'est-àdire une présence accrue d'agents extérieurs qui s'immiscent dans la communauté et une attraction/répulsion de plus en plus ressentie face à la société de consommation - par rapport auxquelles les espérances politiques et économiques des jeunes Tlapanèques de la communauté de Barranca Tigre en sont venus à se définir au cours des dernières années.

\section{BARRANCA TIGRE, UNE COMMUNAUTÉ TLAPANĖQUE de La Montaña du Guerrero}

Même si depuis une décennie les communautés de la Selva Lacandona semblent être au cœur des questions autochtones au Mexique, leur position par rapport à l'intervention étatique - exception faite de la militarisation de la dernière décennie a généralement été beaucoup plus marginale que dans d'autres régions du pays. La colonisation de la Selva s'est peut-être faite sous l'impulsion d'incitatifs gouvernementaux (De Vos 1988), mais comme disent les zapatistes, les communautés autochtones de la région ont plutôt vécu dans «l'oubli » jusqu'en 1994. À cet égard, le cas de la Montagne du Guerrero diverge sensiblement de la dynamique chiapanèque. En considérant le dernier siècle d'histoire de cette région, on constate qu'elle a été parfois plongée dans un oubli pratiquement total et parfois plongée en plein cœur de la tourmente des interventions gouvernementales (Hébert 2002b). Faute d'espace, il est impossible de reprendre ici une discussion approfondie de cette histoire en dents de scie. Cependant, pour comprendre les facteurs les plus immédiats qui pèsent sur la jeune génération de la communauté tlapanèque de Barranca Tigre, il est important de remonter au moins à 1989, c'est-à-dire à la cessation des activités de l'institut mexicain du café (INMECAFÉ) dans la région. Cette fermeture a marqué la fin des programmes gouvernementaux d'aide aux caféiculteurs qui avaient jusqu'alors permis aux producteurs de compter sur un revenu relativement sûr, même dans un contexte de grande insécurité et de fluctuations considérables dans le marché caféier international.

La production commerciale du café à Barranca Tigre est une conséquence directe de l'intervention gouvernementale dans la région au début des années quatre-vingt, alors même que la génération qui accède à des postes politiques aujourd'hui voyait le jour. Plusieurs facteurs ont présidé à l'accélération de la transition d'une économie centrée sur l'agriculture de subsistance vers une économie monétarisée basée sur la commercialisation du café. Parmi ces facteurs on peut noter le bon prix du café à l'époque, des pressions internationales exercées sur le Mexique pour le remboursement de sa dette extérieure, de même que le besoin d'intégrer plus étroitement des communautés rurales potentiellement dissidentes dans les structures de l'État. Mais, très certainement, la qualité des sols et du climat de la Montagne pour la caféiculture ne fut pas une grande préoccupation dans l'implantation de ce cultigène à grande échelle. Un employé d'une entreprise caféière décrit la production locale et son potentiel de mise en marché de la manière suivante :

Les communautés certifiées pour l'exportation sont en très petit nombre. Sur le territoire que nous couvrons, seulement trois communautés produisent du café exportable [...]. Ces communautés ont été rigoureusement choisies par la compagnie Nestlé. Ici, à [Barranca Tigre], les gens produisent du café de bonne qualité, mais celui-ci est exclusivement destiné au marché national. Tout d'abord, à 1570 mètres, la communauté est située au-dessus de la barre des 1500 mètres que nous considérons comme la limite pour la production de café d'exportation. Ici, l'altitude fait que les nuits sont froides et l'alternance de celles-ci avec des journées chaudes est malsaine pour le café. (ma traduction) 
N'eut été de l'intervention massive de l'INMECAFÉ dans la région au début des années quatre-vingt sous forme de services agronomiques, distribution de plants et d'intrants, de même que d'achat des récoltes, il est fort probable que la culture à grande échelle du café n'aurait jamais connu le développement qu'elle a eu dans la région. Il n'est donc pas surprenant que, à peine une dizaine d'années après le début de ses interventions importantes dans la région, la disparition de l'INMECAFÉ ait grandement ébranlé les bases de l'économie des communautés autochtones de la Montagne.

Les contrecoups sociaux de cette déstabilisation économique ont été importants sur plusieurs registres. Dès le début des années quatre-vingt-dix, on notait une augmentation importante de la migration des jeunes hors des municipes les plus affectés (Martínez Rescalvo et Obregón Téllez 1991). On note aussi une concordance entre cette crise productive et un factionnalisme accru dans les communautés qui procède de l'émergence d'une flambée d'utopies politiques et religieuses très polarisées. Ces dernières constituent autant «d'options radicales » venant ouvrir de nouveaux « champs d'option et d'action pour des acteurs sociaux » aux prises avec des horizons de plus en plus fermés (Beaucage 2004 : 45). La crise s'est également matérialisée dans une flambée de banditisme, qui a agi comme catalyseur des espoirs et des désespoirs régionaux. Tous ces facteurs, évidemment, vinrent peser sur la génération montante.

Nous pouvons dire que, depuis le début des années quatrevingt-dix, les habitants de la Montagne du Guerrero se sont trouvés à la fois au cour d'une crise économique, judiciaire et politique. Pierre Beaucage (2004 : 38) a déjà souligné la variété d'utopies agraires, politiques, nativistes et religieuses qui ont surgi dans ce contexte. Les jeunes autochtones adhèrent activement à ces discours et à ces systèmes d'espérance. Mais à mesure que plusieurs de ces utopies s'épuisent (à l'exception possible de l'utopie pentecôtiste, qui semble encore en progression) et que la crise économique persiste, les aspirations de la jeune génération deviennent plus vagues et ressemblent davantage aux images-souhaits diffuses dont parlait Bloch, qu'à de véritables projets collectifs.

Deux modes distincts et complémentaires de l'espérance économique ont pu être relevés chez les habitants de Barranca Tigre que j'ai rencontrés. La répartition de ces modes, comme je l'ai noté pour le cas de Santa Elena, possède une composante générationnelle. Il n'est pas question ici de catégoriser ces espérances strictement selon cet axe, mais il faut relever une différence importante dans les manières qu'ont les jeunes adultes et leurs aînés de parler de ces espérances. À première vue, la génération des aînés adopte souvent un ton qui peut paraître fataliste lorsqu'ils parlent de leurs fortunes et infortunes économiques. Un homme dans la quarantaine, interrogé en 1999 à propos de la réaction des habitants de la communauté face à la baisse dramatique du prix du café à cette époque, me répondait de la manière suivante :

Ici les gens travaillent dur. Ils ne savent rien du prix du café ou de ces choses. Ils coupent leur café, puis ils le vendent en espérant que les revenus seront suffisants pour nourrir leur famille pendant toute l'année.

Il faut faire attention, toutefois, de ne pas trop laisser l'interprétation que nous faisons de cette réponse être obnubilée par l'attitude qu'elle suggère lorsqu'elle est extraite de son contexte de sens. La citation précédente est assez représentative du discours que tenaient les doyens des familles durant la chute dramatique du prix du café au tournant du millénaire. Mais il faut bien tenir compte, ici, des multiples actions qui sont sousentendues par cette "espérance » de subsistance économique de la maisonnée, telle que formulée par cet aîné. Comme je l'ai noté ailleurs (Hébert 2002a), « espérer », chez les Tlapanèques, demande de réaliser certains rituels domestiques qui mettront les esprits (ou les saints) dans de bonnes dispositions favorisant la bonne croissance, la bonne qualité et, depuis l'introduction de cultigènes commerciaux, le bon prix, des produits agricoles. Selon cette vision, si les rites agraires propitiatoires ne sont pas accomplis, une maisonnée n'est pas en droit d'espérer une bonne récolte, car elle a brisé le lien de réciprocité qui l'unissait aux forces surnaturelles régissant la nature nourricière.

Même si la formulation de leurs propres espérances économiques est souvent plus explicite chez les jeunes de Barranca Tigre, cette composante implicite d'actions rituelles continue d'avoir une certaine influence chez ces derniers. Même si elle n'occupe maintenant qu'une place aux côtés d'autres actions plus concrètes comme la migration, la recherche de travail salarié et la poursuite des études, la participation aux rituels de prospérité demeure vivante chez les jeunes Tlapanèques de Barranca Tigre. Même chez les jeunes pentecôtistes, ce mode d'espérance persiste sous la forme des nouvelles pratiques religieuses adoptées: "Les croyants ne vont plus demander la pluie aux divinités du sommet de la montagne, mais, ensemble, ils prient Dieu pour qu'il pleuve. » (Beaucage 2004 : 41)

L'espérance économique, chez la jeune génération, ne vient cependant plus uniquement du lien social entretenu avec les forces obscures régissant la nature. Cette espérance semble associée, aujourd'hui, avec la création d'un capital social d'un autre type, de liens avec un « dehors » prospère.

Il n'est pas très surprenant que, aussi loin que l'on puisse documenter, le monde «extérieur» à la communauté ait toujours existé dans la vie économique des Tlapanèques (Galarza 1972 : 40; Dehouve 1990). Mais depuis une génération, les horizons de l'échange économique se sont élargis de beaucoup et, qui plus est, les chemins de la prospérité ont été dramatiquement redessinés pour les jeunes Tlapanèques d'aujourd'hui. Dans le texte qui suit, un jeune père de famille de 22 ans nommé Antonio (nom fictif) relate sa vision des actions qui mèneront vers la prospérité :

Les gens ne croient pas en leur produit. L'an dernier, j'ai apporté quelques kilos de mon café à un Américain qui tient un café à Mexico et celui-ci m'a donné 130 pesos le kilo [de café moulu] et m'a dit qu'il m'en achèterait quelques sacs [à ma prochaine récolte]. Cet homme m'a dit que le café est une nécessité, c'est la première chose que l'on prend en se levant. Ainsi, notre produit est important et il a de la valeur. [...] Notre produit a de la valeur. Spécialement le café de Guerrero; ici tous les produits ont plus de saveur. Cet homme, à Mexico, m'a dit que le café du Guerrero a un parfum de femme tant il sent bon. (ma traduction)

Dans ce discours, la terre promise est représentée par les terrains même de la communauté. Les récits de fondation tlapanèques insistent sur le fait que le territoire occupé par le groupe est un don qui leur a été fait directement par les dieux et les ancêtres (Hébert 2000a), et, par conséquent, l'idée que la terre de Barranca Tigre puisse donner du café de qualité moyenne ne peut, elle-même, être envisagée que très difficilement dans l'imaginaire local. Pour Antonio la pauvreté et le manque «d'avancées » de la communauté ne peuvent donc venir que des intermédiaires, comme les accapareurs privés et 
même la coopérative caféière autochtone locale, qui s'interposent entre la communauté et les consommateurs de son café.

Limage-souhait d'Antonio se rendant à Mexico pour y vendre son café directement à un restaurateur et revenant dans la communauté avec un revenu de cinq à six fois supérieur à ce que la coopérative autochtone locale lui aurait payé est caractéristique des stratégies économiques individuelles, ou de très petits groupes, qui émergent à Barranca Tigre. Pendant quelques années, les paysans et les chercheurs ont eu l'impression, ou l'espoir, que la participation dans une entreprise collective comme la coopérative caféière autochtone locale (nommée LuzMont) pourrait, malgré les difficultés financières de cette entreprise et son faible pouvoir sur les marchés, apporter des gains politiques appréciables pour les autochtones de la Montagne. Tel fut probablement le cas de 1995 à 1999, alors qu'il fut observé que :

Dans le marché mondial du café traditionnel, la concentration de capital extrêmement mobile entre les mains d'entreprises transnationales comme Nestlé a pratiquement empêché toute compétition sérieuse venant des petits producteurs caféiers. Loin de démobiliser LuzMont, cependant, cette impasse économique a poussée l'organisation vers l'avant sur le chemin du renforcement [empowerment] politique face à l'État mexicain. (Johnson 2001 : 28)

Les impératifs économiques ont cependant rapidement rattrapé les paysans. Il est exact que l'organisation de plus de quarante communautés en une coopérative régionale a constitué un grand pas politique pour les habitants de la Montagne. Lespoir de voir un jour émerger un municipe autonome, qui regrouperait les villages autochtones hors de la domination des chefs-lieux métis, a été, et demeure, une image-souhait très mobilisatrice pour les autochtones de la région. Elle a d'ailleurs donné lieu à des avancées concrètes comme la création d'un conseil autochtone régional et d'un système judiciaire autonome très fonctionnel (Martínez Sifuentes 2001). Mais, dans un contexte de crise qui perdure, les jeunes familles doivent composer avec des impératifs plus immédiats de subsistance. Dans plusieurs cas, les espoirs à court terme des ménages sont tournés vers des stratégies familiales et économiques davantage que collectives et politiques.

Cette configuration des espérances des jeunes ménages est devenue particulièrement apparente lorsqu'un soir j'ai reçu une lettre obscure rédigée par Pedro, l'un de mes informateurs clés âgé de 24 ans. Pedro m'invitait à lui rendre visite le lendemain dans le quartier (colonia) de Barranca Tigre où il habitait. La lettre mentionnait seulement qu'il désirait me parler « d'une question importante ». Je me rendis donc chez lui à la première heure et le trouvai, avec son épouse, qui m'attendaient. Pedro commença par me dire que, ces derniers jours, il avait entrepris de calculer les dépenses qui étaient nécessaires pour le faire vivre, lui et sa jeune famille. Le montant, m'a-t-il dit, s'élève à presque cent pesos par jour pour la nourriture, les vêtements et les frais divers. Le quatre litres de maïs (bote) se vendait alors à huit pesos dans la communauté. Les besoins de sa famille en maiis étaient de trois cent botes par année, qui s'élevaient à une facture de 2400 pesos mexicains. De plus, ajouta-t-il, les autres aliments sont devenus très chers. Par exemple, le kilo de tomates se vendait maintenant vingt-cinq ou trente pesos.

« Le café, poursuivit-il, est très bas cette année, à 5,60 pesos le kilo. Ma production totale ne me rapportera pas six mille pesos cette année, car je ne sortirai pas une tonne métrique de café cette année. »C'est pour cette raison que Pedro et sa femme avaient pris la décision de quitter la communauté pour quelque temps afin de tenter leur chance aux États-Unis, ou même au Canada :

Nous voulons partir aussitôt que le café sera vendu, probablement au mois d'avril. Nous allons fermer la maison et allons laisser les enfants chez la sœur de ma femme pour quelques mois. Pendant ce temps, nous allons tenter d'économiser de l'argent pour vivre jusqu'à la prochaine récolte de café. (ma traduction)

Je n'ai pas rencontré de jeunes Tlapanèques qui parlaient d'une émigration définitive hors de la communauté. Par contre, les projets de migration temporaire, comme celui de Pedro et sa femme, abondent et, dans la plupart des cas, sont expliqués de la même manière : le manque de possibilités économiques. «Lailleurs » est souvent idéalisé et les revenus qu'on peut y gagner sont en général exagérés d'autant. Mais l'attrait de cette utopie économique individuelle que constitue el Norte ne cesse de croître, et c'est maintenant par camionnettes pleines que partent pour la frontière les jeunes Tlapanèques.

\section{LES ESPÉRANCES CROISÉES}

À Santa Elena comme à Barranca Tigre, chaque individu possède des aspirations qui lui sont propres, inscrites à l'intérieur de l'univers social et culturel qui lui est propre. Lespérance politique et l'espérance économique, nous rappelle Ernst Bloch par sa formule « Là où l'on trouve l'espérance, on trouve aussi la religion » (1976: 15), sont proches parentes de l'espérance religieuse et, en un sens, peuvent être aussi intangibles que le sentiment religieux. Comme ce dernier, les images-souhaits de prospérité économique et d'augmentation du pouvoir politique s'accentuent souvent en période de crise. Les fenêtres que j'ai ouvertes ici sur ces espérances telles qu'elles sont entretenues aujourd'hui, ou l'ont été dans un passé récent, chez des jeunes de ces deux communautés autochtones du Mexique, ne doivent, en aucune manière, sembler aplanir cette complexité intrinsèque de l'espérance. Leur juxtaposition tend, au contraire, à faire voir l'importance d'affiner mon analyse de l'action sociale et de s'intéresser à des échelles de discours situées en deçà des discours officiels de mouvements politiques autochtones comme le sont le mouvement zapatiste au Chiapas et le mouvement régional autonomiste ayant émergé de la création de LuzMont dans la Montagne du Guerrero (Hébert 2000a; Johnson 2001).

Cette nécessité d'aborder les communautés engagées dans ces mouvements du point de vue ethnographique est particulièrement sentie lorsque l'on tente de comprendre la participation ou la non-participation des jeunes générations. Dans les deux exemples considérés ici, les jeunes autochtones rencontrés ont souvent atteint l'âge adulte dans un contexte où les mobilisations politiques régionales ont émergé, ont grandi et ont atteint une proéminence en matière de revendications autochtones et ce, en grande partie sans eux. En position d'acteurs «juniors », pour l'instant, les jeunes ne portent pas encore la parole officielle du mouvement et sont souvent peu loquaces sur les raisons de leur participation. Un jour, j'eus un entretien, que j'avais jugé peu fructueux à l'époque, avec un jeune Tzeltal de 15 ans, à peine entré dans la milice zapatiste:

- De quoi veux-tu que je parle? me demanda-t-il.

- De n'importe quoi, de la vie au village, des ARIC, de la situation ici...

- Je ne sais pas quoi dire, je ne sais pas comment le dire, mon espagnol n'est pas très bon. 
- Ça ne fait rien, petit à petit nous y parviendrons.

- Je n'ai pas d'opinion, je ne sais pas vraiment ce qui se passe. [...] Oui, je sais ce qui ce passe, mais je ne sais pas comment l'expliquer.

- Et les zapatistes, pourquoi luttent-ils?

- Les mots me manquent, demande [au capitaine de la milice], lui il sait expliquer ces choses-là. [...] Je ne sais pas expliquer ces choses-là, c'est quelque chose que je ressens dans mon cœur très fort mais que je ne peux pas expliquer... (ma traduction)

Conscient du besoin de maintenir une unité discursive face aux étrangers, mon informateur a préféré, ici, me rediriger vers son supérieur que de donner une "mauvaise réponse » qui l'aurait mis dans l'embarras face au discours officiel très articulé et bien diffusé du mouvement. S'il n'avait pas les mots, en cette occasion, pour exprimer ses motivations, il n'en ressentait pas moins une vive nécessité « dans son cœur » de participer.

Les entretiens avec des jeunes de Santa Elena et de Barranca Tigre dont j'ai rendu compte ici n'ont pas tous été réalisés avec des jeunes militants aussi convaincus que pouvait l'être ce milicien. Les images-souhaits exprimées s'éclairent cependant les unes les autres et évoquent une grande diversité d'aspirations face à la crise perçue dans ces communautés. Certaines de ces aspirations, souvent de nature politique, motivent la génération montante à s'engager dans les mobilisations autochtones régionales. Limage-souhait d'une communauté unifiée autour d'un projet commun est certainement présente dans les deux villages. Une autre image importante pour la mobilisation politique est celle de la prospérité communautaire. Cette prospérité peut être vue comme une utopie agraire (Manuel, Santa Elena) ou plutôt sur le mode du développement industriel (Juan, Santa Elena) ou de l'infrastructure moderne (Marcelo, Santa Elena). Même Antonio (Barranca Tigre), démobilisé face à sa coopérative caféière et enclin à adopter une stratégie individuelle, entretient encore le souhait d'une organisation régionale qui réponde à sa propre utopie coopérative.

Cependant, ce serait bien mal comprendre ces mobilisations régionales que de ne les aborder qu'à travers les imaginaires des gens qui y participent. Pedro (Barranca Tigre) est un jeune père de famille qui s'est révélé à maintes reprises tout à fait en accord avec les idéaux du mouvement autonomiste autochtone de la Montagne; cependant, les piètres performances économiques du secteur caféier en général et de LuzMont en particulier, à qui il vend son café " par principe » même s'il en retire dix centavos de moins le kilo, créent des conditions objectives dans lesquelles ses espérances politiques se trouvent en conflit avec ses espérances économiques. La migration économique vers Mexico ou vers les États-Unis, même si elle est temporaire, l'éloigne de la participation politique régionale. Pour Pedro et pour les jeunes Tlapanèques dans sa situation, l'idée de fonder une famille et de s'installer, traditionnellement conséquente avec celle de faire « avancer la communauté », peut maintenant lui paraître antithétique.

La division que nous avons observée à Santa Elena reflète cette tension entre des aspirations croisées. Même si l'idée de l'harmonie communautaire maya a été identifiée depuis longtemps comme étant un mythe (Nash 1994), l'histoire orale que nous avons recueillie dans cette communauté fait état, à tout le moins, d'une plus grande unité communautaire avant que la question de la lutte armée ne surgisse (voir aussi Legorreta Díaz 1998 : chap. 4). Cette unité perdue est regrettée tant par la faction pro-zapatiste de Santa Elena que par sa faction pro-gouvernementale. Mais cette division est difficilement réconciliable. Elle s'enracine dans des aspirations politiques divergentes qui opposent l'idée de l'autonomie politique à celle de la participation accrue dans le système existant. Elle oppose également des aspirations économiques divergentes qui cherchent soit à répondre à court terme aux besoins pressants de la communauté, soit à envisager l'acceptation d'un niveau de privation plus grand à court terme pour forcer un règlement définitif de la question. Toutes ces options sont ancrées dans les imaginaires locaux et ont un sens pour les jeunes hommes qui deviennent actifs dans la sphère politique et qui tentent de faire face à la crise qui s'impose à eux.

\section{Conclusion}

Dans cet article j'ai tenté de cerner certaines trames de l'imaginaire des jeunes autochtones de deux communautés du Mexique. La perspective « à ras le sol » adoptée introduit beaucoup de variabilité dans les discours, et c'est là l'effet recherché. Il a été noté que la constriction du rôle de l'imagination comme principe politique peut être vue comme une forme de coercition, voire de violence exercée contre le sujet imaginant (Graeber 2004 : 11). Face à la possibilité de cette coercition dans le discours et dans l'imaginaire, l'anthropologue aurait un rôle à jouer en donnant voix aux espérances et aux utopies qui sont détectables sur le plan ethnographique, mais qui n'émergent pas dans les discours politiques officiels et cristallisés. Le mouvement zapatiste et le mouvement autonomiste de la Montagne du Guerrero sont, à n'en pas douter, des mouvements démocratiques. Par contre, les discours et images politiques officielles de ces mouvements peuvent à l'occasion néanmoins noyer des discours émergents, et en particulier les discours des jeunes autochtones exprimant leurs espoirs et désespoirs face à leur avenir politique et économique.

J'ai considéré ici certains de ces discours recueillis à partir des images-souhaits qui leur sont associés. La complexité et la diversité relevées ne tracent pas une ligne d'action politique concrète, mais portent plutôt à la réflexion. Qui plus est, tout en mettant en évidence l'attachement symbolique des jeunes autochtones à leur communauté, aux pratiques traditionnelles et aux idéaux de coopération, cette analyse fait ressortir un fort utopisme rêvant d'un «ailleurs» et d'un «meilleur» à atteindre par des moyens et des actions que les générations précédentes n'ont pas eu à considérer. Peut-être est-ce l'apport principal de la présente exploration. Sans pouvoir, ou vouloir, dire de manière univoque ce qui est espéré par les jeunes Tzeltals de Santa Elena et par les jeunes Tlapanèques de Barranca Tigre, il est clair que, même au cour de la profonde crise sociale, économique, juridique et politique actuelle, c'est l'espoir et non le désespoir qui domine leur imaginaire politique.

\section{Ouvrages cités}

BABB, Sarah, 2001 : Managing Mexico. Economists From Nationalism to Neoliberalism. Princeton University Press, Princeton, New Jersey.

BEAUCAGE, Pierre, 1992: "Crise des subsistances ou crise des modèles explicatifs? À propos d'un mouvement indigène et de ses interprétations ». Anthropologie et Sociétés 16(2) : 67-90.

—, 2004: "Nouveaux imaginaires et conversions au protestantisme chez les Amérindiens du Guerrero (Mexique) ». Recherches amérindiennes au Québec XXXIV(2) : 33-47.

BLOCH, Ernst, 1976 : Le Principe espérance-1. Gallimard, Paris 
BROCKETT, Charles D., 2005 : Political Movements and Violence in Central America. Cambridge University Press, New York.

BURRIDGE, Kenelm, 1969: New Heaven, New Earth. A Study of Millenarian Activities. Basil Blackwell, Oxford.

COLLIER, George A., et Elizabeth Lowery Quaratiello, 1994 : Basta! Land and the Zapatista Rebellion in Chiapas. First Food Books, Oakland, CA.

DEHOUVE, Danièle, 1990 : Quand les banquiers étaient des saints. 450 ans de l'histoire économique et sociale d'une province indienne du Mexique. Éditions du CNRS, Paris.

DESROCHE, Henri, 1973 : Sociologie de l'espérance. Calmann-Lévy, Paris

DE VOS, Jan, 1988: Oro verde. Fondo de Cultura Economica, Mexico.

FEMIA, Joseph, 1975: "Hegemony and Consciousness in the Thought of Antonio Gramsci ». Political Studies (23)1 : 29-48.

GALARZA, Joaquín, 1972 : Lienzos de Chiepetlan : manuscrits pictographiques et manuscrits en caractères latins de San Miguel Chiepetlan, Guerrero, Mexique. Collection Études mésoaméricaines, vol.1, Mission archéologique et ethnologique française, Mexico.

GRAEBER, David, 2004 : Fragments of an Anarchist Anthropology. Prickly Paradigm Press, Chicago.

HARVEY, Neil, 1998: The Chiapas Rebellion. The struggle for land and democracy. Duke University Press, Durham, NC.

HÉBERT, Martin, 2000a: Sous le regard des ancêtres. Anthropologie de la mobilisation socio-politique chez les Tlapanèques du Guerrero. Thèse de doctorat, Université de Montréal, Montréal.

—, 2000b: " A Family Remembers a Zapatista Child Soldier». Peace Review 12(3) : 357-360.

—, 2002a : «L'autre main invisible: Deux rituels domestiques de prospérité chez les Tlapanèques du Guerrero, Mexique ». Recherches amérindiennes au Québec XXXII(1) : 83-92.

—, 2002b : «Whose Utopia? Development, Resistance, and Patterns of Structural Violence in a Mexican Indigenous Region ». Social Justice 3(3-4) : 99-137.

JOHNSON, Jennifer L., 2001 : «What's Globalization Got to Do With It? Political action and peasant producers in Guerrero, Mexico ». Canadian Journal of Latin American and Caribbean Studies 26(52): 267-283.
LEGORETTA DÍAZ, Maria del Carmen, 1998 : Religión, política y guerrilla en Las Cañadas de la Selva Lacandona. Cal y Arena, Mexico.

LINDAU, Juan D., 1996 : «Technocrats and Mexico's Political Elite ». Political Science Quarterly 111(2) : 295-322.

MARTÍNEZ QUEZADA, Alvaro, 1995 : Crisis del café y estrategias campesinas. Universidad Autónoma Chapingo, Mexico.

MARTÍNEZ RESCALVO, Mario O., et Jorge R. OBREGÓN TÉLLEZ, 1991: La Montaña de Guerrero. Economia, historia y sociedad. Instituto Nacional Indigenista, Mexico.

MARTÍNEZ SIFUENTES, Esteban, 2001 : La policia comunitaria. Un sistema de seguridad publica comunitaria en el estado de Guerrero. Instituto Nacional Indigenista, Mexico.

NADAL, Marie-Josée, 1994 : À l'ombre de Zapata. Vivre et mourir dans le Chiapas. La Pleine Lune, Montréal.

NASH, June, 1994: «Global Integration and Subsistence Insecurity ». American Anthropologist 96(1) : 7-30.

-, 2001: Mayan Visions. The Quest for Autonomy in an Age of Globalization. Routledge, Londres.

PRUD'HOMME, Jean-François (dir.) 1995 : El impacto social de las políticas de ajuste en el campo mexicano. Plaza y Valdes, Mexico.

ROY, Jean, 2000 : Le souffle de l'espérance. Le politique entre le rêve et la raison. Bellarmin, Montréal.

TELLO DÍAZ, Carlos, 1995: La rebelión de las Cañadas. Cal y Arena, Mexico.

TOLEDO, Victor, 2000: La paz en Chiapas. Ediciones Quinto Sol, Mexico.

VITE PÉREZ, Miguel Ángel, et Roberto RICO MARTÍNEZ, 2001 : Que solos están los pobres. Neoliberalismo y urbanización popular en la zona metropolitana de la ciudad de México. Plaza y Vlades, Mexico.

WILLIAMS, Heather L., 2001 : Social Movements and Economic Transition. Markets and Distributive Conflict in Mexico. Cambridge University Press, Cambridge.

ZUÑIGA LÓPEZ, Rosa Elva, et Manuel R. PARRA VÁZQUEZ, 1998 : «Una mirada al pasado, tres visiones del futuro. Las rebeliones en Santa Martha, Chenalhó », in M.E. Reyes Ramos, R. Moguel Viveros et G. van der Haar (dir.), Espacios disputados : transformaciones rurales en Chiapas : 277-294. Universidad Autónoma Metropolitana, Mexico. 


\section{Collection Paléo-Québec}

\section{Paléo-Québec 31}

\section{Sommaire}

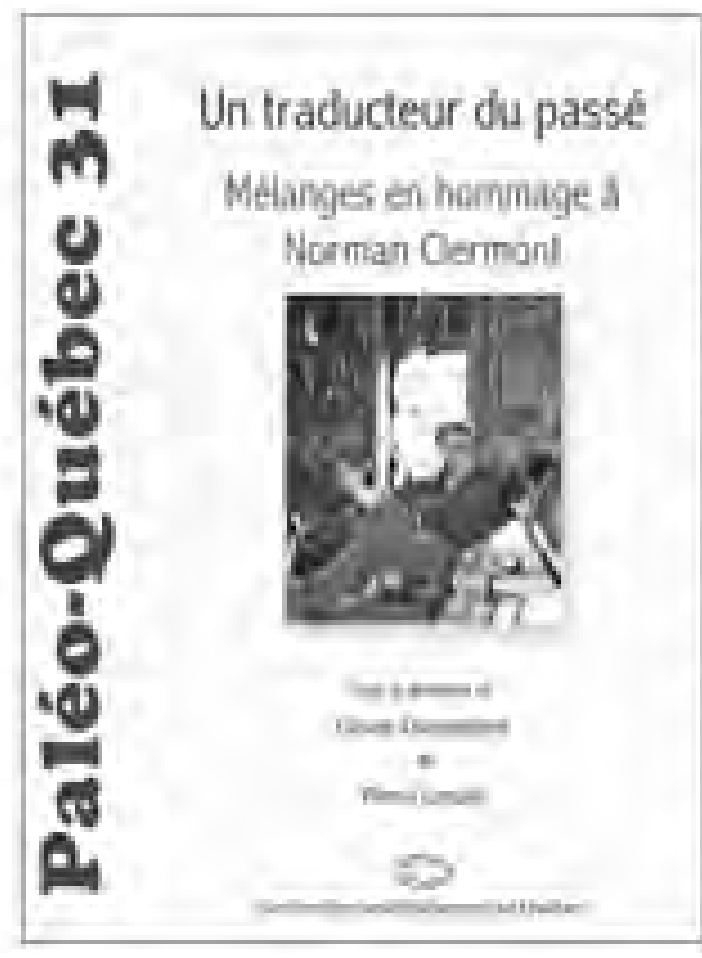

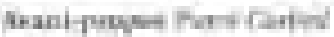

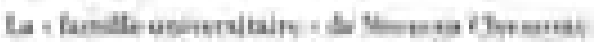

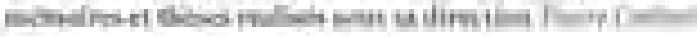

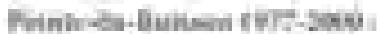

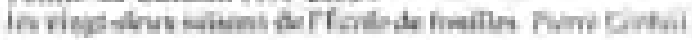

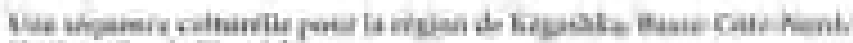

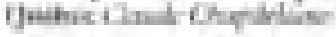

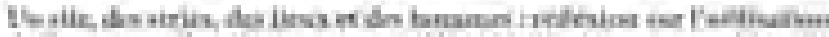

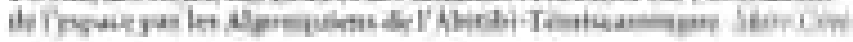

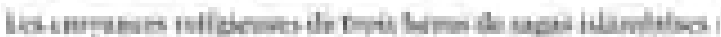

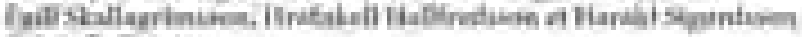

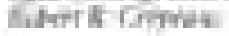

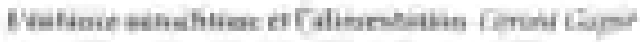

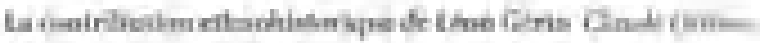

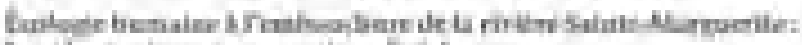

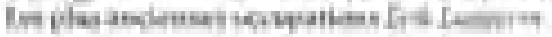

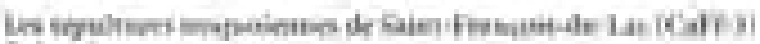
What Irisherin

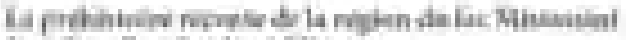

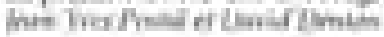

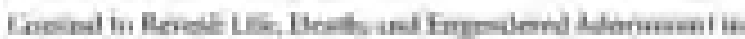

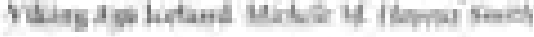

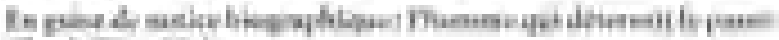

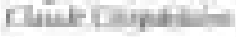

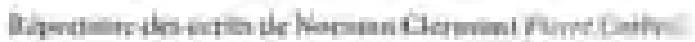

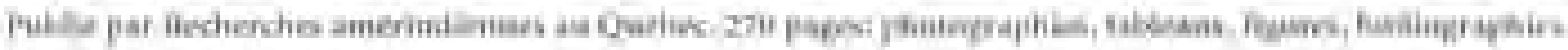

\section{Pour commander un exemplaire :}

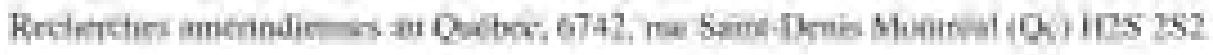

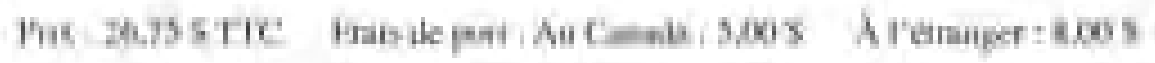




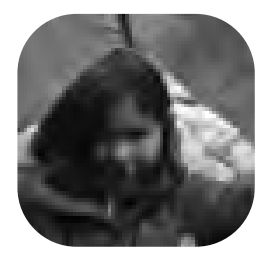

Comment flirter avec la modernité pour conforter son identité Projet éducatif d'une communauté métisse au Manitoba

\section{Thibault Martin}

Université du Québec en Outaouais, Gatineau

et

Brieg Capitaine

Université de Bretagne occidentale, Brest
E 1994, l'École communautaire AurèleLemoine de Saint-Laurent (Manitoba) se joignait à la Division scolaire franco-manitobaine (DSFM). Cet événement clôturait des années d'efforts et de négociations au sein de la communauté métisse mais aussi avec les instances politiques extérieures. Ladhésion à la DSFM permettait à la communauté d'obtenir une école francophone, mais, surtout, elle ouvrait la voie à un projet communautaire holistique dont l'objet était de rapprocher les jeunes de la culture métisse. Dix ans après sa création, le personnel de l'école, les parents et les dirigeants locaux lancent le projet de construire un nouveau bâtiment pour leur école, consacrant ainsi la scission entre les deux institutions scolaires (anglophone et francophone) qui jusqu'à maintenant cohabitent sous un même toit. Cette nouvelle école est étroitement associée à un projet de centre d'interprétation de la culture métisse et à une réalisation importante pour la communauté, l'ouverture du National Museum of the American Indian (NMAI) du Smithsonian Institution à Washington, qui consacre une salle aux Métis de la rivière Rouge, composée d'artefacts et de témoignages récoltés à Saint-Laurent. Cette réalisation représente un événement marquant pour la communauté de Saint-Laurent qui voit ainsi ses efforts pour actualiser son histoire et transmettre la culture métisse reconnus à l'échelle mondiale.

Cette volonté de placer la culture traditionnelle au cour de la vie sociale et économique fait partie de la stratégie développée par les leaders de SaintLaurent pour enrayer le déclin auquel leur communauté est confrontée. En effet, le taux de chômage y est de 36,5 \% (Canada 2001) alors qu'il varie entre $5 \%$ et $8 \%$ pour l'ensemble de la province du Manitoba. Le revenu total médian des familles est de 34000 dollars canadiens contre 51000 dollars pour l'ensemble de la province. La population (1 172 habitants en 2001) vieillit, $32 \%$ des résidants de Saint-Laurent sont des retraités. Les jeunes qui ne trouvent pas d'emploi dans la communauté partent s'installer à Winnipeg. Dans ce projet général de revitalisation, l'éducation et la transmission de la culture sont devenues, pour les leaders communautaires, un objectif majeur, et dans ces circonstances l'école s'est imposée comme une instance fédératrice autour de laquelle s'est développé un véritable projet politique communautaire.

Dans les pages qui suivent, nous allons brièvement situer le contexte sociohistorique des Métis. Ce retour nous apparaît indispensable car le projet éducatif développé à Saint-Laurent vise, dans l'esprit des éducateurs, à reconstruire le sentiment de fierté mis à mal par le système éducatif eurocanadien. Ensuite, nous nous attacherons plus précisément à présenter l'école de Saint-Laurent et sa contribution à l'acculturation de la communauté ainsi qu'à l'émergence d'une modernité homogène. Enfin, nous verrons comment le projet éducatif actuel, à travers la réhabilitation de l'histoire, l'alliance avec la francophonie et la participation aux projets communautaires, est l'instrument d'un projet politique 


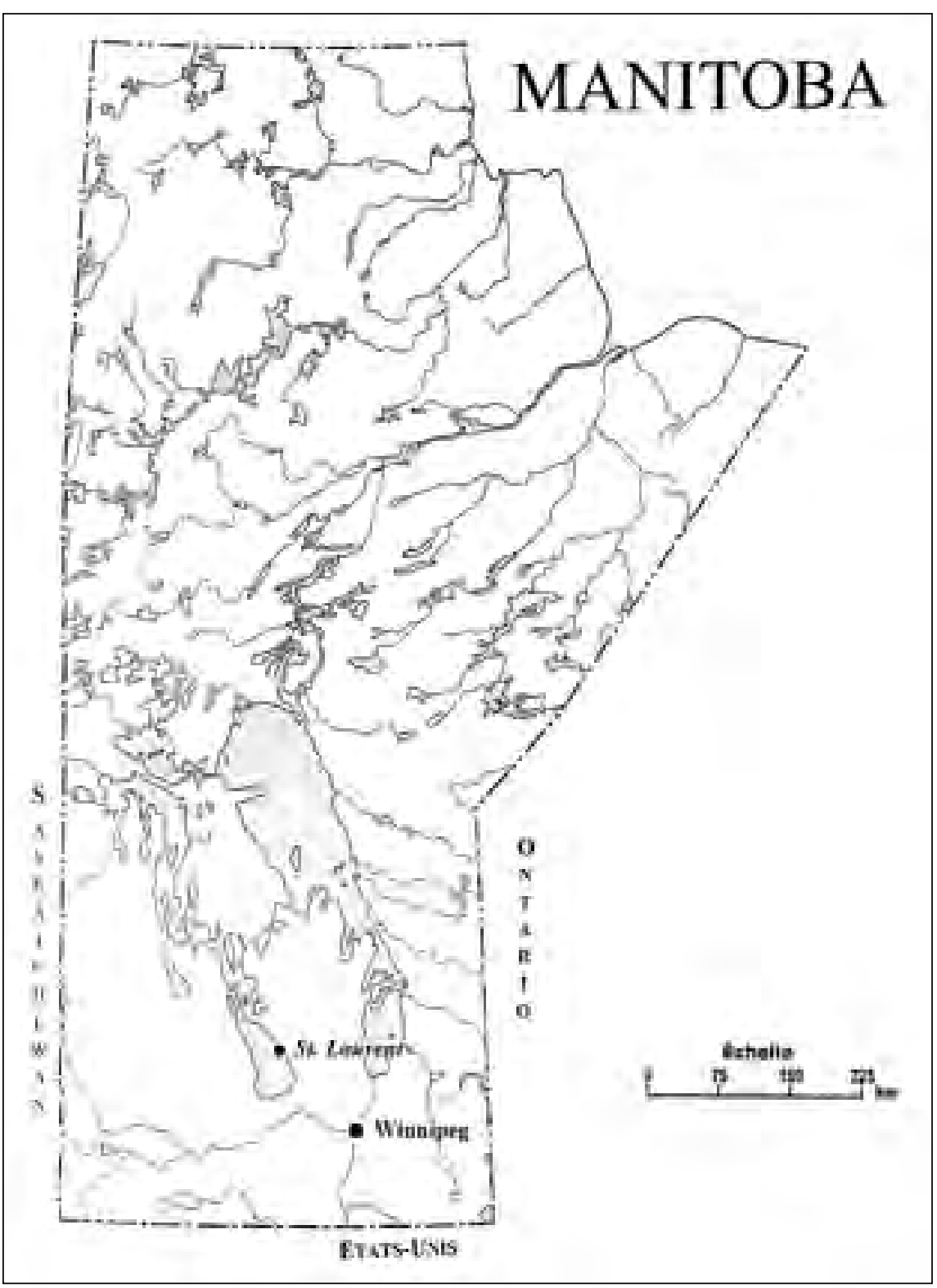

Localisation de la communauté de Saint-Laurent au Manitoba

(Carte réalisée par Brieg Capitaine d'après Atlas du Canada, http://atlas.gc.ca)

alliance entre les « coureurs des bois » français et les Amérindiens des Plaines (Cris et Ojibwas), et celle issue de l'interaction entre des Amérindiens et des Écossais de la « Hudson Bay Company ».

En 1870, l'acte de création du Manitoba scellait l'entrée des Métis dans la confédération canadienne. Malheureusement, le gouvernement fédéral ne devait tenir ni son engagement de respecter l'égalité officielle du français et de l'anglais ni celui de permettre aux Métis de garder leurs terres nécessaires au maintien de leur mode de vie (Stanley 1961 : 119). Non content de ne pas respecter ses engagements vis-à-vis des Métis, Ottawa ne fit rien pour empêcher que s'instaure, à la fin du XIX ${ }^{e}$ siècle et au début du $x^{e}$ siècle, une période que certains historiens qualifient de « règne de la terreur » (Shore 2001), pendant laquelle les Métis perdirent leurs terres, qui furent distribuées aux colons. Si bien qu'en 1886, les Métis ne représentaient plus que $7 \%$ de la population du Manitoba alors qu'en 1870 ils constituaient $83 \%$ de la population totale de la province (De Trémaudan 1984 : 250).

Non seulement ont-ils été dépossédés de leurs terres mais, qui plus est, parce qu'ils ne sont ni «Blancs» ni «Amérindiens», ils furent longtemps méprisés par les Eurocanadiens s'efforçant de "civiliser» leurs mœurs et de purifier leur langue (ibid). Au cours du $\mathrm{XX}^{\mathrm{e}}$ siècle, les projets assimilationnistes de l'État conjugués à ceux de l'Église contribuèrent à leur dispersion et à leur acculturation, si bien qu'aujourd'hui il ne reste plus dans les Prairies canadiennes que quelques communautés métisses structurées.

Cependant, depuis une vingtaine d'années, on assiste chez les Métis à un éveil identitaire et à un projet de reprise en main des institutions sociales, notamment éducatives, considérées comme un

qui vise à léguer la culture métisse en héritage à la nouvelle génération. Ce retour de la tradition, à laquelle on cherche à rattacher les jeunes, contribue au développement d'une culture originale et hybride fondée sur des pratiques et des valeurs à la fois traditionnelles et modernes dont le but est d'offrir aux jeunes un cadre de vie et un avenir attrayants et non pas de les enfermer dans un passé reconstruit artificiellement.

\section{LA SOCIÉTÉ MÉTISSE ET SAINT-LAURENT}

$\mathrm{Au} \mathrm{XVIII}{ }^{\mathrm{e}}$ siècle, en pleine apogée de la traite des fourrures, s'est constituée au sein des Prairies canadiennes une nouvelle société : la nation métisse. Née de l'union biologique et culturelle entre nouveaux venus et autochtones - ce qui a donné naissance à une société originale, fondée sur l'hybridation. Deux traditions métisses virent le jour, celle issue d'une élément clé de la transmission de la culture aux jeunes générations. Ce mouvement de revendication n'est pas particulier aux Métis mais s'inscrit dans le contexte des revendications sociopolitiques des groupes autochtones. Dans le cas des Métis, un des foyers de résistance et de renouveau se situe à SaintLaurent (Manitoba) où nos recherches nous ont conduits.

\section{LE PROJET DE RECHERCHE}

Nos recherches sont le fruit d'une collaboration de plusieurs années avec la communauté de Saint-Laurent qui compte, selon le recensement de 2001, 1172 habitants, dont 675 (58\%) d'origine métisse ${ }^{1}$. Cela dit, ce texte se fonde avant tout sur des données collectées lors de deux terrains d'enquête (décembre 2002 et février et mars 2004) qui ont combiné observation participante et collecte de récits de vie pour le 
premier terrain, et observation participante, entretiens semidirigés et analyse de documents pour le second terrain ${ }^{2}$. Plus précisément, les deux terrains nous ont amenés à assister aux enseignements donnés à l'École communautaire AurèleLemoine ainsi qu'aux différentes manifestations touristiques célébrant la fin de la saison de la pêche. Nous avons aussi réalisé quarante-trois entretiens formels auprès d'enseignants, d'aînés, de pêcheurs métis, de membres de leurs familles et d'acteurs politiques engagés dans le développement communautaire. Ces entretiens ont permis de récolter des données sur l'École communautaire et sur les orientations politiques en termes de développement. Nous tenons à remercier les résidants et les leaders de Saint-Laurent qui nous ont ainsi généreusement donné de leur temps.

\section{L'ÉCOLE AGENT DE CONSTRUCTION D'UNE MODERNITÉ UNIFORME}

$C^{\prime}$ 'est une constante de I'histoire des $\mathrm{XIX}^{\mathrm{e}}$ et $\mathrm{xx} \mathrm{x}^{\mathrm{e}}$ siècles, que tous les États du monde, les uns après les autres, s'emparent de l'école, au travers de laquelle ils visent des objectifs multiples: faire faire à tous les enfants les apprentissages fondamentaux qui feront d'eux des citoyens et des travailleurs compétents, les socialiser dans un projet national plus ou moins imposé par une minorité, plus ou moins démocratique, et par conséquent unifier le pays autour de la culture véhiculée par l'école. (Warnier 1999 : 66)

Ce projet éducatif civilisateur des missionnaires fut repris au $\mathrm{XX}^{\mathrm{e}}$ siècle par l'État qui implanta des programmes scolaires destinés à angliciser et canadianiser les populations immigrantes ou autochtones. Le projet d'homogénéisation de l'école venait s'opposer à l'essence même de la culture métisse fondée sur l'hybridation et sur l'emprunt de valeurs et de pratiques exogènes. L'école fut, pour les Métis, autant un lieu d'acculturation que de résistance. Ainsi lorsque les francophones du Manitoba perdirent en 1916, avec la loi Thornton, les faibles gains linguistiques qu'ils avaient obtenus depuis que le bilinguisme de la province avait été aboli en 1875 (Blay 1987), certaines écoles, surtout les catholiques, furent le théâtre d'un mouvement concerté de résistance passive dont les FrancoMétis profitèrent indirectement. Ce mouvement orchestré par l'Association d'éducation des Canadiens français du Manitoba, encouragea un apprentissage, en cachette, du français, comme nous le rapporte ce témoignage d'un aîné métis de Saint-Laurent :

L'inspecteur venait en auto. D'habitude la maîtresse savait quelle journée l'inspecteur allait venir, ça fait qu'elle nous disait d'emmener tous nos livres de français à la maison [...] pour pas que l'inspecteur voie ça [...] La maîtresse nous avait expliqué: " D'habitude, je sais quand l'inspecteur vient, puis là vous devez emmener vos livres français à la maison. Si jamais l'inspecteur rentre, si vous avez un livre français sur votre pupitre, mettez-le en dessous [vous savez il y avait comme une tablette], cachez votre livre. » Elle parlait seulement anglais quand l'inspecteur venait, je me rappelle qu'il y avait un Mister Friesen qui était inspecteur, ç'avait l'air d'être un homme pas mal gentil. II faisait la classe avec nous pour voir comment on faisait les choses. II faisait l'arithmétique un peu, puis l'histoire du Canada, puis il nous demandait des questions [...], mais il ne demandait pas de questions à propos du français. J'ai entendu dire qu'il savait que les maîtresses nous enseignaient le français mais probablement qu'il fermait les yeux là-dessus.

Malgré cette résistance, le dialecte franco-métis, le mitchif, parlé à Saint-Laurent, connaît un repli important du fait de l'adhésion de certains jeunes aux valeurs de la modernité. Léducation était considérée comme un moyen permettant de s'émanciper d'une condition perçue comme étant insatisfaisante au regard des standards de la modernité, comme l'explique cette aînée :

J'espère que les jeunes gens iront à l'école autant qu'ils pourront, ça aide à développer leur esprit; l'école peut-être bon pour eux pour trouver un bon travail, faire beaucoup d'argent et profiter d'un niveau de vie meilleur que celui que nous avons eu. (cité dans Lavallée 2003 : 34)

Pour d'autres, c'est la stigmatisation de leur culture qui constitue l'élément déclencheur. En effet, comme l'a suggéré Goffman (1975: 17), la critique institutionnalisée d'une culture amène les individus qui en sont membres à rejeter leur identité. C'est ainsi que, durant les années de forte pression contre les métis, de nombreux jeunes cachèrent leur appartenance, cessant de parler mitchif de peur des railleries et abandonnèrent peu à peu les pratiques traditionnelles pour se conformer au modèle dominant occidental, véhiculé par l'école et l'Église.

Parallèlement, à partir des années 1960, différents projets, dont plusieurs réalisés grâce à l'aide de fonds gouvernementaux, virent le jour et permirent à la communauté de Saint-Laurent de découvrir la «passion moderniste», pour reprendre l'expression de Balandier (1985). Dans les années 1970, une usine de mobilier, un abattoir et deux coopératives de pêche et d'agriculture offraient ainsi aux résidants de Saint-Laurent des emplois salariés qui contribuèrent à créer un cadre de vie de plus en plus moderne, comme l'explique cet observateur de l'époque: "Saint-Laurent est une communauté qui est rapidement devenue l'une des plus progressistes du Manitoba » (Sealey 1974: 37). Signe de ce changement, le nombre de pêcheurs décrut rapidement. Parallèlement, plusieurs jeunes dotés d'un capital scolaire élevé quittèrent la communauté pour aller chercher un travail à Winnipeg ou ailleurs.

\section{CONTESTATION DE LA MODERNITÉ ET REDÉFINITION DU MODE D'ÉDUCATION À SAINT-LAURENT}

Si les années 1960-1970 correspondent, à Saint-Laurent, à une période de modernisation rapide, les décennies suivantes vont être celles d'un déclin économique important, conséquence du repli des mêmes activités industrielles ayant contribué à la modernisation. Ainsi, au début des années 1990, presque toutes les usines de Saint-Laurent qui avaient contribué à l'industrialisation de la municipalité sont fermées, seuls l'abattoir et la coopérative de pêche ont résisté. En faisant l'inventaire des services dont disposait la communauté au début des années 1990, un membre de la Corporation de développement communautaire (CDC) de Saint-Laurent nous confiait :

Nos droits avaient été lésés pendant toutes ces années et on avait vraiment abusé de nous. C'est parce qu'on s'est rendu compte qu'on vivait dans des conditions tiers-mondiste qu'on a voulu prendre notre avenir en main et s'occuper de notre développement par nous-mêmes.

La décennie 1985-1995 sera donc celle du bilan pour les Métis. Non seulement constatent-ils l'échec de l'industrialisation de leur communauté mais, de plus, certains commencent à regretter les sacrifices culturels importants consentis pour atteindre un niveau de développement économique qui s'avère 


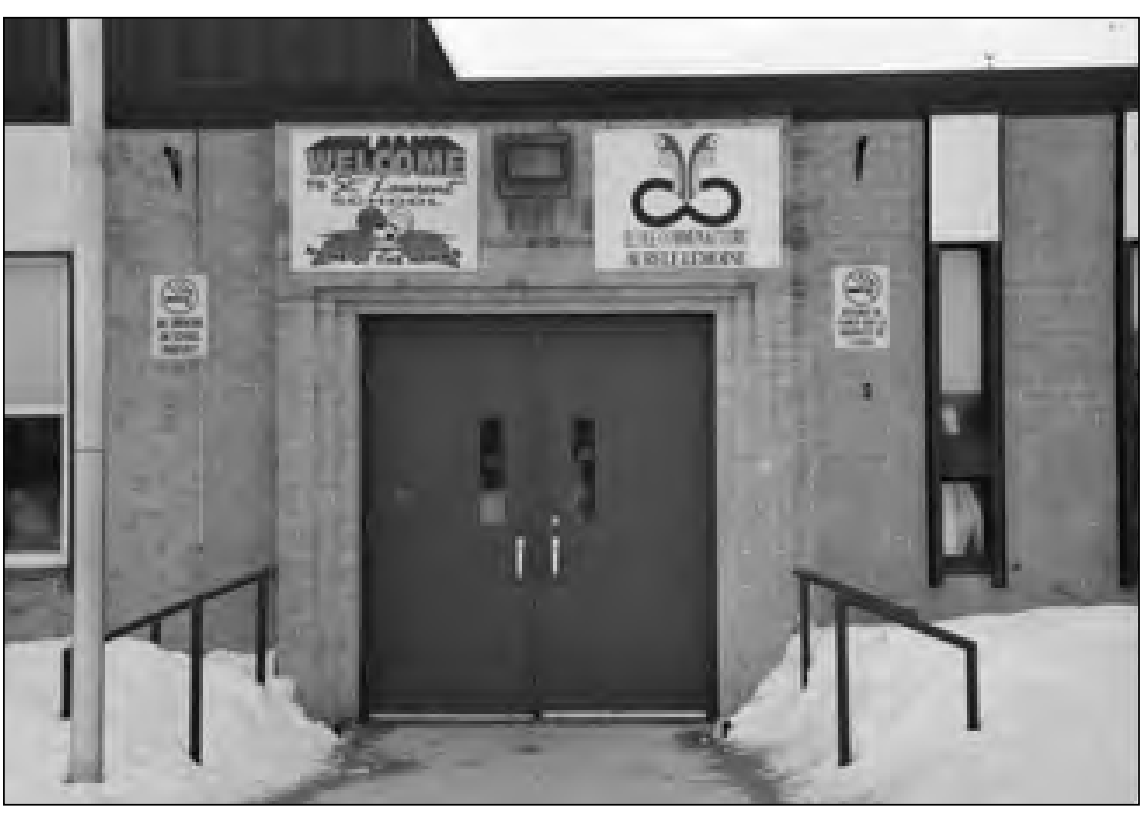

Entrée de l'école Saint-Laurent montrant la division entre les écoles anglophone et métisse (Photo Brieg Capitaine)

finalement insatisfaisant. Comme le raconte ce répondant qui avait quitté la communauté pendant quelques années:

Quand je suis revenu une dizaine d'années plus tard, je me suis rendu compte comment il y avait eu des ravages sur le plan de I'assimilation. Quand je suis allé à l'école c'était toujours la même bonne vieille école avec les mêmes enseignants mais il y avait eu une érosion linguistique qui correspondait pas mal avec ce qui se passait partout ailleurs dans le monde sur le plan de la francophonie que ce soit chez les cajuns en Louisiane ou dans tous les milieux minoritaires. Tout ce patrimoine linguistique qui était en train de s'effriter et de disparaître. J'avais beau savoir que c'était partout pareil quand j'ai vu que dans les corridors les jeunes ne parlaient plus français ni mitchif mais anglais, j'ai été profondément choqué. C'est là que je me suis dit qu'il fallait faire quelque chose, qu'il fallait qu'on travaille le problème à la source : ici même à l'école.

Ce projet de reprendre en main l'éducation, les Métis de Saint-Laurent l'ont développé en s'associant aux francophones de la province. En 1970, le français fut reconnu au Manitoba comme langue d'enseignement mais les écoles françaises restèrent attachées aux commissions scolaires anglophones, ce qui n'était pas satisfaisant pour beaucoup de parents francophones. Ladoption de la Charte canadienne des droits et des libertés par le parlement canadien, en 1982, donna aux minorités linguistiques une assise légale leur permettant d'appuyer devant les tribunaux leurs revendications en matière de contrôle de l'éducation. Ainsi, en 1990, l'arrêt Mahé reconnaissant aux FrancoAlbertains le droit de gérer leurs écoles ouvrit la voie à la création de commissions scolaires francophones au sein des provinces anglophones. Au Manitoba, la DSFM (Division scolaire franco-manitobaine) vit le jour en 1993 et les parents de vingt-deux écoles choisirent de joindre la nouvelle division scolaire. Dans chaque communauté, les débats associés à l'adhésion à la DSFM furent douloureux. En effet, certains Franco-Manitobains avaient peur qu'un tel choix ne les marginalise, d'autres craignaient que d'éduquer leurs enfants en français ne limite leur capacité de s'insérer dans la vie professionnelle.

À Saint-Laurent, les débats furent encore plus passionnés. En effet, contrairement aux autres communautés qui avaient déjà des écoles francophones en place, à Saint-Laurent il n'y avait qu'une école dont la langue d'enseignement était l'anglais. Dans ces conditions, le projet d'adhérer à la DSFM impliquait la création, dans le village, d'une seconde école où les familles francophones pourraient inscrire leurs enfants. Comme on peut le concevoir, ce projet a eu un effet diviseur au sein de la communauté, et de nombreux débats et réunions publiques furent organisés. Lors de ces réunions, les tenants communautaires du projet tentèrent de convaincre les opposants au projet qu'il ne s'agissait pas de diviser la communauté mais de s'associer à un projet émergeant au sein duquel les Métis pourraient prendre leur place. Malgré tout, les Métis de langue anglaise désapprouvèrent la création d'une école francophone, n'y voyant aucune nécessité fondamentale, et ils associaient ce projet au désir de créer un « petit Québec au sein du Manitoba ». Pour plusieurs, aussi, la création d'une nouvelle école signifiait la perte d'élèves dans l'école existante et en conséquence une diminution des subventions et du nombre d'enseignants.

La question de l'alliance avec la francophonie manitobaine était aussi problématique pour certains Métis qui se demandaient en quoi une école francophone serait mieux à même de protéger leur culture qu'une école anglaise. En effet, selon eux, l'histoire avait démontré que les institutions canadiennesfrançaises avaient tout autant contribué à leur acculturation que celles de la majorité anglophone. Les tenants de l'adhésion à la DSFM firent valoir que la proximité linguistique entre Métis et Franco-Manitobains ainsi qu'une commune expérience de minorités justifiait un rapprochement avec la DSFM. De plus, le projet pédagogique de la DSFM, en mettant l'accent sur «le développement de personnes autonomes, épanouies, compétentes, sûres de leur identité et fières de leur langue et de leur culture » (DSFM 2005), correspondait aux demandes identitaires des Métis et permettait d'espérer que la nouvelle école de Saint-Laurent obtienne le support nécessaire pour créer des projets pédagogiques spécifiques aux besoins des Métis.

Finalement, en 1994, l'École communautaire Aurèle-Lemoine voyait le jour, après quatre ans de débats, d'alliances et de déchirements. Cette école ne venait pas remplacer l'école anglophone mais seulement s'y ajouter afin d'offrir aux seules familles qui le souhaitaient un enseignement en français. Pour complexifier encore la situation, l'école française a dû s'installer dans les locaux de l'école anglophone et partager les salles de classe. Rapidement, les francophones réclamèrent une aile entière du bâtiment. Lécole fut donc divisée en deux : une partie française et une anglaise, les deux séparées (ou unies selon le point de vue que l'on adopte) par un couloir. Aujourd'hui, la division spatiale est en voie d'être consommée puisque les dirigeants de l'école Aurèle-Lemoine appuyés par la DSFM demandent la construction d'un nouveau bâtiment qui accueillera leur école. 


\section{L'École communautaire Aurèle-Lemoine}

\section{UN PROJET POLITIQUE À LÉGUER À LA NOUVELLE GÉNÉRATION}

L'École communautaire Aurèle-Lemoine a eu dès le départ le projet d'affirmer et de revaloriser l'identité métisse. Pour cela, l'école n'hésite pas à être engagée, car « toute forme d'éducation est un projet politique », nous confiait le directeur de l'École communautaire Aurèle-Lemoine. Effectivement, une des premières tâches que celui-ci s'est donnée fut l'appropriation, la mise en scène du lieu, tel que son témoignage l'indique :

Un des premiers symboles auquel on s'est attaqué c'est celui du drapeau métis, et ça nous a pris environ deux ans avant d'avoir la permission de pouvoir faire flotter le drapeau métis sur le toit de l'école. [...] J'ai trouvé que le drapeau était un bon cheval de bataille et ça a été notre symbole de lutte pendant plusieurs années. Grâce à cette bataille-là, on a rallié un groupe de parents qui, sur le plan de l'alliance, étaient devenus de solides partenaires dans cette grande odyssée qui était de mettre en œuvre une école française, avec une vocation qui était surtout la promotion, la normalisation de la vie métisse francophone.

Cette affaire du drapeau est devenue à Saint-Laurent un symbole du projet d'affirmation politique et du rôle joué par l'école dans ce projet. Cela n'est d'ailleurs pas sans augmenter les tensions issues de la création même de l'école. En effet, les familles anglo-métisses sont embarrassées par cet épisode puisque le drapeau métis est un symbole qui leur appartient aussi. Lusage politique qu'en fait l'école franco-métisse les irrite, puisque le drapeau qui ne flotte que du côté francophone, suggère ainsi que les Métis francophones se considèrent davantage métis que les autres Métis. Bien sûr, d'un côté comme de l'autre, on tente d'éviter les conflits ouverts et on espère que la création d'une école distincte devrait contribuer à formaliser la rupture, tout en diminuant les tensions au quotidien.

Il est à noter que la nécessité de créer une nouvelle école réside autant dans un souci de qualité pédagogique (davantage d'espace, création d'un environnement linguistiquement homogène) que dans le projet des Franco-Métis d'afficher leur autonomie et de s'ancrer sur un espace qui leur soit propre. Tel que l'indique ce témoignage d'un membre de la direction de l'école :

Maintenant, ce qui est à venir, c'est qu'on va faire l'achat d'un terrain, et un peu dans l'esprit des dernières décisions qui ont été rendues au pays, on veut avoir notre propre bâtiment et faire notre propre gestion dans un pavillon distinct et sur notre lopin de terre à nous.

Cependant, le projet politique de l'école ne se limite pas à des actes d'affirmation tournés vers l'extérieur mais s'accompagne aussi d'une volonté de redéfinir de façon positive l'identité métisse. Pour cela, l'école s'est donnée comme mission d'offrir aux jeunes Métis une lecture de l'histoire davantage conforme à la vision que les Métis ont d'eux-mêmes que celle présentée dans les programmes scolaires de la province. Ainsi, l'histoire enseignée à l'École communautaire Aurèle-Lemoine met l'emphase sur le rôle central joué par les Métis dans l'histoire de la province. Louis Riel est présenté, dans les cours, comme le fondateur de la province du Manitoba et l'emphase est moins mise sur son statut de martyr que sur son action de lutte pour le maintien de la culture et la reconnaissance des droits des Métis. De façon intéressante, l'École communautaire Aurèle-Lemoine illustre ce constat sociologique d'Anne-Sophie Marchand qui observe que, pour les minorités, notamment au Manitoba, il est parfois plus valorisant et constructif sur le plan identitaire de s'attacher à la réaction de son groupe face à l'oppression, qu'à l'oppression elle-même (Marchand 1997 : 43).

Toutefois, la reconquête de l'histoire ne se limite pas à une relecture uniquement théorique du passé. Ainsi, l'école organise chaque année un pèlerinage à Batoche en Saskatchewan, où fut écrasée la résistance métisse. Les aînés accompagnent les élèves sur le site, ils leur racontent la fondation de la nation métisse et leur exposent le rôle central que ceux-ci jouèrent dans la fondation de la province du Manitoba. Le pèlerinage constitue un des temps forts du programme d'histoire et permet de restaurer les liens traditionnels entre les jeunes et les anciens, liens reposant sur l'oralité. Interrogé sur la place de ce voyage à Batoche, le directeur de l'école répondait de façon un peu provocante mais très révélatrice:

Le pèlerinage à Batoche, c'est le but ultime de l'éducation que nous donnons à nos jeunes. Apprendre d'où l'on vient et qui l'on est, c'est aussi important que d'apprendre à lire. L'école ne transmet pas que des savoirs, elle crée des citoyens.

\section{UN PROJET D'ALLIANCE AVEC LA FRANCOPHONIE GLOBALE}

La dénomination «École communautaire Aurèle-Lemoine » est un hommage rendu à un ancien curé de Saint-Laurent, un Canadien français, fervent défenseur du fait français au Canada. D'ailleurs, plusieurs tableaux et autres artefacts datant du temps où les religieuses enseignaient à Saint-Laurent sont exposés à divers endroits de l'école et cohabitent avec des objets métis, notamment ceux qui sont chargés symboliquement, comme la ceinture fléchée. Interrogés sur la raison de la présence de ces objets rattachés à une période douloureuse pour les Métis, une enseignante nous a indiqué que le but était de maintenir le lien avec le passé :

Même si nous sommes aujourd'hui un peu critiques par rapport à l'enseignement que nous donnaient les religieuses, surtout parce qu'elles voulaient nous empêcher de parler mitchif, il faut quand même que nos enfants sachent que, si on parle français, c'est grâce à elles.

La contradiction apparente que recèle ce témoignage révèle la dualité politique du projet de l'école : l'affirmation des stigmates du passé et le rapprochement avec la communauté francophone.

Cette main tendue, la communauté franco-manitobaine l'a saisie. Certes, les débats sur la spécificité de l'école de SaintLaurent et sur la nécessité d'incorporer des contenus traditionnels au sein du programme scolaire ont été vifs. D'ailleurs, selon le témoignage d'un résident de Saint-Laurent :

C'est un commissaire d'origine algérienne qui s'est battu pour convaincre les Franco-Manitobains de nous accepter. On peut dire que c'est grâce à l'internationale francophone qu'on a pu avoir notre école!

Néanmoins, l'École communautaire Aurèle-Lemoine et son équipe pédagogique jouissent, aujourd'hui, d'un respect important au sein de la DSFM ${ }^{3}$. Cela dit le projet métis de se connecter à la francophonie ne se limite pas à l'adhésion à la DSFM. En effet, d'autres initiatives comme, par exemple, le fait que l'école recrute des enseignants au Québec, attestent ce projet d'ouverture sur le monde francophone. De nombreux autres liens entre la communauté et les organismes francophones provinciaux ont aussi été tissés notamment dans le domaine économique. Les liens entre la Corporation de développement communautaire (CDC) et le Conseil de développement économique du Manitoba (CDEM), l'organisme qui chapeaute le 


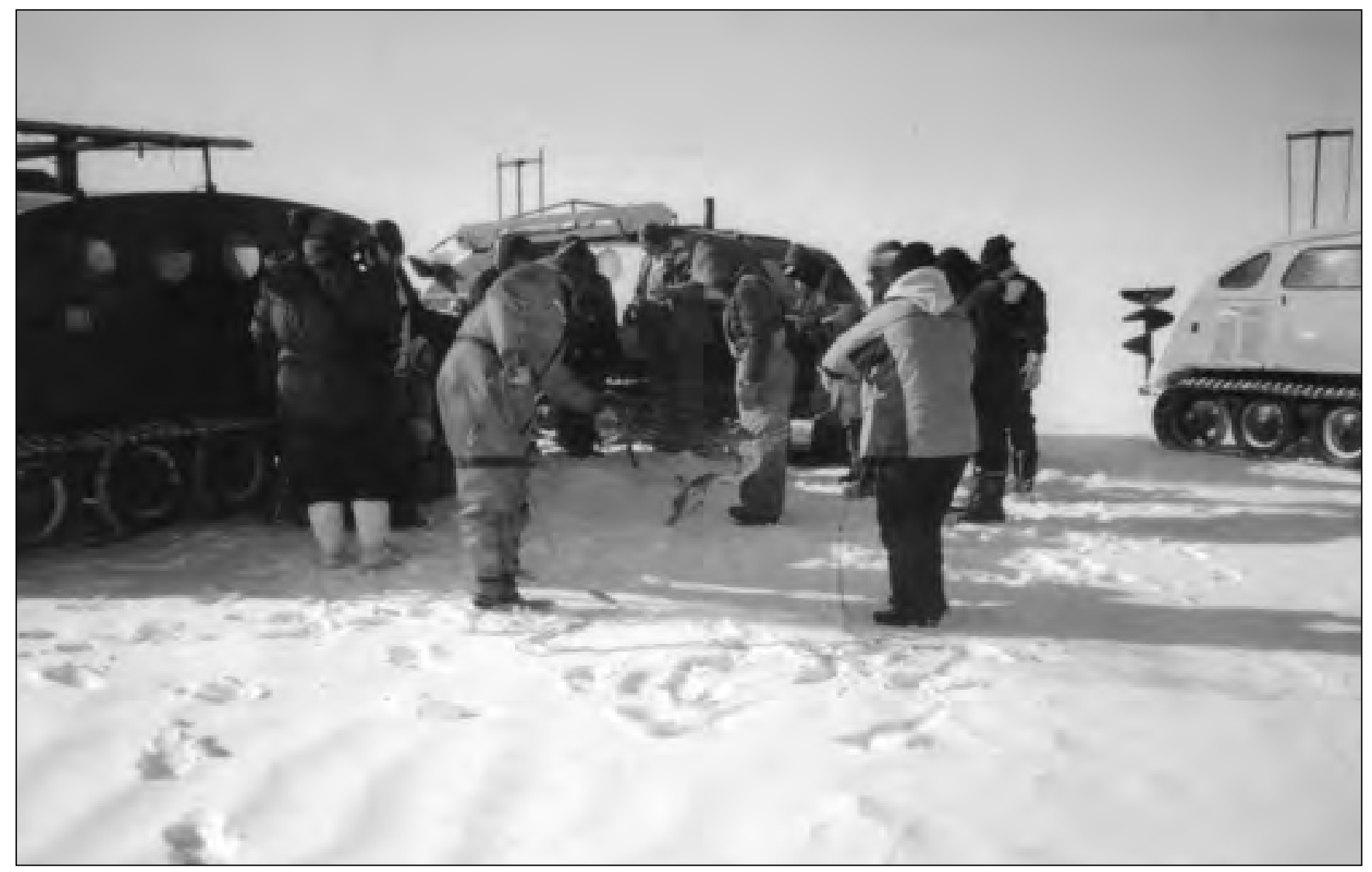

Démonstration de pêcheurs lors du festival Manipogo

(Photo Thibault Martin)

développement socio-économique des collectivités francophones, ont permis à la communauté de développer plusieurs projets culturels, dont plusieurs festivals. Récemment, une délégation d'hommes d'affaires français a été invitée à Saint-Laurent par le CDEM pour tenter d'établir des liens économiques et touristiques entre le Manitoba francophone et la France.

Cependant, cette alliance avec la francophonie augmente la scission avec la composante anglophone de Saint-Laurent. D'ailleurs, il est intéressant de noter que, lors de nos différents séjours dans le village, nos guides, des Métis francophones de la communauté, ne nous ont pas présentés à des Métis anglophones. Pourtant, ces derniers s'investissent eux aussi dans les festivals culturels et les différents projets de mise en valeur de la culture métisse. Leur engagement se fait dans des domaines de la culture tels que la musique ou la danse qui ne mettent pas en jeu la langue, comme l'indique ce commentaire d'un répondant :

Les anglophones, eux, ont trouvé une façon de s'affirmer à partir de la musique, surtout le violon. Ils ne s'affirment plus du tout dans le domaine linguistique, les violons font taire la parole. Et pour eux, la culture métisse ça se résume à ça (la musique). Lorsque tu vas parler de culture, ils ont leurs propres symboles. Toutes leurs références culturelles vont être autres que linguistiques. Mais pour nous, du côté francophone, ce qui est le plus important dans la culture c'est la langue, c'est ce qui nous a isolés, nous a rendus uniques. C'est le mitchif [...]. Pour nous, la question linguistique du mitchif, elle est non négociable.
Cette prédominance attribuée à la langue par les Métis francophones, dans la définition de leur identité, contribue à conforter l'alliance avec la francophonie. Il est important d'indiquer que cette alliance profite, bien que de façon distincte, aux deux parties. En effet, en s'alliant aux Métis, les Franco-Manitobains s'associent à la nation fondatrice du Manitoba et réactualisent ainsi leur alliance historique avec les premières nations. Par contre, du point de vue métis, cet enracinement à la francophonie locale et globale a pour but de donner aux jeunes une ouverture sur le monde, un monde qui n'est pas immédiatement contigu, anglophone, où ils risquent de s'assimiler, mais un univers international, celui de la francophonie. Cette stratégie évite que le retour dans le programme de la tradition enferme les jeunes Métis dans le passé et ne leur donne le sentiment de retourner en arrière. Au contraire le projet de sauver la tradition, notamment à travers la défense de la langue vernaculaire, fait que les Métis s'ouvrent sur le monde. Les succès remportés par cette stratégie, dont le festival Manipogo (festival faisant la promotion de la culture métisse et attirant de nombreuses personnes de Winnipeg), et la création d'une salle Métis au NMAI sont autant de facettes qui contribuent à convaincre les jeunes que leur culture vaut la peine d'être vécue au présent.

Qui plus est, le succès des activités en français n'est pas uniquement identitaire, mais aussi économique. Ainsi la création d'une salle Métis au National Museum of the American Indian (NMAI) ne constitue pas seulement une victoire morale mais est aussi vue comme une source de revenu potentiel. En effet, 
dans la foulée de cet événement, la communauté espère fonder son propre centre d'interprétation qui permettra de créer des emplois. Lapport économique de la culture métisse, notamment dans son expression francophone, ne laisse pas les jeunes indifférents. Si bien qu'aujourd'hui plusieurs d'entre eux se lancent, en français, dans des activités artistiques qui incorporent des éléments de la tradition (théâtre, musique, vidéo). Finalement, le succès de cette approche se traduit par une augmentation remarquable des inscriptions à l'École communautaire Aurèle-Lemoine, dont le nombre d'élèves est passé d'une quarantaine en 1994, à 105 élèves en 2004.

\section{L'ÉCOLE COMMUNAUTAIRE AURÈLE-LeMOINe OU L'HYBRIDATION SOCIALE EN ACTION}

Le XXe siècle aura été, en Amérique du Nord, celui de la généralisation de l'écrit. Malgré la montée d'une seconde oralité, développée à partir des médias modernes (télévision, radio, Internet), l'écrit s'est imposé comme outil de base pour la communication et la transmission du savoir (Ong 1988). Ce phénomène aura engendré, pour les sociétés à tradition orale de ce continent, des impacts de deux ordres. Tout d'abord, la montée de l'écrit a minorisé la culture première et orale de ces groupes et a simultanément entraîné, par manque de maîtrise des outils du pouvoir moderne, une marginalisation sociale, économique et politique des membres de ces communautés. Par ailleurs, les efforts imposés ou consentis par ces minorités pour rejoindre les rangs de la modernité et des peuples lettrés ont provoqué un repli et une désaffection lente de leur culture première. Malgré tous leurs efforts de modernisation pour acquérir les outils de la modernité et malgré l'enrôlement des jeunes de ces communautés dans le système scolaire, le taux d'analphabétisme au sein des communautés autochtones reste largement supérieur à la moyenne nationale (Wagner 2000) en dépit des variations contextuelles.

Pour un sociologue, la problématique de sous-scolarisation, qui prévaut dans ces communautés à tradition orale minorisée, n'est pas uniquement une question d'apprentissage scolaire mais relève également de l'acculturation. Les jeunes de ces communautés souffrent non seulement d'un problème personnel de scolarisation mais sont aussi entraînés dans une spirale collective de perte de culture. En somme, la scolarisation, si elle permet de résoudre certains problèmes, ne résout pas nécessairement tous les problèmes d'intégration de l'individu. D'ailleurs, il apparaît que l'écrit ou la scolarisation pour les jeunes de ces communautés n'a souvent qu'un usage opérationnel, c'est-àdire obtenir un emploi (Lafontant et Martin 2000), et ne sert pas de lien avec la culture et les générations passées, comme le faisait la littérature orale ou comme l'écrit le permet dans les sociétés à tradition écrite. Au contraire, l'écrit et l'éducation scolaire creusent le fossé culturel en ouvrant sur un tout autre univers, celui de la culture de la majorité, et ne répondent donc que partiellement au double problème des jeunes de ces communautés.

En somme, pour les sociétés à tradition orale, la montée du niveau d'éducation formelle se traduit par un recul des récits, des chants et des légendes traditionnelles, ce qui, dans le cas des communautés autochtones, provoque un repli de la langue maternelle. Plus les jeunes de ces communautés s'instruisent à même la culture de la majorité, plus ils s'éloignent de leur culture première. Une fois la langue maternelle oubliée, ils migrent vers les grands centres urbains, et leur communauté d'origine s'étiole, vieillit et, en conséquence, présente de moins en moins d'attrait. Cela veut-il dire que, pour sauvegarder un patrimoine culturel, il faille renoncer à l'éducation ou même plus simplement à la modernité? Non, semblent nous dire les décideurs de Saint-Laurent qui ont choisi de revitaliser leur communauté en investissant dans l'éducation, vue comme le plus sûr moyen de sortir de la marginalité. Cependant, l'école qu'ils mettent en place ne doit pas éduquer uniquement aux valeurs de la majorité mais, au contraire, remettre au cœur de la vie quotidienne des pratiques, un langage, et des récits traditionnels. Lécole devient donc un outil d'hybridation, un moteur d'union entre les pratiques traditionnelles et les ressources modernes.

En fait, ce qui se passe à Saint-Laurent n'est pas unique et se donne à voir dans d'autres communautés autochtones. En effet, à partir des années 1990 s'est développée au Canada une réflexion sur la participation des aînés dans l'éducation et sur le rôle que l'école devrait jouer dans l'instauration d'un leadership autochtone. Aujourd'hui les études portant sur le système scolaire en milieu autochtone sont assez nombreuses, surtout en ce qui concerne l'ouest du pays, principalement en Colombie-Britannique et en Saskatchewan. Ces études revendiquent la mise en place de programmes éducatifs enracinés dans les contextes communautaires (Bashutski et al. 2003; Schissel et Wotherspoon 2002). Plusieurs affirment même que le système scolaire " eurocanadien » est ethnocentrique, hostile, voire raciste, et inadapté au besoin des étudiants autochtones (British Columbia Human Rights Commission 2001). Il faut 
donc, selon plusieurs recherches issues du domaine des sciences de l'éducation, modifier les programmes scolaires afin de faire cohabiter les deux systèmes culturels au sein d'un même système scolaire (Stairs 1994; Friesen et Friesen 2002).

Sur le plan pratique, plusieurs commissions scolaires de l'Ouest mais aussi les ministères de l'Éducation de ces provinces ont mis en place ou sont en train de mettre en place des projets destinés à promouvoir la participation pleine et entière des communautés autochtones au système scolaire (Saskatchewan Department of Education 1991) ou encore favorisent l'intégration des aînés dans les projets scolaires (Saskatchewan School Trustees Association 1993). Les commissions scolaires essaient aussi d'intégrer du contenu pédagogique autochtone dans le programme (Saskatchewan School Trustees Association 1993). Cependant, les initiatives des commissions scolaires auraient pour objet, selon Johnson et Boehm (1995), de légitimer leurs droits d'acculturer les autochtones en rejetant la responsabilité de l'échec de l'institution éducative moderne auprès des jeunes autochtones sur l'héritage des pensionnats. Il s'agirait donc d'un projet politique destiné à « bonifier » le système scolaire afin de le rendre acceptable par les autochtones au lieu de constater l'incompatibilité des fondements de l'école moderne avec les valeurs des premières nations.

Par contre, les projets des autochtones eux-mêmes pour créer une école qui corresponde à leurs valeurs et qui leur permette de redéfinir les relations de pouvoir avec la majorité (Bishop et Glynn 1999) sont très nombreux. Il est d'ailleurs remarquable que le mouvement pour une prise de contrôle de l'éducation traverse tous les contextes autochtones, de la Nouvelle Zélande au Nunavut (Neil 2000); il serait donc impossible d'en dresser ici l'inventaire exhaustif. Toutefois, il est quand même possible de dire que ces projets ont ceci de commun, d'être tous "écologiques », selon la définition que Wagner (2000) donne à ce terme, c'est-à-dire que ces projets sont développés de manière à maintenir l'équilibre social. Ils ne sont donc pas, comme l'école, une institution exogène, mais sont des produits de la culture elle-même et, à cet égard, ils concourent à la pérennisation de la société. D'une manière générale l'éducation écologique en milieu autochtone aide à maintenir une relation significative entre les aînés et les jeunes, permet d'institutionnaliser les savoirs traditionnels et de perpétuer le lien entre la culture et la nature, notamment en étant un véhicule de la spiritualité traditionnelle, et, enfin, elle participe au développement de rapports sociaux non individualistes.

Dans le cas des Métis, l'Institut Gabriel Dumont (situé en Saskatchewan) a grandement contribué à fournir aux communautés métisses des supports leur permettant de penser ou de développer des programmes éducatifs. En effet, la mission de l'Institut est de :

promouvoir le renouvellement et le développement de la culture métisse à travers la recherche, la mise au jour, la collecte et la distribution d'éléments divers, ainsi que le développement et la livraison de services et de programmes éducatifs axés sur les Métis. (Gabriel Dumont Institute 2005, notre trad.)

On le voit, donc, le projet de l'École communautaire AurèleLemoine s'inscrit dans un processus global de volonté de reprise en main de l'éducation par les communautés autochtones. Ce qui distingue sans doute l'expérience de Saint-Laurent, c'est que dans la plupart des cas la mise en place de projets éducatifs communautaires et écologiques nécessite l'établissement de compromis destinés à faire coexister les deux modes d'éducation
(Stairs 1994; Friesen et Friesen 2002). Ces compromis qui altèrent l'intégrité du modèle éducatif écologique et holistique autochtones sont généralement considérés comme un mal nécessaire, tandis que dans le cas des Métis cette coexistence de deux types d'institutions qui finissent par s'interpénétrer est considérée comme positive.

En effet, à l'École communautaire Aurèle-Lemoine, l'hybridation ne se fait pas de façon accidentelle. Au contraire, il s'agit plutôt d'un projet politique dont l'objet est de permettre à la culture métisse de se développer comme elle l'a toujours fait, c'est-à-dire en se construisant à partir des deux univers sociaux dans lesquels elle trouve ses racines, sans renier l'un et sans ignorer l'autre. Ainsi, les connaissances traditionnelles, souvent enseignées par les aînés et fondées sur des observations empiriques, sont apprises parallèlement aux contenus scolaires actuels, fondés sur les connaissances occidentales produites par la science. La nécessité d'inculquer aux élèves les fondements de la rationalité moderne en même temps que le savoir traditionnel nous a été mentionnée, à maintes reprises, par le directeur et les enseignants de l'école, qui reconnaissent que «la société contemporaine est fondée sur le primat de la connaissance scientifique. Il faut que nos élèves aient accès à cette connaissance pour pouvoir survivre dans un monde de plus en plus moderne », tel que l'a souligné une enseignante qui s'est empressée pourtant d'ajouter: «ce n'est pas parce que nous leur apprenons ce que la science dit, par exemple en biologie, en mathématiques, que nous ne leur apprenons pas aussi ce que nous savons d'après nos traditions sur la nature ». En fait, l'imbrication des deux niveaux de savoirs se fait de façon réflexive, la science moderne est enseignée pour son utilité mais ne prend pas systématiquement le pas sur le savoir traditionnel, et inversement. Ainsi, la science historique n'est pas exclue du programme pour être totalement remplacée par des récits mythologiques, comme le voudrait un retour pur et dur à la tradition; au contraire, l'histoire, les textes et les documents d'historiens sont utilisés abondamment en classe; c'est l'interprétation, la lecture de ces événements qui est faite selon une grille de lecture traditionnelle. La manière dont les élites de Saint-Laurent construisent cette école, atteste un travail analytique et réflexif dont le but est de faire émerger une culture viable à partir d'une éducation qui permet de préparer les jeunes au marché du travail sans pour autant les acculturer complètement. Certes, pour cela, la tradition n'est pas transmise en bloc mais par bribes, là où elle est compatible avec la modernité, ou bien elle est mise au premier plan quand cela contribue à renforcer le sentiment de fierté ou d'appartenance. Cette stratégie, empruntant le terme à Giddens (1994), nous l'avons déjà qualifiée de « réflexive » (Martin 2001, 2003).

Ce que nous n'avions pas vu ailleurs, en tout cas pas aussi clairement, c'est que cette réflexivité se construit dans le conflit, ou tout au moins dans l'opposition. En effet, la création de l'école franco-métisse ne s'est pas faite dans l'harmonie mais a conduit les Métis francophones à se distancier des Métis anglophones pour se rapprocher d'une autre solitude, celle des Canadiens français. Ce mouvement leur a permis de s'arrimer à un ensemble culturel global moins acculturant que le monde canadien anglais. Le résultat est que l'École communautaire Aurèle-Lemoine participe à la création d'une communauté qui n'est pas liée au territoire géographique mais à un territoire symbolique dont les limites sont définies par la langue. Cela tend à indiquer qu'au sein de la modernité, l'ethnicité - et les Métis se définissent eux-mêmes comme un groupe distinct - 
est moins une question de filiation biologique que de pratiques culturelles, même si, paradoxalement, la filiation fait partie des éléments que les Métis considèrent eux-mêmes comme essentiels dans la définition de ce qu'ils sont (Martin et Patzer 2004). Mais dans le cas de Saint-Laurent, la proximité généalogique ne suffit pas à maintenir la proximité sociale, celle-ci est avant tout déterminée par la proximité linguistique.

\section{MOdernité Et INSTITUTIONNALISATION DE LA DIFFÉRENCE}

De la fin des années 1980 jusqu'au milieu de la décennie 1990, la communauté métisse de Saint-Laurent qui faisait face à une crise économique importante, conséquence d'un repli des activités industrielles de type moderne, choisit de mettre au centre de ses priorités de développement le redéploiement de la culture traditionnelle. L'école fut choisie comme le lieu privilégié pour entamer ce projet de reconstruction sociale. Toutefois, pour redonner un avenir à la langue mitchif, il fut décider de créer, au sein de la communauté, une nouvelle école qui ne serait pas de langue anglaise. Ce projet de prise en charge de l'éducation, et surtout cette volonté de créer une école spéciale pour les Métis francophones, entraîna un certain nombre de conflits au sein de la communauté et obligea les tenants du projet à en faire un projet politique. Pour faire aboutir ce projet, ces derniers se rapprochèrent d'une autre minorité linguistiquement proche d'eux, les Franco-Manitobains, et décidèrent d'adhérer à la Division scolaire franco-manitobaine (DSFM). Il s'agissait, d'une certaine manière, d'un compromis puisque le mitchif, bien que proche du français, est toutefois considéré par les Métis comme une langue distincte. Cela dit, le compromis fut assez facile puisque la DSFM, soucieuse de s'associer aux descendants des fondateurs de la province, leur laissa une grande liberté dans l'intégration de contenus d'enseignement.

Cette alliance permit aux Métis de réaliser leurs objectifs en termes de valorisation et de maintien de la culture traditionnelle au sein de l'école, sans toutefois les enfermer dans un univers folkloriste puisqu'elle leur a permis de se connecter avec l'univers global de la francophonie. Cette association s'accompagne d'ailleurs de l'adhésion de la communauté à des réseaux provinciaux et internationaux reliés sur lesquels les leaders locaux s'appuient pour obtenir le soutien nécessaire au développement de leurs projets culturels et économiques. Néanmoins cette association avec la francophonie génère des conflits au sein de la communauté dans la mesure où elle institutionnalise, à travers l'école, la séparation préexistante entre Métis anglophones et francophones. Cette division a créé un nouvel espace politique propre aux francophones, que l'école en tant qu'agent de socialisation contribue à transmettre aux jeunes. Ainsi, la modernité définie par les Métis francophones, et dont l'école constitue la pierre angulaire, génère et garantit la reproduction de la différence. En cela, l'école de Saint-Laurent ne fonctionne pas comme une institution moderne.

C'est donc une tout autre vision de la modernité que donne à voir l'étude de l'École communautaire Aurèle-Lemoine. En effet, la perspective sociohistorique choisie dans le cadre de cet article rend nettement visible les ruptures, les ajustements et les reconfigurations caractéristiques de la modernité métisse. Ainsi, celle-ci apparaît comme le produit d'un bricolage social continuel, original qui repose sur l'hybridation, c'est-à-dire sur la dynamique culturelle au coeur du mode de production de la nation métisse et, ce, depuis son émergence au temps de la Prairie.

\section{Notes}

1. Cependant, plusieurs acteurs politiques évaluent la proportion de Métis à $80 \%$ du fait, selon eux, que de nombreuses personnes cachent encore aujourd'hui leur origine métisse.

2. Cette deuxième recherche s'inscrit dans un projet de recherche d'envergure: "Initiative on the New Economy of the Social Sciences and Humanities Research Council of Canada », via Manitoba Research Alliance on Community Economic Development in the New Economy. Ce projet a été financé par le Conseil de recherches en sciences humaines du Canada. Pour plus d'information consulter http://www.brandonu.ca/organi $\downarrow$ zations/rdi/MRA.html

3. L’un des auteurs de ce texte a été représentant élu à la Commission scolaire franco-manitobaine pendant deux ans, et ce constat se fonde sur les commentaires qu'il a relevés lors des multiples débats auxquels il a participé.

\section{Ouvrages cités}

BALANDIER, Georges, 1985: Sociologie des Brazzavilles noires. Presses de la fondation nationale des sciences politiques, Paris.

BASHUTSKI, Barry, et al., 2003: That's A Good Idea - Effective Practices in First Nations and Métis Education. Regina, Saskatchewan School Trustees Association.

BISHOP, Russell, et Ted GLYNN, 1999 : Culture Counts: changing power relations in education. Zed Books, London et New York.

BLAY, Jacqueline, 1987 : L’article 23. Les péripéties législatives et juridiques du fait français au Manitoba 1870-1986. Les Éditions du Blé, Winnipeg.

BRITISH COLUMBIA HUMAN RIGHTS COMMISSION, 2001 : Pathways to Equality: Hearings on Access to Public Education for Aboriginal People. British Columbia Human Rights Commission, Vancouver.

CANADA, 2001 : Enquête auprès des peuples autochtones. Statistique Canada, <http://www.12.statcan.ca/english/profilolaps/high.」 lights.cfm>, (page consultée le 15 janvier 2005).

DE TRÉMAUDAN, Auguste Henri, 1984 [1936] : Histoire de la nation métisse dans l'Ouest canadien, Éditions des Plaines, Saint-Boniface.

DSFM, 2005 : Site Internet de la Division scolaire franco-manitobaine, accessible à l'adresse: <http://www.dsfm.mb.ca>, (page consultée le 14 juillet 2005).

FRIESEN, John W., et Virginia Lyons FRIESEN, 2002 : Aboriginal Education in Canada: A Plea for Integration. Detselig Enterprises Ltd., Calgary.

GABRIEL DUMONT INSTITUTE, 2005 : Mission de l'Institut, Gabriel Dumont Institute, <http://www.gdins.org/AboutGDI.shtml\#. missionstatement>, (page consultée le 14 juillet 2005).

GIDDENS, Anthony, 1994 : Les Conséquences de la modernité. Paris, Éditions de l'Harmattan.

GOFFMAN, Erving, 1975 : Stigmate. Les usages sociaux des handicaps. Les Éditions de Minuit, Paris.

JOHNSON, Geneviève Marie, et Reinhild BOEHM, 1995 : « Aboriginal Canadian University Students: A Comparison of Students Who Withdraw and Students Who Continue », Australian Journal of Adult and Community Education 35(2): 56-141.

LAFONTANT, Jean, et Thibault MARTIN, 2000 : "Lamour de la langue et les langues de l'amour $»$. Recherches sociographiques 41(2) : 479-508

LAVALLÉE, Guy, 2003: The Metis of St. Laurent, Manitoba: Their Life and Stories, 1920-1988. Éditions Guy Lavallée, Winnipeg.

MARCHAND, Anne-Sophie, 1997 : « Louis Riel : « lieu de mémoire » et de métissage des identités culturelles franco-manitobaines ». Cahiers franco-canadiens de l'Ouest, 9(1-2) : 35-53. 
MARTIN, Thibault, 2001 : "The Reflexive Community. Quest for Autonomy as a Coping Strategy in an Inuit Community», in Jorgen Ole Baerenholdt et Nils Aarsaether, Coping Strategies in the North. Local Practices in the Context of Global Restructuring (vol. 2) : 41-69. MOST-UNESCO Copenhagen.

—, 2003 : De la banquise au congélateur. Mondialisation et culture au Nunavik. Unesco et Presses de l'Université de Laval, Paris et Québec.

MARTIN, Thibault, et Jeremy PATZER, 2004 : « Yvon Dumont ou la renaissance du leadership métis ». Revue d'éthique et de théologie morale 12 : 379-404.

NEIL, Roger, 2000: Voice of the Drum: Indigenous Education and Culture. Canadian Social Science and Humanities Research Council, Ottawa.

ONG, Walter J., 1988 : Orality and Literacy: the technologizing of the word. New Accents. New York.

SASKATCHEWAN DEPARTMENT OF EDUCATION, 1991 : Indian and Metis Education Policy from Kindergarten to Grade 12, Saskatchewan Education, Regina.

SASKATCHEWAN SCHOOL TRUSTEES ASSOCIATION, 1993 : Indian and Metis Education: Parents as Partners. SSTA Forum
Report, Saskatoon, Saskatchewan, Canada, October 14-15, 1993. Saskatchewan School Trustees Association, Regina.

SCHISSEL, Bernard, et Terry WOTHERSPOON, 2002 : The Legacy of School for Aboriginal People. Oxford University Press.

SEALEY, Margaret, 1974 : Six Metis Communities. Manitoba Metis Federation Press, Winnipeg.

SHORE, Fred, 2001: "The Emergence of the Métis Nation in Manitoba», in J. BARKWELL, Leah DORION et Darren R. PREFONTAINE (dir.), Métis Legacy: 71-78. Institut Louis Riel et Institut Gabriel Dumont, Winnipeg et Saskatoon.

STAIRS, Arlene, 1994: "Culture and Education: Aboriginal Settings, Concerns, and Insights », Canadian Journal of Education 19(2): 96-121.

STANLEY, George F.G., 1961: The Birth of Western Canada: A History of the Riel Rebellions, University of Toronto Press, Toronto.

WAGNER, Serge, 2000 : «Formation de base écologique chez les autochtones du Canada », in Jean-Paul Hautecoeur (dir.). Éducation écologique dans la vie quotidienne, Ministère de l'Éducation du Québec et Institut de l'UNESCO pour l'éducation, Québec.

WARNIER, Jean-Pierre, 1999 : La Mondialisation de la culture. La découverte, Paris.

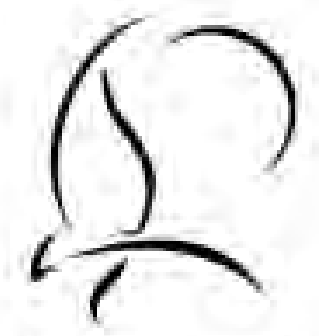

\title{
Présence autochtone 2006
}

\author{
Du 26 mai au 8 juin et du 21 au 25 juin 2006
}

Le grand rendez-vots des Premières Nations ouvre pour une seizième ânnéc la saison des lestivals au coeur de Montréal. Du pare Émilie-Gamelin à la Grande bibliothèque en passant par le Cínéma ONF et la Cinèmathèque québécoise, c'est tout un quartier de la métropole culturelle du Québec quí vibre aux accents, aux rythmes et aux couleurs des artistes, artisans, musiciens, écrivains, cinéastes of danseurs parmi les meilleurs des Premieres Nations des irois Amériques.

En 2006, les cultures et traditions dé l'Iroquoisie seront à l'honneur. www.nativelynx.qc.ca

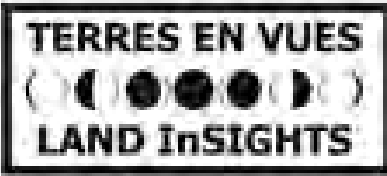

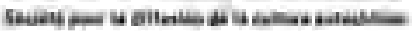




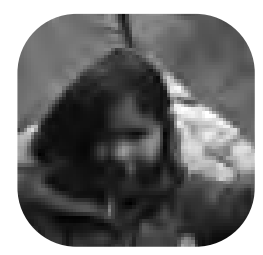

Être jeune et maori aujourd'hui :

l'université comme site de (ré)affirmation et de coexistence $^{1}$

Natacha

Gagné

Unité mixte de recherche Genèse et transformation des mondes sociaux (GTMS), (EHESS-CNRS),

et

Université

d'Ottawa
T 'ANALYSE QUE JE DÉVELOPPERAI dans ces pages s'attache aux expériences des jeunes Maoris comme acteurs dans les institutions de la société dominante en contexte urbain. Ces réalités maories et, en général, autochtones ont longtemps été négligées par les chercheurs. En particulier, il s'agit de comprendre les façons complexes par lesquelles les Maoris découvrent, maintiennent, remodèlent et/ou affirment leurs visions et leurs identités, et s'engagent de façon hétéroglossique à la fois dans les univers de sens maoris et non maoris. Il s'agit de s'attarder sur la manière dont les sens, les identités et les conceptions du monde émergent et se déploient dans la pratique, l'expérience et les interactions entre les personnes et l'environnement. La réalité décrite ici n'est pas considérée comme un domaine unitaire où les relations sociales et les expériences demeurent stables, mais comme un champ d'ambiguité, de pluralité et de lutte de différents ordres. Lexpérience des acteurs est comprise comme coexpérience ou interexpérience qui se module selon les contextes, les relations de pouvoir et les interrelations avec les autres, maoris ou non.

Cette étude s'inscrit dans les débats plus larges sur la décolonisation et l'autodétermination. Elle contribue, entre autres, à montrer comment les personnes et les groupes constituent et s'approprient les lieux, sur le plan physique comme politique, à travers leurs engagements dans ces mêmes lieux. L'université fut un des sites explorés lors d'études de terrain parmi les Maoris d'Auckland en 2001 et
2002. Avant d'entrer dans le vif du sujet, soit la signification de l'université comme lieu de (ré)affirmation, de résistance et de coexistence pour les jeunes Maoris, voyons d'abord quelques précisions quant au contexte de mes recherches ainsi qu'à la situation des Maoris dans le système universitaire néo-zélandais.

Ma première approche du terrain se fit à l'université d'Auckland puisque, dès mon arrivée, je m'y suis inscrite à des cours de langue maorie. C'est de cette façon que je fis la connaissance de plusieurs étudiants. La pause-café devint un moment privilégié pour bavarder. C'est ensuite par leur entremise que je pris part à la vie du marae de l'université : pratiques de kapa haka (danses et chants traditionnels maoris), retraites d'étude, cérémonies de graduation et autres activités.

Le marae est le lieu traditionnel de rassemblement des Maoris. C'est un site cérémoniel dédié à la pratique de rituels ainsi qu'à diverses activités socioculturelles (Salmond 1975 : 31). Ce lieu possède une dimension spirituelle et sacrée et incarne le lien à la terre et aux ancêtres qui y vécurent. C'est de ce lien que les membres des marae tirent leur identité et leurs droits (et obligations) sur le marae comme taangata whenua, c'est-à-dire comme membres du groupe qui entretient une relation spéciale avec le marae, soit à travers des liens généalogiques, soit par adoption. Nous reviendrons sur ce point plus loin. Chaque marae consiste en un ensemble de bâtiments, dont la wharenui (la grosse maison ou maison de rassemblement), 


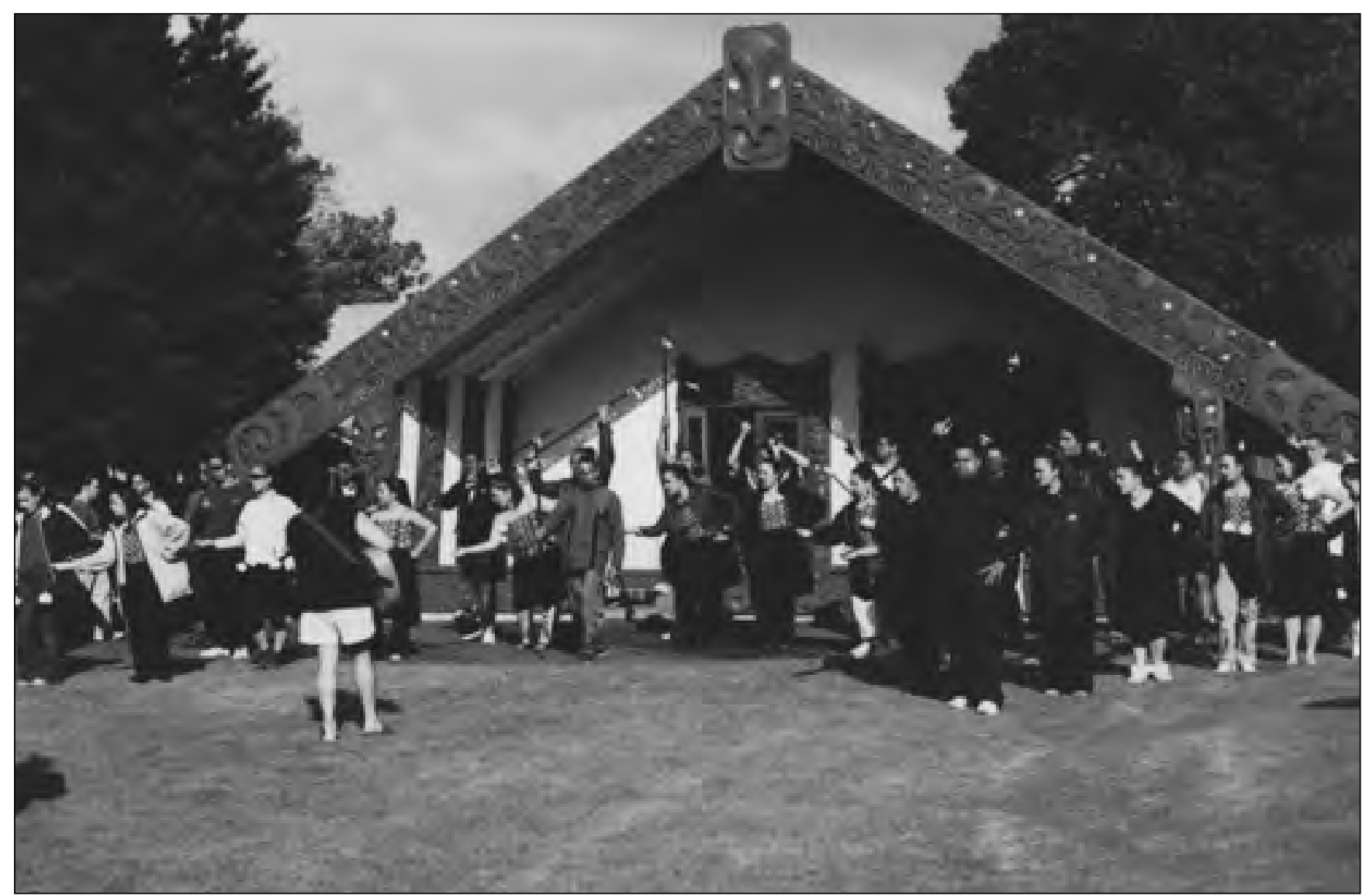

Maison de rassemblement Tanenuiarangi, Waipapa Marae, Université d'Auckland, mai 2002

(Photo Natacha Gagné et reproduite avec la permission de Rangimarie Rawiri, Erana Foster et Valance Smith)

une cuisine et une salle à manger. Le marae est équipé pour recevoir et loger des visiteurs. Il peut aussi comprendre d'autres bâtiments comme un dortoir, une église et une école. Lensemble est habituellement clôturé (pour des détails, voir Tauroa et Tauroa 1986, Salmond 1975).

La wharenui qui peut aussi être appelée whare tipuna (maison ancestrale) est nommée du nom d'un ancêtre. Dès lors, non seulement la maison représente l'ancêtre, mais elle l'incarne et l'on s'adresse à elle comme à une personne vivante. Si la maison est sculptée ou peinte, toutes les formes se rapportent à différentes figures, personnages ou éléments ancestraux. À l'intérieur de la maison, on est donc entouré et protégé par les ancêtres et par les dieux. La maison est un lieu hautement tapu (sacré) par opposition à la cuisine et à la salle à manger, par exemple, qui sont des lieux noa (libres de restrictions religieuses, ordinaires).

Lune des fonctions principales du marae est d'accueillir des visiteurs. Traditionnellement, le terme marae ou marae atea (cour cérémonielle) désignait seulement la portion de terre située en face de la maison de rassemblement. C'est encore aujourd'hui sur le marae atea que sont accueillis les visiteurs et que sont échangés les discours formels lors du powhiri (cérémonie d'accueil). Les gens utilisent de nos jours l'appellation « marae proper» pour distinguer la cour cérémonielle de tout le complexe de bâtiments.

Traditionnellement, les marae étaient soit familiaux, soit tribaux. À partir des années 1960, étant donné l'importante migration urbaine suivant la fin de la Seconde Guerre mondiale, des marae tribaux et pantribaux furent construits en ville. Ces marae urbains permirent aux Maoris qui vivaient trop loin de leur propre marae pour les visiter régulièrement de se rassembler en ville et de tenir les tangihanga (funérailles), un rituel aujourd'hui considéré par plusieurs comme le plus important. Plusieurs institutions scolaires et gouvernementales se dotèrent aussi de marae. Comme nous le verrons, le marae de l'université est un lieu très important pour les étudiants maoris en termes identitaires, en termes de confort ${ }^{2}$, mais aussi comme lieu de discussion et d'échanges.

À l'université d'Auckland, le marae fut inauguré en 1988 après plusieurs années d'hésitation de la part de l'université, étant donné les fonds importants devant être investis. Pour les étudiants et le corps professoral maoris ainsi que pour leurs sympathisants non maoris, cela a signifié plus d'une décennie de mobilisation. Ce projet a d'ailleurs pris une tournure hautement politique et ethnicisée (Webster 1998: 201). En 1983, les étudiants en études maories installèrent des bâches - que les médias ont baptisées « the tent-marae " - devant le bureau du registraire en réaction à l'absence de progrès en vue de la construction du marae alors que l'université se préparait à célébrer son 100e anniversaire (Webster 1998: 194-195). Cette mobilisation, ainsi que l'arrivée de nouveaux fonds et des développements sur la scène nationale concernant les doléances maories et les responsabilités de l'État - et d'institutions 
comme les universités -, a amené l'université à amorcer les travaux en 1984.

Précisons qu'à la suite de revendications maories de plus en plus fortes dans les années 1960 et 1970 - période qu'il est maintenant convenu d'appeler la Renaissance culturelle maorie -, l'État a fait du biculturalisme une politique officielle ${ }^{3}$. Cette politique trouve son fondement dans le Traité de Waitangi signé en 1840 par des chefs maoris et la Couronne britannique. Ce traité - qui a soulevé de nombreux malentendus à travers l'histoire (voir, par exemple, Kawharu 1990) - est interprété aujourd'hui comme reconnaissant un partenariat entre deux peuples, les Maoris et les Pakehas (population aux origines principalement européennes) et, sur cette base, est largement considéré comme le document fondateur de la Nouvelle-Zélande. Après maintes violations, l'importance du Traité de Waitangi fut réaffirmée en 1975 par une loi qui établit le Tribunal de Waitangi. Le tribunal fut chargé d'investiguer les doléances et revendications maories liées à des violations du Traité et de faire des recommandations au parlement en vue d'accords avec les plaignants. Avec cette loi, le Traité de Waitangi est devenu un cadre interprétatif important dans les relations entre Pakehas et Maoris de façon générale, mais aussi entre le gouvernement et les Maoris. Différentes mesures ont été prises à tous les plans de la société pour s'assurer d'une représentativité maorie adéquate selon les termes du Traité et du redressement de certains torts historiques. C'est dans ce contexte que la construction du marae à l'université s'est imposée. Sa construction a ouvert de nouvelles opportunités pour les Maoris du campus, à la fois en termes culturels et en termes d'emplois.

Les universités néo-zélandaise, en particulier l'université d'Auckland, ont été depuis longtemps des sites importants d'affirmation et de résistance pour les Maoris. Dans les années 1960 et 1970, elles ont été centrales dans le mouvement de la Renaissance culturelle maorie. Plusieurs des figures de proues de ce mouvement qui sont devenues par la suite des leaders en éducation et en politique active ont d'ailleurs été formées à l'université d'Auckland. Pensons simplement à Pita Sharples, Graham Hingangaroa Smith et Linda Tuhiwai Smith, tous engagés depuis le début dans le mouvement maori en éducation et la création des écoles maories. Aujourd'hui, Pita Sharples, entre autres fonctions, est professeur à la Faculté d'éducation de l'université d'Auckland, leader d'un marae urbain pan-tribal, député et coleader d'un nouveau parti politique, le Maori Party, fondé en juillet 2004 et qui, depuis les élections de septembre 2005, a quatre membres élus au parlement. Linda Tuhiwai Smith, quant à elle, est professeure à l'université d'Auckland et codirectrice de Ngaa Pae o te Maaramatanga / The National Institute of Research Excellence for Maori Development and Advancement, créé en 2002. Elle est aussi l'auteure du livre Decolonizing Methodologies (1999) qui a acquis une réputation internationale. Quant à Graham Hingangaroa Smith, il est invité à l'université de Colombie-Britannique comme professeur distingué en études autochtones.

Linda Tuhiwai Smith a aussi fait partie, avec des collègues de sa génération tels que Syd et Hana Jackson, Peter Rikys et Donna Awatere (Walker 2004 : 210), du groupe Ngaa Tamatoa ('les jeunes guerriers') qui fut très actif dans les années 1970 et qui avait pour but de remettre en question l'establishment pakeha. Ce groupe, fondé à l'université d'Auckland, a mis sur pied une série de programmes à Auckland et à Wellington. Entre autres, il dispensait de l'aide aux migrants maoris qui arrivaient en ville en quête de travail et des conseils en matière légale aux Maoris appelés à comparaître en cour (Walker 2004 : 210-211). Le groupe a aussi fait pression en faveur de l'inclusion de la langue maorie dans le système d'éducation.

De plus, en 1979, un groupe de protestataires maoris, He Taua ('les vengeurs'), s'en prirent à des étudiants en ingénierie qui parodiaient un haka («danse» guerrière) [Walker 2004: 221-225]. Cette parodie était jouée depuis plus d'une vingtaine d'années lors de la journée de graduation à l'université d'Auckland. Le but de He Taua était de mettre un terme à la moquerie après que plusieurs démarches diplomatiques intentées depuis 1971 eurent échoué. Ce raid fut condamné par la presse comme violent et entraîna onze arrestations. Lévénement donna pourtant lieu à un important mouvement de solidarité et de dénonciation du racisme en Nouvelle-Zélande qui alla bien au-delà des murs de l'université puisque, lors du jugement, étaient présents les parents des accusés, des aînés maoris, des étudiants de l'université, un contingent d'étudiants de l'université Waikato de Hamilton ainsi que les présidents des plutôt conservateurs Maori Council, Maori Women's Welfare League et Auckland District Maori Council. Cet événement, qui devint largement connu comme " the Haka Party incident » (Webster 1998 : 192), accéléra l'approbation du projet d'un marae par les autorités de l'université en 1979 (Webster 1998 : 193).

Au cours des années qui suivirent, les Maoris firent des avancées importantes de façon plus générale en éducation. Les années 1980 ont vu la création des kohanga reo (écoles préscolaires basées sur les principes de la famille étendue et l'utilisation de la langue maorie) qui sont aujourd'hui au nombre de sept cents (Walker 2004: 344) et des kura kaupapa (écoles maories), maintenant au nombre de cinquante-neuf au niveau primaire et de quatre au secondaire (Walker 2004: 344). Quant aux années 1990, elles ont vu la mise sur pied des whare waananga (établissements maoris postsecondaires) qui sont maintenant au nombre de trois. Le taux de fréquentation des institutions postsecondaires par les Maoris est en progression, bien que plutôt lente, passant de 10,7\% en 1994 à 13,0\% en 2000 (Walker 2004: 359). Les initiatives maories en éducation ont fait et font encore beaucoup pour améliorer la perception de l'école parmi les jeunes Maoris, l'absentéisme et le décrochage scolaires étant des problèmes de longue date.

\section{ÊTRE ÉTUDIANTS À L'UNIVERSITÉ D'AUCKLAND AUJOURD'HUI}

À l'université d'Auckland, les étudiants maoris représentent $5,4 \%$ de la population étudiante totale. Cela correspondait à 1905 étudiants sur un total 35085 en $2003^{4}$. Quand je suis arrivée sur le terrain en 2001, les étudiants de mes cours de langue maorie provenaient surtout de la Faculté des arts. Plusieurs étaient des étudiants adultes (c'est-à-dire ne venant pas du secondaire; leur âge allait du début de la vingtaine à la fin de la soixantaine) qui fréquentaient l'université pour la première fois ou qui retournaient à l'université après quelques années sur le marché du travail ou à la maison à s'occuper des enfants. Plusieurs envisageaient sérieusement faire leur diplôme en droit, ce qui n'était pas surprenant puisque plusieurs apprenaient la langue maorie de façon à pouvoir travailler pour les Maoris. Un moyen évident et utile, selon plusieurs des participants à mes recherches, est bien de plaider en leur faveur dans les cours de justice. D'autres étaient inscrits à des programmes avec des composantes en études maories, en histoire, en musique, en anthropologie et dans d'autres disciplines des sciences sociales. Très peu étudiaient en sciences naturelles, 
ingénierie, commerce ou médecine. Cette situation reflétait assez bien le fait que les Maoris sont sous-représentés en général dans ces disciplines (Durie 2001: 9; Equal Opportunities Office 2003), ceux-ci, par exemple, ne représentant que 1,9\% des étudiants de la Faculté d'ingénierie, 2,4\% des étudiants de la Faculté des sciences et 3,2\% des étudiants de la Faculté de commerce et d'économie (University of Auckland 2004). Les étudiants maoris représentent par ailleurs 8,6 \% des étudiants de la Faculté des arts, ce qui équivaut au tiers d'entre eux, soit 33,5\% (University of Auckland 2004). Notons que quelques programmes visent à inciter plus de Maoris à s'inscrire dans les disciplines où ils sont sous-représentés, dont des bourses de soutien pour les étudiants ayant d'excellents résultats et un programme de recrutement s'adressant aux élèves de 12 et 13 ans pour les informer des possibilités dans les secteurs des sciences, technologie, ingénierie, architecture, médecine et beaux-arts, et des pré-requis nécessaires pour accéder à ces programmes ${ }^{5}$. Certains programmes contingentés admettent aussi un nombre limité d'étudiants maoris dont la moyenne est inférieure à la moyenne requise. Ces étudiants bénéficient par la suite d'un suivi particulier. Notons que tous ces programmes s'inscrivent dans le contexte plus large de la politique biculturelle découlant de la reconnaissance du Traité de Waitangi. Lassociation étudiante maorie, Ngaa Tauira Maaori (NTM), a aussi un programme appelé Haerenga ('voyage') [voir http://www.ntm.maori.nz/] dans le cadre duquel, chaque année, un groupe d'étudiants visitent les marae d'une région tribale pour y rencontrer les jeunes et les inciter à poursuivre des études universitaires et les informer des différentes options possibles.

Les étudiants de mes cours, ainsi que les enseignants, furent mes premiers contacts avec les mondes maoris. J'ai été privilégiée de partager avec eux une expérience très importante et plutôt stressante : aller à l'université pour la première fois ou retourner aux études après avoir eu des enfants ou après quelques années sur le marché du travail. Comme nous le verrons, le monde universitaire n'est pas toujours des plus faciles pour les étudiants maoris...

Étant donné cet ancrage à l'université, mon échantillon fut en majorité composé d'étudiants qui apprenaient la langue maorie, qui étaient engagés dans le groupe de kapa haka (danses et chants maoris) et/ou dans l'association étudiante maorie et qui gravitaient autour du marae. Mes analyses sont donc moins représentatives d'étudiants qui avaient peu à voir avec ces sites. La majorité des étudiants qui ont participé à mes recherches étaient directement engagés dans des processus d'apprentissage de la langue et de l'histoire maories et de conscientisation aux problématiques maories. Le fait que la majorité était aussi des étudiants de la Faculté des arts - localisée juste à côté du marae et dont plusieurs des départements ont des affinités plus immédiates avec les études maories ou offrent des cours portant directement sur les Maoris et la Nouvelle-Zélande (comme l'histoire, la sociologie et la science politique) - accentue sûrement l'importance qu'ils accordaient, comme nous le verrons, à la (ré)affirmation de leur identité maorie et aux lieux maoris de l'université. Des étudiants d'autres facultés sont pourtant aussi engagés dans les lieux maoris de l'université et ils figuraient parmi les participants à mes recherches.

Précisons encore que le terme rangatahi est de nos jours souvent utilisé pour parler des jeunes Maoris. Ce terme possède un large éventail de sens et peut référer à différentes catégories de personnes. Muru-Lanning (2004: 4) soulignait d'ailleurs l'ambiguïté entourant le terme, celui-ci pouvant signifier, selon les contextes, « adolescent», « jeune leader», « adulte», "membre d'une cohorte de la génération suivante », « à l'avant-garde », « une classe d'âge » ou « quelqu'un n'ayant pas atteint la pleine maturité ». La définition de cette catégorie varie aussi en fonction des tribus ainsi que des instances gouvernementales ou autres qui utilisent ce concept pour définir à qui s'adressent certains programmes ou activités.

Une personne peut aussi être considérée comme rangatahi, selon les contextes particuliers et les personnes en relation avec elle. À partir d'un certain âge, le niveau de connaissance des protocoles traditionnels et de la langue maorie ainsi que le mana (pouvoir spirituel, autorité, statut, prestige) jouent un rôle significatif quant à savoir si quelqu'un est toujours un rangatahi ou est passé au stade suivant. Tout dépendant des critères retenus et des contextes, la catégorie « rangatahi » peut inclure des personnes âgées de 12 à 50 ans (Muru-Lanning 2004).

Je parlerai ici des étudiants fréquentant l'université comme des jeunes ou rangatahi même si certains des étudiants ne faisaient clairement pas partie de la tranche d'âge à laquelle on réfère généralement en Occident par le terme " jeune ». Ainsi, certains des étudiants un peu plus âgés, soit ceux de la fin trentaine, début quarantaine, seront ici considérés comme des rangatahi ou jeunes parce qu'ils sont dans un processus de formation, parce qu'ils partagent une expérience commune avec leurs plus jeunes collègues étudiants et surtout, selon Muru-Lanning (comm. pers., janv. 2005), parce qu'ils font partie du groupe de rangatahi.

Comme nous le verrons, l'entrée à l'université est vécue par plusieurs comme une étape charnière, celle de la « découverte » et/ou de la (ré)affirmation de leur(s) identité(s) maories. J'entends explorer de façon particulière comment l'université est un lieu important où les jeunes Maoris affirment et négocient diverses identités et où s'expriment et se jouent les rapports aux autres, Maoris et non-Maoris. J'insisterai aussi sur la fluidité et la multiplicité des identités et des engagements maoris dans différents mondes.

\section{L'ENTRÉE À L'UNIVERSITÉ}

Cette période de l'entrée à l'université est souvent très émotive car, pour plusieurs, c'est l'occasion des premiers contacts avec la grande ville avec ce que cela entraîne comme excitation, découvertes, nouveaux espaces de liberté, mais aussi comme moments de solitude et craintes de toutes sortes. Plusieurs font vite l'expérience de l'université comme un lieu étranger, austère et même hostile, un lieu non maori où l'on perd ses repères. Linconfort chez mes collègues étudiants était souvent palpable à travers leur langage corporel. Du lieu lui-même - l'université étant vue et reconnue comme un lieu supérieur, un symbole d'avancement et de savoir - semble se dégager une force extrêmement puissante qui intimide et même qui effraie plusieurs étudiants maoris qui sont souvent les premiers et les seuls de leur famille à fréquenter l'université. Plusieurs des étudiants m’ont aussi parlé de l'université comme d'un lieu colonisé. Ce sentiment n'est sûrement pas étranger au fait que les Maoris ne représentent qu'un faible pourcentage des étudiants. C'est aussi à l'université qu'on est directement confronté à la présence des « deux mondes », l'un pakeha (en référence à la population d'origine européenne) et l'autre maori ${ }^{6}$. En fait, pour plusieurs étudiants maoris, tout semble divisé en deux mondes bien distincts dont on accentue les différences et les incompatibilités. Par exemple, le marae de l'université et le département d'études maories sont souvent décrits comme constituant le 
monde maori alors que « de l'autre côté [du terrain de stationnement] », c'est le monde pakeha. Dans le terrain de stationnement, m'expliquait Margaret (tous les noms des participants sont des pseudonymes), on peut déjà sentir la présence des deux mondes.

Cette vision peut paraître plutôt dichotomique et je ne veux surtout pas réifier ces « deux mondes ». Ce qui est intéressant, c'est plutôt d'analyser les processus rhétoriques et idéologiques qui sont à l'œuvre puisqu'en observant les pratiques des étudiants dans le quotidien, il apparaît que la frontière entre les mondes n'est pas si étanche et que les gens y participent de façon plutôt fluide. Plusieurs étudiants qui s'identifient comme Maoris sont d'ailleurs issus d'unions mixtes (Chapple 2000) et plusieurs sont familiers avec ces « deux mondes ». La frontière n'est pas perçue et vécue de la même façon par tous et cela dépend de plusieurs facteurs, dont les circonstances particulières, le confort personnel et collectif dans les différents mondes, les périodes de la vie, les expériences passées, les réseaux sociaux, les conditions socio-économiques, la classe sociale ainsi que le contexte sociopolitique plus large. Les façons dont ces mondes sont définis varient aussi énormément, ce qui fait qu'il y a plusieurs mondes maoris, comme il y a plusieurs mondes non maoris.

Pour un des responsables de l'association des étudiants maoris, le caractère « étranger » de l'université est aussi attribuable à ce qui est enseigné et à ce qui est valorisé dans l'enseignement :

Il valide un ensemble très particulier de connaissances, une façon très particulière d'apprendre et d'utiliser la connaissance. C'est donc très spécifique. Pour ceux [...] qui ont grandi avec une façon différente de penser, quand tu es forcé à t'insérer dans un système dont tu n'as pas acquis la compréhension, tu es mis dans une position très difficile où tout ce que tu apprends est en conflit avec la façon dont tu as été élevé et c'est ce qui arrive à beaucoup de Maoris. Ce qu'ils ont appris est invalidé dans le système éducatif actuel et ils sont forcés de s'insérer dans quelque chose qui, finalement, finit souvent par leur paraître assez hostile [...] Bien sûr, dans ce cursus monolithique, on retrouve les Maoris et on leur a attribué un rôle, ce que j'appelle un rôle servile, dans lequel être maori équivaut à être mauvais, idiot, pauvre, tu sais, toutes les choses négatives. (Robert)

Cette idée est largement défendue par plusieurs spécialistes en éducation maorie dont Smith L.T. (1999) et Smith G.H. (1997). Le mouvement des écoles maories est d'ailleurs né, en partie, de cette constatation.

La popularité des études maories est un cas éclairant. En effet, en 2003, dix-sept des soixante-seize diplômés maoris du baccalauréat en arts (B.A.) à l'université d'Auckland, soit près du quart, avaient comme première ou seconde majeure les études maories (University of Auckland 2004) ${ }^{7}$. Cette option n'est souvent pourtant pas le premier choix de plusieurs, mais un choix plus ou moins forcé. Avant de s'y rabattre, plusieurs ont fait face à une concurrence presque démesurée dans d'autres programmes sans pourtant que leurs résultats soient si mauvais. Plusieurs n'ont pas non plus les pré-requis nécessaires pour s'inscrire dans d'autres programmes. Pour les étudiants doués, le choix des études maories est aussi fréquemment motivé par le désir d'être à l'abri du racisme. Ils ont souvent eux-mêmes, ou des membres de leurs cercles d'amis ou de leur famille, fait l'expérience de différentes formes de discrimination dans leurs études antérieures. Le racisme et la discrimination sont encore bien présents aujourd'hui et s'expriment de différentes façons dans tout le système scolaire. Pour d'autres, le choix des études maories s'est imposé à cause de talents « naturels » pour tout ce qui a trait à la langue et aux arts maoris, des talents très valorisés dans les écoles maories, dans les événements maoris en tous genres et dans les médias, surtout maoris. Beaucoup d'importance est accordée à ces talents qui servent souvent à distinguer les Maoris « authentiques» des autres dans le cadre de la rhétorique opposant les « vrais » aux soi-disant « faux » Maoris - à laquelle je reviendrai.

Les explications sous-jacentes aux statistiques à propos de la répartition des étudiants maoris dans les différentes disciplines sont donc complexes. Elles relèvent de facteurs socioéconomiques, mais s'inscrivent aussi dans l'histoire des relations entre Maoris et Pakehas et des processus maoris d'affirmation et de résistance. L'histoire du développement des études maories est aussi à mettre en perspective puisque certains stéréotypes ethniques y furent renforcés (voir Webster 1998). Il est donc important de tenir compte de ces contextes complexes dans lesquels se font les choix de disciplines d'études puisqu'en jetant un regard sur le marché du travail, il ne va pas de soi que les études maories soient la meilleure façon de faire une carrière en Nouvelle-Zélande, bien que cette option ouvre certaines possibilités dans le cadre d'organisations maories et de certains programmes de représentativité équitable dans les institutions publiques en lien avec la politique biculturelle en vigueur. Il est à prévoir, par exemple, que le nouveau réseau de télévision maori entré en onde le 28 mars 2004 (Walker 2004: 402) contribuera à orienter les inscriptions vers les études maories, en particulier vers les différents programmes reliés à l'apprentissage de la langue maorie, aux médias et aux arts de la scène. Lors de nouvelles recherches de terrain en 2005, au fil de conversations informelles, une dizaine de jeunes m'ont justement fait part de leur désir de travailler pour ce réseau. Il ressort d'ailleurs d'entrevues avec des représentants maoris des divers médias (secteurs privé et public) un besoin criant de personnes bilingues et formées dans tous les secteurs de cette industrie.

Des programmes existent pourtant pour accueillir les étudiants à l'université et leur permettre d'apprivoiser ce lieu plus facilement comme le programme New Start qui, bien que ne s'adressant pas uniquement à des étudiants maoris, comprend des cours sur les habiletés de base nécessaires pour entreprendre des études universitaires. Plusieurs facultés ont aussi des programmes de parrainage Tuaakana (parents plus âgés, senior) qui jumellent un étudiant « aîné » en termes d'expérience universitaire avec un nouvel arrivant (Equal Opportunities Office 2003). De surcroît, dans les dernières années, l'offre universitaire s'est élargie pour offrir toute une gamme de programmes et de cours directement axés sur des problématiques maories ou touchant les populations du Pacifique.

Lassociation étudiante maorie contacte aussi les étudiants qui se sont identifiés comme Maoris lors de leur inscription pour les inviter à des activités d'accueil et leur faire visiter les locaux de l'association en leur disant que ce lieu leur appartient et qu'il leur revient d'en profiter. Lassociation s'occupe de plus de diffuser des informations sur les services offerts aux étudiants, dont ceux s'adressant spécifiquement à la clientèle maorie. Les étudiants sont aussi invités au marae pour un powhiri (cérémonie d'accueil). Ces activités sont, pour plusieurs, déterminantes pour la rencontre d'autres étudiants et la création d'un premier réseau d'amis. Cependant, Robert, un des leaders de l'association étudiante maorie, précisait que, de tous les Maoris sur le campus, seulement de cent à deux cents participent 
aux activités de l'association étudiante maorie. Le plus difficile pour lui est de constater que certains étudiants maoris complètent leurs études sans même en connaître l'existence.

La même chose est vraie pour la fréquentation des autres lieux maoris comme le marae : seulement une petite proportion les fréquente régulièrement et leur fréquentation varie selon les contextes, les périodes, les besoins personnels et collectifs, les réseaux d'amis et de collègues et le confort dans ces lieux. Ces lieux assurent une présence maorie importante à l'université une institution vue par plusieurs comme plutôt austère et étrangère à ce qu'ils sont - et sont le symbole d'un pouvoir certain. Leur faible fréquentation n'est pourtant pas un indicateur négatif par rapport à l'identité et aux pratiques culturelles maories puisque plusieurs étudiants demeurent solidement rattachés au marae de leur lieu de naissance, à leur tribu et à d'autres lieux maoris en ville. Certains étudiants préfèrent pourtant ne pas fréquenter ces lieux parce qu'ils ne s'y sentent pas à l'aise à cause des lieux eux-mêmes ou à cause des personnes qui s'y rassemblent. D'autres voient leur fréquentation comme pouvant, dans une certaine mesure, les empêcher par la suite de développer d'autres réseaux puisque les gens s'y rassemblant sont parfois vus comme formant une clique relativement fermée ${ }^{8}$. D'autres ne les fréquentent pas parce qu'ils ne leur apparaissent tout simplement pas nécessaires pour le moment ou simplement parce qu'ils manquent d'information à leur égard.

L'entrée à l'université, c'est donc l'occasion de la rencontre avec d'autres Maoris provenant de partout. Ces rencontres sont très enthousiasmantes, mais elles apportent aussi avec elles leur lot de difficultés. Entre autres, la rhétorique très politisée à propos des Maoris « réels » ou « authentiques » ou « vrais » par opposition aux Maoris urbains ou "plastiques » ou « faux » est prégnante. Dans ce contexte, plusieurs sont vite appelés à devoir justifier leur maoritude. À l'université, comme ailleurs, ces dichotomies sont liées aux stéréotypes et aux critères essentialistes sur ce qu'est être maori. Des critères liés à l'apparence physique et aux signes de richesse sont parfois utilisés pour disqualifier quelqu'un comme n'étant pas un «vrai » Maori, mais plutôt un Maori urbain, c'est-à-dire un Maori assimilé, fragmenté, et qui ne sait plus d'où il vient. J'ai souvent entendu des étudiants maoris parler d'autres étudiants en utilisant le terme riiwai (patate), signifiant ainsi que ces personnes sont brunes à l'extérieur et blanche à l'intérieur, et donc tout ce qui est considéré maori à leur propos est la couleur de leur peau. Cette comparaison n'est pas unique aux Maoris puisque les autochtones du Québec - et vraisemblablement d'ailleurs en Amérique du Nord - utilisent le terme bien connu " pomme » à des fins rhétoriques et idéologiques similaires. D’autres expressions sont aussi largement utilisées à l'université et ailleurs telles que « Ngati Tupperware » ou « Ngati Plastic», le préfixe «Ngati » signifiant « de cette personne ». En effet, les groupes traditionnels ont un ancêtre éponyme de qui tout le groupe descend. Par exemple, la tribu descendant de Porou s'appelle Ngati Porou (Waymouth 2003). Ainsi, parler de quelqu'un en termes de "Ngati Tupperware », c'est dire de cette personne qu'elle a perdu son affiliation tribale, qu'elle ne sait pas d'où elle vient, qu'elle n'est personne en termes maoris. " Tupperware » et « Plastic » accentuent davantage le caractère artificiel ou non authentique d'une personne et de son identité comme Maorie. D'autres diront que ces « autres non authentiques » sont pakehafiés. Cela résonne très bien avec l'utilisation de l'adjectif «blanchi » que j'ai entendu au Québec chez les Innus, par exemple.

Cette rhétorique sur ce que signifie être un « vrai » Maori empêche certains d'être à l'aise en ville ainsi que dans les institutions non traditionnellement maories ou occidentales. Ces expressions sont aussi utilisées pour promouvoir des façons particulières d'être ou de faire. J'ai souvent entendu à l'université des commentaires tels que « Regarde-la! Elle est habillée comme une Pakehae!» Ces commentaires s'adressaient à des personnes qui portaient, par exemple, des vêtements à la dernière mode ou encore des bijoux autres que le «traditionnel » pounamou (jade) qui possède une valeur symbolique. En pratique, ne pas avoir un « look maori » ou être considéré comme « trop pakeha » peut être un facteur d'exclusion même si quelqu'un se revendique Maori, possède tous les critères généalogiques lui permettant une telle identification et agit en respectant les valeurs et traditions maories largement reconnues comme importantes.

Il y a plusieurs façons de réagir à pareilles accusations. Plusieurs étudiants, par exemple, accentuent certains marqueurs reconnus de l'identité maorie. D'autres parlent beaucoup en termes de wairua (esprit, émotion émanant d'un lieu, d'événements ou de personnes) et font référence de façon répétée et ouverte à leurs tiipuna (ancêtres), disant que ceux-ci les accompagnent. Selon Keita, «c'est leur façon de dire 'Je suis maori' et d'être crus ». Ce serait aussi une façon d'établir des relations avec les autres Maoris et de faire partie du même monde en entrant dans la whaanau (famille étendue). Il y a pourtant des limites à respecter qui varient selon les contextes, les personnes en présence, les différences tribales, et il n'est pas toujours facile d'en juger. Certaines exagérations peuvent amener différentes formes d'exclusion. Des expressions telles que «try hard», «wannabe » ou «born again Maori » sont souvent utilisées pour décrire ces personnes « qui en font trop ». Tous ces noms provoquent beaucoup de souffrance. Ils touchent souvent à des points sensibles, dont les personnes visées étaient déjà très - ou même trop - conscientes. L'analyse des catégories rhétoriques est pourtant très éclairante, puisqu'on s'aperçoit vite que presque tout le monde - à l'exception de quelques personnes de grand mana (pouvoir spirituel, autorité, statut, prestige) largement reconnues comme les représentantes de la «vraie » maoritude - est la riiwai (patate) de quelqu'un d'autre.

Notons que, historiquement, l'identité maorie était autoproclamée. On était maori parce qu’on se disait maori. Il était seulement nécessaire de pouvoir faire la preuve qu'on avait un ancêtre maori quelque part dans sa généalogie. À la différence des autochtones au Canada, par exemple, il n'y a pas de «statut maori » qui pourrait se comparer au statut d'Indien tel que défini par la loi canadienne. Même aujourd'hui, l'identité maorie demeure plutôt fluide, c'est-à-dire que, chez chaque personne, son importance varie selon les contextes et les périodes de la vie (voir Chapple 2000; Gagné 2004). Cela tend pourtant à être de moins en moins vrai puisqu'on assiste de plus en plus à une essentialisation de cette identité - qui doit être comprise comme un effet de la dynamique des relations plus larges entre la population dominante et la population minorisée - et à son encadrement normatif. En effet, depuis le début des années 1980, le pouvoir de la rhétorique à propos des Maoris « réels » par opposition aux Maoris « urbains » ou « faux » s'est intensifié avec l'enchâssement juridique de la propriété maorie, un processus complexe qui suppose plusieurs éléments: 1) la reconnaissance par l'État néo-zélandais des tribus comme entités corporatives légales et la participation 
active concomitante des représentants des tribus à la retribalisation; 2) le renforcement de l'autorité et de la légitimité des tribus comme héritières des ressources et des connaissances traditionnelles; et 3) le nouvel accent mis sur le sang et l'ascendance comme unique critère d'accès aux propriétés et aux bénéfices. Ce processus d'essentialisation a été davantage marqué à mesure que la retribalisation progressait et qu'en découlèrent de nombreuses revendications devant les tribunaux, et aussi que des moyens et modes traditionnels de production furent mis aux services d'entreprises fonctionnant sur la logique capitaliste et que les généalogies furent bureaucratisées (Rata 2000). Ce processus est aussi renforcé par d'autres pratiques de l'État, par les médias, ainsi que par certaines élites maories qui ont des intérêts économiques et politiques dans les processus décrits ici (Rata 2000 et 2003). Il faut pourtant comprendre que ces élites jouent un rôle extrêmement important dans les relations entre les Maoris et l'État comme gardiennes de l'ordre social et symbolique et par la transmission culturelle qu'elles assurent et qui bénéficie à tous les Maoris. La rhétorique à propos des « vrais » par opposition aux «faux » Maoris est donc le fruit de processus historiques et s'inscrit dans des structures et des contextes sociaux plus larges.

\section{L'UNIVERSITÉ, UN SITE D'AFFIRMATION ET DE RÉSISTANCE}

La transition dans le monde universitaire n'est donc souvent pas facile, et plusieurs abandonnent assez vite. Le taux de rétention des étudiants maoris à l'université d'Auckland était de $70,0 \%$ en 2002-2003 comparativement à 87,6 \% la même année pour l'ensemble des étudiants (Equal Opportunities Office 2003 : 34)9 . Parmi ceux qui restent, pourtant, plusieurs apprécient grandement leur expérience. La perception de l'université comme une expérience positive est étroitement liée au fait qu'ils y trouvent une famille au sens symbolique et donc, un réseau d'entraide, d'amitié et de soutien à plusieurs niveaux.

La majorité des étudiants du groupe de kapa haka (danses et chants maoris) de l'université, par exemple, considèrent ce groupe comme leur whaanau (famille étendue). Matiu expliquait pourquoi :

Spécialement à l'université, il y a beaucoup d'étudiants maoris qui sont déconnectés de leurs liens tribaux et peut-être que le kapa haka est un forum où ils peuvent trouver d'autres Maoris [...] qui peuvent comprendre ce qu'ils ressentent et où ils ne se sentent pas seuls. Le kapa haka est le médium à travers lequel ils peuvent se rassembler et partager des waiata, les chansons maories traditionnelles, et la musique maorie contemporaine comme moyen pour se sentir mieux et pour rétablir et créer un sentiment de whaanau. (Matiu)

Fiona, qui fut engagée dans l'organisation du groupe pendant un certain temps, abondait dans le même sens :

Nous utilisons le kapa haka comme un véhicule permettant aux étudiants maoris de se retrouver ensemble. Le kapa haka, c'est la compétition, mais pour nous, notre kaupapa [principe, mission] a toujours été whanaungatanga, entretenir les relations et agir comme les taangata whenua [groupe ayant les droits ancestraux] du marae de I'université. Et ce sont donc les deux principales raisons pour lesquelles j'ai joint le kapa haka et que j'y ai tenu le rôle de tuteur. De cette façon, il y aura toujours un lieu pour que les étudiants viennent et rencontrent d'autres étudiants maoris et pour leur laisser savoir que le marae de l'université est un lieu pour eux et c'est la raison pour laquelle le marae de l'université est différent d'autres marae tribaux. [...] C'est un lieu pour tous les étudiants durant toutes leurs années universitaires, à l'extérieur du cadre de l'université, à l'extérieur du cadre de la ville et de leur propre marae tribal, qui peut être comme leur chez-eux. (Fiona)

Pour Miiria, le groupe a été une source importante de soutien quand les choses allaient plutôt mal à l'université, avec son copain et sa grossesse. Il a aussi été une source d'entraide puisqu'il a permis à plusieurs membres du groupe de partager les responsabilités par rapport aux enfants. Pour une autre étudiante, le groupe a été une ressource importante dans l'apprentissage des danses et chants traditionnels et dans l'acquisition de connaissances en matières de tikanga (coutumes, règles). Le groupe de kapa haka est donc aussi une courroie de transmission culturelle et une source de soutien à tous les niveaux, à l'université et bien au-delà de ses murs. Faire partie du groupe permet d'être plus confortable de façon générale, mais aussi plus confortable comme étudiant.

Le groupe tient souvent des fins de semaines consacrées à la pratique pendant lesquelles tout le monde vit et dort ensemble au marae, le but principal étant de renforcer les liens en son sein. Il arrive aussi souvent que plusieurs étudiants restent au marae ou au local étudiant maori durant les fins de semaines et les jours de congé. Les maisons ou appartements de certains étudiants deviennent aussi des lieux importants de rassemblement et d'entraide après les cours. Le sentiment d'être une whaanau vient de l'expérience commune d'être étudiants, d'apprendre ensemble, de partager des expériences difficiles et heureuses et d'être côte à côte chaque jour dans les mêmes cours et dans les différents locaux de l'université.

Il est important de mentionner que la whaanau et le whanaungatanga (renforcement et enrichissement des liens familiaux) sont devenues des symboles importants de l' être Maori » au cours des dernières décennies. La plupart des événements maoris ont une période de temps réservée aux activités de whanaungatanga. Limportance de la whaanau était soulignée encore et encore par les participants à cette recherche, mais aussi sur le nouveau réseau de télévision maori et dans les documents et discours publics maoris de toutes sortes. Ces discours ne semblent pourtant pas tenir leur promesse pour tous... Différents jeux de pouvoir sont à l'œuvre et certains sont exclus des whaanau étudiantes et de leurs activités. Je pense, par exemple, à ceux qui ne sont pas assez bons pour les prestations du kapa haka, qui ne sont pas assez libres pour faire la fête étant donné leurs autres obligations à l'extérieur du cercle universitaire, qui ne sont pas assez (ou trop) minces pour donner une bonne image du groupe ou qui ne parlent pas assez la langue maorie.

De surcroît, plusieurs étudiants maoris ressentent un fort attachement au marae de l'université et y sont activement engagés. Au marae se tiennent toutes sortes d'activités telles que les powhiri (cérémonies d'accueil) pour les invités et visiteurs du département d'études maories et de l'université en général, qui s'accompagnent toujours d'un goûter ou d'un repas. Se tiennent aussi au marae des réunions, rencontres et retraites de toutes sortes, des réceptions et des fêtes à l'occasion de levées de fonds pour aider au financement de divers projets, de mariages ou de réalisations exceptionnelles par certains membres maoris de l'université. Le marae accueille aussi les funérailles d'étudiants ou de membres du personnel (actuels ou anciens), les pratiques de kapa haka, les cérémonies de graduation maories. Les différents édifices du marae sont aussi utilisés pour des activités d'enseignement surtout par le département 
d'études maories, mais aussi, à l'occasion, par d'autres départements et facultés.

Comme nous l'avons vu, le marae de l'université joue un rôle très important dans la vie des étudiants et dans leur identité comme Maoris. Il les aide à se sentir chez eux, bien, confortables à l'université et en ville. Les étudiants parlent souvent du marae comme d'un endroit paisible où ils peuvent se retrouver et réfléchir. Le marae de l'université étant pan-tribal, plusieurs étudiants aiment dormir aux pieds de leur tipuna (ancêtre) sculpté sur les poteaux de charpente de la wharenui (grosse maison ou maison de rassemblement) pendant les retraites d'études ou les fins de semaine dédiées aux pratiques de kapa haka. Ce qui est d'ailleurs particulier à la wharenui de l'université d'Auckland, c'est qu'y sont représentés les capitaines et prêtres-navigateurs de tous les canots dans lesquels sont venus en Nouvelle-Zélande les ancêtres des différentes tribus maories au XIVe siècle. Ce lien étroit des jeunes avec leurs ancêtres leur permet d'avoir le sentiment d'« appartenir » à une lignée et à un lieu particulier. La relation aux ancêtres sculptés peut aussi être une façon d'affirmer une identité tribale particulière parmi les collègues universitaires et dans le monde urbain en général, des mondes très diversifiés où les identités tribales et la mémoire des relations intertribales à travers l'histoire jouent encore un rôle important dans les interrelations entre Maoris aujourd'hui, jeunes et vieux.

Plusieurs étudiants maoris considèrent que le marae de l'université est leur tuurangawaewae, c'est-à-dire l'endroit où ils peuvent se "tenir debout », " poser leurs pieds » (traduction littérale) puisque ce lieu est le leur, un des seuls lieux leur « appartenant» vraiment comme lieu incontestablement maori. Cet usage du terme tuurangawaewae dans un contexte urbain est nouveau. Traditionnellement, ce mot réfère à la connexion à la terre à travers la connexion aux ancêtres qui marchèrent et prirent part à la vie du marae et qui y sont maintenant enterrés. C'est cette connexion qui donne les droits (et responsabilités et obligations) aux individus et groupes, comme taangata whenua (gens de la/cette terre), pour participer aux affaires de la communauté, mais aussi pour participer aux décisions quant aux kawa (protocole) du marae, aux événements qui s'y tiennent, aux rôles que chacun y joue. C'est aussi du devoir des taangata whenua de s'assurer que le principe d'hospitalité est bien pratiqué (Tauroa et Tauroa 1986 : 38). Le tuurangawaewae est donc étroitement lié à l'identité des personnes et des groupes à travers les liens étroits à une terre et une lignée particulière. Ces droits peuvent aussi être acquis par adoption. Le tuurangawaewae est ainsi un chez-soi spirituel et un lieu sacré (Tauroa et Tauroa 1986) où l'on peut se tenir debout fièrement en sachant qui on est.

En toute honnêteté, je peux dire que j'ai plus à faire avec le marae de l'université qu'avec mon propre marae tribal. C'est vraiment triste à dire, mais c'est la simple réalité. Et c'est là [...] où je travaille et étant dans le groupe de kapa haka, notre groupe tire son nom du marae de l'université et étant à l'université [...], c'est l'endroit où je veux être. Ce n'est pas mon tuurangawaewae traditionnel, mais quand je suis ici en ville, il me procure une assise grâce à laquelle je peux me tenir debout, ainsi, c'est comme une sorte de tuurangawaewae, mais pas dans un sens traditionnel [...], c'est-àdire dans le sens de la terre à laquelle vous vous identifiez à travers la tribu. Mais, comme j'ai dit, tant que je suis ici en ville, c'est un lieu où je peux être et auquel je peux m'identifier [...] II arrive souvent que j'ai besoin de temps loin d'ici, je vais demander les clés et je vais m'asseoir dans la whare [maison] et je vais juste m'asseoir là et lire, juste pour échapper au cadre de la ville avec toutes les nuisances de la vie urbaine, juste pour retourner à mes racines maories. (Fiona)

Ainsi, pour ceux qui s'identifient au marae de l'université et participent à ses activités, ce lieu, ils le connaissent très bien et savent comment s'y comporter et comment interagir avec les autres qui le fréquentent. Ils s'y sentent confortables et ils sentent qu'ils y ont des responsabilités importantes pour s'assurer que le marae soit un endroit vivant et chaleureux, ce qui n'est pas nécessairement le cas sur le marae traditionnel de leur tribu. Le marae ainsi que d'autres lieux maoris de l'université tels que le département d'études maories et les locaux étudiants constituent des environnements où les étudiants peuvent se ressourcer sur les plans social, culturel et spirituel. C'est là que plusieurs trouvent un « chez-soi » à l'université et, comme expliqué plus haut, une whaanau (famille étendue) au sens symbolique, mais parfois aussi au sens réel puisque plusieurs étudiants se découvrent des relations de parenté. Ces endroits procurent souvent assez de confort pour permettre de se sentir plus à l'aise dans l'université dans son ensemble.

Mais il est bien connu que faire d'un endroit un « chez-soi » est aussi une action qui peut être contestable et qui est souvent contestée (Olwig 1998). Ainsi, certains ne se sentiront jamais chez eux au marae pantribal de l'université ou dans certains locaux maoris parce qu'ils ne se sentent pas à l'aise avec les gens qui s'y trouvent ou avec l'esprit du lieu. Plusieurs ressentent qu'on ne les considère pas « assez » maoris ou qu'ils ne font pas vraiment partie du groupe, qu'ils n'« appartiennent» pas vraiment au lieu parce qu'ils n'ont pas les mêmes antécédents (de classe, de tribu, de style de vie, etc.). La rhétorique au sujet des « vrais » en opposition aux « faux » Maoris est aussi à l'œuvre dans ces lieux.

De plus, l'université est vue sous un éclairage positif par plusieurs parce que c'est un lieu où on apprend sur soi et sur son histoire comme Maori.

C'est important pour moi, l'université m'a donné plus d'information. Je ne connaissais pas l'histoire des Maoris avant que je ne vienne à l'université, je n'avais pas appris à l'école, je ne savais pas... que nous avions été colonisés [...] Je veux dire... je savais parce que j'en avais fait l'expérience, mais je ne savais pas que c'était comme... un système qui avait été mis en place. [...] L'université m'a fait voir les choses différemment. [...] Ce n'est que lorsque je suis venue à l'université que j'ai commencé à comprendre ce qui se passait et à remettre en question les choses autour de moi. [...]

Je pense que c'est la différence entre une institution comme celleci et la réalité de ma vie à l'extérieur d'ici. [...] tu viens ici et tu deviens... tu apprends plus au sujet de toi-même et tu veux t'identifier à cette personne ou à cette culture particulière. (Joanna)

Cette période, pour plusieurs étudiants, est donc celle de la (re)découverte de leur taha (côté) maori et/ou de la (ré)affirmation de celui-ci par toutes sortes de moyens, dont l'inscription à des cours de langue maorie ou à des cours d'histoire ou de culture maories, la participation à diverses activités regroupant des Maoris et la fréquentation de lieux identifiés comme maoris.

Le port sur soi de différents symboles maoris est une autre façon d'affirmer son identité. Certains vont aussi utiliser des symboles d'une identité plus spécifique comme l'appartenance à une tribu, à une famille particulière ou à d'autres groupes. Le port de T-shirts ou de casquettes identifiant son groupe d'appartenance est très répandu. D'autres se font faire des moko 
(tatouages) traditionnels et/ou contemporains qui contiennent des symboles d'affiliations spécifiques.

La langue maorie est un autre aspect important de l'identité maorie et de la reconnaissance par les autres comme Maori. Au marae de l'université, des tensions surviennent souvent entre ceux qui parlent maori et ceux qui ne parlent pas ou ont une maîtrise limitée de la langue, mais aussi entre ceux dont le maori est la langue maternelle ou une langue apprise dans leur tribu à la campagne et ceux qui l'ont apprise à l'université ou dans les ateliers de langue des marae urbains.

Kepa a attiré aussi mon attention sur le fait que même la façon dont ils parlent anglais a un effet sur leur reconnaissance par les autres comme Maoris. Il m'a expliqué : « Il y a ceux qui me regardent et me disent que je ne suis pas un Maori parce que je ne parle pas comme un Maori » (Kepa). Aussi, il découvrit qu'il adaptait sa façon de parler à son audience.

De plus, certaines personnes se présentent différemment, dépendant de leur interlocuteur. Plusieurs utilisent même des noms différents. Je pense à un étudiant qui s'était présenté à moi comme étant John alors qu'il se présentait par la suite à un étudiant maori comme étant Hone. L'entrée à l'université semble aussi marquer une période importante de changement où plusieurs décident d'utiliser un nom maori. Ce nom peut être la traduction de leur nom anglais ou encore un nom maori reçu à la naissance. Ce fut le cas pour Fiona qui utilise maintenant un nom maori. Elle voit son nom, qui est un signe externe, public, comme un véhicule idéal pour dire à tout le monde Maori ou non - qu'elle est maorie. Pour elle, comme pour plusieurs autres étudiants, ce nouveau nom fait partie d'un processus plus large de (re)découverte, ou simplement d'affirmation de son taha (côté) maori.

J'ai remarqué que, parmi les rangatahi (jeunes) surtout — et cela est très évident en milieu universitaire - l'usage du «Bro!» (pour brother) et du «Cous! » (pour cousin) pour interpeller les autres est très répandu. C'est aussi une façon de signifier qui fait partie du groupe, de la whaanau.

D'autres privilégient d'autres voies d'affirmation comme de réussir dans des programmes traditionnellement non fréquentés par les Maoris ou dans des programmes qui leur permettront, dans le futur, de travailler directement avec et en faveur des Maoris.

Tous ces trucs entourant l'obtention de mon diplôme [...], tout ça est lié au fait d'être capable d'être une sorte d'agent de changement [...] pour le groupe plus large. [...] C'est être capable d'utiliser suffisamment mes habiletés pour faire des changements pour ma famille, ma famille immédiate, whaanau [...], et ensuite, les gens avec qui je travaille. [...] C'est pour être capable d'être efficace dans ce que je fais. (Mere)

Limportance de la whaanau est à noter à nouveau ici. Plusieurs étudiants maoris ont des motivations et des buts collectifs sousjacents à leurs études, qui revêtent beaucoup d'importance.

Pour être honnête avec toi, je ne veux pas faire un doctorat, je ne peux pas penser à quelque chose de pire que d'être assise pendant trois ans et d'écrire sur quelque chose. J'aimerais mieux être en vacances $[. .$.$] La raison pour laquelle je vais essayer d'avoir mon$ doctorat est d'être en position de pouvoir aider les gens de mon iwi [tribu] [...] et je sais que c'est mon destin. Je suis sur un bateau et je ne peux pas en descendre [...] Ça m'est juste venu, comme dans un rêve ou quelque chose du genre, ça m'est venu et on $\mathrm{m}^{\prime}$ a dit de le faire. [...] Cette étude [...] ce n'est pas pour moi, c'est pour ma tribu, c'est pour les autres jeunes Maoris [...], c'est pour toutes ces familles qui n'ont pas accès à l'éducation, à la santé, à l'argent et au logement, à des vêtements décents, à de la nourriture décente... Ce doctorat, même si c'est un doctorat, c'est un taonga [trésor], c'est une façon de rendre la vie un peu meilleure. (Aroha)

Pour plusieurs, il est essentiel aussi de soumettre à leur famille leur projet de thèse pour discussion et approbation. Cela est vrai pour ceux qui étudient des questions maories, mais aussi pour des étudiants qui étudient dans des champs complètement différents. Tui résume bien pourquoi il est si important de consulter sa famille ou sa tribu :

Si un ou deux n'aiment pas l'idée, je peux vivre avec ça. S'il y en a plusieurs, non. Alors, je vais essayer de voir comment je peux les satisfaire, je peux voir ce que je peux faire pour faire accepter l'idée ou... je ne ferai pas la recherche. Ça ne vaut pas la peine. Mais je pense que c'est quelque chose d'important [...] Si les gens ne sont pas d'accord avec l'idée, s'ils n'ont pas un bon feeling à propos de la recherche... personne ne va t'aider. Deuxièmement, les gens sont inquiets. Nous sommes inquiets à propos de qui va en bénéficier... Est-ce que ça va bénéficier à la communauté ou que c'est juste par intérêt égoïste? (Tui)

Lappui de la famille est donc essentiel à la poursuite des études et c'est un facteur qui contribue au confort en général à l'université. Les étudiants semblent plus à l'aise avec l'idée qu'ils travaillent pour leur whaanau, leur tribu, et pour améliorer la situation des Maoris dans leur ensemble. Cette idée de vouloir travailler pour leur whaanau ou pour les Maoris en général vient en partie d'un habitus (Bourdieu 1980) où la logique collective est marquée, mais fait aussi partie de la prise de conscience qui est à l'œuvre plus largement en Nouvelle-Zélande et, en particulier, sur les bancs de l'université.

Certains craignent aussi que les choses tournent mal ou qu'adviennent des utu, c'est-à-dire un « retour », bon ou mauvais, par exemple une vengeance ou une punition, en provenance des ancêtres ou du monde surnaturel (Metge 2002), si leur sujet d'étude n'est pas approuvé par la whaanau. Plusieurs sont d'ailleurs attentifs aux signes en provenance du monde surnaturel, comme des rêves, des maladies ou autres sensations qui peuvent leur donner des indications quant à savoir s'ils ont fait les bons choix. Cela ressortait d'ailleurs très bien des propos d'Aroha cités ci-dessus.

Certains étudiants maoris, bien sûr, poursuivent leurs études sans l'avis de leur whaanau ou encore sans que celle-ci ne se sente vraiment concernée par leurs études, soit par manque d'intérêt de celle-ci, soit parce qu'ils considèrent que leurs études sont plus une affaire de choix personnels. D'autres encore iront contre l'avis de leur whaanau. Dans ce dernier cas, il serait surprenant que leur famille les empêche de continuer, mais ils pourraient bien avoir à choisir entre leurs études et leur whaanau, du moins, pour un certain temps.

L'université comme lieu de rencontre donne aussi souvent le goût de s'engager davantage dans les mondes maoris ou dans la société en général. C'est là qu'on discute ce qui a été appris en classe. C'est là qu'on est mis au courant de certains programmes et actions du gouvernement et de leurs répercussions pour les Maoris, mais aussi pour tout le pays. C'est aussi là qu'on discute des problématiques autochtones, de questions de portée générale ou internationale et de divers événements sur la scène mondiale. Certains lieux universitaires sont aussi propices à des discussions sur ce qui est enseigné sur la colonisation et l'histoire de la Nouvelle-Zélande. C'est aussi là qu'on apprend ce qui se passe dans différentes tribus, dans les 
relations intertribales ou intergroupes et dans «le monde maori » en général. C'est là qu'on se fait rappeler que le marae et la place des Maoris à l'université ne sont pas un acquis de longue date et ont été au cœur d'intenses tensions, comme nous l'avons vu.

Cette période de leur vie associée à leurs études universitaires est donc considérée comme déterminante par plusieurs étudiants et ex-étudiants maoris et ce, autant pour leur engagement dans les mondes maoris que dans les mondes autres. Leur expérience de l'université, le soutien et les encouragements maoris - et non maoris - qu'ils y trouvent leur permet de s'engager dans le monde plus large avec plus d'aisance, soit sur le marché du travail, soit dans divers groupes, projets et organisations. Luniversité est le lieu où s'acquièrent des habiletés importantes comme celles d'organisateurs ou de négociateurs puisque plusieurs s'engagent dans les différentes associations étudiantes, maories ou non, tiennent des positions de leadership dans des campagnes de financement, sont bénévoles ou organisateurs d'ateliers lors de diverses rencontres et colloques, gagnent une expérience en enseignement et en recherche. Toutes ces expériences ouvrent parfois sur des engagements à plus long terme ou plus directement politiques, selon les opportunités ou les contextes sociopolitiques. En mai 2004, par exemple, un groupe d'étudiants se mobilisèrent et participèrent à l'hikoi (marche) [Walker 2004: 214-215] en protestation à une loi sur la gestion des plages et des fonds marins sous la bannière de Ngaa Tauira Maaori, l'association des étudiants maoris. À la fin de l'année 2004, une branche étudiante du Maori Party a aussi été fondée.

\section{L'UNIVERSITÉ, UN SITE EN INTERRELATIONS AVEC D'AUTRES}

Il apparaît clairement que l'aisance, la résistance et la (ré)affirmation de différentes identités, incluant des identités maories, la coexistence avec les autres, Maoris et non-Maoris, sont étroitement liées au confort dans certains lieux universitaires où l'on se sent bien et où l'on se sent reconnu comme membre du groupe et soutenu en tant que tel. Ces sites de confort ne sont pas sans liens avec d'autres sites, maoris et non maoris, dans et à l'extérieur de l'université. Ainsi, on peut expliquer que certaines personnes sont plus confortables à l'université parce qu'elles sont à l'aise sur le marae de l'université, ce confort pouvant à son tour être attribuable à leur confort sur leur propre marae tribal ainsi qu'à leur connaissance des protocoles et de la langue maorie. D'autres ont un bon réseau familial qui les soutient à tous les niveaux, y compris dans leurs études. D'autres encore arrivent à trouver un certain confort à l'université parce qu'ils ont d'autres lieux où se ventiler et se ressourcer comme membres d'une équipe de sport, d'une Église ou d'un groupe de hip-hop. Luniversité comme site de (ré)affirmation, de coexistence et de résistance ne doit donc pas être comprise comme un site isolé. C'est un site en interrelations avec un ensemble de sites comme les marae (ruraux et urbains), les autres institutions d'enseignement, les clubs de sports, les salles communautaires et certaines maisons maories en ville qui sont souvent des lieux de rencontre par excellence où sont perpétuées les traditions maories (Gagné 2004). Ce qui se passe à l'université est aussi lié à ce qui se passe dans différents sites urbains et ruraux, maoris et non maoris, municipaux, régionaux, nationaux et internationaux.

Certains aspects inconfortables de la vie à la campagne ou de la vie précédant l'université peuvent aussi expliquer en partie le confort à l'université et dans le nouvel environnement. Pour un bon nombre de participants à mes recherches, l'université et la nouvelle vie en ville signifient la liberté, liberté en termes d'indépendance nouvelle de l'étudiant par rapport à sa famille et sa tribu, mais aussi, pour une minorité, liberté de vivre son homosexualité encore mal acceptée par la famille. Le nouveau contexte peut donc permettre l'affirmation de nouvelles identités ou d'exprimer ses identités avec des nuances différentes. Cependant, l'aisance avec laquelle cela est possible dépend aussi des relations établies dans le nouveau contexte et des ressources personnelles qui permettent (ou non) d'explorer les possibilités du milieu.

Au cours de leur vie, plusieurs des étudiants ont aussi incorporé les façons de faire et d'être - l'habitus (Bourdieu 1980) qui les rendent confortables à l'université et dans d'autres institutions dominantes. Plusieurs sont ainsi déjà habiletés à s'engager dans plusieurs mondes, maoris et pakehas, parce que, par exemple, ils proviennent d'unions mixtes, ont grandi en ville, ont pris part à des activités diverses et fréquenté toutes sortes d'institutions, ont en outre parfois voyagé outre-mer, et leurs parents ou d'autres membres de leur famille ont fréquenté l'université avant eux. On peut qualifier ces étudiants de « biculturels» (Schwimmer 2003 et 2004) puisqu'ils sont bien « outillés » pour faire preuve d'hétéroglossie dans leurs participations aux mondes maoris et pakehas. Ces personnes biculturelles peuvent parfois être dérangeantes pour les autres, car leur aisance dans plusieurs mondes fait qu'elles ne sont pas toujours " clairement » identifiables comme Maoris. On doute alors de leur allégeance et on les accuse de traîtrise dans certaines situations politisées. Encore une fois, personne n'est à l'abri de la rhétorique au sujet des « vrais » et des «faux » Maoris. Et cette rhétorique influence les personnes biculturelles dans leur fréquentation de certains lieux et leurs engagements avec les autres.

Rappelons que ce ne sont pas tous les étudiants maoris qui participent aux activités du marae ou fréquentent les locaux de l'association étudiante maorie et le département d'études maories. Il convient alors de se demander ce qu'il en est des pratiques identitaires des étudiants maoris qui cheminent sans contact avec ces lieux d'appartenance collective. S'insèrent-ils dans d'autres univers, qu'ils soient pakehas, asiatiques, départementaux ou facultaires, marginaux, artistiques? Comment et quand leur identité maorie est-elle un facteur significatif dans les relations ainsi établies? Qu'est-ce qui détermine leur confort dans ces groupes? Voilà des questions qui restent ouvertes à l'exploration. Ajoutons simplement que, pour plusieurs, fréquenter l'université signifie aussi l'occasion d'enrichir leurs cercles d'amis par des amitiés avec des étudiants de diverses provenances avec ce que cela amène en termes de découvertes et de nouveauté.

Enfin, lors d'un retour au terrain au début de 2005, j'ai été invitée à faire une présentation dans une retraite d'écriture regroupant une quarantaine d'étudiants maoris au doctorat venant des quatre coins du pays et de toutes les facultés. Il était clair qu'un sentiment généralisé de fierté se dégageait du groupe puisque très peu de Maoris atteignent ce niveau et qu'ils sont souvent les seuls de leur famille à avoir un diplôme universitaire. Plusieurs ont souligné la nécessité de se soutenir mutuellement, insistant sur les difficultés auxquelles font face les étudiants maoris à l'université, comme Maoris, d'autant plus qu'ils sont souvent les seuls Maoris dans leur programme d'études. Des opportunités telles que cette retraite d'écriture sont donc perçues comme très importantes pour enrichir et 
renforcer les liens familiaux - ici symboliques - avec d'autres doctorants maoris à travers le pays, pour partager leurs expériences et pour s'entraider et s'encourager à persévérer dans l'écriture de la thèse.

De telles rencontres sont aussi l'occasion d'expériences de mise en commun, au-delà des différences tribales, disciplinaires et individuelles. Elles permettent même de surpasser les divisions trop souvent créées par la rhétorique opposant les Maoris "réels » aux soi-disant "faux » Maoris et les frictions intervenant entre ceux qui sont plus actifs dans les mondes maoris (dans et à l'extérieur de l'université) et ceux qui le sont moins. Une conscience nouvelle du «nous» peut alors émerger, permettant au groupe de mieux cerner les champs d'application possibles - tradition, mieux-vivre, vivre-ensemble, défense et promotion des droits, etc. - des diverses connaissances, habiletés et ressources en présence. Les doctorants soulignaient d'ailleurs le fait que leur réussite n'était pas simplement personnelle, mais avait été rendue possible grâce à leur whaanau et à leurs collègues étudiants, surtout maoris. Cette réussite était avant tout perçue comme une réussite pour tous les Maoris et, pour cette raison, comme l'a déclaré l'une des participantes à la retraite, "ils doivent finir la thèse, c'est leur mission politique. Ils n'ont pas le choix ». En effet, la réussite bénéficiera à l'ensemble des Maoris et permettra de garder un « nous » fort, mais elle permettra aussi une participation aux mondes dominants qui, avec le temps, seront davantage pénétrés et donc transformés graduellement. Luniversité apparaît à nouveau clairement comme un site important de résistance, mais aussi d'affirmation et de négociation des rapports aux autres et donc, de la coexistence tant entre Maoris qu'entre Maoris et non-Maoris, dans un espace qui s'étend bien au-delà du campus universitaire.

\section{Notes}

1. Mes recherches ont été facilitées par des bourses du Conseil de recherche en sciences humaines du Canada (CRSH), des Fonds pour la Formation des chercheurs et l'aide à la recherche (FCAR), de l'Université McGill, de l'Université d'Ottawa, du Centre for Society, Development and Technology (STANDD), de la Fédération canadienne des femmes diplômées des universités (FCFDU) et de la Société canadienne d'anthropologie (CASCA).

2. Le concept de « confort » (qui suppose toujours sa contrepartie inconfortable) utilisé tout au long de cet article est polysémique et recouvre plusieurs niveaux de sens qui varient en fonction des contextes dont 1) une mesure d'appui, de soutien, d'aide, de réconfort ; 2) une mesure de satisfaction, d'agrément ; 3) une mesure de bien-être physique, de plaisir, d'aisance, une marge de manœuvre (sens littéral); 4) une mesure d'intimité ; 5) un état émotionnel, un sentiment de sécurité; 6) un état intellectuel, un degré de connaissance; 7) une mesure d'autonomie, une marge de manœuvre (sens métaphorique); 8) une mesure d'affinités culturelles, sociales et politiques ou de convergence / divergence; 9) un état spirituel qui suppose une sensibilité à des sensations bonnes ou mauvaises, de « chaud » ou de « froid » émanant de situations, de lieux, de personnes vivantes ou décédées, de choses ou des mondes surnaturels (voir Rybczynski 1986, Radice 2000 et Gagné 2004). Les Maoris s'expriment régulièrement en termes de confort pour parler de leurs relations avec les autres, maoris ou non, avec leurs ancêtres ainsi que pour qualifier leurs sensations ou émotions dans certains lieux et leurs engagements dans divers mondes.

3. Voir, entre autres, Pearson (1991, 1996), Rata (2000), Schwimmer (2003, 2004), Sharp (1995), Sissons (1995), Webster (1998).
4. Ce chiffre correspond aux étudiants ayant retenu la catégorie « Maori » comme origine ethnique sur leur formulaire d'inscription. 14524 ont retenu la catégorie «Européen », 12259 «Asiatique », 2165 « Insulaire du Pacifique » et 1735 « Autre». 2497 étudiants n'ont retenu aucune catégorie (University of Auckland 2004). Les statistiques quant à la répartition des étudiants en fonction du sexe et de l'âge n'étaient pas disponibles.

5. Notons que le choix des programmes est précontraint par les choix aux niveaux primaire et secondaire, plusieurs programmes exigeant des pré-requis en sciences et en mathématiques. Ces choix précoces sont influencés par plusieurs facteurs autres que l'ethnicité, dont le milieu socioéconomique et la scolarité des parents.

6. Les étudiants maoris sont aussi confrontés à une forte présence asiatique, ceux-ci représentant 34,9\% des étudiants à l'université d'Auckland (University of Auckland 2004). Cette présence est pourtant perçue dans des termes différents, l'immigration asiatique étant un phénomène assez récent (Bouchard 2000; Fleras et Spoonley 1999).

7. À ce nombre, s'ajoutent tous les étudiants qui s'inscrivent seulement à quelques cours ou au volet « langue » ou « arts de la scène », par exemple, tout en étant inscrits à un diplôme autre que le B.A. En 2004, le département d'études maories estimait, en faisant le cumul des inscriptions dans les différents cours, qu'il était fréquenté par l'équivalent d'environ 70 étudiants maoris à temps plein.

8. Des indications seront données plus loin quant à savoir pourquoi. Mentionnons seulement que ces lieux sont plutôt politisés et que divers processus rhétoriques et idéologiques y sont à l'œuvre.

9. Cette différence peut sans doute être expliquée en grande partie par le fait que les étudiants maoris viennent généralement de milieux socio-économiques plus défavorisés. Cette hypothèse n'a pourtant pas pu être vérifiée puisque des statistiques quant aux caractéristiques socio-économiques des étudiants de l'université d'Auckland n'étaient pas disponibles. Luniversité ne recueille que des informations relatives aux écoles secondaires fréquentées par les élèves provenant directement du secondaire, ce qui sert d'indication quant au milieu socio-économique d'origine, mais je n'ai pas eu accès à ces données.

\section{Remerciements}

Je remercie les participants à mes recherches, en particulier les étudiants maoris de l'université d'Auckland. Merci à Claude Bariteau et à Éric Schwimmer pour leurs commentaires sur une version préliminaire de ce texte, ainsi qu'aux évaluateurs de cet article. Merci aussi à Linda Tuhiwai Smith pour la vérification des passages la concernant. Merci enfin à Pamela Moss et David Tippin du Planning \& Quality Office de l'université d'Auckland pour l'accès à certaines statistiques.

\section{Ouvrages cités}

BOUCHARD, Gérard, 2000: Genèse des nations et cultures du nouveau monde: Essai d'histoire comparée. Boréal, Montréal.

BOURDIEU, Pierre, 1980: Le Sens pratique. Les Éditions de Minuit, Paris

CHAPPLE, Simon, 2000: «Maori Socio-Economic Disparity». Political Science 52(2) : 101-115.

DURIE, Mason, 2001 : Mauri Ora. The Dynamics of Maori Health. Oxford University Press, Auckland.

EQUAL OPPORTUNITIES OFFICE, 2003 : Annual Report Summary. University of Auckland, Auckland.

FLERAS, Augie, et Paul SPOONLEY, 1999: Recalling Aotearoa: Indigenous Politics and Ethnic Relations in New Zealand. Oxford University Press, Auckland. 
GAGNÉ, Natacha, 2004: Maori Identities and Visions: Politics of Everyday Life in Auckland, New Zealand. Thèse de doctorat, département d'anthropologie, Université McGill, Montréal.

KAWHARU, I. Hugh, 1990 : « Sovereignty vs. Rangatiratanga: The Treaty of Waitangi 1840 and the New Zealand Maori Council's Kaupapa 1983 », in A. Pawlay (dir.), Man and a Half: Essays in Pacific Anthropology and Ethnobiology in Honour of Ralph Bulmer: 573-581. Mémoire 48, The Polynesian Society, Auckland.

METGE, Joan, 2002 : « Returning the Gift: Utu in Intergroup Relations ». The Journal of the Polynesian Society 111(4): 311-338.

MURU-LANNING, Marama, 2004: "The Emergence of New Waikato-Tainui Subjects and Spaces ». Communication présentée au congrès annuel de l'Association for Social Anthropology in Oceania (ASAO), Hawthorne Hotel, Salem, États-Unis, février.

OLWIG, Karen Fog, 1998 : « Epilogue: Contested Homes: HomeMaking and the Making of Anthropology », in Nigel Rapport et Andrew Dawson (dir.), Migrants of Identity: Perceptions of Home in a World of Movement: 225-236. Berg, Oxford.

PEARSON, David, 1991 : «Biculturalism and Multiculturalism in Comparative Perspective », in Paul Spoonley, David Pearson et Cluny MacPherson (dir.), Nga Take: Ethnic Relations and Racism in Aotearoa/New Zealand: 194-214. Dunmore Press, Palmerston North.

_, 1996: «Crossing Ethnic Thresholds: Multiculturalisms in Comparative Perspective » in Paul Spoonley, David Pearson et Cluny MacPherson (dir.), Nga Patai: Racism and Ethnic Relations in Aotearoa/New Zealand: 247-266. Dunmore Press, Palmerston North.

RADICE, Martha, 2000 : Feeling Comfortable? Les Anglo-Montréalais et leur ville. Les Presses de l'Université Laval, Sainte-Foy.

RATA, Elizabeth, 2000 : A Political Economy of Neotribal Capitalism. Lexington Books, New York.

-, 2003: "Leadership Ideology in Neotribal Capitalism». Political Power and Social Theory 16:43-71.

RYBCZYNSKI, Witold, 1986: Home. A Short History of an Idea. New York, Viking.

SALMOND, Anne, 1975: Hui: A Study of Maori Ceremonial Gatherings. Reed Books, Auckland.
SCHWIMMER, Éric, 2003 : «Les minorités nationales: Volonté, désir, homéostasie optimale. Réflexions sur le biculturalisme en Nouvelle-Zélande, au Québec et ailleurs ». Anthropologie et Sociétés 27(3) : 155-184.

—, 2004: «Making a World: The Mâori of Aotearoa / New Zealand », in John Clammer, Sylvie Poirier et Éric Schwimmer (dir.), Figured Worlds: Ontological Obstacles in Intercultural Relations : 243-274. University of Toronto Press, Toronto.

SHARP, Andrew, 2002 : «Blood, Custom and Consent: Three Kinds of Maori Groups in New Zealand and the Challenges They Present to Government and the Constitution ». University of Toronto Law Journal 51(1) : 9-37.

—, 1995: «Why Be Bicultural? » in Margaret Wilson et Anna Yeatman (dir.), Justice and Identity: Antipodean Practices : 116-133. Allen \& Unwin, Wellington.

SISSONS, Jeffrey, 1995 : « Tall Trees Need Deep Roots: Biculturalism, Bureaucracy and Tribal Democracy in Aotearoa/New Zealand ». Cultural Studies 9(1) : 61-73.

SMITH, Graham Hingangaroa, 1997 : The Development of Kaupapa Maori: Theory and Praxis. Thèse de doctorat, département d'éducation, University of Auckland, Auckland.

SMITH, Linda Tuhiwai, 1999 : Decolonizing Methodologies: Research and Indigenous Peoples. Zed Books et University of Otago Press, Dunedin et Londres.

TAUROA, Hiwi, et Pat TAUROA, 1986: Te Marae: A Guide to Customs \& Protocol. Reed Books, Auckland.

UNIVERSITY OF AUCKLAND, 2004: Student Statistics 2003. Planning \& Quality Office, Auckland, mai.

WALKER, Ranginui, 2004: Ka Whawhai Tonu Matou: Struggle Without End. $2^{e}$ édition, Penguin, Auckland.

WEBSTER, Steven, 1998 : Patrons of Maori Culture: Power, Theory and Ideology in the Maori Renaissance. University of Otago Press, Dunedin.

WAYMOUTH, Lyn, 2003 : «The Bureaucratisation of Genealogy ». Ethnologie comparée (6), [http://alor.univ-montp3.fr/cerce/」 revue.htm]. 


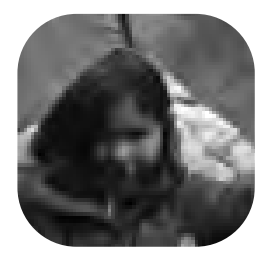

L'espérance passe de la forêt au milieu scolaire Clivage et continuité dans les valeurs entre générations de Dènès Tha

Jean-Guy A. Goulet

Faculté des sciences humaines, Université Saint-Paul, Ottawa

et

\section{Kim Harvey-} Trigoso

ChangePoint, Inc. Portland, Oregon
D) ANS CET ARTICLE, nous explorons la manière dont les Dènès Tha construisent, dans le temps et dans l'espace, un mode de vie inspiré par une attitude positive face à la vie. La réponse donnée à ce que signifie être un jeune Dènè Tha aujourd'hui repose sur une analyse qualitative et quantitative de données recueillies par les auteurs entre 1979 et 1999, ainsi que celles rendues publiques par les Dènès Tha (Dene Tha Women's Society 1990, Dene Tha Community School Testing Information 1996 et Dene Tha' Nation 1997). C'est par leur comportement, leurs récits et leurs dessins, dont huit sont reproduits dans les pages qui suivent, que des enfants Dènès Tha nous communiquent comment ils perçoivent leur milieu et comment ils y vivent en plus ou moins grande continuité avec les valeurs de leurs aînés et ancêtres.

Dans cette présentation et analyse des données, nous procédons en deux parties. En un premier temps, nous identifions à quel point le mode de vie des Dènès Tha du Nord-Ouest albertain s'est transformé depuis un siècle, transformation qui conduit depuis une quarantaine d'années à un clivage important entre certains jeunes et aînés quant aux valeurs et aux comportements. Nous proposons une compréhension de ce phénomène dans le cadre d'une comparaison des principes épistémologiques et éthiques qui sous-tendent des pratiques éducatives distinctives, chez les autochtones d'une part, et chez les Occidentaux d'autre part. Dans un deuxième volet, nous examinons à quel point la continuité sociale entre générations est étroitement liée au type de socialisation en bas âge. Nous y démontrons comment l'enfant en milieu scolaire qui a une attitude positive face à la vie et s'oriente vis-à-vis de ses compagnons et compagnes de classe en fonction de valeurs communautaires est celui qui participe le plus aux activités traditionnelles de chasse, pêche et cueillette avec des membres de sa parenté. Nous voyons ainsi l'espérance passer de la forêt au milieu scolaire. Au terme de cette analyse, une conclusion s'impose : les activités traditionnelles constituent le contexte privilégié au sein duquel les Dènès Tha produisent et reproduisent des perceptions et des valeurs issues d'une expérience millénaire en terre amérindienne - leur pays natal.

\section{Situation des DènÈs Tha hier ET AUJOURD'HUI}

Les Dènès Tha sont connus dans la littérature anthropologique comme les Esclaves (Asch 1981), les Indiens Castors de Hay River (Mills 1982), la branche Dènè Tha des Indiens Castors (Smith 1987), ou les Dènès de Hay Lakes en Alberta (Helm 1994) - nom donné à la bande par le gouvernement fédéral en 1953. Les Dènès Tha vivent aujourd'hui majoritairement à Chateh, aussi appelé Assumption. Selon Statistique Canada, on y comptait une population autochtone de 773 en 1991, 838 en 1996 et 905 en 2001. Les trois noms, Hay Lakes, Assumption, et Chateh, représentent autant de repères historiques incontournables à qui veut saisir les conditions de vie et les aspirations des jeunes Dènès Tha d'aujourd'huil ${ }^{1}$. 


\section{LA PRÉSENCE DU GOUVERNEMENT, DES COMMERÇANTS ET DES MISSIONNAIRES}

En 1900, les Dènès Tha signent le traité numéro 8 (Fumoleau 1994). À leur insu, ils sont désormais assujettis à la Loi sur les Indiens. On dresse une liste des membres de la bande. Ces derniers ont droit de vivre dans la réserve. On donne à tous un patronyme. Les membres adultes mâles élisent parmi eux ceux qui seront chef et membres du Conseil de bande. Ces élus constituent ainsi un relais administratif important entre la bande et les responsables des affaires indiennes au sein du gouvernement canadien. À l'époque, les Dènès Tha vivent et chassent sur un vaste territoire, depuis le nord-est de la ColombieBritannique, le nord de l'Alberta et le sud des Territoires du Nord-Ouest. Hay Lakes constitue alors un important lieu de rassemblement saisonnier. Au printemps et à l'automne, on y trouve de la nourriture en abondance (poisson, chevreuil, orignal, castor, rat musqué, canard et oie) ainsi que des prés pour faire paître les chevaux. La fin de l'automne signale le moment de la grande dispersion, chaque famille étendue se rendant sur son propre territoire afin d'y passer l'hiver.

Après la signature du traité numéro 8 les échanges entre Eurocanadiens et Dènès sont peu nombreux. Ils se limitent à la visite occasionnelle au poste de traite de la Hudson Bay Company situé en territoire cri, au Fort Vermillion, sur les rives de la rivière Athabasca. Les Dènès Tha y vendent leurs fourrures et y achètent des victuailles tels la farine, le lard, le thé, le sucre ainsi que des munitions et des pièges pour la prochaine saison de piégeage. De cette manière, les autochtones contribuent à l'économie de marché tout en subvenant à leurs propres besoins sans qu'il en coûte quoi que ce soit au gouvernement. Le gouvernement appuie cette participation des Indiens à la traite des fourrures puisqu'il n'entretient aucun projet d'envergure en vue d'exploiter les ressources naturelles de la région.

Après les commerçants, ce sont les missionnaires qui interviennent le plus dans la vie des Dènès Tha. Ils cherchent à les convertir à la foi chrétienne en les visitant à domicile. En 1917, le père Joseph Habay, o.m.i., construit une chapelle en bois rond à Hay Lakes, site qui en 1953 fut nommé Habay en sa mémoire. Il fut suivi du père Arbet, o.m.i., qui y construisit une deuxième chapelle en 1927-1928. Au début des années 1980, les anciens aimaient raconter comment il se rendait chez eux en traîneau à chiens afin d'administrer les sacrements et de les instruire dans la foi chrétienne.

En 1938 et à nouveau en 1948 les Dènès Tha réclament une école résidentielle ainsi qu'un hôpital. Dans une lettre en date du 17 juillet 1948, le chef de bande écrivait au ministre des Mines à Ottawa ce qui suit:

Nous les Indiens de Hay Lakes prenons sur nous de vous écrire et de vous soumettre quelques questions. Nous sommes des Indiens, pauvres, ignorants, et nous commençons à nous rendre compte de notre situation par rapport aux Blancs. Nous sommes des trappeurs en Alberta et en partie en Colombie-Britannique. Mais la trappe devient si peu profitable que certains d'entre nous auront probablement à trouver du travail parmi les Blancs. Seulement six d'entre nous parlent l'anglais après avoir fréquenté l'école au Fort Vermillion. (Notre traduction ${ }^{2}$ )

Dans sa lettre, le chef insiste sur le besoin d'un hôpital local, compte tenu des dix-sept décès survenus l'année précédente, trois en raison du vieil âge, un à la naissance, un de pneumonie, et douze autres de tuberculose. Le chef termine sa lettre en soulignant que la bande souhaite une école résidentielle,
« puisqu'il est impossible pour nous d'envoyer nos enfants à une école de jour » et qu'il est « très difficile, sinon impossible de transporter sur des distances supérieures à 80 milles, en traîneaux à chiens, des victuailles en quantité suffisante pour subvenir aux besoins de grandes familles ».

Suivant cette pétition, on construisit une école résidentielle non pas à Habay mais sur un site qu'on nomma Assumption, situé à treize kilomètres de là. Les missionnaires jugèrent que le site était plus à l'abri des inondations saisonnières et plus approprié pour une école résidentielle puisqu'on pourrait y établir une ferme qui alimenterait la mission. C'est sur ce site qu'en février 1951 l'école résidentielle recevait soixantequatorze enfants ( 28 garçons et 46 filles). Le mois suivant, seize nouveaux enfants s'y ajoutèrent. De 1951 à 1969, le nombre d'étudiants en résidence varia entre 72 à 125, ce chiffre maximum ayant été atteint en 1960. Tout au cours de ces années un nombre croissant de familles construisirent leurs cabanes en bois rond à proximité de la mission et de l'école résidentielle, ce qui permettait des visites plus régulières auprès de leurs enfants. À l'été de 1966 et de 1967, on comptait cent vingt Dènès Tha résidant à Habay et cinq cent soixante-quinze résidant à Assumption. C'est en y établissant un cimetière et en y vivant en plus grand nombre que les gens de Habay devinrent les gens d'Assumption. Le lien étroit entre l'église locale (l'ancienne chapelle de l'école résidentielle), le cimetière et la population dènèe s'exprime aujourd'hui le plus clairement à l'occasion des funérailles. Léglise est alors bondée de gens qui suivent ensuite le cercueil jusqu'au cimetière.

Un tournant historique important survint en 1965 lorsqu'on découvre d'importants gisements de gaz naturel et d'huile à l'ouest de la réserve. On y crée une nouvelle ville, Rainbow Lake, qu'on relie à la ville voisine de High Level à quelque quatrevingts kilomètres de distance. C'est ainsi qu'éclate au grand jour l'écart entre les conditions de vie des Blancs et celles des Indiens tant pour la qualité des habitations et des modes de transport, de l'emploi et du chômage, que des services en eau courante et en électricité. Les nouveaux venus, des immigrants en terre dènèe, jouissent d'un niveau de vie qui est hors d'atteinte de tous les Dènès. C'est aussi à partir de 1965, qu'on voit de plus en plus souvent à Assumption des Dènès Tha en état d'ébriété. Ce phénomène est un des premiers symptômes de la restructuration profonde de leur monde qui entraîne de nouvelles souffrances (Dene Tha Band of Assumption 1990 et Dene Tha Women's Society 1990).

Les Dènès Tha ne sont plus les acteurs principaux sur leurs terres ancestrales. Les ressources qui rendaient possible leur mode de vie sont menacées. Les adultes sont écartés de la nouvelle économie, mis en marge d'un nouvel ordre social au sein duquel ils deviennent tout au plus une main-d'œuvre non spécialisée à bon marché. Dans ce contexte, la solidarité sociale ancestrale est rompue et les divisions se multiplient, entre générations et genres. Hommes et femmes, jeunes et vieux, sont entraînés dans de nouvelles dynamiques socio-économiques et culturelles. Les tensions et les frustrations s'intensifient. Elles se manifestent dans la vie des individus, les hommes en particulier, qui en état d'ébriété laissent libre cours à leur rage dans leur milieu, détruisant les biens familiaux et communautaires et allant malheureusement, dans certains cas, jusqu'à s'enlever la vie ou à prendre celle de leurs proches. Ce sont ces faits qui trop souvent font la manchette des journaux et qui définissent aussi pour une bonne part la vie familiale et sociale d'une nouvelle génération de Dènès Tha, incluant ces enfants dont nous 
reproduisons dans la deuxième partie de cet article quelques dessins qu'ils ont faits de leur communauté.

En 1965, les hommes de la bande décident de se rendre à Edmonton et d'y organiser une manifestation afin de revendiquer des emplois et des meilleures conditions de vie. En réponse le gouvernement fédéral construisit de nouvelles demeures à l'Assumption. Ces résidences de deux à trois chambres à coucher faites en contreplaqué sont érigées à côté des maisons en bois rond que les Dènès Tha y avaient construites une génération plus tôt (photos 1 et 2). Le gouvernement fédéral décida aussi de construire une école de jour à l'Assumption. Celle-ci ouvre ses portes en 1969. La même année, les missionnaires ferment les portes de leur école résidentielle et les membres de la bande adoptent un nouveau nom pour leur communauté: Assumption devint Chateh en honneur du chef qui signa le Traité numéro 8. Ce changement de nom survient dans le contexte d'une mobilisation politique globale des peuples autochtones. À l'époque, dans les Territoires du Nord-Ouest, les autochtones adoptent le vocable « Dènè » pour se désigner eux-mêmes. Cette affirmation de soi va de pair avec la promotion d'une nouvelle forme de gouvernement autochtone dans les Territoires du NordOuest, la Denendeh, 'la terre des Dènès', qui devait coexister avec le Nunavut, 'notre terre'. C'est dans ce contexte qu'au Canada les Indiens Esclaves d'Assumption deviennent les Dènès Tha de Chateh ${ }^{3}$.

Depuis cette date, ce sont les compagnies pétrolières et forestières qui influent le plus sur le territoire et le peuple dènè. Leur territoire est aujourd'hui entrecoupé d'un important réseau de routes empruntées par les compagnies de l'industrie forestière et pétrolière. Les Dènès Tha empruntent ces mêmes routes pour se rendre en forêt pour les activités de subsistance traditionnelle (AST). En raison de la pollution des lacs et des cours d'eaux entraînée par cette activité indus-

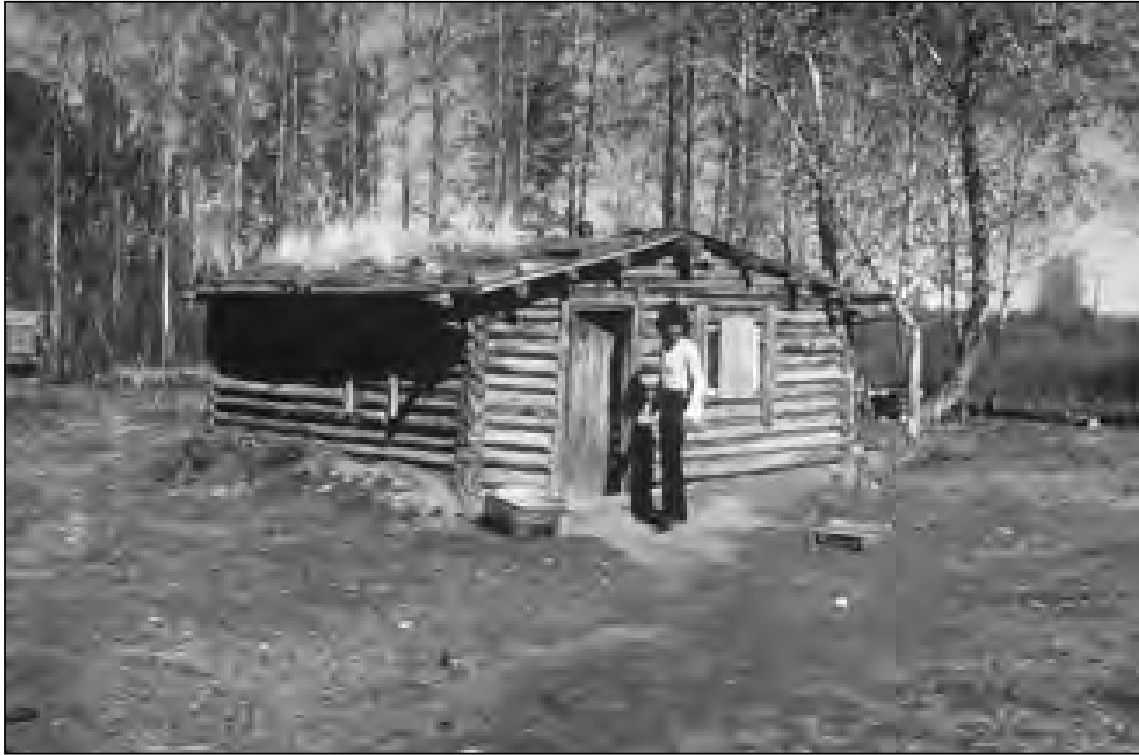

Photo 1

Domicile dènè tha, 1940

(Photo Jean-Guy Goulet)

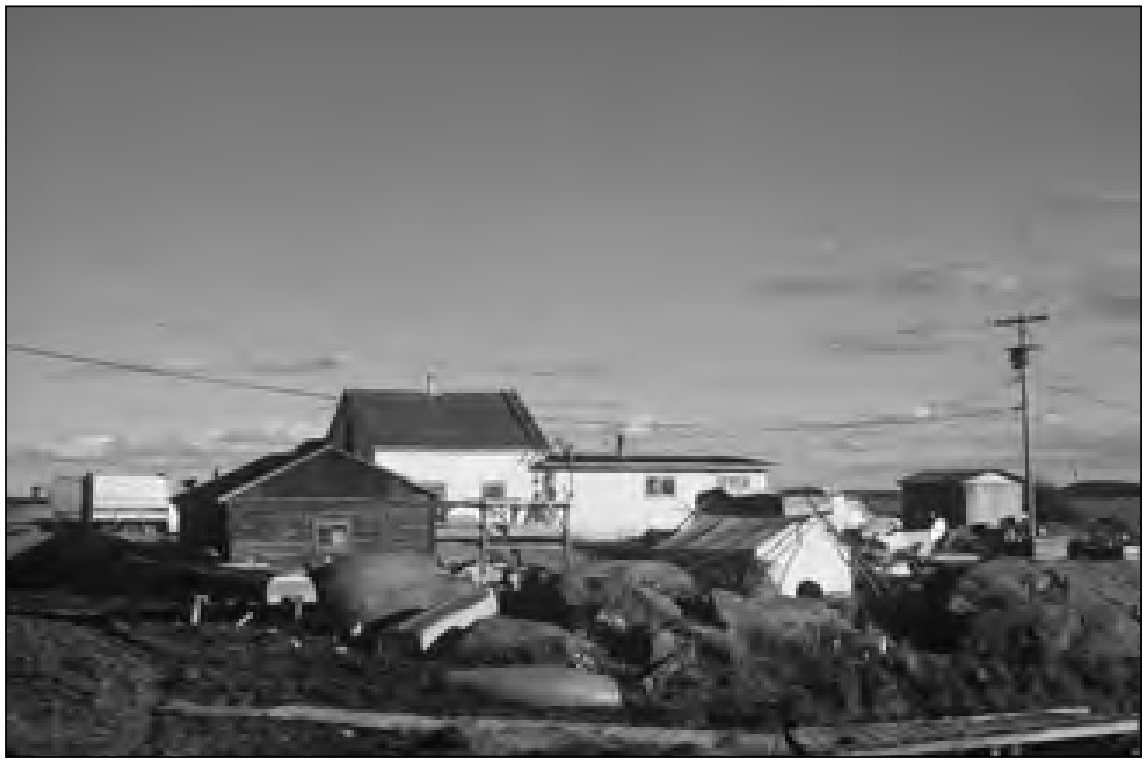

Photo 2

Domicile dènè tha, 1980

(Photo Jean-Guy Goulet) trielle, on doit emporter avec soi des bouteilles d'eau potable lorsqu'on se rend en forêt. Afin de s'orienter dans l'espace, on parle ainsi couramment du «Chemin Mobil», du «Chemin Esso », ou du «Chemin Husky ». En plus d'être mis à contribution comme main d'œuvre saisonnière dans le secteur industriel, les hommes piègent, pêchent et chassent le gros et le petit gibier. Les femmes chassent le petit gibier, vont à la pêche et cueillent aussi les petits fruits saisonniers. Ce sont aussi les femmes qui dépècent la viande du gibier, la sèchent et l'entreposent. C'est dans ce contexte qu'une majorité d'enfants, garçons et filles, apprennent à devenir compétents dans un monde où les valeurs du respect et de l'autonomie sont tout aussi importantes que celles du partage et de la coopération. Les enfants développent dans la forêt des attitudes positives face à la vie, qui se traduisent dans la manière dont ils se représentent dans leur milieu et agissent entre eux en salle de classe.

Comme nous le verrons dans la deuxième partie de cet article, tout en participant aux AST, les Dènès Tha valorisent l'ensemble des connaissances qui y sont associées. L'école de jour a son propre programme de "Culture et langue dènèe tha » auquel les anciens et les anciennes participent activement, favorisant ainsi un lien vital entre les générations. La direction de l'école tolère les absences des enfants en période de chasse ou d'activités familiales en forêt. Dans la cour d'école, les 


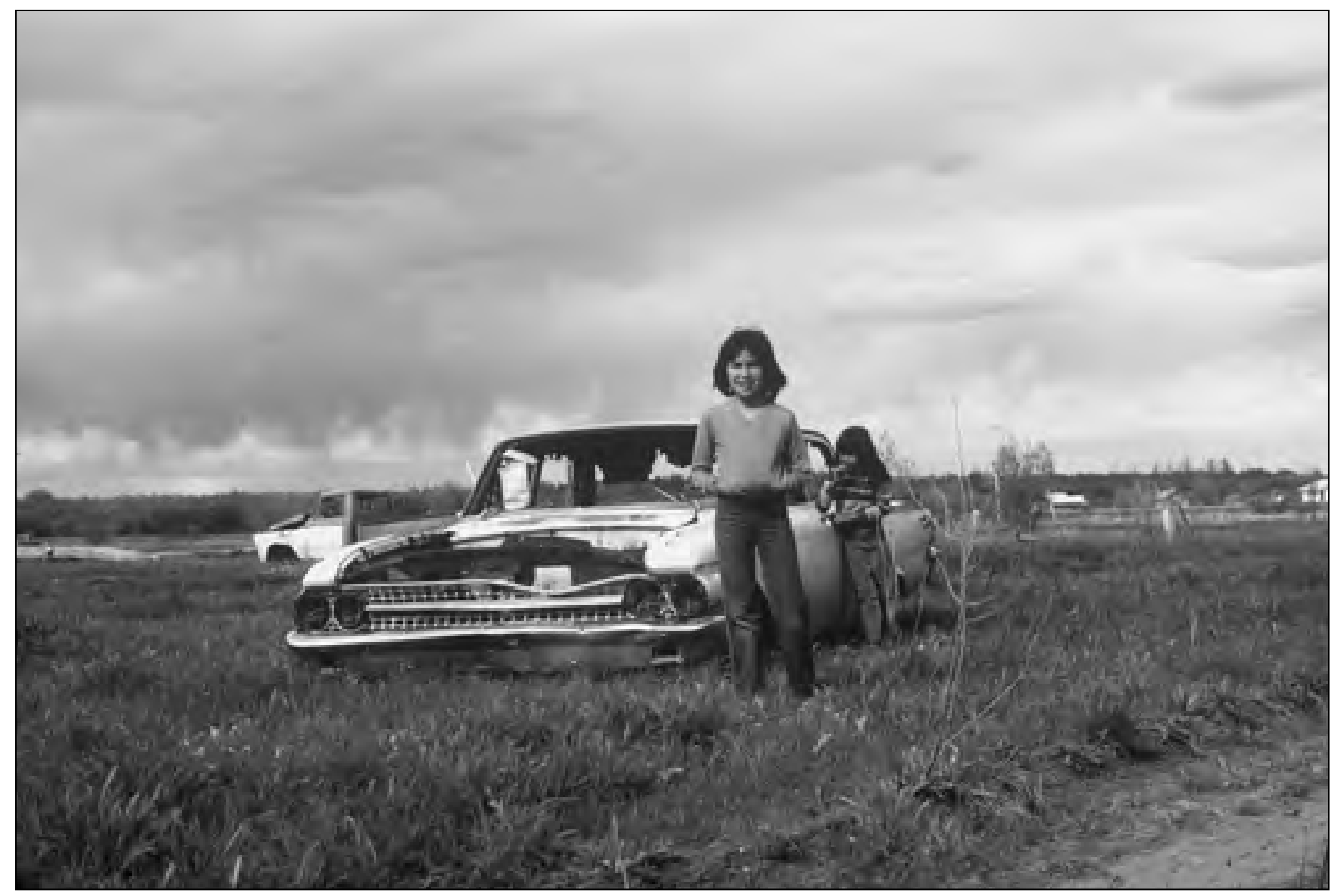

\section{Photo 3}

Les carcasses d'automobiles sont plus nombreuses que les canots

(Photo Jean-Guy Goulet)

enfants jouent dans leur langue. Ce sont ces enfants qui, en 1991, montraient à Kim Harvey-Trigoso, en particulier à partir de leurs dessins, comment ils se distinguaient les uns des autres selon leur degré d'adhésion au « conservatisme » et au « collectivisme »- au sens défini dans les pages qui suivent.

\section{Trois PRojets de ReCherChe PARMI les Dènès Tha}

J'ai eu l'occasion de décrire ailleurs mon propre cheminement comme chercheur parmi les Dènès Tha du Nord-Ouest albertain entre juillet 1979 et juillet 1985, à raison de plusieurs séjours d'une durée de six mois par année à partir de janvier 1980 (Goulet 2001, 2004). Je cherchais à comprendre leur monde social tel qu'ils le construisaient après un siècle d'interaction avec des allochtones. En quel sens étaient-ils encore autochtones? À quel point avaient-ils été transformés par les étrangers? Les commerçants et les missionnaires, les agents de la Couronne et les bureaucrates, les policiers et les juges, les éducateurs, les médecins et les infirmières, n'étaient-ils pas tous venus, tour à tour, avec l'intention avouée de mettre un peu plus d'ordre dans leur monde afin qu'ils puissent jouir d'une vie plus évoluée parce que moderne? J'ai découvert parmi les Dènès ce que d'autres chercheurs ont trouvé parmi les Innus, les Cris, les Anishinaabes, ou les Atikamekw. Encore de nos jours, il existe chez ces autochtones une perspective sur les pouvoirs humains et autres qu'humains, sur ce qu'il est permis d'espérer et de faire, qui est étrangère à la culture eurocanadienne dominante.

C'est afin de donner une voix à cette perspective qu'en 1995 et 1996 les Dènès Tha participaient à une étude menée par l'Arctic Institute of North America afin de consigner par écrit les connaissances des anciens au sujet des sites qu'ils considéraient comme importants du point de vue religieux, historique et économique. Cette étude fut menée dans l'espoir que les politiciens et les hommes d'affaires les prendraient en considération au moment de décider du développement économique régional (Dene Tha' Nation 1997). C'est dans la foulée de cette étude que Kim Harvey-Trigoso entreprit en 1998 et 1999 une recherche portant sur les valeurs des enfants dènès tha, particulièrement en ce qui a trait à leur attitude face à leur milieu social et naturel, telle que définie par leur degré de participation aux activités de subsistance traditionnelles (AST) et leur familiarité avec le savoir traditionnel véhiculé à travers les récits et les rituels des anciens. Sur la base de cartes géographiques locales dessinées par les enfants, de récits qu'ils ont faits d'événements vécus sur ce territoire, ainsi que de données recueillies à l'occasion d'entrevues avec leurs parents et aînés, Kim Harvey-Trigoso a mis en lumière deux valeurs, le conservatisme et le collectivisme.

Nous faisons nôtre la définition du terme « conservatisme » comme étant ce « qui tend à maintenir l'ordre social existant », 
tel que fournie par le Le Petit Robert, tout en tenant compte du fait que dans le monde dènè cet « ordre social » inclut les personnes humaines et les personnesautres-qu'humaines ${ }^{4}$ ainsi que les pratiques gouvernant les rapports avec les animaux et le gibier. Nous utilisons le vocable «collectivisme» dans le sens d'une attitude positive assumée par un ensemble d'individus motivés à atteindre un but commun ${ }^{5}$. Dans le cas des Dènès Tha, ce but consiste à maintenir sur un territoire ancestral un ensemble d'activités de subsistance traditionnelles et de valeurs en vue du développement de relations interpersonnelles respectueuses du droit à l'autonomie et au respect ${ }^{6}$. Ces valeurs qui sont au coeur de la culture des Dènès Tha, se retrouvent dans les populations de chasseurs et de cueilleurs, quel que soit l'endroit où on les rencontre (Tanner 1979; Woodburn 1982; Cox 1987; Gardner 1991; Wenzel 1991; Ridington 1992; Barnard 2004).

$\mathrm{Au}$ sein des AST, les relations de coopération et de réciprocité constituent la matrice au sein de laquelle chaque personne trouve l'occasion de se réaliser comme homme ou femme. Même si le mode de production contemporain des Dènès Tha représente un mélange d'activités traditionnelles en forêt et de participation périodique au marché du travail, nous postulons que la participation aux activités de chasse, pêche et cueillette favorise la transmission des valeurs traditionnelles associées avec la culture dènèe. La recherche parmi les chasseurs et cueilleurs conçoit les activités de subsistance non pas uniquement comme des « conditions de la vie matérielle des chasseurs » mais comme « des valeurs qui intègrent les relations économiques des chasseurs dans leur vie quotidienne » (Wenzel 1991 : 57). Dans l'étude de la question des relations qu'entretiennent les populations autochtones avec la société occidentale dominante dans la région subarctique, Tanner (1979), Ridington (1992) et Goulet (2004) notent que si celles-ci ont adopté la vie sédentaire, la nouvelle technologie et certains aspects du système économique dominant, elles le font non pas en vue de s'assimiler à la société blanche, mais en vue d'incorporer dans leur monde des éléments qui leur facilitent la vie. Au lieu de l'assimilation tant attendue de l'Indien, nous constatons plutôt un processus d'adaptation créatif qui évolue à la lumière d'un ensemble de valeurs autochtones (Goulet 1998a: 83). Celles-ci ont un effet structurant important dans la vie familiale et communautaire tel que nous le verrons dans la deuxième partie de cet article. Tout cela étant dit, il reste que, près des demeures dènès tha, on trouve aujourd'hui de moins en moins de canots et de plus en plus d'automobiles abandonnées qui servent de dépôts de pièces de rechange pour les nombreux jeunes mécaniciens qui passent des heures à réparer leurs véhicules. C'est d'ailleurs plus souvent en automobile ou camion qu'à pied qu'on se rend aux cérémonies d'offrandes présidées par les prophètes dènès (Goulet) [photos 3 et 4]

\section{LES VALEURS D'AUTONOMIE ET DE RESPECT DE SOI ET DES AUTRES}

La recherche parmi les chasseurs-cueilleurs constate un phénomène social caractéristique de ces sociétés, soit celui de

la valeur de «l'autonomie individuelle» dans le contexte d'un mode de production communautaire. Cette autonomie est particulièrement manifeste parmi les Athapascans septentrionaux dans leur acquisition de savoir et de pouvoir au moyen de ce qu'il est convenu d'appeler la quête d'un animal ou d'un esprit titulaire (Guédon 1994; Goulet 1994). Les récits racontés par les anciens ainsi que les cérémonies d'offrandes, à la maison, en forêt ou au site communautaire de la danse des prophètes (Goulet 2001), sont intimement associés au thème de la survie grâce à l'aide d'entités plus puissantes que l'être humain. Tout enfant apprend ainsi l'importance des rêves et des visions grâce auxquels les animaux confèrent aux élus un " pouvoir » pour améliorer sa fortune et la qualité de vie de ses proches (Moore et Wheelock 1990; Goulet 1998). Lindividu obtient de son esprit titulaire des pouvoirs de guérison qu'il fait connaître au groupe et qu'il doit utiliser pour le bénéfice des autres. Dans ce contexte comme dans tous les autres, les parents imposent très peu de restrictions aux enfants, leur laissant libre cours afin qu'ils puissent se rendre en forêt et y apprendre d'eux-mêmes les conditions pour devenir autonomes par la confiance en euxmêmes et par la compétence personnelle (Goulet 1998a : 39).

Il en est de même dans la prise de décision par les enfants dènès par rapport à tous les aspects de la vie. Du point de vue dènè, imposer à l'enfant une intervention dont il ne veut pas équivaudrait à lui enlever son autonomie et la possibilité de faire l'expérience de sa vie telle qu'il entend la vivre. Cette attitude parentale surprend l'observateur allochtone. Je me souviens d'une infirmière et d'un médecin qui demandaient à voir un enfant qu'ils soupçonnaient susceptible de contracter la tuberculose d'une grand-mère qui en était atteinte. Ils adressaient leur demande à des adultes réunis dans la salle communautaire dans laquelle jouaient plusieurs enfants, dont la jeune fille en question. Tous les adultes dènès savaient que l'enfant avait entendu la question et comprenait le but de la visite du médecin et de l'infirmière. Les minutes passèrent en silence. La question fut posée une autre fois, puis une troisième fois. Personne ne répondant, le médecin et l'infirmière quittèrent les 
lieux. Murray L. Wax rapporte une scène fort semblable qui s'est déroulée parmi les Sioux de Pine Ridge. Des parents expliquèrent à leur enfant de six ans que l'école souhaitait qu'il soit vacciné. L'enfant leur demandait de quoi il s'agissait, et les parents lui répondaient en lakota à la lumière des explications qui leur avaient été données par le directeur de l'école. «Informé, l'enfant refusait le vaccin et les parents abondaient dans son sens », respectant ainsi sa volonté, à l'encontre des allochtones qui voyaient dans cette attitude des parents « la marque d'un manque évident de sens des responsabilités » (Wax 2003 : 168).

Rien ne remplace l'observation en milieu naturel afin de saisir la distance qui sépare le monde familial dènè du monde familial eurocanadien. Les quelques scènes qui suivent, tirées de Goulet 2001, serviront à démontrer la véracité de cette affirmation et nous permettront de dégager ensuite les principes éthiques et épistémologiques qui sous-tendent l'ensemble de la vie sociale dènèe. Imaginez pour un moment la scène qui suit. Cinq adultes et sept enfants sont présents dans la grande salle de la maison. Au centre de cette salle, un poêle à bois répand sa chaleur dans toute la pièce. Latmosphère est calme, bien que la veille au soir un voisin en état d'ébriété ait lancé une pierre à travers la grande vitre qui donne sur la façade de la maison. La fenêtre brisée laisse pénétrer le vent par un espace irrégulier dans lequel plongent des lames de verres aux formes irrégulières. Un enfant d'environ un an qui sait à peine marcher pousse devant lui une chaise en direction de la table. Celle-ci repose directement sous la fenêtre brisée. Rendu à la table, l'enfant se hisse sur la chaise, s'y tient debout et monte à quatre pattes sur la table. Sitôt fait, il se dirige vers le trou dans la fenêtre et glisse son bras entre les morceaux de verre. Il sent l'air frais à l'extérieur, retire sa main pour la replonger aussitôt dehors. Il répète le geste plusieurs fois sans jamais toucher le verre brisé. Tout au cours de son exploration, ses parents et grands-parents l'observent du coin de l'œil tout en poursuivant calmement leurs activités. Pendant ce temps, je sens la tension monter en moi à la pensée qu'à tout moment l'enfant se coupera la main ou le bras. Laccident redouté ne survient toutefois pas. L'enfant satisfait de son exploration se laisse déjà glisser de la table à la chaise et se retrouve sain et sauf sur le plancher.

Un peu plus tard, dans la même salle un enfant de six ans s'approche du poêle en tenant une lourde casserole en fonte. Il la dépose sur le poêle. D'une voix à peine audible le père dit «attention, c'est très chaud». L'enfant poursuit sa démarche. Il fait fondre du beurre dans la poêle et y brise trois oufs qu'il dépose dans une assiette, une fois cuits. Il les saupoudre généreusement de sel et de poivre sans qu'aucun adulte ne dise « attention n'en met pas trop ». Il les mange et en fait cuire trois autres et les mange pour satisfaire sa faim! Toujours sans commentaires de la part des adultes ou de réactions de la part de ses compagnons de jeux, ils sont nombreux, qui poursuivent leurs activités dans la maison sans dire «moi aussi j'en veux! » Dans cette famille, comme dans toutes les autres familles dènès tha, personne ne dira jamais « le souper est servi » ou «venez manger, la nourriture est prête ». Non, chaque membre de la famille puisera dans le garde-manger ou le réfrigérateur ce qu'il a le goût de manger au moment où il le veut, à moins qu'il ne trouve déjà sur le poêle un succulent plat qui mijote, préparé par un adulte et mis à la portée de tous et chacun au moment opportun.

Un tel style de vie ne signifie pas que chacun ne vit que pour soi. Au contraire, chaque personne sait bien qu'elle vit avec d'autres, en leur présence. C'est d'ailleurs en se donnant l'occasion de s'observer mutuellement dans presque toutes leurs activités que les autochtones multiplient les occasions d'« apprendre par observation». Le plus souvent c'est en effet sans instruction qu'on apprend comment se fait telle ou telle chose. Plus encore, parents et enfants s'adonneront ensemble au piégeage, à la chasse et à la cueillette afin de ramener de la nourriture et des fourrures à la maison. De même, les grandsparents rempliront de victuailles le réfrigérateur de la maisonnée dont ils font partie en dépensant au magasin local leur chèque de pension de vieillesse que leur verse le gouvernement fédéral. Tous et chacun prendront part à la bonne marche de la famille, mais toujours sans demander ou commander aux autres quoi faire.

J'ai vu des septuagénaires et octogénaires, hommes et femmes, prendre jusqu'à cinq minutes pour se hausser à bord d'une camionnette, perdant parfois pied, mais se reprenant tout aussitôt, afin de se rendre à leur siège d'eux-mêmes, bien que tout en sueur et à bout de souffle. Tout au long de cette difficile ascension, les passagers adultes qui occupent déjà la camionnette poursuivent leur conversation comme si de rien n'était. Il eut été si simple d'aider ces personnes âgées à monter à bord. Spontanément, je voyais cette absence d'aide comme un manque de respect. À l'inverse, les Dènès auraient vu un tel geste comme une atteinte à l'autonomie d'autrui, une façon de dire à la personne âgée « tu n'es pas capable » ou « nous sommes trop pressés pour te laisser faire par toi-même ce que nous pouvons faire encore nous-mêmes sans efforts ».

Si nous différons tellement dans nos réactions spontanées devant ces enfants et ces aînés, c'est entièrement dû à notre éducation première. Selon moi - ainsi que pour la grande majorité des Euro-Nord-Américains -, il aurait fallu intervenir et prendre l'enfant avant qu'il ne parvienne à la fenêtre afin de le protéger d'un accident possible. Il aurait aussi fallu rappeler à l'enfant que deux œufs suffisaient et qu'il fallait penser aux autres. Mais selon les Dènès Tha de telles interventions priveraient les enfants de leur droit à l'autonomie. Ils sont d'avis que chacun est capable d'apprendre par observation et de s'engager avec compétence dans les activités qu'il veut accomplir. En milieu dènè, c'est d'ailleurs tout au cours de sa vie qu'une personne observe autrui faire l'expérience de ses capacités et du respect inconditionnel de l'autonomie de chacun. C'est ainsi qu'à tous les âges de la vie on retrouve parmi les Dènès Tha un des phénomènes importants de la psychologie communautaire, le «développement du pouvoir d'agir des personnes et des collectivités » ainsi que «l'espoir appris » (learned hopefulness, selon Zimmerman 1990) [Le Bossé et Dufford 2001 : 49].

Lorsque nous disons aux parents dènès : " mais vous devriez intervenir », ou «vous devriez expliquer », les Dènès nous regardent de façon incrédule. Lorsque nous décrivons leurs modes d'apprentissage comme se déroulant sans intervention ou directives de la part d'autrui, nous signalons l'absence des éléments qui chez nous sont constitutifs d'un bon enseignement ou d'une bonne attitude parentale. Décrivant leur monde tel qu'il apparaît à nos yeux et non aux leurs, nous passons sous silence les aspects positifs de l'apprentissage en milieu dènè, apprentissage qui repose sur un sens aigu de l'observation, une dextérité maintes fois éprouvée et améliorée et un mimétisme mille fois récompensé par la satisfaction d'une réussite anticipée et voulue. Dans ce monde le silence est d'or et la parole est d'argent. Les Dènès souscriraient entièrement aux propos des Warlpiris au sujet des Blancs : « Tout ce qu'ils désirent c'est parler... Ce jacassement me donne vraiment mal à la tête. 
Les Blancs parlent trop, comme les oiseaux!» (cité par Dussart 2004 : 74). Chez les Dènès comme chez les Warlpiris, et comme chez tous les peuples chasseurs, " apprendre c'est une affaire d'observation, non de questions » (Dussart 2004 : 74).

Compte tenu de ces postulats épistémologiques autochtones, il saute aux yeux que le système scolaire eurocanadien opère sur la base de tout autres principes. Il y a plus de trente ans, McClellan (1972 : xvi) notait qu'indépendamment de la matière enseignée, dans la scolarisation des enfants indiens, c'est « l'accent mis de plus en plus fort sur l'instruction verbale dans les salles de classe avancées qui contribue chez les enfants plus âgés au retrait du système scolaire qui fait si souvent l'objet des commentaires des Blancs ». Le même constat apparaît dans une étude récente des difficultés en milieu scolaire des enfants dènès tha de Chateh :

Nos évaluations révèlent que les enfants dènès tha ont des forces importantes dans plusieurs domaines. Ils ne sont pas stupides, incapables, ou déficients. Presque tous les étudiants ont manifesté une grande dextérité ainsi qu'une habileté à apprendre en imitant ce qu'ils observaient. Cependant, ces mêmes étudiants sont faibles lorsqu'il s'agit d'apprendre par le biais de l'exposé oral. C'est dans l'ensemble de la population étudiante que l'on retrouve ce patron d'apprentissage visuel fort et d'apprentissage verbal faible. Ce patron sera un facteur déterminant négatif dans le milieu scolaire qui dispense les connaissances selon les méthodes conventionnelles d'exposés magistraux. (Dene Tha Community School Testing Information $1996: 5)$

En d'autres mots, si le rapport constate que dans le milieu scolaire les enfants dènès tha sont désavantagés, il ne fait aucune recommandation quant aux manières dont la pédagogie pourrait être modifiée afin de mettre à profit le mode d'apprentissage privilégié par les Dènès.

Dans ce contexte la question qui s'impose à nous est la suivante: comment apprendre en faisant appel le moins possible à l'échange de questions et réponses entre la personne qui enseigne et celle qui apprend? Aux yeux de Kroeber, ce refus amérindien de donner des explications à celui qui en demande constituait « une preuve des déficiences de la part des Yuroks au niveau de l'intellectualisation » (1976: 331, in Buckley 2002 : 105). À l'inverse les Yuroks interprétaient les questions de la part de Kroeber comme «manquant de politesse et ne produisant pas une véritable compréhension » (Buckley 2002 : 105) Au dire de Robert Spott, un Yurok fort respecté : «Les Blancs expliquent trop ». Spott soutenait qu'il avait tenté en vain d'instruire Kroeber qui en bout de ligne s'était révélé tout à fait « incapable d'apprendre » (Buckley 2002 : 106). N'est-ce pas la même conclusion à laquelle arrivent les nombreux enseignants eurocanadiens qui se trouvent en présence d'élèves autochtones récalcitrants? Une profonde disparité sur les plans épistémologique et pédagogique entre autochtones et allochtones explique l'impasse dans laquelle ils se retrouvent en milieu scolaire. Les uns ne trouvent pas un milieu propice à leur mode d'apprentissage par observation, les autres cherchent en vain des élèves capables de maîtriser le programme qui leur est proposé par le biais d'exposés verbaux.

\section{De LA fORÊt À LA SALLE DE CLASSE}

Dans les pages qui suivent, nous analysons les données relatives à deux groupes d'enfants, ceux chez qui on note un haut taux de participation aux activités de subsistance traditionnelle (HTPAST) et, d'autre part, ceux chez qui on relève un faible taux de participation aux activités de subsistance traditionnelle
(FTPAST). La comparaison des deux groupes nous permet de mettre en lumière des différences importantes entre ces deux groupes en ce qui a trait à leur attitude face à la vie et à leur milieu social.

Lhypothèse envisagée était la suivante: plus les enfants assimilent le savoir traditionnel, plus leur attitude vis-à-vis du milieu naturel (conservatisme) et de leur communauté (collectivisme) est positive ${ }^{7}$. Létude prédit que le collectivisme et le conservatisme vont de pair puisqu'il existe un lien intime entre les deux attitudes chez les peuples autochtones. Tel que noté plus haut, le conservatisme consiste en l'usage efficace des ressources en lien avec une préservation de l'environnement; le collectivisme consiste en une attitude bienveillante vis-à-vis de la communauté fondée sur la coopération et la réciprocité. Tel que nous le démontrons plus loin, le degré de participation des enfants aux AST, soit la variable indépendante, est identifié par le biais d'entrevues centrées ou guidées avec les adultes ayant à charge les enfants participant à l'étude. La variable dépendante, elle, soit le degré de collectivisme et de conservatisme, est définie par des éléments trouvés dans les dessins des enfants, les associations verbales et les types de comportements sociaux. $\mathrm{Au}$ terme d'une analyse d'un ensemble de données (récits, comportements et dessins), une conclusion s'impose: plus l'enfant en milieu scolaire participe aux activités traditionnelles de chasse, pêche et cueillette avec des membres de sa parentèle, plus il s'oriente dans la vie de façon positive en fonction de valeurs communautaires et de conservation des ressources naturelles.

Les méthodes choisies dans cette étude ont permis de cueillir des données sur le degré de participation des enfants dans les AST ainsi que sur les connaissances qui leur sont transmises au sujet de leur milieu naturel et social. Des indices de cette transmission apparaissent dans les dessins individuels des enfants (des cartes de leur communauté et d'espaces privilégiés entourant leur communauté), dans leurs associations verbales à ces dessins et dans l'interaction des enfants réalisant collectivement des cartes géographiques de leur communauté. Aux données cueillies auprès des enfants s'ajoutent celles, complémentaires, obtenues des parents ou adultes qui en avaient la charge. L'expérience décrite dans les pages qui suivent démontre à quel point il est profitable, au-delà de la simple observation des comportements des enfants, de leur poser des questions concernant leurs dessins tout en prenant note des associations verbales spontanées qu'ils expriment à leur propos 8 .

\section{ÉCHANTILLONNAGE DES ENFANTS et CARACTÉRISTIQUES DE LEURS DESSINS}

C'est dans la salle de classe qu'en 1998 et 1999, dans le cadre d'un projet de recherche inscrit au sein d'un programme de maîtrise en anthropologie que Kim Harvey-Trigoso rencontre à Chateh les élèves dènès tha qui participeront à son étude. Elle postule que les enfants de 8 à 11 ans ont déjà eu l'occasion de participer aux activités de subsistance traditionnelle (AST), chasse, pêche et cueillette, en compagnie de leurs aînés et que, chez ces enfants, l'intériorisation des valeurs traditionnelles est déjà faite (Fine et Sandstrom 1988). Lidentité sociale étant déjà formée chez les enfants de ce groupe d'âge situé entre l'enfance et l'adolescence (Erikson 1950), on considère comme probable qu'ils participeront aisément à l'étude de leurs valeurs et de leurs comportements. Compte tenu de ces postulats, l'école représente le milieu idéal dans lequel rencontrer ces enfants.

$\mathrm{Au}$ début de l'enquête, 82 enfants inscrits à l'école correspondent à ce groupe d'âge. De ce nombre, 56 participent à 


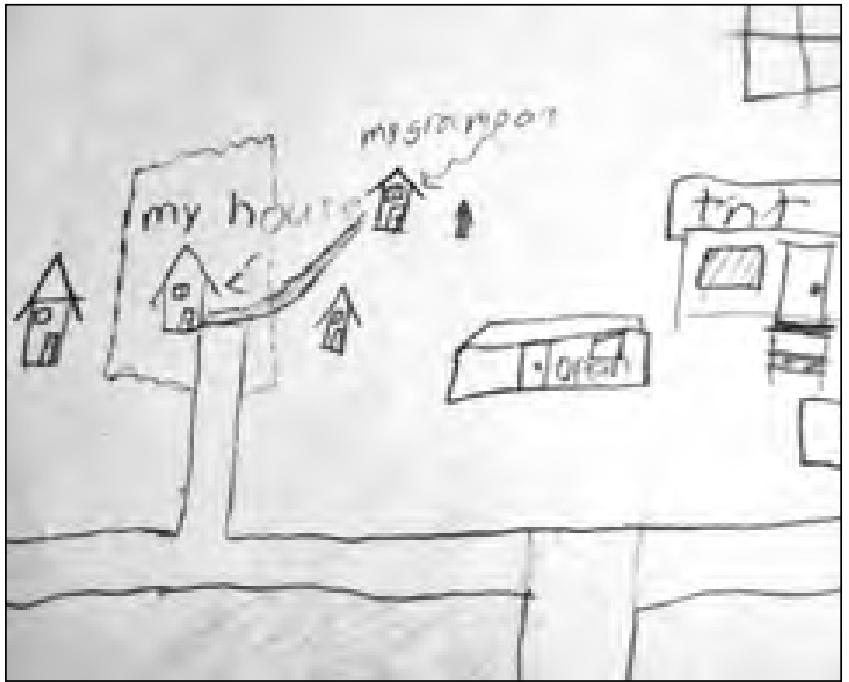

Figure 1

Le sentier entre le domicile de l'enfant et celui de son grandpère indique le lien qu'ils entretiennent (collectivisme fort) [Dessin de Willie, 4e - 5e année]

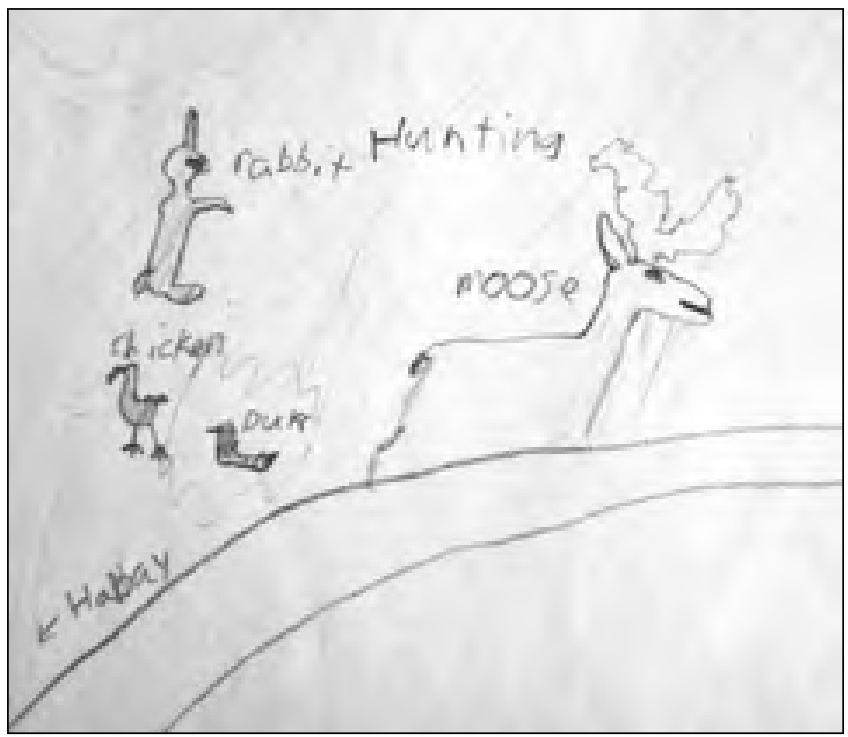

Figure 2

Plusieurs formes de gibier (conservatisme fort)

[Dessin de Willie, 4e - 5e année]

l'étude. La non-participation des 26 autres enfants s'explique par un ensemble de facteurs : le déménagement de cinq enfants, l'absence répétée de trois autres, le faible taux de participation de cinq autres qui doivent souvent quitter la classe, et la décision de cinq autres de se retirer de l'étude. Les données qui proviennent de l'échantillon des 56 enfants (32 filles et 24 garçons) qui ont participé à l'étude ainsi que des entrevues avec les adultes qui en avaient la charge, ont conduit à l'identification de 27 enfants ayant un HTPAST et de 29 enfants ayant un FTPAST.

Les dessins qui apparaissent dans cet article varient selon qu'ils sont l'œuvre d'enfants qui participent beaucoup ou peu aux AST. Le degré de conservatisme et de collectivisme est plus élevé chez les enfants qui ont un HTPAST (voir

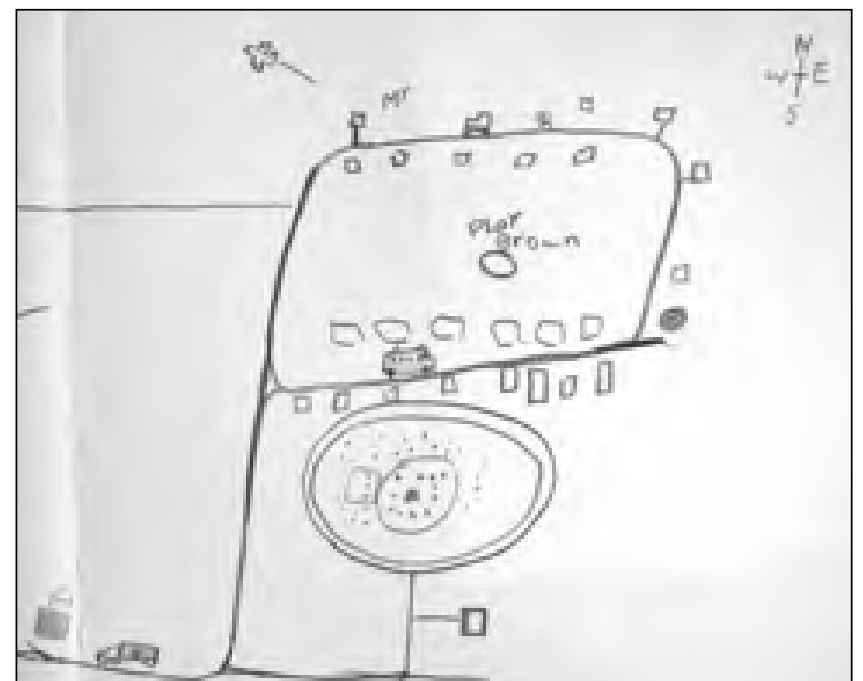

Figure 3

Terrain de jeu juxtaposé au Cercle de la Danse des prophètes (aussi appelée Tea Dance en anglais) - au sein du Cercle les personnes sont représentées par des points (collectivisme fort) [Dessin de Gary, 3e année]

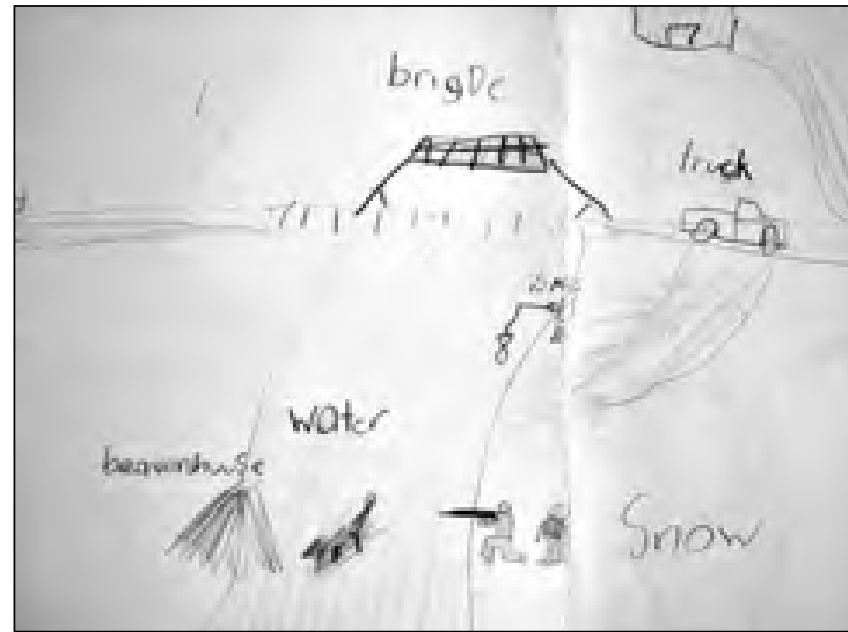

Figure 4

Enfant à la pêche en compagnie de son frère qui regarde le père chasser à sa droite (conservatisme fort)

[Dessin de Daniel, 3e année]

dessins 1, 2, 3 et 4). Ceux-ci font référence plus souvent aux institutions qui offrent des services à la communauté ainsi qu'aux activités qui font appel à la coopération entre les membres de la communauté. Ils dessinent davantage de scènes en forêt, y inclus des terrains de chasse à l'intérieur et à l'extérieur de la communauté. Ces enfants parlent de la chasse et du piégeage comme de moyens de subsistance, même si en réalité leurs parents vivent de leur salaire plus que de la forêt. Dans leurs dessins, ces enfants qui font l'expérience d'un HTPAST se représentent eux-mêmes en train de chasser. Ils parlent aussi de la chasse et de la cueillette comme étant des activités qui réunissent les gens. À l'opposé, les enfants qui participent peu aux AST tendent à dessiner le village ou la ville ainsi que des scènes en milieu de travail (voir les dessins 5, 6, 7 et 8). Ils représentent très peu d'interaction entre personnes ou de mises 


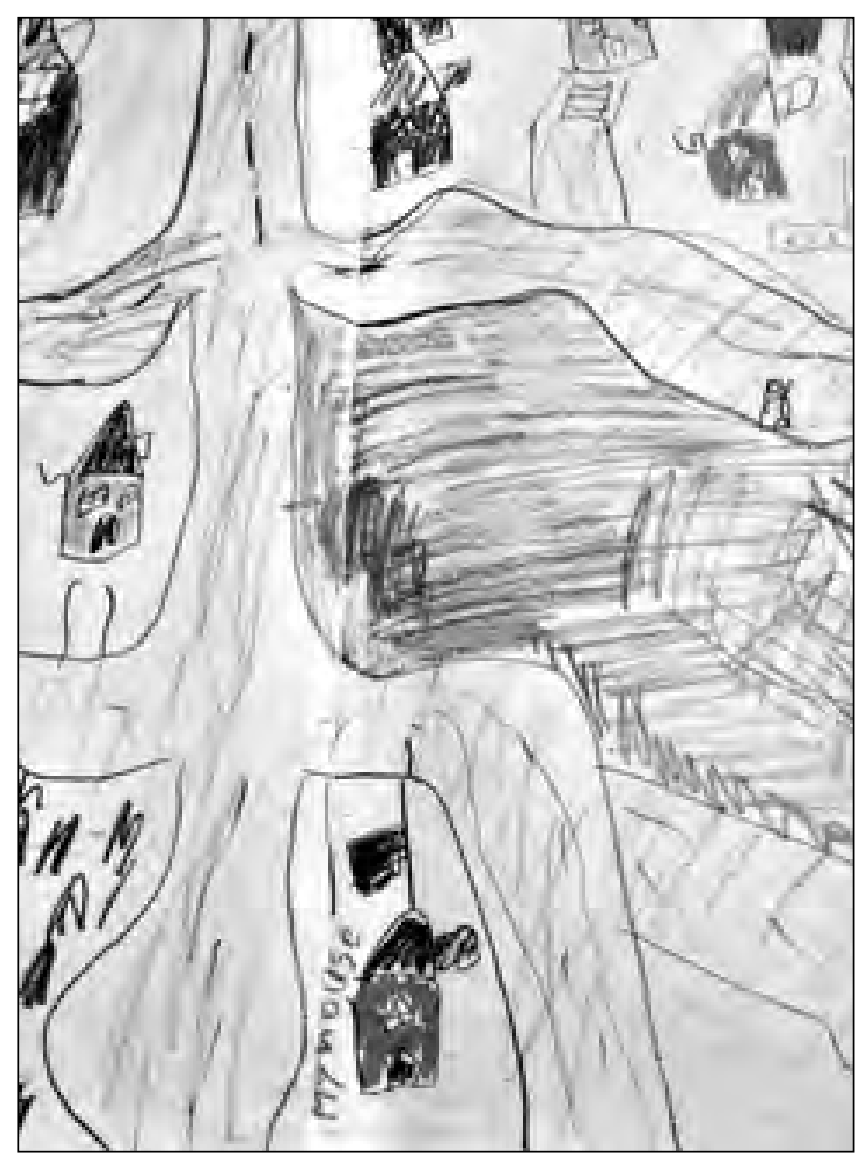

Figure 5

Domicile d'un enfant sans relation à d'autres demeures (pas de sentier) [collectivisme faible]

[Dessin de Sarah, 3e année]

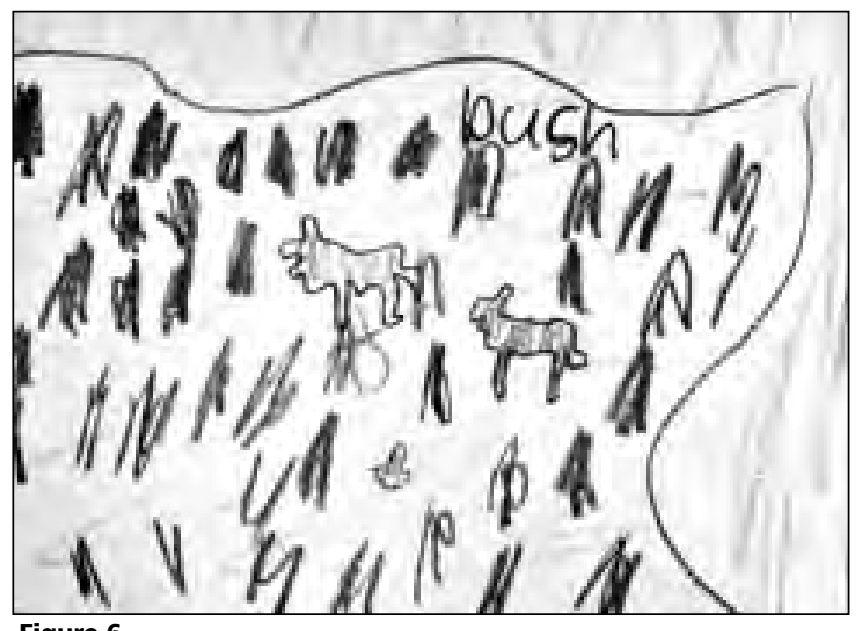

Figure 6

Des animaux qui ne sont pas associés à la chasse

(conservatisme faible)

[Dessin de Sarah, 3e année]

en scène de chasse en forêt. Leur degré de conservatisme et de collectivisme est donc faible.

Les dessins des enfants furent codifiés de la manière suivante suivant les variables de conservatisme et de collectivisme.

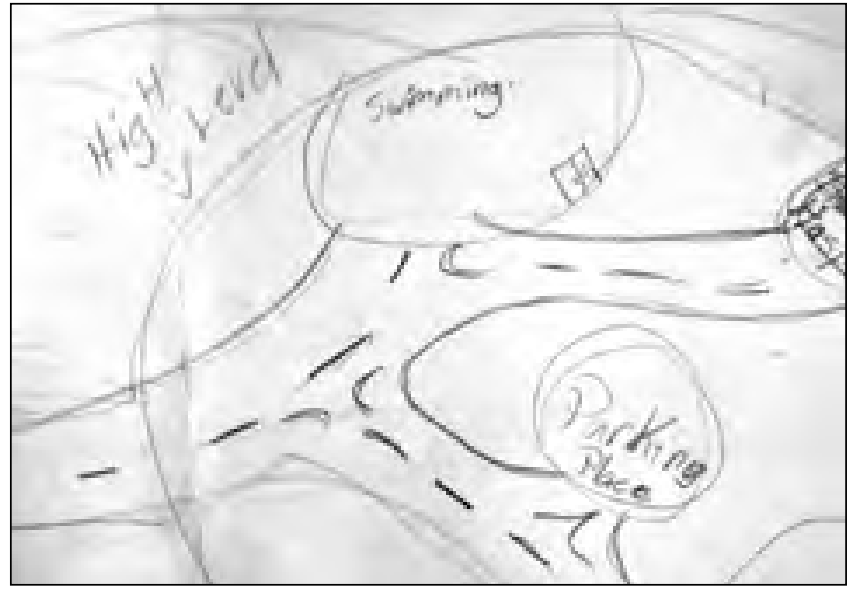

Figure 7

Lieux externes à la communauté de l'enfant, trouvés dans la ville voisine de High Level, sans relation à qui que ce soit (collectivisme faible)

[Dessin de Marianne, $3^{e}$ année]

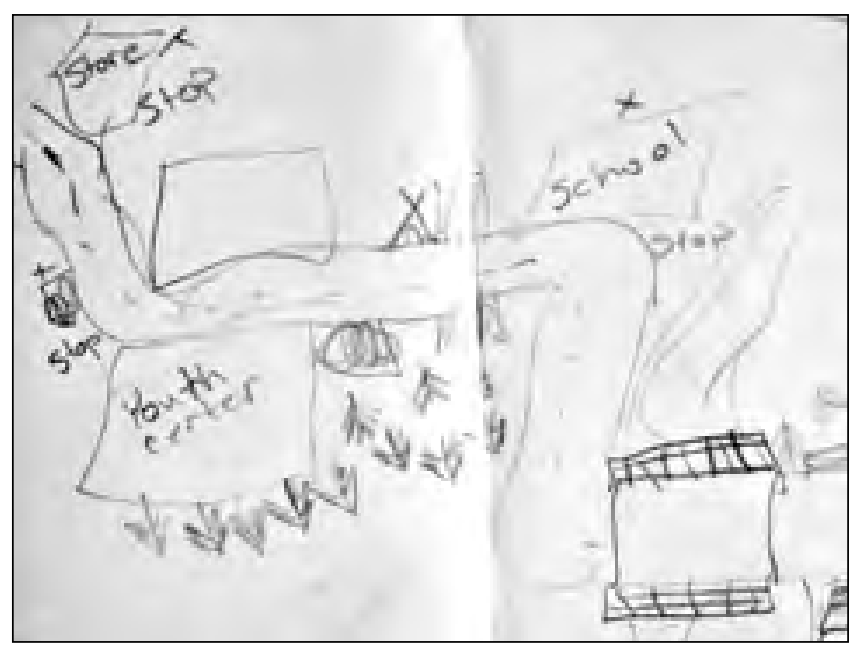

Figure 8

Milieu urbain et matériaux. Absence de personnes ou de relations, pas d'AST, peu d'éléments naturels (conservatisme faible et collectivisme faible)

[Dessin d'Ingrid, 6e année]

Pour le conservatisme, furent codifiés tous les éléments dessinés ou écrits concernant des éléments naturels, tels des créatures, des plantes, des phénomènes géophysiques ainsi que les activités impliquant une utilisation de ces éléments naturels. Furent aussi codifiés les associations verbales ainsi que les commentaires concernant ces éléments. Pour le collectivisme furent codifiés tous les dessins ou commentaires verbaux au sujet d'une interaction sociale concernant un lieu, une créature, un objet ou une activité qui entraînait une association volontaire entre individus à l'extérieur du domicile. Furent prises aussi en note les associations verbales indiquant si l'enfant avait aimé l'activité.

En bout de ligne, la corrélation entre les scores obtenus concernant les AST et le degré de conservatisme et de collectivisme se révéla être de l'ordre de $r=.67$ et .59 respectivement $(p<.01)$. Ces corrélations sont très significatives. C'est en effet $45 \%$ de la variance concernant le conservatisme et $35 \%$ de la variance concernant le collectivisme qui se trouve expliquées 
par les AST. Tel que nous le verrons plus bas, ces données quantitatives appuient fortement les données qualitatives obtenues au cours des entrevues avec les adultes ayant les enfants à charge.

\section{DESSINER COMME ACTIVITÉ SOCIALE}

Les enfants de chaque année scolaire furent divisés en groupe selon leur niveau de participation aux AST. Les cinq groupes au HTPAST et les cinq groupes au FTPAST furent observés durant l'exécution d'un exercice qui consistait à dessiner ensemble une carte géographique de leur communauté. La différence entre les deux types de groupe s'exprime à trois niveaux: dans le type de leadership qui émerge dans le groupe, le style de communication privilégiant soit la coopération ou la discorde, ainsi que dans les manières dont les enfants envisagent les problèmes sociaux de leur milieu.

Dans les groupes d'enfants ayant un HTPAST, on s'affirme sans devenir autoritaire ou dominant, sans donner des ordres aux autres et sans chercher à leur imposer ses idées. La participation est fluide, évidente à la vue de la coopération spontanée entre enfants. Dans une classe de la sixième année, le leadership s'exerce par émulation. La fillette qui prend l'initiative de dessiner la carte explique aux autres ce qu'elle dessine. Apparemment stimulés par l'intérêt de la première fillette, les autres membres du groupe collaborent immédiatement au dessin. Celle-ci ne s'impose jamais, laissant à tous et chacun une liberté entière dans l'exécution du projet. Lorsqu'un garçon arrête de dessiner, elle n'intervient pas pour le ramener à la tâche. Dans un autre groupe, le leader communique sans cesse avec ses coéquipiers, les aidant au besoin et évaluant l'évolution du travail de l'équipe au fur et à mesure que chacun y apporte sa contribution. Par son comportement, ce leader stimule les autres à contribuer au projet, favorisant ainsi des interactions positives constantes.

De même, dans un groupe d'enfants de troisième année ayant un HTPAST, deux enfants seulement travaillent à la carte parce que les deux autres membres du groupe doivent s'absenter. Cette situation donne lieu à un partage du leadership, chacun menant selon son expertise. Gary, qui semble plus confiant quand il s'agit d'art graphique, dessine la carte. Jamie l'accompagne dans l'exécution du dessin par ses commentaires verbaux. Lorsque Gary invite Jamie à dessiner des canards, ce dernier prend une position de yoga, ferme les yeux et prétend méditer en silence. Il sort subitement de sa méditation et dit à Gary : «Voici, il doit y avoir un orignal juste ici. » Gary s'exécute. Jamie reprend l'attitude du yogi et dit avoir une vision d'un loup, que Gary dessine là où Jamie l'indique. Dans cet exemple, Jamie met à profit sa connaissance de la tradition dènèe et simule la réception de « visions » qui décident des endroits où dessiner les animaux sur la carte. En guise de commentaire, Gary dit: "Vous avez un jeune qui en sait beaucoup [et] un jeune qui raconte [révèle] beaucoup. » À l'opposé, les leaders des groupes ayant un FTPAST sont plus autoritaires et critiquent facilement les membres du groupe. Dans un groupe de sixième année au FTPAST celle qui dirige le groupe perturbe les activités. Elle critique les dessins de ses coéquipiers et efface ce qu'ils dessinent en affirmant que ce qu'ils avaient fait était « trop gros» ou « trop loin». Ce type de leader conduit les autres à se retirer du processus de création.

Le style de communication privilégié varie aussi dans les groupes selon le degré de participation des enfants aux AST. Les enfants ayant un HTPAST se consultent souvent. Cette consultation prend la forme d'échanges sur l'endroit précis où situer les éléments de la carte, de commentaires expliquant ce qu'ils s'apprêtent à dessiner, et d'ouverture à corriger les erreurs s'il s'en trouve. Ces enfants s'écoutent et répondent positivement aux commentaires des uns et des autres. C'est par la négociation et les compromis que les conflits sont résolus avant même qu'ils n'accaparent la vie du groupe. C'est l'opposé de ce que l'on observe dans les groupes d'enfants ayant un FTPAST. Les enfants tendent à dessiner indépendamment les uns des autres. Les échanges verbaux sont négatifs; on s'échange régulièrement des critiques, des plaintes et des insultes. Les différences d'opinions s'enveniment rapidement. Dans ces groupes, ces enfants corrigent les dessins des uns et des autres sans consultation et sans dialogue. Par exemple, lorsque deux enfants ne s'entendent pas sur un élément du dessin, ils se fâchent et commencent à dessiner sur le dessin de l'autre. Cet épisode se clôt par le départ d'un des enfants. Tous ces comportements conduisent à la confusion et la frustration, diminuant d'autant le goût de participer à la tâche collective.

La coopération généralisée dans les groupes d'enfants à HTPAST prend différentes formes. Les enfants s'aident mutuellement en situant les éléments de la carte les uns par rapport aux autres, en épelant correctement les mots, et en allant même jusqu'à dessiner les uns pour les autres. Ces enfants s'encouragent aussi à dessiner ensemble la carte de leur communauté. Dans un groupe de troisième année, c'est par le recours à l'humour qu'un garçon entraîne son compagnon à l'aider à dessiner la communauté plutôt que les régions avoisinantes. «Ne dessine pas seulement les buissons... ce n'est pas tout le monde qui a besoin de tant d'air frais!» lui dit-il. Aussitôt dit, on éclate de rire, et le compagnon se rallie au projet collectif.

Pas étonnant que dans les groupes ayant une HTPAST les enfants manifestent de la confiance en soi et en autrui. Ils cherchent visiblement à dessiner une carte géographique réaliste de leur communauté. Dans un de ces groupes, on trace des liens entre Chateh et deux communautés dènès voisines, celle de Meander et Bushe. Les enfants disent que ces deux réserves voisines font partie de leur communauté. On ne voit rien de semblable dans les groupes ayant un FTPAST. Dans ces groupes, la coopération est moindre, et les enfants tendent à s'engager dans des activités parallèles. L'interaction, lorsqu'elle survient, tend à être faible et négative. Les enfants deviennent défensifs lorsqu'ils s'entendent dire par leurs compagnons qu'ils sont « paresseux » ou que leurs dessins sont « laids». Les critiques mutuelles de ce qui est dessiné inhibent l'interaction et la coopération et diminuent l'enthousiasme pour la tâche confiée au groupe. Les membres du groupe ont moins confiance en eux et ils effacent souvent ce qu'ils viennent de dessiner. Si les cartes de ces enfants contiennent certains éléments naturels, on ne représente jamais des AST. De même, on rencontre rarement dans leurs cartes les lieux où se célèbre la Danse des prophètes.

Enfin, les enfants des deux groupes ont recours à des récits et à des fantaisies afin de traiter des problèmes sociaux de leur milieu, en particulier ceux qui sont liés à l'abus de l'alcool et à la violence qui en découle. Dans les deux types de groupe, le thème de la vie et de la mort domine. On dessine des accidents de circulation causés par la conduite en état d'ébriété. On représente le cimetière qui apparaît comme un symbole important sur leurs cartes. On raconte souvent, en les illustrant, des récits de personnes mortes d'hypothermie par suite de chutes à l'extérieur, l'hiver, lorsqu'elles étaient en état d'ivresse. Bien 
qu'il s'agisse là d'événements relativement fréquents au sein de leur communauté, les enfants diffèrent dans la manière de traiter ces phénomènes. La conclusion des récits des enfants de groupes au FTPAST est invariablement triste. Dans un de ces groupes, on discute même de la possibilité de faire sauter la communauté et d'entraîner ainsi un déménagement général dans la ville voisine de High Level. À l'encontre, dans les récits des enfants des groupes ayant un HTPAST, des expériences négatives se transforment en expériences positives. Par exemple, un enfant dit qu'il a été blessé par une balle de fusil, mais qu'il est toujours en vie. Dans un autre récit, un garçon rapporte qu'un enfant est tombé dans la rivière. Une fillette du groupe enchaîne aussitôt en disant que quelqu'un l'a sauvé, ce qu'elle représente immédiatement sur la carte. Dans son étude des dessins des enfants cris de Mashkeko, George Fulford (1994) rapporte des thèmes similaires importants. Il note que les enfants paraissent s'identifier avec les bonnes tortues ninja dans des combats contre des personnages sinistres qui représentent les problèmes sociaux de leur milieu (l'alcool, la violence, le gambling)

\section{LES ACTIVITÉS DE SUBSISTANCE TRADITIONNELLE DANS LE CONTEXTE FAMILIAL}

Les entrevues ont permis aussi d'identifier le type d'éducation familiale dont les enfants font l'expérience selon que les parents ou les adultes ayant les enfants à charge disent passer du temps avec les enfants, leur enseigner des attitudes positives face à la nature et à la communauté et les protéger ou non des effets néfastes de la boisson. À tous ces niveaux, il n'y a pas de différence significative dans le résultat des entrevues des membres de familles ayant un haut ou un faible taux de participation aux AST.

De même, toutes les personnes rencontrées en entrevue s'entendent pour dire que c'est par l'observation et l'imitation que les enfants apprennent à participer aux AST. Cette participation n'est pas imposée, mais survient lorsque l'enfant manifeste de l'intérêt pour ces activités. Plus l'intérêt de l'enfant est grand, plus on lui donne l'occasion de s'adonner aux AST en compagnie des aînés. Par exemple, une grand-mère remarque que sa petite-fille n'est toujours pas intéressée à enfiler des perles afin de confectionner des colliers. La grand-mère ajoute qu'il en avait été de même au même âge avec sa fille, la mère de l'enfant. Lintérêt pour ce genre de travail étant venu plus tard chez la mère de la fille, il était normal d'attendre que cet intérêt se manifeste éventuellement chez la petite-fille. Dans une autre famille, une mère mentionne que sa fille « observe » lorsqu'elle (la mère) et la grand-mère traitent les peaux d'orignal. Une autre mère dit qu'elle pense que ses deux garçons savent comment sécher de la viande parce qu'ils l'ont observée lorsqu'elle le faisait. De même, dans une autre famille, une mère dit que son plus jeune garçon qui ne sait pas encore chasser accompagne son frère aîné et «l'observe ». Les parents racontent aussi comment les enfants tentent d'exécuter certaines tâches, tels la cuisson, le dépeçage des animaux ou le traitement de la viande, sans les maîtriser complètement.

Il ressort aussi des entrevues que le genre est une dimension importante de la socialisation dans le cadre des AST. C'est en effet généralement avec une femme qu'une fillette participe aux AST et avec un homme que le garçon fait de même. Ce que les enfants observent et apprennent diffère ainsi selon le genre. Le garçon se rend à la chasse et au piégeage; la fille s'adonne à la préparation des peaux et de la nourriture ainsi qu'aux différentes formes d'artisanat. On note toutefois une plus grande flexibilité des rôles dans les familles caractérisées par un HTPAST. Dans ces familles, il n'est pas rare de rencontrer des femmes qui ont chassé l'orignal ainsi que des filles intéressées à faire de même. Parmi les jeunes filles des familles à HTPAST, trois d'entre elles avaient chassé et plusieurs autres accompagnaient leurs parents à la chasse. Cette pratique de la part de ces jeunes filles indique-t-elle un affaiblissement du tabou athapascan traditionnel concernant les menstruations et l'interdiction faite aux femmes de participer aux expéditions de chasse en période de menstruation? Pas nécessairement, car il est possible que ces jeunes femmes sachent restreindre leurs déplacements en forêt en raison précisément de ces tabous. Nous n'avons pas de données qui nous permettent de trancher dans un sens ou dans l'autre. La deuxième possibilité nous semble la plus probable ${ }^{9}$

Dans les familles caractérisées par un FTPAS, les filles s'adonnent tout au plus à la pêche et à la cuisson de viandes sauvages. Dans ces familles, les adultes disent que les filles sont moins intéressées que les garçons à se rendre en forêt. Les filles se trouvent ainsi exclues de la chasse. Un père de famille reconnaît que sa fille ne l'accompagne pas à la chasse avec ses frères, précisément parce qu'elle est une fille. Le père s'explique en disant que «La coutume pour les autochtones veut que les filles demeurent à domicile et fassent les travaux domestiques et que les hommes demeurent à l'extérieur pour chasser; mais maintenant tout a changé ».

Les entrevues avec les parents ou les adultes ayant à charge les enfants mettent aussi en lumière à quel point on associe les AST avec le maintien de liens étroits avec la famille étendue et la communauté. Dans toutes les familles intensément engagées dans les AST, on manifeste un grand intérêt à ce que les enfants passent du temps avec les grands-parents. Après une chasse ou une cueillette réussie, par exemple, l'enfant distribue une partie des victuailles aux membres de sa parentèle. Une mère raconte comment, à son retour de chasse, son fils apporte de la viande sauvage aux anciens « parce qu'il aime les nourrir ». En retour, les anciens lui parlent et l'instruisent. Dans les familles caractérisées par un HTPAST la famille étendue joue un rôle important dans la transmission de la tradition. Lorsque Kim Harvey-Trigoso demande à une mère célibataire qui lui avait enseigné à chasser, elle mentionne son oncle, ses cousins et ses beaux-frères. Dans plusieurs cas ce sont les grands parents et d'autres aînés qui initient très tôt l'enfant aux AST. Dans ce contexte les aînés transmettent aussi des récits pertinents qu'ils ont eux-mêmes reçus de leurs ancêtres.

C'est tout le contraire dans les familles qui participent peu aux AST. La majorité des enfants de ces familles ne s'engagent pas dans des activités avec leurs grands-parents. Moins du tiers des enfants de ces familles accompagnent les grands-parents dans des AST. Une mère raconte comment sa fille aimerait entendre des récits de sa grand-mère, mais en vain parce que la grand-mère est toujours absente de la maison. Une autre mère note que si son enfant se rend à la demeure des grands-parents ce n'est pas pour passer du temps avec eux mais pour jouer avec les enfants qui s'y trouvent. Dans les familles peu engagées dans les AST, les enfants passent peu de temps avec les grandsparents, même si ces derniers vivent dans la même demeure.

La connaissance de la langue autochtone favorise la transmission des connaissances entre grands-parents et petits-enfants. Lacquisition des connaissances et des habiletés reliées aux AST se fait en milieu familial, et dans ce milieu les grands-parents 
jouent un rôle important. Dans ces familles, on parle d'ailleurs le dene dhàh. Certaines mères disent parler anglais avec leurs enfants en précisant que ces derniers parlent dene dhàh avec leurs grands-parents. La connaissance de cette langue est valorisée. Un père de famille affirme qu'il est important « d'être en lien avec qui l'on est... [ainsi qu'avec] ce qui définit sa culture ». À l'opposé, dans les familles qui participent peu aux AST, même si l'on affirme souhaiter le bilinguisme, on délaisse la langue autochtone. À ce sujet, les propos suivants d'un père de famille parlant de sa fille d'âge scolaire sont très clairs : « De nos jours, l'anglais [peut l'aider] davantage à l'école [et] elle comprend [cela]. Les habitudes sociales meurent lentement; nous perdons... lentement nous les perdons. De fait, je lui parle en anglais à la maison. » Même dans ces circonstances la grande majorité des enfants issus de ces familles comprennent le dene dhàh et ils sont encore nombreux à pouvoir le parler. Afin d'entraver l'érosion du savoir traditionnel, l'école a introduit un programme "Culture et langue dènèe ». On y consacre quatre classes de quarante minutes par semaine, auxquelles s'ajoutent quelques sorties en forêt durant l'année scolaire. Dans le cadre de ce programme, l'enseignement se fait généralement de manière didactique plutôt qu'expérientielle, opérant ainsi à l'encontre des postulats épistémologiques traditionnels. Lécole joue donc un rôle minime dans l'initiation des enfants aux AST.

Enfin, les familles participant beaucoup aux AST et celles qui y participent peu se distinguent de deux autres manières quant à leur manière de voir la communauté et le milieu naturel. Dans les deux types de familles, on affirme que les AST sont importantes et bénéfiques pour la communauté. Nonobstant ces propos, l'écart est manifeste entre la pratique et ce que professent ces parents au sujet des AST. Dans les familles ayant un HTPAST, les personnes semblent attachées émotionnellement à ces activités, ce qui peut expliquer qu'elles continuent à s'y engager activement. Ces personnes tiennent pour évident qu'il vaut la peine de transmettre aux enfants tout ce qui concerne les AST. «Elles ont toujours fait partie de notre vie. Elles nous ont été transmises de génération en génération », dit un homme. "C'est ce que les gens font à l'année longue ", ajoute-t-il. Lattachement aux AST est implicite dans l'affirmation suivante d'une mère :

Parce qu'il y a très longtemps c'est ainsi que les gens vivaient, avant I'arrivée de tous ces produits vendus en magasin. C'est ce dont vivaient nos ancêtres. Je me souviens, lorsque j'étais petite fille... Mes grands-parents se rendaient dans la forêt... au mois d'août pour revenir en septembre. Et nous vivions de la viande sauvage, de la bannique, et du thé. II était rare de voir une pomme.

Dans les familles s'engageant beaucoup dans les AST, celles-ci sont perçues comme une manière efficace de réduire la consommation d'alcool et de prévenir d'autres mauvaises habitudes. Ces idées se retrouvent dans une série d'affirmations, telles les suivantes, entendues au cours des entrevues : "J'avais l'habitude d'amener mes enfants en forêt et d'aller à la chasse... afin d'éviter qu'ils se retrouvent dans les pépins. » "Les jeunes devraient surtout aller chasser au lieu de passer leur temps à courir aux alentours... et à boire. » Ce sont les AST qui font que « la famille se retrouve ensemble».

Les familles s'engageant peu dans les AST les considèrent néanmoins comme une manière de combler la perte éventuelle d'aide financière de la part des gouvernements. Dans ces familles, certains parents affirment qu'ils initieront leurs enfants aux AST lorsqu'ils seront plus âgés. D'ailleurs, dans ce type de famille au FTPAST, les adultes semblent apprécier la forêt davantage que les enfants. Sur ce plan, un clivage important s'est opéré entre les générations. Dans plusieurs cas les enfants n'aiment pas la forêt. S'ils aiment vivre à proximité de leur famille étendue, ils sentent fortement l'attrait de la ville. Dans ces familles, les enfants expriment le désir d'y vivre afin d'éviter les problèmes sociaux de la communauté. Plus de la moitié des adultes de ces familles partagent leur avis.

Dans les familles ayant un HTPAST, on sent au contraire la confiance dans les habiletés déjà développées chez les enfants. Dans ces familles, les adultes tendent à enseigner très spécifiquement aux enfants comment exprimer le respect dû à la nature, par exemple en offrant du tabac lorsqu'on cueille des plantes. Ce type de connaissance n'est pas souvent enseigné dans les familles à FTPAST. Aussi les parents remarquent-ils avec fierté que leurs enfants ont une attitude positive par rapport à la forêt, qu'ils ne craignent pas de s'y rendre, savent comment se protéger des dangers. Pour les enfants de ces familles, la survie en forêt ne fait pas problème lorsqu'ils s'y aventurent d'euxmêmes ou lorsqu'ils accompagnent leur famille dans des expéditions de chasse ou de pêche. En discutant des attitudes par rapport à la communauté, plusieurs adultes dans les familles à HTPAST indiquent que leurs enfants aiment vivre avec leurs proches et préfèrent leur communauté à la ville. Ces enfants sont néanmoins touchés par les problèmes sociaux touchant la communauté, tels l'alcool et la violence. Ce qu'ils ont appris en forêt ce sont « des savoir-faire, des savoir-être et des éco-savoirs - Une façon d'être en lien avec les éléments naturels dans la pensée d'A. Moneyron» (Nicolas 2004 : 4, en italiques dans l'original).

\section{Conclusion}

Les données recueillies sur le terrain démontrent qu'il existe un lien étroit entre les AST et les valeurs sur le plan du collectivisme et du conservatisme. Des différences significatives dans la participation aux AST se traduisent par des indices clairs dans les comportements, dans les dessins et les interactions des enfants dènès tha. Les enfants les plus engagés dans les AST tendent à manifester une attitude plus positive à l'égard de la communauté. Les valeurs qui guident leurs comportements dans la salle de classe sont acquises dans le milieu familial, particulièrement dans le contexte des AST.

Les enfants qui participent peu en bas âge aux AST ont des liens moins étroits avec la communauté et l'environnement. Cette perte sur le plan du collectivisme et du conservatisme se manifeste dans leurs dessins et dans leur interaction en salle de classe, ainsi que dans le peu d'espoir qu'ils ont face aux problèmes sociaux de la communauté. C'est dans cette cohorte que l'on observe la manifestation d'un clivage important entre générations en ce qui a trait aux valeurs et aux comportements. A l'inverse, on observe une profonde continuité intergénérationnelle dans les familles qui privilégient les AST et s'y adonnent activement. L'enfant qui participe en bas âge à ces activités apprend à vivre positivement dans son environnement ; il développe des relations étroites avec les membres de sa famille immédiate et étendue; il partage les fruits des AST avec les membres de sa communauté.

Tous ces facteurs, auxquels s'ajoute la maitrise passive et active de sa langue maternelle, permettent à l'enfant d'avoir accès direct aux anciens et au précieux héritage culturel dont ils sont porteurs. D'où, semble-t-il, une attitude plus positive face à la communauté et un espoir appris face à l'avenir. Bien que touchés par les nombreux problèmes qui accablent trop souvent 
leurs proches et les membres de leur communauté, bien qu'exposés aux médias et aux systèmes éducatifs et religieux implantés chez eux par les Eurocanadiens, ces enfants puisent toujours dans le réservoir de leurs connaissances et de leurs pratiques traditionnelles afin de s'orienter dans la vie selon une perspective bien à eux, qui provient de leurs ancêtres, dans leur pays.

\section{Notes}

1. Le survol historique suivant est tiré en partie de Goulet 1998a, 1998b, 2004 et de Harvey-Trigoso 1999.

2. Dans cet article, toutes les traductions de l'anglais au français, y compris la traduction de la deuxième partie de l'article rédigée par Kim Harvey-Trigoso, sont de Jean-Guy Goulet.

3. Voir Goulet 2004 pour un compte rendu des développements locaux et régionaux les plus importants sur les plans économique et politique au cours des dix dernières années.

4. Cette expression traduit la notion de other-than-human-person introduite en anthropologie par Hallowell (1976 : 405) afin de désigner les animaux perçus comme des êtres conscients et savants avec lesquels il faut entretenir des relations interpersonnelles fondées sur le respect et la réciprocité.

5. Cette définition que nous donnons au mot collectivisme va dans le sens de la signification plus précise définie par Le Petit Robert comme «doctrine représentant un socialisme non étatiste et non centralisant ».

6. Ce respect de l'autonomie de chacun va de pair avec l'absence de relation de subordination ou de domination parmi les peuples chasseurs et cueilleurs. À ce sujet, Niezen applique aux athapascans de l'Amérique du Nord ce que Montesquieu affirmait au sujet des peuples qui ne cultivent pas la terre : " If a leader wanted to take their liberty away from them, they would immediately go and seek it with another leader or withdraw into the woods to live there with their family. Among these peoples, the liberty of the man is so great that it necessarily brings with it the liberty of the citizens. » (Montesquieu [1989] 1748 : 292, in Niezen 2003 : 122)

7. À ce sujet, il est intéressant de noter qu'à Paris aussi l'espérance passe de la forêt à la salle de classe. C'est ce que constate Philippe Nicolas, enseignant en zone d'éducation prioritaire. Après des sorties de classe en forêt et en bordure de rivières, des changements importants se sont manifestés chez les enfants, à la grande surprise des parents et des enseignants. "Ces confrontations élémentales tavec la forêt et la rivière] ont contribué à une émancipation globale de la plupart des enfants » qui ont grandi dans un environnement « outrageusement bétonné ». En effet, après ces sorties dans la forêt, les enfants exposent à l'école les « objets glanés lors des sorties », installent des aquariums dans leur salle de classe, empruntent à la bibliothèque municipale, sans qu'on leur suggère, « tous les ouvrages spécialisés en aquariophilie », et pour comble, à la grande surprise de leurs parents se mettent à lire « le soir des revues, des livres sur le domaine avant de se coucher » (Nicolas 2004: 4). Ces observations de la part de Nicolas nous donnent à penser que chez les enfants dènès tha c'est aussi l'expérience de soi dans la nature qui est primordiale par rapport à la formation d'une attitude positive face à la vie.

8. Deux études antérieures, l'une en études urbaines (Lynch 1977), l'autre en anthropologie (Fulford 1994) ont aussi recours à des dessins d'enfants afin de saisir leur connaissance de leur communauté et de leur culture.

9. Sur ce tabou parmi Athapascans, voir Goulet 1998a: 95-97 pour les Dènès Tha du Nord-Ouest albertain, ainsi que Lanoue (1994: 119) pour les Sekanis de la Colombie-Britannique chez qui «Les femmes chassent souvent quand leurs époux sont absents ».

\section{Remerciements}

Je remercie Achiel Peelman qui a lu et donné ses commentaires sur la toute première version de la première partie de cet article, ainsi que les deux évaluateurs anonymes pour leurs commentaires sur une version ultérieure de ce texte. Mes remerciements vont également à Christine Hanssens pour sa lecture attentive et ses suggestions. Mes remerciements s'adressent à Madame Marcelle Roy pour la révision linguistique de cet article. Je remercie aussi Laurent Jérôme pour l'invitation à présenter les grandes lignes de cette recherche au colloque "Réalités et défis pour les jeunes en milieux autochtones contemporains : faut-il parler des problèmes ou des projets? » organisé par le CIÉRA (Centre interuniversitaire d'études et de recherches autochtones), à l'Université Laval, les 14 et 15 avril 2005. C'est à ce colloque que j'ai fait l'heureuse rencontre de Philippe Nicolas qui m'a ouvert les yeux sur les liens entre ma recherche parmi les Dènès Tha et la sienne en milieu parisien (voir note 7 ).

\section{Ouvrages cités}

ASCH, Michael, 1981 : "Slavey », in June Helm (dir.), Subarctic. Handbook of North American Indians, vol. $6: 338-349$. Smithsonian Institution, Washington DC.

BARNARD, Alan (dir.), 2004: Hunter-Gatherers in History, Archaelogy and Anthropology. Berg Publishers, Oxford.

BUCKLEY, Thomas, 2002: Standing Ground. Yurok Indian Spirituality, 1850-1990. University of California Press, Berkeley.

COX, Bruce A., (dir.), 1987 : Native People, Native Lands: Canadian Indians, Inuit and Metis. Carleton University Press, Ottawa.

DENE THA BAND OF ASSUMPTION, 1990: Submission to the Task Force on the Criminal Justice System and Its Impact on the Indian and Metis People of Alberta. Manuscrit, 18 avril.

DENE THA COMMUNITY SCHOOL TESTING INFORMATION, 1996 : Manuscrit. 3 avril.

DENE THA' NATION, 1997 : Dene Tha' Traditional Land-Use and Occupancy Study. Arctic Institute of North America, Calgary.

DENE THA WOMEN'S SOCIETY, 1990 : Submission to the Task Force on the Criminal Justice System and Its Impact on the Indian and Metis People of Alberta. Manuscrit, 18 avril.

DUSSART, Françoise, 2004: «Montrer sans partager, présenter sans proférer. Redéfinition de l'identité rituelle chez les interprètes rituelles warlpiri ». Anthropologie et Sociétés 28(1) : 67-88.

ERIKSON, Erik H., 1950: Childhood and Society. W.W. Norton, New York.

FINE, Alan, et Kent L. SANDSTROM, 1988: Knowing Children: Participant Observation with Minors. Sage Publishers, Newbury Park, California.

FULFORD, George T., 1994 : Children's Drawings in a Mashkeko ('Swampy Cree') Community. Ph. D. Dissertation. McMaster University, Hamilton.

FUMOLEAU, René, 1994 : Aussi longtemps que le fleuve coulera : La Nation Denee et le Canada. Éditions du Septentrion, Sillery.

GARDNER, Peter M., 1991 : «Foragers' Pursuit of Individual Autonomy ». Current Anthropology 32(5) : 543-572.

GOULET, Jean-Guy A., 1994: « Dreams and visions in Other Lifeworlds », in D. E. Young and J.-G. Goulet (dir.), Being Changed by Cross-Cultural Encounters. The Anthropology of Extraordinary Experiences : 16-38. Broadview Press, Peterborough, Ontario.

-, 1998a: Ways of Knowing: Experience, Knowledge, and Power Among the Dene Tha. University of Nebraska Press, Lincoln.

—, 1998b: «Le savoir anthropologique sur les Dènès et son rapport avec le savoir dènè ». Recherches amérindiennes au Québec 28(3) : 109-115. 
—, 2001 : «Vivre ensemble! Chacun pour soi? Gérer les conflits avec les autochtones / vivre en relation entre autochtones». Sciences pastorales / Pastoral Studies 20(1) : 45-72.

_, 2004: "The Dene Tha of Chateh: Continuities and Transformations », in R. B. Morrison et C. R. Wilson (dir.), Native Peoples: The Canada Experience: 157-177. Third Edition. Oxford University Press, New York.

GUÉDON, Marie-Françoise, 1994: «Dene Ways and the Ethnographer's Culture », in Jean-Guy Goulet et D. Yong (dir.), Being Changed by Cross-cultural Encounters. The Anthropology of Extrarodinary Experiences: 39-70. Broadview Press, Peterborough, Ontario.

HALLOWELL, Irwing (dir.), 1976: «Ojibwa World View and Disease », in Contributions to Anthropology: Selected Papers of A. Irwing Hallowell : 37-390. University of Chicago Press, Chicago.

HELM, June, 1994 : Prophecy and Power among the Dogrib Indians. University of Nebraska Press, Lincoln.

LANOUE, Guy, 1994 : « La mythification et la marchandise dans le Nord canadien. La représentation télévisée du pouvoir (Note de recherche)». Anthropologie et Sociétés 18(3) : 113-123.

LE BOSSÉ, Yann, et Francine DUFORT, 2001 : « Le cadre paradigmatique de la psychologie communautaire: Vers une épistémologie du changement social », in Francine Dufort et Jérôme Guay (dir.), Agir au cour des communautés. La psychologie communautaire et le changement social: 33-74. Les Presses de l'Université Laval, Québec.

LYNCH, Kevin, 1977 : Growing Up in Cities: Studies of the Spatial Environment of Adolescence in Cracow, Melbourne, Mexico City, Salta, Toluca, and Warsaw. MIT Press, Cambridge, Mass.

McCLELLAN, Catharine, 1972: The Girl who Married the Bear. National Museum of Man Publications in Ethnology, No. 2. Musées nationaux du Canada, Ottawa.

MILLS, Antonia, 1982 : The Beaver Indian Prophet Dance and related movements among North American Indians. Ph.D. dissertation. Harvard University.

MONEYRON, Anne, (dir.), 2003: Transhumance et Éco-savoir. Reconnaissance des alternatives écoformatrices. L'Harmattan, Paris.
MONTESQUIEU, Charles-Louis de Secondat, 1989 [1748]: The Spirit of the Laws. Traduit et édité par Anne Cohler, Basia Miller et Harold Stone. Cambridge University Press, Cambridge, England.

MOORE Patrick, et Angela WHEELOCK, 1990 : Wolverine myths and visions: Dene traditions from northern Alberta. University of Alberta Press, Edmonton.

NICOLAS, Philippe, 2004 : «Pensée critique de l'enseignement en Zone d'éducation prioritaire à la lumière de l'écoformation. Qu'est-ce qui peut accompagner en Z.E.P. les enfants dans leur existence à venir? » Manuscrit, $9 \mathrm{p}$.

—, 2005: "La complainte des eaux vives». Plaisirs Pêche. Le Magazine des pêcheurs à la mouche 96 : 60-65.

NIEZEN, Ronald, 2003 : The Origins of Indigenism. Human Rights and The Politics of Identity. University of California Press, Berkeley.

RIDINGTON, Robin, 1992 : Little Bit Know Something: Stories in a Language of Anthropology. University of Iowa Press, Iowa City.

SMITH, James E., 1987 : « The Western Woods Cree: Anthropological Myth and Historical Reality ». American Ethnologist 14: 434-448

TANNER, Adrian, 1979 : Bringing Home Animals: Religious Ideology and Mode of Production of the Mistassini Cree Hunters. St. Martin's Press, New York.

WAX, Murray, 2003: «La salle de classe comme théâtre: une recherche de terrain chez les Sioux oglala». Anthropologica 45(1) : 163-173.

WENZEL, George W., 1991 : Animal Rights, Human Rights: Ecology, Economy and Ideology in the Canadian Arctic. University of Toronto Press, Toronto.

WOODBURN, James, 1982 : «Egalitarian Societies ». Man 17(3): 431-445.

ZIMMERMAN, M.A., 1990: «Toward a Theory of Learned Hopefulness: A Structural Model Analysis of Participation and Empowerment ». Journal of Research in Personality 24 : 71-86. 


\section{Lucie Sauvé, Hélène \\ Godmaire, \\ Marie Saint- \\ Arnaud, \\ Renée \\ Brunelle et \\ Françoise \\ Lathoud}

Chaire de

recherche du

Canada en éduca-

tion relative à

I'environnement,

Université du

Québec à

Montréal,

Montréal $\complement^{\prime}$ EST À PARTIR des préoccupations particulières de notre champ de recherche et d'action sociale, celui de l'éducation relative à l'environnement, que nous nous intéressons aux cultures autochtones. D'une part, nous y trouvons un riche univers cosmologique, comme point de références pour repenser notre propre rapport au monde. D'autre part, l'exploration des approches, des pratiques, des stratégies et des significations autochtones en ce qui concerne une certaine « éducation » relative à l'« environnement » (termes qui doivent être eux-mêmes redéfinis en contexte) permet de repérer des éléments essentiels à l'enrichissement de ce champ théorique et pratique.

À travers différents projets de recherche en milieu autochtone (projets de type « recherche-intervention », où l'investigation est associée à l'action éducative), nous avons été confrontées au défi de mettre en ouvre ou d'accompagner des projets d'éducation relative à l'environnement appropriés à divers contextes culturels. Une telle tâche s'est avérée exigeante mais féconde en observations et, surtout, elle a suscité chez nous des questions et des réflexions fondamentales. En particulier, comment contribuer à favoriser chez les jeunes autochtones un processus d'appropriation de leur milieu de vie, en lien avec une démarche d'affirmation identitaire et de reconstruction des relations sociales au sein de leur communauté comme à l'extérieur? Comment l'éducation formelle, jusqu'ici souvent aliénante, peut-elle se transformer pour accompagner les jeunes autochtones dans la construction et l'expression de leur monde? Comment peut-elle devenir un creuset de clarification de leurs inquiétudes, attentes, espoirs, désirs? Comment peut-elle valoriser leur voix et contribuer à la faire entendre et reconnaître? Comment des expériences de vie en contexte d'éducation non formelle ou informelle peuvent-elles concourir à une telle émancipation, en complémentarité avec l'école?

Des transformations majeures et prometteuses vers l'autogouvernance sont en cours au sein des systèmes politiques, socio-économiques et institutionnels des populations autochtones. À cet égard, comme l'observe entre autres Sheila Watt-Cloutier (2000), l'école mérite une attention particulière parce qu'elle est un relais culturel privilégié. C'est précisément dans cette perspective d'un monde en changement, où se transforment les rapports aux autres et à l'environnement, que nous avons souhaité contribuer à l'exercice d'imaginer un pont entre recherche et éducation, entre école et milieu de vie, entre nature et culture, entre nos visions du monde et celles des peuples autochtones, entre jeunes et avenir.

\section{UN CADRE DE RÉFÉRENCE INITIAL EN ÉDUCATION RELATIVE À L'ENVIRONNEMENT}

Il y a déjà plus de vingt ans, dans un numéro de Recherches amérindiennes au Québec consacré à l'éducation et l'autogestion (1983, vol. XIII, n 1), Léonard Paul identifiait trois écueils essentiels à 
éviter: l'un d'ordre idéologique (ethnocentrisme), l'autre concernant la «néo-folklorisation » et un troisième, relatif aux problèmes de l'instrumentation, du choix d'un médium d'intervention qui ne soit pas approprié au contexte culturel. De telles mises en garde nous ont incitées à clarifier dès le départ nos points d'ancrage afin de mieux prendre conscience de leur influence sur nos choix et nos trajectoires. Nous adoptons en effet une épistémologie de recherche dite "interprétative », qui reconnaît l'importance de la subjectivité comme « filtre » d'appréhension du monde. Et en toute cohérence, nous favorisons une approche d'intervention éducative qui valorise la clarification de la dimension subjective comme levier de développement identitaire et de re-construction du rapport au monde.

L'éducation ne peut être associée étroitement à l'« instruction », soit à la transmission d'informations pour une formation prédéterminée. Nous considérons plutôt que l'éducation est un processus d'actualisation des potentialités de chaque personne, au sein de son groupe social et en fonction de son cadre culturel de référence. Cela implique aussi pour chacun le développement d'une capacité à prendre une distance critique à l'égard des réalités socioculturelles qui construisent l'identité et déterminent l'agir personnel et collectif.

L'éducation relative à l'environnement est cette dimension essentielle de l'éducation fondamentale qui concerne notre relation au milieu de vie, à cette «maison de vie» partagée. Elle a pour but d'optimaliser notre rapport à l'environnement, dont la trame est celle de la vie elle-même, à la jonction entre nature et culture. Sur le plan personnel, elle vise à développer une appartenance au milieu de vie, un sens à l'être au monde, une culture de l'engagement. À l'échelle des communautés, puis à celle de réseaux de solidarité élargis, elle vise à induire des dynamiques sociales favorisant l'approche collaborative et critique des réalités socio-environnementales et une prise en charge autonome et créative des problèmes qui se posent et des projets qui émergent. Parce qu'elle concerne des réalités collectives, l'éducation relative à l'environnement est un processus politique et, par conséquent, essentiellement participatif. S'ouvrir à la dimension politique implique de ne pas rester indifférent, de prendre partie face aux réalités sociales et environnementales, explique Francisco Gutiérrez (2002). Il s'agit de s'éduquer à la participation par la participation à un projet socio-politique, à un projet créateur et constructif, à travers l'action partagée.

En raison de la complexité des objets abordés, l'apprentissage tout comme la recherche en matière d'environnement fait de préférence appel à une approche interdisciplinaire et collaborative. Les savoirs sollicités ou produits sont de divers types ou encore, ils intègrent des éléments issus d'approches épistémologiques différentes: savoirs disciplinaires, savoirs d'expérience, savoirs locaux, savoirs traditionnels, etc. Au-delà de la description des situations ou phénomènes, la recherche est axée sur la réflexivité et la quête de significations; elle se préoccupe de changement social. Enfin, un tel type de recherche trouve un contexte privilégié au cour d'un projet d'action ou d'intervention éducative: la recherche est alors étroitement arrimée à la réflexion sur et dans l'action, et elle devient un creuset d'apprentissage pour tous les participants.

\section{ÉDUCATION ET ENVIRONNEMENT EN MILIEU AUTOCHTONE : UNE PREMIÈRE EXPLORATION}

Notre première étape de travail a été de tenter de cerner les principaux repères de ce qu'il est convenu d'appeler « l'éducation autochtone » et d'examiner divers projets d'« éducation relative à l'environnement » (ou de nature similaire) qui ont été menés dans de tels milieux. Notre recension des écrits a d'abord mis au jour diverses manières d'approcher la question éducative. En effet, la littérature, tant d'origine autochtone qu'en provenance des champs de l'anthropologie, de l'éducation, des sciences sociales ou autres, aborde le sujet soit en tant que tel (indigenous pedagogy, aboriginal education), soit sous l'angle des connaissances (traditionnelles ou non), des savoirs et de leur transmission. Quoi qu'il en soit, il s'agissait d'y trouver des critères de pertinence et des sources d'inspiration pour les projets que nous souhaitions accompagner. Nous avons également pu confronter nos fondements de départ (voir plus haut) à des propositions éducatives issues du milieu autochtone ou conçues pour un tel contexte.

\section{UN REPÈRE : L'ÉDUCATION TRADITIONNELLE EN MILIEU AUTOCHTONE}

L « éducation autochtone » prend forme et signification particulières dans différents contextes, reflétant et renforçant la diversité des cultures; elle s'inscrit dans la mouvance de l'histoire et de la trajectoire des groupes sociaux. Pour tenter une première approche générale du phénomène de l'éducation en milieu autochtone, différents auteurs (dont Briggs 1983, Cajete 1994, Blanchard 1999, Brant Castellano et al. 2000, Ongtooguk 2000, Baskin 2002, Berger 2002, Pardo 2002, McKay 2005) nous mettent sur la piste de traits convergents qui permettent de caractériser sommairement ce qui apparaît à travers leurs propos comme des éléments fondamentaux d'une « éducation traditionnelle en milieu autochtone ».

Celle-ci est basée sur une ontologie qui situe la Nature dans l'ordre du sacré et célèbre l'interdépendance des êtres, des éléments et des phénomènes (Berkes 1989). À titre d'exemple, signalons la conception qu'adoptent, entre autres, les peuples autochtones du Yukon, de ce que nous nommons «la santé »: un état d'harmonie entre le corps, l'âme et le territoire (ou la Terre, the Land), entre la personne et sa communauté (Van Oostdam et al. 1999). En cohérence avec un tel univers cosmologique, l'épistémologie est axée sur une forme de savoir holistique (qui reconnaît le tout dans chaque partie et saisit les relations des éléments entre eux); un tel savoir est ancré dans l'expérience et la quête de sens, au sein de la communauté. La source véritable du savoir se trouve en chacun et dans les êtres de la Nature. Le savoir n'est pas un cumul d'informations, il s'intègre à travers différents niveaux de conscience et de compréhension $^{1}$ : il est sagesse qui se déploie dans la maturité, comme le « isuma» des Inuits (Briggs 1983).

Léducation traditionnelle en milieu autochtone témoigne de ces fondements ontologiques et épistémologiques. Gregory Cajete (1994 : 29-31) identifie quarante-deux principes d'une telle éducation. Entre autres, selon cet auteur, il s'agit essentiellement d'une activité sociale communautaire, d'une éducation tout au long de la vie, qui enseigne des façons d'être et de faire permettant de soutenir tant les personnes que la communauté, leur permettant de répondre à leurs besoins de base; elle leur fournit une « carte du monde » qui oriente la trajectoire de vie de chacun. Chaque personne possède en elle-même ce qui est nécessaire à son bien-être et à son développement; elle est respectée dans sa façon d'être, de faire, de comprendre. Léducation développe le pouvoir-faire personnel; elle renforce la confiance en soi en aidant à mieux comprendre qui l'on est et en favorisant le déploiement de son propre potentiel. 
Lapprentissage se fait à travers l'expérience, avec le corps et l'âme, comme avec l'esprit. Il s'appuie sur l'observation et la réflexion, sur la stratégie de l'« essai et erreur». Il amène à reconnaître qu'il y a diverses facettes aux réalités, qui interagissent entre elles et qui sont évolutives, en perpétuel changement. L'éducation autochtone reconnaît également le pouvoir de la pensée (en particulier, la pensée symbolique) et du langage pour créer le monde où nous vivons. Le langage, comme l'art, apparaît comme une expression du « souffle de l'âme ». Lart et le conte deviennent des stratégies éducatives privilégiées pour mieux ancrer le savoir. Le jeu, associé à l'humour, confronte, consolide et complète l'apprentissage. Laccompagnement des aînés, détenteurs du savoir ancestral, est fondamental. Celui qui enseigne est un guide à travers l'expérience d'apprentissage, et non le "maître » du développement du jeune. Enfin, parmi les attitudes sollicitées et développées chez ce dernier, on retrouve l'ouverture, la curiosité, la flexibilité, la patience, l'écoute, le respect, le partage, le courage, la détermination.

Michelle Rae McKay (2005 : 3) ajoute que l'un des principes de la pédagogie autochtone communs aux diverses sociétés est celui de l'unité avec la nature : l'harmonie de/avec/dans la nature est perçue comme sacrée. « Le rôle de la nature traverse le processus d'apprentissage », affirme-t-elle. «Dans les sociétés traditionnelles autochtones, les enfants apprennent l'intégration, la relation, l'interconnexion' des choses, des êtres et des phénomènes, la circularité des processus puisque tout est relié. »

Ainsi, nature et culture sont indissociables : elles correspondent à deux modes d'expression de la vie sur un territoire. Gregory Cajete (1994 : 84) parle de la « géopsyché » des populations : celles-ci sont imprégnées de leur territoire, comme en retour, elles façonnent ce dernier. Restaurer ou préserver la culture ne peut se faire sans restaurer aussi la « Terre », le territoire. Rétablir le lien ancestral avec la Nature, avec le territoire, est un moyen fondamental de guérison dans le contexte actuel des transformations que vivent les peuples autochtones.

\section{L'ÉDUCATION EXOGÈNE : UNE RUPTURE}

S'il est ainsi possible de tracer les grands contours de l'éducation traditionnelle en milieu autochtone, il importe également de mettre en lumière la rupture actuelle entre celle-ci et les systèmes d'éducation exogènes qui perdurent dans plusieurs milieux et qui ont accueilli et accueillent encore les jeunes autochtones; ces derniers s'y sentent intimidés et en perte d'identité (Ongtooguk 2000, Penashue 2000, Berger 2002).

En réponse à une éducation décontextualisée et aliénante, Sheila Watt-Cloutier (2000), comme Gregory Cajete (1994) et plusieurs autres auteurs, insiste sur la nécessité d'une nouvelle éducation autochtone qui intégrerait une dimension de critique sociale. Cette auteure observe que la patience et la confiance, des valeurs fondamentales des cultures autochtones, ont finalement piégé les communautés qui ont fait confiance aux « autres» : ceux qui ont pris le contrôle de leur destinée, ont imposé leurs institutions et ont enseigné des choses qui n'avaient pas de signification pour les autochtones. Cette situation aurait entraîné la perte de pouvoir-faire et de liberté, de même que la dépendance et l'aliénation. Selon Watt-Cloutier, il importe donc de se réapproprier l'apprentissage de l'autonomie et de la liberté, en marge de l'école, en attendant de pouvoir changer l'institution. Il ne suffit certes pas d'introduire des cours de langue autochtone ou d'artisanat pour réintégrer la culture autochtone à l'école. Il faut retourner à la Terre (the Land), apprendre à vivre d'elle, avec elle et avec l'Autre, pour trouver la sagesse, la compréhension holistique du monde, la force et le pouvoir-faire d'affronter les défis de ce monde en changement.

Léducation devient ici un projet politico-pédagogique, un processus de dénonciation des rapports de pouvoir aliénants et de reconstruction identitaire et communautaire. Cela fait appel à des stratégies pédagogiques particulières, qui pourraient s'inspirer de la pédagogie critique de Paulo Freire (1972), et que le courant de la critique sociale en éducation relative à l'environnement (Robottom et Hart 1993, Sauvé 1997) pourrait avantageusement appuyer.

\section{L'ÉDUCATION AUTOCHTONE EN TRANSITION}

Mais au-delà de la dénonciation de l'école exogène, il importe de reconnaître également que l'éducation en milieu autochtone est entrée dans une phase de transition. Différents curriculums et programmes ont été développés et proposés, visant à transformer les systèmes d'éducation néo-colonialistes et à mettre en œuvre une éducation qui intègre les principes fondamentaux de l'éducation traditionnelle en milieu autochtone, tout en favorisant l'adaptation aux changements qui émergent dans le contexte actuel d'une certaine "modernité ».

Plusieurs exemples témoignent de cette transition: par exemple, le Dene Kede Curriculum des Territoires du Nord-Ouest (Collectif 1993) qui a été développé dans un processus collaboratif par des éducateurs, des parents et des aînés des communautés concernées. Ce curriculum adopte une perspective essentiellement communautaire, valorise la langue et la culture, mais, au-delà, il insiste sur le développement d'attitudes et d'habiletés liées au territoire (land skills): celui-ci est porteur de vie (life giving) et doit être respecté et conservé. Lapprentissage est holistique et « en spirale » (expériences répétées et approfondies), il s'inscrit dans une trajectoire de vie. Parmi les stratégies proposées: la rencontre avec les aînés, l'écoute de légendes et d'histoires, l'enquête auprès des membres de la communauté, des activités et expériences de terrain (parcours, camps, feux, etc.), des groupes de discussion, des activités artistiques, la production de vidéos, des rencontres avec d'autres jeunes autochtones, etc.

On retrouve des caractéristiques semblables dans divers autres curriculums, programmes et projets développés par et pour des groupes autochtones, comme le curriculum «Inuuqatigiit» (Collectif 1996). Le curriculum « organique» proposé par Rosemary Ann Blanchard (1999) est également axé sur une dynamique communautaire; il inclut une dimension historique, qui retrace l'origine du mode de vie de la communauté et recherche les facteurs du changement actuel, dont l'influence d'une certaine science et technologie; il aborde aussi les questions économiques et politiques globales, et les enjeux contemporains de la Nation et de la communauté qui y sont reliés.

Notre exploration nous a aussi menées à repérer des projets éducatifs proposés au milieu autochtone (comme le projet Contaminants Found $\mathrm{Me}$ du Yukon Contaminants Committee, 2000) qui, tout en se préoccupant de tenir compte des « savoirs traditionnels autochtones ", adoptent fondamentalement des approches et stratégies qui relèvent plutôt d'une culture éducationnelle valorisant la science positiviste et la rationalité analytique.

Cette exploration des fondements et des enjeux de l'éducation autochtone et celle de diverses propositions éducatives nous permettent d'identifier des points de convergence avec notre propre conception de l'éducation relative à l'environnement et aussi de cerner certains écueils à éviter. 


\section{Des CONVERGENCES THÉORIQUES}

L'éducation traditionnelle en milieu autochtone, axée sur la construction d'une vision holistique du monde, de même que sur l'importance de la Terre (ou territoire) comme source d'apprentissage, rejoint de façon très évidente deux courants qui ont été développés dans le champ de l'éducation relative à l'environnement et que nous adoptons généralement de façon complémentaire dans nos travaux, soit les courants biorégional et holistique (Sauvé 2003). Le courant biorégional est axé sur la redécouverte du milieu de vie (où s'entremêlent nature et culture), en lien avec la re-construction de l'identité. Quel est ce milieu de vie où nous sommes immergés? Quelle est l'histoire écologique et culturelle de ce lieu? Quelle est ma relation à ce milieu, comment interagissons-nous entre nous et avec ce milieu? De quelles significations cette relation est-elle porteuse? Comment harmoniser nos rapports éco-logiques et éco-nomiques à ce milieu? Le courant holistique, pour sa part, enrichit de façon transversale une démarche éducative de type biorégional: il valorise l'unicité des multiples dimensions de notre être et de notre rapport au monde et se préoccupe de stimuler les diverses façons complémentaires d'apprendre de ce dernier et de s'y relier (approches cognitive, affective, spirituelle, kinésique, etc.). Il adopte une épistémologie dialectique et dialogique, qui reconnaît la spécificité et la contribution de divers types de savoirs.

Par ailleurs, il nous apparaît que le courant de la critique sociale développé dans le champ de l'éducation relative à l'environnement, offre une avenue intéressante en milieu autochtone. Au-delà du constat des problématiques socio-environnementales, la question du «pourquoi » se pose avec acuité. Entre autres : qui décide quoi? pour qui? pourquoi? Il s'agit de mettre au jour les valeurs, les intérêts, les intentions qui sont en jeu dans les rapports sociaux, d'être attentifs aux rapports de pouvoir et à l'aliénation qu'ils engendrent, et de travailler ensemble à transformer les situations qui posent problème. Une telle approche « critique » s'associe particulièrement bien à un projet d'investigation et d'action communautaire.

De façon générale, nous rejoignons Leanne Simpson (2002) qui propose, pour sa part, une éducation relative à l'environnement autochtone pour la survie culturelle (indigenous environmental education for cultural survival) comme moyen de renforcer les cultures, de promouvoir la protection de l'environnement, de construire des économies locales « durables» et d'aider les jeunes et les éducateurs dans leur processus de décolonisation et de guérison (recherche de l'équilibre). Pour ce faire, affirme cette auteure, l'éducation doit se baser sur les systèmes de connaissances autochtones, systèmes de connaissances qui comptent parmi les éléments clés de la participation des autochtones aux débats environnementaux touchant leurs communautés et territoires.

Ces convergences nous ont inspirées pour la conception et l'analyse des projets dont nous témoignons à la section suivante. Il nous est apparu a priori que la culture autochtone était de nature à renforcer l'approche holistique et à « colorer » de façon particulière l'approche biorégionale du rapport à l'environnement. Quant à l'approche critique, nous verrons que sa mise en œuvre soulève certaines questions au regard des cultures autochtones, a priori peu compatibles avec une certaine rationalité critique.

\section{DES PROJETS DE RECHERCHE - INTERVENTION}

Les observations et réflexions exposées aux points précédents ont été amorcées au départ de nos travaux, mais elles se sont également enrichies de nos expériences d'immersion dans différents projets en milieu autochtone. Nous avons eu en effet l'occasion de participer à diverses initiatives d' « éducation relative à l'environnement » associées à de plus larges processus de résolution de problématiques socio-environnementales: en particulier, nous nous pencherons ici sur deux expériences, dont la première est centrée sur la question des contaminants au Labrador, et la deuxième, sur celle de l'exploitation forestière en Abitibi-Témiscamingue. Chacun de ces projets émerge d'une situation de collaboration particulière et s'ancre dans une réalité socio-environnementale unique. Nous verrons qu'ils partagent toutefois certaines stratégies et qu'il en résulte des observations et un questionnement convergents.

Nous nous attarderons plus spécifiquement à un « moment » de ces projets, celui qui met en œuvre une stratégie d'exploration du milieu de vie (selon une triple approche holistique, biorégionale et critique) qui amène notamment les jeunes à capter en images (photos) certains éléments de l'environnement qui les touchent particulièrement et à expliciter les raisons de leur choix.

\section{Chez les Innus du Labrador}

Au Labrador, nous avons été invitées à participer à une vaste recherche interdisciplinaire, écosystémique et participative sur la contamination du milieu (en particulier, par le mercure) et ses effets sur la santé humaine. Notre mandat, tel que nous l'avons redéfini, consistait à induire une dynamique éducative en vue de stimuler la participation de la communauté à une démarche d'investigation visant à une meilleure compréhension et appropriation de la problématique.

Il faut dire que jusqu'à tout récemment, le discours scientifique relatif au mercure (et autres contaminants) et à ses effets néfastes sur la santé a soulevé et soulève encore des inquiétudes au sein des communautés autochtones.

II y a un historique de communication inadéquate concernant les problématiques des contaminants chez les Inuits; cette situation a conduit à un certain degré d'inquiétude, d'appréhension et de méfiance. Les principaux obstacles à une communication efficace ont été la langue et la culture. (Ukpatiku 2001 : 342)

En réponse à cette situation, il était primordial de mettre l'accent non seulement sur la communication et la compréhension de la dynamique de communication (Poirier et Brooke 2000) mais aussi sur la participation de la communauté et sur l'intégration des savoirs autochtones à la démarche de recherche scientifique (Loring 2001, Ukpatiku 2001). C'est dans une telle perspective, enrichie d'une visée éducative, que nous avons envisagé un projet en collaboration avec la communauté innue.

Au départ, des moments d'échange et de discussion avec les représentants de la communauté ont permis de valider, d'orienter et de compléter une proposition initiale d'éducation relative à l'environnement, dont un volet s'adressait à la communauté en général et l'autre, aux jeunes de l'école. Dans la communauté, avec des membres de divers groupes d'âge et rôles sociaux, cette démarche a consisté à explorer les réalités socioenvironnementales locales en adoptant une approche critique, à tenter de comprendre les causes et les effets des problèmes qui les préoccupent (plus spécifiquement ceux qui ont trait aux contaminants, dont le mercure) et, enfin, à rechercher ensemble des solutions appropriées, à la fois scientifiquement fondées et culturellement et socialement acceptables. Il nous apparaissait en effet évident qu'aborder un sujet aussi 
spécifique que celui du mercure allait être peu signifiant pour les gens de la communauté. Il importait donc de situer cette problématique isolée dans l'ensemble de la situation socioenvironnementale du milieu. Il s'agissait de pouvoir répondre à nos objectifs de recherche dans la mesure du possible, tout en induisant une démarche qui soit de nature à tenir compte des véritables préoccupations de gens de la communauté. Le choix d'une approche collaborative avec ces derniers répondait à cette exigence éthique, et la « politique de recherche » que s'est donné la communauté offrait un cadre structurant à cet égard.

Notre désir de rejoindre les jeunes nous a menées à l'école secondaire. Avec eux, comme avec les gens de la communauté, nous avons abordé l'ensemble des réalités socioenvironnementales du milieu sans nous limiter à la question du mercure. Une première activité a consisté à explorer la signification des termes qui « traduisent » ou « évoquent» en langue innue les idées de "nature », d' « environnement», de « pollution », de «santé », de "rapport au monde », dans le but de mieux saisir les représentations sociales relatives aux réalités abordées, d'échanger des savoirs et de faciliter le dialogue. Puis une activité d'exploration du milieu a suivi. En petites équipes, les jeunes ont parcouru leur milieu dans une perspective de re-connaissance et y ont saisi des images en photos. Ces photos témoignaient des éléments jugés positifs («ce que je souhaite préserver») ainsi que des problèmes repérés («ce que je souhaite améliorer »). Chaque équipe a ensuite fabriqué une grande carte murale du milieu de vie (représentant le village et les alentours) pour y disposer les photos recueillies. Puis les élèves ont présenté et commenté leur montage graphique: chaque photo a été interprétée en contexte, de façon à en faire ressortir la signification. Une discussion de groupe a ensuite eu lieu autour de murales collectives: commentaires, rires, questions, constats, plaisirs, échanges. Le nombre d'éléments positifs (incluant le visage d'un petit frère, une paire de raquette, une épinette...) surpassait celui des problèmes parmi lesquels apparaissait par exemple la question des déchets, dont celle des produits toxiques. Certains objets, comme la motoneige, ont soulevé des débats: avantages pour les uns, nuisances pour les autres. Lactivité a suscité beaucoup d'intérêt, comme en témoigne la personne chargée de l'enseignement des sciences auprès de ces jeunes à l'école:

L'activité a suscité chez les élèves une conscientisation à l'environnement, à leur chez soi, à l'école, à la communauté et à la nature [...] les amenant à poser un regard plus critique sur le milieu de vie. La confection des murales a stimulé leur créativité et l'interprétation de celles-ci leur a permis d'exprimer leur vision de leur monde, sans même avoir besoin de traduire. Les élèves ont mis beaucoup d'attention, de fierté et de plaisir à compléter leur travail. Les discussions qui ont accompagné les murales ont porté sur le " bon », le " mauvais », là où les choses vont (sur la murale et dans la vie) et les enjeux de responsabilité. Des discussions d'une telle qualité n'arrivent pas assez souvent à l'école [...] Les élèves sont plus conscients, ils ont davantage d'idées et désirent les partager. Ma classe de sciences au secondaire a poursuivi l'activité. Les élèves ont fait une recherche sur un problème de leur choix [...] II y a eu des discussions encore plus intéressantes pendant le processus de recherche et de confection d'affiches. J'ai découvert que les jeunes ont de bonnes idées sur les façons de changer les choses dans leur environnement.

La suite devait consister à présenter la murale et les affiches en activité parascolaire aux membres de la communauté afin de les inviter à découvrir le regard des jeunes. Le but était de favoriser la réflexion et le dialogue intergénérationnel pour l'interprétation collective des réalités du milieu et l'identification de solutions et d'actions visant à l'amélioration de la qualité de vie au village. Il s'agissait au bout du compte de cheminer vers le développement d'un désir et d'un sentiment de « pouvoir-faire » quelque chose ensemble. Mais auparavant, les murales ont été présentées à des représentants de la communauté afin de valider le travail collaboratif. Certains d'entre eux se sont montrés très enthousiastes à l'égard du travail des jeunes, mais l'un des représentants, particulièrement en rupture avec l'école, y a vu un risque de mettre au jour et de diffuser des problèmes internes à la communauté. Il a demandé de mettre un terme au projet. Le processus a donc ainsi été interrompu, sans possibilité d'en discuter. Les jeunes se sont tus; nous ne pouvons pas rapporter leurs propos. Plusieurs constats et hypothèses peuvent être avancés pour expliquer l'avortement du projet, malgré le succès qu'il connaissait à l'école :

Rupture école-communauté : parmi les membres de la communauté, certains n'acceptaient pas l'école du village, une école exogène, non autochtone. Toute initiative de rapprochement entre l'école et la communauté leur paraissait a priori défavorable.

Tensions méthodologiques au sein du groupe de recherche universitaire : le manque d'harmonisation des pratiques de recherche des différentes équipes " de terrain » associées au projet global (soit une étude de cas multidisciplinaire), plus spécifiquement en ce qui a trait à la mise en œuvre d'une « approche participative », a fragilisé le travail de notre équipe chargée du volet « éducation ».

Présence insuffisante : un financement restreint ne nous permettait que quelques visites de courte durée par année; cela s'est avéré nettement insuffisant pour comprendre la dynamique sociale et installer la confiance.

Bien qu'inachevé, ce projet a toutefois laissé des traces intéressantes. La démarche d'investigation du milieu, dans la double visée d'appropriation de ce dernier et de diagnostic pour le choix de projets environnementaux pertinents et signifiants, s'est poursuivie à l'école après notre départ, à travers diverses initiatives qui ont continué à insuffler énergie et motivation. Par ailleurs, les membres de notre équipe de recherche, de même que certains enseignants et la direction de l'école, en ont retiré des réflexions de nature à enrichir le travail professionnel de chacun.

\section{Chez les Algonquins de l'Abitibi-Témiscamingue}

En septembre 2001, à la demande du Conseil des Anicinapek ${ }^{2}$ de Kitcisakik ${ }^{3}$ (Grand-Lac-Victoria en Abitibi-Témiscamingue), nous avons entrepris l'élaboration d'un projet de recherche collaborative, visant à caractériser une foresterie dite « amérindienne » (Parsons et Prest 2003) qui soit adaptée au contexte socio-environnemental de la communauté. Pour les Anicinapek de Kitcisakik, la question forestière est particulièrement préoccupante. Depuis 1960, plus de $40 \%$ des superficies forestières productives ont été récoltées sur le territoire ancestral de la communauté. La foresterie industrielle ainsi qu'un ensemble d'autres facteurs associés à la modernité ont contribué à la transformation du mode de vie des gens de Kitcisakik et à un changement dans leur rapport à l'environnement. Ces changements se sont généralement traduits par une perte de pouvoir et d'autonomie de la communauté, une désintégration du tissu social et la persistance de frustrations et de conflits au regard de la dégradation de l'environnement (Leroux et al. 2004). 


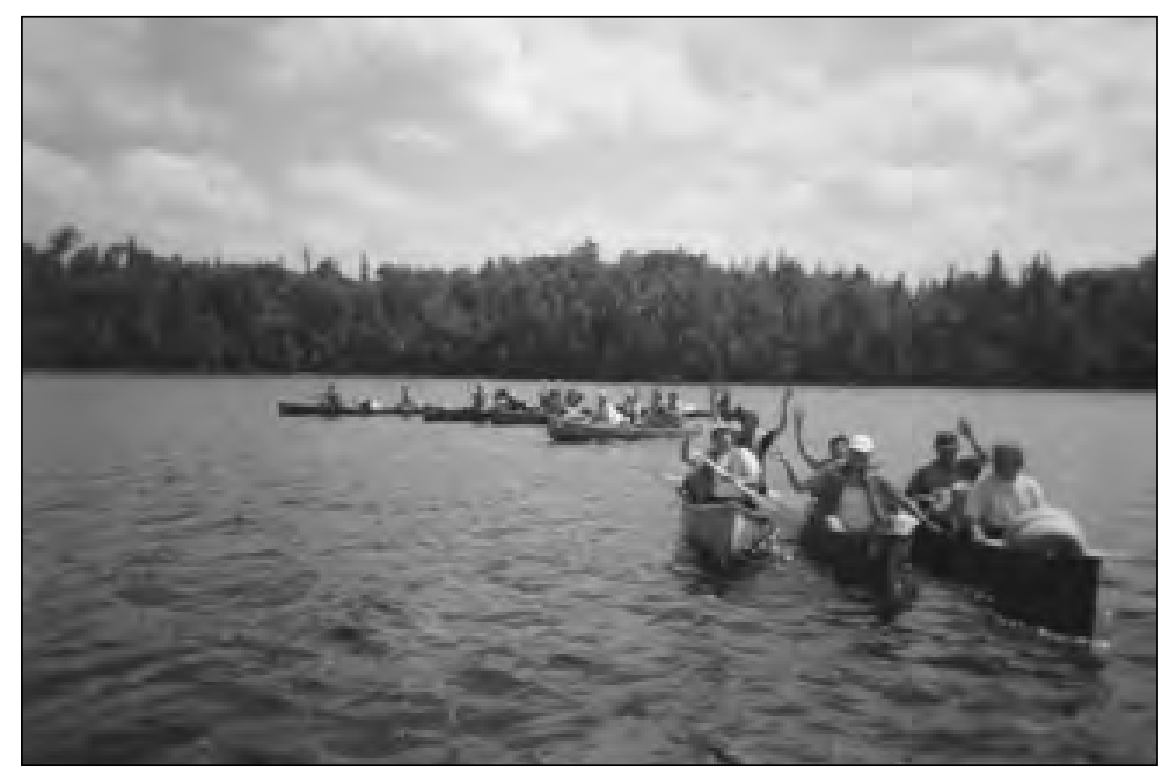

Le rassemblement de départ

«Cela me fait pleurer de joie parce que les Anicinapek ont toujours respecté leur tradition. Un grand rassemblement pour partir sur notre long séjour [...] J'aime que les gens s'entraident. » (Rodney Papatie, 2004)

Au Québec, les changements apportés en 2001 à la Loi sur les forêts (L.R.Q.l, c. F-4) ont introduit l'obligation de consulter les communautés autochtones sur les grands enjeux de la foresterie dans leur région. Toutefois, les différentes expériences de consultation menées à ce jour montrent que ces dispositions législatives nécessiteront la mise en place de conditions propices au dialogue, l'émergence d'une nouvelle culture de participation chez les autochtones (et chez les industriels) et le renforcement des capacités locales en foresterie. Faisant le même constat, le récent rapport de la Commission d'étude sur la gestion de la forêt publique québécoise (Gouvernement du Québec 2004) insiste sur la nécessité d'investir dans l'éducation relative au milieu forestier de manière à favoriser la participation des citoyens aux décisions en matière d'aménagement.

Le premier volet de notre démarche visait à caractériser les éléments dominants des systèmes de représentations (savoirs, attitudes, valeurs, préoccupations, aspirations) qui concernent la relation qu'entretiennent les Anicinapek avec la forêt et la foresterie. Cette étape nous est apparue essentielle pour amorcer un dialogue avec la communauté avant d'entreprendre une réflexion sur les pratiques d'aménagement et les scénarios souhaitables d'exploitation/conservation des forêts.

Une approche multi-méthodologique et adaptative a caractérisé notre démarche de recherche. Ainsi, nous avons saisi l'occasion de la participation d'un groupe de jeunes à une expédition sur le territoire ancestral pour documenter la relation à l'environnement forestier et pour susciter une dynamique participative. En juillet 2004, quinze jeunes Algonquins âgés de 16 à 28 ans, accompagnés de deux aînés et de trois animateurs communautaires, ont entrepris un périple de neuf jours en canot, au cours duquel ils ont parcouru $150 \mathrm{~km}$ sur les lacs et rivières du territoire (photo 1 ). Ce voyage, organisé chaque été à Kitcisakik, comporte un objectif thérapeutique (pour certains participants aux prises avec des problèmes de toxicomanie) et aussi un objectif éducatif, celui de permettre aux jeunes d'expérimenter un contact étroit avec la nature et de renouer avec leur culture.
En collaboration avec les intervenants du milieu, nous avons développé un dispositif de recherche-intervention, invitant les jeunes à adopter une démarche réflexive au cours de l'expédition. Nous avons offert à chaque participant une pochette comprenant une caméra et un « calepin de journaliste ». Chacun avait le mandat de rapporter des images (deux photos par jour) et des commentaires à partir de ses observations du milieu, notamment en vue de préparer une exposition de photos qui serait présentée à la communauté à leur retour. Certaines questions ont été formulées dans le but de préciser la démarche photographique : 1) Qu'est-ce que la forêt pour un Anicinabe? 2) Quelles sont vos préoccupations ou vos inquiétudes en ce qui concerne Nopomuk (la forêt) et Aki (la Terre, l'environnement)? 3) Pouvez-vous imaginer un rêve, un projet pour Kitcisakik, Aki et sa forêt?

Nous avons recueilli plus de cent cinquante photographies illustrant l'environnement « nature », l'environnement « milieu de vie », l'environnement « culture », l'environnement « désastre », etc. Au retour, nous avons demandé à chaque participant de choisir deux photos qui représentaient le mieux son expérience. Deux questions ont été formulées: 1) Pourquoi as-tu choisi cette photo? 2) Quelles sont tes impressions, tes sentiments, tes émotions à l'égard de cette photo? Quelques semaines plus tard, les participants ont préparé ensemble un diaporama qu'ils ont choisi d'intituler : « Les sentiers vers nos ancêtres». Il a été convenu qu'une première présentation s'adresserait à un groupe restreint d'amis et de membres de leurs familles. La formule « souper-causerie » qui a été retenue a connu un grand succès. Limportance de telles activités pour perpétuer le « mode de vie anicinapek » a été soulignée à plusieurs reprises. Une deuxième présentation, s'adressera à toute la communauté. Ce sera l'occasion d'animer un groupe de discussion intergénérationnel portant sur la question forestière.

De nombreux jeunes ont exprimé leur appréciation de cette expérience de solidarité et d'immersion dans le territoire et leur culture traditionnelle. Selon nos observations et l'analyse des témoignages, le « projet canot» a permis de renforcer le sentiment d'appartenance au milieu de vie. Il a permis l'expression de la conscience aiguë qu'ont les jeunes de la situation environnementale, au-delà du paysage et de l'image (photo 2). Plusieurs commentaires traduisent le désir d'affirmation identitaire des jeunes et attestent leur capacité d'établir des relations solidaires entre eux. Un sentiment de fierté anime les jeunes qui ont relevé un défi important, contribuant au renforcement de l'estime de soi et au désir de partager l'expérience. La randonnée en canot comportait en effet des difficultés et faisait appel à des habiletés physiques, techniques et sociales qui interpellent le « patrimoine culturel » de la communauté. Les commentaires recueillis témoignent de l'attachement à un mode de vie traditionnel (photo 3) et du respect des ancêtres et des aînés. La dimension culturelle de cette expérience d'exploration du 
milieu s'est avérée dominante, comme en témoigne cet extrait du journal d'une participante

$8^{\mathrm{e}}$ journée - Je suis très touchée par ce que les aînés ont partagé au cours de l'expédition. Ils ont fait part de plusieurs aspects du mode de vie des Anicinapek comme l'entraide, les traditions, la culture, les familles, les territoires, la nature, surtout nos ancêtres. Aujourd'hui, on a vu où trouver l'eau potable, les endroits que les Anicinapek ont fréquentés, et un aîné nous a raconté des légendes. J'ai aussi observé que la nature se détériore beaucoup. L'aîné remarque que les gens d'aujourd'hui sont moins intéressés à transmettre leurs savoirs à leurs générations futures. Pourtant, les jeunes sont intéressés à suivre le mode de vie des Anicinapek, [...] mais sans oublier la modernité. [...] Et à notre tour, comment pourrons-nous transmettre les savoirs aux plus jeunes? (Johanne Papatie, 2004)

En attendant la construction d'une école à Kitcisakik, les jeunes doivent fréquenter les institutions de Val-d'Or, à plus de $100 \mathrm{~km}$ de leur village, et demeurer en famille d'accueil pendant la semaine. Pourtant, depuis toujours, la communauté s'appuie sur des expériences d'éducation informelle et sur les modes traditionnels de transmission des savoirs pour assurer la survie de sa culture. La randonnée en canot, en tant qu'expérience éducative, répond à cette préoccupation. Elle a aussi été l'occasion de réfléchir individuellement et collectivement sur la question forestière. D'autres expériences semblables pourront stimuler la mobilisation des gens de Kitcisakik pour clarifier leurs souhaits et désirs d'avenir. On assiste ainsi depuis quelques années à une diversification des occasions d'apprentissage au niveau local et à la valorisation d'une éducation s'inspirant de la tradition autochtone. Les différentes stratégies mises de l'avant par notre équipe de recherche sont de nature à renforcer cette tendance dynamique et pourront éventuellement se greffer au curriculum de la future école de Kitcisakik.

\section{ENJEUX, ÉCUEILS, ESPOIRS : DES CONVERGENCES}

Lexploration des écrits, l'analyse de propositions (projets, programmes) relatives à l'éducation autochtone ainsi que l'analyse réflexive de nos propres expériences nous ont permis de confirmer ou de clarifier certains enjeux, écueils et possibilités pour une éducation relative à l'environnement en milieu autochtone.

\section{D'ABORD, UNE CONFIRMATION}

Dans les milieux où se sont déroulés nos projets, et en dépit des efforts consentis (dont certains méritent certes d'être

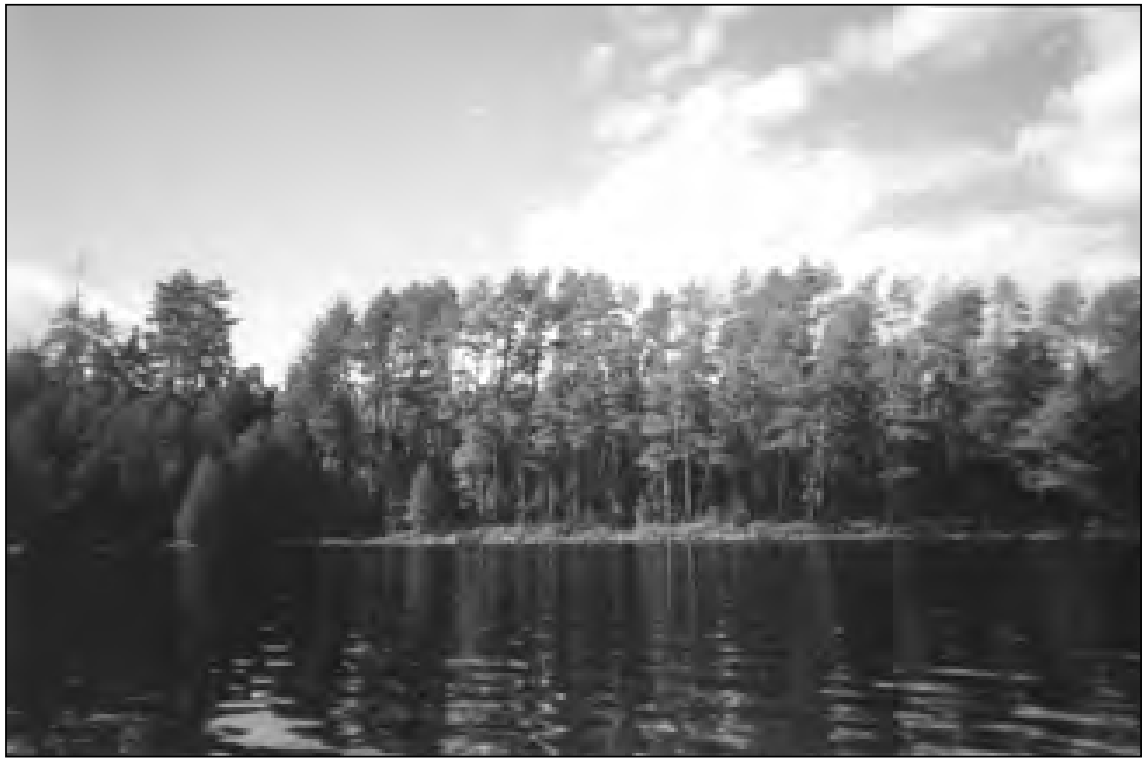

Une lisière boisée en bordure d'un cours d'eau faux, car si vous regardez derrière les arbres, c'est vide et défriché. Même la forêt est en train de

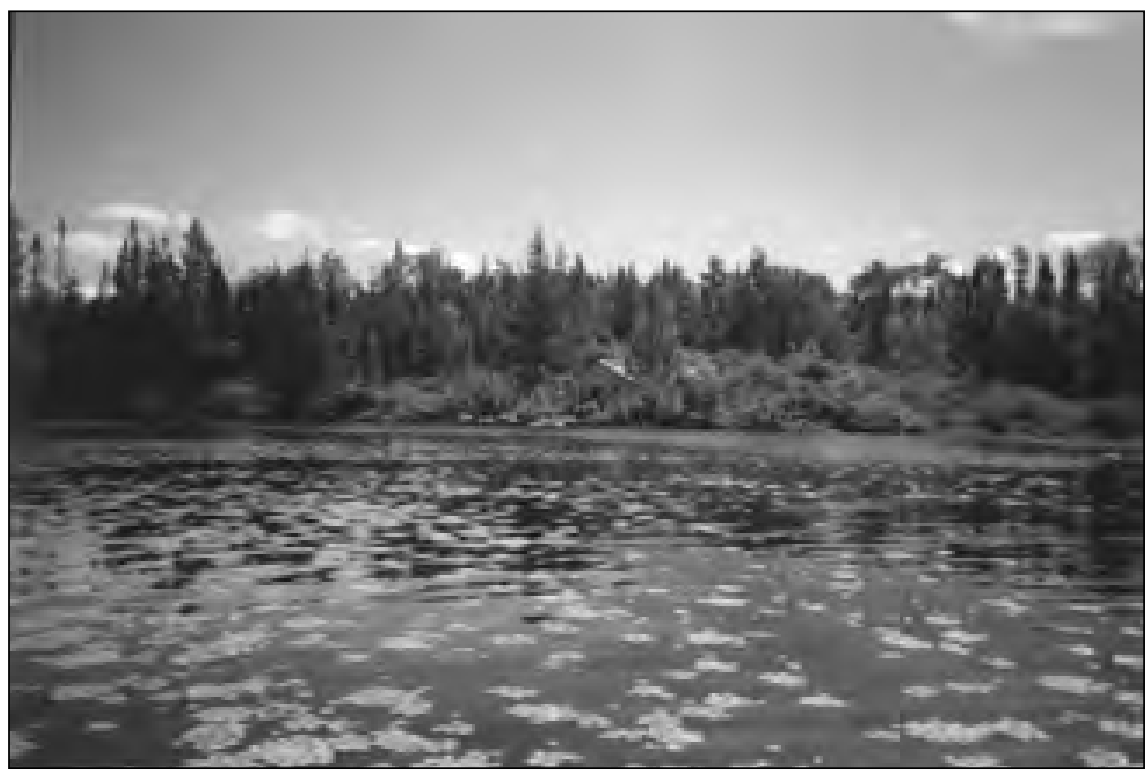

maison abandonnée par la joie de penser qu'il y avait des gens qui vivaient là, des gens de ma culture qui résidaient ici, dans un endroit plaisant et calme. " (Vince Papatie, 2004)

valorisés), l'école formelle est en rupture avec la communauté. Il y a peu d'enseignants autochtones. Les enseignants du Sud s'y sentent généralement isolés; ils sont peu formés pour travailler dans un milieu culturel si différent du leur; ils vivent des tensions internes à l'école et entre l'école et la communauté. Le curriculum est celui d'une autre culture, il ne répond pas ou peu aux besoins spécifiques des communautés. Leffort de contextualisation culturelle consiste le plus souvent à insérer des cours de langue et des contenus relatifs aux "savoirs autochtones », mais sans tenir compte des processus culturels 
de production et d'échange de ces savoirs. Certains enseignants sont conscients du manque de pertinence des programmes, du curriculum et du calendrier, mais ils se sentent impuissants à changer les choses.

Les taux d'absentéisme et de décrochage des jeunes sont élevés. Les parents participent généralement très peu. Beaucoup d'entre eux éprouvent de l'amertume et de la méfiance à l'égard de l'école, dont ils gardent le mauvais souvenir d'une institution colonisatrice. On observe toutefois chez certains un « désir d'école » : celle-ci est vue malgré tout comme une voie de solution privilégiée pour changer les choses, surtout si la communauté parvient à s'approprier l'école. On reconnaît l'importance de concevoir une école autochtone qui soit reliée à sa communauté. Ainsi l'éducation informelle (au sein des expériences de vie et des interactions sociales) prend-elle une grande importance. Il y a un désir de transformer l'école pour y inclure des activités en milieu naturel (manifestement très appréciées des jeunes) et aussi pour y intégrer mieux et davantage la culture autochtone.

\section{L'EXPLORATION DU MILIEU : UN POINT D'ANCRAGE}

Dans un tel contexte, il nous est apparu qu'une certaine « éducation relative à l'environnement » axée sur l'appropriation collective du milieu de vie et des réalités de la communauté pouvait contribuer à intégrer à l'école actuelle plusieurs dimensions d'une «éducation autochtone » de nature à rendre l'apprentissage plus signifiant, plus pertinent. En particulier, la stratégie de l'exploration du milieu apparaît comme un creuset d'apprentissage privilégié, invitant à la découverte ou redécouverte de cet « espace de vie partagée », ce lieu « habité » par les êtres et les choses. L'exploration du milieu de vie sollicite les différents « capteurs sensibles» de l'environnement (d'ordre physique, kinésique, affectif, spirituel, cognitif, etc.) pour une approche expérientielle et holistique des réalités. Tout au long de l'itinéraire, et au fil des observations, le questionnement devient aussi le déclencheur d'une découverte de soi-même, d'une prise de conscience chez les participants de leurs propres savoirs, croyances, attitudes, valeurs, agir, etc., en somme des différents aspects de leur rapport au monde. Plus encore qu'un intérêt ou une curiosité, nous avons constaté une fierté chez les jeunes autochtones à parcourir, observer et « interroger» leur milieu, avec ses dimensions bien concrètes mais aussi symboliques. Nous avons aussi observé un enthousiasme chez les adultes, enseignants ou accompagnateurs. Nos expériences nous permettent de croire qu'une telle stratégie peut favoriser la création de liens entre les jeunes, et entre l'école et la communauté, perçue comme «communauté éducative». L'école « hors les murs » peut devenir le centre d'une dynamique communautaire pour l'éducation relative à l'environnement, incluant la participation des aînés à l'accompagnement des jeunes dans ce monde en changement.

\section{UN DÉFI CULTUREL}

Nos projets chez les Innus du Labrador et les Algonquins de l'Abitibi-Témiscamingue (comme d'autres projets auxquels nous avons été associées ou que nous avons analysés) ont soulevé d'importants défis d'ordre culturel. La signification des réalités environnementales auxquelles sont confrontées les communautés ne peut être appréhendée en effet que dans le contexte d'univers culturels spécifiques. Nous avons conscience que l'idée même d'une « éducation relative à l'environnement » doit être repensée de façon particulière en milieu autochtone, où le concept d'« éducation » est souvent associé à la mémoire d'une triste scolarisation exogène et où le concept d'« environnement» vient d'ailleurs. Ainsi, on ne trouve pas de correspondance exacte pour «environnement» ni chez les Innus interrogés, ni chez les Algonquins. De même, les mots « forêt » et «foresterie » ne se traduisent pas textuellement en langue algonquine. Le concept de Nopomuk, généralement utilisé pour «forêt », renvoie aux parties de la forêt qui sont loin des lieux habités. Il s'agit, en réalité, d'une partie de la terre habitée par les Amérindiens, elle-même nommée Aki (Jacques Leroux, comm. pers., 2005). Par ailleurs, selon les rédacteurs du projet Contaminants Found Me (YCC 2000), les langues des premières nations du Yukon ne comportent pas de mots pour «poison», « déchet », « pollution », « toxique » ou « contaminant». Une exploration du langage devient une bonne stratégie pour créer des ponts permettant la communication interculturelle. Les mots ne sont pas que des formes : ils traduisent une vision du monde qu'il importe de ne pas trahir en imposant des mots venus d'ailleurs et qui véhiculent (trop souvent inconsciemment) d'autres univers représentationnels, relevant d'autres cosmologies.

Ainsi, comment nommer l'« éducation relative à l'environnement » en milieu autochtone? Quels liens peut-on établir entre l'idée de "responsabilité environnementale » (au sens fondamental de responsabilité d'être et d'agir) et celle de " gardiens de la Terre », familière aux peuples autochtones? En ce sens, n'y a-t-il pas un « viol culturel ${ }^{4} »$ à imposer, à travers l'éducation ou des modes de gestion, le projet politico-économique nordoccidental d'un « développement durable » aux communautés autochtones, en tentant de les convaincre que leurs traditions en ont toujours été porteuses? S'ouvre ainsi un univers de recherche fascinant pour nous, du domaine de l'éducation relative à l'environnement, essentiellement préoccupée de reconstruire le « rapport au monde », dans un souci d'intégrité et d'intégralité.

En lien avec la recherche de fondements appropriés, nos projets ont impliqué également un questionnement relatif aux approches et stratégies pédagogiques en milieu autochtone. Il nous apparaît que les pratiques d'éducation relative à l'environnement axées sur la démarche systématique de résolution de problèmes et l'analyse systémique pour la « gestion rationnelle de l'environnement» ne sont pas à privilégier ici. Certains entretiens réalisés à Kitcisakik ont montré les limites de notre rationalité instrumentale, qui nous amène à analyser et distinguer les éléments d'un tout pour résoudre un problème, qu'il soit d'ordre éducatif, social ou environnemental. Par exemple, une question qui invite à prioriser un problème environnemental ou à identifier un indicateur peut se heurter à une approche holistique des réalités chez les autochtones, parce que « tout est important», parce que «tout est relié ».

Par ailleurs, des questions fondamentales émergent en ce qui concerne la dimension « critique » de l'éducation relative à l'environnement en milieu autochtone. Sur le plan épistémologique, est-il pertinent de promouvoir une rationalité critique dans de tels contextes culturels? Sur le plan social, l'approche critique risque-t-elle de susciter ou d'exacerber des conflits dans la communauté? Les chercheurs et les enseignants sont-ils vraiment outillés et engagés pour assumer les conséquences des conflits qu'ils pourraient susciter? Quel sens prend ici l'approche critique? Quelles stratégies adopter à cet égard? Chez les autochtones, on trouve une culture de l'observation, de l'immersion, on apprend par l'expérience, qui comprend à la fois des aspects positifs et négatifs. Les curriculums autochtones 
analysés incluent une certaine réflexivité et une quête de sens dans l'expérience.

Certes, nos trajectoires de projets ont confirmé la nécessité d'une éducation qui favorise la prise de conscience des différentes formes d'aliénation, de même que l'émancipation et le développement d'un « pouvoir-agir ». Mais l'approche critique des réalités ne devrait-elle pas prendre une « couleur» culturelle particulière, moins centrée sur la seule rationalité, sur la seule déconstruction par l'argument logique? Pourrait-elle, par exemple, prendre la forme d'une résistance active qui s'appuie sur l'affirmation identitaire? "Résister, c'est créer », selon la proposition de Florence Aubenas et Michel Benasayag (2004). Ainsi, il ne s'agirait pas tant de "lutter contre», mais de construire ailleurs, autrement, des projets signifiants qui prennent forme dans un agir dialogique au sein de la communauté (Habermas 1987).

Enfin, toujours à propos de l'approche critique, signalons qu'à travers nos explorations, projets et expériences, nous avons développé une sensibilité accrue à l'égard de deux pièges idéologiques associés à la rhétorique de la protection de l'environnement : d'un côté la « néo-folklorisation » du rapport holistique et cosmologique des autochtones à la nature, et de l'autre, la volonté de réhabiliter l'autochtone acculturé qui a brisé son lien filial à la Terre. Ici, c'est le chercheur qui doit confronter son propre système de représentations (incluant des stéréotypes et des préjugés favorables ou défavorables) à une réalité qu'il importe de nuancer à la lumière d'un examen critique et de l'interprétation des réalités par les personnes et groupes concernés.

Enfin, en lien avec les défis culturels auxquels nous avons été confrontées, il importe de souligner également les enjeux éthiques et stratégiques qui découlent plus spécifiquement de la mise en œuvre d'une approche participative. Ceux-ci mériteraient certes un espace d'explicitation.

\section{ApPorts au Champ de l'Éducation Relative À L'ENVIRONNEMENT}

Nos expériences de recherche-intervention en milieu autochtone confirment l'intuition initiale de trouver dans un tel contexte des pistes pour la valorisation ou le renforcement d'une dimension d' éducation relative à l'environnement * dans l'éducation autochtone, mais aussi, tel que le suggère Michelle Rae McKay (2005), des éléments de théorie et de pratique transférables à d'autres contextes, enrichissant ainsi le champ de l'éducation relative à l'environnement en général, et plus encore, fournissant des pistes pour rendre nos systèmes d'éducation plus pertinents.

La pédagogie autochtone met en lumière l'importance d'une approche globale (holistique) et expérientielle des réalités et elle valorise la dimension communautaire du processus d'apprentissage. Elle s'appuie sur une vision du monde qui nous invite à repenser notre rapport à l'environnement, à remettre en question la rupture entre le corps et l'esprit, entre l'humain et la nature, entre l'environnement et la culture. En ce sens, Gregory Cajete (1994: 194) signale que l'éducation autochtone est essentiellement « environnementale ». Ici, l'idée de développement n'a de sens que si cela consiste à libérer les gens des entraves à l'actualisation de leur culture propre. De tels fondements sont certes de nature à inspirer des pratiques d'éducation relative à l'environnement qui vont au-delà de l'approche instrumentale de la «modification de comportements civiques » (comportements isolés et individuels), pour se pencher sur le sens de notre "être-au-monde », ensemble, ici et maintenant, dans ce milieu de vie partagé.

En particulier, une approche interculturelle d'éducation relative à l'environnement serait à privilégier. Par exemple, «le projet canot » avec les jeunes Algonquins a montré leur sensibilité particulière à saisir « l'esprit du lieu» ou « le sens du lieu». Il serait avantageux de mettre à profit cette « compétence » dans le cadre d'un échange invitant des jeunes d'autres cultures à « développer la conscience du mélange complexe des enjeux culturels et environnementaux qui contribuent à façonner un endroit, un paysage », comme le relate Alistair Stewart (2004) dans son analyse d'une expérience éducative australienne d'expédition en canot.

Certes, les projets dont témoigne cet article restent isolés et inachevés, mais au fil des boucles d'expérience, de tels projets sont destinés justement à ne jamais prendre fin : l'école comme un projet de vie, le milieu de vie comme un projet d'apprentissage, la communauté comme un projet culturel, en mouvance, en évolution. Léducation immergée dans les situations de vie, ici et maintenant, entre nous, ne fait pas que « capter les rêves »; elle aide à en construire, à les réaliser, à les ancrer dans une identité affirmée, capable d'altérité saine. Enfin, notre propre projet d'apprentissage se poursuit: les questions soulevées ne sont certes pas encore résolues. Elles sont le moteur d'un cheminement continu de recherches collaboratives et participatives qui devront aussi s'inscrire dans le partage et le croisement des savoirs (disciplinaires et autres) pour la construction d'un espace fécond de métissage épistémologique et pédagogique.

\section{Notes}

1. Dans une étude récente portant sur l'accès au savoir environnemental dans les communautés autochtones du Nord québécois, Carole Lévesque et al. (2004) font état de la multiplicité des définitions et catégorisations des systèmes de savoirs. Selon ces chercheurs, «La plupart des auteurs [...] qui ont étudié les savoirs autochtones et ont tenté de les définir en fonction de composantes ou de catégories clés s'entendent néanmoins pour dire que l'interprétation et la compréhension des systèmes de savoirs autochtones et des liens entre différentes catégories, nécessitent une connaissance holistique appréhendant les dimensions éthique et cosmologique » (p. 68-69)

2. Les Algonquins se désignent dans leur langue par le terme Anicinapek, au pluriel, et Anicinabe, au singulier.

3. Kitcisakik est le terme algonquin qui signifie «à la grande embouchure » pour désigner le Grand lac Victoria, un élargissement de la rivière des Outaouais, sur les rives duquel se trouve le site de rassemblement estival de la communauté (Leroux et al. 2004).

4. Aminata Traoré (2002) traite du «viol de l'imaginaire »dans son ouvrage où elle dénonce les «mots d'ordre » des organisations internationales qui contraignent les populations en leur imposant un langage et un projet politico-économique exogène, les empêchant ainsi de se donner un véritable projet social qui convienne à leur culture et à leurs aspirations.

\section{Ouvrages cités}

AUBENAS, Florence, et Miguel BENASAYAG, 2004 : Résister, c'est créer. La Découverte, Paris.

BASKIN, Cyndy, 2002 : «Re-Generating Knowledge: Inclusive Education and Research ». Communication présentée à la Annual Conference of the Canadian Indigenous and Native Studies Association (CINSA), 29-31 mai 2002, Toronto. 
BERGER, Paul, 2002 : " Adaptations of Euro-Canadian Schools to Inuit Culture in Selected Communities in Nunavut ». Communication présentée au 14th Annual Meeting of the Arctic Research Consortium of the United States (ARCUS) and Arctic Forum, mai 2002, Arlington, Virginia.

BERKES, Fikret, 1989 : Sacred Ecology. Traditional Ecological Knowledge and Resource Management. Taylor and Francis, Philadelphia.

BLANCHARD, Rosemary Ann, 1999: «Forging a Civic Relationship between Native Youth and Their Indigenous Nation: A Tribally-Specific, Tribally Formed Social Studies Curriculum », in Ray Barnhardt (dir.), Indigenous Education Around the World, p. 15-22. Center for Cross-Cultural Studies, University of Alaska Fairbanks, Fairbanks, Alaska.

BRANT CASTELLANO, Marlene, Lynne DAVIS et Louise LAHACHE (dir.), 2000: Aboriginal Education: Fulfilling the Promise. UBC Press, Vancouver.

BRIGGS, Jean L., 1983 : « Le modèle traditionnel d'éducation chez les Inuits: Différentes formes d'expérimentation face à l'inconnu ». Recherches amérindiennes au Québec 13(1) : 13-25.

CAJETE, Gregory, 1994: Look to the Mountain: An Ecology of Indigenous Education. Kivaki Press, Durango, Colorado.

COLLECTIF, 1993 : Dene Kede Education: A Dene Perspective. Department of Education, Culture and Employment, Yellowknife, Northwest Territories.

COLLECTIF, 1996: Inuuqatigiit: The Curriculum from the Inuit Perspective. Department of Education, Culture and Employment, Yellowknife, Northwest Territories.

FREIRE, Paulo, 1972: Pedagogy of the Oppressed. Herder and Herder, New York.

GOUVERNEMENT DU QUÉBEC, 2004 : Commission d'étude sur la gestion de la forêt publique québécoise. Gouvernement du Québec, Québec.

GUTIÉRREZ, Francisco, 2002: Educación como praxis política. Siglo Veintiuno, México.

HABERMAS, Jürgen, 1987 : Théorie de l'agir communicationnel. A. Fayard, Paris.

LEROUX, Jacques, Roland CHAMBERLAND, Edmond BRAZEAU et Claire DUBÉ, 2004 : Au pays des peaux de chagrin: Occupation et exploitation territoriales à Kitcisakik (Grand-Lac-Victoria) au XXe siècle. Presses de l'Université Laval et Musée canadien des civilisations, Québec/Hull

LÉVESQUE, Carole, Christiane MONTPETIT, Isabelle POULIN, Dominic BABEUX et Ginette LAJOIE, 2004: Éléments d'une stratégie visant à promouvoir et à faciliter l'accès au savoir environnemental dans les communautés autochtones du Nord québécois. Université du Québec - INRS Culture, urbanisation et société, et Administration régionale crie, Montréal.

LORING, Eric, 2001 : «Training and Support for Regional Contaminant Coordinators », S. Kalhok (dir.), Synopsis of Research Conducted under the 2000-2001 Northern Contaminants Program, p. 320-322. Ministère des Affaires indiennes et du Nord Canada, Ottawa.

McKAY, Michelle Rae, 2005 : Indigeous Pedagogy: Can this Concept be Successfully Implemented Within the Current Curriculum? <http://uregina.ca/ mckaymm/ip.html>, (page consultée le 25 janvier 2005)

ONGTOOGUK, Paul, 2000: «Aspects of Traditional Inupiat Education ». Sharing Our Pathways: A Newsletter of the Alaska Rural Systemic Initiative 5(4): 8-12.

PARDO, Thierry, 2002 : Héritages buissonniers : Éléments d'ethnopédagogie pour l'éducation relative à l'environnement. Éditions de Babio, La Caunette.

PARSONS, Reginald, et Gordon PREST, 2003: «Aboriginal Forestry in Canada ». Forestry Chronicle 79(4) : 779-784.

PAUL, Léonard, 1983 : «La résistance culturelle des Montagnais et le cas de Betsiamites ». Recherches amérindiennes au Québec 13(1): 5-12.

PENASHUE, Elizabeth, 2000 : «Like the Gates of Heaven », in Nympha Byren et Camille Fouillard (dir.), It's Like the Legend: Innu Women's Voices, p. 157. Gynery Books, Charlottetown.

POIRIER, Sylvie, et Lorraine BROOKE, 2000 : « Inuit Perceptions of Contaminants and Environmental Knowledge in Salluit, Nunavik ». Arctic Anthropology 37(2) : 78-91.

ROBOTTOM, Ian, et Paul HART, 1993 : Research in Environmental Education. Engaging the Debate. Deakin University Press, Deakin, Victoria, Australie.

SAUVÉ, Lucie, 1997 : "L'approche critique en éducation relative à l'environnement : origines théoriques et applications à la formation des enseignants ». Revue des sciences de l'éducation 23(1): 169-189.

—, 2003 : Courants et modèles d'interventions en éducation relative à l'environnement. Module 5. Programme d'études supérieures Formation en éducation relative à l'environnement - Francophonie internationale. Les Publications ERE-UQAM, Université du Québec à Montréal - Collectif ERE-Francophonie, Montréal.

SIMPSON, Leanne, 2002 : «Indigenous Environmental Education for Cultural Survival». Canadian Journal of Environmental Education 7(1): 13-25

STEWART, Alistair, 2004 : « Canoeing the Murray River (Australia) as Environmental Education: A Tale of Two Rivers ». Canadian Journal of Environmental Education 9 : 136-147.

TRAORÉ, Aminata, 2002: Le Viol de l'imaginaire. Fayard/Actes Sud, Paris

UKPATIKU, Eric, 2001 : «Kivalliq Inuit Association Regional Contaminants Coordinator: a Five-Year Strategy of Communication and Action on Contaminant Issues in the Canadian Arctic », in S. Kalhok (dir.), Synopsis of Research Conducted under the 2000-2001 Northern Contaminants Program, p. 342-344. Ministère des Affaires indiennes et du Nord Canada, Ottawa.

VAN OOSTDAM, J. , et al., 1999 : « Human health implications of environmental contaminants in Arctic Canada: a review ». The Science of the Total Environment 230: 1-82.

WATT-CLOUTIER, Sheila, 2000 : « Honoring our Past, Creating our Future: Education in Northern and Remote Communities», inAboriginal Education: Fulfilling the Promise

YUKON CONTAMINANTS COMMITTEE, 2000 : Contaminants Found Me: A Science Curriculum for All Yukoners. Yukon Contaminants Committee, Whitehorse, Yukon. 


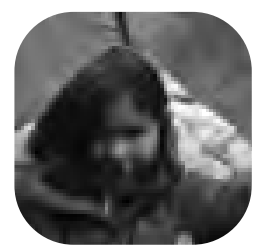

L'importance de l'aspect relationnel

dans l'auto-(re)présentation de jeunes Innus de la communauté de Uashat mak Mani-Utenam

Karoline Truchon

Département de sociologie et d'anthropologie, Université

Concordia, Montréal

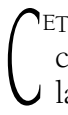
T ARTICLE PRÉSENTE LES FONDEMENTS de ce que je nomme « le relationnel dans la technique ». Plusieurs mentionnent que la production artistique des autochtones foisonne d'imageries émanant de la relation symbolique et symbiotique qu'ils entretiennent avec la terre et la nature (Jacknis 1996; Leuthold 1998; Roessel 1996; Worth et Adair 1997). D'autres ajoutent que les photographes et vidéastes autochtones feraient autant dans le documentaire que dans l'artistique et utiliseraient les éléments techniques comme des métaphores (Leuthold 1998 : 125).

Néanmoins, ce qui caractériserait la production artistique des autochtones ne relèverait pas de ses aspects techniques. Le facteur principal de différenciation serait plutôt d'ordre relationnel, ce que Troy (1992: 50) appelle « la fusion de la fiction et du fait, du subjectif avec l'objectif, du photographe avec ce qui est photographié » et qui serait « une scission complète du 'Je-Eux' de l'anthropologie visuelle » jusqu'à présent. Ainsi, même si dans cet article je présente une quinzaine de photos prises et commentées par des jeunes autochtones et illustrant dans une certaine mesure leur quotidien, mon but premier n'est pas de cerner les identités des jeunes Innus, mais de montrer l'interdépendance de l'objet (la photo) et du sujet (la (re)présentation) comme caractéristique influençant grandement les identités actuelles et futures des jeunes Innus rencontrés pendant cette recherche, et peut-être, cela reste à confirmer, de la jeunesse autochtone contemporaine ${ }^{1}$.
Interpellée par les problématiques sociales soulevées par les scientifiques et les journalistes, j'ai voulu vérifier comment les jeunes autochtones vivaient leur quotidien. La majorité des représentations de ce segment de population étant effectuées par des adultes experts, il m'est apparu important de proposer une forme de recherche où les jeunes autochtones ne seraient pas que sujets mais aussi partenaires dans leurs représentations. Plus d'une soixantaine de jeunes Innus de la communauté de Uashat mak ManiUtenam, une communauté de la CôteNord, au Québec, ont répondu à mon invitation de photographie collaborative, projet intitulé « La photographie comme bâton de parole ${ }^{2} »$. D'un espace d'expression pour ces jeunes, ce projet de photographies commentées s'est transformé en un lieu de rencontres et de dialogues inter-pairs, inter-générationnels et interculturels grâce, entre autres, à la publication d'une quinzaine d'articles dans le mensuel autochtone Innuvelle et à la conception d'une exposition itinérante.

Le mandat des participants était de photographier ce qu'ils aimaient ou aimaient moins de leur communauté. La trentaine de participants de l'exposition ont principalement proposé des commentaires positifs de leur quotidien pour les images qu'ils ont captées (Truchon 2005a, 2005b). Outre ce résultat qui remet en question les représentations de souffrance et de misérabilisme véhiculées fréquemment par certains leaders autochtones et par plusieurs Québécois non autochtones au sujet des jeunes autochtones, un fait maintes fois répété 
pendant ma recherche m'a particulièrement intriguée. Pendant nos discussions à propos de leurs photos, les jeunes participants en ont rarement souligné les multiples ratés techniques. Non seulement ne se sont-ils pas offusqués de ce que plusieurs adultes considéreraient être des photos manquées, mais ils ont choisi ces photos pour faire partie de l'exposition, présentée depuis aux gens de leur communauté, à d'autres autochtones ainsi qu'à un public de non-autochtones. C'est au fil de mes conversations avec ces jeunes Innus de la communauté de Uashat mak Mani-Utenam que j'ai découvert que l'important pour eux était ce qui figurait sur la photo, c'est-à-dire le lien les unissant à ce qu'ils ont (re)présenté.

La prochaine section explique le cadre d'analyse des données récoltées pendant ce projet, qui seront présentées en deuxième partie de l'article. Cette théorisation de l'art comme système relationnel s'avère la pierre d'assise dans la compréhension de la sélection d'images et de commentaires dits « positifs » par les jeunes participants innus. Les photographies, résultat du médium utilisé, y sont considérées comme une forme d'expression et, donc, d'art.

Le cadre conceptuel est aussi formulé à partir d'une lecture d'universalisation plutôt que de particularisation des communautés autochtones. Deux raisons justifient la combinaison d'exemples d'autres nations à ceux des Innus pour proposer une explication des choix des photos commentées par les jeunes de ce projet. Dans un premier temps, un seul ouvrage, à ma connaissance, présente des photos commentées par des Innus sans néanmoins aborder les aspects de représentation et de relations entre producteur (photographe) et sujets (Innus) [Jauvin 1993].

Dans un deuxième temps, mon intention est de proposer que, pareillement au processus d'objectivation de la culture où un savoir-être est évacué au profit d'une image construite d'un savoir à posséder (Susan Wright, citée par Poirier 2004 : 9), les rapports entre producteur, sujet et récepteur de recherches et de documents visuels sont souvent influencés par ce que Martin Buber (cité par Asch 2005) nomme le « Je-Ça », qui en fait des relations transactionnelles, c'est-à-dire des relations fondées sur ce qu'il y a à gagner plutôt qu'à partager. Dans une relation centrée sur un partage commun, Sylvie Poirier souligne que :

la tolérance et une rhétorique d'ouverture à l'Autre sont loin d'être suffisants, encore faut-il vouloir ou pouvoir valoriser une logique relationnelle, et penser ontologiquement (mais aussi épistémologiquement) le multiple et la multiplicité, ainsi que la fluidité et la perméabilité des frontières. (Poirier 2004 : 11)

Buber (cité par Asch 2005) définit cette «logique relationnelle » par le «Je-Tu», qui est une façon d'être avec les autres commandant non seulement une acceptation de l'autre, mais aussi une confirmation inconditionnelle de l'autre sans se perdre à travers cet autre (Friedman 1983). Dans cette conception de la rencontre, l'aspect relationnel, basé sur le savoir-être, transcenderait l'aspect transactionnel, ce dernier tablant sur les différences entre nations, tribus, individus. De là le cadre de référence proposé qui favorise l'intégration des façons d'être d'autochtones à l'égard du visuel plutôt qu'une dichotomisation statique par nation du rôle technique du visuel pour eux. En universalisant l'expérience de la production artistique des autochtones, nous pouvons en proposer des fondements qui, dans cet article, prennent la forme de l'art comme système relationnel, une caractéristique devenant un lien entre les nations.
Cette approche d'universalisation, opposée à la particularisation de la culture, est d'ailleurs empruntée par certains auteurs pour exposer des causes communes à des expériences similaires entre les nations (Wesley-Esquimaux et Smolewski 2004).

\section{Le RELATIONNEL DANS LA TECHNIQUE}

Contrairement aux Américains ou Canadiens non autochtones qui sépareraient et polariseraient la nature et la culture, ces dimensions seraient interreliées pour les autochtones (Leuthold 1998). Par exemple, là où les non-autochtones définiraient les conflits concernant les terres en termes juridiques et de propriété, les autochtones estimeraient que ces chicanes sont d'ordre culturel, plus que structurel, et même de l'ordre de la moralité (ibid. : 125-129). Linterprétation de la fonction de l'art et des œuvres qui en résultent s'avère aussi un aspect provoquant des schismes importants entre les deux groupes.

\section{DE « L'ART ORIENTÉ VERS L'OBJET » À « L'ART COMME SYSTÈME RELATIONNEL "}

Apparue en Occident pendant le siècle des Lumières et la période romantique au XIX ${ }^{e}$ siècle, la conception automatiste des Américains ou des Canadiens non autochtones contraste avec l'expérience d'une esthétique autochtone ancrée dans l'amalgame des relations sociales. Si, dans une vision automatiste, l'art est unique, non utilitaire, innovateur, sans règle, valide en soi, fait pour être vendu ou exposé comme objet sans lien avec une communauté précise et centré sur l'ego et les intentions de l'artiste, la conception de l'art autochtone s'inscrit à l'opposé de ces principes. Une des distinctions des cultures autochtones serait que ces dernières n'identifient pas leurs œuvres expressives comme de l'art (Leuthold 1998 : 7). Le mot « art » serait même absent du lexique de toutes les nations autochtones en Amérique du Nord. Cette conception de l'expression serait plutôt une invention européenne (Feest 1992: 9). Contrairement aux préceptes de la vision automatiste de l'art, l'expression matérielle des peuples des premières nations serait guidée par des règles sociales strictes. Les objets et les événements fabriqués par cette expression seraient orientés vers le service à la communauté. Ces formes matérielles d'expression doivent être à la fois belles et utiles. Leur beauté serait tributaire de leur fonctionnalité. Les fabricants de formes matérielles, ou « artistes », ne seraient pas séparés ou supérieurs au reste de la société et aucune pression ne s'exercerait sur eux afin qu'ils innovent à tout prix (Leuthold 1998: 7). Cette divergence conceptuelle entre les deux groupes amènerait les non autochtones à interpréter la production artistique d'autochtones différemment de ses producteurs, notamment en regard du concept de « romanticisation » et du choix de certains aspects picturaux.

Les critiques non autochtones reprochent aux autochtones de reproduire des clichés romantiques avec les images de la nature (Leuthold 1998). Les longues prises de vue des territoires, les hommages à ce qui est considéré comme traditionnel et les thèmes de la spiritualité et de la famille forment autant d'éléments que les vidéastes non autochtones considèrent comme intrinsèques à l'époque de la romanticisation des «Indiens». Dans cette optique, le romantisme implique un rejet du monde contemporain nourri par une vision esthétique incapable de subsister dans la modernité. Cette vision serait ethnocentrique, selon Roy Big Crane, un vidéaste autochtone du Montana, car elle négligerait d'incorporer la relation intemporelle qu'auraient les autochtones avec la terre (cité dans Leuthold 1998 : 120). 
Le problème serait donc de savoir ce que "romantisme » signifie pour les autochtones. Cette notion a-t-elle ou non une résonance pour eux? Le fait d'imposer une perspective critique non autochtone impliquerait que les systèmes esthétiques et éthiques qui en diffèrent peuvent être abandonnés et donc devenir un vocabulaire réductionniste au même titre que les images l'ont été dans le passé (Leuthold 1998 : 120). Le débat demeure ouvert : l'incorporation de ce type d'imageries romancées résulte-t-elle de la relation symbiotique que vivraient les autochtones avec la terre ou témoigne-t-elle de leur acculturation? Ces deux optiques pourraient toutefois refléter davantage l'appartenance identitaire de celui qui les véhicule que l'idéologie réelle qui motive ce choix. Ainsi, la première proposition émanerait principalement des autochtones, tandis que la deuxième, de non autochtones scolarisés (ibid. : 124). Si l'imagerie évoquant la période du romantisme est décriée par les modernistes et post-modernistes prisant le high art, il n'en demeure pas moins que le public n'a jamais renié cette vision, bien au contraire ${ }^{3}$.

La démarche d'interprétation des choix techniques et artistiques est aussi subjective et aléatoire, comme le révèle le prochain exemple. La propension des Navajos à inclure des gens qui marchent dans leur film a fait sourciller Worth et Adair (1997). Dans leurs notes, les chercheurs interprétaient ce choix des participants navajos comme l'aveu qu'ils ne savaient quoi faire. C'est seulement au retour de leur terrain que les chercheurs ont réalisé qu'en voulant trouver la différence dans la présentation et la conception des images à travers les catégories « Eux » et « Nous », ils avaient éliminé la signification de ce choix éditorial et pictural des apprentis navajos, pour qui « marcher » mettait en relief leur façon de percevoir leur univers et que le choix de montrer des images de gens qui marchent était délibéré, alors que pour les chercheurs, cet acte ne signifiait rien. « Nous n'avons fait attention qu'à ce à quoi nous accordions de l'importance », ont révélé Worth et Adair.

Nous cherchions des "mythes », des "trames narratives » en rapport avec les légendes [que nous connaissions de cette nation], des mines d'argent, etc. C'est seulement quand nous avons revu les films, analysé nos entrevues et relu la littérature sur les Navajos que nous avons compris que l'acte de marcher possède une qualité mythique pour ces derniers. Pour les Navajos, marcher est un événement en soi et non pas strictement une façon de se rendre quelque part. (ibid. : 146, les italiques sont des auteurs)

Cette transmission visuelle de l'univers des Navajos suggère que les notions de « réalité » et de « vérité » des productions autochtones pourraient s'inscrire dans un espace-temps différent de celui qui est connu de la majorité des non autochtones et qui est appris et retransmis par eux. La valeur d'une image serait dans ce qu'elle dépeint (des liens, j'argue), et cette dernière perdrait son identité moderne d' «image » et postmoderne de « reflet », car son action serait d'être en relation avec un système de valeurs où une certaine vérité existe, demandant à être révélée. Les photos doivent être «vraies » pour les photographes, les sujets et leur culture (Lippard 1992 : 23).

Suivant cette actualisation de vérité subjective intemporelle, la façon de représenter la nature, par exemple, ne serait pas strictement réaliste, mais aussi symbolique de "l'essence et de la pulsion de vie». La nature s'inscrirait dans le domaine du poétique pour beaucoup de peintres autochtones, estimait le regretté Blackbear Bosin, un artiste comanche-kiowa (cité par Leuthold 1998 : 128). Selon celui-ci, en maniant des pinceaux, les enfants non autochtones chercheraient à reproduire ce qu'ils voient comme création de Dieu, contrairement aux jeunes autochtones qui tendraient à vouloir saisir l'essence humaine dans leur expression picturale. Leurs bagages culturels - la langue, le langage et l'humour - leur enseigneraient cette façon d'être à l'opposé de celles des non autochtones qui tableraient sur le procédé figuratif. Les jeunes autochtones aspireraient à peindre les symboles qui les entourent, soit les pulsions et les essences de vie de leurs réalités.

Ces exemples suggèrent que, pour mieux apprécier cette façon plus holistique de voir la vie, l'interprétation de la production d'autochtones exigerait une transition de «l'art orienté vers l'objet » vers «l'art comme système relationnel » (Leuthold 1998: 7). Cette distinction peut aussi s'appliquer à l'acte photographique et à ses résultats.

\section{LE SACRÉ DES LIENS QUOTIDIENS}

La photographie deviendrait un acte conjoint quand « l'Indien derrière la caméra est connecté à l'Indien devant la caméra. Ils doivent êtres des égaux, ils doivent être des collaborateurs. Ils doivent absolument se faire confiance (Troy 1992: 564)». Là ou les autochtones penseraient en fonction de l'interconnexion et du respect (ibid), la conception des non autochtones serait d'ordre juridique et éthique (Brumbaugh 1996: 36; Jacknis 1996 : 6-7). La métaphore du Kwikwilyaqa, un clown katsina qui ressemble à une caméra, illustre les dangers de sortir de la connexion quand on photographie quelqu'un (Jacknis 1992 : 2; Lippard 1992 : 21). En geste d'amitié, ce personnage se colle à tous ceux qu'il rencontre, mais il finit par leur faire ombrage. Le Kwikwilyaqa avertit du dommage qu'un photographe peut causer aux autres par ses photographies.

Cette interrelation humaine a une incidence sur ce qui peut être public, donc partagé, ou privé, c'est-à-dire pour soi ou les siens. Selon le photographe Tuscora Richard Hill (cité par Troy 1992 : 56), la plupart des photographes des premières nations, amateurs ou professionnels, ne s'imposeraient pas lors de cérémonies. Leur façon de travailler s'appuierait sur la nécessité de relations interpersonnelles plutôt que sur des intrusions forcées, contrairement aux non autochtones qui se trouveraient dans des situations similaires (Lippard 1992; Maxwell 1999). En simplifiant beaucoup, nous serions devant deux conceptions du savoir. Le savoir fondé sur une philosophie européenne valoriserait la circulation de l'information comme une fonction libératrice pour tous, tandis que, selon la cosmologie autochtone, certains savoirs devraient rester entre les mains de leurs détenteurs, car leur diffusion pourrait être dangereuse (Jacknis 1996 : 5-6; Eric Michaels, cité par Chalfen 1997). Ainsi, la polyphonie des voix de même que la communication ouverte ne seraient pas toujours indiquées, car, dans plusieurs nations, des normes strictes, différant de ces philosophies, régissent la circulation de l'information (Chalfen 1997: 320). Même aujourd'hui, l'utilisation de la caméra serait restreinte pour protéger les droits entourant les danses et chansons exclusives de certaines familles autochtones (Marr 1996 : 57). La distinction entre propriété privée ou publique est aussi au cour des débats. Par exemple, en 1960, les Zunis contemplaient l'idée d'un musée autant pour les touristes que pour les gens de leur communauté ; ils estiment aujourd'hui que leur savoir doit être exclusif à leur communauté (Holman 1996 : 104).

Les liens familiaux constitueraient autant des marqueurs identitaires que des normes de fonctionnement communautaire. Chez les Aborigènes d'Australie, le réseau d'affiliation 
familiale dicterait les manières de communiquer et de produire entre les Warlpiris. Ce type de réseau influencerait toute activité médiatique. Exempte de ce système, toute opération serait plutôt d'appartenance et de fonctionnement européens (Michaels, cité par Chalfen 1997 : 320). Les photos de la collection privée du photographe crow Richard Throssel, prises au début du siècle dernier, suscitent encore aujourd'hui de vives réactions chez les membres de cette nation, précisément parce qu'ils reconnaissent leurs ancêtres ou des personnes de leur bande (Albright 1997: 67). En documentant les affiliations familiales, les photographies d'ancêtres serviraient, entre autres, de liens entre les générations (Marr 1996 : 59; Troy 1992 : 56). En ce sens, la caméra deviendrait un outil de survie des histoires personnelles, familiales et communautaires, et les photographies assureraient la continuité du passé dans le présent (Semchuk, citée par Troy 1992 : 57).

Une photographie ne serait pas la reproduction d'une réalité sociale, mais d'une réalité intime. Le Navajo Monty Roessel aurait montré à une dame de sa communauté la photo d'une famille devant le drapeau américain, et celle-ci aurait commenté de la façon suivante: "Cette image, pour moi, ce n'est pas une image de cette famille, mais une image de ma tante ou de mon oncle avec leur bébé.»(Roessel 1996: 86, mes italiques) Pour certains autochtones, l'identité publique serait donc primordiale, et la quantité phénoménale de photographies sans identification ou autres renseignements pertinents rappellerait encore aujourd'hui le manque de respect des non autochtones qui, à la fin du $\mathrm{XIX}^{\mathrm{e}}$ et au début du $\mathrm{XX}^{\mathrm{e}}$ siècle, cherchaient à mettre en marché une indianité sans relief ni contraste (Marr 1996: 625). Cette pratique déshumanisait les gens (Brumbaugh 1996: 39). Si, dans l'imaginaire de plusieurs colons, voyageurs, missionnaires, anthropologues et agents gouvernementaux et muséaux, les « Indiens » semblaient tous pareils et se valaient, il n'en aurait pas été ainsi pour les autochtones. Ces derniers ne se seraient pas considérés comme une entité collective ou des artefacts de collection, mais comme des êtres humains interreliés.

Cette connexion entre les êtres humains serait possible en parlant de ce que l'on connaît et non de ce que l'on étudie (Francis 1992: 2). Même si les images candides des photographes crow Richard Throssel et kwakiutl George Hunt ont été possibles parce qu'ils photographiaient de l'intérieur (Albright 1997; Jacknis 19926), des non autochtones ont aussi produit des photographies illustrant l'intimité partagée entre eux et leurs sujets. Après avoir effectué plusieurs projets avec des communautés autochtones au Québec, le photographe Serge Jauvin a vécu un an avec la famille innue Mark en 1982. Grâce à son travail, plus de 25000 photos rappellent le quotidien de cette famille. La complicité entre Jauvin et ses sujets est visible dans sa production photographique (1993). D'une part, ses photos présentent des activités du quotidien effectuées autant par les femmes, les hommes que les enfants. Contrairement aux photos figées des photographes professionnels et amateurs non autochtones de la fin du XIX et du début du XX ${ }^{e}$ siècle, la plupart des gens photographiés par Jauvin continuent de vaquer à leurs occupations. Certains semblent heureux de lui sourire, de le regarder et d'être devant son objectif. Par ailleurs, nous voyons une famille qui utilise des outils contemporains dans la poursuite de son mode de vie que d'aucuns qualifieraient de traditionnel: des motoneiges tirent des traîneaux chargés de nourriture, des radios à ondes courtes servent à communiquer avec les gens restés au village, des scies mécaniques servent à couper le bois de chauffage pour les poêles des campements de toile. D'autres photos montrent, notamment, des jeunes jouant au baseball et d'autres accompagnant à vélo des adultes qui chassent. Ces photos, commentées par la famille Mark et des aînés innus, n'effacent pas les traces de modernité du mode de vie de cette famille autochtone afin qu'elle soit considérée comme plus « traditionnelle ${ }^{7}$. Cet exemple montre que ce ne serait pas l'appartenance locale qui favoriserait la connexion entre le photographe et ses sujets (James C. Faris, cité par Chalfen 1997; Troy 1992). Ce serait plutôt l'intention de la personne qui se tient derrière la caméra qui permettrait ou non d'entrer en relation avec l'autre (Worth et Adair 1997).

Le bio-documentaire, tel que conceptualisé par Sol Worth, est une approche intéressante pour aborder l'importance de l'aspect relationnel dans la production visuelle. Le chercheur en communication affirmait qu'à la différence d'un document réalisé par un anthropologue qui souhaite conserver, interpréter, commenter et documenter ce qu'il a observé et étudié, le bio-documentaire effectué par un non-professionnel présente comment celui-ci se sent dans le monde dans lequel il vit. Plutôt que d'être axé sur l'opérationnalité du document, le bio-documentaire accentuerait l'intention de la personne qui utilise la caméra, subjectivité qui se communiquerait au récepteur invité à faire contraster ses valeurs avec celles projetées par le réalisateur. Le bio-documentaire exprimerait la culture de son producteur, car ce type de document n'exige aucune conscience de l'art, ni des capacités techniques précises, ni paradigmes de pensée comme certaines formes d'art. Le plus important aspect du bio-documentaire serait son lien intime avec la cosmologie du moment du réalisateur (Worth et Adair 1997 : 25-26). Le documentaire «interprétatif » Hopiit du Hopi Victor Masayesva comporte une trame narrative informelle et des espaces-temps juxtaposés plutôt que présentés de façon linéaire: ce film serait donc un bio-documentaire (Leuthold 1998 : 118).

Cette perspective nous amène à considérer que l'une des caractéristiques des documents autochtones pourrait être leur nature personnelle, selon Masayesva (cité par Leuthold 1998 : 122, mes italiques) :

J'insiste sur l'accès à des histoires à propos des premières nations, par les premières nations. Je reconnais l'Amérique... aussi, mais il y a un autre point de vue. [...]

Mais nous en avons assez de cela. Nous avons d'abord une responsabilité envers nous-mêmes. Nous avons besoin de prendre soin de nous. [...]

[...] Nous voulons participer et développer une esthétique autochtone. Il existe une esthétique autochtone et elle débute dans le sacré.

Masayesva comparerait l'esthétique autochtone non pas à un discours truffé d'éléments formels d'expression (comme le veulent souvent, volontairement ou non, les non-autochtones à travers des normes documentaires), mais à une quête du spirituel interpersonnel communautaire (Leuthold 1998 : 122). Cette quête transcenderait les genres, les techniques et les normes dans un engagement envers la recherche du «sacré » dans la vie quotidienne. Le sacré ne serait pas une osmose parfaite, ni magique, du désir d'un individu et de la réalité empirique de celui-ci. Dans le cas de l'expression ou de l'art, j'avance que le sacré résiderait dans la relation (même imparfaite) avec le sujet présenté plutôt que dans la représentation (parfaite) du sujet « objectifié ». Le sacré dans l'acte photographique serait donc 


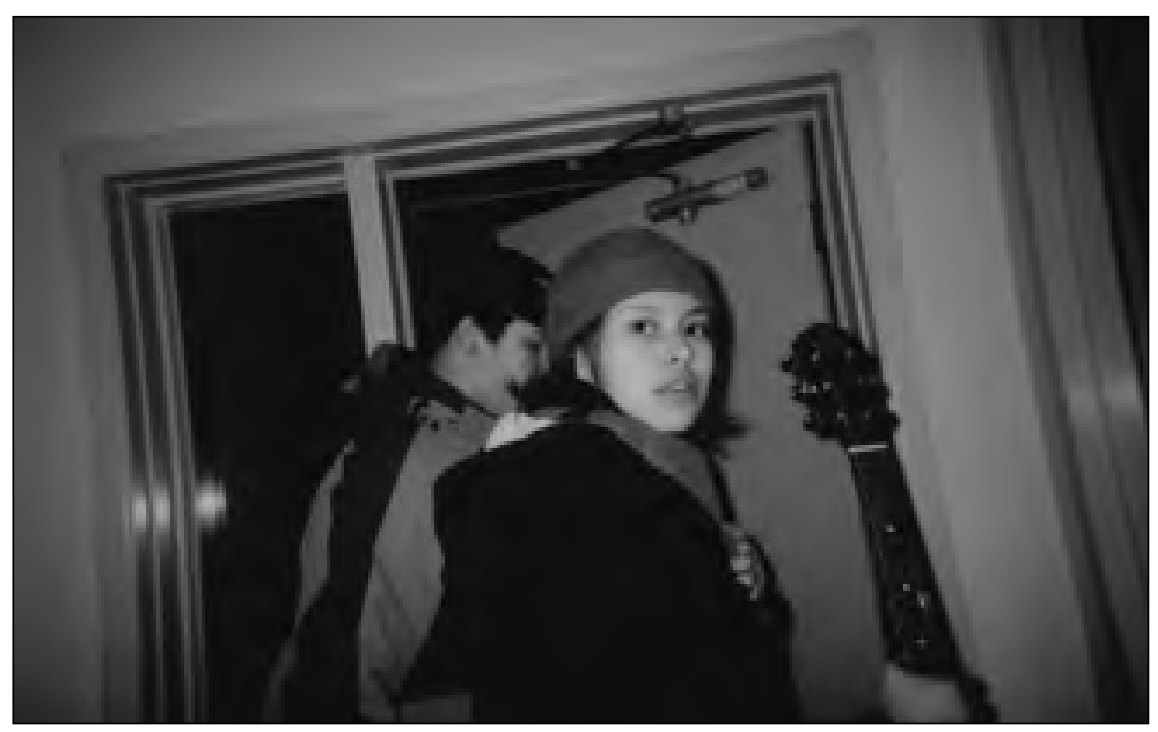

Photo 1

"C'est Christine et Shandy dans le cadre de porte. J'aime la guitare. J'aime jouer de la guitare. J'aimerais apprendre à en jouer toute seule. "

(Rosy Vollant, 13 ans, Mani-Utenam, 2004)

d'entrer en relation avec l'autre plutôt que de le prendre en photo. La photographie deviendrait ainsi un acte d'amour (Berger 2003 : 26) plutôt que de possession où la connexion avec l'autre efface notre ego (Francis $1992: 2$ ).

Nous venons de voir comment l'interprétation des différences à travers les prismes de sa propre lorgnette culturelle, délibérément ou non, entraîne des visions réductrices et ethnocentriques de nos sujets de recherche. À vouloir posséder un savoir, nous construirions de nouvelles images, miroirs des objets conceptualisés désirés. Nous serions alors en « transaction » plutôt qu'en « rela-

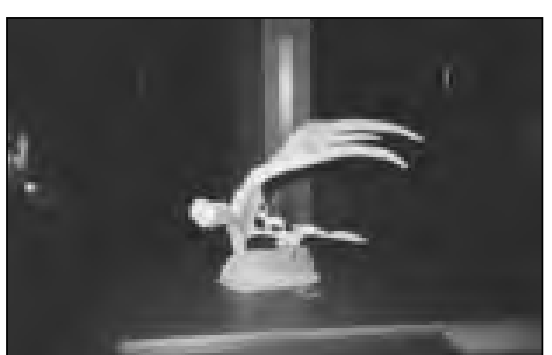

Photo 2

" J'ai pris cette photo-là parce qu'elle me rappelait le premier caribou que j'ai étripé avec mon père dans le bois cet hiver. "

(Shannon Vollant-Pinette, 13 ans,

Mani-Utenam, 2003)

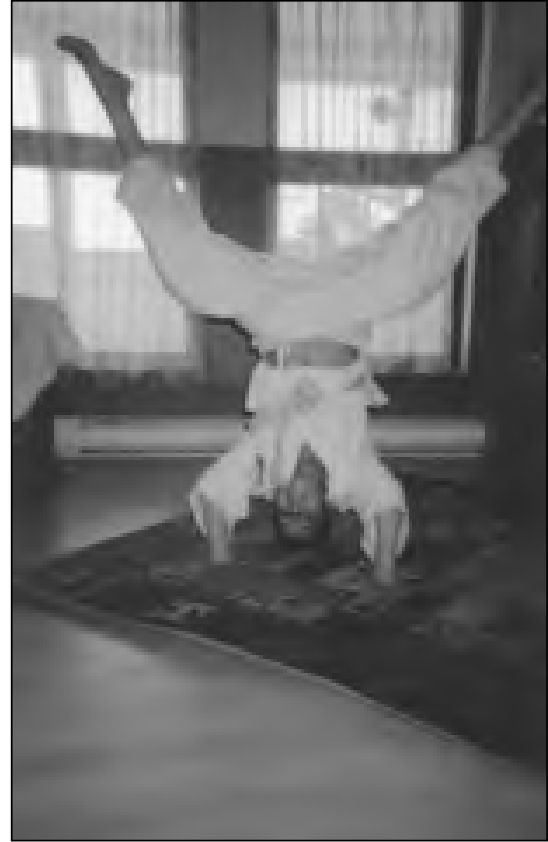

Photo 3

“ C'est Sylva en train de tenir le monde. " (Christopher Fontaine, 12 ans,

Mani-Utenam, 2004)

soixante jeunes âgés de 6 à 16 ans de la communauté de Uashat mak ManiUtenam, une communauté innue installée près de Sept-Îles sur la Côte-Nord, ont participé au projet «La photographie comme bâton de parole ». Dans le cadre de ce projet, des ateliers de photos commentées ont été organisés conjointement avec les jeunes et les adultes de cette communauté. Une vingtaine de participants ont collaboré à des articles de la page « Jeunesse », créée par le mensuel autochtone Innuvelle pour mettre en tion» (Martin Buber, cité par Asch 2005), reniant ainsi une « logique relationnelle » (Poirier 2004) voilée par les frontières de nos référents culturels. Nous avons aussi considéré que l'expression matérielle autochtone puisse s'affirmer comme révélatrice des liens sacrés du quotidien, systèmes relationnels autour desquels s'articulerait une esthétique autochtone. La prochaine section montre comment le passage de la photographie d'objet à système relationnel s'est articulé dans le quotidien de jeunes Innus de la communauté de Uashat mak Mani-Utenam, et pourra ainsi déclencher, je l'espère, une réflexion des répercussions possibles sur les identités contemporaines de la jeunesse autochtone par la surabondante utilisation actuelle d'images de souffrance et de misérabilisme, fréquemment générées par certains adultes, au demeurant souvent bien intentionnés.

\section{« LA PHOTOGRAPHIE COMME BÂTON DE PAROLE " : DE L'EXPRESSION PRIVÉE À LA PRODUCTION ET MOBILISATION PUBLIQUES}

Entre juillet 2002 et juin 2004, pendant ma recherche de maîtrise en anthropologie à l'université Concordia, plus de valeur les jeunes de ce projet. Une trentaine de jeunes ont accepté de participer à une exposition présentée au musée Shaputuan et au festival Présence autochtone en juin 2004, de même qu'au festival Innu Nikamu de Mani-Utenam en août 2004 et dans les écoles primaires et l'école secondaire de la communauté en novembre et décembre 2004 (Innuvelle 2004, La Presse 2004). Les autres, une dizaine, ont jugé leurs photos « trop personnelles » pour en parler avec moi ou ont signifié avoir perdu l'appareil photo remis pour le projet ${ }^{8}$. Une dizaine de participants ont voulu utiliser plus d'un rouleau de films.

La moitié des participants a été recrutée dans des lieux officiels tels que les maisons de jeunes et les foyers de groupes pour enfants et adolescents. Lautre moitié m'a abordée lors de mes déplacements dans la communauté. Les participants ont reçu la consigne de photographier, avec un appareil jetable de 27 poses, ce qu'ils aimaient ou aimaient moins de leur vie et de leur communauté. Ils m'ont ensuite expliqué la signification de leurs photos, moments que j'ai nommé «partages», car ce mot respecte l'esprit du langage entendu dans la communauté lors de mes huit visites, variant entre deux semaines et deux mois, nécessaires à l'achèvement du projet. J'ai concentré mon 


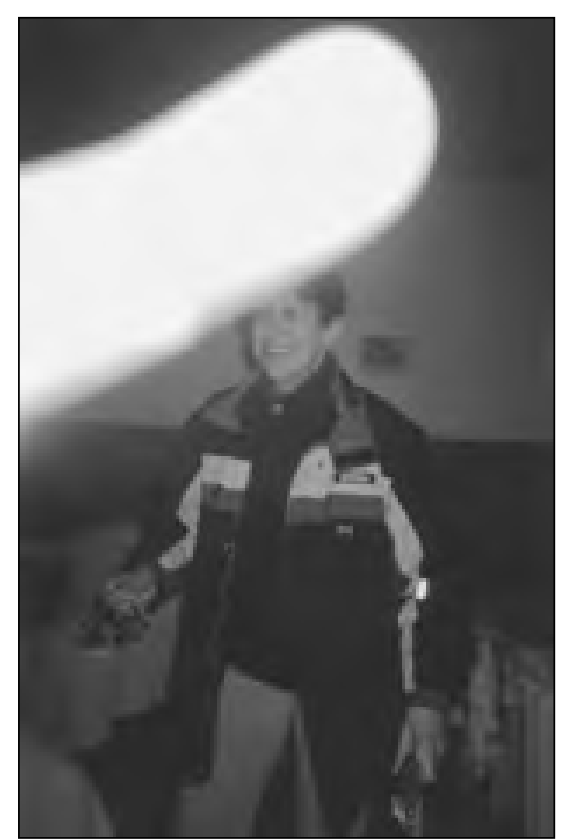

\section{Photo 4}

“C'est Lise. " (Jean-Philippe Pinette, 10 ans, Mani-Utenam, 2004)

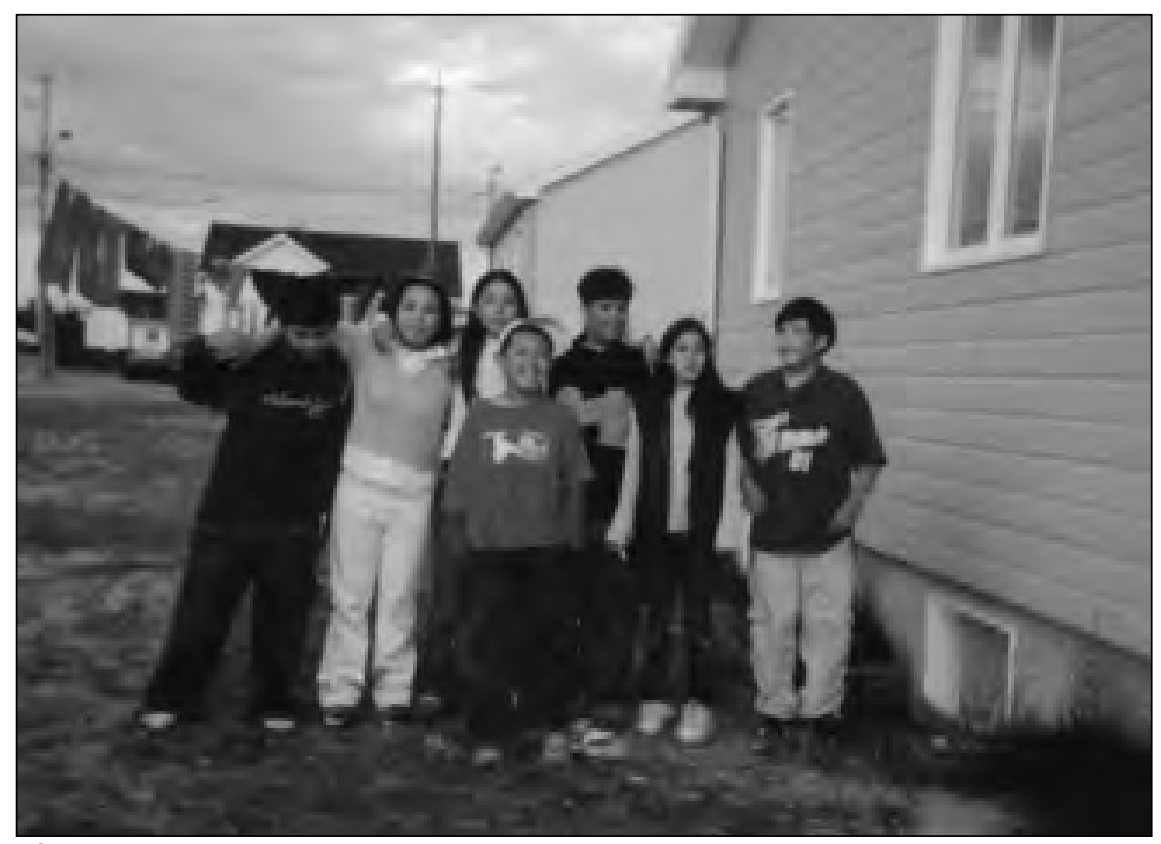

\section{Photo 6}

“ Eux autres, c'est toute ma famille. On est toujours ensemble. On s'aime. "

(Rocko Vollant-Pilot, 10 ans, Mani-Utenam, 2003)

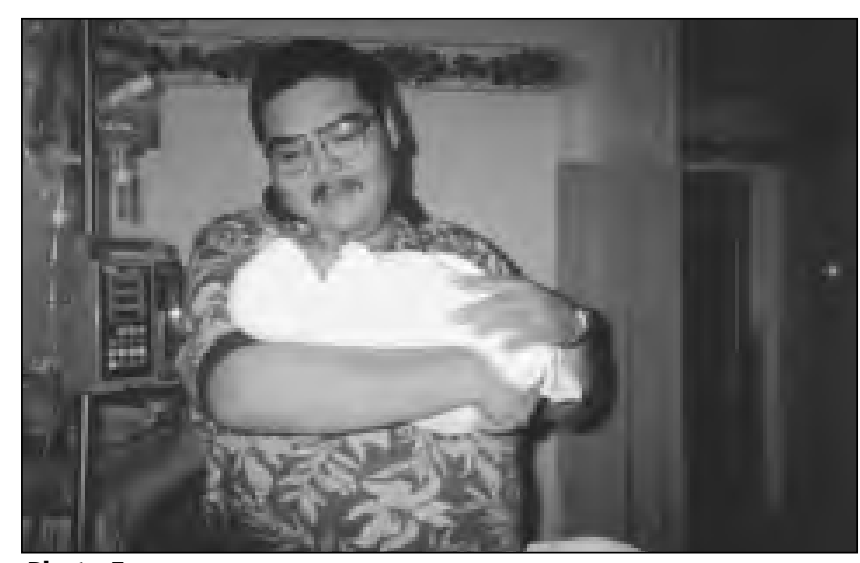

Photo 5

“ J'aime mon père et ma petite sœur. J'aime que mon père prenne soin de ma petite sœur. "

(Myranda Vollant, 9 ans, Mani-Utenam, 2003)

analyse de ce projet aux trente et un participants qui ont accepté de présenter leurs visions à l'exposition itinérante «Eshi uapatamat eshi mishkutshipanit anite nitinniniunnat/Notre vision du changement dans notre mode de vie/Our vision of change in our new lifestyle », appartenant maintenant au musée Shaputuan de la communauté de Uashat mak Mani-Utenam. Dans la mesure du possible, l'équilibre a été respecté entre les genres et la provenance des deux communautés: 15 filles et 16 garçons ont participé à l'exposition, 14 participants provenaient de Uashat (six filles et huit garçons), et 17 de ManiUtenam (neuf filles et huit garçons).

J'ai souligné ailleurs, et j'y reviendrai avant de conclure, que sans nier les réalités plus difficiles de leur quotidien, les participants de ce projet ont majoritairement préféré capter et commenter des images positives de leur vie - la famille, les amis, les jeux, les fiertés, les personnes admirées, les institutions

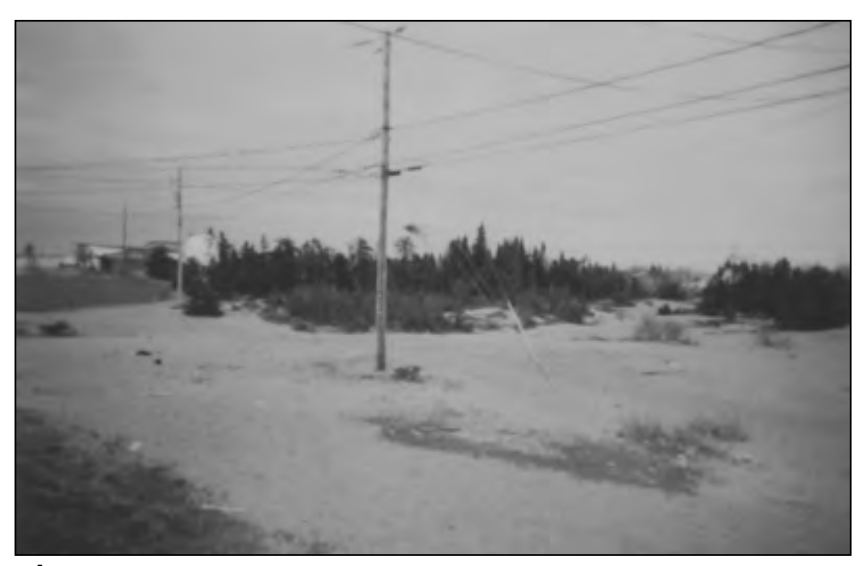

Photo 7

" La forêt. J'aime ça quand je me promène dans la forêt. J'aime ça quand je regarde la forêt. "

(Marie-Soleil Joseph, 8 ans, Uashat, 2004)

respectées et événements appréciés, contrairement à ce que pourraient laisser présager les réalités misérabilistes et victimisantes souvent dépeintes par plusieurs journalistes des médias de masse ou des scientifiques de revues savantes, ou encore des leaders autochtones et Euroquébécois dans leurs discours (Truchon 2005a, 2005b). Le deuxième constat émergeant de ce projet nous renvoie à la perception de leur vie, entretenue par les participants en regard de leurs relations quotidiennes avec leurs pairs et des contacts intergénérationnels et interculturels à travers des personnes, des objets, des institutions et des événements.

\section{DES BIO-DOCUMENTAIRES RÉVÉLATEURS DE LIENS IMPORTANTS POUR LES JEUNES PARTICIPANTS INNUS}

Lintention première de ce projet de photos commentées était que chaque participant présente des parcelles de sa vie 
à un moment précis de son histoire. Que des histoires d'autochtones soient racontées par des autochtones, selon le souhait de Victor Masayesva (cité dans Leuthold 1998: 122), car très peu de ces narrations sont disponibles dans l'espace public. En ce sens, les participants ont réalisé des bio-documentaires (Worth et Adair 1997: 25-26) sans trames narratives précises, outre la consigne de photographier ce qu'ils aimaient et aimaient moins de leur communauté. Leurs réalisations sont des illustrations personnelles et temporelles reflétant comment ils se sentaient dans leur vie aux moments de la prise de photos et du partage des significations de ces dernières.

Bien qu'ils puissent interpréter avec un cadre sémiologique (Tomaselli 1996) ou analytique contextuel (Collier 2001), les anthropologues referont-ils jaillir en surface le punctum, le détail inconscient qui remonte à la conscience de la personne qui a créé cette photo (Barthes 1980; Garrigues 2000)? Par exemple, Rosy Vollant ${ }^{9}$ a sélectionné une photo qui, selon moi, représentait ses deux amis, surpris au moment de sortir de leur domicile. Pour Rosy, cette photo montre une guitare, un instrument de musique qu'elle voudrait « apprendre à jouer toute seule» (photo 1). Shannon VollantPinette explique que la sculpture des bois du caribou lui rappelle le premier caribou qu'il a étripé avec son père, et Christopher Fontaine a photographié son ami Sylva qui « tient le monde entre ses mains» (photo 2 et photo 3 ). Ces exemples montrent que l'acte d'attribution du sens est ambigu et qu'une photo peut se prêter à plusieurs interprétations (Hall 1997 : 228).

Lors des partages des participants, l'aspect technique des photos était rarement évoqué. Quand il l'a été, c'était pour reconnaître une compétence inconnue « J'suis bon! C'est vrai hein Madame?» (Shannon Vollant-Pinette, comm. pers. 2003) Ou encore pour affirmer que malgré le non-respect des normes visuelles généralement acceptées socialement, les photos étaient néanmoins valables (photo 4). Le choix de Jean-Philippe de présenter une photo qui ne semble pas réussie techniquement n'est pas unique. Si, au premier regard sur les photos des participants, les défauts techniques de plusieurs d'entre elles ou un manque d'intérêt apparent m'ont laissée perplexe, ces notions réductrices se sont évaporées quand, en plus d'écouter, j'ai aussi entendu les partages des participants. Il est relativement facile d'écouter ce que l'on veut recevoir ou d'orienter une conversation en ce sens, comme l'ont reconnu Worth et Adair (1972). Le fait d'entendre ce qui est partagé du point de vue de la personne qui s'exprime est autrement plus subtil (Roberts 2000) et demande de

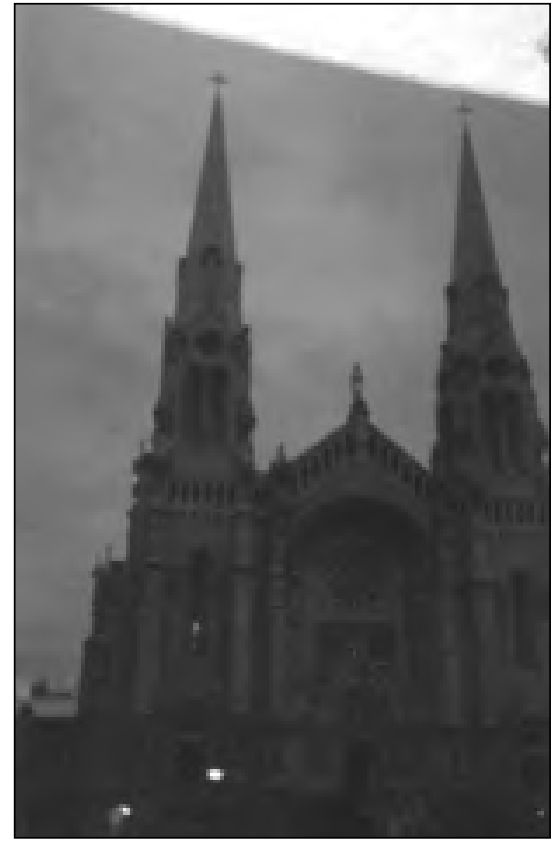

Photo 10

" La photo que j'ai eu beaucoup de difficulté à prendre et qui me tient à cœur. " (Myriam Vollant, 14 ans, Uashat, 2003)

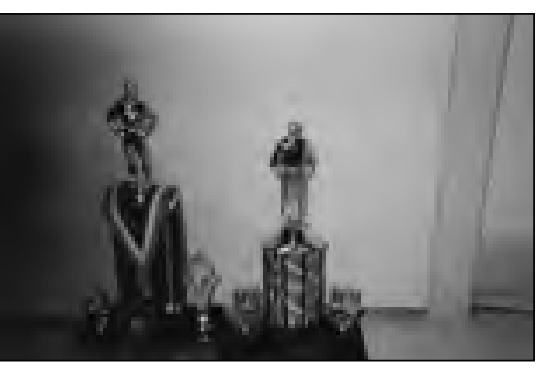

Photo 11

" J'aime les trophées. Je suis ceinture orange en judo. J'ai eu une médaille dans une compétition à Baie-Comeau. J'ai été 'judoka du mois' en février 2003. ”

(Jean-Yves Gabriel, 14 ans,Uashat, 2003) ce sport et j'en fais souvent. s'engager dans une logique relationnelle (Poirier 2004). Ce mode plus relationnel que transactionnel (Martin Buber, cité par Asch 2005) m'a permis de réaliser que ce qui importait pour ces jeunes était ce qui figurait sur les photos, ou plutôt le lien qui les unit, comme individus-photographes, avec ce qu'ils ont représenté. Cette interconnexion, ce lien interrelationnel, est du même ordre que les propos susmentionnés de la dame navajo qui affirmait que, sur la photo qui lui était présentée, ce n'était pas une famille, mais son oncle, sa tante et leur bébé (Roessel 1996 : 86). Ce constat de réalité intime plutôt que de représentation sociale orientée vers la perception des autres s'est principalement actualisé par la prise de photos qui montrent les liens avec la famille (photo 5), les amis (photo 6), la 


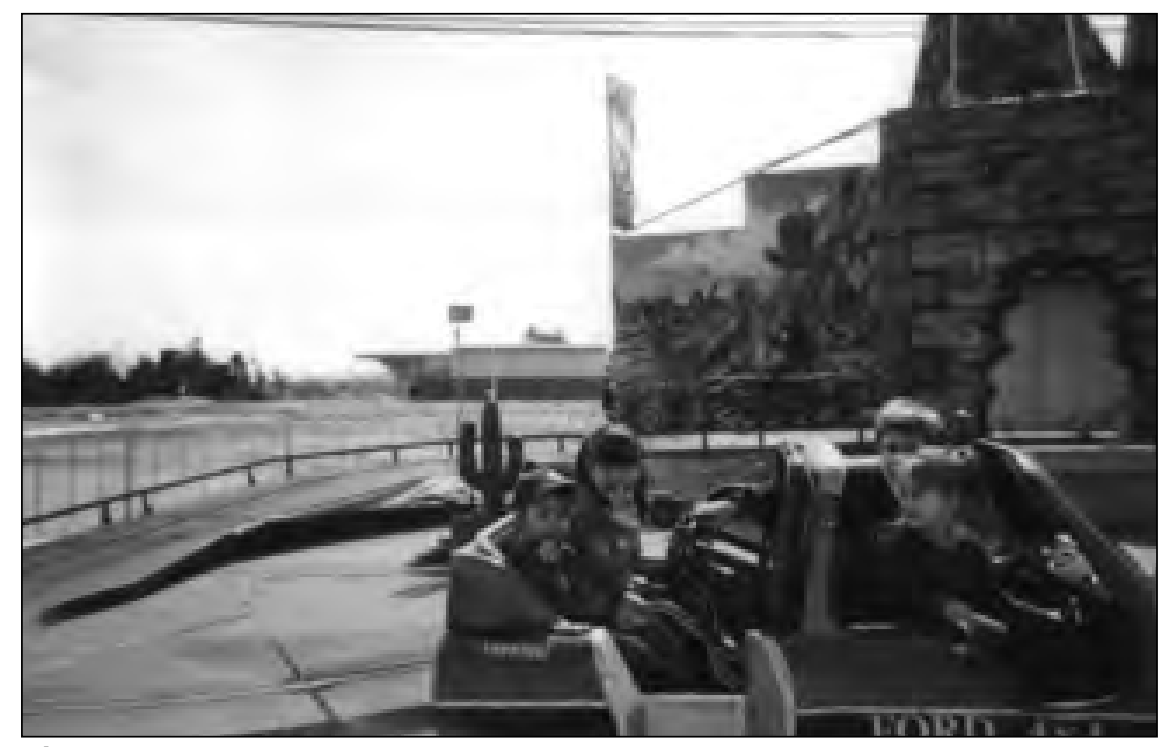

Photo 12

" C'est moi et mon p'tit frère, Jack. On voulait le siège en avant, mais il y avait déjà des personnes. C'est ma mère qui a pris la photo au carnaval. "

(Éloise Fontaine, 6 ans, Uashat, 2003)

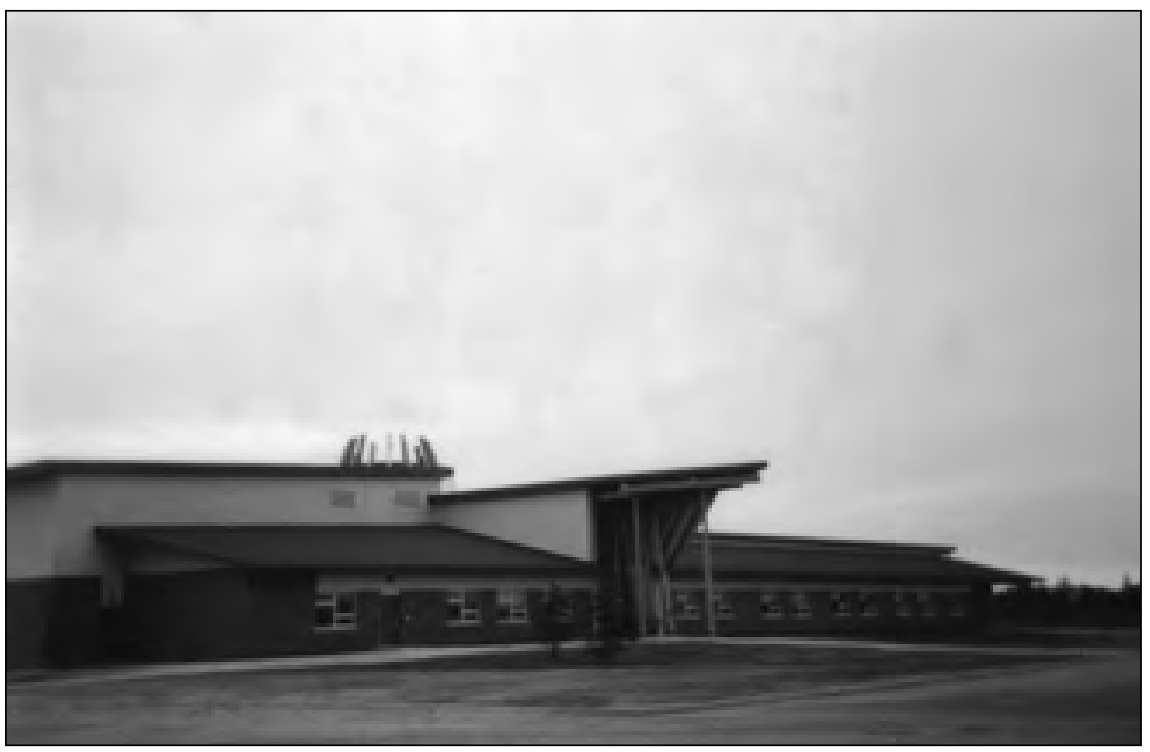

Photo 13

" L'école de mon p'tit frère. II aime ça y aller. "

(Éric Jean-Pierre, 16 ans, Uashat, 2003)

nature (photo 7), les adultes significatifs de la communauté et de l'extérieur (photo 8), les jeux (photo 9), les fiertés (photos 10-11), les institutions respectées et les événements appréciés (photos 12-13).

Les trames narratives des photos, telles qu'exprimées par les jeunes Innus qui ont participé au projet, deviennent des outils pour la survie des histoires personnelles, communautaires et familiales (Semchuk, cité par Troy 1992: 57) en permettant l'esquisse de portraits de personnes de confiance et la connaissance des ingrédients qui composent certaines valeurs. Par exemple, un parent aimé s'occupe de ses enfants (Myranda Vollant, 9 ans, comm. pers. 2003) ou prend soin de son enfant en lui achetant des vêtements, tout en faisant l'impossible pour combler ses besoins élémentaires (Rocko Vollant-Pilot, 10 ans, comm. pers. 2003). Un parent respecté essaie de changer des habitudes de vie qui compromettent le bien-être de ses enfants (Lana Vollant Pinette, 13 ans, comm. pers. 2003). Si les parents doivent nécessairement procurer de l'amour et des soins de base à leurs enfants, les amis et les adultes significatifs seraient plutôt, selon les participants, des accompagnateurs dans leur cheminement.

Quant à l'ami, c'est quelqu'un qui nous remonte le moral (Myriam Vollant, 14 ans, comm. pers. 2003), qui garde nos secrets (Caroline Vollant, 13 ans, comm. pers. 2003), qui ne chiale pas (Marie-Ève Ambroise, 13 ans, comm. pers. 2003) et qui, par le lien de confiance établi, devient un membre de la famille (Rocko VollantPilot, 10 ans, comm. pers. 2003). S'il s'agit de professionnels travaillant avec les jeunes, une caissière de dépanneur qui se transforme en compagne de jeu est encouragée (Janice Régis-Michel, 12 ans, comm. pers. 2003); une éducatrice appréciée est une personne souriante et gentille (Jean-Louis Andrew, 16 ans, comm. pers. 2004); un entraîneur estimé encourage à persévérer (Alexanne Grégoire, 13 ans, comm. pers. 2003); un professeur influent diversifie ses activités d'apprentissage pour maintenir l'intérêt de ses élèves (Étienne Andrew, 13 ans, comm. pers. 2003); et une anthropologue valorisée "montre de nouvelles choses aux enfants » (Rocko Vollant-Pilot, 10 ans, comm. pers. 2003) et fait confiance à ces derniers en leur passant son appareil photo «qui vaut cher » (Caroline Vollant, 13 ans, comm. pers. 2003).

Les commentaires accompagnant les photos soulignaient également des valeurs importantes. Notons le courage, que Justine Hervieux a personnifié sous les traits de Dark Angel, une fille robot qui « est forte » et qui « ne fuit pas ses problèmes » (comm. pers. 2003) (photo 14). J'en tire la conclusion que les qualités ou valeurs énumérées ne sont pas l'apanage exclusif d'une seule catégorie de lien parent, ami ou adulte significatif - mais plutôt un assemblage nécessaire au mieux-être des enfants qui ont participé au projet. En ce sens, les participants semblent indiquer qu'ils veulent des adultes aptes à prendre soin d'eux et de leurs besoins élémentaires, tout en étant doux, encourageants et stimulants; bref ils recherchent, et plusieurs se rapprochent, des personnes engagées à les faire grandir sainement. À maintes occasions, j’ai entendu des jeunes exprimer à quel point ils détestaient se « faire chialer» parce qu'ils ne faisaient pas ce que les autres leur demandaient. Je déduis donc qu'ils recherchent le respect 


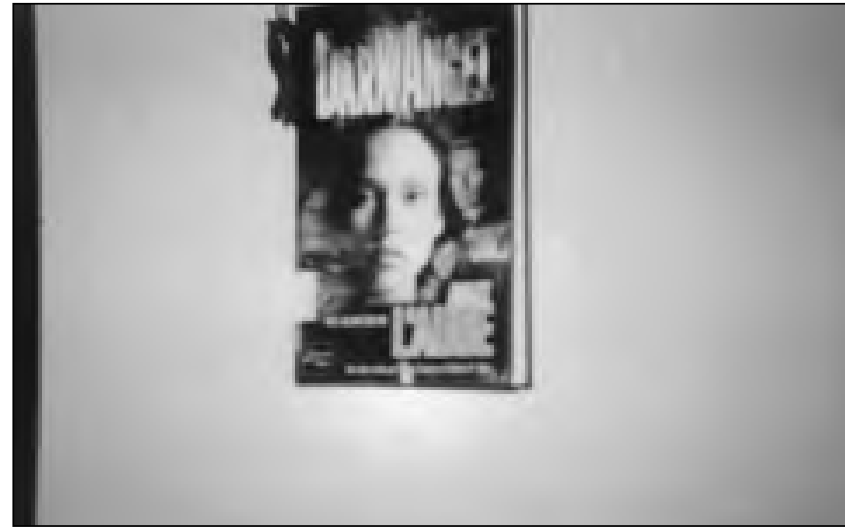

Photo 14

« Ça, c'est parce que j'aime lire. C'est Dark Angel, l'ange noire. J'ai d'autres livres de ça. C'est l'histoire d'une fille-robot qui a en arrière de sa nuque un numéro de série. Elle est forte, la fille. Elle se bat comme un gars. Elle s'est sauvée d'un endroit à 14 ou 15 ans, elle était jeune. Elle ne fuit pas ses problèmes et elle s'organise pour les régler elle-même. "

(Justine Hervieux, 15 ans, Uashat, 2003)

de leur intégrité, tout en étant guidés et accompagnés dans leur exploration des multiples facettes de la vie.

Les aspects plus positifs du quotidien des communautés autochtones, tels les éléments présentés par les jeunes de ce projet, sont rarement offerts en contrepartie aux reportages, articles et discours axés sur les problématiques en milieux autochtones. La prochaine section aborde les impacts d'une stratégie exclusivement axée sur les revendications de droits humains pour les autochtones sans égard aux aspects plus positifs de leur vie.

\section{REMISE EN QUESTION DE LA REPRÉSENTATION PUBLIQUE DU MISÉRABILISME}

Tels les «photographes de l'intérieur » qu'ont été Richard Throssel et George Hunt au début du Xxe siècle, les participants de ce projet de photos ont documenté leur quotidien, très différent de celui qu'une majorité d'images misérabilistes véhiculent publiquement dans plusieurs médias de masse, revues savantes et discours de leaders autochtones et nonautochtones. Le passage des images des jeunes Innus du privé (projet de photos avec une étudiante en anthropologie) au public (exposition itinérante et articles de journaux) permet de douter qu'un seul type de représentation, celui d'images de souffrance, puisse rendre compte des réalités des jeunes autochtones contemporains. Certes, les participants de ce projet de photos et des productions matérielles en résultant (expositions et articles de journaux) ont mentionné certains aspects plus négatifs de leur vie, tels l'alcool, la violence, la mort ou la pollution (photo 15). Néanmoins, la majorité des images commentées donnent à penser que leur quotidien leur apporte de beaux moments, pourtant notoirement absents de l'espace public (photo 16). John Berger (cité dans Williams 2003: 30) suggère que les photographies prises pour une utilisation privée sont moins « aliénées » de leur contexte de production et de réception que celles prises dans un but commercial ou public. Les photos commerciales n'auraient rien à voir avec les récepteurs. Ces photos seraient de l'information, mais une information déconnectée de la signification originale de l'expérience (ibid).

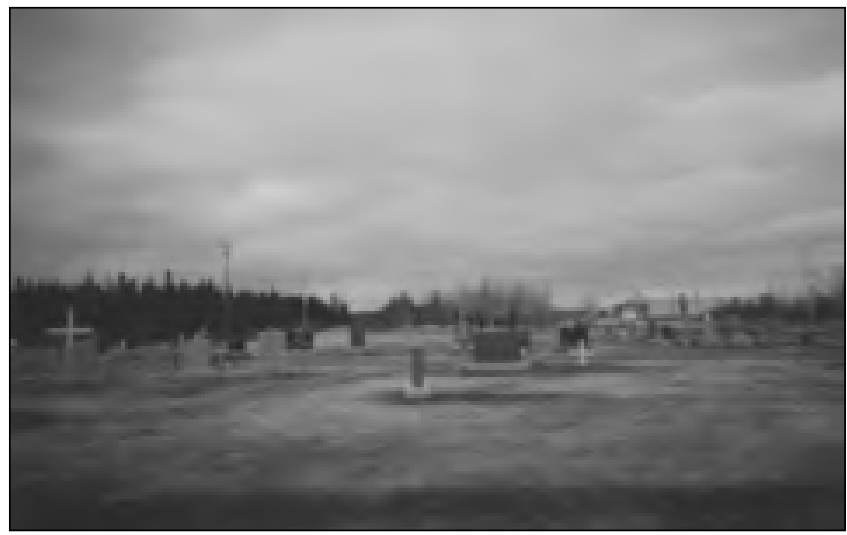

Photo 15

« Mon professeur était malade. J'ai eu de la tristesse pendant son enterrement. Elle s'appelait Thérèse Ambroise. Elle était l'enseignante de maternelle à Johnny Pilot. " (Guylaine Joseph, 6 ans, Uashat, 2003)

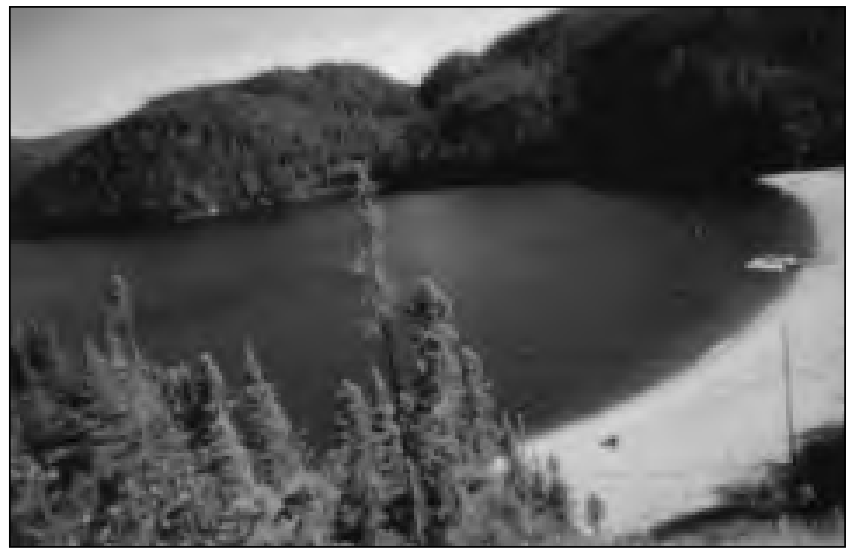

Photo 16

" J'ai pris cette photo de la fenêtre de la cafétéria. Je trouve que c'est beau. On voit la piscine, à droite en haut, avec les câbles. On allait de là jusqu'à la balloune rouge à la nage. Un jour, j'étais sur le bord de l'eau et je me suis dit : 'C'est quoi ça? Des panaches!' On pouvait voir un poisson dans la bouche de I'orignal. J'avais oublié mon appareil. "

(Samuel Jourdain, 13 ans, Uashat, 2003)

Quoi qu'il en soit, dans le cadre du projet « La photographie comme bâton de parole », ce passage du privé au public met en valeur des participants qui ont fait d'abord le choix de montrer publiquement leurs visions et, ensuite, la sélection des photographies qu'ils voulaient montrer tout en expliquant leurs significations pour eux à cette période. Dans ce contexte d'auto-(re)présentation de jeunes Innus, je suggère que ce passage du privé au public a contribué à équilibrer les représentations contemporaines à leur sujet, qui trop souvent les victimisent, en ajoutant à ce répertoire des images de loisirs, de fiertés, d'amitiés et d'amours, aspects qui font partie de la vie des jeunes Innus rencontrés pendant ce projet.

Je pose donc comme hypothèse que des plaisirs similaires doivent aussi composer les réalités de jeunes autochtones d'autres communautés et que ces réalités sont occultées, comme je viens de l'exposer, par les regards que leur jettent certains adultes. C'est comme si cette souffrance pouvait devenir une expérience « utilisée comme un produit, une marchandise, 
et qu'à travers cette représentation culturelle de la souffrance, [cette] expérience est re-fabriquée, allégée et déformée » (Kleinman 1997 : 2).

Loin de moi l'idée de nier les difficultés vécues en milieux autochtones ou de faire preuve d'une complaisance s'apparentant à du néocolonialisme. Mon propos cherche plus à mettre en garde contre la surabondance d'images de misérabilisme et à remettre en question les impacts de cette stratégie et les motivations sous-jacentes de certains fabricants de ces images et discours. Tel que l'illustre la métaphore du Kwikwilyaqa, sortir de la connexion peut faire ombrage à ceux dont nous pensons, ou disons vouloir, améliorer le sort.

\section{Conclusion}

Les photos de petits frères et de petites sœurs, d'amis, de parents, de personnes appréciées, de jeux, de fiertés, de difficultés, etc. qu'ont présentées les participants de ce projet seraient avant tout des preuves de connexion entre celui ou celle qui est derrière la caméra et ceux qui sont devant. À l'instar des Navajos pour qui marcher serait un acte mythique en soi, et non un simple moyen de se rendre à destination (Worth et Adair 1997: 146), photographier, pour les jeunes Innus de la communauté de Uashat mak Mani-Utenam de ce projet, pourrait être envisagé comme un acte d'engagement dans la recherche du sacré grâce aux liens qu'ils entretiennent dans leur vie quotidienne. Cet acte photographique évacuerait tout critère basé sur la technique, les genres et les normes (Victor Masayesava, cité dans Leuthold 1998 : 122). Ainsi, en cette ère dite post-moderne où toute vérité serait fragmentée (Clifford 1986), ce projet de photos commentées laisse percevoir que «le fragment de vérité » d'une photo ne serait pas ce qu'elle représente, mais les liens qu'elle présente entre le producteur, le sujet et le récepteur (Lippard 1992).

En faisant basculer la photo d'« objet » à « relation », nous pouvons entrevoir que certains regards d'adultes posés sur les jeunes autochtones révéleraient peut-être plus les intentions disciplinaires de ces derniers que la totalité des réalités quotidiennes de leur objet d'études. Si les photos des jeunes Innus de ce projet montrent des environnements qui pourraient susciter de la part d'étrangers des questions sur les conditions dans lesquelles ils vivent, jamais ces jeunes n'en n'ont-ils fait mention ou n'ont semblé mal à l'aise de cette situation. Ils me semblaient plutôt concentrés sur ce qui les reliait au sujet de la photo, à ce moment précis. En perpétuant surtout des images négatives pour faire avancer les choses dans les communautés autochtones et parce que ces images limitent les attentes ainsi que les réalités fluctuantes des autochtones, se pourrait-il que des décideurs, chercheurs, professionnels et journalistes favorisent, sans en être conscients, l'instauration « d'une prophétie autoréalisatrice » (Cheshire et Kawamoto 2003: 83)?

Dans ce contexte, je crois que l'intégration des visions des jeunes eux-mêmes qui, en présentant les liens qu'ils affectionnent, réfutent dans ce cas-ci l'assertion voulant que leur quotidien ne soit que misères, permettrait d'enrayer la cassure que la rencontre, anthropologique ou quotidienne, peut occasionner : celle du «Nous » et de l'« Eux » (Troy 1992). Ma recherche avec de jeunes Innus de la communauté de Uashat Mak ManiUtenam m'amène à estimer que ce n'est pas la tentative des adultes de représenter les jeunes autochtones qui est problématique, mais plutôt les regards largement «victimisants » qu'ils leur jettent et les intentions qui sous-tendent ces regards, et ce, sans chercher à intégrer ou à élargir les interprétations de ceux qui sont au cour des débats.

Les Navajos nomment l'être humain bila' 'ashdla' li, 'la personne aux cinq doigts'. Pour refléter une personne adéquatement, les photographies doivent montrer ses multiples facettes, toute la profondeur de cet être humain, son entièreté (Roessel 1996: 89). Mais ce postulat ne signifie pas la connaissance absolue de l'Autre. Il suggère la reconnaissance que l'Autre est aussi un être humain, avec qui nous entrons en relation, et non pas un objet imaginé. Ainsi, lors des partages avec les participants ceux-ci m'ont transmis que « ceci n'est pas une photo. Ceci est mon père... ma petite sour... mon professeur... mon bicyk...». Les jeunes Innus nous rappellent donc qu'une photo n'est pas qu'une photo, ou la représentation d'une photo (Foucault 1973). Ce morceau de papier ne prend vie que parce qu'il présente le fruit des représentations entre le producteur, son sujet et le récepteur grâce à leurs relations et interactions, aussi imparfaites soient-elles.

\section{Notes}

1. La « représentation » ici s'inspire de la définition proposée par Hall (1996). Selon ce dernier, le préfixe « re » serait problématique, car il annoncerait une remise en spectacle d'un événement déjà survenu. Je soulève donc ce point en écrivant ce concept «(re)présentation ». Cet article aborde ainsi, indirectement, la relation entre la mise en scène et l'action spontanée à un moment précis. Comme initiatrice de cette auto-(re)présentation, j'ai aussi été la médiatrice, créant des impacts difficilement quantifiables et qualifiables sur les résultats (Truchon 2005a). Cet aspect mériterait d'être éventuellement mieux compris.

2. Historiquement, les Innus de Uashat montaient en canot à leurs territoires par la rivière Sainte-Marguerite, et ceux de ManiUtenam, par la Moisie. La réserve de Uashat a été créée en 1925 et celle de Mani-Utenam en 1949. À la demande du gouvernement provincial, les autorités de la ville de Sept-îles ont tenté de relocaliser les Innus de Uashat à Mani-Utenam afin que le gouvernement du Québec puisse faire des représentations auprès des instances gouvernementales fédérales chargées du dossier des autochtones. Néanmoins, la plupart des Innus de Uashat ont résisté.

3. En 2004, une Innue scolarisée que je connais affichait chez elle une photo prise par le controversé Edward Curtis, tout comme le laboratoire d'informatique des étudiants de premier cycle de sociologie et d'anthropologie de l'université Concordia.

4. Bien qu'elle évoque les autochtones, cette citation s'applique, selon moi, à tous les photographes, sans distinction ethnique. Tous peuvent être connectés à leur sujet. Les conceptions de la vie des autochtones ne sont pas nécessairement appliquées par les autochtones, et vice versa pour les caractéristiques associées aux non-autochtones. Loin de vouloir encastrer la condition autochtone dans une perspective "nouvel âge», ou encore diaboliser les non-autochtones, je cherche à proposer une piste de compréhension des fondements relationnels tels qu'ils sont explicités par les différents auteurs, autochtones ou non.

5. Par exemple, l'anthropologue Alfred Kroeber n'écrivait pas les noms des autochtones même s'il les savait (Jacknis 1996 : 25), contrastant ainsi avec la pratique du Kwakiutl George Hunt qui notait tout en anglais, en incluant des termes kwakiutl (Jacknis 1992: 147). Nous pourrions nous demander si la conservation de l'anonymat qui caractérise fréquemment la pratique anthropologique d'hier à aujourd'hui contreviendrait, dans certains cas, à la vision qu'auraient certains autochtones de leurs propriétés identitaires.

6. Plusieurs aspects des photographies personnelles de Richard Throssel et de George Hunt diffèrent de leurs corpus professionnel et commercial (Albright 1997; Jacknis 1992). Les 
photos prises par Throssel et Hunt pour leur plaisir montrent principalement des scènes du quotidien de leur communauté ainsi que des cérémonies et rituels. Si, dans la sphère publique, Richard Throssel est surtout connu pour ses trente-neuf photos de la série Western Classics From the Land of the Indian (soit $4 \%$ de sa production totale, une série destinée à un public d'acheteurs blancs, friands des clichés reproduits), George Hunt a effectué des photographies anthropométriques alors qu'il assistait Franz Boas.

7. Nombre de photographes non autochtones ont utilisé la recherche de la « tradition » comme moyen visuel pour camper les autochtones dans un passé composé les excluant de toute participation contemporaine civique (Maxwell 1999).

8. Le financement de la réalisation des photos a été possible grâce à une petite subvention du Secrétariat aux affaires autochtones. La production des articles dans Innuvelle a été financée par le journal, et l'exposition a nécessité la participation financière du musée Shaputuan, du Centre de santé et des services sociaux de la communauté de Uashat mak Mani-Utenam, de la radio CKAU et du conseil de bande (Innu Takuaikan Uashat mak Mani-Utenam). La traduction des textes en innu a été défrayée par l'Institut culturel et éducatif montagnais (ICEM).

9. Pour contrer l'anonymat et la déshumanisation des photos du passé (voir Brumbaugh 1996 : 39; Marr 1996 : 62), les noms des participants, avec leur consentement, apparaissent dans cet article.

\section{Remerciements}

Je remercie Laurent Jérôme, pour son invitation à participer à ce numéro thématique, ainsi que les évaluateurs anonymes et Michel Lavoie, codirecteur de la revue, pour leurs commentaires pertinents. Je tiens aussi à remercier Éric Chalifoux de Recherches amérindiennes au Québec pour avoir facilité la coordination de ce premier article scientifique. Je remercie également Michelle Blaquière et Marcelle Roy pour la révision linguistique de cet article. De plus, sans les personnes suivantes, cet article, tiré, de mon mémoire de maîtrise, ne serait pas le même : Christine Jourdan, Bernard Roy et Bob White ont été des membres de comité de mémoire des plus attentionnés; Lyse Mérineau m'a souligné que j'utilisais la photographie comme un bâton de parole et Alexandrine BoudreaultFournier m'a fait comprendre que mon projet de maîtrise permettait aux participants innus de passer du privé au public. Finalement, sans la collaboration immédiate des personnes suivantes de la communauté de Uashat mak Mani-Utenam, ce projet n'aurait pu prendre forme : Jean-Marie Caron, directeur du Centre de santé et des services sociaux de la communauté, et Suzanne Régis, rédactrice en chef d'Innuvelle. Outre la soixantaine de participants, plus de soixante-quinze personnes, autochtones et non autochtones, se sont ajoutées à eux en cours de route, mais les nommer ici serait trop long.

\section{Ouvrages cités}

ALBRIGHT, Peggy, 1997: Crow Indian Photographer. The Work of Richard Throssel. University of New Mexico Press, New Mexico.

ASCH, Michael, 2005: "Towards a Relational Anthropology». Communication présentée lors de la conférence annuelle de la Société canadienne d'anthropologie (CASCA), du 3 au 8 mai 2005, Merida, Mexique.

BARTHES, Roland, 1980: La Chambre claire: note sur la photographie. Cahiers du cinéma, Paris.

BERGER, John, 2003: "Introduction», in David Lee Strauss, Between the eyes: essays on photography and politics: vii-xv. Aperture, New York.

BRUMBAUGH, Lee Philip, 1996: « Shadow Catcher or Shadow Snatchers? Ethical Issues for Photographers of Contemporary Native Americans». American Indian Culture and Research Journal 20(3) : 33-49.
CHALFEN, Richard, 1997 : «Afterword to the Revised Edition », in Sol Worth et John Adair (dir.), Through Navajo Eyes. An Exploration in Film Communication and Anthropology: 275-341. University of New Mexico Press, Albuquerque.

CHESHIRE, Tamara C., et Walter T. KAWAMOTO, 2003 : « Positive Youth Development in Urban American Indian Adolescents », in Francisco A. Villaruel et al. (dir.), Community Youth Development. Programs, Policies, and Practices: 79-89. Sage Publications, Thousand Oaks, California.

CLIFFORD, James, 1986 : « Partial Truths », in James CLIFFORD et George E. MARCUS (dir.), Writing Culture. The Poetics and Politics of Ethnography: 1-26. University of California Press, Berkeley.

COLLIER, Malcolm, 2001: "Approaches to Analysis in Visual Anthropology », in Theo van Leewen et Carey Jewitt (dir.), Handbook of Visual Analysis : 35-60. Sage Publications, Londres.

FEEST, Christian F., 1992 : Native Arts of North America. Thames and Hudson, New York.

FOUCAULT, Michel, 1973: «Nulle part, il n'y a de pipe ». <http://dubhe.free.fr/gpeint/magritte6.html> (page visitée le 18 avril 2005)

FRANCIS, Daniel, 1992: The Imaginary Indian: The Image of the Indian in Canadian Culture. Arsenal Pulp Press, Vancouver.

FRIEDMAN, Maurice, 1983: The Confirmation of Otherness in Family, Community and Society. Pilgrim Press, New York.

GARRIGUES, Emmanuel, 2000 : L'Écriture photographique: Essai de sociologie visuelle. L'Harmattan, Paris.

HALL, Stuart, 1997 : "The Spectacle of Others », in Stuart Hall (dir.), Representation: cultural representations and signifying practices : 223-290. Sage, Londres.

HOLMAN, N., 1996: "Photography as Social and Economic Exchange: Understanding the Challenges Posed by Photography of Zuni ». American Indian Culture and Research Journal 20(3): 93-109.

JACKNIS, Ira, 1992 : « George Hunt, Kwakiutl Photographer », in Elizabeth Edwards (dir.), Anthropology and Photography. 18601920 : 143-151. Yale University Press, Londres.

—, 1996: «Preface», in Ira JACKNIS (dir.), Special Edition: American Indian Culture and Research Journal, 20(3): 1-14.

JAUVIN, Serge, 1993: Aitnanu: la vie quotidienne d'Hélène et de William-Mathieu Mark. Libre Expression, Montréal.

KLEINMAN, Arthur, et Joan KLEINMAN 1996 : " The Appeal of Experience, the Dismay of Images ». Daedalus 125(1): 1-23.

LEUTHOLD, Steven, 1998 : Indigenous Aesthetics. Native Art, Media and Identity. University of Texas Press, Austin.

LIPPARD, Lucy R., 1992 : «Introduction », in Lucy R. Lippard (dir.), Partial Recall : 13-33. The New Press, New York.

MARR, Carolyn J., 1996 : « Marking Oneself: Use of Photographs by Native Americans of the Southern Northwestern Coast ». American Indian Culture and Research Journal 20(3) : 51-65.

MAXWELL, Anne, 1999: Colonial Photography \& Exhibition. Representations of the 'Native' and the Making of Europe Identities. Leicester University Press, London.

MICHEL, Yvette, 2004 : « Le projet La photographie comme bâton de parole. Exposition de photos des jeunes ». Innuvelle, 4 juillet 2004.

PERREAULT, Laura-Julie, 2004 : « Dans l'œil de jeunes Innus ». La Presse, 15 juin, Cl-2.

POIRIER, Sylvie, 2004 : «Présentation : Réflexions sur un concept pluriel ». Anthropologie et Sociétés 28(1) : 3-21.

ROBERTS, Helen, 2000: «Listening to Children and Hearing Them », in Pia Christensen et Allison James (dir.), Research with Children. Perspectives and Practices: 225-240. Falmer Press, London. 
ROESSEL, Monty, 1996 : « Navajo Photography ». American Indian Culture and Research Journal 20(3) : 81-91.

TOMASELLI, Keyan G., 1996 : Appropriating Images: the Semiotics of Visual Representation. Intervention Press, Højbjerg, Denmark.

TRUCHON, Karoline, 2005a: L'Anthropologie qui "laisse des traces ». La photographie comme agent d'empowerment : une ethnographie avec des Innus de Uashat mak Mani-Utenam. Mémoire de maîtrise, département de sociologie et d'anthropologie, université Concordia, Montréal.

—, 2005b : «Représentations d'autochtones d'hier à aujourd'hui : questionner l'image de victime ». Communication présentée lors de la conférence annuelle de la Société canadienne d'anthropologie (CASCA), du 3 au 8 mai 2005, Merida, Mexique.
TROY, Timothy, 1992: «Anthropology and Photography: Approaching a Native American Perspective ». Visual Anthropology $1(1)$ : 43-61.

WESLEY-ESQUIMAUX, Cynthia C., et Magdalena SMOLEWSKI, 2004: Traumatisme historique et guérison autochtone. Fondation autochtone de guérison, Ottawa.

WILLIAMS, Carol, 2003 : Framing the West: Race, Gender, and the Photographic Frontier in the Pacific Northwest. Oxford University Press, New York.

WORTH, Sol, et John ADAIR, 1997 [1972] : Through Navajo Eyes. An Exploration in Film, Communication and Anthroppology. University of New Mexico Press, New Mexico.

Affiquets, matachias et vermillon

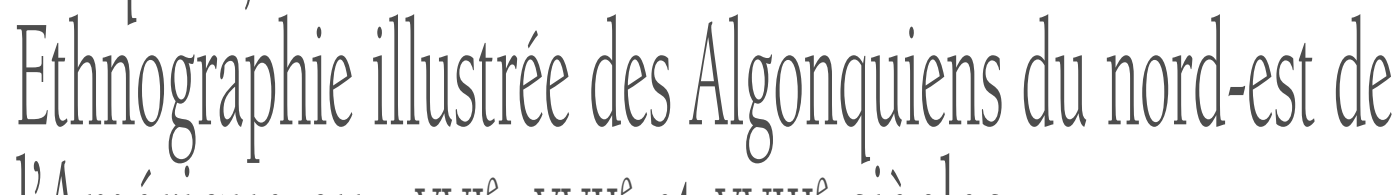

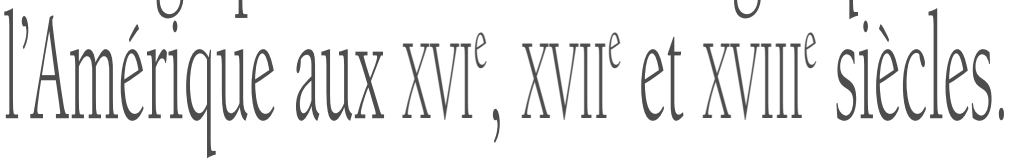

par Marc Laberge

\section{Illustrations de François Girard}

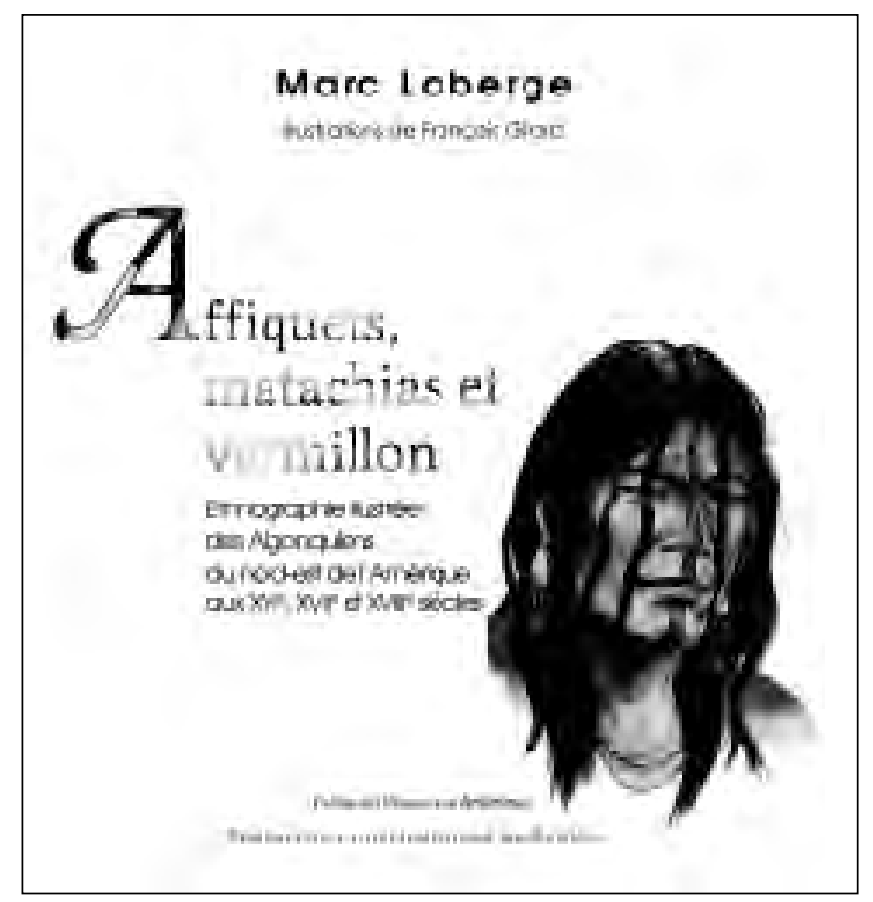

À quoi ressemblaient les Algonquiens lorsque Champlain a fondé Québec en 1608? Comment étaient-ils vêtus, coiffés, maquillés ? Quels types d'ornements et de décorations portaient-ils ? Comment vivaient-ils ?

Marc Laberge et François Girard ont joint leurs recherches et leurs talents pour répondre à ces questions et tenter de créer une nouvelle iconographie documentée des Algonquiens de la Nouvelle-France.

Un volume de 227 pages contenant plus de 120 illustrations.

Collection «Signes des Amériques ", $\mathrm{n}^{\circ} 11$ 30 \$ (tps et frais de port inclus)

Faire parvenir votre commande accompagnée d'un chèque à :

Recherches amérindiennes au Québec 6742 rue Saint-Denis Montréal QC H2S 2S2 


\section{In Memoriam}

\section{JAMES B. PETERSEN \\ (1954-2005) \\ L'inspirant respect d'une étoile filante}

I A NOUVELlE A FRAPPÉ de plein fouet la communauté archéologique l'été dernier, trop soudaine pour y croire mais trop perçante pour en douter. Elle arrivait de loin, précisément d'Iranduba, à quelques kilomètres au sud de Manaus, en Amazonie brésilienne. Le soir du samedi 13 août, James B. Petersen, archéologue et professeur à l'université du Vermont, décédait à la suite d'un cambriolage dans un restaurant d'Iranduba où il se trouvait en compagnie de quelques collègues. Jim avait 51 ans.

Né au Connecticut en 1954, Jim a fait ses études de maîtrise à l'université du Vermont, puis il a poursuivi son doctorat à l'université de Pittsburgh où il a soutenu sa thèse en 1983. Il a ensuite été professeur à l'université du Maine à Farmington où il a mis sur pied et dirigé l'Archaeology Research Center, un organisme universitaire d'expertise en archéologie professionnelle. En 1997, il est revenu à l'université du Vermont, cette fois à titre de professeur associé et directeur du département d'anthropologie.

Jim était au faîte de sa carrière. Passionné par la préhistoire des Amériques, il conduisait des recherches au sein de trois grandes aires culturelles, ce qui lui permettait de contribuer à l'avancement de l'archéologie à une échelle continentale. Dans les Caraïbes, Jim a travaillé à Montserrat puis à Anguilla. Au Brésil, il conduisait des recherches en pleine Amazonie où lui et son équipe concentraient leurs efforts sur l'association entre les sites villageois et la terra preta, ce sol organique et riche, d'origine anthropique et qui apparaît de façon sporadique dans la jungle. Mais c'est dans le Nord-Est américain que Jim débuta sa carrière avec l'étude de la céramique préhistorique de la Nouvelle-Angleterre, un champ de recherche qu'il n'abandonnera jamais. Ses intérêts ne se sont toutefois pas limités à cet aspect, et il a contribué de façon éclairante à une foule d'aspects du passé amérindien de cette région du continent, que ce soit la relation entre les Paléoindiens récents et les premiers groupes de l'Archaïque, la perception de l'identité culturelle à travers la technologie des fibres textiles, les sites submergés du golfe du Maine, la richesse et la variabilité des cultures archaïques anciennes, les premières apparitions des cultigènes en Nouvelle-Angleterre et la présence des Iroquoiens du Saint-Laurent au Maine et au Vermont, pour ne nommer que ceuxlà. Il ne fait pas de doute que l'archéologie lui doit en grande partie un portrait élargi du Nord-Est, où la NouvelleAngleterre fait figure, comme il se doit, de joueur aussi important que l'État de New-York.

En plus de nager dans cet éclectisme professionnel, Jim était également un être ouvert, attachant et généreux de sa personne. Il portait toujours un intérêt sincère pour les travaux et les idées des collègues. Jim a également travaillé étroitement avec les Abénaquis, témoignant en leur faveur dans le combat qu'ils mènent au Vermont pour leur reconnaissance auprès des autorités. Dans son vaste réseau de travail, Jim avait eu l'occasion de tisser des liens avec beaucoup de collègues québécois et canadiens. Il nous ouvrait grandes les portes en ce qui a trait aux données de la NouvelleAngleterre et nous invitait à la moindre occasion à venir partager ses connaissances. Il était de ceux qui mettent en pratique une science archéologique faisant abstraction des frontières politiques et linguistiques. En somme, nous avons perdu un grand archéologue, respectueux et respecté. Pour ceux d'entre nous qui ont eu la chance de te connaître Jim, tu nous manqueras profondément.

\section{Roland Tremblay}

\section{PIERRE DUMAIS (1951-2006)}

Nous apprenons avec une grande tristesse le décès de Pierre Dumais, survenu lundi le 9 janvier 2006. Pierre Dumais avait 54 ans. La communauté perd un de ses archéologues les plus prolifiques. Le prochain numéro de la revue présentera un hommage à sa mémoire. Pierre a été un précieux collaborateur de Recherches amérindiennes au Québec pendant de nombreuses années et nous offrons nos sincères condoléances aux membres de sa famille.

Léquipe de Recherches amérindiennes au Québec
Le monde autochtone

\section{en chiffres}

On dit souvent qu'une image vaut mille mots. Un chiffre en vaut parfois tout autant. Loin de croire que les chiffres puissent s'interpréter d'eux-mêmes, mais loin également de l'idée que l'interprétation puisse toujours se passer de repères statistiques ou chiffrés, nous présentons aujourd'hui une nouvelle chronique intitulée "Le monde autochtone en chiffres», qui vise à stimuler la réflexion autour d'enjeux contemporains pour lesquels existent des données quantifiées.

La Rédaction

\section{AUTOCHTONES CANADIENS : COMBIEN SONT-ILS ?}

Pierre Trudel

Cégep de Vieux Montréal

\section{LES SOURCES D'INFORMATION}

Deux sources de données servent à établir le nombre d'autochtones au Canada: le recensement du gouvernement canadien et le Registre du ministère des Affaires indiennes et du Nord canadien. Le Registre comptabilise le nombre d'Indiens inscrits. Puisque la loi définit le statut d'Indien, il est facile d'en déterminer le nombre. La situation n'est pas la même dans le cas du recensement.

En effet, jusqu'en 1996, le nombre d'autochtones était estimé au moyen de questions sur les origines des Canadiens. En 1996, l'ajout de « l'origine ethnique canadienne » a pu avoir un effet sur les résultats, en ce qui concerne les Autochtones, car certains d'entre eux ont pu choisir de répondre qu'ils sont d'« origine canadienne ». Par contre, depuis 1996, le recensement contient une question qui renvoie directement à l'identité. La voici : «Cette personne est-elle un Autochtone, c'est-à-dire un Indien d'Amérique du Nord, un Métis, ou un Inuit (Esquimau)?»Le répondant peut préciser s'il détient une identité autochtone mixte, ce qu'il ne pouvait faire précédemment.

Il est difficile de déterminer si les méthodes précédentes - celles d'avant 1996 - ont provoqué une sous-estimation ou une surestimation du nombre d'autochtones. Une chose est certaine: ces méthodes manquaient de précision. 
Notons que 1319980 personnes ont déclaré en 2001 avoir une ascendance autochtone, alors que 976000 affirmaient détenir une identité autochtone (Statistique Canada 2004a). Une autre source d'information chiffre à 1066500 pour le nombre de personnes ayant une identité autochtone (Statistique Canada 2005). Certaines réserves ont refusé, en tout ou en partie, de participer au recensement. Il s'agit de 77 réserves qui représentent 43600 personnes. En 2001, la marge d'erreur engendrée par ce dénombrement incomplet des réserves a été estimée à 10,3\%, une réduction de $4 \%$ par rapport au recensement de 1996. Pour ce qui est de la population autochtone hors réserve, la marge d'erreur est de $2,9 \%$, pourcentage semblable à celui de la population en général.

Le recensement contient une question sur l'inscription ou non des autochtones au Registre. Le MAINC explique l'écart important - il est de 175626 personnes - entre le nombre d'Indiens inscrits dans son Registre et ceux des données du recensement par le fait qu'une partie de ces Indiens sont à l'extérieur du pays, que d'autres sont en prison et que des réserves refusent de participer au recensement. De plus, en raison de différents facteurs, dont des déclarations tardives de décès et de naissances, le nombre d'Indiens inscrits apparaissant au Registre est ajusté en ajoutant $2 \%$.

\section{COMBIEN SONT-ILS ?}

D'après le recensement de 2001, 3,3\% de la population canadienne, soit 976000 personnes, dit avoir une identité autochtone (fig. 1) : $48 \%$ de cette population réside en milieu urbain, $33 \%$ vit dans les réserves et le reste se trouve en milieu rural (MAINC 2005). Statistique Canada ventile ce nombre de la façon suivante : 505000 seraient des Indiens inscrits et 104000 des Indiens non inscrits. Les Inuits seraient 45000 et formeraient $5 \%$ des autochtones tandis que les Métis, au nombre de 292 000, en représenteraient 30 \% (Statistique Canada 2004b). Environ 30000 personnes ayant déclaré avoir une identité autochtone ne peuvent être classées dans l'une ou l'autre de ces catégories. Pour l'année 2004, le MAINC indique qu'il y a 733626 Indiens inscrits (MAINC 2005).

En ce qui concerne les Métis, il importe de mentionner que des personnes s'associent à l'identité métisse sur une base individuelle, c'est-à-dire parce qu'elles sont nées de parents dont l'un est autochtone et l'autre non, mais que d'autres s'identifient plus clairement à un peuple qui s'est formé au cours de l'histoire du Canada. Les données du recensement ne semblent pas permettre de distinguer les deux catégories, quoique l'information selon laquelle la moitié des Métis réside dans les Prairies peut servir d'indicateur quant au nombre de gens qui forment le peuple métis. Le Métis National Council, organisation qui représente la portion du peuple métis qui réside au Canada - le territoire ancestral du peuple métis se trouve au cour de l'Amérique du Nord, de part et d'autre de la frontière entre le Canada et les États-Unis - estime, quant à lui, que entre 350000 et 400000 Canadiens forment le peuple métis du Canada. Lorganisation affirme sur son site (metisnation.ca) que le recensement canadien a toujours sous-estimé le nombre de Métis.

La difficulté de connaître le nombre exact d' "Autochtones » vivant au Canada peut aussi être illustrée par le fait que 6600 immigrants ont déclaré être « autochtones »...

D'après le recensement de 2001, c'est dans les Prairies que se trouve la majorité des autochtones et c'est l'Ontario qui, comme province, en compte le plus, soit 267 700. Nous avons vu plus haut que $33 \%$ des autochtones vivent dans des réserves, mais pour ce qui est des Indiens inscrits, c'est la majorité (60\%) qui opte, au début des années 2000, pour ce lieu de résidence. Notons que $60 \%$ des réserves ont moins de 500 habitants, $11 \%$ en comptent entre 1000 et 2000, et $6,5 \%$ seulement en ont plus de 2000 (fig. 2) La réserve la plus populeuse est Six Nations of the Grand River dans le sud de l'Ontario avec 22086 personnes, suivie d'Akwesasne qui en compte 10 052. Kahnawake, au Québec, arrive au quatrième rang avec 9275 personnes.

Selon Statistique Canada (2005a), il y a 96400 autochtones au Québec, soit 1,3\% de la population de cette province. Notons que le site du Secrétariat aux affaires autochtones du Québec ne donne aucune information sur les Métis et les Indiens non inscrits. Le Secrétariat établit qu'en 2005, il y a 72770 Indiens inscrits et 10054 Inuits, ce que l'organisme gouvernemental considère être la totalité des autochtones du Québec. LAlliance Autochtone du Québec affirme sur son site Internet (www.aaqnaq.com) qu'elle représente environ 26000 membres d'ascendance autochtone, soit des
Métis, des Indiens sans statut ou vivant hors réserve.

\section{VARIATIONS RÉCENTES ET PROJECTIONS}

La Loi sur les Indiens a été modifiée en juin 1985 (Projet de loi C-31) afin de corriger une discrimination sexiste qui retirait le statut d'Indienne aux femmes qui épousaient un non-inscrit. Présentement, $16 \%$ des Indiens inscrits l'ont été en raison de ce correctif à la loi. Des enfants et des petits-enfants de ces femmes ont été inscrits parce qu'elles-mêmes ont demandé de retrouver leur statut. Il s'agit de 113254 personnes. Bien qu'ayant connu une forte poussée au cours des années qui ont suivi la modification de la loi, la croissance de cette population, dite de «C-31», est maintenant ralentie (elle était de $48 \%$ en 1988, mais de $1 \%$ seulement en 2002). On estime qu'en 2021, 126700 personnes auront été inscrites en vertu de cette modification à la Loi sur les Indiens.

Entre 1996 et 2001, la population autochtone a augmenté de $22 \%$. Les naissances ont contribué à près de la moitié de cette croissance (10\%) tandis que des « changements dans les déclarations » seraient responsables du reste (12\%) [Statistique Canada 2004a]. Statistique Canada avance que «des facteurs non démographiques » font que davantage de gens affirment leur identité autochtone. Ce sont les Métis qui ont montré la plus forte augmentation avec une croissance de $43 \%$ (Statistique Canada 2004b). Et cette croissance a eu lieu plus particulièrement au centre et dans l'est du pays.

Il est encore possible qu'un enfant perde son statut d'Indien lorsqu'il naît d'un mariage mixte (c'est-à-dire entre un Indien inscrit et une personne non inscrite, autochtone ou pas). S'il s'agit de la première génération d'un mariage mixte, l'enfant conserve bien son statut d'inscrit, mais il relève alors du paragraphe 6(2) du projet de Loi C-31. Et si ce « 6(2)» se marie à son tour avec un non-inscrit, ses enfants n'auront pas le statut d'Indien.

$\mathrm{Ne}$ pas déclarer un père inscrit peut aussi être à l'origine d'une perte de statut. Si la mère, elle-même née de deux parents inscrits, ne déclare pas l'identité du père, ce qui est assez courant chez les jeunes femmes, le père sera considéré comme non-inscrit. Les petits-enfants de cette femme risquent donc de perdre leur 
statut. Entre avril 1985 et décembre 1999, 19 \% des enfants nés de mère inscrite en vertu du paragraphe 6(1) du projet de Loi C-31 - c'est-à-dire dont les deux parents sont inscrits - n'ont pas eu de pères déclarés (MAINC 2003: 3). Il s'agit de 37300 enfants, dont 17381 (46,6 \%) sont nés de mères n'ayant pas 15 ans (fig. 3). Lorsque la mère est inscrite en vertu du paragraphe 6(2) parce que l'un de ses parents n'était pas inscrit -, ce sont ses enfants eux-mêmes qui perdent leur statut si leur père n'est pas déclaré. Pour la même période, le ministère des Affaires indiennes et du Nord canadien estime à 13000 le nombre d'enfants qui se sont trouvés dans cette situation. C'est au Manitoba et en Saskatchewan que l'on trouve les taux les plus élevés de non-déclaration du père. En 2001 ce phénomène a affecté respectivement $27,7 \%$ et $27,1 \%$ des enfants de mères inscrites en vertu du paragraphe 6(1). Notons que le Québec se distingue nettement des autres provinces avec un taux de 3,8\% de naissances sans pères déclarés.

Dans les réserves, la transmission du statut d'Indien est présentement de $99 \%$. Or, en 2021, elle sera de $87 \%$. Hors réserve, le taux de mariage mixte étant élevé, la transmission du statut passera de $79 \%$ à $52 \%$ (MAINC, s. d.). La diminution projetée du nombre d'Indiens inscrits vivant hors réserve est sans doute liée à ce phénomène.

En 2017, la population autochtone canadienne sera d'environ 1400000 personnes. Au Québec, elle sera de 123000 personnes. La proportion entre les trois groupes restera sensiblement la même. Il semble que la tendance sera, pour les Indiens inscrits, de résider surtout dans les réserves. Alors qu'en 2000, $40 \%$ d'entre eux vivaient hors réserve, en 2021 ce chiffre tomberait à $25 \%$. La fécondité de la population des réserves est supérieure à celle de la population vivant hors réserve et Statistique Canada estime qu'un mouvement migratoire (migration annuelle nette) contribuera à la croissance de la population des réserves (MAINC 2004 : 5). L'organisme ne donne pas d'explication à ce phénomène qui apparaît étonnant, étant donné le manque d'emplois dans les réserves et la très forte croissance de la population active. Lidée selon laquelle il y aurait migration vers les centres urbains constitue un mythe, selon Statistique Canada

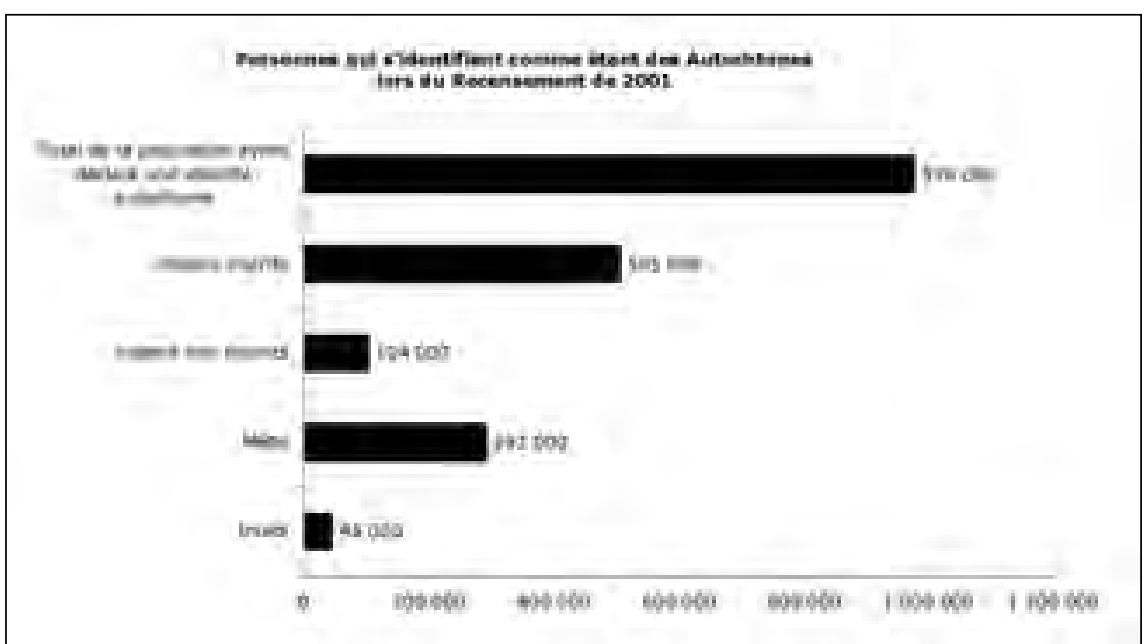

Figure 1

Environ 30000 personnes ( $3 \%$ de la population ayant déclaré une identité autochtone) se sont identifiées à plus d'un groupe autochtone ou ont déclaré être des Indiens inscrits ou des membres d'une bande indienne qui ne s'identifiaient pas comme étant des Autochtones. (Tiré de Statistique Canada 2004a : 3)

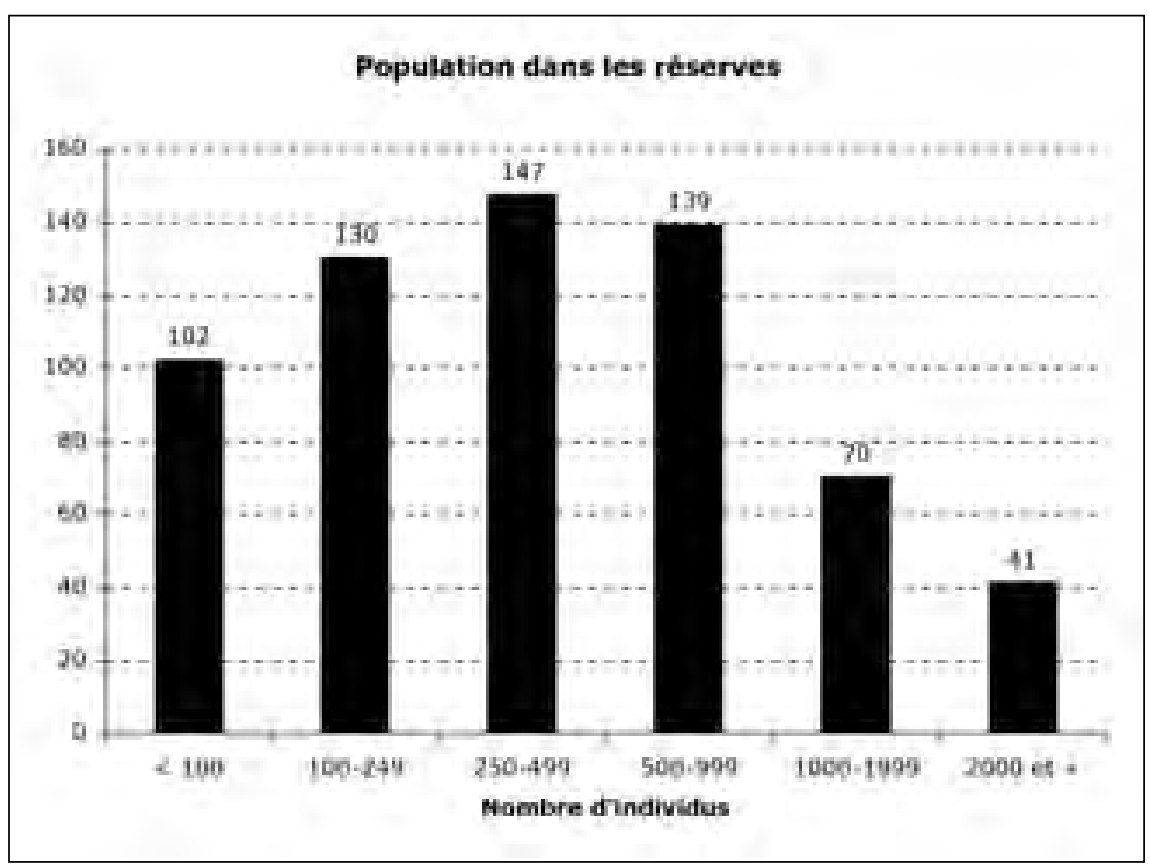

Figure 2

(Tiré de MAINC 2005a : xii, données au 31 décembre 2004)

qui estime que, en 2021, il y aura 939666 Indiens inscrits et que 236477 d'entre eux seulement résideront hors réserve. Ce sont les Inuits qui ont le taux de natalité le plus élevé. Les Métis, dont le taux de fécondité se rapproche de celui de l'ensemble des Canadiens, auront la plus faible croissance parmi les autochtones. Lorganisme fédéral précise que ces projections comportent une marge d'erreur du fait qu'elles portent sur une petite population et aussi parce qu'elles ne tiennent pas compte de l'issue des revendications territoriales et politiques.

Présentement $60 \%$ de la croissance de la population canadienne est due à l'apport de l'immigration. Les autochtones y contribuent pour $7 \%$. Or, en 20162017, la contribution des autochtones à la croissance de la population canadienne sera de $10 \%$ mais ce groupe ne formera qu'environ $4 \%$ de la population totale. 


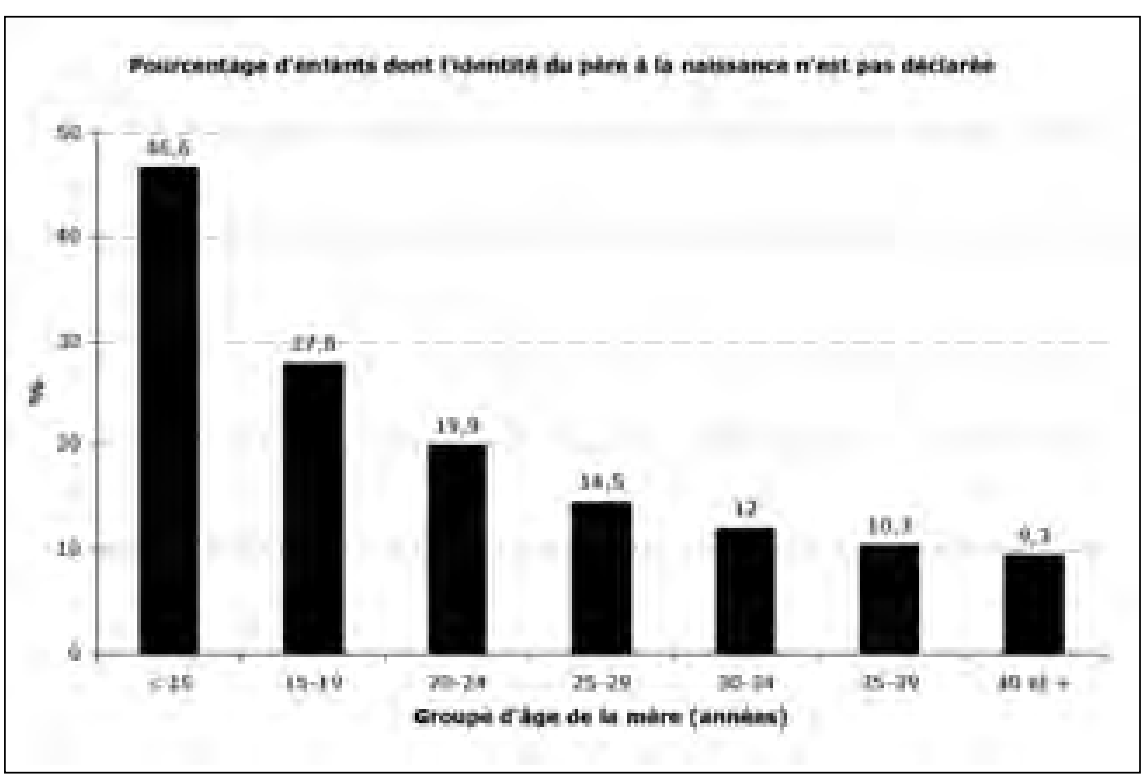

Figure 3

(Tiré de MAINC 2003 : 4, fig. 2; estimation réalisée à partir de données couvrant les années 1985 à 1999)

\section{IMPACTS SOCIAUX DE LA CROISSANCE DÉMOGRAPHIQUE}

En 2017, la population autochtone âgée de 20 à 29 ans sera de 40 \% supérieure à celle d'aujourd'hui (Statistique Canada 2005b). La population des réserves passera de 407300 à 703200 personnes. Il s'agira d'une pression considérable sur l'emploi, le logement et les services sociaux. La qualité de vie des enfants qui résident présentement dans les réserves constitue un déterminant important pour l'avenir de l'ensemble de cette population. Et pour longtemps. Maintenir le statu quo quant aux conditions socio-économiques actuelles multipliera les problèmes sociaux et de santé publique.

Des démographes estiment que le Canada dans son ensemble devra faire face à une véritable crise suscitée par le faible taux de natalité : parce qu'il y aura moins de contribuables et de travailleurs, les services publics seraient alors menacés et les entreprises manqueraient de main-d'œuvre. Nous avons vu que $60 \%$ de la croissance de la population canadienne repose sur l'immigration. Nous avons vu également qu'en 2017, les autochtones pourraient fournir $10 \%$ de cette croissance. Il importe, à mon avis, de voir à ce que, forts de leur nombre, ils puissent contribuer à combler les besoins en main-d'œuvre qui se feront sentir dans les régions. Il apparaît évident, en effet, que la création d'emploi dans les bande qui, au chapitre des services publics, discriminent entre eux et le reste de la population de la réserve.

\section{Ouvrages cités}

Statistique Canada, 2004a : Profil de la population des Indiens de l'Amérique du Nord au Canada ayant le statut d'Indien inscrit. Document disponible sur Internet : <http://www.aboriginalroundtable.ca/ sect/stscan/naistus_f.html> (consulté le 21 novembre 2005 ).

_, 2004b: Population métisse du Canada. Document disponible sur Internet $<$ http://www.aboriginalroundtable.ca/ $\downarrow$ sect/stscan/Metis_f.pdf> (consulté le 21 novembre 2005).

-, 2005a: Projections des populations autochtones, Canada, provinces et territoires, 2001 à 2017. Publication 91-547XIF. Document disponible sur Internet : http://www.statcan.ca:80/francais/ freepub/91-547-XIF/91-547-XIF200ـ 5001.htm (consulté le 21 janvier 2006).

_, 2005b: « La population autochtone du Canada en 2017 ». Le Quotidien, 28 juin 2005. Disponible sur Internet: $<$ http://www.statcan.ca:80/Daily/Franc ais/050628/q050628d.htm> (consulté le 24 janvier 2006).

MAINC (Ministère des Affaires indiennes et du Nord Canada), 2003: Facteurs contribuant à la non-reconnaissance de la paternité. Direction générale de la recherche, Ottawa.

\section{PROBLÈME RELATIF À LA PERTE DU}

\section{STATUT D'INDIEN INSCRIT}

D'autre part, le nombre croissant de résidants des réserves qui va perdre leur statut d'Indien inscrit créera de nouvelles tensions internes comme celles qui ont été provoquées par l'existence des «C-31», car ces « enfants d'inscrits qui ne seront plus inscrits » n'auront pas droit aux mêmes services publics que les « inscrits».

Lobligation faite aux femmes « indiennes inscrites » de déclarer le père « inscrit » de leurs enfants afin que ces derniers ne subissent pas de préjudice quant à leur statut juridique, et afin que les enfants de ces enfants n'en subissent pas non plus, constitue, à mon avis, une atteinte grave aux droits humains fondamentaux. Larticle 67 de la Loi canadienne sur les droits de la personne fait que la Loi sur les Indiens a préséance sur la Loi canadienne sur les droits de la personne; les Indiennes inscrites n'ont, par conséquent, aucun recours contre cette forme de discrimination qui les oblige à déclarer le père de leurs enfants. Pour la même raison, les non-inscrits, ainsi que les « C-31», n'ont aucun recours, eux non plus, contre les politiques gouvernementales, ou celles des conseils de

—, 2004: Données ministérielles de base 2003. Section des statistiques sur les Premières Nations et le Nord. Ministre de Travaux publics et Services gouvernementaux, Ottawa.

—, 2005 : Population indienne inscrite selon le sexe et la résidence. Section des statistiques sur les Premières Nations et le Nord. Ministre de Travaux publics et Services gouvernementaux, Ottawa.

— , s. d. : Projection de la population indienne inscrite pour le Canada et les régions 2000-2021. Document disponible sur Internet : <http://www.ainc-inac.gc.ca/ $\downarrow$ pr/sts/ipp_f.pdf> (consulté le 24 janvier 2006). 
Chronique juridique

\section{QUELQUES RÉFLEXIONS AU SUJET DE LA FUTURE POLITIQUE DE CONSULTATION DES PEUPLES AUTOCHTONES DU QUÉBEC}

Paul Dionne, avocat

\section{LE CONTEXTE}

Lors d'une conférence à Vancouver, j'ai été frappé par la mine sombre des avocats qui avaient plaidé avec succès la cause des Autochtones dans les affaires Nation Haïda et Taku River ${ }^{1}$. Ils doutaient tout simplement que le gouvernement de Colombie-Britannique accepte de réaménager ses relations avec les Autochtones pour respecter les arrêts de la Cour suprême. "After Haïda, disait l'un d'eux, I haven't seen any damage to the status quo. »Curieusement, les avocats qui avaient représenté le gouvernement et l'industrie me parurent plus optimistes. C'étaient eux qui s'efforçaient de mettre en lumière les gains réalisés par les autochtones depuis l'enchâssement constitutionnel des droits ancestraux et issus de traités.

Lattitude de ces avocats reflète bien l'humeur contrastée des autochtones et des gouvernements depuis que la Cour suprême a souligné, il y a quinze ans, que l'enchâssement constitutionnel des droits ancestraux et issus de traités exige le respect de ces droits ${ }^{2}$. Du côté autochtone, on désespère du caractère non contraignant des jugements qui confirment les droits ancestraux et issus de traités, convaincu qu'on est de la ferme intention des gouvernements d'y échapper. Pour illustrer ce sentiment, un chef autochtone affirmait naguère devant une assemblée de fonctionnaires: "When we win, we lose; when you lose, you win. » Du côté gouvernemental, on jure que chaque arrêt portant sur l'article 35 de la Loi constitutionnelle de 1982 provoque une véritable effervescence chez les fonctionnaires. On parle d'une « nouvelle culture » au sein de la bureaucratie. On souligne les progrès, et on invite les autochtones à être patients.

Il y a là sans doute plus qu'une simple différence de perception. Chose certaine, le rapport de force inégal entre l'État et les peuples autochtones marque profondément chaque action en justice sur les droits ancestraux et issus de traités. Ce déséquilibre se traduit, après le prononcé du jugement final, par une exécution laissée au bon plaisir du Souverain. À la suite des arrêts Adams et Côté ${ }^{3}$, par exemple, l'Assemblée nationale a légiféré afin de permettre au gouvernement du Québec de conclure des ententes avec les Autochtones pour concilier leurs activités « alimentaires, rituelles ou sociales » avec la gestion de la faune (article 24.1 de la Loi sur la conservation et la mise en valeur de la faune). Elle s'apprête à légiférer de nouveau, cette fois pour permettre la conclusion d'ententes visant à concilier ces activités avec la gestion des terres publiques (projet de loi no 122 , Loi modifiant la Loi sur les terres du domaine de l'État et d'autres dispositions législatives, présenté le 19 octobre 2005 par le ministre des Ressources naturelles et de la Faune). Cette façon arbitraire de donner suite aux arrêts de la Cour suprême est perçue comme statique et minimaliste par les autochtones (voir le mémoire de l'Assemblée des premières nations du Québec et du Labrador présenté le 17 novembre 2005 à la Commission de l'économie et du travail). Ceux-ci reprochent notamment au gouvernement d'évacuer l'aspect économique de ses démarches visant à mettre en œuvre la jurisprudence sur l'article 35 de la Loi constitutionnelle de 1982.

Loccasion se présente peut-être de rétablir l'équilibre. Les arrêts récents Nation Haïda, Taku River et Mikisew Cree First Nation 4 de la Cour suprême confirment en effet l'obligation constitutionnelle pour l'État de consulter et, s'il y a lieu, d'accommoder les peuples autochtones qui revendiquent des droits ancestraux ou qui détiennent des droits issus de traités, avant d'autoriser tout projet potentiellement attentatoire à ces droits. Ces arrêts font voir en outre que la transgression de l'obligation de consulter peut entraîner la nullité des permis d'utilisation du territoire ou de prélèvement des ressources que l'entreprise privée reçoit du gouvernement. C'est la première fois que des tiers risquent vraiment d'être affectés par le refus ou l'omission de l'État de se conformer à un jugement final portant sur l'article 35 de la Loi constitutionnelle de 1982. L'entreprise privée a donc intérêt à ce que l'État respecte ses obligations envers les peuples autochtones intégralement. Quant à l'État, il a intérêt à donner satisfaction à l'entreprise privée pour des raisons économiques et sociales.

C'est dans ce contexte que doit être examiné le projet du gouvernement du Québec de se donner une politique de consultation des peuples autochtones, pour se conformer aux arrêts récents de la Cour suprême, et de consulter les peuples autochtones avant d'adopter cette politique (voir le communiqué du 4 juillet 2005 du Secrétariat aux Affaires autochtones : « Le ministre Geoffrey Kelly annonce que le Québec définira une politique de consultation des autochtones »).

\section{L'APPROCHE}

Comment le gouvernement du Québec doit-il consulter les peuples autochtones au sujet d'une politique de consultation des peuples autochtones? En développant avec eux le cadre de référence et en les associant à la formulation de cette politique. Dans une lettre qu'ils adressaient à tous les chefs autochtones de la province le printemps dernier, les responsables des trois grandes associations autochtones de Colombie-Britannique écrivaient: « Premier Campbell [...] acknowledged that the unilateral development and implementation of the Provincial Consultation Policy after the British Columbia Court of Appeal decisions in Haïda clearly did not work and he expressed interest in "doing it right" this time. » Dans le document de travail conjoint annexé à cette lettre, on précisait que le cadre de référence des politiques provinciales concernant les autochtones serait dorénavant préparé par un comité de gestion formé de représentants des premières nations et de hauts fonctionnaires provinciaux.

Le Québec doit choisir une approche bilatérale semblable pour au moins trois raisons. D'un point de vue pratique, une politique de consultation ni conçue, ni appliquée en fonction de la nature des rapports entre le consultant et le consulté ne fonctionne tout simplement pas, comme en témoigne l'expérience récente de la Provincial Consultation Policy en Colombie-Britannique ou celle, au Québec, de la consultation des peuples autochtones au moyen d'une politique adoptée en vertu de l'article 211 de la Loi sur les forêts. D'un point de vue constitutionnel, les politiques de consultation des peuples autochtones adoptées en vase clos «n'équivalent pas à une consultation [...] et ne peuvent la remplacer. » (Nation Haïda, par. 78). Enfin, d'un point de vue statutaire, le ministre délégué aux Affaires autochtones du 
Québec ne respecterait pas sa mission en élaborant une politique de consultation des peuples autochtones sans «prendre en compte les aspirations, priorités et demandes des nations et communautés autochtones " (Loi sur le ministère du Conseil exécutif, articles 3.43 et 3.44).

À quel moment le gouvernement du Québec doit-il consulter les peuples autochtones au sujet de cette politique? De même que l'obligation constitutionnelle de consulter les peuples autochtones doit être mise en œuvre à l'étape de la «planification stratégique » (Nation Haïda, par. 76), la consultation au sujet d'une future politique québécoise de consultation des peuples autochtones devrait avoir lieu avant que le gouvernement ne prenne des décisions quant aux orientations ou quant au contenu de cette politique. La raison en est que, dès que l'appareil étatique a pris des décisions préliminaires au sujet d'un projet, celles-ci poussent le projet dans une direction qu'il est ensuite difficile de changer ${ }^{5}$. Par conséquent, le gouvernement devrait éviter que les travaux préparatoires du groupe de travail interministériel annoncés dans son communiqué de presse du 4 juillet 2005 ne débouchent sur des décisions préliminaires au sujet de la politique de consultation des peuples autochtones. Autrement dit, au moment pour le gouvernement de rencontrer les peuples autochtones au sujet de cette politique, tout devrait être sur la table.

Dans quel cadre la consultation sur la future politique doit-elle être menée? Depuis 1985, le gouvernement du Québec promet de créer un «forum parlementaire permanent » qui servirait de lieu d'échanges et de discussions entre les élus à l'assemblée législative et les chefs autochtones (voir la Résolution de l'assemblée nationale du 20 mars 1985 et les Orientations du gouvernement du Québec en matière autochtone de 1998). Une nouvelle instance de discussion politique paritaire, regroupant certains ministres clés du gouvernement du Québec et des représentants des chefs autochtones du Québec, a été créée récemment. Le «Conseil conjoint des élus », comme on l'appelle, constitue peut-être un bon cadre pour amorcer la discussion sur les orientations et la portée de la future politique de consultation des peuples autochtones. Toutefois, dans la mesure où ce conseil ne constitue encore que l'embryon du « forum parlementaire permanent» tant attendu, le gouvernement du Québec devrait s'assurer que les nations autochtones qui préfèrent désigner leurs propres représentants pour l'exercice de consultation, puissent le faire. En établissant la méthodologie de l'exercice, les parties pourront charger un comité technique conjoint de leur proposer le cadre de référence de la future politique de consultation ou d'en préciser certaines modalités.

\section{LES PRINCIPES}

Que tout soit sur la table au moment de la première rencontre entre le gouvernement du Québec et les peuples autochtones devrait inciter les parties à énoncer, en début d'exercice, les principes qui guideront la consultation et qui sous-tendront le cadre de référence de la future politique. Certains de ces principes s'infèrent de l'expérience et du sens commun. D'autres s'imposent à la lecture des arrêts de la Cour suprême. En voici quelques-uns qui nous paraissent particulièrement importants.

\section{Principes concernant l'exercice DE CONSULTATION SUR LA FUTURE POLITIQUE DE CONSULTATION DES PEUPLES AUTOCHTONES}

1. Objectif

Lexercice de consultation aura pour objectif de dégager un consensus sur les orientations et la portée de la future politique de consultation des peuples autochtones. Pour favoriser cet objectif, le cadre de référence de cette politique sera défini conjointement par le Québec et les peuples autochtones.

2. Bonne foi

Les parties veilleront à créer et à maintenir une ambiance propice à l'exercice de consultation. Elles favoriseront toute action susceptible de raffermir la confiance durant l'exercice. À l'inverse, elles éviteront toute action susceptible de créer la suspicion ou de semer le doute.

3. Représentativité

Les parties se donneront des assurances mutuelles sur la représentativité et la portée du mandat de chacun des participants à l'exercice de consultation.

4. Méthodologie, calendrier

Les parties établiront ensemble une méthodologie et un calendrier réaliste et flexible pour le déroulement de l'exercice de consultation.
5. Ressources humaines et financières Le Québec pourvoira au financement nécessaire pour que les peuples autochtones puissent participer utilement à l'exercice de consultation.

\section{Principes concernant le cadre} DE RÉFÉRENCE DE LA FUTURE POLITIQUE DE CONSULTATION DES PEUPLES AUTOCHTONES

1. Véhicule de mise en ouvre de l'obligation constitutionnelle de consulter

Lobligation constitutionnelle de consulter les peuples autochtones exige un processus distinct et cohérent. Lobligation sera donc mise en œuvre au moyen d'une nouvelle politique de consultation des peuples autochtones, plutôt qu'au moyen de stipulations législatives disséminées dans les lois du Québec décrétant la consultation de divers groupes d'intérêt.

2. Examen des stipulations législatives de consultation

Les stipulations législatives et les politiques pré-existantes de consultation des peuples autochtones seront examinées à la lumière de la nouvelle politique. Le cas échéant, elles seront modifiées ou abrogées à des fins de conformité.

3. Projets-déclencheurs

Les projets-déclencheurs de l'obligation de consulter ne sont pas limités aux activités physiques susceptibles d'un effet immédiat. Ils peuvent aussi comprendre des politiques, des programmes, des lois ou des règlements, de même que tout projet dont l'effet sur les Autochtones est moins évident, plus éloigné dans le temps ou s'additionne aux effets de projets antérieurs.

4. Permanence du processus

Tout processus de consultation et d'accommodement débute au stade de la «planification stratégique » du projet concerné et se poursuit au-delà de l'autorisation principale, tant que le projet nécessite une intervention gouvernementale ou ministérielle.

5. Intégration

Lintervention de deux ou plusieurs ministères ou organismes gouvernementaux et/ou de deux ou plusieurs Premières Nations concernés par un même projet doit, dans toute la mesure du possible, être intégrée en un seul processus de consultation.

6. Contenu de l'obligation de consulter Le contenu de l'obligation de consulter 
est établi au début de tout processus de consultation, avant même que soient établis la méthodologie et le calendrier du processus. Ce contenu varie en fonction de l'évaluation, à première vue, de la force de la revendication et de la gravité des effets potentiels du projet, mais il doit toujours refléter l'idée que la consultation doit être menée de bonne foi, dans l'intention de tenir compte réellement des préoccupations des peuples autochtones.

7. Aide extérieure

La possibilité de solliciter l'aide d'un tiers pour circonscrire le contenu de l'obligation de consulter, doit être considérée. La possibilité de créer un tribunal spécialisé à cette fin pourra aussi être considérée 6 .

8. Obligation d'accommoder

Seule une consultation adéquate permet de vérifier, au terme du processus, s'il y a obligation d'accommoder.

9. Contenu de l'obligation d'accommoder Parmi les mesures d'accommodement, doit être envisagée la possibilité de modifier ou d'abandonner le projet.

10.Évaluation et modification de la politique La politique de consultation des peuples autochtones sera évaluée périodiquement par le gouvernement et les peuples autochtones. Les peuples autochtones seront invités à participer à toute modification de la politique.

11. Ressources humaines et financières Il est essentiel que les parties puissent disposer de toutes les ressources humaines et financières nécessaires pour participer utilement à un processus de consultation et, le cas échéant, d'accommodement visé à la politique. Les parties s'entr'aideront mutuellement dans toute démarche nécessaire pour obtenir l'engagement du gouvernement fédéral à cette fin.

\section{AU-DELÀ DES PRINCIPES}

Le paragraphe 35(1) de la Loi constitutionnelle de 1982 n'établit que les normes constitutionnelles minimales que doivent respecter les gouvernements dans leurs rapports avec les peuples autochtones relativement aux droits ancestraux et issus de traités. Sous réserve des limites imposées par la Constitution, il est loisible aux gouvernements d'aller au-delà des normes fixées par le paragraphe 35(1) [R. c. Côté, par. 83].
En ce qui concerne plus particulièrement l'obligation de consulter les peuples autochtones, « il est loisible aux gouvernements de mettre en place des régimes de réglementation fixant les exigences procédurales applicables aux différents problèmes survenant à différentes étapes, et ainsi de renforcer le processus de conciliation et réduire le recours aux tribunaux » (Nation Haïda, par. 51). Par contre, une politique de consultation des peuples autochtones ne constitue pas un régime de réglementation (Nation Haïda, par. 51). Elle ne le devient que si elle s'intègre dans un cadre législatif ou réglementaire.

Il serait souhaitable que la future politique québécoise de consultation des peuples autochtones soit intégrée à une loi qui aurait préséance sur les autres lois de la législature. L'enchâssement législatif de la politique de consultation des peuples autochtones serait un gage de permanence et d'application rigoureuse de la politique. De plus, le caractère pré-éminent de la loi d'enchâssement permettrait aux parties de déroger aux lois québécoises dans le cadre des processus de consultation et d'accommodement visés à la politique. Ainsi élevée au rang des lois fondamentales, la politique de consultation des peuples autochtones favoriserait le dialogue plutôt que les discours, et la conciliation plutôt que les litiges.

\section{Conclusion}

Dans le rapport de la Commission d'étude que le gouvernement de Robert Bourassa avait mise sur pied après l'échec de l'Accord du lac Meech pour examiner les conditions de l'accession du Québec à la souveraineté, on s'efforçait de démontrer que les peuples autochtones du Québec étaient mieux lotis que ceux des autres provinces canadiennes. Cela ne devrait pas faire oublier le chemin qu'il reste à parcourir, notamment pour mettre en œuvre plus diligemment et plus objectivement les jugements portant sur l'article 35 de la Loi constitutionnelle de 1982.

La Cour suprême pourrait tenter de remédier elle-même à la situation en introduisant par interprétation, dans l'article 35 de la Loi constitutionnelle de 1982, un processus « indépendant, efficace et objectif » pour mettre en œuvre ses jugements, comme elle l'a fait dans le paragraphe $11 \mathrm{~d}$ ) de la Charte canadienne pour la rémunération des juges des cours provinciales ${ }^{7}$. Mais une solution négociée est toujours préférable à une solution imposée de l'extérieur. Le gouvernement du Québec et les peuples autochtones devraient donc saisir l'occasion que leur fournit la future politique québécoise de consultation des peuples autochtones, dans la foulée des arrêts Nation Haïda, Taku River et Mikisew, de rendre plus crédible le processus de mise en œuvre des jugements portant sur l'article 35 de la Loi constitutionnelle de 1982. Dans cette perspective, le gouvernement et les peuples autochtones devraient formuler ensemble le cadre de référence de cette politique au cours d'un exercice présidé par une personne impartiale ${ }^{8}$, où ils se présenteront sans conclusion ou échéancier prédéterminé, en acceptant de se laisser guider par des principes dont la valeur s'impose d'emblée.

[10 janvier 2006]

\section{Notes}

1 Haïda Nation v. British Columbia (Minister of Forests), 2004 C.S.C. 73; Taku River Tlingit First Nation v. British Columbia (Project Assessment Director), 2004 C.S.C. 74.

2 R. c. Sparrow, [1990] 1 R.C.S. 1075, p. 1119.

3 R. c. Adams, [1996] 3 R.C.S. 101; R. c. Côté, [1996] 3. R.C.S. 139.

4 Mikisew Cree First Nation v. Canada (Minister of Canadian Heritage), 2005 C.S.C. 69.

5 Voir, par analogie, l'arrêt Squamish Indian Band v. British Columbia, [2005] 1 C.N.L.R. 347, par. 74 et 83.

6 Nation Haïda, par. 37; voir aussi l'arrêt Paul c. British Columbia (Forest Appeals Commission), 2003 C.S.C. 55.

7 Renvoi relatif à la rémunération des juges de la Cour provinciale de l'île-duPrince-Édouard, [1997] 3 R.C.S. 3; Association des juges de la Cour provinciale du Nouveau-Brunswick c. NouveauBrunswick, 2005 C.S.C. 44.

$8 \mathrm{Au}$ moment de compléter ce texte, l'auteur a pris connaissance de la nomination par le gouvernement du Québec du juge Réjean F. Paul à titre de conciliateur "afin de rapprocher les parties gouvernementale et autochtone et de favoriser une réconciliation de leurs intérêts respectifs dans le cadre des travaux d'élaboration d'une politique de consultation des Autochtones »: voir le décret no 1228-2005 du 7 décembre 2005 , G.O.Q., partie II, 28 déc. 2005, p. 7512. 


\section{Comptes rendus}

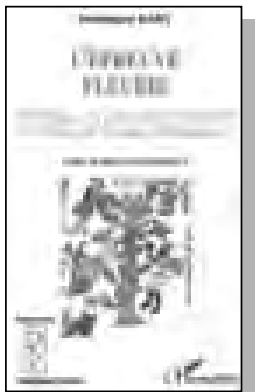

L'Épreuve fleurie. Symboliques du genre dans la littérature des Nahua du Mexique préhispanique Dominique Raby. Collection Recherches Amériques latines, L'Harmattan. Paris, 2003, 350 pages.

TSSU DE LA THĖSE de doctorat de l'auteure, ce document de 350 pages constitue 1 un ouvrage fort bien documenté, fruit d'une recherche méticuleuse des sources historiques et d'une quête constante de témoignages visant à faire émerger les conceptions des Nahuas quant au genre.

Une tendance assez répandue en anthropologie consiste à masquer la variabilité intraculturelle, soit pour des raisons méthodologiques ou théoriques, soit par souci de généralisation et de simplification. Heureusement, de nombreuses recherches n'hésitent pas à traiter des exceptions et des données contradictoires, qui constituent autant de preuves de l'existence de conceptions parallèles, porteuses non seulement de changements mais aussi de richesses intrinsèques. Le livre de Dominique Raby s'inscrit dans cette seconde approche.

Inspirée par le structuralisme, l'auteure se donne pour objectif de dégager les conceptions liées aux genres dans les représentations symboliques et littéraires. Il s'agit d'analyser le discours sur les sexes, leurs rôles respectifs et leurs relations dans différents styles littéraires selon la valeur accordée à chacun d'eux dans la hiérarchie sociale.

La première partie du livre, intitulée «Un imaginaire à recouvrer », situe d'emblée le contexte de l'étude. Le cadre théorique du discours sur le genre est évalué dans le premier chapitre à l'aune des approches culturalistes, marxistes, féministes et structuralistes.

Le second chapitre s'attarde aux sources (surtout des codex, chroniques, relations et compilations de la période postérieure à la Conquête), à leur validité et fiabilité. En effet, l'influence catholique dans l'interprétation et la transcription des codex, ou dans la compilation et la traduction des traditions préhispaniques, a eu tôt fait de transformer une jeune fille en vierge, avec tout ce que cela comporte comme glissement de sens et jugements de valeurs. Puis, la méthode employée est décrite: une sélection des mythes, sortilèges et poèmes est opérée sur la base de la présence d'un personnage féminin. Trois ensembles de discours ont été retenus pour examen: le mythe (teotlahtolli), associé aux autorités civico-religieuses plutôt masculines; le sortilège (nahuallahtolli), style en usage chez les guérisseuses et les sorciers des classes populaires; enfin, la poésie (cuicatl), employée par les nobles dans un contexte profane.

Le troisième chapitre brosse un tableau de la situation de la femme nahua en fonction de trois paramètres: le statut social, la sexualité et la représentation symbolique du genre. Cette revue de la littérature nous apprend, entre autres, que les femmes et les hommes du Mexique central, sans être égaux, n'étaient pas considérés en termes hiérarchiques mais complémentaires et que la sexualité, bien que soumise à la notion d'équilibre, n'était pas associée à l'idée de péché; on voit aussi que les distinctions de genre au sein du panthéon ne pouvaient être trop strictes, puisqu'il y avait interpénétration des aspects féminins et masculins chez toute divinité.

«La version officielle » est le nom donné à la deuxième partie du document. Comme son sous-titre l'indique, « symbolique du genre selon les autorités civico-religieuses », il s'agit d'expliquer le contrôle exercé par l'élite nahua sur les récits mythiques, chants et traités officiels. Lanalyse des versions de mythes issus des cycles chichimèque, toltèque et aztèque y est présentée. Le quatrième chapitre est consacré à la rencontre de Mixcoatl et de Chimalman; l'auteure y illustre avec brio l'efficacité de la méthode structuraliste. La démonstration de la part essentielle jouée par la femme dans la guerre (sur le plan social) et dans le sacrifice au soleil (sur les plans cosmique et religieux) prouve qu'il ne saurait être question de hiérarchie, mais plutôt de complémentarité entre les sexes. C'est l'enfant à naître qui constitue pour la femme le silex donneur de mort du guerrier mâle, et l'accouchement représente le champ de bataille où la guerrière est sacrifiée pour nourrir l'astre du jour et le porter dans sa course quotidienne entre zénith et couchant. Mythe cosmologique puisque c'est là la rencontre primordiale des hommes et des femmes - et la fixation de leurs rôles respectifs sur les plans sexuel, social et cosmique -, des chasseurs nomades et des agricultrices sédentaires, qui se trouvent à jeter les bases structurelles et fonctionnelles de l'univers.

Le cinquième chapitre traite des relations ambivalentes de Xochiquetzal et Tezcatlipoca et de leurs implications dans des événements aussi distincts que la chute de Tollan ou le cycle du maïs. La présentation des versions illustre la tentative chrétienne d'associer ces divinités nahuas au récit d'Adam et Ève. Le sixième chapitre s'arrête sur les mythes associés aux sœurs de Huitzilopochtli.

La troisième partie de l'ouvrage cerne «Les opinions divergentes », soit le point de vue des spécialistes du sortilège et des poétesses. Au septième chapitre, on aborde le monde magique, c'est-à-dire ce monde parallèle où le discours hégémonique se relâche, où une vision du monde différente se déploie dans un univers caractérisé par la dualité et la complémentarité. Au huitième chapitre, c'est la poésie amoureuse qui occupe l'avant-scène et Xochiquetzal en est la protagoniste principale. Ces chapitres offrent de nouvelles visions face aux aux conceptions émanant des autorités civicoreligieuses. Il ressort clairement que les femmes jouent un rôle fondamental dans la construction de la symbolique de genre au sein de la littérature nahua, littérature caractérisée par une forte dose de variation et de complexité.

Toute personne ayant fait du terrain dans cette région du monde reconnaît que si le discours public est dominé par les hommes, le discours privé est largement dominé par les femmes. En somme, l'ouvrage de Raby semble démontrer les racines anciennes et l'ancrage classique de ce phénomène.

En scrutant la parole des guérisseuses, sages-femmes et prêtresses, ce n'est pas seulement le discours des femmes nahuas sur elles-mêmes qui nous parvient comme un écho lointain mais combien éclairant de la condition féminine en Mésoamérique ancienne, mais aussi des 
indices de la transformation des mythes et des sociétés par-delà l'âme bicéphale des Nahuas, déchirés entre leur origine nomade et leur devenir sédentaire, et le millénaire de militarisme qui secoue la Mexique post-classique.

Que le peuple dominant de la Mésoamérique de 1520, avec la société complexe qu'on lui connaît, ait tenu différents discours sur des questions aussi centrales que le genre ne doit étonner personne. Pourtant, peu d'avancées ont été faites en cette direction jusqu'à aujourd'hui.

La contribution de Dominique Raby démontre l'ampleur des possibilités de recherche, ainsi que le chemin qui reste à parcourir dans nos connaissances sur cette société si proche de nous dans le temps et l'espace, et encore méconnue.

Paul Roy anthropologue, Cégep du Vieux-Montréal

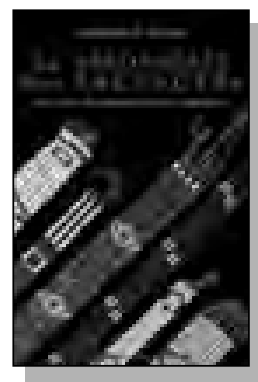

La " monnaie des Sauvages ". Les colliers de wampum d'hier à aujourd'hui

Jonathan C. Lainey. Septentrion, Sillery, 2004, $283 p$.

TONATHAN LAINEY aborde un sujet à la fois fascinant et controversé, en tentant $\int$ d'éclaircir les origines mystérieuses des six colliers de wampum conservés au Musée de la civilisation de Québec. Lanalyse de Lainey s'inscrit dans le contexte plus large de l'historiographie relative aux wampums et explore les usages et les origines historiques ainsi que les interprétations contemporaines des colliers de wampum en général. En intégrant dans son analyse presque toutes les facettes de l'étude des wampums, Lainey a produit une source précieuse qui a pour effet de relier multiples approches, interprétations et éléments des wampums avec la mythologie, la diplomatie et les collections muséales. Bien que son ouvrage puisse être considéré comme une tentative courageuse ayant pour but de théoriser l'analyse des wampums dans son ensemble, on peut s'interroger, cependant, sur les applications plus étendues de ses interprétations en rapport avec ces six colliers.

Lainey commence par retracer les origines et les routes commerciales empruntées par les perles blanches et violettes produites à partir des coquillages de la côte atlantique, pour ensuite s'intéresser plus particulièrement, dans le second chapitre, aux colliers de wampum eux-mêmes. Après avoir documenté la présence ancienne de différents colliers dans la littérature de voyage, Lainey examine attentivement les origines mythiques et spirituelles des wampums dans le récit fondateur des Iroquois, un intérêt, admet-il, qui émane du fait que les Cinq Nations soient les « inventeurs des wampums utilisés à des fins diplomatiques » (p. 35).

Ce deuxième chapitre, toutefois, signale ce qui apparaît rapidement comme étant la principale faiblesse de l'ouvrage, c'est-àdire l'absence de la mythologie huronne, ce qui n'est pas sans importance, puisque trois des six colliers de la collection du Musée de la civilisation sont d'origine huronne. La confédération huronne devait sans doute posséder son propre assemblage de récits mythiques en rapport avec la diplomatie et la spiritualité ainsi que de nombreuses applications réservées aux colliers et aux cordes de wampum, considérant que leur utilisation était fort répandue. Peut-être que les sources mythologiques huronnes sont-elles plus difficiles à repérer, mais leur substituer la mythologie iroquoise implique que tous les peuples autochtones souscrivent invariablement à des structures mythiques similaires, ce qui simplifie grandement la nature des traditions autochtones. Certes, est-il possible de tisser des liens entre les mythologies huronnes et iroquoises, ainsi que dans l'utilisation des wampums, voire même y trouver un certain nombre de similitudes, mais de privilégier l'une par rapport à l'autre, c'est négliger un aspect crucial de la collection des six colliers du Musée de la civilisation.

La suite du second chapitre traite de l'utilisation des wampums dans la diplomatie et fait état d'un nombre impressionnant de façons dont les Amérindiens et les Européens ont employé les colliers de wampum. L'examen par l'auteur des wampums comme objet relevant du sacré plutôt que du commun est particulièrement fascinant: «ce n'est pas nécessairement l'entente sous-jacente à l'échange des colliers qui est sacrée, mais plutôt le fait que les colliers aient été échangés au cours d'un rituel qui a été élaboré par les ancêtres fondateurs, comme l'attestent les récits mythologiques» (p. 57). En concluant que les colliers qui représentaient «des éléments et des symboles mythologiques et fondateurs » (p. 57) constituaient des colliers sacrés et que les autres demeuraient simplement ordinaires (p. 59), Lainey propose une solution originale au problème de discerner lesquels, parmi les milliers de colliers échangés, demeuraient les plus significatifs. Ainsi, l'analyse pénétrante de Lainey lui permet d'expliquer pourquoi certains colliers ont été minutieusement préservés, alors que d'autres ne l'ont jamais été; lorsqu'un collier était donné uniquement pour soutenir la parole, « il n'était pas nécessaire de le préserver au fil des générations, [parce que] son discours, la parole qui l'accompagn[ait], [avait] une importance à plus court terme » (p. 60).

En dépit de quelques analyses captivantes, une partie de la démonstration de Lainey sur les protocoles entourant l'échange de wampums lors de conseils est quelque peu généralisée, les conclusions se fondant uniquement sur une poignée d'exemples: "Le nombre de colliers devait donc être le même que le nombre de propositions originales » (p. 45), ou encore, "Si aucun échange de wampums ou d'autres présents n'avait lieu, les paroles prononcées n'étaient point considérées » (p. 52). Alors que ces suppositions corroborent sans aucun doute l'historiographie sur la question, Lainey minimise l'évolution du protocole entourant l'échange de wampums et insiste uniquement sur les façons dont les Européens ont profondément modifié l'étiquette et l'utilisation des colliers eux-mêmes. Pour arriver à conclure de façon convaincante sur l'utilisation des wampums dans les activités diplomatiques, plusieurs autres exemples doivent être utilisés et comparés systématiquement les uns aux autres, sur la longue durée historique, afin de déterminer exactement dans quelles circonstances Européens et Amérindiens utilisaient les colliers de wampum, en quelle quantité, quand et pourquoi étaient-ils remplacés par des pelleteries, ainsi de suite. Sans doute ce projet comparatif se situe-t-il à l'extérieur de la grille d'analyse de l'auteur. Néanmoins, l'enthousiasme démontré par Lainey pour examiner toutes les facettes des wampums dans la diplomatie est 
dilué dans des interprétations trop générales. Ce que nous savons cependant, et que Lainey démontre habilement, c'est que les colliers de wampum ont été de maintes façons au cour de la diplomatie nord-américaine jusqu'au début du XIX ${ }^{e}$ siècle.

Le deuxième chapitre se conclut sur les façons dont les colliers, autrefois si nombreux, ont décliné, particulièrement à cause des destructions intentionnelles des Amérindiens eux-mêmes (brûlés lors du sacrifice iroquois du Chien-Blanc ou simplement défaits pour mettre les perles au service du protocole matrimonial), ou par les tentatives méthodiques des autorités canadiennes et américaines visant à confisquer et effacer les souvenirs du passé amérindien. Cette dernière section favorise encore l'expérience iroquoise, laissant le lecteur perplexe vis-à-vis du statut des wampums chez les Hurons des XIXe et $x^{e}$ siècles. Peut-être que, dans l'intérêt de donner une voix aux Hurons, aurait-il été utile d'interroger les anciens pour faire surgir l'histoire du déclin et de la résurgence de la tradition des wampums dans la communauté.

La deuxième partie de l'ouvrage présente un récit intrigant de l'histoire des wampums conservés au Musée de la civilisation. Elle commence par l'examen des cinq colliers que Joachin Desrivières Tessier a donné au Musée de l'Université Laval, en 1931, ainsi que de celui conservé au Musée du Québec. Lainey brosse un intéressant portrait de Tessier et d'autres personnalités impliquées dans l'acquisition, fort populaire au tournant du xxe siècle, d'artefacts amérindiens. Par l'entremise d'un remarquable travail d'enquête, Lainey montre, par des années de communications personnelles, par des archives muséales et des photographies, les origines possibles de la collection de wampums du Musée de la civilisation. Il conclut avec certitude que trois de ceux-ci sont des colliers hurons vendus par différentes familles huronnes. Le doute entoure toujours les trois colliers restants, bien qu'il soit possible qu'il s'agisse aussi de colliers hurons. Quelles que soient leurs origines, ces colliers ont sans aucun doute fait l'objet d'une vente, affirme Lainey, parce qu'ils « perdent leur utilité, les messages qu'ils contiennent ne sont plus répétés, ils deviennent inutiles, de propriété privée, au même titre que les autres objets historiques, pour être finalement vendus au plus offrant » (p. 137). Cela est certainement le cas pour certains des six colliers, puisque Lainey a découvert une transaction entre Tessier et Paul Picard, un Huron qui a vendu des artefacts familiaux pour payer ses dettes et faire instruire ses enfants. Il convient cependant de demeurer prudent lorsque l'on s'approprie les interprétations de collectionneurs qui affirmaient dans des échanges épistolaires que les colliers avaient perdu toute signification, puisque ces dilettantes tentaient d'obtenir à tout prix des artefacts autochtones et que cet irrésistible engouement aurait fort bien pu teinter leurs propos.

Lainey développe cette idée plus avant dans le quatrième chapitre et discute de la quête d'artefacts amérindiens par les collectionneurs du tournant du $\mathrm{XX}^{\mathrm{e}}$ siècle. Encore ici, il présume que tous les colliers de wampum avaient perdu leur signification et, ainsi, les communautés amérindiennes pouvaient s'en défaire plus facilement. Dans une tentative pour différencier les collectionneurs américains et canadiens, Lainey prétend que les Indiens américains, particulièrement les Iroquois, «cédèrent leurs wampums aux mains des musées et des conservateurs [qui avaient] une vocation scientifique et préservative envers les objets » (p. 144). D'un autre côté, des hurons vendaient les artefacts uniquement pour leur «valeur marchande » (p. 144). Lainey ferme cependant les yeux sur l'activité très répandue du commerce sans scrupule des artefacts amérindiens des deux côtés de la frontière. En fait, à la fin du XIX ${ }^{e}$ siècle, un mouvement s'est enclenché chez les Iroquois de Grand River (que Lainey cite sans élaborer), où les chefs traditionnels, le Secrétariat aux Affaires indiennes et des anthropologues de renom firent campagne pour assurer le retour de onze colliers qui appartenaient, selon eux, à la Ligue iroquoise, mais qui avaient été vendus par des individus. Certes, des colliers de wampum ont quitté des communautés amérindiennes pour se retrouver dans des musées. À titre d'exemple, les Onnontagués ont volontairement désigné le New York State Museum comme le conservateur officiel de leurs colliers. D'autres colliers, qui avaient perdu toute signification, ont été vendus légalement par des individus, ce qui semble être le cas pour les colliers acquis par le collectionneur Tessier. D'autres, enfin, furent vendus illégalement au grand désespoir des chefs traditionnels qui pouvaient ou non les interpréter, tels ceux de Grand River. La question n'est pas aussi simple que semble l'affirmer Lainey; si ses conclusions collent bien aux six colliers détenus par le Musée de la civilisation, elles ne devraient pas être systématiquement généralisées à tous les colliers de wampum qui sont parvenus entre les mains des collectionneurs ou des musées.

Le manque de contexte huron est aussi évident dans le quatrième chapitre, alors que Lainey traite du rôle des chefs dans la conservation des colliers de wampum et des messages qu'ils véhiculent. Comprendre la place du wampum dans la mythologie et dans la société huronne peut servir à éclairer son déclin en tant qu'instrument social et politique, ainsi qu'à identifier les responsabilités précises de ceux qui étaient chargés de conserver les annales. Bien que Lainey décrive comment des chefs ont réussi à protéger des colliers pour leur collectivité, il néglige d'explorer et d'expliquer leurs responsabilités uniques vis-à-vis des colliers. Est-ce que le fait qu'un gardien des wampums ne soit pas désigné, si cela était le cas, reflète une moindre importance des wampums dans la mythologie huronne? Si les wampums sont effectivement moins présents dans la mythologie huronne, cela peut-il expliquer, sur la longue durée, la perte de signification? Se trouvent-ils d'autres colliers de wampum préservés chez les Hurons? Si oui, pourquoi certains colliers auraient-ils conservé leur signification, alors que d'autres l'auraient perdue? Ces questions de grand intérêt ne peuvent trouver réponse que dans une solide étude sur la place des wampums dans la mythologie huronne elle-même.

Une excellente analyse des symboles et des couleurs les plus présentes sur les colliers de wampum forme en grande partie le cinquième et ultime chapitre de l'ouvrage. Habilement, Lainey émet des hypothèses sur les interprétations possibles des motifs ornant les six wampums du Musée de la civilisation, concluant que «l'interprétation des wampums est difficile, voire impossible pour plusieurs raisons » (p. 191). Cela est certainement le cas pour les colliers conservés au Musée de la civilisation, alors que l'absence de tradition orale impose la spéculation sur l'interprétation des motifs, ce qui rend la tâche de décodage ardue, voire impossible, comme le démontre Lainey. Dans certains cas, cependant, la tradition orale rattachée aux wampums est demeurée relativement intacte, permettant aux colliers de «parler» et ce, à 
cause des inextricables liens entre l'appareil mnémonique et l'articulation du message verbal. Bien que Lainey fournisse de brèves explications sur la tradition orale dans son chapitre final, il l'identifie comme une source, au même titre que les sources d'archives, ce qui implique qu'elle ne soit pas une partie intégrante du wampum. Le collier ne veut rien dire sans le message oral, comme Lainey l'a si bien démontré avec la collection du Musée de la civilisation. En outre, le message oral n'acquiert aucune légitimité sans le collier. Lainey a parfaitement raison lorsqu'il souligne que « nous devrions nous en remettre davantage à la tradition orale puisqu'un wampum est supposé trouver son sens dans la tradition orale qu'il supporte, et non dans l'analyse des documents historiques » (p. 200). Mais la question demeure entière: où se trouve l'analyse de la tradition orale dans l'ouvrage de Lainey?

Ce livre représente une magnifique tentative d'illustrer littéralement toutes les nuances qui se rattachent à la recherche sur les wampums. Il se trouve peu de sujets sur lesquels l'auteur ne se penche pas. Et si certains de ceux-ci sont trop généralisés, il n'en demeure pas moins que le livre de Lainey représente une lecture incontournable pour quiconque s'intéresse à l'étude des wampums. Lauteur conclut son ultime chapitre en affirmant que «même les wampums qui ne parlent plus, ceux sans voix qui se trouvent dans les musées, continuent de demeurer signifiants, car rester muet veut toujours dire quelque chose » (p. 214). Ultimement, Lainey démontre que ces pièces fascinantes de la culture matérielle resteront toujours vivantes, même si certains de leurs messages sont perdus dans les annales du temps. Que ce soit en tant qu'objet non réclamé dans les musées ou comme un puissant rappel nationaliste pour les communautés autochtones, les colliers de wampum continueront de parler, d'une façon ou d'une autre, à la fois aux autochtones et aux non-autochtones.

Kathryn V. Muller candidate au doctorat en histoire Université Queen's, Kingston traduit de l'anglais par Michel Lavoie

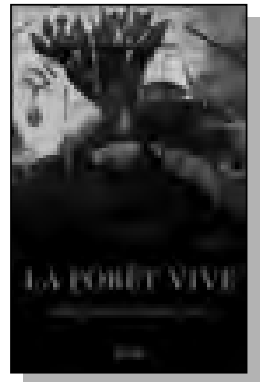

\section{La forêt vive. Récits fondateurs du peuple innu}

Rémi Savard. Montréal, Boréal, 2004, 218 pages.

Ans Ce Petit ouvRage consacré à la mythologie des Innus du Québec, Rémi Savard poursuit son étude rigoureuse des récits oraux que lui et son équipe ont recueillis dans les années 1970 lors de plusieurs séjours dans les communautés de la Basse-Côte-Nord. Les récits proviennent ici d'un seul conteur, François Bellefleur (1903-1978), connu aussi sous le nom de Penashue Pepine.

Louvrage est divisé en quatre parties, auxquelles s'ajoutent une introduction et une conclusion en deux morceaux. Le livre se clôt avec deux annexes didactiques: l'une sur l'aire algonquienne et la famille algique, l'autre sur les différentes variantes retenues. Lintroduction dresse d'abord une brève histoire ainsi qu'un tableau récapitulatif de la situation contemporaine des 15000 Innus qui vivent dans le nord-est de l'Amérique. Le texte s'achève avec la présentation du conteur, originaire de la communauté d'Unaman-shipit (La Romaine). Savard évoque la vie quotidienne dans les réserves, qu'il qualifie, avec humour et perspicacité, de «cliniques biodégradables, dont l'existence ne devait pas dépasser la durée nécessaire à la déprogrammation socioculturelle de leurs bénéficiaires autochtones » (cf. note 1). Heureusement, les traditions des Innus tiennent le choc, et le magnifique atanukan rapporté par l'auteur (ces récits oraux sont transmis aux gens par des entités autres qu'humaines, p. 22), en témoigne.

Le cour de l'ouvrage se compose de quatre récits, tous présentés en italique. Chaque récit fait l'objet d'un commentaire extensif par l'auteur, qui en examine les éléments saillants et les plus pertinents pour un auditoire non indien. Ici et là, des liens sont établis avec des variantes qui circulent dans d'autres groupes autochtones de l'Amérique du Nord. Le Tshakapesh des Innus, par exemple, ressemble comme deux gouttes d'eau au Tsa'kap des Kutenais de l'Ouest canadien et à Edechewe des Achumavis de Californie. Ces analogies conduisent Savard à voir dans ce personnage " un héros légendaire continental » (p. 67). À cet égard, il vaudrait la peine de comparer le mythe de Tshakapesh à celui de Kiviuq des Inuits. Mais Savard voit encore plus loin, étendant sa comparaison aux sociétés situées de l'autre côté du Pacifique (Chine ancienne ou moderne, Sibérie, Moyen-Orient).

Dans le premier récit, le lecteur découvre comment le héros Tshakapesh met en place des éléments clés de la cosmologie des Innus et fait de la chasse le mode principal de subsistance. Ce premier épisode offre au structuraliste que l'on connaît l'occasion de proposer une nouvelle lecture des rites funéraires des Innus. En s'appuyant sur des témoignages ethnographiques recueillis entre le milieu du XVII ${ }^{e}$ siècle et la période contemporaine, l'auteur relie ainsi rites de chasse et rites funéraires, les sépultures aériennes prenant tout leur sens à la lumière de la verticalité du cosmos algonquien (cf. les deux photographies p. 60-61). Comme les restes de gibier doivent être suspendus en hauteur ou placés sur des échafauds de bois, Savard suggère d'associer les arbres de la forêt boréale à de véritables rampes de lancement. Celles-ci permettraient à la vie de quitter le monde d'ici-bas, de s'élancer vers la lumière où séjourne Tshakapesh, pour finalement revenir sur terre sous forme d'étoiles filantes et compléter de la sorte le cycle de réincarnation (p. 67). Tel que le raconte le mythe des bébés qu'on trouve dans les souches, les ancêtres se réincarneraient donc en nourrissons. Cette lecture mériterait d'être testée plus à fond. Certains mythes sénécas évoquent également cette fonction salvatrice des arbres, des astres et de la lumière (cf. le récit $\mathrm{n}^{\circ}$ 100: l'étoile du matin et la femme cannibale). Les Inuits associent eux aussi les étoiles aux défunts et la Voie lactée au chemin des morts, mais contrairement à leurs voisins de la forêt, ils ne disposent pas de telles rampes. S'ils placent bien leurs défunts sur des collines ou sur des lieux particulièrement ensoleillés en bord de mer, il faut rappeler que la réincarnation des humains ne s'opère pas de cette manière, mais par la transmission des noms, la circulation des chairs correspondant davantage au cycle de réincarnation des animaux.

Les trois autres récits présentés par Savard portent sur les diverses règles que les Innus doivent respecter pour ne 
pas que leur monde tombe dans le chaos. Intitulé «L'enfant abandonné: l'origine de l'été », le deuxième épisode permet à l'auteur de revenir sur le rituel de «la tente agitée », selon l'expression introduite jadis par Jacques Rousseau. Pour Savard, cette terminologie est plus proche du terme innu de kushapatshikan, (littéralement 'ce qui sert à voir loin'). Ce terme s'avère donc plus adéquat que celui de «tente tremblante» lequel, tiré de l'anglais, ne rend pas compte des secousses violentes que provoquent les figures non humaines lorsqu'elles répondent « présentes » à la convocation de l'officiant, le kakushapatak (p. 98-99). Au personnage de Mistapeu qui dispense du gibier aux humains et occupe "l'empyrée chaud et lumineux », l'auteur oppose l'entité dangereuse et anthropophage d'Atschen qui dévore les humains et siège dans les profondeurs «froides et obscures » de la terre. Cette opposition verticale évoque une fois de plus une cosmologie étagée. Car, dans la tente agitée, les entités siégeant en haut sont perçues comme favorables contrairement à celles du bas, plutôt terrifiantes. Dans le jargon de la discipline, Savard souligne le rôle de Mistapeu qui inaugure « la reproduction du personnel et des ressources indispensables au bon fonctionnement de l'unité de production domestique » (p. 91)! Il s'ensuit quelques propos bien argumentés sur l'importance de la dichotomie générale hiver-été et sur les saisons (quatre, cinq ou six selon les ethnographies) dans les classifications zoologiques et les pratiques.

Intitulé « Être la bru de son mari : la fin de l'été », le troisième récit offre à l'auteur l'occasion de conduire son lecteur dans l'univers des êtres maléfiques et dangereux pour les humains: insectes, vers et animaux à cornes s'y bousculent. Ethnographies et dictionnaires à la main, Savard fait de nouveau ressortir cette cosmologie verticale des Innus. Il montre en particulier le rôle de grand protecteur que joue un peu partout en Amérique du Nord la figure de l'oiseau-tonnerre, continuelle source d'inspiration chez de nombreux artistes autochtones. Thunderbird occuperait le pôle céleste de cet axe vertical dont l'extrémité chthonienne est, elle, associée au serpent cornu, une figure néfaste également très répandue dans les mythologies amérindiennes.

Dans le quatrième et dernier récit intitulé « Le gendre de son fils : la chute aux enfers », Savard traite de la disparition sous la terre de Tsheshei (le héros incestueux) ou, selon d'autres variantes, de sa transformation en crapaud immortel. Cette partie me semble plus touffue que les autres et moins réussie. En quelques pages et au prix de nombreux raccourcis, le lecteur se promène de la côte nord du golfe du Saint-Laurent au pays de Gilgamesh, des textes de l'Ancien Testament à ceux de la Grèce ancienne.

Dans la section suivante qui fait office de première conclusion, Savard prend du recul pour mettre en perspective certaines traditions des Innus avec celles de plusieurs peuples eurasiens. Avec brio, il fait apparaître la nécessité de rattacher le Nouveau Monde au continent eurasiatique. Savard utilise prudemment la notion « d'échos » qui implique à la fois distorsion et répétition, mais les pièces versées à ce dossier ont de quoi convaincre. Trois principaux éléments sont retenus. Laxe vertical du monde se retrouverait d'abord chez les Algonquiens et dans de nombreuses sociétés eurasiatiques où de gros oiseaux-tonnerre ou des oiseaux-serpents sont un peu partout associés à une fonction protectrice et où, à l'inverse, des insectes plus terrifiants les uns que les autres incarnent des puissances maléfiques. Comme Savard mentionne le rôle d'un chien protecteur parmi des précisions qu'il obtint un jour de François Bellefleur (p. 93, deuxième récit), il pourrait ajouter aux pièces à conviction cette vaste répartition du thème du chien psychopompe. Lui aussi se trouve présent des deux côtés du Pacifique, associé à l'ancestralité d'une part, et à la garde du royaume des défunts d'autre part, un rôle qui le place donc au début comme à la fin de la vie humaine (voir Kretschmar 1938). Le deuxième élément qui permet de rattacher ces traditions se situe au niveau des rituels de chasse et du motif de la prothèse, selon l'expression que Savard emprunte à K. Tuite (p. 157). La scapulomancie (soit l'utilisation d'une omoplate animale que l'on fait craquer dans le feu à des fins divinatoires) et que l'auteur retrouve tant chez les Innus qu'en Chine et chez les Toungouzes constitue le troisième élément. Speck ne l'avait repéré que chez deux groupes de langue athapascane, mais cette forme de divination paraît encore plus répandue des deux côtés du Pacifique si l'on intègre ses variantes, comme le makittarniq des Inuits.

Avec ce bel ouvrage, Savard fait des deux rives du Pacifique une véritable aire culturelle qui reste à mieux comprendre. Lauteur partage ce point de vue avec d'autres, comme Lydia Black ou Rémi Mathieu, dont les travaux sur le corbeau (Mathieu 1984) auraient d'ailleurs pu être utilisés pour donner encore plus de poids à l'argumentation. Qui sait, l'histoire des Amériques fera peut-être un jour partie à part entière d'une histoire eurasiatique qui reste à écrire?

Dans sa deuxième conclusion sur le droit, la souveraineté et les arbres, l'auteur procède peut-être un peu vite. Le Savard militant dépasse le structuraliste rigoureux. Rien à dire sur l'équation établie entre l'axe vertical du monde et l'arbre de vie (p. 168), mais les paragraphes suivants sur le droit demeurent plus discutables. Sur la base du récit de Tschakapesh et en s'appuyant sur des auteurs comme Grammond, l'auteur défend l'idée qu'il existe de « véritables traditions juridiques » chez les Innus. Je ne doute pas de la portée normative de certains récits mythologiques mais l'utilisation des mêmes termes rend les différentes conceptions en présence encore plus difficiles à saisir. N'est-il pas excessif de parler de droit et de jurisprudence, comme s'il y avait dans les mythes un corpus de lois prêt à être lu et appliqué et des conceptions identiques à celles de l'Occident moderne et judéo-chrétien? Au contraire, il me semble que de nombreuses sociétés autochtones tantôt s'accommodent du droit occidental en se l'appropriant ou en le tournant à leur avantage, tantôt s'en méfient et rejettent sa rigidité, se reconnaissant davantage dans des systèmes où l'on suit strictement des règles locales et familiales, autrement dit, des règles avant tout relationnelles et non décrétées valides et applicables de façon universelle. Le passage d'un système à l'autre est évidemment possible avec de multiples combinaisons, mais ce processus implique un travail de traduction où néologismes et malentendus abondent. En somme, il ne s'agit pas de placer encore les sociétés amérindiennes à l'envers du Blanc, ni de nier l'émergence contemporaine d'un droit autochtone ou encore de considérer ces sociétés comme étant incapables de gouverner ou de changer. Il s'agit plutôt de reconnaître, autant que possible, leurs différences et la valeur de leurs perspectives à une époque où le rouleau compresseur des gouvernements et des idéologies individualistes modernes n'a pas renoncé à son projet de nivellement. Car, comme le fait bien observer Savard, la résistance des Innus est manifeste, encore faut-il se mettre à l'écoute et savoir la lire. Comme 
on le sait, la difficulté majeure tient au fait que les autochtones entendent à la fois participer à la modernité et faire valoir leurs différences, un message ambivalent que les Blancs ne parviennent pas à accepter.

Un autre critique que l'on peut adresser à l'auteur est de ne pas aborder la réception du christianisme par les Innus, comme si les conceptions de jadis exposées par le mythe n'avaient pas changé avec l'irruption de ces nouvelles idées. Selon certains récits, Atshen par exemple, n'a-t-il pas été vaincu par les missionnaires et n'a-t-il pas eu tendance, ensuite, à incarner la figure démoniaque? Dans l'ouvrage, seule la note 27 (p. 195) fait référence au pèlerinage de SainteAnne, un sujet pourtant fort bien traité par Gagnon (2003) et d'autres chercheurs. Ce choix se défend en raison du sujet choisi, mais il reste paradoxal, car si tout le livre laisse plutôt apparaître des continuités culturelles, la dernière conclusion fait soudainement référence à ces changements. Enfin, Savard aurait pu utiliser davantage les travaux de ses collègues car ni le Grateful Prey de R. Brightmann (1993), ni les travaux de P. Armitage (1992), par exemple, ne sont cités.

En dépit de ces quelques points, ce livre bien écrit reste novateur et très inspirant. Toute sa richesse repose sur ce jeu de zoom qui fait de la cosmologie verticale des Innus une conception à la fois très particulière et finalement assez commune (voir aussi certaines cosmologies autochtones du Mexique). Lauteur montre de façon remarquable à ceux et celles qui en douteraient encore, combien les récits oraux des Innus comme ceux d'autres groupes autochtones doivent être pris au sérieux pour être placés sur le même plan que les grands récits classiques qui constituent une partie substantielle du patrimoine de l'humanité.

\section{Ouvrages cités}

ARMITAGE, Peter, 1992: «Religious Ideology Among the Innus of Eastern Quebec and Labrador ». Religiologiques $6: 64-110$.

BRIGHTMAN, Robert, 1993 : Grateful Prey. Rock Cree Human-Animal Relationships. University of California Press, Berkeley, Los Angeles, Oxford.

GAGNON, Denis, 2003 : Deux cents ans de pèlerinages: les Mamit Innuat à Musquaro, Sainte-Anne-de-Beaupré et Sainte-Anne-d'Unamen-Shipu (18002000). Thèse de doctorat, département d'anthropologie, Université Laval, Sainte-Foy.
KRETSCHMAR, Freda, 1938: Hundestammvater und Kerberos. Vol. I-II, Frankfurt, A.M.

MATHIEU, Rémi, 1984: «Le corbeau dans la mythologie de l'ancienne Chine ». Revue d'histoire des religions 201(3) : 281-309.

Frédéric Laugrand Département d'anthropologie Université Laval

\section{Publications québécoises récentes}

\section{Chee Chee:}

A Study of Aboriginal Suicide.

Al Evans. McGill-Queen's University

Press, Montréal et Kingston, 2004,

202 pages. $33 \$$.

Le problème très préoccupant du suicide qui sévit gravement chez plusieurs communautés amérindiennes fait l'objet de ce livre, un essai basé sur une enquête menée par Alvin Evans, ancien officier de la GRC, professeur retraité en études religieuses à l'université de Waterloo et spécialiste des études sur le suicide. Après une longue mise au point dans laquelle l'auteur sent la nécessité de justifier qu'un non-Amérindien ait écrit un tel livre, Evans raconte la vie et les circonstances du suicide de l'artiste ojibwa Benjamin Chee Chee, qu'il n'a pas connu mais dont il appréciait le talent. Puis il tente de comprendre cet acte dramatique dans son contexte socioculturel, notamment à l'aide d'interviews avec la mère de Chee Chee, des amis proches et d'autres artistes. L'objectif principal de l'auteur est toutefois de présenter une évaluation critique de l'impact négatif de la société «blanche» sur la vie et la culture des Amérindiens de ce pays afin, ultimement, de susciter chez ses concitoyens une prise de conscience de l'existence du problème, de sa gravité et de l'urgence d'y remédier.

\section{Contributions to the Study of the Dorset Palaeo-Eskimos.}

Patricia D. Sutherland (dir.). Mercury Series, Archaeology Paper 167. Canadian Museum of Civilization, Gatineau, 2005, 180 pages. $30 \$$.

Cet ouvrage collectif regroupe neuf contributions présentées par des chercheurs en préhistoire de l'Arctique provenant du
Canada, des États-Unis et du Danemark. Chacun des articles regroupés ici présente les résultats de recherches récentes abordant notamment les perpétuels problèmes de chronologie, de typologie et de continuités/discontinuités en archéologie préhistorique, mais aussi la question des affinités biologiques entre populations à l'aide d'analyses de l'ADN mitochondriale et d'analyses craniométriques, de même que les problèmes d'interprétation de la variabilité des industries lithiques et osseuses, des modalités d'exploitation des carrières de stéatite, du sens de l'art rupestre et de la dimension sociale des maisons-longues dorsétiennes. Le court essai présenté en guise d'introduction par Surtherland permet de situer ces contributions dans un contexte historique plus large. On appréciera la qualité des illustrations et le nouveau design graphique de la collection Mercure, bien connue des archéologues.

\section{Dictionnaire encyclopédique} et historique des coureurs des bois. Alain Messier. Guérin, Montréal, 2005, 512 pages. $22 \$$.

Ce livre est plus qu'un simple dictionnaire; c'est aussi une histoire des coureurs des bois en Amérique du Nord en six chapitres, de même qu'un " hommage aux pères jésuites ». Lauteur, qui est historien, adopte une approche ethnohistorique pour y explorer en particulier le thème de la rencontre des civilisations amérindienne et européenne. On y retrouve aussi l'étrange histoire et des extraits d'un récit écrit en 1805 par l'explorateur et coureur des bois FrançoisAntoine LaRocque dans les montagnes Rocheuses. Une liste des principaux forts, emplacements stratégiques et postes de traite en Amérique du Nord est aussi donnée, ainsi que de longues citations isolées en début d'ouvrage, des extraits de documents d'archives qui parsèment la partie correspondant au dictionnaire proprement dit et une traduction des nombres en langue algonquine qui servent d'ailleurs à numéroter les pages liminaires du livre..

Ethnography and Development: The Work of Richard F. Salisbury. Marilyn Silverman (dir.). Fontanus Monograph Series XV, McGill University Libraries, Montréal, 2004, 408 pages. 60 \$.

Richard S. Salisbury (1926-1989) a enseigné l'anthropologie durant plusieurs années à l'Université McGill. Chercheur 
productif et influent, il était également un enseignant dévoué, et quatre de ses anciens étudiants de McGill (Harvey Feit, Henry J. Rutz, Colin H. Scott et Marilyn Silverman) lui rendent ici hommage. Un hommage qui ne prend pas la forme d'un banal festschrift; les quatre auteurs ont plutôt rassemblé un certain nombre d'articles de Salisbury (18 au total, dont certains inédits) qu'ils jugent représentatifs de sa contribution en anthropologie politique, en anthropologie économique, en anthropologie du développement, en anthropologie appliquée et en anthropologie de la Nouvelle-Guinée. De courts essais introductifs contextualisent ces écrits, et les auteurs tentent également d'y montrer la pertinence de l'œuvre de Salisbury dans la pensée anthropologique contemporaine. Louvrage se termine par une bibliographie complète des publications de Salisbury.

Ghost Brothers. Adoption of a French Tribe by Bereaved Native America: A Transdisciplinary Longitudinal Multilevel Integrated Analysis Rony Blum. McGill-Queen's University Press, Montréal et Kingston, 2005, 464 pages. $80 \$$.

Rony Blum est chercheure de niveau postdoctorat au département d'études sociales de la médecine à l'Université McGill. Dans ce livre, elle tente d'expliquer le «succès» des premières relations et alliances franco-amérindiennes, principalement avec les Algonquiens et les Hurons (Wendats). Elle en trouve l'explication dans la «médiation par les fantômes », un processus sociopsychologique de compréhension et de rapprochement transgénérationnel et transculturel. Ainsi, l'état de deuil quasipermanent chez les Amérindiens du Nord-Est causé par les maladies infectieuses transmises par les premiers Européens, trouvait écho auprès des colons Français - mais nullement, apparemment, auprès des Espagnols ou des Anglais - qui avaient subi en France des épidémies semblables (bien que de moindre envergure), en plus d'être fortement nostalgiques de la mère-patrie. Cette étude constitue aussi une exploration originale de l'« amérindianisation » (Nativization) des Français de la Nouvelle-France. À noter que l'ouvrage contient pas moins de 84 pages de notes, une bibliographie de 100 pages et un index.
Paroles et pouvoir de femmes des Premières Nations. Manuel pour prise de parole sur la vie, la santé... le diabète.

Bernard Roy et Katia Fecteau. Les Presses de l'Université Laval, Québec. 2005. 228 pages. $35 \$$.

Les communautés amérindiennes du Québec connaissent des taux de diabète inquiétants et les femmes sont généralement plus touchées que les hommes. Ce guide d'animation de groupes de discussion, écrit par deux anthropologues de la santé de l'Université Laval, en collaboration avec la Commission de la santé et des services sociaux des premières nations du Québec et du Labrador, s'adresse aux professionnels de la santé œuvrant en milieu autochtone. Refusant les « stratégies culpabilisantes et normatives » du discours médical habituel, les auteurs proposent une approche novatrice qui reconnaît l'expertise et valorise le savoir des femmes amérindiennes (à qui il incombe traditionnellement de veiller sur la santé des membres de leur famille) et qui vise l'intégration et la participation de ces femmes dans la planification d'activités de prévention du diabète et de promotion de la santé de manière générale. La parole est donc largement donnée aux femmes (attikameks, micmacs et innues) qui se racontent dans ce manuel et dont les témoignages sont regroupés autour de six grands thèmes faisant l'objet d'autant de chapitres.

\section{Le rêve et la forêt. Histoires de chamanes nabesna.}

Marie-Françoise Guédon. Les Presses de l'Université Laval, Québec, 2005, 616 pages. $40 \$$.

Lanthropologue Marie-Françoise Guédon enseigne au département des études anciennes et des sciences des religions de l'Université d'Ottawa. Spécialiste des populations autochtones de l'aire circumpolaire, elle présente ici les récits chamaniques que lui ont raconté les Nabesnas, un petit groupe athapascan d'environ 500 personnes habitant la frontière entre l'Alaska et le Yukon. Lauteure cherche d'abord à valider l'hypothèse voulant que «... les pratiques chamaniques, comme la mythologie, comme les rituels, forment souvent le noyau, durable, résistant, du contexte culturel de la société qui les porte ». Elle tente ensuite de montrer comment les Nabesnas ont élaboré une variante locale du complexe chamanique athapascan, lui-même distinct de ceux des cultures voisines. Sa perspective circumpolaire lui permet, finalement, de suggérer une certaine correspondance entre la délimitation des complexes chamaniques et les frontières linguistiques. Par ailleurs, il est à noter que chacun des sept chapitres s'ouvre avec une histoire Nabesna et que le livre est parsemé de dessins et de photographies réalisés par l'auteure.

\section{Something New in the Air: The Story of First Peoples Television Broadcasting in Canada.}

Lorna Roth. McGill-Queen's University Press, Montréal et Kingston, 2005, 320 pages. $30 \$$.

Cet ouvrage retrace l'histoire de la télévision amérindienne au Canada, dont le point culminant fut la mise sur pied de Television Northern Canada (1992-1999), précurseur de l'actuel Aboriginal Peoples Television Network (APTN), le réseau de télévision le plus développé du genre dans le monde. Lauteure, professeure au département de communications de l'Université Concordia, tente en parallèle de montrer le rôle de la télévision dans l'affirmation et la promotion des droits et de la culture des Amérindiens dans une perspective d'autonomie de développement et de gouvernance. De nombreuses cartes et photographies accompagnent le texte, de même que de courts extraits des nombreuses interviews menées par l'auteure.

Christian Gates St-Pierre

\section{Thèses et mémoires}

BERGERON, Odile, 2005 : " Religion is not Inuit ». Expérience, identité et ontologie religieuses contemporaines chez les jeunes Inuit d'Iqaluit. Mémoire de maîtrise, département d'anthropologie, Université Laval.

BERNIER, Hélène, 2005 : Étude archéologique de la production artisanale au site Huacas de Moche, côte nord du Pérou. Thèse de doctorat, département d'anthropologie, Université de Montréal.

DE CANCK, Alexis, 2005: Convenance(s) des histoires. De l'évolution de ce qui est "convenable" en appartenance identitaire. Histoire et éducation entre Amérindiens et Eurocanadiens du Québec, 
l'exemple de la nation atikamekw. Mémoire de maîtrise, Faculté de théologie, d'éthique et de philosophie, Université de Sherbrooke.

DESROSIERS, Pierre, 2005 : L'archéomuséologie, un modèle conceptuel interdisciplinaire. Thèse de doctorat, département d'histoire, Université Laval.

GADOUA, Marie-Pierre, 2005 : Chasse aux mammifères marins et identité ethnique : le rôle du harpon au sein de la culture thuléenne. Analyse comparative des sites Clachan et de l'île Skraeling. Mémoire de maîtrise, département d'anthropologie, Université McGill.

GARRARD, Margaret, 2005 : Towards an Understanding of Tradition in Cree Women's Narratives, Waskaganish, James Bay. Mémoire de maîtrise, département d'anthropologie, Université McGill.

HENDERSEN, Lyle, 2005 : Thule and Caribou Inuit Subsistence Strategies: Re-evaluating the Origins of the Caribou Inuit. Mémoire de maîtrise, Département d'anthropologie, Université McGill.

POULIN, Isabelle, 2005 : Des femmes mettent la main à la pâte: pratiques quotidiennes de cuisines populaires a Villa Maria del Triunfo, Lima (Pérou). Mémoire de maîtrise, département d'anthropologie, Université McGill.

TARGÉ, Anne-Pascale, 2005 : Transmission des savoirs oraux dans les écoles inuit. Étude du cas de la communauté d'Arviat, Nunavut. Mémoire de maîtrise, département d'anthropologie, Université Laval.

TEASDALE, Guillaume, 2005 : La politique autochtone du gouvernement canadien dans l'Ouest à la fin du XIX e siècle. Une analyse de l'interdiction de la Danse du Soleil. Mémoire de maîtrise, Faculté de théologie, d'éthique et de philosophie, Université de Sherbrooke.

Christian Gates St-Pierre

\section{Livres reçus}

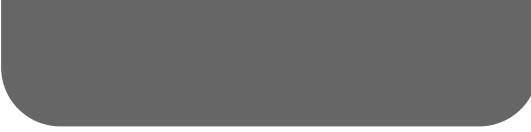

Citizen Indians: Native American Intellectuals, Race, and Reform Lucy Maddox. Cornell University Press, Ithaca, New York, 2005, 220 p.

Le Cercle de la vie. Histoires et sagesse du peuple sioux

Joseph Marshall III. Terre indienne, Albin Michel, Paris, 2005, 248 p.

Entre Dios y el Diablo. Magia y poder en la costa norte del Perú

Hiroyasu Tomoeda, Tatsuhiko Fujii et Luis Millones (dir.). Instituto Francés de Estudios Andinos, Pontificia Universidad Católica del Perú, 2004, 209 p.

El Dios Yabireri y su cargado Yayenshi France-Marie Renard-Casevitz. (Edición bilingüe Matsiguenga-Español). Instituto Francés de Estudios Andinos, Lluvia Editores, 2004, $161 \mathrm{p}$.

Los Andes y el reta del espacio mundo. Homenaje a Olivier Dollfus Jean-Paul Deler et Évelyne Mesclier (dir.). Instituto Francés de Estudios Andinos, 2004, $419 \mathrm{p}$.

Guía Etnográfica de la Alta Amazonía Fernando Santos et Frederica Barclay. Volume IV, Instituto Francés de Estudios Andinos, Instituto Smithsonian de Investigaciones Tropicales, 2004, 368 p.

\section{El Reto fisiológico de vivir} en los Andes

Carlos Monge C. et Fabiola León-Velarde S. (dir.). Instituto Francés de Estudios Andinos, Universidad Peruana Cayetano Heredia, 2003, $435 p$.

Retisser nos liens : Comprendre les traumatismes vécus dans les pensionnats indiens par les Autochtones Deborah Chansonneuve. Fondation autochtone de guérison, 2005, 114 p. et annexes.

La Raison du corps : idéologie du corps et représentations de l'environnement chez les Miraña d'Amazonie colombienne

Dimitri Karadimas. Langues et sociétés d'Amérique traditionnelle 9, SELAF 412, Éditions Peeters, Paris, 2005, 451 p.
Antropología de la Naturaleza

Philippe Descola. Instituto Francés de Estudios Andinos, Lluvia Editores, 2003, $91 \mathrm{p}$.

Dictionnaire encyclopédique et historique des coureurs des bois Alain Messier. Guérin, Montréal/Toronto, 2005, $367 p$.

Chee Chee: A Study

of Aboriginal Suicide

Al Evans. McGill-Queen's University Press, Montréal et Kingston, London, Ithica, 2004, 178 p.

Ghost Brothers. Adoption of a French Tribe by Bereaved Native America: A Transdisciplinary Longitudinal Multilevel Integrated Analysis Rony Blum. McGill-Queen's University Press, Montréal et Kingston, London, Ithica, 2005, $448 p$.

Ethnography and Development: The Work of Richard F. Salisbury Marylin Silverman (dir.). Fontanus Monograph Series XV. McGill University Libraries, Montréal, 2004, 398 p.

Something New in the Air: The Story of First Peoples Television Broadcasting in Canada Lorna Roth. McGill-Queen's University Press, Montréal et Kingston, London, Ithica, 2005, $300 \mathrm{p}$.

In the Way of Development. Indigenous Peoples, Life Projects and Globalization

Mario Blaser, Harvey A. Feit et Glenn McRae (dir.). Zed Books en collaboration avec International Development Research Center, London et New York, 2004, $362 p$.

Le rêve et la forêt. Histoires de chamanes nabesna

Marie-Françoise Guédon. Les Presses de I'Université Laval, Québec, 2005, 586 p.

Contributions to the Study of the Dorset Palaeo-Eskimos

Patricia D. Sutherland (dir.). Collection Mercure, Archaeology Paper 167, Musée canadien des civilisations, Hull, 2005, 165 p.

Le Soleil-Dieu et le Christ. La christianisation des Indiens du Mexique Guy Stresser-Péan. Recherches Amériques latines. L'Harmattan, Paris, 2005, 568 p. et DVD du film « La Fête du Maïs ». 
Droit, territoire et gouvernance des peuples autochtones

Ghislain Otis (sous la direction de). Les Presses de l'Université Laval, Québec, 2004, $197 p$.

Face à l'arbre sacré. Mémoires d'un indien sioux

John G. Neihardt. Terre Indienne, Albin Michel, Paris, 2005, 346 p.

Hinóno'éínoo3ítoono. Arapaho Historial Traditions (Told by Paul Moss)

Andrew Cowell et Alonzo Moss, Sr. (dir.). Collections de la Société d'édition de textes algonquiens. The University of Manitoba Press, Manitoba, 2005, 531 p.

\section{Colloques et congrès}

Colloque annuel du Centre interuniversitaire d'études et de recherches autochtones (CIÉRA)

Date : 20-21 avril 2006

Thème: Regards sur les arts autochtones: Créations, productions et réappropriations

Lieu: Pavillon La Laurentienne,

Université Laval, Québec

Renseignements :

http://www.ciera.ulaval.ca/

\section{Northeast Anthropological Association}

Date : 5-7 mai 2006

Thème: 46th Annual Meeting Lieu : Albany, New York (USA)

Renseignements : Sean Rafferty

(rafferty@albany.edu)

\section{4e Congrès annuel de I'ACFAS}

Date : 15-19 mai 2006

Thème: Le savoir, trame de la modernité Lieu : Université McGill, Montréal Renseignements :

http://www.acfas.ca/congres/

\section{3th Annual Stabilizing Indigenous} Languages Symposium

Date : 25-28 mai 2006

Thème: And Together Our Minds Are One

Lieu: Buffalo State College, SUNY Buffalo

Renseignements : http://jan.ucc.nau.edu/」 jar/SIL9brochure.html

\section{Congrès CASCA 2006}

Date : 9-14 mai 2006

Thème: Nature humaine, identité humaine : reconsidérations

Lieu : Université Concordia, Montréal Renseignements

http://artsandscience.concordia.ca/ $\downarrow$ socanth/casca2006/FR/index.htm

\section{Association canadienne d'archéologie}

Date : 24-27mai 2006

Thème: 39 e congrès annuel

Lieu: Université de Toronto, Ontario

Renseignements : http://caa2006.cana」

dianarchaeology.com/indexfr.html

\section{Association des archéologues du Québec}

Date : 25-28 mai 2006

Thème: $25^{\mathrm{e}}$ Congrès annuel

Lieu : Chicoutimi, Québec

Renseignements : erik_langevin@uqac.ca

The Omohundro Institute of Early American History and Culture

Date : 9-11 juin 2006

Thème: Twelfth Annual Institute

Conference

Lieu : Québec, Québec

Renseignements

http://www.wm.edu/oieahc/conferences/」 index.html

\section{$52^{\mathrm{e}}$ Congrès international des américanistes}

Date : 17-26 juillet 2006

Thème: Peuples et Cultures

des Amériques

Lieu: Séville, Andalousie (ES)

Renseignements : http://www.52ica.com/

\section{Résumés \\ Abstracts}

Les jeunes Algonquins sont-ils biculturels?

Modèles de transmission et innovations dans quelques réserves Marie-Pierre Bousquet

Les jeunes autochtones sont souvent présentés dans le discours populaire comme étant « pris entre deux mondes », ce qui justifie les images d'individus «à problèmes » qu'on projette sur eux. Seraient-ils donc biculturels? À l'aide de discours d'Algonquins nés depuis 1970, l'article montre l'importance des représentations spatiales, juridiques et générationnelles dans la définition de leur identité culturelle. Entre eux et les aînés, derniers semi-nomades, une génération assure une médiation culturelle, celle de leurs parents qui ont vécu les transitions du pensionnat, de la sédentarisation et qui ont créé de nouveaux modèles. Les jeunes, pour leur part, investissent de repères l'univers de la réserve, leur lieu de référence, et innovent pour « faire évoluer la culture ». Ainsi, ils élaborent une culture algonquine moderne. Mais la Loi sur les Indiens crée des tensions, venant renforcer l'illusion d'une bipartition du monde.

\section{Are Young Algonkins Bicultural?}

Models of Transmission and Innovation in some Algonkin Reserves Marie-Pierre Bousquet

Young people from First Nations are often portrayed as caught "between two worlds", justifying the image projected upon them as "problem" individuals. But can they really be considered bi-cultural? In this article, the importance of spatial, legal and generational representations in defining the cultural identity of Algonkins born after 1970 is explored. A transitional generation, that of their parents - who lived through the changes brought about by residential schools, sedentarisation and the creation of new models - mediates between young Algonkins and the elders, who lived a semi-nomadic life. The universe of the reserve, their source of reference, is invested with meaning by young people as they innovate to "make the culture evolve". In their own way they are striving to create a modern Algonkin culture but the Indian Act creates tensions that reinforce the illusion of a bipartite division of their world.

Musique, tradition et parcours identitaire de jeunes Atikamekw La pratique du tewehikan dans un processus de convocation culturelle Laurent Jérôme

À travers l'expérience d'un groupe de joueurs de tambour de la communauté atikamekw de Wemotaci, cet article montre comment de jeunes autochtones négocient le changement dans un contexte social et culturel en pleine mutation. La pratique contemporaine du tambour est révélatrice de transformations et de persistances culturelles véhiculées par la jeunesse: valorisé dans de nombreuses communautés, le tambour s'inscrit dans un processus plus large de convocation culturelle qui a pris naissance 
dans la communauté de Wemotaci dans les années 80. À côté de la responsabilité culturelle que cette pratique implique, les membres du groupe revendiquent aujourd'hui une responsabilité professionnelle intervenant tout autant dans leur expérience et dans leur représentation de ce que signifie être jeune et atikamekw aujourd'hui. Les dispositions musicales, les lois du marché, le respect des contrats, les négociations et les conciliations liées à la professionnalisation se positionnent donc aux côtés des savoirs locaux persistant dans le contexte contemporain.

\section{Music, tradition and the identity route of young Atikamekw The evoking of cultural processes through the Tewehikan \\ Laurent Jérôme}

Through the experience of a drumming group of the Atikamekw community of Wemotaci, I show in this article how young aboriginal people are negotiating social and cultural transformation in a changing context. The contemporary drum practice reveals these cultural transformations carried by and for the youth; the drum, as a cultural element valued in numerous communities, is part of a larger process of eliciting cultural forms that emerged in Wemotaci during the 1980's. Today, drumming reflects not only a cultural responsibility, but also a professional responsibility intervening in the drummer's experience and interpretation of what it means to be young and Atikamekw today. Musical arrangements, market laws, necessity to fulfill contracts, negotiations and conciliations bound to the professionalization of the drumming practice are now part of the drumming experience, together with local knowledge.

\section{Les chansons et musiques populaires innues}

Contexte, signification et pouvoir dans les expériences sociales de jeunes Innus Véronique Audet

Cet article propose une compréhension des expressions musicales populaires innues en tant que voix - et voies d'affirmation identitaire et de revitalisation sociale et culturelle. Ces chansons et musiques en langue innue, inspirées des courants country, rock, folk, pop, etc., ainsi que des traditions innues, panamérindiennes, chrétiennes et québécoises, se pratiquent, se créent et se renouvellent depuis la deuxième moitié $\mathrm{du} \mathrm{xx}^{\mathrm{e}}$ siècle, accompagnant les divers événements, mouvements et transformations souvent radicales qui ont marqué la vie sociale et culturelle des Innus au cours de ces années. Les jeunes contemporains, comme ceux des quelques décennies précédentes, sont les principaux porteurs de ce mouvement d'expression, qu'ils investissent pour participer à la vie de leurs communautés, affirmer leurs identités, leurs expériences et leurs préoccupations, contribuant ainsi à la revitalisation du monde et du «mode d'être » innu. La présentation de quelques chanteurs, groupes et chansons, représentatifs de l'expression musicale des jeunes Innus contemporains et ayant un impact significatif au sein de leur société, offre une compréhension sensible du contexte, de la signification et du pouvoir de ces expressions.

\section{Popular Innu Songs and Music: Context, Meaning and Power in Young Innu Social Experiences Véronique Audet}

This article presents popular Innu musical expressions as voices that serve to reaffirm Innu identity and revitalize Innu society and culture. These songs in the Innu language are inspired by various musical trends such as country, rock, folk and pop, as well as Innu, Pan-Indian, Christian and Quebecois traditions. They have been created, practiced and renewed since the second half of the twentieth century, accompanying the often radical events, movements and transformations that have marked Innu social and cultural life. Contemporary Innu youth, like their predecessors, are the primary conveyers of this expressive movement which they use to participate in the daily life of their communities, and reaffirm their identity, their experiences and their preoccupations, thus contributing to the revitalization of their world and the Innu "way of being". We present some of the singers, groups and songs that are representative of contemporary Innu musical expression and who have had a significant impact within their society. This provides a sensitive understanding of the context, the significance and the power of these expressions.

\section{L'espérance politique et économique chez les jeunes Tzeltals et Tlapanèques du Mexique Martin Hébert}

À partir de données ethnographiques recueillies dans les États du Chiapas et du Guerrero, cet article tente de poser quelques jalons pour ce qui pourrait être nommé une anthropologie des imagessouhaits chez les autochtones du Mexique. Dans un contexte de crise rurale prononcée et de mobilisations particulièrement dynamiques, l'auteur a constaté que les discours politiques et économiques des jeunes membres des communautés visitées étaient fortement marqués par l'idée d'un « ailleurs » ou d'un « futur» meilleurs. Quels sont les contours de cette espérance sociopolitique complexe? Comment des aspirations économiques et des aspirations politiques parfois cohérentes et parfois contradictoires entre elles, viennent-elles jouer dans le processus de mobilisation collective?

\section{Political and Economic Hope Among Young Tzeltals and Tlapanecs of Mexico.}

\section{Martin Hébert}

Using ethnographic data collected in Chiapas and Guerrero, this article aims to lay down some reference points for what could be termed an anthropology of wishimages among the indigenous peoples of Mexico. In a context of marked rural crisis and political mobilisations, it was observed that political and economic discourses of young adults living in indigenous communities were strongly influenced by the idea of a better "elsewhere" or a better "future". What is the shape of this complex social and political hope? How can economic and political aspirations that are sometimes both coherent and contradictory work in the process of mobilisation for collective action?

\section{Comment flirter avec la modernité pour conforter son identité : projet éducatif d'une communauté métisse au Manitoba}

Thibault Martin et Brieg Capitaine

En 1994, une école francophone était créée au sein de la communauté métisse de Saint-Laurent (Manitoba). La création de cette école institutionnalisait la division préexistante entre Métis francophones et Métis anglophones. Cet article propose une analyse du projet éducatif mis au point par l'école métisse francophone. Selon l'auteur, ce projet est à la fois culturel, destiné à préserver la langue vernaculaire des Métis, le mitchif, mais aussi politique, destiné à redonner aux Métis la capacité de définir eux-mêmes leur futur. Sur le plan pédagogique, le projet scolaire tente d'intégrer savoirs modernes et savoirs autochtones et cherche à créer des liens entre les aînés et les jeunes ainsi qu'entre l'école et la communauté. Ses fondements théoriques et idéologiques s'inscrivent dans un courant qui traverse aujourd'hui un certain nombre de collectivités autochtones. La 
particularité du projet réside dans une alliance stratégique entre la communauté métisse et les Franco-Manitobains, et par-delà cette alliance, dans une tentative de relier la communauté aux réseaux globaux, via la francophonie.

\section{How to flirt with modernity to secure one's identity in a Manitoba Metis school \\ Thibault Martin et Brieg Capitaine}

In 1994, a francophone school was established in the heart of the Metis community of St Laurent, Manitoba. The creation of the school institutionalized a pre-existing divide between the Francophone and Anglophone Métis populations. This article provides an analysis of the educational project developed by the Francophone Métis community. We will see that the project is a cultural and political enterprise, which aims both to preserve the traditional vernacular of the Métis, the Michif language, as well as to reclaim the community's capacity for selfdetermination. The pedagogical model of the project integrates elements of modern knowledge and traditional Aboriginal knowledge, and aims to strengthen social ties both between elders and youth and between the school and the larger community. The theoretical foundations of this model are part of a trend observable in a number of Aboriginal communities. However, the specific feature of the project is in the strategic alliance forged between the Métis and FrancoManitoban communities and, beyond this alliance, in attempts at connecting the Métis with global francophone networks.

\section{Être jeune et maori aujourd'hui : l'université comme site de (ré)affirmation et de coexistence Natacha Gagné}

Luniversité constitue un site important d'affirmation de diverses identités maories, et c'est là que s'expriment et se négocient les rapports aux autres, maoris et non maoris. L'entrée à l'université, c'est l'occasion d'une confrontation directe avec les « deux mondes », l'un occidental et l'autre maori. Plusieurs font alors vite l'expérience de l'université comme un lieu étranger, un lieu non maori. Cependant, l'entrée à l'université signifie aussi la rencontre de jeunes Maoris provenant de toute la Nouvelle-Zélande. Ces rencontres sont enthousiasmantes, mais elles peuvent aussi être stressantes et décevantes puisque la rhétorique très politisée concernant les « vrais » Maoris en opposition aux « faux » Maoris est très prégnante, et plusieurs sont vite appelés à devoir justifier leur maoritude. La transition n'est donc souvent pas facile. Pour ceux qui décident de poursuivre leurs études, cependant, cette période de leur vie associée à leurs études universitaires est considérée comme déterminante, et ce, autant pour leurs engagements dans les mondes maoris que dans la société plus large. Cette période est d'ailleurs vécue par plusieurs comme une étape charnière, celle de la " découverte » et/ou de la (ré)affirmation de leur identité maorie par toutes sortes de moyens, dont un attachement particulier aux lieux distinctement maoris de l'université.

\section{Being Young and Maori Today: University as a Site of (Re-)Affirmation and Coexistence Natacha Gagné}

The university constitutes an important site of (re-)affirmation for diverse Maori identities, where relationships among Maori and between Maori and non-Maori are negotiated and shaped. Higher education entails a direct confrontation between "two worlds", one Western and the other Maori. Many students experience the university as an alien location, as it is a place deemed non-Maori. However, this site can also be an opportunity for Maori students to meet other young Maori from all over New Zealand. Such encounters are exciting, but they can also be stressful or disappointing due to the prominent and highly politicised rhetoric about "real" in contrast to "false" Maori identities. In such a context, many are soon asked to justify their Maori-ness. The transition is thus not always easy. For those who decide to pursue their studies, however, their university years are considered determining ones, shaping their engagements as much in the Maori worlds as in society in general. Attending university is experienced by many as a turning point; it is a time of "discovery" and/or (re-)affirmation of their Maori identities. This is made possible through a variety of means including a particular attachment to distinctly Maori sites at the university.

\section{L'espérance passe de la forêt au} milieu scolaire : clivage et continuité dans les valeurs entre générations de Dènès Tha

\section{Jean-Guy A. Goulet}

et Kim Harvey-Trigoso

Que signifie être un jeune Dènè Tha du Nord-Ouest albertain aujourd'hui? Sur la base de données recueillies entre 1979 et 1999, les auteurs notent un clivage important dans les valeurs sociales et les comportements parmi les enfants dènès tha. Au terme de l'analyse d'un ensemble de données (récits, comportements et dessins), une conclusion s'impose : plus l'enfant en milieu scolaire participe aux activités traditionnelles de chasse, pêche et cueillette avec des membres de sa parentèle, plus il s'oriente dans la vie en fonction de valeurs communautaires et plus il a une attitude positive face à la vie. Les auteurs situent ces résultats dans le cadre d'une présentation des principes épistémologiques et éthiques différents qui sous-tendent les pratiques éducatives autochtones et occidentales.

\section{Hopefulness is carried from the bush to the classroom: continuity and discontinuity in values between generations of Dene Tha Jean-Guy A. Goulet et Kim Harvey-Trigoso}

What does it mean today to be a young Dene Tha in northwestern Alberta? The authors attempt to answer this question on the basis of data gathered between 1979 and 1999. The authors note an important difference between children at the level of social values and behaviour patterns. A careful analysis of drawings, stories and behavior of Dene Tha children leads to one conclusion: the more a child of school age has already participated in traditional subsistance activities, hunting, fishing and gathering, the more likely his or her orienttion in life is based on collective values and the more he or she has a positive attitude to life. The authors discuss these results in light of the contrasting epistemological and ethical principles that underlie Dene traditional education and the school's educational practices.

\section{Regards croisés sur une éducation relative à l'environnement en milieu autochtone Lucie Sauvé, Hélène Godmaire, Marie Saint-Arnaud, Renée Brunelle et Françoise Lathoud}

Comment favoriser chez les jeunes autochtones un processus d'appropriation de leur milieu de vie en lien avec une démarche d'affirmation identitaire et de reconstruction des relations sociales? Cette question préoccupante est examinée ici par les auteures dans le cadre de projets d' « éducation relative à l'environnement ", en particulier chez les Innus du Labrador et les Algonquins de l'AbitibiTémiscamingue. Comment l'éducation formelle, jusqu'ici souvent aliénante, peutelle se transformer pour accompagner 
les jeunes autochtones dans la construction et l'expression d'une vision de leur monde? Comment peut-elle devenir un creuset de clarification de leurs inquiétudes, attentes, espoirs, désirs? Comment peut-elle légitimer et valoriser leurs voix et contribuer à les faire entendre et reconnaître? À partir de ce questionnement, les auteures se sont penchées sur divers écrits et propositions éducatives (programmes, projets) ayant trait à l'« environnement » en contexte autochtone, pour en analyser les fondements et en examiner les stratégies. Par ailleurs, elles portent un regard attentif sur leurs propres expériences éducatives en milieu autochtone. Ces analyses critiques ont permis de mettre en évidence ou de confirmer des enjeux, des écueils, mais aussi des possibilités et des voies à privilégier.

\section{Perspectives on environmental education in indigenous contexts}

Lucie Sauvé, Hélène Godmaire, Marie Saint-Arnaud, Renée Brunelle et Françoise Lathoud

How can the process of appropriating one's own living place, in relation with identity strengthening and reconstruction of social relations, be supported among young aboriginal people? This important question is examined here by the authors in the context of environmental education projects collaboratively developed with Innu (Labrador) and Algonquin (Abitibi-Témiscamingue) communities. How can formal education, previously a somewhat alienating experience, evolve in such a way as to contribute to the construction and expression of young aboriginal's identity and world view? How can it be transformed to enable the clarification of their expectations, concerns, needs and hopes? How can it legitimate and value their voices and contribute to their expression and recognition? Based on such questions, the authors first focus on a literature review and a study of educational proposals (programs, projects) related to the "environment" in indigenous contexts. Theoretical bases and pedagogical strategies are examined. Furthermore, researchers also reflect on their own educational experiences in indigenous communities. Such critical analyses help to highlight and confirm issues and constraints, but also opportunities and promising educational paths.

\section{L'importance de l'aspect relationnel dans l'auto-(re)présentation de jeunes Innus de la communauté de Uashat mak Mani-Utenam \\ Karoline Truchon}

Cet article présente les fondements de ce que l'auteure nomme « le relationnel dans la technique ». La trentaine de jeunes Innus de Uashat mak Mani-Utenam qui ont participé à ce projet ont principalement photographié et commenté des aspects qu'ils aimaient de leur quotidien plutôt que d'accentuer ce qu'ils aimaient moins. Ce résultat contraste avec les images de victimisation et de misérabilisme fréquemment véhiculées à leur sujet dans l'espace public par les adultes autochtones et non autochtones. Selon l'auteure, pour ces jeunes, l'acte photographique ne sert pas à fabriquer des photos, mais à présenter les liens les unissant aux personnes, aux objets et aux événements photographiés. L'acceptation de ce constat amène donc à réfléchir sur les impacts d'une surabondance d'images de souffrance des jeunes autochtones. Se pourrait-il qu'un manque d'équilibre dans le type de représentation de leur quotidien puisse favoriser une intériorisation de ces propos négatifs et ainsi participer au déploiement d'une prophétie autoréalisatrice?

\section{The role of relationality in self-(re)presentation of Innu youth from Uashat mak Mani-Utenam community Karoline Truchon}

This article presents the underlying principles of what the author call "the relational of the technique". More than 30 young Innus from Uashat mak Mani-Utenam who participated in this project, photographed and discussed positive aspects of their lives rather than putting emphasis on the negative ones. This result constrasts with the majority of images circulated by native and non-native adults about them in the public sphere in which they are victimised and appear to live a miserable life. It is suggested that for these youth the purpose of photography is not to fabricate photographs per se but rather to present the links with who and what they photograph. This rationale brings us to reflect on what could be the impacts of a quasi-total representation of commodified suffering about First Nations' youth. Could this lack of balance in representation of their daily lives forecast the internalisation of these negative portrayals and then contribute to a self-fulfilling prophecy?
Notes bio-bibliographiques

Véronique Audet a réalisé sa maîtrise en anthropologie à l'université Laval et son mémoire porte le titre «Innu nikamu. Expression musicale populaire, affirmation identitaire et guérison sociale en milieu innu contemporain» (2005). Outre son intérêt pour le monde innu en général, ses champs de recherche portent sur les expressions musicales et artistiques, les mouvements et processus d'affirmation identitaire, de revitalisation et de guérison autochtones, les relations interculturelles et les transformations des modes d'être au monde en contexte post-colonial. Elle a donné plusieurs conférences sur le thème des musiques populaires innues et a publié « Anguille sous roche... La pêche à l'anguille de Sillery" (La Charcotte - Bulletin de la Société d'histoire de Sillery, vol. 16, $\mathrm{n}^{\circ} 1$, 2002 : 6-16), ainsi que Diapason: Contes et musiques au Burkina Faso (incluant un CD; en coll. avec A. Courchesne, 2001). Courriel : penonik@hotmail.com / veronique.audet@ciera.ulaval.ca

Marie-Pierre Bousquet détient un doctorat en anthropologie de l'Université Laval (Québec) et de l'Université de Paris-X Nanterre. Professeure adjointe à l'Université de Montréal depuis 2002, membre du CIÉRA, elle s'intéresse aux Amérindiens contemporains de l'Est canadien, en particulier aux Algonquins. Ses champs de recherche portent sur les transformations vécues par les sociétés algonquiennes à partir de la fin du $\mathrm{XIX}^{\mathrm{e}}$ siècle, du point de vue culturel, politique, patrimonial, spirituel et social, de même que sur la notion de patrimoine, la protection du territoire, les changements religieux. Au nombre de ses publications: "Les Algonquins ont-ils toujours besoin des animaux indiens? Réflexions sur le bestiaire contemporain » (Théologiques, vol. 10, $\mathrm{n}^{\circ} 1,2002$ : 63-87); «La production d'un réseau de sur-parenté : histoire de l'alcool et désintoxication chez les Algonquins » (Drogues, santé et société, vol. 3, n² 2, 2005 : 63-85). Courriel : marie-pierre.bousquet@umon`」 treal.ca

Renée Brunelle possède une formation en sciences de l'environnement, en coopération internationale et en écologie 
végétale. Elle mène des activités professionnelles dans le domaine de l'ERE et de la gestion environnementale de projets dans un contexte international. Dans le cadre de la Chaire de recherche du Canada en éducation relative à l'environnement, elle est associée à divers projets, notamment sur la place de l'ERE dans les réformes éducatives contemporaines. Parmi ses publications récentes, en collaboration avec Lucie Sauvé et Tom Berryman, mentionnons: "Environnement et développement : la culture de la filière ONU » (Education relative à l'environnement - Regards, Recherches, Réflexions, 4, 2003: 33-55).

Courriel : brunelle.renee@uqam.ca

Brieg Capitaine est titulaire d'une mâ̂trise en ethnologie soutenue en 2001 à l'Université de Bretagne occidentale (France) et il réalise présentement une thèse de doctorat au département de sociologie de l'Université de Bretagne occidentale sur le développement économique de la communauté innue de Uashat mak Mani-Utenam. Il s'intéresse aux relations externes qui forcent le devenir de cette communauté autochtone et particulièrement aux rapports de dépendance. Il occupe actuellement un poste d'assistant de recherche et d'enseignement en sociologie de l'aménagement à l'Institut de géoarchitecture de Brest. Courriel : brieg.capitaine@univ-brest.fr

Natacha Gagné a terminé en 2004 une thèse de doctorat en anthropologie à l'université McGill intitulée « Maori Identities and Visions: Politics of Everyday Life in Auckland, New Zealand ». Elle est présentement chercheure postdoctorante à Paris à l'Unité mixte de recherche Genèse et transformation des mondes sociaux (GTMS) de l'École des hautes études en sciences sociales et du Centre national de recherche scientifique. Elle est aussi rattachée au département d'anthropologie de l'Université de Lund en Suède. Elle sera professeure adjointe à l'Université d'Ottawa à partir de janvier 2006. Courriel : natgagne@uottawa.ca

Hélène Godmaire est titulaire d'un doctorat en écologie aquatique de l'Université de Toronto. Elle est également diplômée $\mathrm{du}$ programme court de $2^{\mathrm{e}}$ cycle en éducation relative à l'environnement à l'UQAM. Elle a récemment publié « La recherche participative: croisement de savoirs et de pratiques. » (Collection Les Cahiers scientifiques de l'ACFAS, 140, 2005: 227-234) et «Explorer, comprendre et agir ensemble : une recherche collaborative avec les Innus $\mathrm{du}$ Labrador » (Éducation relative à l'environnement - Regards, Recherches, Réflexions, 4, 2003: 147-162) en collaboration avec Lucie Sauvé

Courriel : godmaire.helene@uqam.ca

Jean-Guy A. Goulet est professeur titulaire rattaché au programme de maîtrise en études de conflits à la Faculté des sciences humaines de l'université SaintPaul. Rédacteur en chef de la revue Anthropologica de 1998 à 2002, il est l'auteur de nombreux articles sur les Dènès Tha ainsi que d'une monographie intitulée Ways of Knowing: Experience, Knowledge and Power Among the Dene Tha (University of Nebraska Press, Lincoln, 1998). Il est coauteur de Being Changed by Cross-Cultural Encounters: The Anthropology of Extraordinary Experiences (Broadview Press, Peterborough, 2e édition, 1998), ainsi que de Transformations in the Field. An anthropology of cross-cultural encounters (University of Nebraska Press, Lincoln, à paraître en 2006).

Courriel : jggoulet@ustpaul.uottawa.ca

Kim Harvey-Trigoso (MA, Université de Calgary) est une anthropologue americano-péruvienne. Elle a fait du terrain parmi les Dènès Tha de Chateh et, en collaboration avec les Confederated Tribes de l'Orégon, a produit un film documentaire intitulé Growing Up Native: Four Life Stories. En 2002, cette cuvre s'est mérité une subvention de l'Oregon Council for the Humanities, afin d'en augmenter la diffusion. Dans le cadre d'un projet financé par la Robert Wood Johnson Foundation, elle travaille présentement au développement et à la mise sur pied d'un programme de traitement pour l'abus de l'alcool et des drogues parmi les adolescents hispanophones. Courriel : harvey_trigoso@yahoo.com

Martin Hébert est professeur adjoint au département d'anthropologie de l'université Laval, à Québec. Sa thèse de doctorat (Université de Montréal, 2001) portait sur les bases imaginaires de l'action sociopolitique chez les Tlapanèques du Guerrero. Il a également travaillé dans des communautés tzeltales du Chiapas où il s'est intéressé au processus de mobilisation qui a conduit au soulèvement zapatiste de 1994. Outre plusieurs articles dans Recherches amérindiennes au Québec, il est l'auteur, entre autres, de «Whose Utopia? Development, Resistance, and Patterns of Structural Violence in a Mexican Indigenous Region » (Social Justice: Anthropology, Peace and Human
Rights 3(3-4) 2002 : 99-137) et de « Ni la guerre, ni la paix: Campagnes de 'stabilisation' et violence structurelle chez les Tlapanèques de la Montaña du Guerrero (Mexique)» (Anthropologica, vol. $48, n^{\circ} 1$ ). Il est membre du Centre interuniversitaire d'études et de recherches sur les autochtones (CIÉRA), du Groupe de recherche Régions et développement au Mexique (GREDEM) et du Groupe de recherche sur les imaginaires politiques en Amérique latine (GRIPAL). Courriel : martin.hebert@ant.ulaval.ca

Laurent Jérôme est chercheur-étudiant au CIÉRA (Centre interuniversitaire d'études et de recherches autochtones) à l'université Laval et à l'ERASE (Équipe de recherche en anthropologie et sociologie de l'expertise) à l'université PaulVerlaine, de Metz. Candidat au doctorat en anthropologie dans le cadre d'une cotutelle de thèse France-Québec, il s'intéresse aux pouvoirs des pratiques culturelles et rituelles dans les processus d'affirmations identitaires en milieux autochtones. Son expérience de terrain chez les Atikamekw de Wemotaci l'a conduit à s'intéresser plus particulièrement aux expériences sociales et individuelles des jeunes autochtones. Sur ce thème, il a notamment présidé le comité d'organisation du colloque annuel 2005 du CIÉRA et coorganisé avec Natacha Gagné deux sessions de réflexion à la conférence annuelle de la Société canadienne d'anthropologie (CASCA) (Mérida, Mexique, Yucatan).

Courriel : laurent.jerome@ciera.ulaval.ca

Françoise Lathoud est actuellement doctorante en sciences de l'environnement à l'UQAM. Elle s'intéresse à l'éducation relative à l'environnement en contexte autochtone et est activement engagée dans le domaine, particulièrement avec des Atikamekw et des Inuits. Elle a récemment publié « Exploitation des ressources forestières, enjeux culturels et participation. Le cas des Cris de la Baie James. » (GLOBE, revue internationale d'études québécoises, vol. 8, no 1, 2005 : 155-173; et « Paradigmes socio-culturels associés au territoire forestier de la Baie James. (Vertigo, Vol. 6 no 1, 2005, Dossier - Les savoirs locaux et l'environnement). Courriel : ecrismoia@hotmail.com

Thibault Martin détient un doctorat de l'université Laval en sociologie (2001) pour lequel il a obtenu le Prix d'excellence de la Faculté des sciences sociales (meilleure thèse de doctorat). Il est l'auteur de plusieurs articles sur les 
questions autochtones et de l'ouvrage De la banquise au congélateur: mondialisation et culture au Nunavik (UNESCO et Presses de l'Université Laval, 2003) pour lequel il a obtenu le Prix du jeune sociologue, décerné tous les quatre ans par l'Association internationale des sociologues de langue française à l'auteur du meilleur premier ouvrage de sociologie. Après avoir enseigné la sociologie des questions autochtones à l'Université de Winnipeg pendant quatre ans il est aujourd'hui professeur de sociologie à l'Université du Québec en Outaouais. Courriel : thibault.martin@uqo.ca

Marie Saint-Arnaud est biologiste et elle a une formation en anthropologie et en ethnobotanique. Elle effectue présentement des recherches en foresterie sociale dans le cadre de ses études de doctorat en sciences de l'environnement à l'UQAM. Son projet, mené avec la collaboration de la communauté des Anicinapeks de Kitcisakik, porte sur la définition d'une foresterie amérindienne à travers une collaboration avec la communauté des Anicinapek de Kitcisakik.

Courriel : st-arnaud.marie@uqam.ca
Lucie Sauvé est professeure au département d'éducation et pédagogie de l'Université du Québec à Montréal (UQAM). Au sein de cette institution, elle est titulaire de la Chaire de recherche du Canada en éducation relative à l'environnement, membre de l'Institut des sciences de l'environnement et directrice du Programme d'études supérieures en éducation relative à l'environnement (ERE). Elle dirige des projets de recherche principalement axés sur l'institutionnalisation de l'ERE, sur la formation des enseignants et autres éducateurs, et sur l'intervention en milieu communautaire, tant au Québec qu'en contexte de coopération internationale. Elle co-dirige la revue internationale de recherche Éducation relative à l'environnement Regards, Recherche, Réflexions. Au nombre de ses publications, "Enjeux et défis de la globalisation en éducation relative à l'environnement » (La revue POUR, 187, 2005: 67-75); en collaboration avec Hélène Godmaire, « Environmental Health Education: A Theoretical and Praxical Proposal » (Ecohealth, déc. 2004 35-46) et « Explorer, comprendre et agir ensemble: une recherche collaborative avec les Innus du Labrador » (Éducation relative à l'environnement - Regards, Recherches, Réflexions, 4, 2003 : 147-162). Courriel : sauve.lucie@uqam.ca

Karoline Truchon est titulaire d'un baccalauréat en communication publique et elle a complété sa maîtrise en anthropologie à l'université Concordia de Montréal en avril 2005. Intitulé Lanthropologie qui 'laisse des traces'. La photographie comme agent d'empowerment: une ethnographie avec des Innus de Uashat mak ManiUtenam, son mémoire a permis la participation de jeunes, de leur famille et d'adultes significatifs d'une communauté innue de la Côte-Nord. En 2005, elle a également donné deux communications dans le cadre de la conférence annuelle de la Société canadienne d'anthropologie (CASCA) qui se tenait à Merida, Mexique. En 2006, elle entreprend un doctorat sur la décolonisation de l'anthropologie visuelle en milieu autochtone.

Courriel : karoline.truchon@gmail.com

\section{« JOURNÉE DE RÉFLEXION SUR LES NOUVELLES ENTENTES ENTRE Le Québec et les Autochtones, QuéBeC, 20 MARS 2003 ॥}

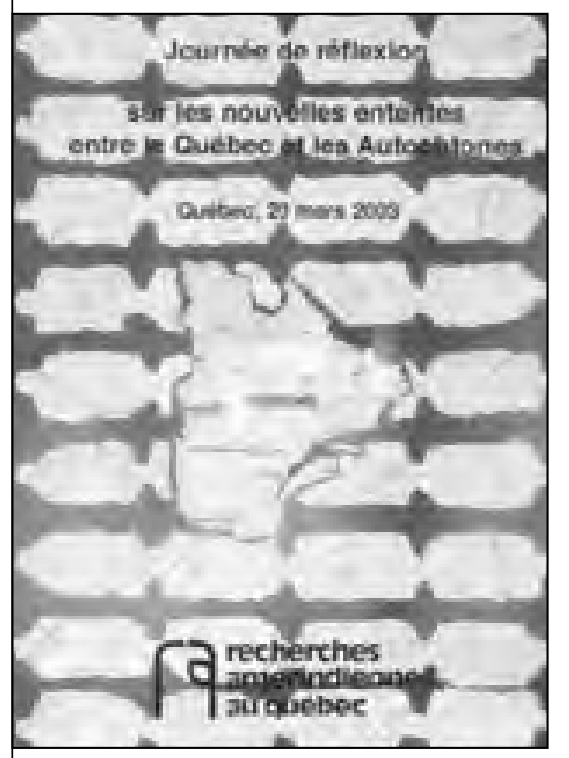

\section{Un document audiovisuel (format VHS et DVD) qui marque la création d'une nouvelle collection de documents audiovisuels Montage vidéo (VHS et DVD) : Maurice Bulbulian Réalisation DVD : Laurent Girouard}

Les années 2002 et 2003 ont été marquées par d'âpres discussions autour des projets d'entente entre le Québec et certaines communautés innues. Le 20 mars 2003, en dehors de toute arène politique, vingt-cinq conférenciers et plus de 250 participants ont pu s'exprimer sur l'avenir des relations entre le Québec et les Autochtones.

Le captage vidéo de l'ensemble des discussions de cette journée est présenté sous forme de cinq DVD (ou de trois bandes VHS). Un outil essentiel pour mieux comprendre les négociations qu'entreprendront les communautés innues après la signature de l'Entente de principe avec le gouvernement du Québec.

DVD (coffret de 5 DVD - avec un menu de navigation permettant un visionnement sélectif de chaque atelier ou de chaque conférencier) : $149,85 \$(+10,49 \$$ TPS, $12,05 \$$ TVQ et $5 \$$ poste $)$.

VHS (coffret de 3 VHS) : 89,65 \$ (+6,29\$ TPS, 7,21\$ TVQ et $6 \$$ poste).

Faites parvenir votre commande accompagnée d'un chèque à :

Recherches amérindiennes au Québec

6742, rue Saint-Denis, Montréal, (Québec) H2S 2S2

Tél : (514) 277-6178 - reamqu@globetrotter.net

www.recherches-amerindiennes.qc.ca 
RECHERCHES

AMERINDIENNES

AU QUEBEC

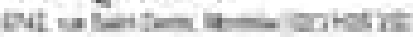

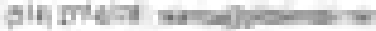

\begin{tabular}{|c|c|c|}
\hline \multicolumn{3}{|c|}{ 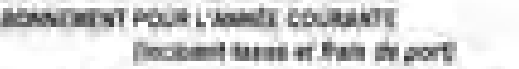 } \\
\hline $4 \mathbf{m}$ & ENi: & Ete \\
\hline $4=$ & ElII & 당 \\
\hline usas & - III & $4 h i$ \\
\hline Inlin & Eal & nal \\
\hline
\end{tabular}

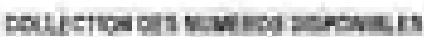

nict in

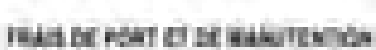

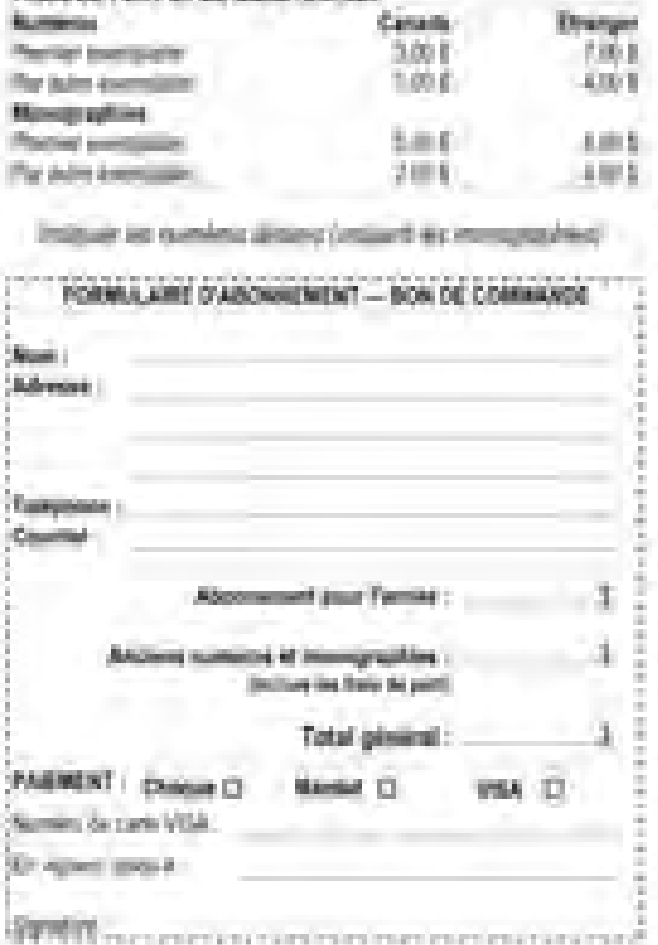

Mintibing

(n)

inth

iar

inhis

月国

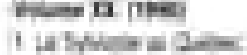

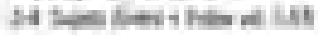

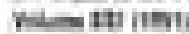

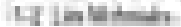

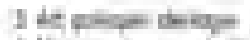

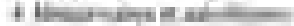

Hatrith

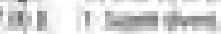

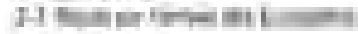

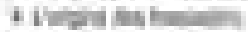

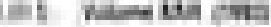

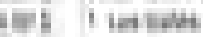

PHinthron

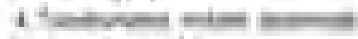

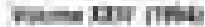

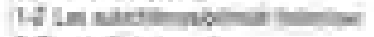

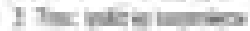

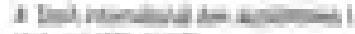

1내 |HA

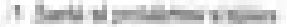

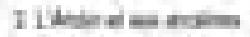

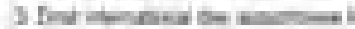

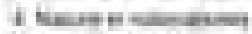

Himmith

1 inding

1 L

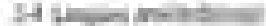

thes if int

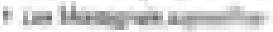

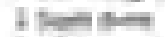

iHth
How in whin

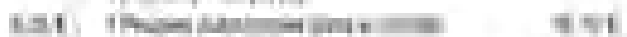

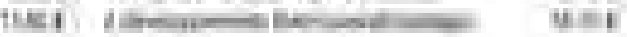

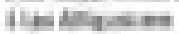

Pill filment int

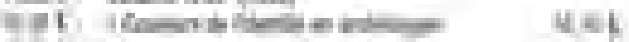

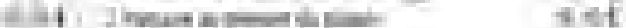

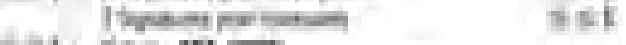

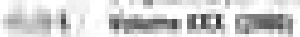

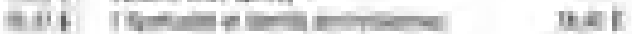

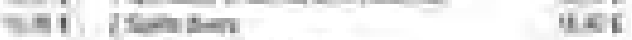

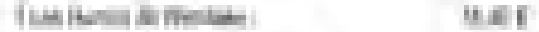

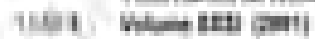

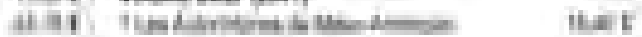

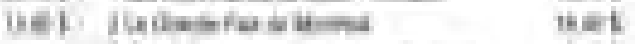

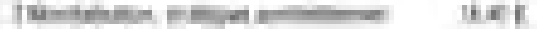

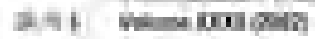

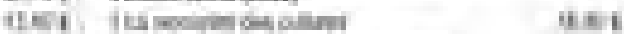

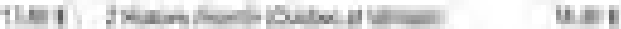

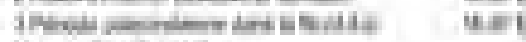

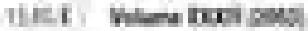

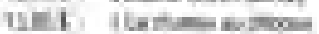

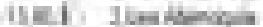

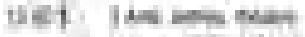

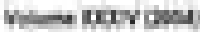

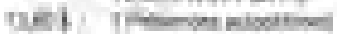

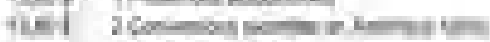

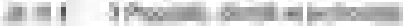

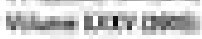

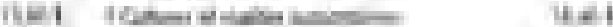

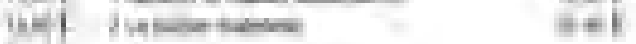

Ha

1+1

19.1

int

int

HII inimolum

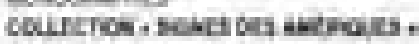

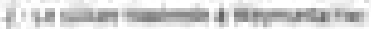

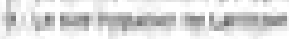

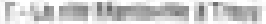

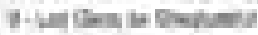

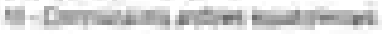

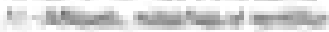

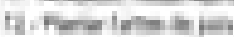

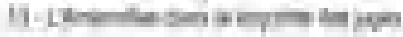

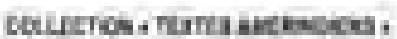

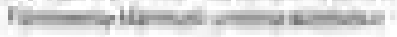

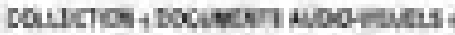

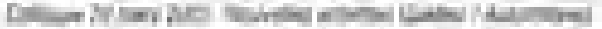

-

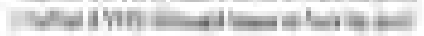

Ifrillt

IIIIIII

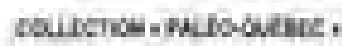

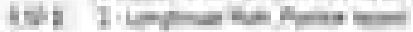

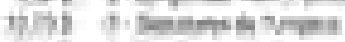

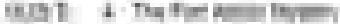

Hat iterimind

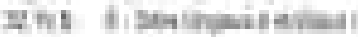

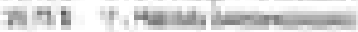

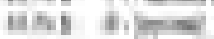

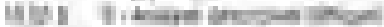

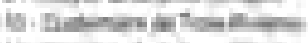

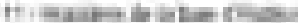

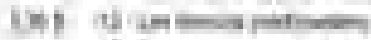

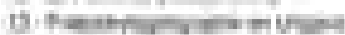

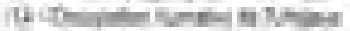

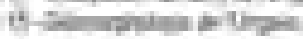

Hith Larill

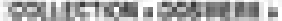

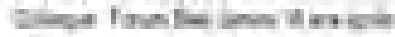

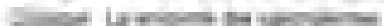

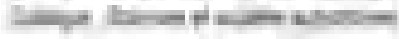

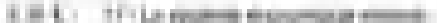

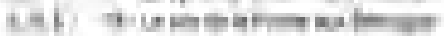

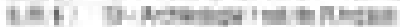

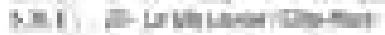

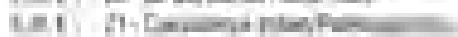

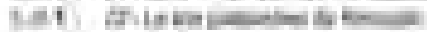

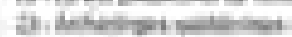

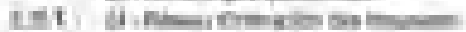

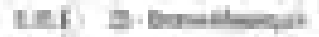

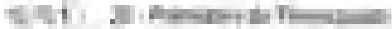

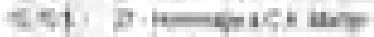

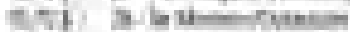

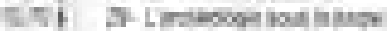

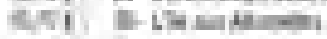

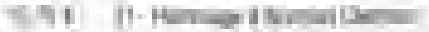

initi

Hit

in 1 in

새

다리

개물

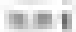

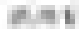

Thi

내대

Hat

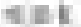

곤

tin

at

표드

패 
Voir notre site Internet pour les directives aux auteurs: www.recherches-amerindiennes.qc.ca

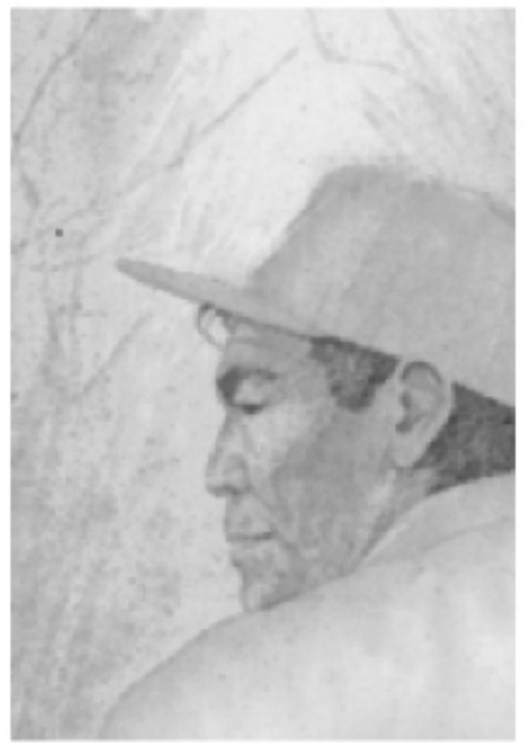




\section{Jeunes autochtones}

\section{Espaces et expressions d'affirmation}

VOL. XXXV, N 3, 2005

Sous la direction de Laurent Jérôme

\section{$\overline{\text { Présentation }}$}

Jeunes autochtones. Espaces et expressions d'affirmation Laurent Jérôme

Les jeunes Algonquins sont-ils biculturels ? Modèles de transmission et innovations dans quelques réserves Marie-Pierre Bousquet

Musique, tradition et parcours identitaire de jeunes Atikamekw : la pratique du tewehikan dans un processus de convocation culturelle Laurent Jérôme

Les chansons et musiques populaires innues : contexte, signification et pouvoir dans les expériences sociales de jeunes Innus

Véronique Audet

L'espérance politique et économique chez les jeunes Tzeltals et Tlapanèques du Mexique Martin Hébert

Comment flirter avec la modernité pour conforter son identité : projet éducatif d'une communauté métisse au Manitoba

Thibault Martin et Brieg Capitaine

Être jeune et maori aujourd'hui : I'université comme site de (ré)affirmation et de coexistence Natacha Gagné

L'espérance passe de la forêt au milieu scolaire : clivage et continuité dans les valeurs entre générations de Dènès Tha

Jean-Guy A. Goulet et Kim Harvey-Trigoso

Regards croisés sur une éducation relative à l'environnement en milieu autochtone Lucie Sauvé, Hélène Godmaire, Marie Saint-Arnaud, Renée Brunelle et Françoise Lathoud

L'importance de l'aspect relationnel dans I'auto-(re)présentation de jeunes Innus de la communauté de Uashat mak Mani-Utenam Karoline Truchon 